\title{
OPTIMIZACIÓN HEURÍSTICA DE PÓRTICOS DE EDIFICACIÓN DE HORMIGÓN ARMADO
}

\author{
Ignacio Payá Zaforteza
}

2007

\author{
Tesis doctoral dirigida por: \\ Prof. Dr. Fernando González Vidosa \\ Prof. Dr. Víctor Yepes Piqueras
}

\begin{abstract}
Universidad Politécnica de Valencia
Departamento de Ingeniería de la Construcción y de Proyectos de Ingeniería Civil
\end{abstract}



A mis padres, familia, amigos

y, muy especialmente, a todos los que estudian y trabajan al mismo tiempo, jánimo! 



\section{Prefacio.}

La presente Tesis Doctoral, que tiene por título "Optimización heurística de pórticos de edificación de hormigón armado", ha sido elaborada por Ignacio Payá Zaforteza y forma parte de los requisitos para la obtención del título de Doctor Ingeniero de Caminos, Canales y Puertos en el programa de doctorado del Departamento de Ingeniería de la Construcción y Proyectos de Ingeniería Civil de la Universidad Politécnica de Valencia.

Los trabajos han sido dirigidos por el Profesor Titular de Universidad D. Fernando González Vidosa y por el Profesor Asociado de Universidad D. Víctor Yepes Piqueras, ambos del Departamento de Ingeniería de la Construcción y Proyectos de Ingeniería Civil de la Universidad Politécnica de Valencia.

\section{Agradecimientos.}

Esta Tesis Doctoral ha sido posible gracias a la dedicación prestada por sus directores, los doctores Fernado González Vidosa y Víctor Yepes Piqueras. Mi más sincero agradecimiento por su gran atención, guía y sugerencias recibidas. Una parte de la misma se elaboró durante una estancia del autor en el Fachgebiet Massivbau de la Technische Universität Berlin. Sirvan estas líneas para expresar mi gratitud hacia el profesor Dr. Mike Schlaich y su equipo de colaboradores por la amabilidad, simpatía, generosidad y confianza con que me acogieron. También quedo en "deuda" con el Dr. Antonio Hospitaler Pérez, por la inestimable ayuda prestada en mis primeros pasos en el ámbito de la optimización multiobjetivo.

Deseo resaltar el apoyo y ánimo que en todo momento he recibido por parte de mis compañeros de la Unidad Docente de Edificación y Prefabricación y del Grupo de Procedimientos de Construcción, Optimización y Análisis de Estructuras. Finalmente, una mención especial para Virginie, mi familia y mis amigos por su comprensión ante los tiempos no dedicados; sin su soporte, comprensión y cariño no hubiera sido posible llegar hasta aquí. 



\section{Resumen.}

PAYÁ ZAFORTEZA, IGNACIO. (2007). Optimización heurística de pórticos de edificación de hormigón armado. Tesis Doctoral. Escuela Técnica Superior de Ingenieros de Caminos, Canales y Puertos. 295 páginas. igpaza@cst.upv.es

El objetivo de esta Tesis es el diseño de algoritmos robustos y flexibles que permitan automatizar el diseño óptimo de los pórticos de hormigón armado habitualmente empleados en edificación y extraer conclusiones generales sobre las estructuras optimizadas. El trabajo define un esquema general para la optimización monoobjetivo (coste económico) y multiobjetivo de estas estructuras que es aplicado a pórticos planos con un máximo de 153 variables. Entre ellas figuran seis calidades diferentes de hormigón.

Para minimizar el coste económico se prueban cinco métodos heurísticos: una Estrategia de Saltos Múltiples Aleatorios (RW), el Gradiente First Best (FB), la Cristalización Simulada (SA), la Aceptación por Umbrales (TA) y los Algoritmos Genéticos (GA). Estas técnicas se utilizan en una primera fase para optimizar un pórtico de dos vanos y cuatro plantas sometido a acciones verticales y horizontales. La versión desarrollada de SA proporciona el diseño de mayor calidad, cuyo coste es de $3473.06 €$. Los mejores proyectos obtenidos mediante las variantes creadas de TA, FB, GA y RW tienen costes mínimos superiores en un $0.52 \%, 5.74 \%, 8.69 \%$ y un $124.6 \%$ respectivamente. Por estos motivos se elige SA para, en una segunda fase, optimizar económicamente otros pórticos de dos vanos y dos, seis y ocho plantas.

Los resultados obtenidos permiten proponer reglas para el predimensionamiento de las estructuras optimizadas y automatizar la elección de los parámetros del algoritmo SA, lo que evita largos procesos de ensayo y error. Se comprueba que los estados límites habitualmente empleados en el diseño de esta tipología estructural son también suficientes para comprobar la seguridad de las estructuras optimizadas.

Asimisimo se investiga la repercusión económica del empleo de un único tipo de hormigón (un HA-25 con resistencia de proyecto a compresión igual a $25 \mathrm{MPa}$ ) y de la utilización de vigas planas en lugar de descolgadas. En el caso del pórtico de ocho plantas, el uso exclusivo de HA-25 supone incrementar su coste un $3.02 \% \mathrm{y}$, utilizar además vigas planas, lo encarece un $46.7 \%$. 
La Tesis presenta finalmente el estudio de la optimización simultanea del coste de la estructura y de objetivos relacionados con su facilidad constructiva, su sostenibilidad ambiental y su seguridad. Para ello se emplea el método de Suppapitnarm para la optimización multiobjetivo mediante Cristalización Simulada (SMOSA). Los resultados muestran que los criterios entran en conflicto y no es posible encontrar un diseño que sea óptimo, a la vez, en los tres objetivos. No obstante, SMOSA es capaz de proporcionar al proyectista un conjunto amplio de alternativas entre las que elegir una solución de compromiso.

Palabras clave: optimización estructural, optimización heurística, optimización multiobjetivo, pórtico de edificación, hormigón armado, Cristalización Simulada, SMOSA, sostenibilidad ambiental. 


\section{Abstract}

PAYÁ ZAFORTEZA, IGNACIO. (2007). Heuristic optimization of reinforced concrete buiding frames. Doctoral thesis. School of Civil Engineering. 295 pages. igpaza@cst.upv.es.

The objective of this thesis is to design robust and flexible algorithms that allow to automate the optimal design of reinforced concrete frames, commonly used in building construction, as well as to draw general conclusions about the optimized structures. This work outlines a general framework for the single-objective (economic cost) and the multiobjective optimization of these structures which is applied to planar frames with a maximum of 153 variables. Among them we find six different types of concrete grades.

Five heuristic methods are tested in order to minimise the economic cost: Random Walk Strategy (RW), First Best Gradient (FB), Simulated Annealing (SA), Threshold Accepting (TA), and Genetic Algorithms (GA). These techniques are used in a first phase in order to optimise a portal frame made up of two bays and four floors subject to vertical and horizontal forces. The SA version developed provides the highest quality design, whose cost is $3473.06 €$. The best projects obtained through the created versions of TA, FB, GA and RW have minimum costs exceeding it by $0.52 \%, 5.74 \%, 8.69 \%$ and $124.6 \%$, respectively. For this reason, SA is chosen to economically optimize other building frames made up of two bays and two, six and eight floors.

The results obtained allow to suggest rules for the pre-dimensioning of the optimized structures and to automate the selection of parameters for the SA algorithm, thus avoiding long trial-and-error processes. Besides, it is verified that the limit states usually checked in this structural typology are also enough in order to guarantee the safety of the optimized structures.

Likewise, the research comprises an analysis of the economic impact derived from the use of a single type of concrete ( $\mathrm{HA}-25$ with project resistance to compression of $25 \mathrm{MPa}$ ), as well as of the use of flat beams instead of overhanging beams. Regarding the eight-floor portal frame, the exclusive use of HA-25 implies a cost increase of $3.02 \%$ and, in case flat beams are also employed, it raises by $46.7 \%$. 
Finally, the thesis includes a study of the simultaneous optimization of the cost of the structure and of the objectives related to its ease of construction, environmental sustainability and safety. For this, the Suppapitnarm Multi-objective Simulated Annealing (SMOSA) method is used. The results show that the different criteria come into conflict, and so it is not possible to find an optimal design for all three objectives simultaneously. Nevertheless, the SMOSA method provides the project designer with a sufficiently wide set of alternatives among which to choose a compromise solution.

Keywords: structural optimisation, heuristic optimization, multi-objective optimization, building frame, reinforced concrete, Simulated Annealing, SMOSA, environmental sustainability. 


\section{Resum}

PAYÁ ZAFORTEZA, IGNACIO. (2007). Optimització heurística de pòrtics d'edificació de formigó armat. Tesi doctoral. Escola Tècnica Superior d'Enginyers de Camins, Canals i Ports. 295 pàgines. igpaza@cst.upv.es

L'objectiu d'aquesta tesi és el disseny d'algorismes robustos i flexibles que permeten automatitzar el disseny òptim dels pòrtics de formigó armat emprats habitualment en edificació i extraure conclusions generals sobre les estructures optimitzades. El treball defineix un esquema general per a l'optimització monoobjectiu (cost econòmic) i multiobjectiu d'aquestes estructures que s'aplica a pòrtics plans amb un màxim de 153 variables. Entre aquestes, figuren sis qualitats diferents de formigó.

Per a minimitzar el cost econòmic, es proven cinc mètodes heurístics: una estratègia de salts múltiples aleatoris (RW), el gradient First Best (FB), la cristal-lització simulada (SA), l'acceptació per llindars (TA) i els algoritmes genètics (GA). Aquestes tècniques s'utilitzen en una primera fase per a optimitzar un pòrtic de dues obertures i quatre plantes sotmès a accions verticals i horitzontals. La versió desenvolupada de SA proporciona el disseny de major qualitat, el cost de la qual és de $3.473,06 €$. Els millors projectes obtinguts mitjançant les variants creades de TA, FB, GA i RW tenen costos mínims superiors en un 0,52\%, $5,74 \%, 8,69 \%$ i un $124,6 \%$, respectivament. Per aquests motius, es tria SA per a optimitzar econòmicament, en una segona fase, altres pòrtics de dues obertures i dues, sis i vuit plantes.

Els resultats obtinguts permeten proposar regles per al predimensionament de les estructures optimitzades i automatitzar l'elecció dels paràmetres de l'algorisme SA, fet que evita llargs processos d'assaig i error. Es comprova que els estats límit habitualment emprats en el disseny d'aquesta tipologia estructural són també suficients per a comprovar la seguretat de les estructures optimitzades.

Així mateix, s'investiga la repercussió econòmica de l'utilització d'un únic tipus de formigó (un HA-25 amb resistència de projecte a compressió igual a $25 \mathrm{MPa}$ ) i de la utilització de bigues planes en lloc de despenjades. En el cas del pòrtic de vuit plantes, l'ús exclusiu de HA-25 suposa incrementar-ne el cost un 3,02\%, i utilitzar a més bigues planes, l'encareix un 46,7 \%. 
La tesi presenta, finalment, l'estudi de l'optimització simultània del cost de l'estructura i d'objectius relacionats amb la seua facilitat constructiva, la sostenibilitat ambiental i la seguretat. Per a això, s'empra el mètode de Suppapitnarm per a l'optimització multiobjectiu mitjançant cristal-lització simulada (SMOSA). Els resultats mostren que els criteris entren en conflicte i no és possible trobar un disseny que siga òptim, alhora, en els tres objectius. No obstant això, SMOSA és capaç de proporcionar al projectista un conjunt ampli d'alternatives entre les quals es pot triar una solució de compromís.

Paraules clau: optimització estructural, optimització heurística, optimització multiobjectiu, pòrtic d'edificació, formigó armat, cristal-lització simulada, SMOSA, sostenibilitat ambiental. 


\section{Índice.}

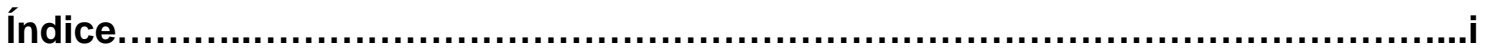

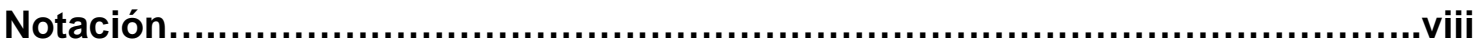

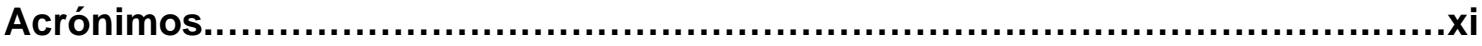

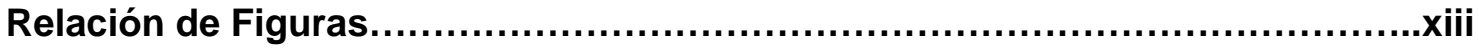

Relación de Tablas........................................................................

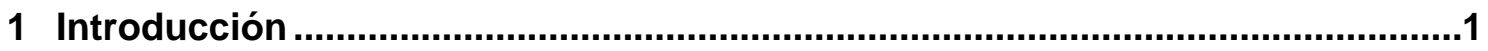

1.1 Planteamiento general del problema de optimización......................................3

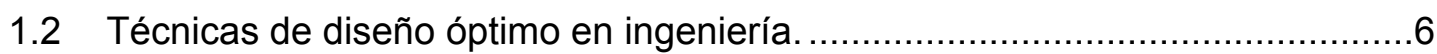

1.3 Técnicas aproximadas empleadas en la optimización monoobjetivo de

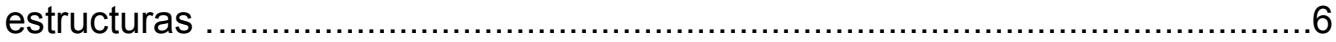

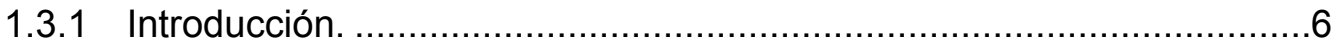

1.3.2 Algoritmos que generalizan la búsqueda secuencial por entornos........9

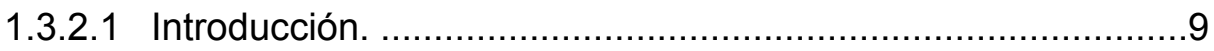

1.3.2.2 Métodos que realizan una aceptación voraz de la solución con un operador simple.

1.3.2.3 Métodos que realizan una aceptación estratégica admitiendo una degradación acotada de la solución

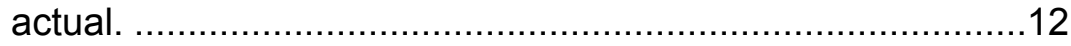

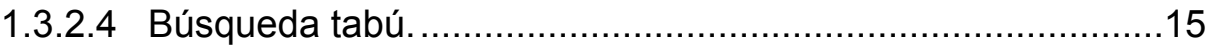

1.3.2.5 Optimización por Colonia de Hormigas. ............................15

1.3.2.6 Aplicaciones.................................................................16

1.3.2.7 Descripción general. .....................................................16

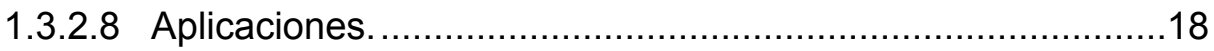

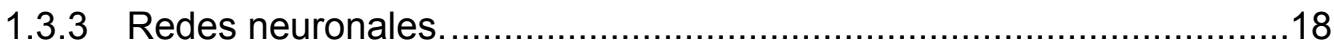

1.4 Técnicas aproximadas empleadas en la optimización multiobjetivo de

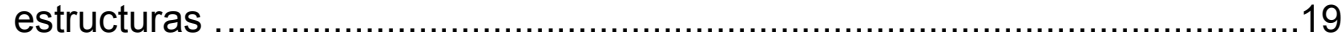

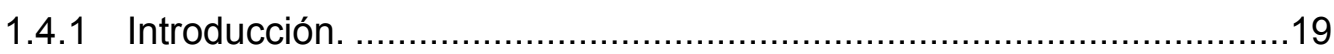


1.4.2 Óptimo de Pareto

1.4.3 Métodos basados en la heurísticas que generalizan la búsqueda secuencial por entornos.

1.4.4 Métodos basados en los algoritmos evolutivos ............................... 21

1.5 Los pórticos en las estructuras de edificación. .......................................... 23

1.6 Objetivos, contribuciones y estructura de esta Tesis................................ 27

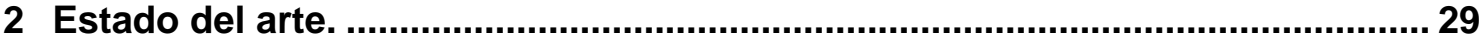

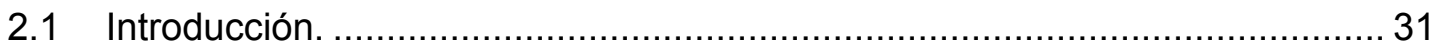

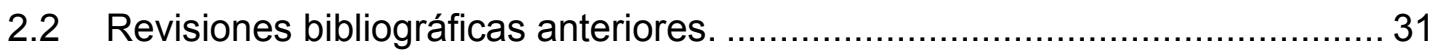

2.3 Técnicas exactas empleadas en el diseño óptimo de estructuras................... 32

2.4 Técnicas aproximadas empleadas en la optimización monoobjetivo de

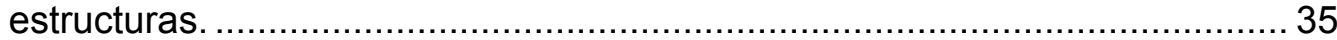

2.4.1 Algoritmos que generalizan la búsqueda secuencial por entornos.... 35

2.4.1.1 Estructuras de acero. ..................................................... 35

2.4.1.2 Estructuras de hormigón. .................................................. 36

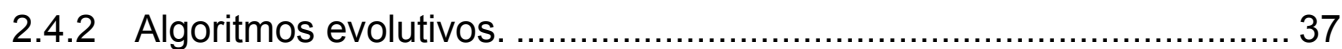

2.4.2.1 Estructuras de acero. .................................................... 37

2.4.2.2 Estructuras de hormigón. ............................................... 39

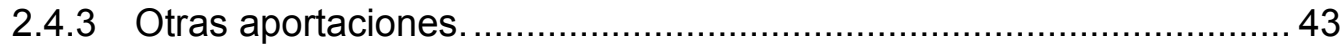

2.5 Técnicas aproximadas empleadas en la optimización multiobjetivo de estructuras.

2.5.1 Estrategias basadas en heurísticas que generalizan la búsqueda secuencial por entornos. ............................................................... 43

2.5.2 Estrategias basadas en los algoritmos evolutivos........................... 45

2.5.2.1 Estructuras de acero. .................................................... 45

2.5.2.2 Estructuras de hormigón. ............................................. 46

2.5.2.3 Otros trabajos.......................................................... 47

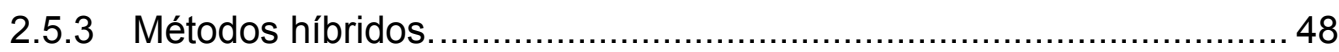

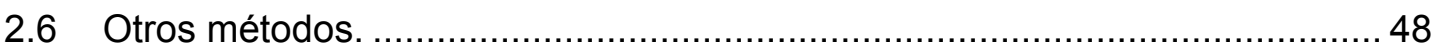

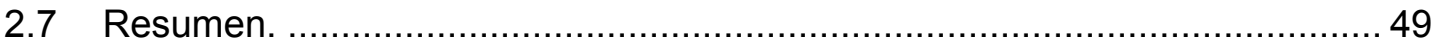

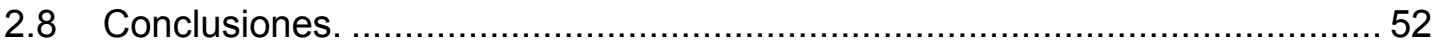

3 Definición del problema de optimización. .......................................................... 55

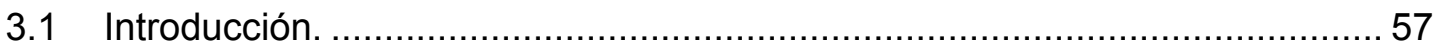

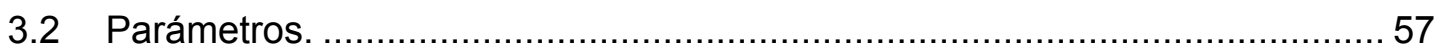

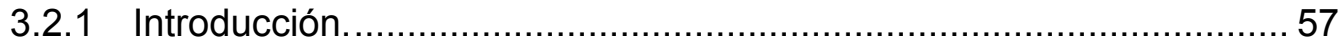

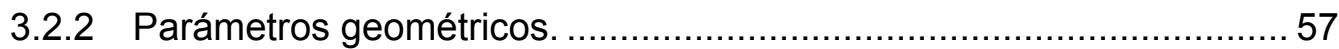

3.2.3 Parámetros relativos a las acciones............................................ 59 
3.2.4 Parámetros relativos a los coeficientes de seguridad y al grado de exposición de la estructura.

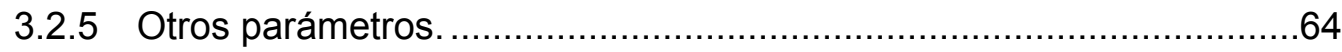

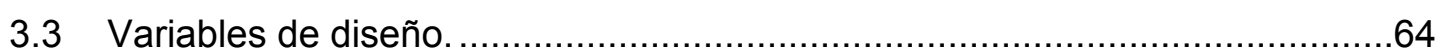

3.4 Restricciones de comportamiento y de diseño...........................................68

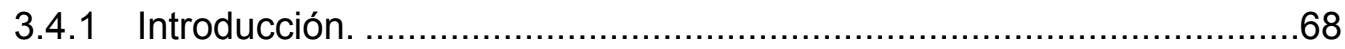

3.4.2 Principios básicos del "Método de los Estados Límite"........................68

3.4.3 Acciones consideradas en el cálculo. Combinación de acciones........69

3.4.4 Verificación de Estados Límites.......................................................69

3.4.4.1 ELS de fisuración en vigas. ..............................................70

3.4.4.2 ELS de deformación en vigas. ...........................................70

3.4.4.3 ELU de agotamiento frente a solicitaciones normales en vigas y pilares............................................................... 71

3.4.4.4 EL de Agotamiento frente a cortante. ..............................72

3.4.4.5 EL de Inestabilidad. ....................................................... 72

3.4.4.6 Disposiciones relativas a las armaduras.............................73

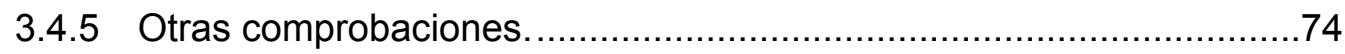

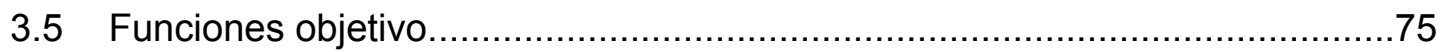

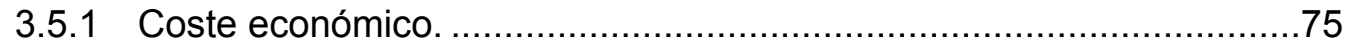

3.5.1.1 Definición de las unidades de obra existentes.....................75

3.5.1.2 Función de coste económico. .............................................76

3.5.2 Facilidad constructiva. ......................................................... 78

3.5.3 Sostenibilidad ambiental. .................................................... 79

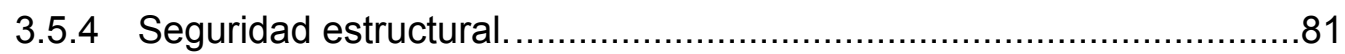

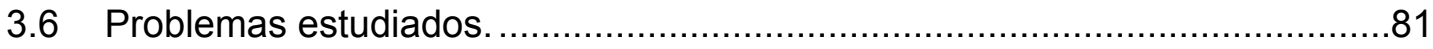

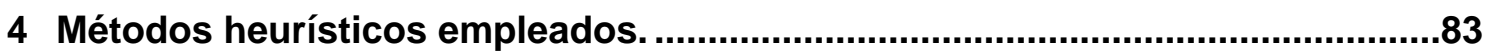

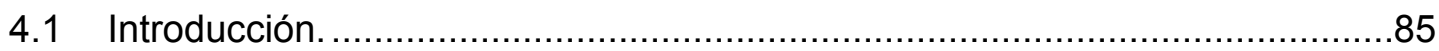

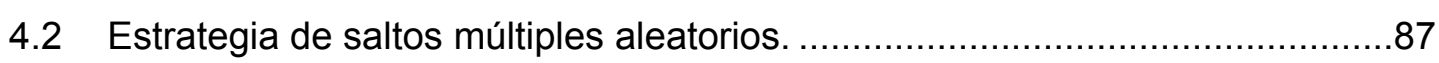

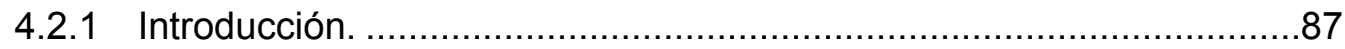

4.2.2 Aplicación del algoritmo al problema planteado. .............................88

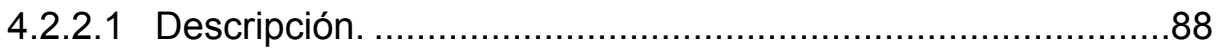

4.2.2.2 Horquillas empleadas en la definición del armado de

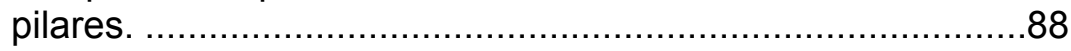

4.2.2.3 Horquillas empleadas en la definición del armado de

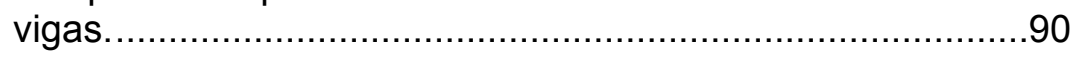

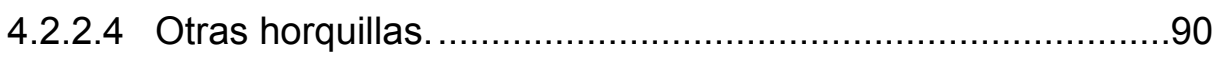

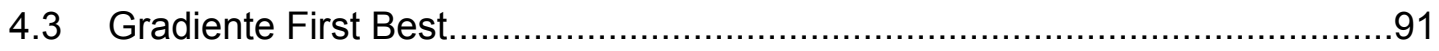

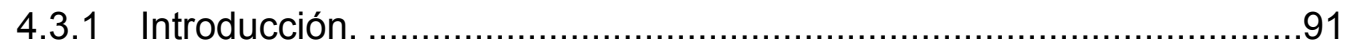




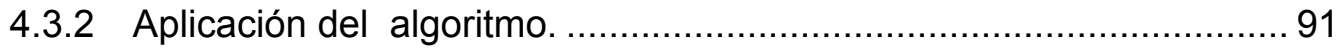

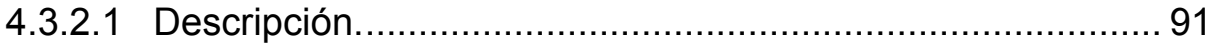

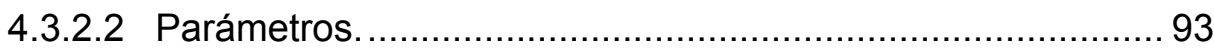

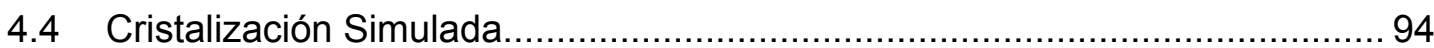

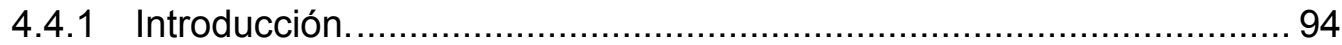

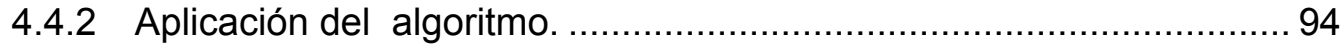

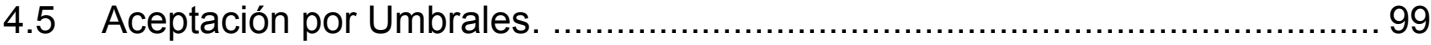

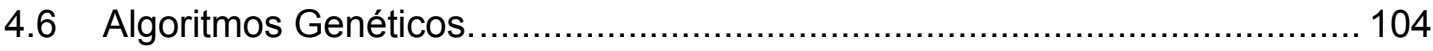

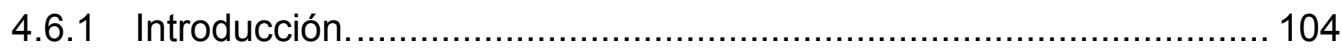

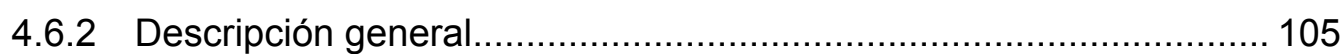

4.6.2.1 Terminología genética y representación de soluciones. ..... 106

4.6.2.2 Funciones de penalización y aptitud de un individuo.......... 107

4.6.2.3 Evaluación de la aptitud de un individuo.......................... 107

4.6.2.4 El operador "Selección".................................................. 108

4.6.2.5 El operador "Cruzamiento" o "Crossover". ........................... 108

4.6.2.6 El operador "Mutación”" .................................................... 110

4.6.2.7 El operador "Elitismo"...................................................... 110

4.6.3 Modificaciones del GA básico................................................... 111

4.6.3.1 Introducción.............................................................. 111

4.6.3.2 Algoritmos meméticos............................................... 111

4.6.3.3 Nuevos modos de aplicar el operador "cruzamiento" ......... 112

4.6.4 Definición completa del algoritmo. ............................................... 115

4.6.4.1 Forma de representación. ................................................ 116

4.6.4.2 Tamaño de la población. .................................................... 116

4.6.4.3 Funciones de penalización............................................. 116

4.6.4.4 Forma de aplicar el operador "selección" .......................... 117

4.6.4.5 Criterio de parada en la mejora local con FB................... 117

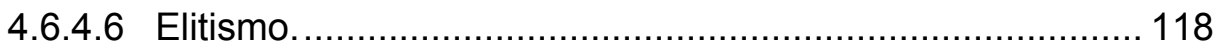

4.6.4.7 Criterio de parada.......................................................... 118

4.7 Método de Suppapitnarm para la optimización multiobjetivo mediante la Cristalización Simulada....................................................................... 118

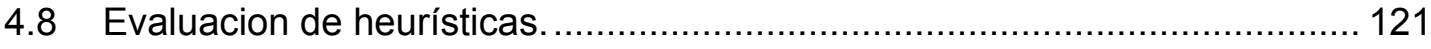

4.9 Programa para el cálculo y optimización de pórticos planos....................... 122

5 Resultados de la optimización económica de un pórtico por diferentes

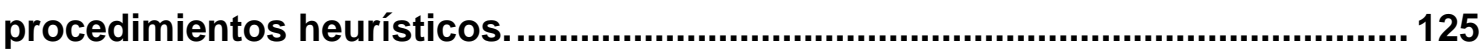

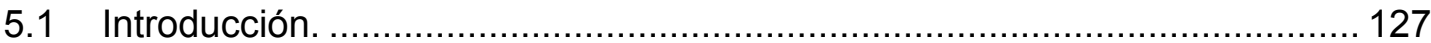

5.2 Estrategia de Saltos Múltiples Aleatorios.............................................. 128 


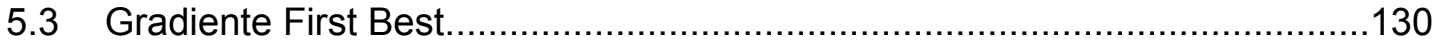

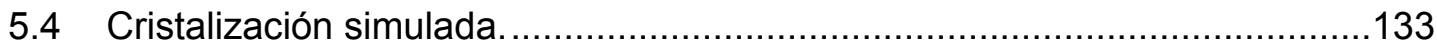

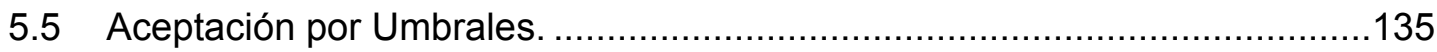

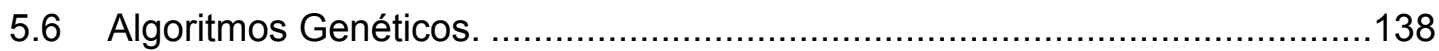

5.7 Justificación del número de ensayos realizados con cada heurística...........140

5.8 Comparación de los resultados. conclusiones. ..........................................141

6 Estudio paramétrico con pórticos de varias alturas. ......................................145

6.1 Optimización de pórticos de dos vanos y dos, cuatro, seis y ocho alturas. ..147

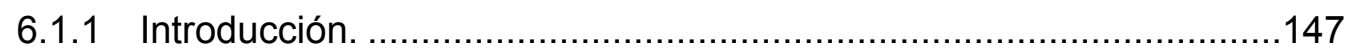

6.1.2 Pórtico de dos vanos y dos alturas. Problema $P \_2 v 2 h-C_{E S T} \ldots \ldots \ldots \ldots . . .147$

6.1.3 Pórtico de dos vanos y seis alturas. Problema $P \_2 v 6 h-C_{E S T} \ldots \ldots \ldots \ldots . . . .149$

6.1.4 Pórtico de dos vanos y ocho alturas. P_2v8h.1-C EST .....................151

6.2 Análisis de las heurísticas que proporcionan las estructuras óptimas. .........154

6.3 Análisis de los resultados de las estructuras optimizadas. ..........................156

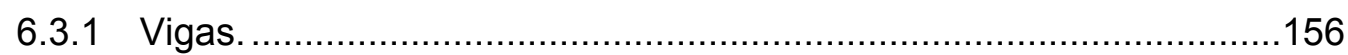

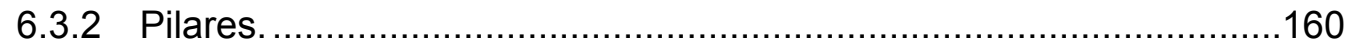

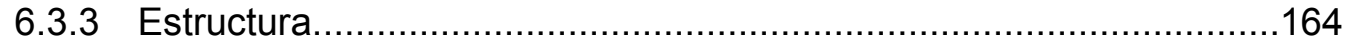

6.3.4 Comparación con los resultados obtenidos o empleados por otros

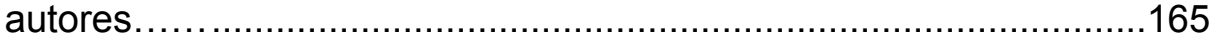

6.4 Análisis del coste de las estructuras optimizadas. ...................................166

7 Resultados adicionales de optimización económica.......................................171

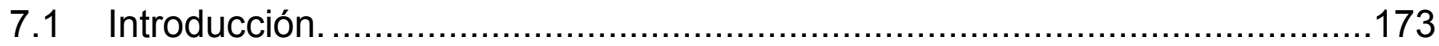

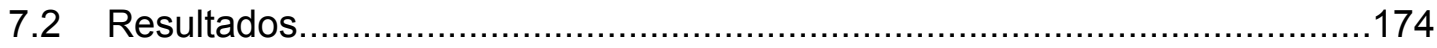

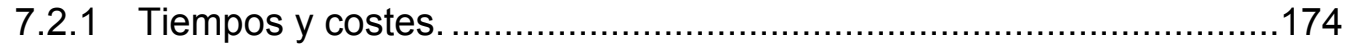

7.2.2 Características de las vigas de las estructuras óptimas para los pórticos de los problemas $P_{-} 2 v 8 h .3-C_{E S T}$ y $P_{-} 2 v 8 h .4-C_{E S T}$. Reglas de predimensionado. .............................................................. 175

7.2.3 Características de los pilares de las estructuras óptimas.................177

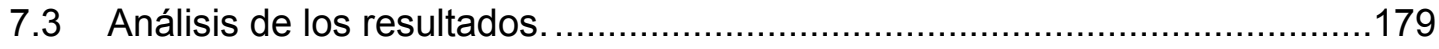

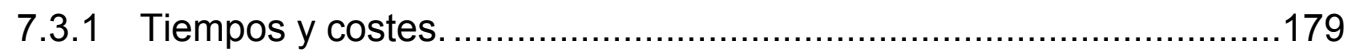

7.3.2 Reglas de predimensionamiento. ...........................................180

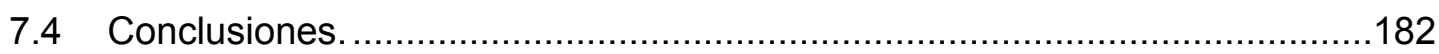

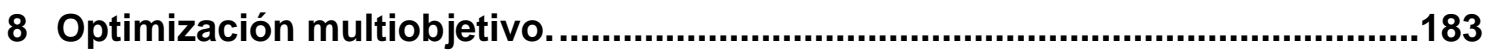

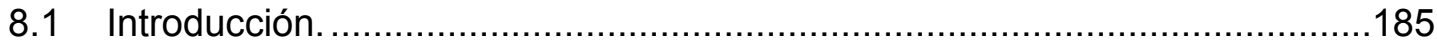

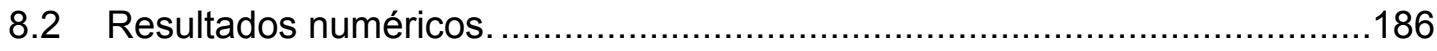




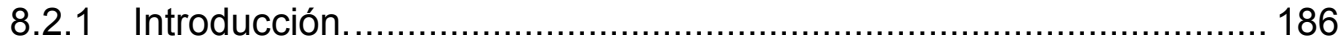

8.2.2 Coste frente a facilidad constructiva. .......................................... 187

8.2.3 Coste frente a sostenibilidad ambiental ......................................... 189

8.2.4 Optimización simultanea de coste económico, facilidad constructiva y sostenibilidad ambiental......................................... 190

8.2.5 Coste frente a seguridad......................................................... 191

8.2.6 Estudio de sensibilidad.............................................................. 192

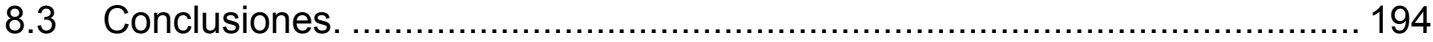

9 Conclusiones y futuras líneas de investigación. ........................................... 195

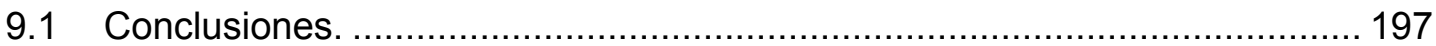

9.2 Futuras líneas de investigación. .......................................................... 202

9.2.1 Métodos heurísticos y funciones objetivo..................................... 202

9.2.2 Modelos de cálculo adoptados.................................................. 203

9.2.3 Tipología estructural y acciones.............................................. 203

9.2.4 Difusión y aplicabilidad de los resultados..................................... 204

9.2.5 Otros aspectos. ......................................................................... 204

\section{Apéndices:}

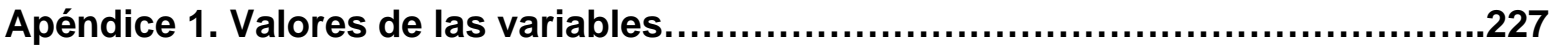

Apéndice 2. Mediciones del pórtico tipo. .................................................251

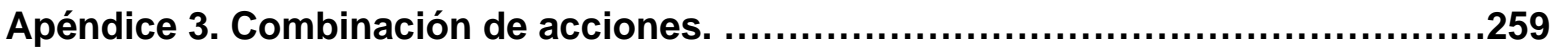

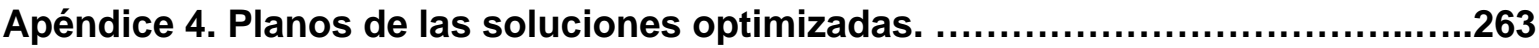




\section{Notación.}

\section{Caracteres romanos.}

B $400 \mathrm{~S}=$ acero para armar con un límite elástico de proyecto de $400 \mathrm{MPa}$.

B $500 \mathrm{~S}=$ acero para armar con un límite elástico de proyecto de $500 \mathrm{MPa}$.

$\mathrm{C}_{\mathrm{EST}} \quad=$ coste total de un pórtico.

$\mathrm{C}_{\mathrm{EST}, \mathrm{M} 2}=$ coste total de un pórtico por metro cuadrado de forjado.

CS $=$ coeficiente de seguridad.

CV $\quad=$ coeficiente de variación.

EC $=$ coste ambiental de una estructura.

$g_{\mathrm{i}} \quad=$ función que define la restricción “i”.

$\mathrm{f}=$ función objetivo.

f' $\quad$ = función objetivo penalizada .

HA-25,HA-30... hormigón con una resistencia de proyecto a compresión de $25 \mathrm{MPa}$, $30 \mathrm{MPa} .$.

$\mathrm{L} \quad \quad=$ luz de un vano de un pórtico.

Lm = longitud de la Cadena de Markov en un algoritmo de Cristalización Simulada.

NCsmmax = número máximo de ciclos sin mejora en una heurística de Aceptación por Umbrales.

NCMsm = número de cadenas de Markov sin mejora de la solución optimizada.

NCMsmmax = número máximo de cadenas de Markov sin mejora de la solución optimizada.

$\mathrm{n}$ = número de individuos que forman la población de un GA. 


$$
\begin{aligned}
& \mathrm{n}_{\mathrm{sb}} \quad=\text { número de barras de la armadura base de una viga. } \\
& \mathrm{n}_{\mathrm{se}} \quad=\text { número de barras de la armadura de refuerzo de una viga. } \\
& \mathrm{n}_{\mathrm{sl}} \quad=\text { número de barras de la armadura longitudinal de un pilar. } \\
& \mathrm{N}_{\mathrm{s}} \quad \text { = número de barras de la armadura longitudinal de un pórtico. } \\
& \text { NGen = número de generación } \\
& \mathrm{Np} \quad \text { = número de plantas de un pórtico. } \\
& \text { Nsol = número de soluciones planteadas en la ejecución de una heurística } \\
& \text { tipo Random Walk. } \\
& \text { Nv } \quad=\text { número de vanos de un pórtico. } \\
& \text { Nit } \quad=\text { número de iteración. } \\
& \text { Nitc = número de iteraciones de un ciclo en el que el umbral de aceptación } \\
& \text { de una heurística TA permanece constante. } \\
& \text { Nitsm = número de iteraciones sin mejora. } \\
& \text { Nitsmmax = número máximo de iteraciones sin mejora. } \\
& \text { Nvar } \quad=\text { número de variables que se modifican en un movimiento en una } \\
& \text { heurística que emplea la búsqueda secuencial por entornos. } \\
& \text { NumVar = número de variables que definen un pórtico. } \\
& \mathrm{P}(\mathrm{i}) \quad \text { = individuos que definen la población de una generación "i” en un } \\
& \text { algoritmo evolutivo. } \\
& \mathrm{p}_{\mathrm{c}} \quad=\text { probabilidad de aplicar el operador cruza en un algoritmo GA. } \\
& \mathrm{p}_{\mathrm{m}} \quad \text { = probabilidad de aplicar el operador mutación en un algoritmo GA. } \\
& \mathrm{P}_{\text {tor }} \quad=\text { probabilidad de que el individuo más apto gane en una selección por } \\
& \text { torneo en un GA. } \\
& r=\text { coeficiente de enfriamiento en una heuristica SA o TA. } \\
& r^{\prime} \quad=\text { multiplicador de las penalizaciones en un GA. }
\end{aligned}
$$




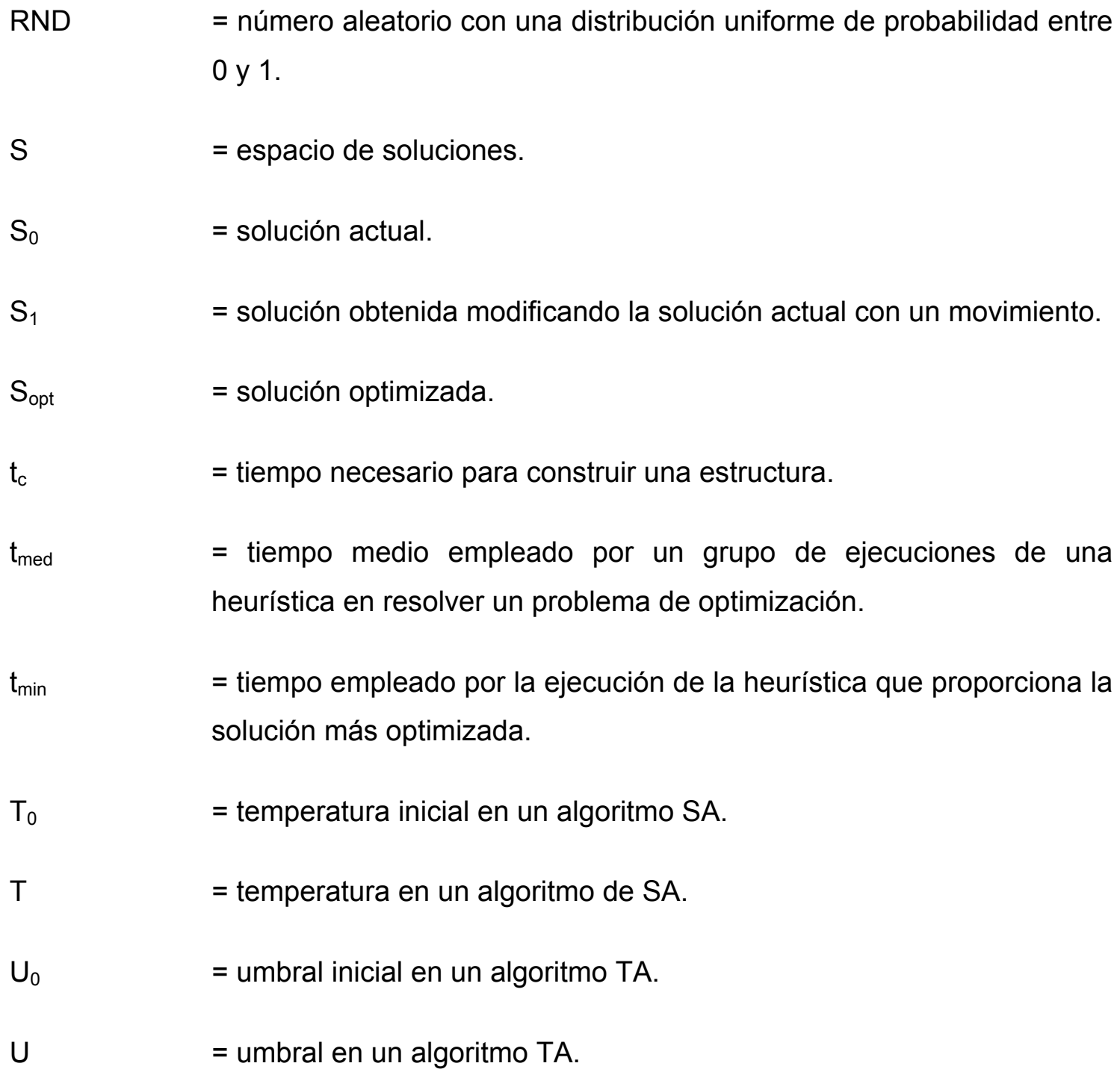

\section{Caracteres griegos.}

$\Delta \quad=$ diferencia entre el valor objetivo de la función objetivo del candidato y el de la solución actual.

$\Phi_{\mathrm{i}} \quad$ = función de penalización asociada a una restricción "i”.

$\sigma \quad=$ desviación típica. 


\section{Acrónimos.}

Concepto

Armadura Base

Armadura de Cortante

Armadura de Refuerzo

Optimización por la Colonia de Hormigas o Ant Colony Optimization

Augmented Simulated Annealing

Coeficiente de Seguridad

Código Técnico de la Edificación

Descenso Aleatorio Adaptativo

Descenso Determinista con Múltiples

Operadores

Descenso Local Aleatorio

Descenso Probabilista con Múltiples Operadores

Estado Límite

Estado Límite de Servicio

Estado Límite Último

Evolutionary Algorithm

Evolutionary Multiobjective Optimization

Estrategias Evolutivas $\mathrm{O}$ Evolution Strategies

Algoritmo Genético o Genetic Algorithm

Gradiente Global Best

Gradiente First Best

Great Deluge Algorithm

Guided Local Search

Grupo de Procedimientos de Construcción, Optimización y Análisis de Estructuras
Acrónimo

$A B$

AC

AR

ACO

ASA

CS

CTE

DAA

DDMO

DLA

DPMO

EL

ELS

ELU

EA

EMOO

ES

GA

GB

FB

GDA

GLS

GPRC 
Concepto

Acrónimo

Greedy Randomized Adaptative Search Procedure

Iterated Local Search

Large Neighborhood Search

Evaluación del Ciclo de Vida o Life Cycle Analysis

Algoritmos Meméticos o Memetic

Algorithms

Multi-Start Local Search

Multi Objective Combinatorial Optimization

Multiobjective Optimization by Simulated Annealing

Redes Neuronales o Neural Networks

Nondominated Sorting Genetic Algorithm

Path Relinking

Randomized Local Search

Estrategia de Saltos Múltiples Aleatorios o Random Walk

Simple Genetic Algorithm

Cristalización Simulada o Simulated Annealing

Scatter Search

Método de Suppapitnarm para la optimización multiobjetivo mediante la cristalización simulada

Strength Pareto Genetic Algorithm

Structural Topology and Shape Annealing

Aceptación por Umbrales o Threshold Accepting

Búsqueda Tabú o Tabu Search

Problema del Viajante de Comercio o

Traveling Saleman Problem

Variable Neighborhood Search
ILS

LNS

MLS

GRASP

MA

MOCO

MOSA

NN

NSGA

PR

RLS

RW

SGA

SA

SS

SMOSA

SPGA

STSA

TA

TS

TSP

VNS 


\section{Relación de figuras.}

1.1. El proceso del diseño estructural.

1.2. Taxonomía de estrategias empleadas en la resolución aproximada de problemas de optimización combinatoria sobre la base de soluciones iniciales.

1.3. Diagrama de flujo de los métodos que generalizan la búsqueda secuencial por entornos.

1.4. Recorrido por el espacio de soluciones en una búsqueda por gradiente en la que se minimiza la función $f$.

1.5. Comparación entre los resultados obtenidos empleando un criterio First Best y uno Global Best en un espacio de soluciones.

1.6. Comparación entre la solución óptima alcanzable mediante una estrategia con un criterio de aceptación voraz y mediante un criterio de degradación estratégica.

1.7. Valor de la probabilidad $p$ de aceptar una solución de mayor coste (factor de Boltzmann) en función de la temperatura $T$.

1.8. Definición de Óptimos de Pareto.

1.9. Pórticos con forjados unidireccionales. Definición de elementos.

1.10. Construcción de un forjado unidireccional parcialmente prefabricado de los habitualmente empleados en España.

1.11. Forjado reticular (1.11.a). Apartamentos DeWitt-Chestnut en Chicago (USA). Estructura tipo tubo. Ing. F. Kahn (1.11.b).

1.12. Tipología de pórticos planos estudiados en esta Tesis: Vista 3D.

3.1. Definición de los parámetros geométricos en una sección y en una planta de una estructura con pórticos y forjados unidireccionales.

3.2. Acciones sobre un pórtico plano. Casos de carga considerados.

3.3. Armaduras longitudinales en vigas.

3.4. Armadura de cortante en vigas.

3.5. Historial de cargas considerado.

3.6. Ejemplo de diagrama de interacción de la sección de un pilar.

3.7. Sección de un forjado con viga plana. Dimensiones máximas de la viga.

3.8. Definición de derivas.

4.1. Número de soluciones distintas para un pórtico simétrico de dos vanos y dos, cuatro, seis y ocho plantas. 
4.2. Diagrama de flujo de la heurística RW empleada en este trabajo.

4.3. Diagrama de flujo de la heurística FB empleada en este trabajo.

4.4. Diagrama de flujo de la heurística SA empleada en este trabajo.

4.5. Esquemas de reducción del umbral empleados por Dueck y Scheuert y en este trabajo.

4.6. Diagrama de flujo de la heurística TA empleada en este trabajo.

4.7. Diagrama de flujo de un GA básico.

4.8. Terminología y ejemplos de representación de soluciones en GA.

4.9. Algoritmo de aplicación de la técnica de selección de la ruleta.

4.10. Cruzamiento de un punto.

4.11. Cruzamiento de dos puntos.

4.12. Cruzamiento uniforme.

4.13. Diagrama de flujo de la heurística MA empleada en este trabajo.

4.14. Cruzamiento 1 en vigas.

4.15. Cruzamiento 1 en pilares y en pilares y vigas. Cruzas 2 y 3 .

4.16. Algoritmo de aplicación de la técnica de selección por torneo probabilístico.

4.17. Diagrama de flujo del algoritmo SMOSA empleado en este trabajo.

4.18. Comparación de heurísticas.

4.19. Diagrama de flujo del programa Optipor.2007.

5.1. Definición geométrica del pórtico a optimizar.

5.2. Ejemplo de los resultados de la aplicación de una heurística RW.

5.3. Evolución del coste de la solución actual en una ejecución típica del algoritmo FB.

5.4. Evolución de la temperatura y del coste de la solución actual en una ejecución típica del algoritmo SA.

5.5. Evolución del umbral y del coste de la solución actual en una ejecución típica del algoritmo TA.

5.6. Evolución del coste de la solución óptima con el número de generaciones en una ejecución típica del GA modificado.

5.7. Resultados de las mejores heurísticas RW, FB, SA, TA y GA.

5.8. Óptimos de Pareto correspondientes a los resultados de las mejores heurísticas RW, $\mathrm{FB}, \mathrm{SA}, \mathrm{TA}$ y $\mathrm{GA}$

6.1. Problema $P \_2 v 2 h-C_{E S T}$. Óptimos de Pareto y punto de coste mínimo proporcionado por SA3. 
6.2. Problema P_2v6h- $C_{E S T}$. Óptimos de Pareto.

6.3. Heurística híbrida RW-FB empleada para la obtención de soluciones factibles en problema P_2v8h.1-C $C_{E S T}$.

6.4. Problema $P \_2 v 8 h .1-C_{E S T}$. Óptimos de Pareto.

6.5. Relación entre el número de variables que definen un pórtico y la Longitud de la cadena de Markov.

6.6. Relación entre el número de variables que definen un pórtico y el tiempo medio de cálculo de una ejecución del algoritmo que proporciona el mejor óptimo local.

6.7. Nomenclatura empleada en la exposición de los resultados de las vigas optimizadas.

6.8. Cuantías de armadura longitudinal (en $\mathrm{Kg} / \mathrm{m}^{3}$ de hormigón) en pilares de la estructura optimizada de seis plantas de menor coste.

6.9. Relación entre el número de plantas de la estructura y el coste total del pórtico optimizado.

6.10. Relación entre el número de plantas de la estructura y el coste total del pórtico por $\mathrm{m}^{2}$ de forjado en estructuras optimizadas.

6.11. Porcentaje del coste total que representa cada una de las unidades de obra en los pórticos optimizados.

6.12. Distribución de la armadura pasiva en los pórticos optimizados.

7.1. Definición de grupos.

7.2. Descomposición del coste de los pórticos optimizados que emplean HA-25.

8.1. Problema P_2v4h-C $\mathrm{EST}_{\mathrm{E}} / \mathrm{N}_{\mathrm{S}}$. Evolución de la Frontera de Pareto en una ejecución típica del algoritmo SMOSA2.

8.2. $\mathrm{N}_{\mathrm{s}}-\mathrm{SMOSA} 1$ y $\mathrm{N}_{\mathrm{s}}-\mathrm{SMOSA}$ 2. Frontera de Pareto final.

8.3. SMOSA1-EC y SMOSA2-EC. Frontera de Pareto final.

8.4. SMOSA- $\gamma_{\text {os }}$. Frontera de Pareto final.

9.1. Redondeo de la ley de momentos flectores en un nudo de un pórtico. 


\section{Relación de tablas.}

2.1. Resumen de los trabajos de optimización de estructuras de hormigón armado más representativos por su relación con esta investigación.

3.1. Parámetros geométricos.

3.2. Parámetros relativos a las acciones.

3.3. Parámetros relativos a los coeficientes de seguridad de las resistencias de los materiales y al grado de exposición de la estructura.

\subsection{Otros parámetros}

3.5. Número de valores diferentes que puede adoptar cada variable. Número de variables de un pórtico con Nv vanos y Np plantas.

3.6. Precios unitarios de las unidades de obra.

3.7. Puntuación ambiental de los materiales empleados en un pórtico.

3.8. Definición de problemas de optimización monoobjetivo.

3.9. Definición de problemas de optimización multiobjetivo.

4.1. Intervalos máximos de variación de las variables.

5.1. Variables del pórtico simétrico de dos vanos y cuatro plantas.

5.2. Heurísticas RW ensayadas. Costes mínimos y tiempos de cálculo asociados.

5.3. Resultados de la heurística RW4.

5.4. Parámetros definitorios de las heurísticas FB. Costes mínimos de las mejores soluciones encontradas.

5.5. Resultados de la heurística FB6 con Nitsmmax $=5000$.

5.6. Parámetros definitorios de las heurísticas SA. Costes mínimos de las mejores soluciones encontradas.

5.7. Resultados de la heurística SA7 con $r=0.8$.

5.8. Parámetros definitorios de las heurísticas TA. Costes mínimos de las mejores soluciones encontradas.

5.9. Resultados de la heurística TA2 con Nvar=Hasta $3, r=0.8$ y NCsmmax $=2$.

5.10. Parámetros definitorios de las heurísticas GA. Resultados obtenidos.

5.11. Resultados de la heurística GA4.

5.12. Problema $P_{-} 2 v 4 h-C_{E S T}$. Comparación de los resultados obtenidos con 9 y con 99 ensayos de la heurística SA7 con $r=0.8$. 
5.13. Comparación de heurísticas.

6.1. Número de variables y dimensión del espacio de soluciones en pórticos simétricos de dos vanos.

6.2. Problema $P \_2 v 2 h-C_{E S T}$. Parámetros definitorios de heurísticas SA.

6.3. Problema $P_{-} 2 v 2 h-C_{E S T}$. Resultados de heurísticas.

6.4. Problema $P \_2 v 6 h-C_{E S T}$. Parámetros definitorios de heurísticas SA.

6.5. Problema $P \_2 v 6 h-C_{E S T}$. Resultados de heurísticas.

6.6. Problema $P \_2 v 8 h .1-C_{E S T . .}$ Parámetros definitorios de heurísticas SA.

6.7. Problema P_2v8h.1-C $C_{E S T}$. Resultados de heurísticas.

6.8. Parámetros de las heurísticas SA que proporcionan las estructuras optimizadas de coste mínimo.

6.9. Coeficientes de seguridad mínimos en estructuras optimizadas correspondientes a la verificación del ELS de deformaciones en vigas.

6.10. Coeficientes de seguridad en estructuras optimizadas correspondientes a la verificación del ELU de agotamiento por solicitaciones normales en vigas.

6.11. Coeficientes de seguridad mínimos en estructuras optimizadas correspondientes a la verificación del ELS de fisuración en vigas.

6.12. Coeficientes de seguridad mínimos en estructuras optimizadas correspondientes a la verificación de las cuantías geométricas y mecánicas mínimas de la armadura longitudinal en vigas.

6.13. Coeficientes de seguridad mínimos en estructuras optimizadas correspondientes a la verificación del ELU de agotamiento frente a cortante. Zona izquierda de las vigas.

6.14. Coeficientes de seguridad mínimos en estructuras optimizadas correspondientes a la verificación del ELU de agotamiento frente a cortante. Zona derecha de las vigas.

6.15. Coeficientes de seguridad mínimos en estructuras optimizadas. ELU de agotamiento frente a cortante. Zona central de las vigas.

6.16. Coeficientes de seguridad mínimos en estructuras optimizadas. ELU agotamiento por solicitaciones normales, EL Inestabilidad.

6.17. Coeficientes de seguridad mínimos en estructuras optimizadas. ELU agotamiento por solicitaciones normales, EL Inestabilidad y cumplimiento de cuantías geométricas mínimas. Pórtico de ocho plantas. Pilares de fachada.

6.18. Coeficientes de seguridad mínimos en estructuras optimizadas. ELU agotamiento por solicitaciones normales, EL Inestabilidad y cumplimiento de cuantías geométricas mínimas. Pórtico de ocho plantas. Pilares centrales. 
6.19. Coeficientes de seguridad mínimos de las armaduras mínimas y máximas.

6.20. Coeficientes de seguridad mínimos en estructuras optimizadas correspondientes a la verificación del ELU de agotamiento frente a cortante. Pilares.

6.21. Coeficientes de seguridad mínimos relativos al cumplimiento de las derivas y desplazamientos horizontales máximos.

7.1. Ampliación del estudio de pórticos de dos vanos y ocho plantas.

7.2. Optimización de pórticos de dos vanos y ocho plantas. Datos.

7.3. Optimización de pórticos de dos vanos y ocho plantas. Resultados.

7.4. Dimensiones de vigas en estructuras optimizadas.

7.5. Cuantías de las vigas óptimas ( $\mathrm{Kg}$ acero $/ \mathrm{m}^{3}$ de hormigón).

7.6. Escuadrías de pilares en estructuras optimizadas.

7.7. Cuantías mecánicas de la armadura longitudinal $(\omega)$ de los pilares de las estructuras optimizadas.

7.8. Cuantías geométricas de la armadura longitudinal de los pilares de las estructuras optimizadas ( $\mathrm{Kg}$ acero $/ \mathrm{m}^{3}$ de hormigón).

7.9. Escuadrías de pilares recomendadas por Rodríguez (2001).

8.1. Problemas $P \_2 v 4 h-C_{E S T}, P \_2 v 4 h-N_{S}$ y $P \_2 v 4 h-E C$. Solución inicial y resultados de la optimización monocriterio mediante $\mathrm{SA}$.

8.2. Problema P_2v4h-C $\mathrm{EST}_{\mathrm{T}} / \mathrm{N}_{\mathrm{S}}$. Resultados.

8.3. Problema P_2v4h-C $\mathrm{EST}_{\mathrm{T}} / \mathrm{EC}$. Resultados.

8.4. Optimización simultanea de $\mathrm{C}_{\mathrm{EST}}, \mathrm{N}_{\mathrm{s}}$ y EC.

8.5. Estudio de sensibilidad. 



\section{CAPÍTULO 1. INTRODUCCIÓN.}




\section{Objetivos.}

Esta Tesis investiga los métodos que permiten automatizar el diseño óptimo de pórticos de hormigón armado empleados habitualmente en edificación y las características de las estructuras optimizadas. Para ello, este primer capítulo responde a las preguntas siguientes:

- ¿Cuál es el planteamiento general del diseño de una estructura y de un problema de optimización?.

- ¿Qué métodos de optimización se emplean en la ingeniería estructural?.

- ¿Cuáles son las características generales de la tipología estructural cuya optimización es objeto de estudio?.

- ¿ ¿Cuáles son las contribuciones de este trabajo?. 
"La Tierra dispone de suficientes recursos para satisfacer las necesidades de todos sus habitantes, pero no para saciar sus avaricias"

M. Gandhi

\subsection{PLANTEAMIENTO GENERAL DEL PROBLEMA DE OPTIMIZACIÓN.}

La metodología habitual del proyecto de estructuras supone un proceso iterativo que parte de la realización por parte del ingeniero de un predimensionamiento (o definición de unas dimensiones y de unos materiales para los elementos estructurales) a partir de su experiencia y de reglas sancionadas por la práctica. Posteriormente, el análisis de la estructura a partir de los principios de la resistencia de materiales y disciplinas afines, de modelos de comportamiento de los materiales y de las prescripciones de la normativa vigente, permite terminar de definirla y comprobar su idoneidad. Normalmente es necesario realizar varios ajustes y correcciones sobre el proyecto inicial hasta obtener un diseño suficientemente seguro y, por tanto, apto para su ejecución. En este proceso, que se ilustra en la Figura 1.1 (ver Hernández, 1990), la experiencia del proyectista tiene una gran influencia en la calidad del resultado final y en el tiempo empleado para obtenerlo, al existir múltiples soluciones para un mismo problema.

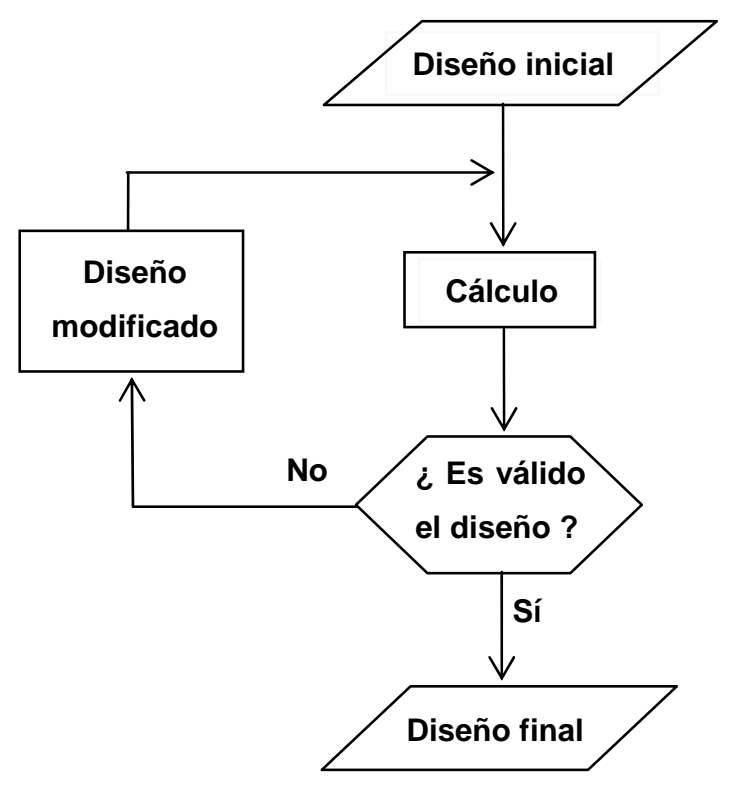

Figura 1.1. El proceso del diseño estructural. 
Frente a la multiplicidad de soluciones para resolver un problema estructural, algunos autores han planteado la obtención de la estructura óptima, entendiendo como tal aquella para la que una función - llamada función objetivo - adopta su valor más adecuado ( ver Moragues, 1980). La función objetivo representa de un modo u otro la dificultad para construir la estructura y, en la práctica, ha representado varios conceptos como su peso o su coste económico. No obstante, la sociedad demanda cada vez más que un diseño, además de la economía, considere aspectos como su sostenibilidad ambiental o su plazo de ejecución. Por ello, es de gran interés el estudio de la optimización simultánea de varias funciones objetivo.

Para plantear un problema de optimización en la ingeniería estructural es necesario que, además de las funciones objetivo, se definan:

1) Unos parámetros o elementos de valor constante y que, por tanto, no varían a lo largo del proceso de optimización.

2) Unas variables de diseño o elementos cuyo valor es susceptible de ser modificado a lo largo del proceso de optimización. Los diferentes valores que pueden adoptar las variables de diseño para unos parámetros dados, definen un conjunto denominado espacio de soluciones (en adelante " $S$ ").

3) Unas restricciones o condiciones de comportamiento y de diseño cuyo cumplimiento es imprescindible para que una solución del problema pueda considerarse realmente como tal porque:

a) Cumple las condiciones de seguridad, nivel de servicio y durabilidad que establece la normativa vigente.

b) Tiene un sentido físico o geométrico.

Los diferentes estados límites últimos y de servicio que debe satisfacer cualquier estructura de acuerdo con una normativa de referencia adoptada, son ejemplos de restricciones del problema.

Los diseños que cumplen todas las restricciones reciben el nombre de soluciones factibles.

En este contexto, el problema general de la optimización de una estructura teniendo en cuenta varias funciones objetivo se enuncia de forma analítica general como: 
Optimizar $\vec{f}=\left(f_{1}(\vec{x}), \ldots, f_{l}(\vec{x})\right)$

Sujeto a $g_{i}(\vec{x}, \vec{p}) \leq 0, \quad i=1, \ldots, k$

Donde:

- $\quad \vec{f}$ es un vector con las diferentes funciones objetivo.

- $\vec{x}=\left(x_{1}, \ldots, x_{n}\right)$ es un vector con las variables de diseño. En el caso general $\vec{x} \in S \subset R^{n}$.

- $\quad \vec{p}=\left(p_{1}, \ldots, p_{m}\right)$ es un vector con los parámetros.

- Cada función $g_{i}$ representa una de las restricciones del problema.

El problema de optimización es monoobjetivo o monocriterio cuando el vector $\vec{f}$ tiene una única componente y multiobjetivo o multicriterio en caso contrario. En esta situación, las funciones objetivo suelen estar en conflicto unas con otras, por lo que el término "optimizar" quiere decir encontrar una solución que proporcione valores aceptables por el proyectista para todas ellas (ver Osyczka, 1985).

Cuando las variables de diseño son discretas, $S$ está formado por una cantidad finita de elementos. La búsqueda de óptimos es, entonces, tarea de una disciplina llamada Optimización Combinatoria que se relaciona con campos como la investigación de operaciones, la teoría de algoritmos y la ingeniería artificial. Para conseguir su objetivo, los algoritmos de optimización combinatoria reducen el tamaño efectivo de $S$ y, además, lo exploran eficientemente. 


\subsection{TÉCNICAS DE DISEÑO ÓPTIMO EN INGENIERÍA.}

Las metodologías que pueden emplearse para resolver un problema de optimización pueden clasificarse en dos grandes grupos:

- Técnicas exactas o métodos que proporcionan la solución óptima del problema. A este grupo pertenecen estrategias como la programación matemática lineal y no lineal (linear and nonlinear programming) o los métodos basados en los criterios de optimalidad (optimality criteria). Una descripción de los mismos y aplicaciones a la ingeniería estructural pueden encontrarse en Moragues (1980), Fletcher (2001) y Hernández y Fontan (2002).

- Técnicas heurísticas y metaheurísticas o métodos aproximados que proporcionan una buena solución del problema aunque no tiene por qué ser la óptima. A este grupo pertenecen técnicas como los Algoritmos Genéticos (ver Goldberg, 1989), la Cristalización Simulada ( Kirckpatrick et al., 1983), la Búsqueda Tabú (Glover y Laguna, 1997 ) y las Colonias de Hormigas (ver Dorigo y Stützle, 2004). Se trata de algoritmos sencillos e intuitivos, pero que requieren un gran esfuerzo computacional pues precisan que la función objetivo y las restricciones del problema sean evaluadas un gran número de veces. Estas estrategias, que son las empleadas en esta Tesis, se describen brevemente en los apartados 1.3 y 1.4 (optimización mono y multiobjetivo respectivamente) y con una mayor profundidad en el capítulo 4.

Una descripción detallada de los trabajos más representativos por su relación con esta investigación se proporciona en el capítulo 2.

\subsection{TÉCNICAS APROXIMADAS EMPLEADAS EN LA OPTIMIZACIÓN MONOOBJETIVO DE ESTRUCTURAS .}

\subsubsection{Introducción.}

Una forma de resolver los problemas de optimización combinatoria es realizar una búsqueda exhaustiva en el espacio de soluciones: se evalúa la función objetivo para todos los valores posibles de las variables de diseño y se elige posteriormente la solución factible con mejor valor de la función objetivo. Sin embargo, este procedimiento es inviable a partir de un determinado número de posibles valores para las variables del problema. Por ejemplo, 
en el clásico problema del viajante de comercio (Travel Salesman Problem, TSP) que consiste en determinar la ruta de menor recorrido que permite a un comerciante visitar un determinado número de ciudades a) sin pasar dos veces por ninguna de ellas y $b$ ) empezando y terminando el trayecto en la misma ciudad, un ordenador que tardase un segundo en calcular la longitud de 20 billones de rutas necesitaría cinco siglos para resolver un problema con 25 poblaciones.

Ante esta dificultad surgen estrategias de búsqueda de óptimos, las heurísticas y las metaheurísticas, que no precisan de la evaluación de todo el espacio de soluciones y proporcionan soluciones satisfactorias a los problemas de optimización combinatoria dedicando un esfuerzo de cálculo razonable.

Los procedimientos heurísticos son métodos sencillos que aportan soluciones a un problema dado mediante algoritmos específicos como los métodos constructivos, de mejora local o combinaciones de ambos. Estas técnicas explotan las características propias del problema para buscar soluciones satisfactorias, por lo que no son aplicables a problemas diferentes a aquel para el que fueron diseñadas (ver Yepes, 2002).

En cambio, las metaheurísticas hacen referencia a métodos generales aplicables a amplios conjuntos de problemas, que emulan estrategias eficientes empleadas por la naturaleza o conceptos basados en la inteligencia artificial (evolución biológica, funcionamiento del cerebro...) y que sirven para guiar el buen funcionamiento de las heurísticas o de otros procedimientos específicos. Una descripción general de estas técnicas y de algunas de sus aplicaciones se puede encontrar en Dreo et al. (2006).

En el contexto de esta Tesis y siguiendo a otros autores (Díaz et al., 1996; Yepes, 2002) se englobarán todas las técnicas heurísticas y metaheurísticas bajo el concepto de optimización heurística. Las principales razones para emplear estas técnicas de optimización son:

- Pueden formar parte de procedimientos globales que garanticen la obtención del óptimo del problema, bien porque proporcionan una buena solución de partida, bien porque participan en un paso intermedio del algoritmo de búsqueda del óptimo global.

- Se trata en general de métodos muy intuitivos y, por lo tanto, fáciles de comprender. 
- Son más flexibles que los métodos exactos, permitiendo la incorporación de condiciones de difícil modelización.

Por el contrario, su principal limitación estriba en que normalmente no garantizan el hallazgo del óptimo global y, en algunos casos, tampoco proporcionan una medida de lo cerca que se encuentra una solución factible particular del óptimo global.

Yepes (2002) ha agrupado las técnicas metaheurísticas en tres grandes conjuntos (véase la Figura 1.2):

- Algoritmos que generalizan la búsqueda secuencial por entornos.

- Algoritmos evolutivos.

- Redes neuronales artificiales.

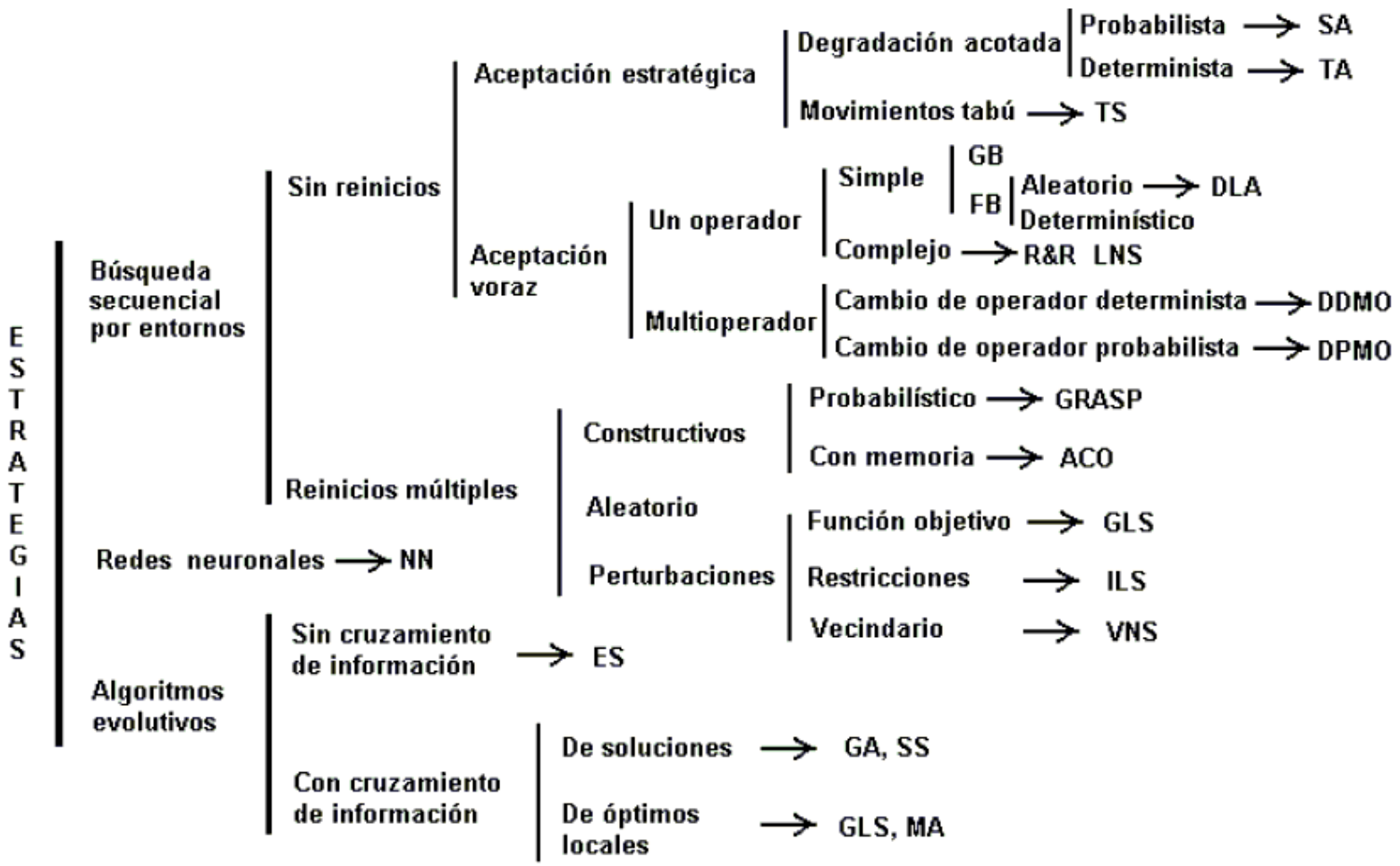

Figura 1.2. Taxonomía de estrategias empleadas en la resolución aproximada de problemas de optimización combinatoria sobre la base de soluciones iniciales. (Yepes, 2002).

Se describen a continuación de forma sucinta estos tres grupos. Una visión más detallada puede consultarse en Yepes (2002). La explicación es de carácter general y no centrada en la ingeniería estructural. Por ello, no se hacen consideraciones respecto a la factibilidad de la solución que son de aplicación únicamente a los problemas de optimización con restricciones. Estos aspectos se examinan con detalle en el Capítulo 4. Además, se supone que se desea minimizar la función objetivo $f$. En caso de buscar su maximización 
bastaría hacer el cambio $z=-f$.

\subsubsection{Algoritmos que generalizan la búsqueda secuencial por entornos.}

\subsubsection{Introducción.}

Estos métodos parten de una solución inicial $S_{0}$ a partir de la cual se obtiene una nueva $S_{1}$ modificando en una pequeña magnitud el valor de una o varias de las variables que la definen. $S_{1}$ es evaluada $y$, si se cumple un criterio de aceptación, se adopta como nueva solución inicial (actualización de la solución). El proceso se ilustra en la Figura 1.3 y se repite hasta que se cumple un criterio de parada. El mecanismo que permite transformar una solución en otra ligeramente diferente recibe el nombre de movimiento. Al conjunto de soluciones a las que se puede llegar aplicando un movimiento a una solución determinada se le conoce como entorno de esa solución.

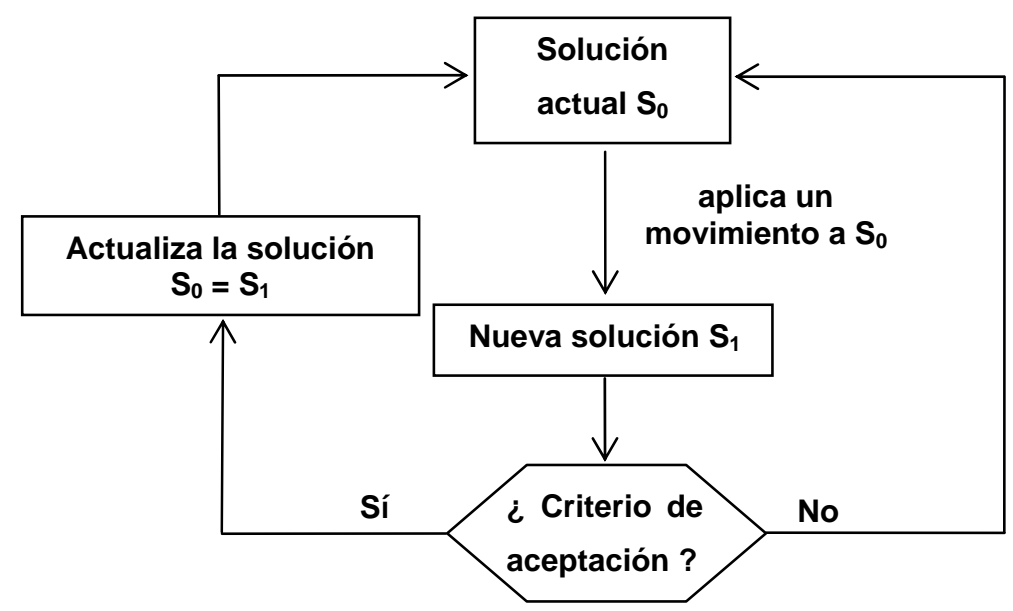

Figura 1.3. Diagrama de flujo de los métodos que generalizan la búsqueda secuencial por entornos.

En estas técnicas es fundamental que los movimientos sean pequeños. De este modo no se pierden las características positivas de la solución de partida, sino que su calidad se mejora de forma gradual y progresiva. Estas heurísticas presentan dos debilidades (ver Yepes, 2002) que se han puesto de manifiesto también en el desarrollo de esta Tesis: a) la obtención de un óptimo local se consigue tras un ajuste delicado de los parámetros que definen la búsqueda y b) precisan de un elevado esfuerzo computacional.

Se describen seguidamente las heurísticas de esta familia más empleadas en la optimización estructural. 


\subsubsection{Métodos que realizan una aceptación voraz de la solución con un operador simple.}

Una manera de explorar el espacio de soluciones consiste en buscar en el entorno de la solución de partida otra solución que la mejore. En caso de buscar mínimos, este proceso repetido sucesivas veces puede asimilarse a realizar un recorrido por el espacio de soluciones en trayectorias descendentes, es decir, de gradiente negativo (como ilustra la Figura 1.4. Por ello, a este tipo de técnicas se las denomina de búsqueda por gradiente.

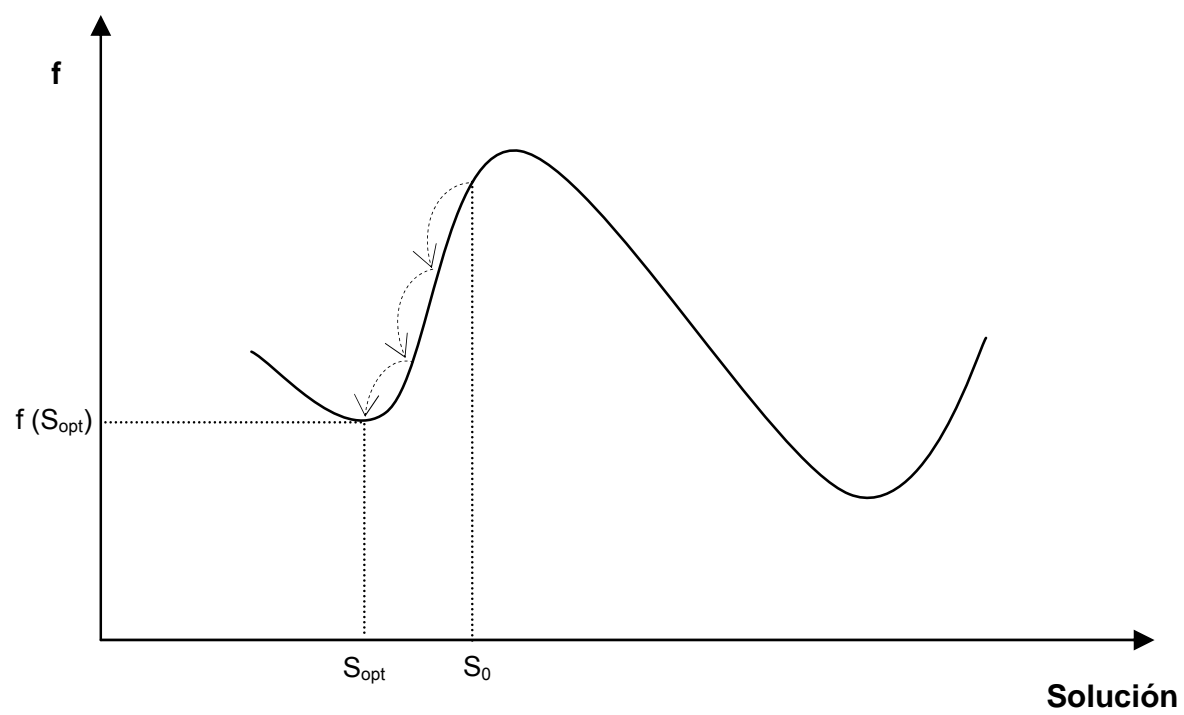

Figura 1.4. Recorrido por el espacio de soluciones en una búsqueda por gradiente en la que se minimiza la función $f$.

En esta estrategia la solución inicial es mejorada a medida que avanza el proceso hasta que no es posible mejorarla más. Se realiza una aceptación voraz de una nueva solución, pues un nuevo diseño sólo es admitido como nueva solución si perfecciona el mejor valor de la función objetivo encontrado hasta ese momento.

El algoritmo se puede describir del modo siguiente:

1) Generar una solución inicial $S_{0}$.

2) Mientras no se cumpla un criterio de parada:

a) Aplicar un movimiento a $S_{0}$ para obtener una nueva solución $S_{1}$.

b) Si $S_{1}$ mejora a $S_{0}$ la reemplaza como solución actual: $S_{0}=S_{1}$. 
c) Volver al paso a).

Dos opciones son posibles para elegir vorazmente:

1) El criterio del "descenso aleatorio". En este caso se selecciona al azar una solución que mejora a la actual, pudiéndose elegir la primera mejora en la exploración del entorno (criterio First Best - FB en lo sucesivo- ) o bien la mejor de un conjunto de soluciones.

2) El criterio del "mayor descenso" (criterio Global Best - GB en adelante -). La nueva solución en este caso es la que produce la mayor mejora de la solución actual tras una exploración exhaustiva de su entorno.

Algunas investigaciones (ver Laguna, 1994) muestran que el criterio GB no siempre conduce a las mejores soluciones finales, pues el proceso puede encajonarse en mínimos locales próximos a la solución inicial. En efecto, el ejemplo de la Figura 1.5, muestra como, partiendo de una solución $S_{0}$, un criterio de elección de soluciones del tipo GB puede conducir a soluciones de menor calidad que un criterio FB.

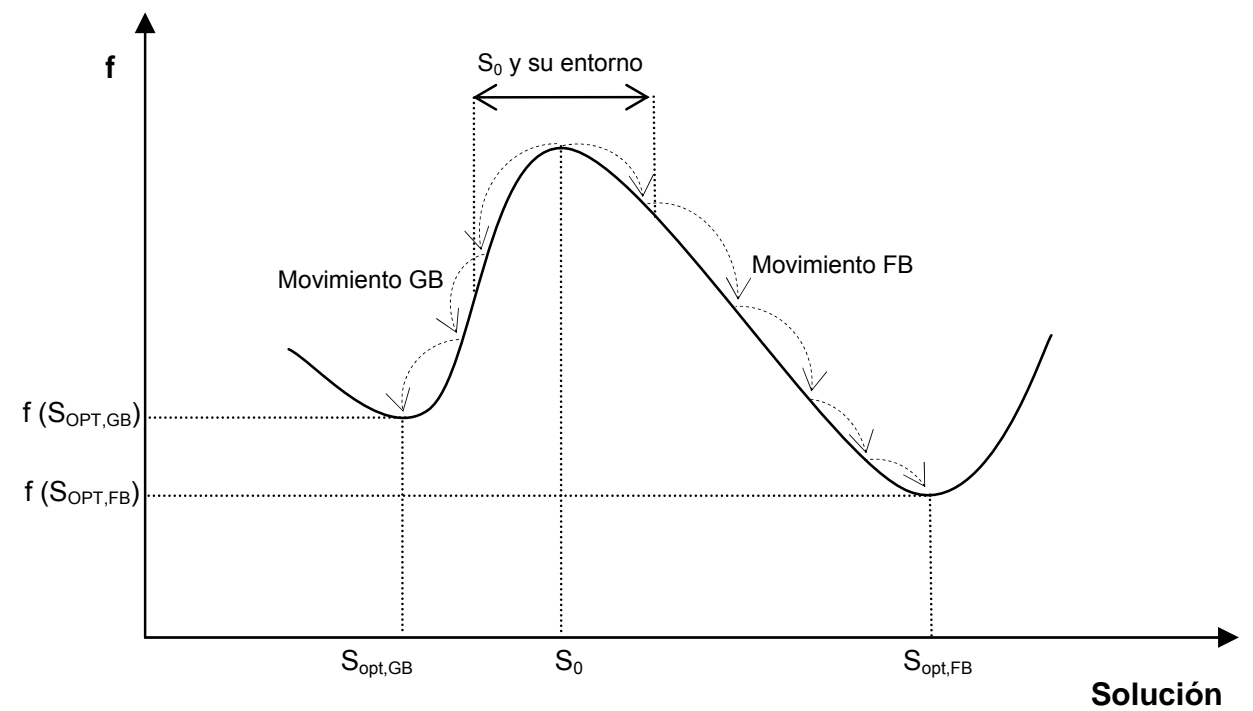

Figura 1.5. Comparación entre los resultados obtenidos empleando un criterio First Best y uno Global Best en un espacio de soluciones. 


\subsubsection{Métodos que realizan una aceptación estratégica admitiendo una degradación acotada de la solución actual.}

Los métodos de gradiente anteriormente descritos aceptan una nueva solución sólo si mejora a la actual. Este criterio puede conducir al encajonamiento prematuro de una solución en un óptimo local (véase la Figura 1.6). Para evitarlo, se han desarrollado otro grupo de heurísticas que permiten una degradación acotada de la solución actual, es decir, permiten que una nueva solución sustituya a la actual aunque la empeore. Para ello, un criterio de aceptación debe satisfacerse y sus características definen diferentes tipos de heurísticas. En la Cristalización Simulada o Simulated Annealing -SA - es de tipo probabilista y en la Aceptación por Umbrales -TA - es determinista.

SA es una estrategia propuesta por Kirpatrick, Gelatt y Vecchi (1983) a partir de los trabajos de Mecánica Estadística de Metrópolis et al. (1953). Se basa en establecer una analogía entre la energía de un sistema termodinámico y la función objetivo $f$ de un problema de optimización combinatoria. En ambos casos se trata de evolucionar de un estado a otro de menor energía o valor de $f$ a través de un parámetro denominado temperatura $(T)$. Lundy y Mees (1986) han demostrado que, con independencia de la solución inicial, el algoritmo converge estadísticamente a la solución óptima aunque puede precisar un tiempo infinito para ello.

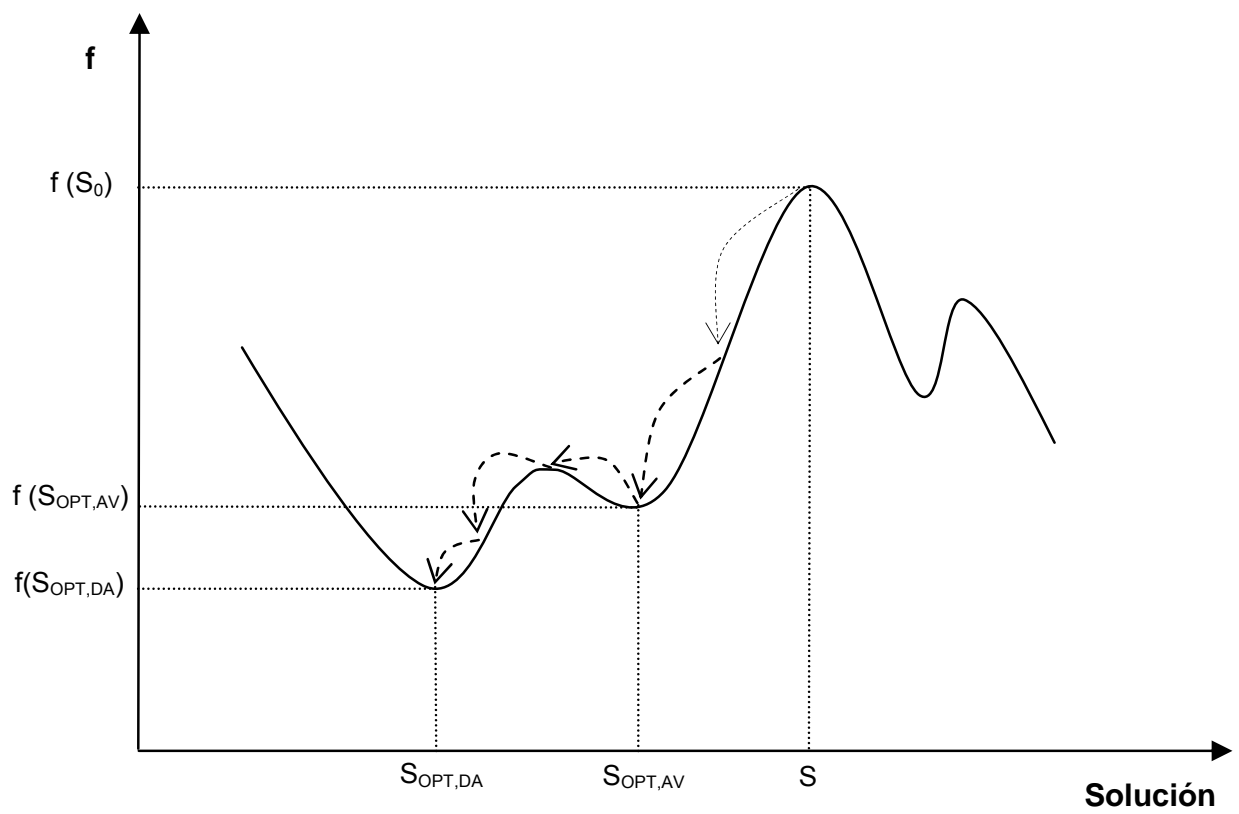

Figura 1.6. Comparación entre la solución óptima alcanzable mediante una estrategia con un criterio de aceptación voraz ( $\left.S_{O P T, A v}\right)$ y mediante un criterio de degradación estratégica ( $S_{O P T, D A}$ ).

SA sigue los pasos siguientes: 
1) Generar una solución inicial $S_{0}$ y elegir una temperatura inicial, $T>0$.

2) Mientras no se cumpla un criterio de parada:

a) Aplicar un movimiento a $S_{0}$ para obtener una nueva solución $S_{1}$

b) Si $S_{1}$ mejora a $S_{0}$, la reemplaza como solución actual $\left(S_{0}=S_{1}\right)$. En caso contrario, $S_{1}$ reemplaza a $S_{0}$ con una probabilidad que viene dada por el factor de Boltzmann (1.3):

$$
p=\frac{1}{\mathrm{e}^{\Delta / \mathrm{T}}} \text {, si } \Delta>0
$$

donde:

$$
\Delta=\left|f\left(S_{1}\right)-f\left(S_{0}\right)\right|
$$

Para aplicar la probabilidad expresada por la ecuación (1.3), se genera un número aleatorio en el intervalo $(0,1)$ con una distribución uniforme de probabilidad. Si el número generado es inferior al valor obtenido en (1.3) entonces la nueva solución es aceptada.

c) Transcurrido un número predefinido de iteraciones, llamado Cadena de Markov, reducir $T$.

d) Volver al paso a).

Se destaca que la forma de la expresión (1.3) hace que la probabilidad de aceptar soluciones de menor calidad disminuye conforme avanza el proceso de optimización (véase la Figura 1.7). En ella se observa como, por ejemplo, un empeoramiento de $f$ de 2 unidades tiene una probabilidad de ser aceptado de 0.905 si la temperatura del proceso es de 20 y de tan sólo 0.018 si la temperatura es de 0.50 . 


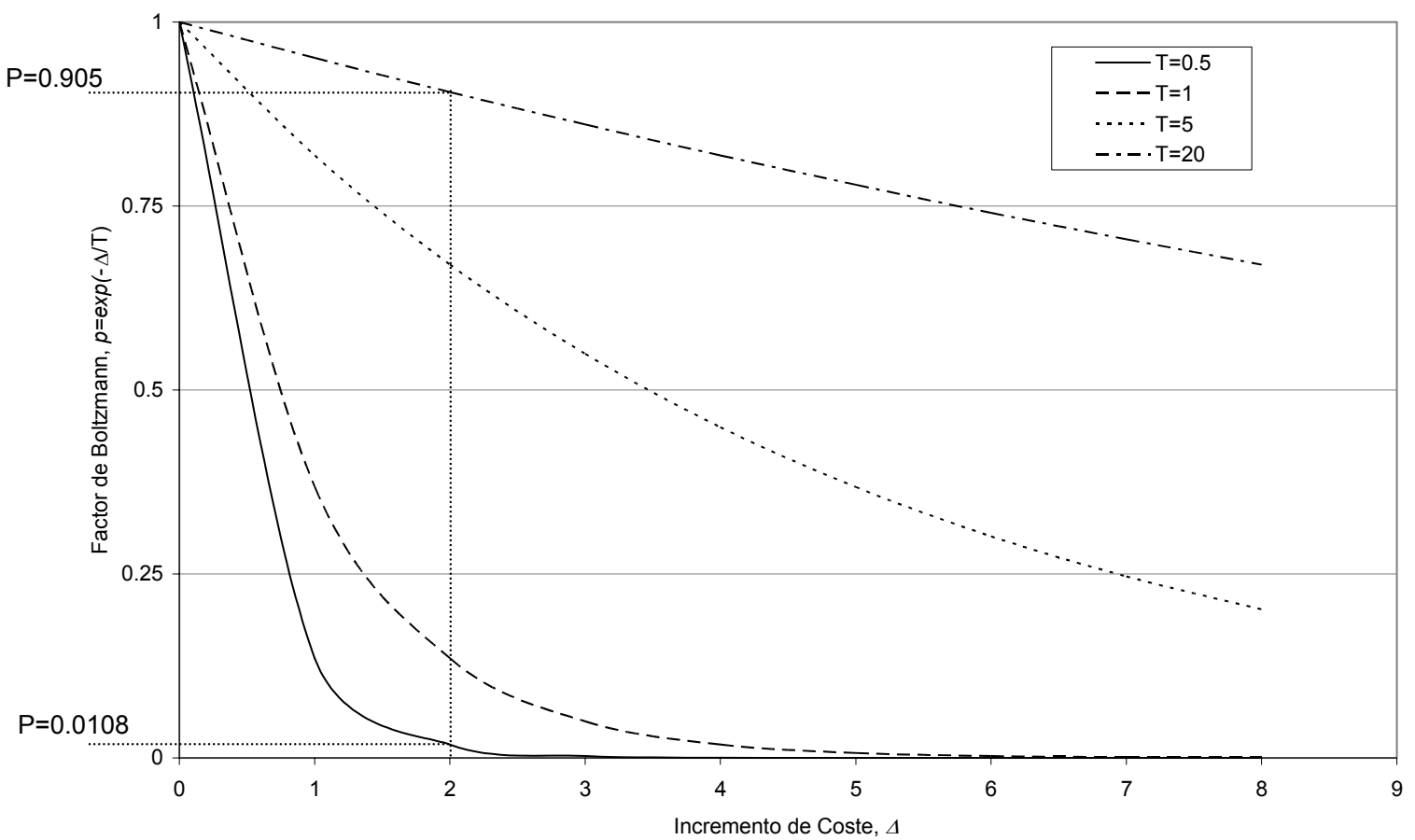

Figura 1.7. Valor de la probabilidad "p" de aceptar una solución de mayor coste (factor de Boltzmann) en función de la temperatura " $T$ ".

Por su parte, el algoritmo TA sigue el esquema siguiente:

1) Generar una solución inicial $S_{0}$ y elegir un umbral inicial, $U>0$ (el umbral inicial es un parámetro del algoritmo TA).

2) Mientras no se cumpla un criterio de parada:

a) Aplicar un movimiento a $S_{0}$ para obtener una nueva solución $S_{1}$.

b) Si $S_{1}$ mejora a $S_{0}$, la reemplaza como solución actual. En caso contrario, $S_{1}$ reemplaza a $S_{0}$ si $f\left(S_{1}\right)-f\left(S_{0}\right)<U$.

c) Si no se consigue ninguna tras un cierto número de iteraciones, disminuir $U$.

d) Volver al paso a). 
Tanto SA como TA pueden adoptar como solución actual una que empeore el valor de la función objetivo. Por ello es necesario almacenar en todo momento el mejor diseño encontrado, pues la solución actual al final del proceso de optimización no tiene por qué coincidir con él.

\subsubsection{Búsqueda tabú.}

La Búsqueda Tabú o Tabu Search (TS) fue propuesta por Glover en 1986 y se basa en guiar la búsqueda hacia zonas del espacio de soluciones que no han sido exploradas. Para ello, TS clasifica como prohibidos un determinado número de los movimientos más recientes que no se pueden repetir durante un cierto número de iteraciones.

Como explica Coello (2007), TS trata de de emular el comportamiento de la persona: "Es bien sabido que los humanos poseemos un avanzado mecanismo de intuición que nos permite operar aún con información mínima o nula, pero por lo general solemos introducir un elemento aleatorio (probabilístico) en dichas decisiones (...). La tendencia resultante en estos casos suele desviarnos de una cierta trayectoria preestablecida, lo cual algunas veces puede ser una fuente de errores, pero en otras ocasiones puede llevarnos a una solución mejor. La TS intenta imitar este mecanismo fundamental (...) asumiendo que no hay razón para escoger un movimiento que nos lleve a una peor solución, a menos que estemos tratando de evitar una ruta que ya se examinó previamente. Con esta sola excepción, la técnica buscará, a cada paso, el mejor movimiento posible (...). Esto hace que la búsqueda se dirija directamente hacia un óptimo local, pero eso no importa porque la búsqueda no se detendrá allí sino que se reinicializará manteniendo la capacidad inicial de identificación del mejor movimiento posible. Además, se mantiene información referente a los movimientos más recientes en una o más listas tabú, a fin de que evitar que una cierta trayectoria previamente recorrida se repita".

Más información sobre esta técnica se encuentra en Osman y Kelly (1996) y en Glover y Laguna (1997).

\subsubsection{Optimización por Colonia de Hormigas.}

La Optimización por Colonia de Hormigas o Ant Colony Optimization (ACO) fue propuesta originalmente por Dorigo et al. (1996). Se basa en el comportamiento colectivo de las hormigas en la búsqueda de alimento, que les permite encontrar el camino más corto entre su hormiguero y la fuente de comida. Inicialmente las hormigas exploran las cercanías de su hormiguero en busca de sustento de modo aleatorio. Una vez una de ellas ha 
encontrado alimento, evalúa su cantidad y calidad y toma una muestra con la que regresa al hormiguero. Por el camino, deja una señal odorífera al depositar una sustancia llamada feromona que permite que otras hormigas puedan seguir la misma trayectoria. Tras un tiempo, el camino hacia la comida se indicará por un rastro odoroso que crece con el número de hormigas que pasan por él y que va desapareciendo en caso contrario. El resultado final es la optimización del trabajo de todo el hormiguero en su búsqueda de comida.

La analogía de este comportamiento con la optimización se realiza del modo siguiente (ver Yepes, 2002):

- La búsqueda de alimento es equivalente a la exploración de las soluciones factibles en un problema de optimización combinatoria.

- La cantidad de alimento es similar al valor de la función objetivo.

- $\quad$ El rastro de feromona es la memoria adaptativa del método.

\subsubsection{Aplicaciones.}

Las primeras aplicaciones de estos métodos a la ingeniería estructural emplearon SA ( Salama et al., 1988; Balling 1991) para optimizar la posición de actuadores y minimizar el coste de estructuras de acero. En hormigón armado, las trabajos pioneros se deben a Balling y Yao (1997) y a Ceracnic et al. (2001), que utilizaron SA para optimizar pórticos de edificación y muros ménsula de contención de tierras respectivamente. Otros trabajos recientes han optimizado mediante ACO estructuras metálicas ( Camp y Bichon, 2004) y plegables (Kaveh y Shojaee, 2007).

Actualmente son dignos de mención los trabajos realizados por el Grupo de Procedimientos de Construcción, Optimización y Análisis de Estructuras de la Universidad Politécnica de Valencia (GPRC) que ha aplicado FB, GB, SA, TA, TS y ACO al diseño de muros ménsula, pórticos y marcos de carretera, pórticos de edificación, bóvedas de carretera y pilas de puentes (Alcalá et al.,2005; Carrera et al. 2005, González Vidosa et al., 2005; Carbonell, 2005; Payá et al. 2006, Perea et al. 2006a, 2006b, 2006c, Yepes et al. 2006, Martínez et al., 2006).

\subsubsection{Descripción general.}

Los algoritmos evolutivos o Evolutionary Alghorithms (EA) constituyen una familia de técnicas basadas en la idea de que una población de soluciones puede evolucionar hacia 
individuos de mayor calidad aplicando los mecanismos que rigen la evolución de las especies. Para ello a) se asigna a cada individuo una aptitud que normalmente es proporcional al valor de la función objetivo y b) se emplean operadores que guían la búsqueda como la selección, la reproducción y la mutación.

El esquema general de un algoritmo evolutivo es el siguiente:

1) NGen=1 (NGen es el número de la generación).

2) Crear una población inicial de soluciones $P(1)$ y evaluar la aptitud de los individuos de $P(1)$.

3) Mientras no se cumpla un criterio de parada:

a) Seleccionar individuos de $P$ (NGen) como padres de individuos de la generación NGen+1 (operador selección).

b) Combinar los padres seleccionados para crear nuevos individuos -hijos(operador reproducción).

c) Modificar los hijos (operador mutación).

d) Evaluar la aptitud de los hijos.

e) NGen=NGen+1.

f) Crear la nueva población $P(N G e n)$ seleccionando individuos entre los elementos de $P(N G e n-1)$ y sus hijos modificados (operador selección).

El empleo o no de los operadores de búsqueda y la manera en la que se aplican da lugar a los diferentes EA.

Las primeras técnicas evolutivas fueron la Programación Evolutiva (Fogel, 1966) y las Estrategias Evolutivas (ES) desarrolladas por H.P. Schwefel e I. Rechenberg en la Technische Universität Berlin (Schwefel, 1965; Rechenberg, 1973). No obstante, la estrategia más empleada en el campo de la optimización estructural son los Algoritmos Genéticos (GA) propuestos por Holland (1975). Goldberg (1989) ha realizado una 
descripción muy detallada de su funcionamiento que ha contribuido enormemente a su difusión, mientras que una introducción a los EA con especial énfasis en los GA se encuentra en Coello (2004).

\subsubsection{Aplicaciones.}

La primera aplicación de una EA a un problema de ingeniería civil se debe a Goldberg (1983), mientras que los trabajos pioneros con GA en el campo de la ingeniería estructural se deben a Goldberg y Samtani (1986) - estructuras de acero-, Coello (1997) estructuras de hormigón armado- y Leite y Topping (1998) - estructuras de hormigón pretensado -. Trabajos posteriores han empleado GA a otros campos como los programas de mantenimiento y rehabilitación de puentes (Elbehairy et al., 2006), las presas de gravedad (Fairbairn et al., 2004) e incluso para minimizar los efectos de un ataque terrorista sobre una infraestructura (Jeong et al., 2006). Por su parte, las ES se han empleado para la optimización de estructuras metálicas (Cai y Thierauf, 1996) y la identificación de sistemas estructurales (Franco et al., 2004).

Una extensa revisión de artículos sobre las aplicaciones de los algoritmos evolutivos a la ingeniería estructural puede encontrarse en Kicinger et al. (2005).

\subsubsection{Redes neuronales.}

Las redes neuronales (NN) artificiales aparecieron al tratar de comprender el cerebro humano, considerándolo como un sistema de procesamiento de la información altamente complejo, no lineal y en paralelo. Su propiedad más interesante es su capacidad de aprendizaje, que hace que hayan sido aplicadas con éxito a problemas tan diversos como el reconocimiento de patrones, la optimización y la planificación.

McCulloch y Pitts (1946) presentaron el primer modelo artificial de neurona, siendo Hopfield y Tank (1985) y Adeli y Yeh (1989) los pioneros en aplicar las NN a problemas de optimización combinatoria y de ingeniería civil respectivamente. Una revisión de sus aplicaciones a la ingeniería civil hasta el año 2000 puede encontrarse en Adeli (2001). Posteriormente, Hadi (2002) las ha empleado para optimizar vigas biapoyadas de hormigón armado y vigas de hormigón reforzadas con fibras, Cladera (2003) para estudiar la resistencia a cortante de elementos lineales fabricados con hormigón de alta resistencia y Hmadahkanlou et al. (2005) para optimizar losas de hormigón armado. 


\subsection{TÉCNICAS APROXIMADAS EMPLEADAS EN LA OPTIMIZACIÓN MULTIOBJETIVO DE ESTRUCTURAS .}

\subsubsection{Introducción.}

De modo análogo a como sucede con la optimización monoobjetivo, los métodos de optimización combinatoria multiobjetivo (también llamados MOCO - Multi-Objective Combinatorial Optimization -) pueden ser divididos en dos grandes grupos: los métodos exactos ( que son capaces de proporcionar un conjunto de soluciones óptimas) y los métodos heurísticos (que proporcionan un conjunto de soluciones del problema que no tienen por qué ser necesariamente las óptimas empleando tiempos de cálculo razonables). Estos últimos pueden ser clasificados en dos bloques, los que emplean estrategias que generalizan la búsqueda secuencial por entornos y los que se basan en los EA.

Puesto que la mayoría de los métodos emplean el concepto de Óptimo de Pareto, esta noción es introducida en el epígrafe 1.4.2. Debido a que este trabajo se centra en los métodos heurísticos, éstos se describen someramente en los epígrafes 1.4 .3 y 1.4.4. Una revisión general de los métodos exactos y heurísticos se encuentra en Ehrgott y Gandibleux (2000).

\subsection{2 Óptimo de Pareto.}

En los problemas MOCO es frecuente que los objetivos entren en conflicto. Por ello, rara vez una única solución es mejor que las demás en todos los criterios. Para ayudar a la toma de decisiones, se emplean los óptimos de Pareto que pueden definirse del modo siguiente (ver Coello, 1996):

"En un espacio de múltiples dimensiones, cada una de las cuales representa a un criterio, un punto " $x$ " es un Óptimo de Pareto de un conjunto de puntos si no existe ningún otro punto que sea capaz de mejorar a " $x$ " en un criterio sin empeorarlo al menos en otro."

Para aclarar esta noción, en la Figura 1.8 se han representado los valores para seis soluciones de dos funciones objetivo, $f_{1}$ y $f_{2}$, que se desea minimizar. El punto "1" es un Óptimo de Pareto. Ello es debido a que ninguno de los demás puntos disminuye su valor de $f_{2}$, sin aumentar $f_{1}$. Análogo razonamiento se puede realizar con los puntos " 2 ", " 3 "y " 4 ". Los puntos " 5 " y "6" son diferentes, pues tanto "2" como " 3 " tienen menores valores de ambas funciones objetivo. Por ello se dice que " 5 " y " 6 " son dominados por " 2 " y " 3 ". Al conjunto de los Óptimos de Pareto se les conoce como Frontera de Pareto (Pareto Set). 


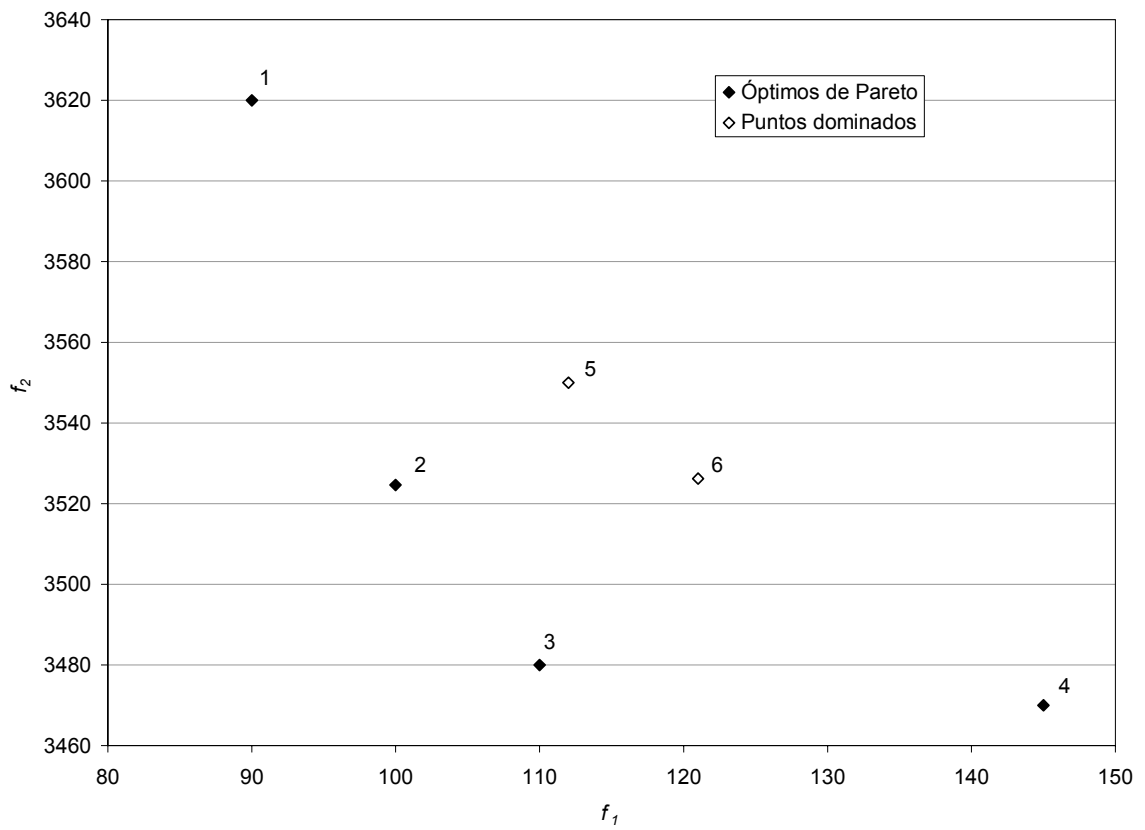

Figura 1.8. Definición de Óptimos de Pareto.

Otra manera de definir un Óptimo de Pareto es la siguiente (ver Coello, 1996):

"Dado un problema de optimización con "l" funciones objetivo $f_{1}, f_{2}, \ldots . . f_{\text {, , se dice que }}$ un vector de variables de decisión $\vec{y}$ que pertenece al conjunto de las soluciones factibles (F) es un Óptimo de Pareto si no existe ningún otro vector $x \in F$ que cumpla simultáneamente las dos condiciones siguientes (asumiendo que se pretenden minimizar todas las funciones objetivo):
1) $f_{i}(\vec{x}) \leq f_{i}(\vec{y})$ para $i=1, \ldots, I$
$y$
2) para al menos un $j, f_{j}(\vec{x})<f_{j}(\vec{y})$ ".

\subsubsection{Métodos basados en la heurísticas que generalizan la búsqueda secuencial por entornos.}

Puesto que SA es capaz de buenas soluciones a problemas de optimización monoobjetivo empleando razonables tiempos de cálculo, algunos investigadores han empleado esta técnica para resolver problemas multiobjetivo. Para ello, se define una función objetivo que representa un compromiso entre los diferentes criterios buscados y se 
optimiza mediante SA. Esta estrategia fue empleada por primera vez en ingeniería estructural para la optimización de estructuras de acero por Bennage y Dhingra (1995), no conociéndose ninguna aplicación a las estructuras de hormigón.

La aproximación anteriormente descrita tiene el problema de que el algoritmo debe ejecutarse varias ocasiones para obtener en cada una de ellas un posible punto de la Frontera de Pareto. El deseo de salvar este obstáculo ha dado lugar a los algoritmos MOSA -Multiobjective Optimization by Simulated Annealing -. Los primeros esbozos de la extensión del SA se deben a Serafini (1985), pero los primeros algoritmos MOSA completos y comprobados en problemas tipo se deben a Czyak et al. $(1994,1998)$, Ulungu et al. (1995) y a Engrand (1997). Una versión modificada del algoritmo de Engrand conocida como SMOSA - Suppapitnarm Multiobjective Optimization by Simulated Annealing, Método de Suppapitnarm para la Optimización Multiobjetivo mediante la Cristalización Simulada - fue aplicada con éxito por Suppapitnarm et al. (2000) a la optimización de a) una función matemática tipo, b) un panel rígido de un vehículo espacial, c) una estructura metálica articulada de diez barras en la que se buscaba minimizar su peso y sus deformaciones y maximizar su frecuencia fundamental de vibración.

\subsubsection{Métodos basados en los algoritmos evolutivos}

Como señala Coello (1996), las técnicas basadas en los algoritmos evolutivos (también conocidas como EMOO - Evolutionary Multi-Objective Optimization-) son especialmente apropiadas para la resolución de problemas multiobjetivo pues:

1) Trabajan con poblaciones de soluciones, lo que les permite encontrar varios miembros del Frontera de Pareto en una única ejecución del algoritmo, mientras que los métodos matemáticos precisan de una ejecución múltiple del algoritmo de optimización para poder definir esta frontera.

2) Son mucho menos susceptibles que los métodos exactos a la forma 0 continuidad del Frontera de Pareto, pudiendo, por ejemplo, abordar sin problemas Frentes de Pareto cóncavos o discontinuos.

Estos métodos pueden ser clasificados en tres grandes grupos: 
1) Aquellos que combinan las diferentes funciones objetivo para definir una única función escalar (aggregating functions methods) que se someterá a optimización mediante un algoritmo genético. Las principales metodologías son:

a) La Combinación Lineal de Pesos (Weighted Sum).

b) La Reducción a un Único Objetivo (Reduction to a Single Objective).

c) La Programación de Metas (Goal Programming).

d) La Logro de Metas (Goal Attainment).

e) El Método $\varepsilon$-constraint ( $\varepsilon$-constraint method).

2) Aquellos que no incorporan de modo directo el concepto de dominancia de Pareto o de Óptimo de Pareto. Ejemplos de estos algoritmos son:

a) El Ordenamiento Lexicográfico (Lexicographic Order).

b) El Vector Evaluated Genetic Algorithm (VEGA).

c) La Teoría de Juegos (Game Theory).

d) El Método Min-Max Ponderado (Weighted Min-Max).

3) Aquellos que hacen uso de la jerarquización de Pareto (Pareto based approaches), asignando en el proceso de selección de los padres de una generación una aptitud más alta a aquellos individuos no dominados y más baja a los dominados. A este grupo pertenecen los siguientes algoritmos:

a) El Multiple Objective Genetic Algorithm (MOGA).

b) El Non-dominated Sorting Genetic Algorithm (NSGA y NSGA-II).

c) El Niched Pareto Genetic Algorithm (NPGA).

d) El Strength Pareto Genetic Algorithm (SPGA).

e) El Pareto Archived Evolution Strategy (PAES).

f) El Pareto Enveloped Selection Algorithm (PESA).

g) El Multiple Objective Messy Genetic Algorithm (MOMGA).

h) El Micro Genetic Algorithm for Multiobjective Optimization (Micro GA).

Una descripción general y crítica de estos métodos así como referencias a algunas de sus aplicaciones se encuentra en Coello $(1996,1999,2005)$ y en Deb $(1999,2001)$, mientras que en Coello y Lamont (2004) pueden consultarse explicaciones muy detalladas 
de aplicaciones de los EMOO a algunos de los principales ámbitos de la ingeniería y de la ciencia.

La primera aplicación en el ámbito de las estructuras metálicas se debe a Hajela y Lin (1992) quienes emplearon el Método Min-Max Ponderado para minimizar el peso de una celosía metálica plana de 10 barras sometida a cargas estáticas y el desplazamiento de uno de sus nudos. En hormigón armado, fueron Koumousis y Arsenis (1998) los precursores al aplicar la Combinación Lineal de Pesos a vigas continuas considerando varios criterios relacionados con la simplicidad de su armado. Otros estudios han empleado las EMOO para investigar la rehabilitación de tableros de una red de puentes (Liu et al., 1997), el diseño conceptual de edificios (Park y Grierson, 1999) o los materiales compuestos con fibras (Savic et al., 1999).

\subsection{LOS PÓRTICOS EN LAS ESTRUCTURAS DE EDIFICACIÓN.}

El Código Técnico de la Edificación (Ministerio de Vivienda, 2006) - CTE en lo sucesivo - establece las condiciones que deben cumplir los edificios en España relacionadas con su seguridad y habitabilidad. Respecto a la seguridad estructural las exigencias básicas son:

1) Resistencia y estabilidad frente a las acciones e influencias previsibles durante las fases de construcción y uso previstas, garantizándose que los eventos extraordinarios no producirán consecuencias desproporcionadas respecto a la causa original.

2) Aptitud al servicio de acuerdo con el uso previsto, de modo que no se produzcan deformaciones inadmisibles, se limite a un nivel aceptable la probabilidad de un comportamiento dinámico inaceptable y no aparezcan degradaciones o anomalías inadmisibles.

El número de sistemas estructurales que permite satisfacer todas las condiciones anteriores es enormemente variado, $\mathrm{y}$, convencionalmente, se han clasificado en dos grandes grupos según sean primordialmente aptos para resistir acciones verticales $u$ horizontales. Calavera (1999) hace una descripción de ellos, siendo el de pórticos paralelos enlazados entre sí por forjados o losas unidireccionales (figuras 1.9 y 1.10) uno de los más empleados. 


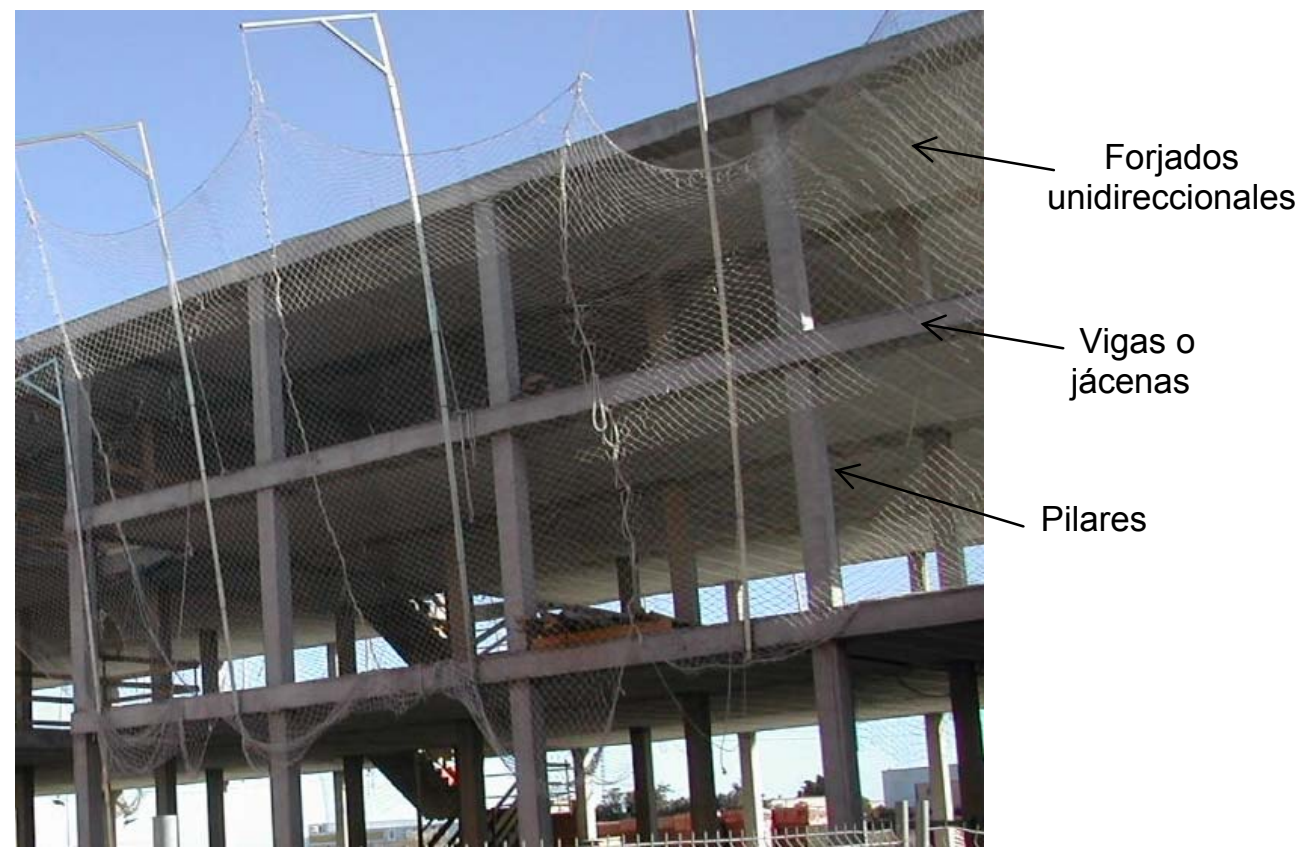

Figura 1.9 Pórticos con forjados unidireccionales. Definición de elementos.

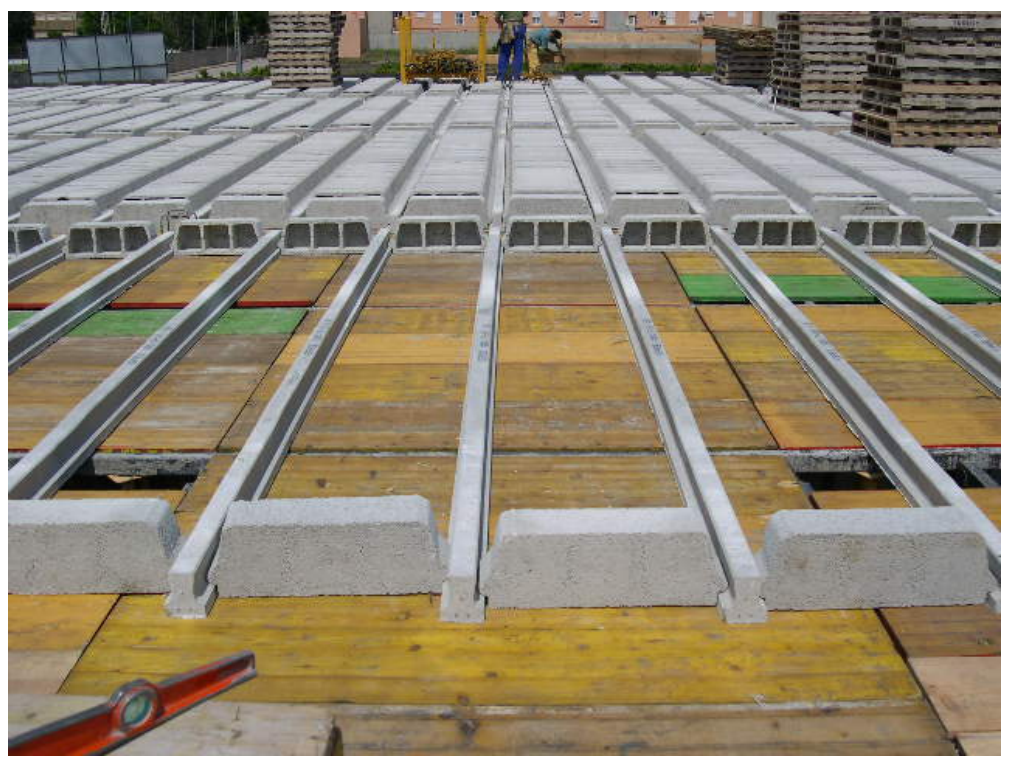

Figura 1.10. Construcción de un forjado unidireccional parcialmente prefabricado de los habitualmente empleados en España.

En esta tipología estructural, los forjados (o elementos que reciben directamente las acciones verticales del edificio) descansan en cada planta sobre elementos llamados pórticos que, a su vez, transmiten las cargas a la cimentación. Los pórticos están formados por elementos horizontales o vigas sobre los que se apoyan los forjados y por elementos verticales o pilares sobre los que descansan las vigas. En edificios con grandes luces y/o cargas, los pórticos se cruzan en los dos sentidos y los forjados unidireccionales se 
transforman en placas macizas o aligeradas (Figura 1.11.a).

Los sistemas de pórticos están ideados para soportar fundamentalmente acciones verticales, por lo que si las acciones horizontales cobran importancia evolucionan hacia otras tipologías (pantallas y pórticos solidarizados por los forjados, sistemas de tubos....). Una de ellas se muestra en la Figura 1.11.b) pudiendo encontrarse en Taranath (1997) y en Gunel e llgin (2006) una explicación detallada de las mismas.
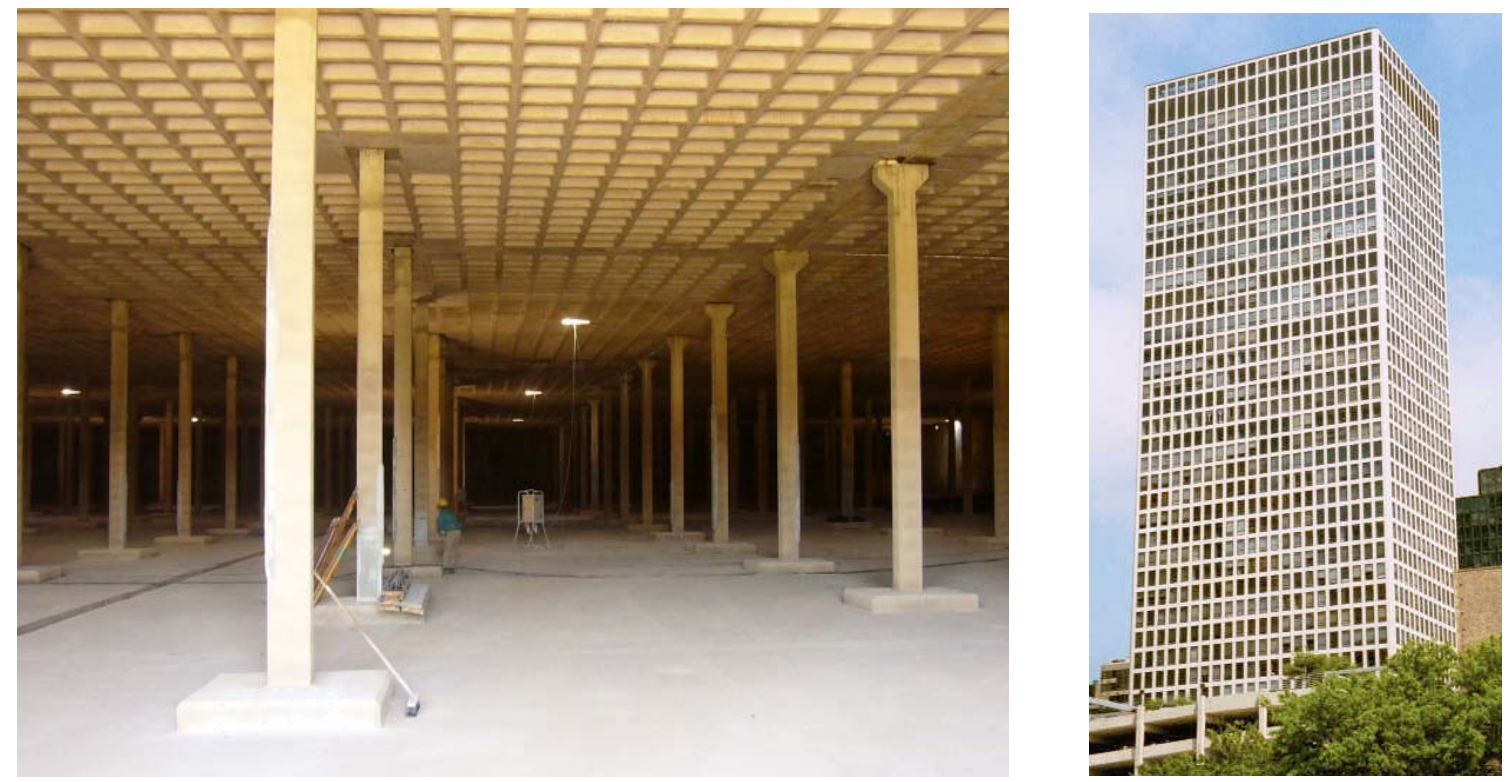

Figura 1.11. Forjado reticular (1.11.a). Apartamentos DeWitt-Chestnut en Chicago (USA). Estructura tipo tubo. Ing. F. Kahn (1.11.b).

De los sistemas estructurales anteriormente descritos, este trabajo se centra en los pórticos de hormigón armado ejecutados "in situ" con forjados unidireccionales. Esta elección se fundamenta en el hecho de que gran parte de los edificios que se construyen en España sigue esta tipología (Gómez, 1998).

El análisis realizado en este trabajo parte de las siguientes consideraciones:

- Los pórticos se encuentran sometidos únicamente a cargas contenidas en el plano de la estructura. Se trata, pues, de pórticos planos.

- Existen juntas de dilatación a distancia adecuada, de forma que los efectos de la retracción del hormigón y de las variaciones de temperatura son despreciables.

- Los forjados del edificio son forjados unidireccionales y todos los pórticos del edificio son de la misma rigidez, de modo que se pueden obtener las cargas actuantes sobre cada pórtico a través de un reparto isostático de las mismas 
(por ámbitos de actuación). Este reparto se efectúa tanto para las acciones verticales como para las horizontales (viento).

- Las condiciones de apoyo de los cerramientos en la estructura hacen que pueda admitirse que las cargas del viento se transmiten a la estructura al nivel del forjado de cada planta.

- No se incluye el estudio de la cimentación por la gran diversidad de soluciones que puede tener dependiendo de las características del terreno de apoyo. Su consideración complicaría de forma innecesaria el estudio y podría distorsionar sus resultados.

- Además, de la enorme variedad de tipologías de pórticos existentes sólo se consideran aquellas que no tienen voladizos y en las que además todos los pilares son continuos desde la cimentación hasta la cubierta del edificio (Figura 1.12).

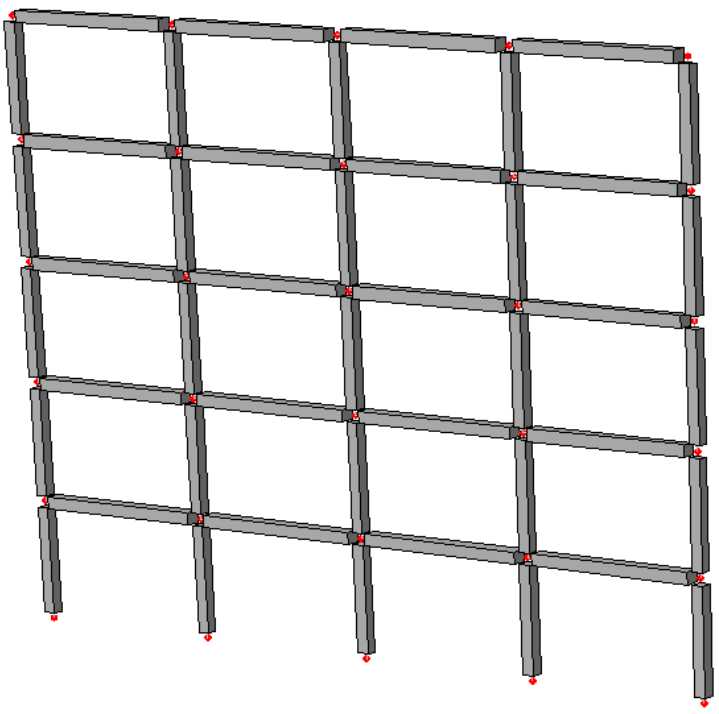

Figura 1.12. Tipología de pórticos planos estudiados en esta Tesis: Vista 3D. No se representa la cimentación.

En cualquier caso, la metodología desarrollada en esta investigación es general y podría adaptarse para considerar condiciones diferentes de las anteriormente descritas. 


\subsection{OBJETIVOS, CONTRIBUCIONES Y ESTRUCTURA DE ESTA TESIS.}

Actualmente, y a pesar de su importancia, los pórticos de edificación de hormigón armado se diseñan según el proceso descrito en la Figura 1. Esta filosofía de proyecto tiene inconvenientes claros pues, como ha señalado el profesor Calavera (1999), "errores apreciables en el predimensionamiento conducen a repeticiones costosas del cálculo". Este autor añade que, para evitar este problema, "es evidente que la experiencia y la habilidad del proyectista son las mejores armas" aunque "los métodos simplificados y aproximados (para el diseño de estructuras) suponen una ayuda muy importante".

Por tanto, sería deseable encontrar procedimientos capaces de "imitar" la sabiduría y olfato que un buen técnico adquiere con la experiencia de muchos proyectos. Y si se quiere dar un paso más, no debería pensarse solamente en economía, sino que criterios como la facilidad constructiva o la sostenibilidad medioambiental deberían también tenerse presentes.

Por ello, los objetivos principales de esta Tesis son:

1) Diseñar algoritmos robustos y flexibles que permitan optimizar el diseño de estructuras aporticadas habitualmente empleadas en edificación, considerando uno o varios objetivos, en un tiempo de cálculo razonable.

2) Estudiar las características generales de las estructuras optimizadas y proponer reglas para su predimensionamiento.

Anteriormente, la optimización de estas estructuras se ha abordado por métodos exactos y heurísticos, pero sin considerar muchas de las variables y restricciones del problema, sin automatizar el proceso, sin proporcionar características generales de las estructuras optimizadas y sin considerar varios objetivos al mismo tiempo.

Por ello, las principales contribuciones que de este trabajo son:

- Presentación de un modelo general para la optimización heurística de pórticos de hormigón armado de edificación. 
- Propuesta de un algoritmo para el diseño óptimo automatizado de estas estructuras, tanto para el problema monoobjetivo (coste económico) como para el multiobjetivo.

- Análisis de las características más importantes (estados límites críticos, dimensiones, cuantías de armadura) de los pórticos optimizados.

El contenido de esta Tesis está organizado de la forma siguiente. El capítulo 2 contiene un estado del arte de la optimización estructural con un énfasis especial en las técnicas heurísticas y en los elementos de hormigón armado. El capítulo 3 presenta la definición completa del problema de optimización, mientras que los algoritmos empleados para resolverlo (FB, SA, TA y GA) se explican en el capítulo 4, junto con la metodología seguida para comparar los resultados.

Las estrategias anteriores se aplican en el capítulo 5 a la minimización del coste económico de un pórtico de dos vanos y cuatro alturas. De la evaluación de los resultados obtenidos se deduce que la versión aquí desarrollada del algoritmo SA es la estrategia más apropiada para optimizar los pórticos. Por ello se emplea en el capítulo 6 para minimizar el coste de estructuras de dos vanos y dos, seis y ocho alturas. Esto permite extraer conclusiones generales relativas al algoritmo y a los diseños optimizados.

En el capítulo 7 se minimiza el coste de un pórtico de dos vanos y ocho alturas considerando la calidad de hormigón habitual en este tipo de estructuras en España (HA-25) y dos variantes para la estructura (vigas descolgadas o planas). Del análisis de los resultados se obtienen reglas de predimensionamiento.

La resolución del problema multicriterio - considerando como objetivos el coste económico e índices relativos a la facilidad constructiva, la sostenibilidad ambiental y la seguridad estructural - se efectúa en el capítulo 8. Finalmente, el capítulo 9 resume las conclusiones del trabajo realizado y apunta futuras líneas de investigación. 


\section{CAPÍTULO 2. ESTADO DEL ARTE.}




\section{Objetivos.}

En este capítulo:

- Se presentan los trabajos más relevantes realizados hasta el momento en relación con el contenido de esta Tesis.

- Se identifican los vacíos existentes en la investigación realizada hasta el momento que deben ser cubiertos por esta investigación. 


\subsection{INTRODUCCIÓN.}

La revisión bibliográfica es una parte esencial de toda Tesis Doctoral pues identifica los aspectos relacionados con el tema estudiado que no han sido tratados en investigaciones anteriores, permitiendo establecer la idoneidad de un tema.

El estado del arte aquí realizado parte de trabajos similares publicados por otros autores (punto 2.2) y cubre los estudios de optimización estructural relacionados con esta Tesis más importantes por la tipología estructural y/o metodología de optimización empleadas. La presentación sigue la clasificación de las técnicas de optimización descrita en el Capítulo $1 \mathrm{y}$, por tanto, abarca la optimización monoobjetivo por métodos exactos (punto 2.3) y aproximados (punto 2.4) y las estrategias aproximadas para la optimización multicriterio (punto 2.5). Asimismo, contempla otros trabajos relacionados con el diseño económico de pórticos de edificación de hormigón que no emplearon ninguna técnica específica de optimización (punto 2.6). Las conclusiones de esta parte de la investigación se proporcionan en el epígrafe 2.7 junto con un cuadro resumen de las principales aportaciones de este trabajo en relación a lo anteriormente publicado.

\subsection{REVISIONES BIBLIOGRÁFICAS ANTERIORES.}

Los primeros trabajos de optimización estructural se deben a Maxwell (1854) y a Michell (1904), habiéndose publicado desde entonces y hasta 1994 unos 150 libros y 2500 artículos sobre el tema (ver Cohn y Dinovitzer, 1994). Estos autores elaboraron un catálogo de los problemas propuestos y resueltos hasta ese momento y señalaron que la investigación en optimización estructural se caracterizaba porque:

- Existía un gran hueco entre los avances en las teorías de optimización y sus aplicaciones en ingeniería, estando la mayor parte de las publicaciones más centradas en los aspectos matemáticos de la optimización que en los estructurales.

- La mayoría de los problemas se resolvían mediante métodos exactos implementados en software comercial. Los algoritmos genéticos eran mencionados como un método con un gran potencial pero con pocas realizaciones. 
- El 92\% de las estructuras resueltas eran estructuras de acero. Un 4\% eran de hormigón y el $4 \%$ restante mixtas.

- Un $88 \%$ de los ejemplos se referían a estructuras sometidas a uno o dos casos de carga estáticos y un $12 \%$ estudiaban cargas dinámicas. En muy raras ocasiones las acciones se combinaban para obtener envolventes de esfuerzos con las que diseñar las estructuras.

- La optimización monobjetivo (considerando el coste o el peso de la estructura como funciones a minimizar) estaba mucho más representada que la multiobjetivo.

- Era necesario resolver ejemplos prácticos con condiciones reales de geometría, carga y estados límites, de modo que las técnicas de optimización, implementadas en sistemas informáticos fáciles de usar, fueran una herramienta más accesible a los ingenieros.

Años más tarde, Sarma y Adeli publicaron estados del arte sobre la optimización de estructuras de hormigón (Sarma y Adeli, 1998) y de acero (Sarma y Adeli, 2000a). En sus conclusiones, insistieron en la necesidad de a) resolver estructuras reales y b) considerar como objetivo no el peso de la estructura sino su coste a lo largo de toda su vida útil.

\subsection{TÉCNICAS EXACTAS EMPLEADAS EN EL DISEÑO ÓPTIMO DE ESTRUCTURAS.}

En esta línea de investigación se reseñan los trabajos siguientes:

- Moragues (1980) aplicó los métodos exactos al diseño de estructuras aporticadas de hormigón armado considerando como función a minimizar el coste de la estructura. En su trabajo estudió de forma sucesiva la forma de optimizar secciones, vigas y pilares rectangulares de hormigón armado y, finalmente, estructuras aporticadas. Para ello, empleó diferentes métodos que iban desde la búsqueda del mínimo de una función continua de una sola variable -en el caso del estudio de la sección- hasta una adaptación del método directo de Powell (1968) con funciones de penalización - en el caso del pórtico -. Finalmente proporcionó algunos criterios generales de predimensionado basados en los resultados obtenidos. Como restricciones del problema señalar que a) consideraba únicamente la verificación del ELU de agotamiento por solicitaciones normales, despreciando la influencia del cortante en la búsqueda de óptimos, b) No consideraba la verificación de ningún ELS ni 
del ELU de Inestabilidad (pandeo). Como variables adoptó un máximo de siete en el caso de estructuras aporticadas (canto útil de las vigas -el mismo para todas independientemente de su altura-, canto útil del pilar extremo y de los pilares centrales -el mismo para todos los pilares intermedios- y los coeficientes de variación con la altura del ancho y del canto de los pilares extremos y centrales). Las estructuras estaban sometidas únicamente a acciones gravitatorias.

- Gasch (1991) desarrolló un método multinivel para la optimización de una planta de forjado reticular sometida exclusivamente a acciones verticales. En una primera fase optimizaba la topología (número de soportes) permaneciendo constantes las dimensiones de las secciones o valores relacionados con ellas (esbeltez del forjado). En una segunda fase fijaba la topología y optimiza la geometría (posición de los soportes) y las dimensiones de forjados y soportes. El número máximo de variables considerado es de 21.

- Moharrami y Grierson (1993) emplearon un criterio de optimalidad para minimizar el coste de un pórtico de hormigón de un vano y cinco alturas y de una estructura tipo tubo de cinco plantas. El primer ejemplo tenía 11 variables y el segundo 30, todas ellas continuas y referentes a la geometría y al armado. No consideron la calidad del hormigón como una variable más y, al no adoptar valores discretos de las variables, no proporcionan planos constructivos de las estructuras ni se verifican las restricciones relativas a algunos estados límites como la fisuración o las disposiciones constructivas de las armaduras.

- Balling y Yao (1997) abordaron la optimización de pórticos de edificación de hormigón armado mediante tres estrategias: 1) un método exacto basado en técnicas del gradiente y que consideraba como variables las dimensiones del pórtico y la cuantía de armadura de flexión en las secciones críticas, 2) un método multinivel que empleaba SA para la optimización de las dimensiones del pórtico y una exploración exhaustiva del espacio de soluciones para la definición de las armaduras de flexión las cuales representaron mediante esquemas reales de armado y no mediante cuantías, 3) una versión del primer método, que eliminaba las áreas de acero como incógnitas y que partía de suponer que las restricciones relativas a las cuantías mínimas de armadura y al cumplimiento del ELU de agotamiento por solicitaciones normales rigen los diseños óptimos. En los ejemplos presentados, el tercer método es mucho más rápido que el multinivel (del orden de 280 veces) y que el método exacto (del orden de dos veces). Las soluciones optimizadas encontradas mediante los 
métodos primero y tercero son un $1.7 \%$ más caras que las del método multinivel que proporciona las soluciones más económicas. Su análisis no incluye la resistencia del hormigón y no considera directamente el cálculo de las armaduras de cortante si bien se realizan ciertas comprobaciones para asegurar que la sección de hormigón es suficiente para resistir este esfuerzo.

- Torrano (2003) ha desarrollado un algoritmo de programación cuadrática sucesiva para optimizar el coste de: a) secciones de hormigón armado con forma arbitraria y cualquier distribución de armado y b) elementos estructurales de ese mismo material. Todas las variables son continuas y el algoritmo se aplica a la optimización de dos secciones convencionales (cuadrada y en L) sometidas a flexión compuesta esviada, una sección rectangular con un agujero centrado solicitada por flexión compuesta recta, una viga biapoyada de sección variable sometida a una carga vertical uniformemente repartida y una sección, que representa un sistema de pantallas, sometida a flexión compuesta esviada. El número máximo de variables es 42 y no considera algunas disposiciones constructivas como las relativas a las separaciones de la armadura de cortante ni la verificación de la fisuración. Concluye que el método propuesto es una técnica sencilla y eficiente que permite obtener diseños más económicos que los obtenidos por procedimientos convencionales.

- Rodrigues et al. (2005) han estudiado la optimización de los pilares de edificios altos sometidos a acciones horizontales y verticales. Emplean un método multinivel que en una primera fase obtiene las dimensiones de los pilares y en una segunda su armado. No consideran como variables la geometría de las vigas ni la calidad del hormigón a pesar de la influencia que estos elementos tienen en la rigidez del edificio. Como restricciones consideran el desplazamiento horizontal máximo y las derivas de cada uno de los pisos, el ELU de inestabilidad en los pilares y sus cuantías máximas. No se verifica el ELU de agotamiento por esfuerzo cortante ni se realiza un diseño detallado del armado de los pilares. El método es aplicado a un edificio de 16 alturas considerando las mismas dimensiones de los pilares en toda la altura del edificio y una, dos o tres reducciones de su sección. Del estudio realizado concluyen que ahorros significativos en el coste de la estructura pueden obtenerse cuando se emplean secciones diferentes de los pilares en función de la planta en la que se ubican. 


\subsection{TÉCNICAS APROXIMADAS EMPLEADAS EN LA OPTIMIZACIÓN MONOOBJETIVO DE ESTRUCTURAS.}

\subsubsection{Algoritmos que generalizan la búsqueda secuencial por entornos.}

Salama et al. (1988) fueron los primeros en aplicar un algoritmo de búsqueda local (el SA) a la optimización estructural al resolver un problema de posicionamiento de sensores en estructuras articuladas. Posteriormente se destacan los trabajos siguientes.

\subsubsection{Estructuras de acero.}

- Balling (1991) ha optimizado mediante SA pórticos tridimensionales de acero.

- Tzan y Pantelides $(1996,2000)$ han propuesto modificaciones al SA que reducen la dimensión del espacio de soluciones. Estas mejoras están específicamente pensadas para casos de carga dinámicos en estructuras de acero, no proporcionando buenos resultados en casos de carga estáticos. El algoritmo se emplea para optimizar un pórtico de acero de un vano y diez alturas y otro de dos vanos y dos alturas.

- Hasancebi y Erbatur (2002) han aplicado SA para la optimización de estructuras espaciales de acero incluyendo en el proceso la definición de su topología (definición del número de barras y de la posición de los nudos). La técnica se utiliza para resolver dos problemas tipo: una pirámide espacial y una estructura articulada plana.

- Shea y Zaho (2004) han empleado una variante de SA llamada Structural Topology and Shape Annealing (STSA) para optimizar el peso de una celosía metálica en voladizo que se ha construido en Londres, considerando variables relacionadas con la topología de la estructura.

- Camp y Bichon (2004) han utilizado ACO para resolver estructuras articuladas planas y espaciales de acero. Los resultados son comparados con los proporcionados por otros autores empleando métodos exactos y GA. En todos los casos ACO proporciona iguales o mejores resultados que los obtenidos mediante otras técnicas.

- Camp et al. (2005) han empleado ACO para optimizar tres pórticos metálicos planos de nudos rígidos (el mayor de tres vanos y 24 alturas). Los resultados se comparan con los obtenidos por otros autores que utilizaron GA, proporcionando ACO mejores resultados. 
- Shea y Smith (2006) han empleado STSA para minimizar el peso de estructuras de barras para torres eléctricas de alta tensión. El cálculo de la estructura se realiza conforme a normas vigentes en Suiza (L'Energie de I'Oest de Suisse, 1997) partiendo de un diseño inicial en el que el algoritmo puede modificar la posición de los nudos e incluso crear nuevas configuraciones estructurales.

\subsubsection{Estructuras de hormigón.}

- Balling y Yao (1997), como ya se ha comentado con anterioridad, han usado un método multinivel para la optimización de pórticos de hormigón armado que combina SA con una exploración intensiva del espacio de soluciones.

- Ceracnic et al. (2001) han empleado SA para la optimización económica de muros ménsula de contención de tierras de hormigón armado. Consideran siete variables en su análisis todas ellas relacionadas con la geometría del muro.

- Alcalá et al. (2005) y Yepes et al. (2006) han optimizado muros ménsula de hormigón armado mediante FB, TA y SA, obteniendo mediante SA los mejores resultados. En su trabajo emplean 22 variables para caracterizar un muro entre las que se encuentran la calidad del acero y del hormigón. Los muros optimizados son más esbeltos de lo que es la práctica habitual de estas estructuras, por lo que es necesario verificar sus deformaciones en cabeza. El hormigón es de la mínima calidad admitida por la normativa y las cuantías de acero son muy elevadas. Además proporcionan reglas para el predimensionamiento de estas estructuras.

- Carrera (2004) y Carrera et al. (2005) han utilizado FB, SA y TA para optimizar pórticos de carretera de hormigón armado considerando 28 variables. Los pórticos optimizados presentan cuantías de acero y esbelteces superiores a las habituales en este tipo de estructuras. Por ello es necesario verificar los estados límites de deformaciones y de vibraciones, lo que normalmente no se realizaba en este tipo de estructuras. Los mejores resultados se obtienen con SA.

- Perea (2006) y Perea et al. (2006a, 2006b, 2006c) han empleado FB, SA, TS, TA y una variante de este último (el Old Bachelor Accepting -OBA-) para optimizar marcos de carretera de hormigón armado. Para caracterizar estas estructuras se emplean 44 variables entre las que se encuentran dos tipos 
diferentes de hormigón. Los marcos optimizados tienden a grandes esbelteces y fuertes armados, lo que hace necesaria la consideración de dos estados límites normalmente no considerados en este tipo de estructuras: deformaciones y fatiga. Los mejores resultados se consiguen empleando SA, TA y TS. Presentan además la aplicación de la metodología a un falso túnel construido en Mallorca de $900 \mathrm{~m}$. de longitud que constituye la mayor estructura de hormigón optimizada construida hasta el momento.

- Carbonell (2005) ha aplicado una búsqueda exhaustiva de máximo gradiente, SA y TA a la optimización de bóvedas de hormigón armado para pasos de carretera. El problema tiene 21 variables entre las que se encuentran tres tipos de hormigones. Los mejores resultados se obtienen con SA que es capaz de disminuir un $7.6 \%$ el coste económico de un diseño realizado por una oficina de cálculo experimentada.

- Martínez et al. (2006) han empleado GFB, TA y ACO para optimizar el alzado y la cimentación de pilas rectangulares huecas de hormigón armado. En su estudio se consideran 95 variables entre las que se incluyen diferentes calidades de hormigón. Los mejores resultados se obtienen mediante ACO.

\subsubsection{Algoritmos evolutivos.}

Describimos seguidamente las principales aplicaciones de los EA a la ingeniería estructural.

\subsubsection{Estructuras de acero.}

Las primeras aplicaciones de los EA se deben a Goldberg y Samtani (1986) que abordaron la optimización de una celosía articulada de 10 barras mediante GA. A esta aplicación, que se ha convertido en el problema más empleado para verificar la calidad de un algoritmo en ingeniería estructural, siguieron las de Hajela (1989, 1990), Jenkins (1991a, 1991b) y Rajev y Krishnamoorty (1992). En estos trabajos se aplicaba el GA descrito en el libro de Goldberg (1989) y conocido como Simple Genetic Algorithm (SGA) a sencillos esquemas estructurales.

Otros trabajos reseñables son:

- Grierson y Pak (1993) han optimizado pórticos de edificación en los que consideran simultáneamente las secciones transversales y la topología de la 
estructura. El método desarrollado es aplicado a tres ejemplos, siendo el de mayor entidad un pórtico de dos plantas y un número de vanos variable con una longitud total de $16 \mathrm{~m}$.

- Adeli y Cheng (1993) han investigado diferentes formas de realizar el operador genético cruzamiento y lo han aplicado a la optimización de tres celosías espaciales con un máximo de 72 barras. Las barras se agrupan de modo que como máximo se consideran 16 variables.

- Adeli y Cheng (1994a, 1994b) han desarrollado un GA híbrido que emplea el Método Lagrangiano Aumentado (Augmented Lagrangian Method) y lo han aplicado a la optimización de estructuras de gran tamaño (una celosía espacial de 72 barras, una cúpula geodésica espacial, una torre en celosía de 26 alturas y otra torre de 35 ). Con la finalidad de disminuir el número de variables, las barras también se agrupan, de modo que el número máximo de grupos es 72 . Como restricciones se consideran las tensiones máximas en las barras de acero (sin tener en cuenta el efecto del pandeo) y, los desplazamientos horizontales en los puntos más altos de las torres y vertical en el punto más alto de la cúpula. La agrupación de las barras se realiza por los autores. Para los cálculos emplean supercomputadoras trabajando en paralelo.

- Cai y Thierauf (1996) han presentado la aplicación de ES a la optimización de estructuras planas y espaciales de acero. El algoritmo se implementa en paralelo en varios ordenadores.

- Rajev y Krishnamoorty (1997) han presentado dos métodos basados en GA para la optimización de celosías metálicas incorporando la definición de la topología de la estructura al problema.

- Sheresta y Gamboussi (1998) han planteado un método basado en GA para la optimización de celosías metálicas planas y espaciales considerando, como en el caso anterior, la topología también como variable. El método es aplicado a dos celosías planas de un sólo vano.

- Camp et al. (1998) han optimizado estructuras reticulares planas de edificios considerando a) tablas de perfiles comerciales, b) estados de carga indicados en el Uniform Building Code (1994), c) los ELS y ELU indicados por la norma norteamericana AISC (1989) y d) diferentes estrategias para los operadores genéticos de selección y cruza. El número máximo de variables empleadas es de 12 y los resultados son comparados con los proporcionados por métodos de optimización exactos. 
- Xu y Gong (2001) han aplicado GA al diseño de celosías planas de acero y presentan dos ejemplos reales de aplicación correspondientes a vigas de cubierta de 97.6 y $58.1 \mathrm{~m}$. de luz.

- Krishnamoorthy et al. (2002) han desarrollado una aplicación informática en entorno windows para la optimización de estructuras planas y espaciales articuladas de acero. En este artículo se proponen diferentes mejoras al SGA, se incorpora una estrategia elitista y la agrupación de las barras se realiza de forma automática por el programa a partir de un número máximo de grupos definido por el usuario. Una herramienta similar pero específicamente diseñada para el proyecto de puentes de ferrocarril ha sido presentada por Pandia y Kalyanaraman (2005).

- Ali et al. (2003) han desarrollado una aplicación para la optimización de estructuras metálicas articuladas planas y espaciales integrando un programa general de cálculo de estructuras (Ansys, 2004) con módulos de preproceso, postproceso y GA programados en Matlab (Matlab, 1999). Aplican el sistema a la optimización de seis problemas tipo, teniendo 44 variables el de más tamaño. Los resultados en términos de calidad de la solución son buenos, pero los tiempos de cálculo resultan muy elevados, lo que se debe a los tiempos empleados en el análisis de la estructura y en el proceso y posproceso de los datos.

- Lagaros et al. (2005) aplican ES al diseño óptimo de una estructura tipo tubo de 36 plantas.

\subsubsection{Estructuras de hormigón.}

La primera aplicación de los GA a estructuras de hormigón se debe a Coello et al. (1997) quienes aplicaron el SGA con cruzamiento de dos a puntos a la optimización de una viga biapoyada de hormigón armado. En su trabajo experimentaron con diferentes sistemas de codificación y consideraron como variables el canto y ancho de la viga y el área de su armadura inferior. Posteriormente, se destacan los trabajos siguientes:

- Rafiq y Southcombe (1998) han aplicado el SGA para optimizar el armado de pilares de hormigón sometidos a un esfuerzo axil y a momentos flectores en los dos ejes del pilar. Las dimensiones del pilar y la calidad del hormigón no son consideradas como variables sino como parámetros.

- Rajev y Krishnamoorty (1998) han presentado una metodología para optimizar pórticos planos de hormigón armado basada en el SGA y la aplican a un pórtico 
de tres vanos y cuatro plantas y a otro de seis plantas y dos vanos sometidos a un único caso de carga. En este artículo no se consideran como variables la armadura de cortante ni la calidad del hormigón y del acero. Además, vigas y pilares son agrupados con la finalidad de reducir el número de variables, de modo que, en el pórtico de seis plantas, el número de variables es de 29.

- Chau y Albermani (2003) han presentado una aplicación informática para el diseño optimizado de depósitos rectangulares de hormigón armado que emplea el programa Abaqus para el cálculo estructural. El análisis es muy simplista pues considera únicamente tres variables: canto de las losas de hormigón, diámetro de la armadura y separación entre barras, adoptando los mismos valores de las variables para todos los elementos (losa de cimentación y alzados) del depósito.

- Leps y Sejnoha (2003) han presentado un algoritmo que combina SA y GA y lo aplican a la optimización de una viga continua simétrica de hormigón armado de dos vanos con armadura de cortante y de flexión con 21 variables. La técnica, conocida como Augmented Simulted Annealing, es similar a un SA en el que: a) se trabaja con una población de soluciones en lugar de con una única solución y b) las nuevas soluciones se obtienen a partir de las existentes mediante la aplicación de los operadores genéticos y no mediante el concepto de movimiento (como se realiza en el SA). En una primera fase, se ejecuta el algoritmo 10 veces para obtener un conjunto de 1000 soluciones de calidad. En una segunda fase, estas 1000 soluciones se emplean como población inicial para otra ejecución del algoritmo. Los autores justifican el empleo de este método ante la imposibilidad de resolver la estructura de forma satisfactoria mediante un GA. Señalamos que: a) las calidades del hormigón y del acero no son consideradas como variables en el análisis, b) la armadura longitudinal superior e inferior es constante a lo largo de toda la longitud de la viga, c) EI coste de la estructura lo obtienen considerando el precio del acero (coronas checas $/ \mathrm{Kg}$ ) y el coste del hormigón (coronas checas $/ \mathrm{m}^{3}$ ), pero no se considera el coste del encofrado ni el de las cimbras, d) consideran un único estado de carga y no todas las combinaciones requeridas por la normativa.

- Lee y Ahn (2003) han optimizado pórticos planos de hormigón armado empleando un SGA al que incorporan una estrategia elitista y considerando las casos de carga de la normativa norteamericana (International Conference of Building Officials, 1997). En su estudio no consideran a) la armadura de cortante ni las resistencias del hormigón y del acero como variables, b) los 
estados límites de fisuración y de deformaciones en vigas. Parten de una base de secciones de hormigón armado para vigas y pilares, lo que reduce la dimensión del espacio de soluciones pues sólo aquellas secciones que cumplen cuantías mínimas y máximas y separaciones máximas y mínimas entre las barras son consideradas. No obstante esa base es muy incompleta pues a) considera en cada sección un único diámetro de la armadura, b) la sección y, por tanto, la armadura es constante en todas las vigas de una planta y de un extremo a otro, lo cual no refleja la práctica habitual de construir con una armadura base constante y otra de refuerzo y resulta antieconómico. El ejemplo de mayor magnitud es un pórtico de tres vanos y veinte alturas en el que consideran 13 variables y en el que la dimensión del espacio de soluciones es $1.14 \times 10^{46}$.

- Camp et al. (2003) han optimizado diversas estructuras planas de hormigón armado mediante una versión del algoritmo genético desarrollado por Carroll (1997). En su análisis a) no se consideran el ELS de fisuración y las flechas se verifican a través de la imposición de un canto mínimo b) la resistencia del hormigón y del acero no son adoptadas como variables, c) la armadura de positivos y de negativos es considerada constante en todas las vigas de una planta y d) todos los pilares de una misma alineación son iguales independientemente de la planta en la que se ubiquen. Entre las estructuras resueltas se encuentra el pórtico de dos vanos y seis alturas estudiado por Krishnamoorty, para el que consideran un espacio de soluciones con $1.26 \times 10^{21}$ elementos y emplean un tiempo de cálculo de 13 horas.

- Kong (2004) ha utilizado SGA para minimizar el peso de edificios de hormigón armado sometidos a acciones horizontales. Considera fijados el ancho de los pilares y las dimensiones de las vigas, quedando como variables únicamente el canto de los pilares. Como única restricción considera la deriva entre dos plantas y no realiza ningún cálculo del armado de la estructura. Este aspecto invalida su modelo pues pueden darse como buenas dimensiones de pilares en las que no sea posible encajar posteriormente un armado.

- Ceracnic y Frier (2004) han desarrollado una aplicación Windows para el diseño automatizado de vigas, placas y pórticos planos de hormigón armado. En su articulo no explican cuales son las variables consideradas en cada uno de los casos y únicamente una viga continua de tres vanos es optimizada. Además, los resultados de la estructura óptima no se proporcionan. 
- Sahab et al. $(2004,2005)$ han optimizado estructuras de edificación en las que los forjados son losas de hormigón. Su estudio incluye la optimización de las luces de los vanos y reduce el problema estructural tridimensional a uno bidimensional empleando el método de los pórticos virtuales. La optimización se realiza en tres niveles: 1) obtención del número óptimo de pilares mediante una búsqueda exhaustiva, 2) dada una configuración de pilares, búsqueda de las dimensiones óptimas de la estructura mediante un GA híbrido y 3) fijadas unas dimensiones de la estructura, búsqueda del armado óptimo mediante una búsqueda exhaustiva. El problema es abordado para diferentes configuraciones (cruzas, funciones de penalización...) del GA. No obstante a) no consideran el hormigón como una variable más en el análisis, b) no consideran la acción de fuerzas horizontales sobre la estructura, c) plantean únicamente cinco esquemas de armado diferentes para los pilares y no se proporcionan datos sobre los esquemas de armado de las losas y d) no se proporcionan resultados de los armados de las losas ni los tiempos absolutos de cálculo. En sus artículos estudian tres edificios distintos y establecen conclusiones referentes a los parámetros del GA y, en un caso, la repercusión económica de igualar variables que resulta ser del orden del $0.7 \%$. El número máximo de variables con las que GA trabaja son 36 .

- Govindaraj y Ramasamy (2005) han estudiado la optimización de vigas continuas con un diseño detallado de su armado. Consideran como variables el canto de la viga en cada uno de sus vanos y un ancho que suponen el mismo en todos los vanos, por lo que el número total de variables es $N_{v}+1\left(N_{v}\right.$ es el número de vanos de la viga). Las armaduras de la viga no se consideran como variables, sino que, dadas unas dimensiones de la viga, el programa determina en primer lugar el armado de flexión óptimo mediante una búsqueda exhaustiva en un catálogo de armados y, posteriormente, el armado de cortante óptimo entre otro catálogo de armados. Su análisis a) no considera la calidad del hormigón como una variable, b) al analizar por separado la armadura de flexión y la de cortante, no tiene en cuenta la relación que existe entre ambas armaduras, que hace que, en algunos casos, pueda resultar más económico armar más a flexión y reducir la armadura de cortante o viceversa. La búsqueda de las dimensiones óptimas de la viga se realiza empleando un SGA. La técnica es aplicada a una viga de un vano y a dos vigas de dos vanos.

- Rafiq et al. (2006) han desarrollado una aplicación informática interactiva para la optimización de pilares sometidos a momentos en las dos direcciones y a 
una carga axil. La aplicación permite al usuario decidir en qué zonas del espacio de soluciones debe centrarse la búsqueda para conseguir diseños más adecuados a sus preferencias.

\subsubsection{Otras aportaciones.}

- Manoharan y Shanmuganathan (1999) han aplicado tres procedimientos metaheurísticos (SA, GA y TS) y un método exacto (branch-and-bound algorithm) a la optimización de tres estructuras metálicas articuladas planas con un máximo de 10 barras. Los resultados muestran que: a) el algoritmo branchand-bound no es apropiado para resolver problemas estructurales de una cierta entidad, b) los resultados de SA, GA y TS son similares en términos de coste, empleando para ello TS menos tiempo seguido de SA y, finalmente, GA.

- Schmidt y Thierauf (2005) han combinado TA con un algoritmo evolutivo y lo han aplicado a la resolución de dos problemas extraídos de la bibliografía: la optimización de un pilar de acero y de una estructura articulada de 10 barras. El algoritmo trabaja con una población de soluciones que en cada iteración son mejoradas localmente mediante TA y posteriormente modificadas con los operadores del algoritmo evolutivo. Los resultados son comparados con los proporcionados por otros autores, mostrando el algoritmo propuesto un comportamiento robusto y una buena velocidad de cálculo.

\subsection{TÉCNICAS APROXIMADAS EMPLEADAS EN LA OPTIMIZACIÓN MULTIOBJETIVO DE ESTRUCTURAS.}

\subsubsection{Estrategias basadas en heurísticas que generalizan la búsqueda secuencial por entornos.}

Los éxitos de SA en la optimización monoobjetivo hizo que algunos investigadores empleasen esta técnica también para resolver problemas multiobjetivo. Para ello, definían una función objetivo que representaba un compromiso entre los diferentes objetivos buscados y la optimizaban posteriormente mediante SA. Esta estrategia ha sido empleada en estructuras de acero por los autores siguientes:

- Bennage y Dhingra (1995) emplean la Teoría de Juegos para definir una función objetivo que representa un compromiso entre la minimización del peso, de las deformaciones de ciertos nudos y la maximización de la frecuencia 
fundamental de vibración de celosías planas y espaciales. Las tres funciones objetivo son consideradas igualmente importantes y no se proporciona ninguna Frontera de Pareto, sino solamente una solución del problema.

- Shea y Cagan (1997) han optimizado cúpulas geodésicas (Fuller, 1954) considerando una función objetivo obtenida mediante una combinación lineal ponderada de los objetivos de mínimo peso, mínimo número de secciones distintas, mínima superficie de la cúpula, máximo volumen interior y máxima uniformidad visual. El método permite obtener tanto diseños similares a los tradicionales como otros innovadores.

- Venanzi y Materazzi (2006) han minimizado los desplazamientos y el espacio en planta ocupado por mástiles atirantados empleados como soportes de antenas telefónicas. Para ello, ambas funciones objetivo han sido combinadas linealmente para definir una única función que se optimiza mediante SA.

Las aproximaciones anteriormente descritas tienen el problema de que el algoritmo debe ejecutarse varias ocasiones para obtener en cada una de ellas un posible punto de la Frontera de Pareto. El deseo de salvar este obstáculo ha dado lugar a los algoritmos MOSA cuyos primeros esbozos se deben a Serafini (1985). Como aplicaciones de estos algoritmos cabe comentar las siguientes:

- Suppapitnarm et al. (2000) mejoran el algoritmo propuesto por Engrand a) intoduciendo una nueva función de probabilidad que incorpora una temperatura asociada a cada uno de los objetivos a optimizar y b) una estrategia de reinicio, de modo que cada cierto número de iteraciones, el algoritmo se reinicia empleando como solución inicial uno de los puntos de la Frontera de Pareto escogido de forma que se potencie la exploración del espacio de soluciones y la diversidad de puntos en la Frontera de Pareto. El algoritmo es aplicado con éxito a la optimización de a) una función matemática tipo, b) un panel rígido de un vehículo espacial, c) una estructura metálica articulada de diez barras en la que se buscaba minimizar su peso y sus deformaciones y maximizar su frecuencia fundamental de vibración. Este algoritmo se conoce como SMOSA.

- Suman (2004) ha aplicado los algoritmos propuestos por Czyak, Ulungu y Suppapitnarm junto con dos algoritmos por él crados a la optimización de cuatro problemas multiobjetivo referentes a a) el proceso de una refinería de petróleo, b) una función matemática con una Frontera de Pareto convexa, c) un sistema de mezclado de $\mathrm{N}$-etapas, d) un sistema de mezclado redundante y e) 
una función matemática. Llega a la conclusión de que todos ellos son capaces de resolver satisfactoriamente los problemas planteados y de proporcionar un amplio número de puntos que cumplan la condición de ser Óptimos de de Pareto.

\subsubsection{Estrategias basadas en los algoritmos evolutivos.}

\subsubsection{Estructuras de acero.}

- Hajela y Lin (1992) emplearon el Método Min-Max Ponderado para a) minimizar el peso de una celosía metálica plana de 10 barras sometida a cargas estáticas y el desplazamiento de uno de sus nudos y b) minimizar el peso de la estructura de un ala de un avión y maximizar la suma de sus dos primeras frecuencias naturales.

- Sandgren (1994) ha utilizado la Programación por Metas acoplada con algoritmos genéticos para optimizar celosías metálicas planas.

- Galante (1996) ha empleado la Combinación Lineal de Pesos para optimizar una celosía de acero plana de 10 barras y otra espacial de 160 , considerando como objetivos a minimizar a) el peso de la estructura y b) el número de perfiles diferentes de acero. Además introduce el operador "rebirth" consistente en la generación de una nueva población desde la que el algoritmo se reinicia a partir de su mejor individuo. Esta nueva población se crea de forma aleatoria, pero limitando el rango de variación de las variables, cuando el algoritmo genético converge.

- Coello y Christiansen (1999) han aplicado una variación del Método Min-Max Ponderado a la optimización de una viga metálica biapoyada con sección transversal en forma de I considerando como objetivos minimizar su peso y su deformación máxima.

- Liu et al. (1998) han aplicado la Combinación Lineal de Pesos para optimizar la forma y la posición de un actuador en una estructura metálica plana de 45 barras.

- Coello y Christiansen (2000) proponen una variante del Método Min-Max Ponderado para optimizar una celosía metálica plana de 200 barras y una espacial de 25 buscando minimizar a) su peso, b) sus desplazamientos y c) las tensiones. En su artículo comparan los resultados obtenidos con métodos 
matemáticos y con otros EMOO y concluyen que a) su técnica proporciona mejores soluciones de compromiso y b) que los GA pueden ser empleados como una adecuada herramienta de optimización en problemas multiobjetivo.

- Ruy et al. (2001) plantean una técnica basada en un algoritmo MOGA combinado con otro llamado Structural Genetic Algorithm for handling Design Alternatives (StrGA_DeAl) para la optimización multicriterio (peso y desplazamientos) de estructuras de barras de acero. El algoritmo permite estudiar la topología más adecuada para resolver un problema (diseño conceptual) y es aplicado a una estructura de tres barras y a otra de diez.

- Botello et al. (2004) han propuesto una modificación del algoritmo PAES y la han aplicado a la optimización de estructuras metálicas de barras planas y espaciales en las que se buscaba minimizar el peso de la estructura y el desplazamiento de sus nudos.

- Greiner et al. (2004) aplican tres estrategias de algoritmos evolutivos a la optimización de pórticos planos de acero. Realizan una optimización monoobjetivo (minimizar el peso de la estructura) y también una optimización multiobjetivo que tiene por objetivos minimizar a) el peso de la estructura y b) el número de perfiles. Emplean una variante del algoritmo NSGA en la que introducen un nuevo operador genético, el "auto-adaptative-rebirth" que mejora los resultados de la aplicación del algoritmo.

\subsubsection{Estructuras de hormigón.}

- Koumousis y Arsenis (1998) han realizado una optimización multiobjetivo de vigas de hormigón armado empleando la Combinación Lineal de Pesos. En su artículo parten de unas dimensiones de armado y de unas cuantías de armadura que suponen proporcionadas por una optimización previa y consideran una única función objetivo que es una suma ponderada de tres funciones objetivo relacionadas con los criterios siguientes: a) minimizar el peso de la armadura pasiva de las vigas, b) uniformizar al máximo los diámetros de las barras y c) minimizar el número de barras. Esta función es optimizada empleando un algoritmo genético y diferentes valores de los pesos de las funciones objetivo elementales. El algoritmo genético es aplicado a una viga de tres vanos y, aunque en su trabajo se menciona la posibilidad de obtener una Frontera de Pareto proporcionando diferentes ponderaciones a los pesos de las funciones que representan los diferentes objetivos, no se obtiene dicha 
Frontera. Por cada vano de la viga consideran tres variables y el diámetro de la armadura es el mismo en todas las secciones, variando únicamente el número de barras de unas secciones a otras, lo que resulta irreal y poco práctico desde el punto de vista económico. A modo de ejemplo, el número de variables consideradas en una viga de tres vanos es de 9 y el número de posibles soluciones es de $7^{9}$.

- Leps (2005) emplea una variante de un GA acoplada con un algoritmo SPGA para minimizar a) el coste y b) las flechas en vigas de hormigón armado. Su análisis es muy simplista pues: a) sólo estudia dos vigas isostáticas (una en voladizo y otra biapoyada), b) no considera la armadura de cortante, c) un único diámetro es adoptado como armadura inferior y superior de la viga y esa armadura es constante a lo largo de toda la longitud de la viga -solamente varía el número de redondos en sus caras superior e inferior-, d) las deformaciones son obtenidas mediante un método elástico sin tener en cuenta las singularidad que el cálculo de flechas tiene en las estructuras de hormigón armado y e) los rangos de variación de algunas variables son muy reducidos (por ejemplo, sólo considera tres posibles valores para el canto de las vigas y cuatro para su ancho).

\subsubsection{Otros trabajos.}

- Liu et al. (1997) han aplicado el MOGA a la rehabilitación de los tableros de una red de puentes a lo largo de un período de tiempo buscando minimizar a) la inversión en rehabilitación y b) el deterioro medio por $\mathrm{m}^{2}$ de los tableros.

- Park y Grierson (1999) han empleado el algoritmo MOGA para el diseño conceptual de edificios buscando: 1) minimizar un coste combinado que engloba el coste de la estructura resistente y el del solar y 2) maximizar la flexibilidad en la disposición del espacio.

- Savic et al. (1999) han utilizado un algoritmo genético con cuatro operadores (selección, cruzamiento, mutación y permutación) para la optimización mono y multiobjetivo del diseño de materiales compuestos con fibras.

- Liu y Frangopol (2005) emplean el NSGA para priorizar las actuaciones anuales en el mantenimiento de puentes de hormigón armado a lo largo de su vida útil teniendo como objetivos a) maximizar el buen estado de los puentes, b) 
maximizar su menor índice de seguridad y c) minimizar el coste de las actuaciones del mantenimiento.

- Kim y Roschke (2006) han aplicado el algoritmo NSGA-II al diseño de aisladores sísmicos de base buscando minimizar a) los desplazamientos horizontales de la base y b) las aceleraciones de la superestructura soportada por el aislador.

\subsubsection{Métodos híbridos.}

- Sarma y Adeli (2000b, 2002, 2005) han creado un método de optimización de estructuras de acero laminado basado en la lógica difusa (Fuzzy Logic) y los GA. Como objetivos consideran la minimización de a) el coste de la estructura, b) su peso, c) del número de secciones diferentes y d) del perímetro total de las secciones. Este último concepto se considera representativo del coste de mantenimiento de la estructura, pues el coste de su pintado es proporcional a este valor.

- Shelokar et al. (2004) han aplicado ACO, en el que han introducido los operadores genéticos de mutación y cruzamiento junto con criterios de selección procedentes del SPGA, a la optimización multiobjetivo de problemas de definición de procesos de ingeniería química.

\subsection{OTROS MÉTODOS.}

Gómez (1998) realiza el cálculo de la estructura de hormigón armado de nueve tipologías de edificios empleando diferentes calidades de hormigones y armaduras pasivas. A partir del análisis de los resultados extrae conclusiones sobre las cuantías de los materiales empleados y los costes asociados a cada una de las opciones posibles. Los principales condicionantes de su trabajo son:

- Plantea el estudio de edificios con un número mínimo de nueve plantas.

- Considera una única calidad de hormigón y acero en cada edificio.

- Cada tipología es analizada en base a criterios propuestos por otros investigadores (como por ejemplo Moragues, 1980) sin emplear ninguna técnica específica de optimización.

- Considera la influencia de la existencia de huecos, brochales y voladizos en la edificación. 
- Considera en el coste de la estructura el precio del espacio ocupado por los pilares.

- No incluye la optimización de los forjados en su análisis.

- Emplea como marco normativo la instrucción EH-91 (1991).

\subsection{RESUMEN.}

Para facilitar la comprensión de los estudios realizados hasta el momento más representativos por su relación con esta Tesis, se adjunta una síntesis de los mismos en la Tabla 2.1. En ella figuran:

- Autores de la publicación.

- Método empleado.

- $\quad$ Tipo de problema.

- Número de variables y dimensión del espacio de soluciones cuando ha sido posible conocer esos datos.

- Simplificaciones o aspectos no tratados que se han incorporado a esta investigación (ver página siguiente). 


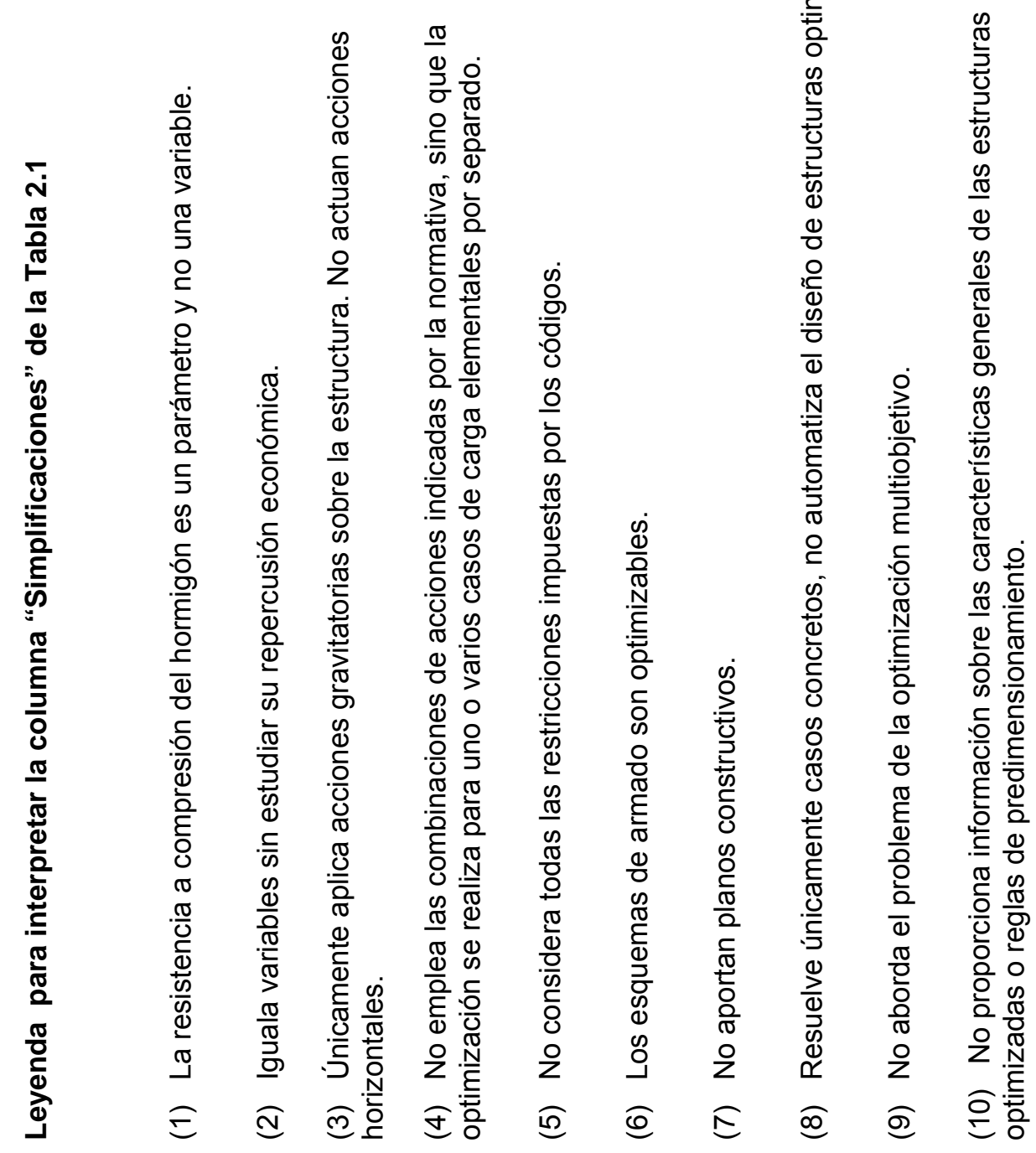


Tabla 2.1. Resumen de los trabajos de optimización de estructuras de hormigón armado más representativos por su relación con esta investigación.

\begin{tabular}{|c|c|c|c|c|c|}
\hline Autores & Método & Problema & $\begin{array}{l}\text { Dimensión } \\
\text { espacio de } \\
\text { soluciones }\end{array}$ & $\begin{array}{l}\text { Número de } \\
\text { variables }\end{array}$ & Simplificaciones \\
\hline Moragues (1980) & $\begin{array}{l}\text { Método directo de } \\
\text { Powell (exacto) }\end{array}$ & $\begin{array}{l}\text { Varias estructuras. Las más } \\
\text { complejas pórticos de edificación. }\end{array}$ & - & 7 & $(1),(2),(3),(5),(7),(8),(9)$ \\
\hline Coello (1997) & GA & Viga biapoyada & - & 3 & (1), (5), (6), (7), (8), (9), (10) \\
\hline Balling y Yao (1997) & $\begin{array}{l}\text { Exacto, multinivel y } \\
\text { exacto simplificado }\end{array}$ & $\begin{array}{l}\text { Pórticos de edificación, el mayor con } \\
\qquad N_{v}=2, N_{p}=2\end{array}$ & - & Máximo 20 & $(1),(2),(5),(7),(8),(9),(10)$ \\
\hline $\begin{array}{c}\text { Rajev y } \\
\text { Krishnamoorty } \\
(1998) \\
\end{array}$ & GA & $\begin{array}{l}\text { Pórticos de edificación }\left(N_{v}=3, N_{p}=4 ;\right. \\
\left.\qquad N_{v}=2, N_{p}=6\right)\end{array}$ & - & Máximo 29 & (1), (2), (4), (5), (7), (8), (9), (10) \\
\hline $\begin{array}{c}\text { Koumosis y Arsenis } \\
(1998)\end{array}$ & GA & Vigas continuas (aplican para $\mathrm{N}_{\mathrm{v}}=3$ ) & $7^{9}$ & 9 & (1), (5), (8), (10) \\
\hline $\begin{array}{l}\text { Leps y Sejnoha } \\
(2003)\end{array}$ & $S A+G A$ & Viga continua de dos vanos simétrica & - & 21 & $(1),(4),(5),(8),(9),(10)$ \\
\hline Lee y Ahn (2003) & GA & $\begin{array}{l}\text { Varias estructuras. La mayor un } \\
\text { pórtico con } \mathrm{N}_{\mathrm{v}}=2, \mathrm{~N}_{\mathrm{p}}=20\end{array}$ & $1.14 \times 10^{46}$ & 13 & (1), (2), (5), (6), (7), (8), (9), (10) \\
\hline Camp et al. (2003) & GA & $\begin{array}{l}\text { Varias estructuras. Entre ellas el } \\
\text { pórtico de Krishnamoorty }\left(\mathrm{N}_{\mathrm{V}}=2, \mathrm{~N}_{\mathrm{p}}=6\right)\end{array}$ & $1.26 \times 10^{21}$ & - & $(1),(2),(5),(6),(7),(8),(9),(10)$ \\
\hline $\begin{array}{l}\text { Sahab et al. (2004, } \\
\text { 2005) }\end{array}$ & $\begin{array}{c}\text { GA híbrido + } \\
\text { exploración exhaustiva }\end{array}$ & Edificios con forjados tipo losa & - & 36 & $(1),(3),(7),(8),(9),(10)$ \\
\hline $\begin{array}{c}\text { Govindaraj y } \\
\text { Ramasamy(2005) }\end{array}$ & $\begin{array}{l}\text { GA + exploración } \\
\text { exhaustiva }\end{array}$ & Vigas contínuas $\left(\mathrm{N}_{\mathrm{v}}=1,2\right)$ & - & $\mathrm{N}_{\mathrm{v}}+1$ & $(1),(4),(5),(7),(8),(9),(10)$ \\
\hline Leps (2005) & $\mathrm{GA}+\mathrm{SPGA}$ & Viga biapoyada y en voladizo & 132 & 3 & $(1),(2),(4),(5),(6),(7),(8),(10)$ \\
\hline
\end{tabular}




\subsection{CONCLUSIONES.}

El estado del arte realizado ha permitido constatar los aspectos siguientes refrentes a la optimización monoobjetivo:

- La mayoría de la investigación se centra en estructuras de acero, aunque existen cada vez más aplicaciones de los métodos heurísticos a elementos de hormigón. Ello es debido a que el análisis y diseño de estructuras de acero, tal y como está planteado en las publicaciones, es más sencillo que el de las estructuras de hormigón.

- Ninguno de los artículos sobre estructuras de acero incluye el proyecto de las uniones y rigidizadores o una estimación de su coste a pesar de su importancia. En lo que respecta a estructuras de hormigón, pocos de los trabajos publicados constituyen diseños suficientemente detallados como para ser construidos.

- En general, los estudios previos realizados con pórticos de hormigón armado:

a) Emplean numerosas simplificaciones relativas a los estados límites y esquemas de armado.

b) No realizan un cálculo de las deformaciones en vigas teniendo en cuenta las singularidades del hormigón armado, sino que exigen unas esbelteces mínimas de las jácenas.

c) No consideran la posibilidad del empleo de vigas planas (común en España).

d) No acotan los incrementos del coste debidos a agrupar miembros para reducir el número de variables del problema

e) No contemplan diferentes calidades del hormigón dentro de la estructura. Es más, en todos ellos la resistencia del hormigón es considerada como un parámetro y no como una variable.

f) No analizan las características de las estructuras óptimas ni automatizan el procedimiento ed su obtención.

Excepciones a algunos de los puntos anteriores son las investigaciones de Moragues (1980), Gómez (1998) y Sahab et al. (2004, 2005). 
- A excepción de los trabajos realizados por el GPRC, las investigaciones con estructuras de hormigón armado trabajan según uno de los dos modos siguientes:

a) Con catálogos de secciones en los que unas dimensiones de la sección transversal llevan asociados unos armados.

b) Siguiendo el orden convencional de diseño según el cual, definida una geometría, se dimensionan las armaduras longitudinales para el ELU de flexión para seguidamente comprobar flechas y fisuración y finalmente obtener las armaduras de cortante sin alterar la armaduras de flexión.

Esta forma de proceder es efectiva al reducir la dimensión del espacio de soluciones, pero obvia posibilidades que el modelo de optimización aquí planteado tiene en cuenta como, por ejemplo, eliminar armadura de cortante mediante incrementos de la armadura de flexión o del canto.

- Los trabajos previos realizados muestran la capacidad de los procedimientos heurísticos para resolver satisfactoriamente complejos problemas de optimización combinatoria para diferentes materiales y configuraciones estructurales.

- Existen en la literatura (Shea y Zaho, 2004; Perea et al, 2006c) ejemplos de estructuras construidas diseñadas mediante técnicas heurísticas.

La revisión bibliográfica referente al problema multiobjetivo pone de manifiesto que:

- La investigación realizada en optimización multicriterio en el ámbito estructural es escasa, centrada en estructuras de acero y emplea mayoritariamente métodos basados en los algoritmos evolutivos.

- En el caso de estructuras de hormigón armado los trabajos realizados son simplistas porque:

a) Los modelos estructurales estudiados (vigas "in situ" isostáticas o contínuas) no reflejan la práctica habitual de las estructuras de la edificación.

b) En el caso de Leps (2005), alguno de los métodos de cálculo empleados son manifiestamente mejorables.

c) En el caso de Koumousis y Arsenis (1998) presentan una técnica multiobjetivo, pero los resultados se centran en el algoritmo genético empleado para minimizar una única función objetivo y no en el análisis 
multicriterio. De hecho, su trabajo no contiene ninguna gráfica con Fronteras de Pareto.

Por todo ello, se plantea en esta Tesis la obtención de un procedimiento que permita el diseño automatizado de pórticos optimizados económicamente de acuerdo con las restricciones y esquemas de armado habituales. Además, se proporcionarán las características de las estructuras optimizadas, reglas para su predimensionado y se abordará el problema multicriterio.

Señalar finalmente que la dimensión del problema que se desarrolla en esta investigación es mayor que la de trabajos anteriores similares. Como se mostrará en el epígrafe 6.1, el número máximo de variables y de posibles soluciones de los problemas aquí tratados es de 153 y $10^{232}$ respectivamente. Los datos recopilados en la Tabla 2.1 muestran que otros estudios con estructuras de edificación habían trabajado con un máximo de 36 variables y un espacio de soluciones con $1.14 \times 10^{46}$ elementos. 


\section{CAPÍTULO 3. DEFINICIÓN DEL PROBLEMA DE OPTIMIZACIÓN.}




\section{Objetivos.}

En este capítulo se realiza una definición completa del problema de optimización de un pórtico plano de hormigón armado. Para ello:

- Se definen sus parámetros, variables y restricciones.

- Se formulan las funciones objetivo a optimizar.

El planteamiento se realiza tanto para el problema monoobjetivo como para el multiobjetivo. 


\subsection{INTRODUCCIÓN.}

Un pórtico es un sistema estructural formado por elementos horizontales (vigas) y verticales (pilares), capaz de transmitir de forma segura a su cimentación las cargas debidas a las acciones verticales ( peso de los elementos estructurales y constructivos y sobrecargas de uso) y horizontales (viento y sismo) que actúan sobre él, manteniendo durante su vida útil unas condiciones adecuadas de servicio. Si todas las directrices de los dinteles y pilares que definen el pórtico, así como las cargas que actúan sobre él, están contenidas en un plano, entonces recibe nos encontramos ante un pórtico plano.

Como se explicó en el capítulo 1, el proceso de optimización de un sistema estructural de estas características requiere la adopción de unos parámetros, unas variables de diseño, unas restricciones y una o varias funciones objetivo. Estos aspectos se abordan de modo general en los epígrafes 3.2 a 3.5, dejándose la definición de los problemas concretos investigados en este trabajo para el apartado 3.6.

\subsection{PARÁMETROS.}

\subsubsection{Introducción.}

Los parámetros definitorios de un pórtico pueden ser relativos a su geometría, a las acciones que actúan sobre él, a sus coeficientes de seguridad, a su grado de exposición ambiental o a otras de sus características. Se describen a continuación.

\subsubsection{Parámetros geométricos.}

Estos parámetros se indican en la Tabla 3.1 y en la Figura 3.1 junto con los valores concretos adoptados en este estudio. 
SECCIÓN A-A. ALZADO
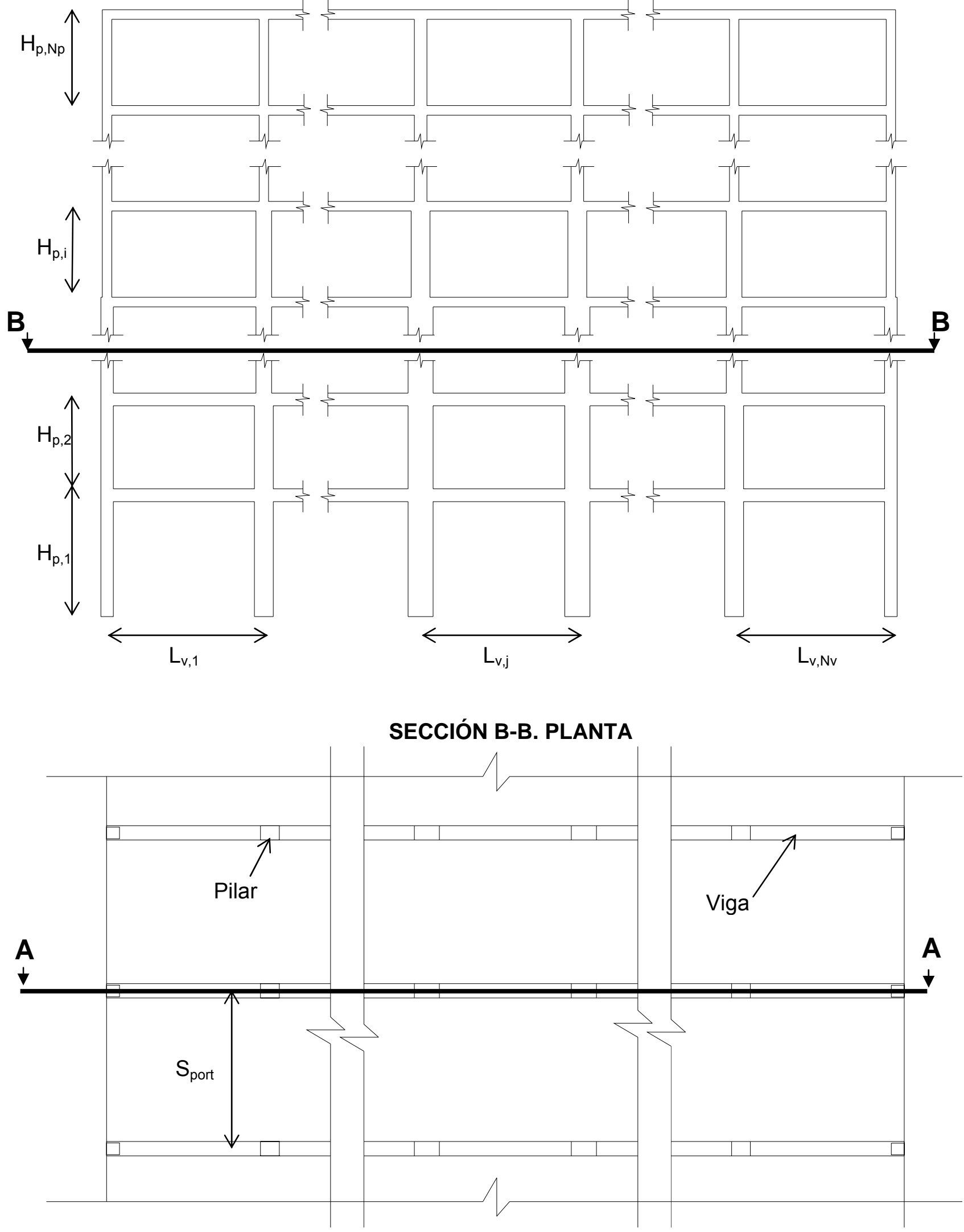

Figura 3.1. Definición de los parámetros geométricos en una sección (figura superior) y en una planta (figura inferior) de una estructura con pórticos y forjados unidireccionales. Las distancias $H_{p, i}$ se miden entre caras superiores de forjados y las $L_{v, j}$ entre ejes de pilares. 
Tabla 3.1. Parámetros geométricos.

\begin{tabular}{lcc}
\hline Descripción & Parámetro & $\begin{array}{c}\text { Valor en este } \\
\text { estudio }\end{array}$ \\
\hline Número de vanos & $\mathrm{N}_{\mathrm{v}}$ & 2 \\
\hline Número de plantas & $\mathrm{N}_{\mathrm{p}}$ & $2,4,6,8$ \\
\hline Altura de cada planta $(\mathrm{m})$ & $\mathrm{H}_{\mathrm{p}, \mathrm{i}}$ & 3 \\
\hline Luz de cada vano $(\mathrm{m})$ & $\mathrm{L}_{\mathrm{v}, \mathrm{i}}$ & 5 \\
\hline Separación entre pórticos $(\mathrm{m})$ & $\mathrm{S}_{\mathrm{port}}$ & 5 \\
\hline Tipo de vigas en dinteles & $\mathrm{T}_{\text {viga }}$ & $\begin{array}{c}\text { Descolgadas, } \\
\text { planas }{ }^{1}\end{array}$ \\
\hline Canto de forjados $(\mathrm{m})$ & $\mathrm{h}_{\text {for }}$ & 0.29 \\
\hline${ }^{1}$ Las vigas planas son aquellas cuyo canto es igual al del forjado que se apoya sobre ellas. \\
\hline
\end{tabular}

\subsubsection{Parámetros relativos a las acciones.}

Se llama acción a cualquier deformación impuesta, carga o conjunto de ellas capaz de producir estados tensionales en una estructura. Ejemplos de acciones a considerar en el cálculo de la estructura de un edificio son el peso de sus forjados, de sus tabiquerías o de las personas que lo habitan.

Las acciones dependen del tipo de estructura, de su uso y de su localización, viniendo definidas por las correspondientes normativas. En el caso de la edificación en España están recogidas en el CTE que las clasifica en:

1) Acciones permanentes.

- Peso propio (peso propio de la estructura, de los elementos embebidos, accesorios y del equipamiento fijo).

- $\quad$ Pretensado.

- $\quad$ Acciones del terreno.

2) Acciones variables. 
- Sobrecargas de uso.

- Acciones sobre las barandillas y elementos divisorios.

- Viento.

- Acciones térmicas.

- Nieve.

3) Acciones accidentales.

- Acciones Sismicas.

- Incendio.

- Impacto.

En esta investigación se han adoptado los parámetros relativos a las acciones que se indican en la Tabla 3.2.

Tabla 3.2. Parámetros relativos a las acciones.

Descripción

Cargas permanentes en forjados por planta $\left(\mathrm{KN} / \mathrm{m}^{2}\right)$

Sobrecargas de uso en forjados por planta $\left(\mathrm{KN} / \mathrm{m}^{2}\right)$

Altitud topográfica del emplazamiento del edificio $(m)$

Zona eólica del emplazamiento del edificio

Situación topográfica del edificio

Porcentaje que suponen el peso de lo elementos no estructurales sobre las cargas permanentes totales (\%)

Edad de la estructura cuando se descimbra (días)

Edad de la estructura cuando se aplican las cargas muertas y la sobrecarga cuasi-permanente (días)

Edad de la estructura para el cálculo de las flechas (días)
Valor en este estudio

Planta tipo: $4 \mathrm{KN} / \mathrm{m}^{2}$

Cubierta: $6 \mathrm{KN} / \mathrm{m}^{2}$

Planta tipo: $3 \mathrm{KN} / \mathrm{m}^{2}$

Cubierta: $1 \mathrm{KN} / \mathrm{m}^{2}$

$0 \mathrm{~m}$.

$\mathrm{H}_{\text {top }} \quad 0 \mathrm{~m}$.

$Z_{e}$

X

$\mathrm{S}_{\text {top }}$

Normal

$P_{\text {nest }}$

Planta tipo: $\quad 25 \%$

Cubierta: $\quad 50 \%$

$t_{\text {desc }} \quad 14$ días

$\mathrm{t}_{\mathrm{cm}} \quad 28$ días

$t_{f}$

8.000 días 
Los datos de la tabla anterior permiten definir las acciones actuantes sobre un pórtico plano teniendo en cuenta que:

1) Se analizan únicamente los pórticos interiores del edificio y no los pórticos extremos colindantes con una calle o con otro edificio. Por este motivo, las cargas totales actuando sobre los dinteles de los pórticos debidas a las acciones gravitatorias en forjados se obtienen multiplicando la separación entre pórticos por el valor de la acción gravitatoria por unidad de superficie de forjado.

2) En cada vano del pórtico, la sobrecarga de uso actúa en toda su área tributaria o no está presente, dando lugar a tres hipótesis de carga diferentes: sobrecarga en vanos alternos (dos posiciones) y sobrecarga en todos los vanos (véase la Figura $3.2)$.

3) A efectos de las cargas de viento se supone que la planta baja del edificio se sitúa a cota 0 sobre el nivel del mar.

4) La carga del viento actúa a nivel de los forjados de cada planta y su valor se obtiene empleando el método explicado en la norma "NTE ECV-88. Viento" (1988). Se ha tomado este valor y no el procedente del CTE debido a que en el momento de aprobarse esta norma una gran parte de esta investigación ya estaba realizada.

5) Existen juntas de dilatación suficientemente próximas como para que se puedan despreciar los efectos de las acciones térmicas y reológicas.

6) Se supone que los asientos diferenciales en la cimentación del edificio son despreciables.

7) El edificio se encuentra en zona no sísmica y, por lo tanto, no es necesario considerar los efectos de un terremoto sobre el mismo. 
Cargas permanentes

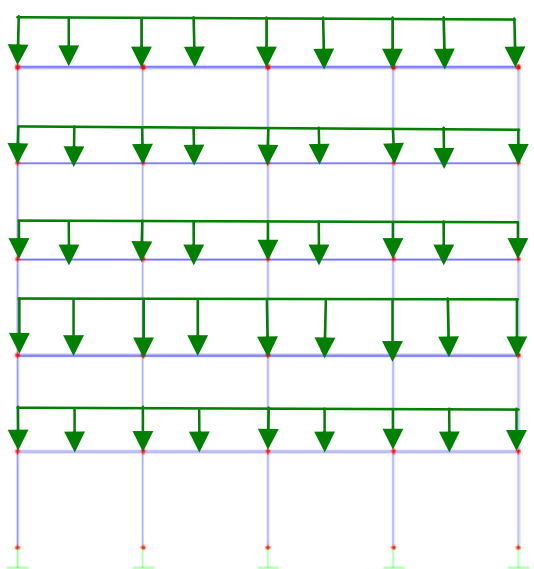

Sobrecarga de uso en vanos alternos (posición 2)

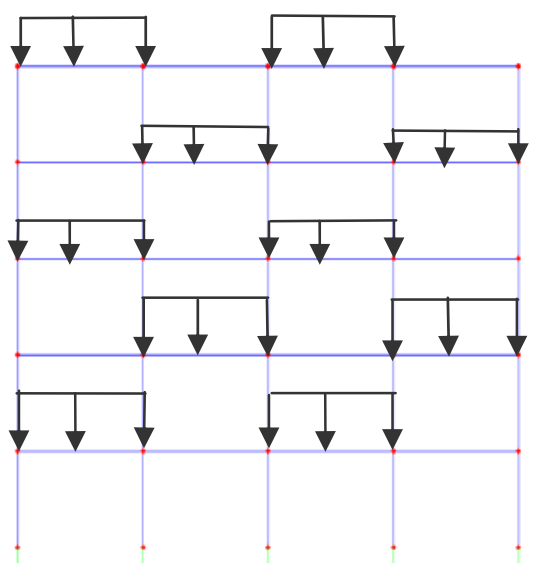

Viento izquierda - derecha

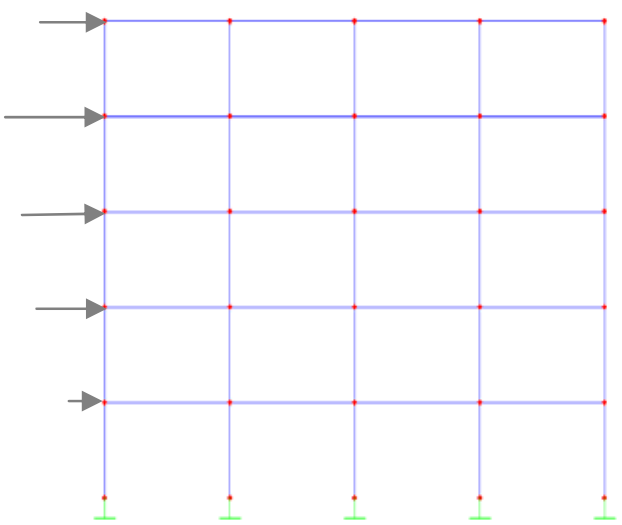

Sobrecarga de uso en vanos alternos (posición 1)

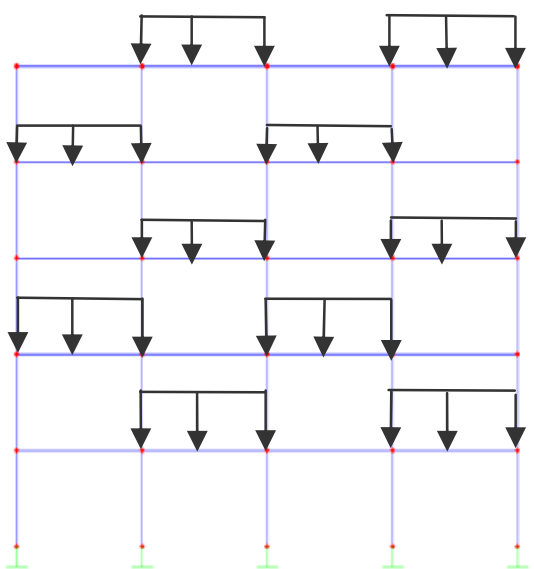

Sobrecarga de uso en todos los vanos

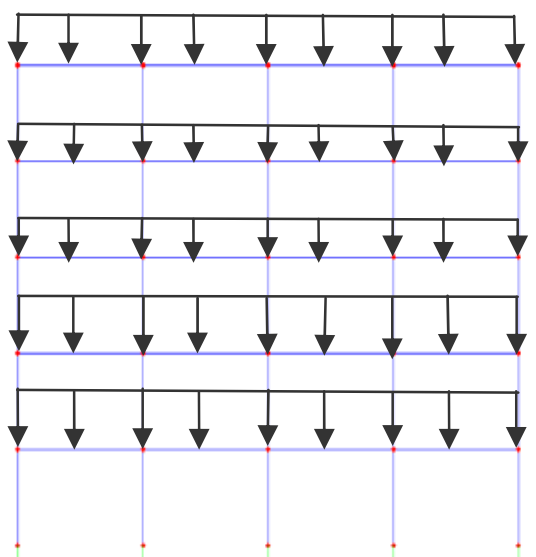

Viento derecha - izquierda

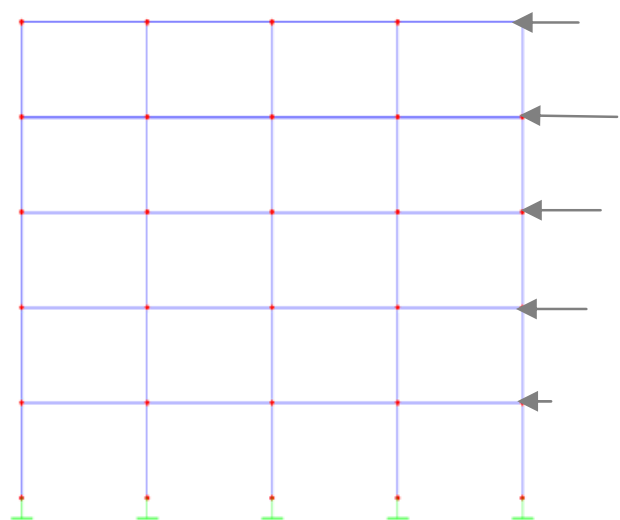

Figura 3.2. Acciones sobre un pórtico plano. Casos de carga considerados.

Se señala que dada la altura máxima de los edificios situados y su número de plantas no se ha considerado la acción de la nieve. Para tenerla en cuenta, y quedando del lado de la seguridad, podría añadirse el valor de esta acción al de la sobrecarga de uso. 
En cualquier caso, las hipótesis asumidas no afectan a la generalidad de la metodología planteada en esta investigación.

\subsubsection{Parámetros relativos a los coeficientes de seguridad y al grado de exposición de la estructura.}

El cálculo y la comprobación de la estructura de hormigón armado de un pórtico plano se realiza de acuerdo con el "Método de los Estados Límite", tal y como prescribe la Instrucción de Hormigón Estructural (EHE) en su Título $1^{\circ}$.

La aplicación de éste método requiere del empleo de coeficientes de minoración de resistencias de los materiales $\left(\gamma_{c}, \gamma_{s}\right)$ y de mayoración de acciones $\left(\gamma_{f}\right)$. La Tabla 3.3 indica los parámetros relativos a estos coeficientes. Los valores de $\gamma_{f}$ no se proporcionan directamente porque dependen del nivel de control de ejecución de la obra adoptado. En la tabla figura también el "Tipo de ambiente". Este dato es necesario para verificar el Estado Límite de Servicio de Fisuración.

Tabla 3.3. Parámetros relativos a los coeficientes de seguridad de las resistencias de los materiales y al grado de exposición de la estructura.

\begin{tabular}{lcc}
\hline \multicolumn{1}{c}{ Descripción } & Parámetro & $\begin{array}{c}\text { Valor en este } \\
\text { estudio }\end{array}$ \\
\hline Coeficiente de minoración de resistencias (hormigón) & $\gamma_{\mathrm{c}}$ & 1.5 \\
\hline Coeficiente de minoración de resistencias (acero) & $\gamma_{\mathrm{s}}$ & 1.15 \\
\hline Nivel de Control de la Ejecución & $\mathrm{NCE}$ & Normal \\
\hline Tipo de ambiente & $\mathrm{A}$ & $\mathrm{I}$ \\
\hline Recubrimiento nominal de las armaduras $(\mathrm{cm})$ & $\mathrm{r}_{\mathrm{nom}}$ & $30 \mathrm{~mm}^{2}$ \\
\hline${ }^{2}$ En el caso de que la resistencia a compresión del hormigón de un elemento sea igual o superior a $40 \mathrm{MPa}$, \\
$\mathrm{r}_{\text {nom se ha reducido en } 5 \mathrm{~mm} \text {. (Tabla } 37.2 .4 \text { de la EHE). }}$ &
\end{tabular}

Se ha considerado un ambiente tipo I por estar la mayoría de los elementos situados en el interior del edificio y por considerar que aquellos elementos estructurales que podrían tener un contacto con el exterior (cara superior de las vigas de cubierta y cara exterior de los 
pilares de fachada) se encuentran suficientemente protegidos de la intemperie (por el material de la cubierta y por los cerramientos respectivamente).

\subsubsection{Otros parámetros.}

Finalmente, la Tabla 3.4 recoge el resto de parámetros necesarios para optimizar un pórtico plano de hormigón armado.

Tabla 3.4. Otros parámetros.

\begin{tabular}{lcc}
\hline \multicolumn{1}{c}{ Descripción } & Parámetro & $\begin{array}{c}\text { Valor en este } \\
\text { estudio }\end{array}$ \\
\hline Tamaño máximo del árido & Tma & $2.0 \mathrm{~cm}$ \\
\hline $\begin{array}{l}\text { Distancia máxima entre la cara superior del forjado de } \\
\text { una planta y la cara inferior de las vigas de la planta } \\
\text { superior }(\mathrm{m})\end{array}$ & Gmax & $2.45 \mathrm{~m}$ \\
\hline
\end{tabular}

\subsection{VARIABLES DE DISEÑO.}

Previamente a la definición de las variables es necesario hacer las siguientes puntualizaciones:

- Por motivos de facilidad constructiva:

a) Se ha considerado que la calidad del hormigón es la misma para todos los pilares de una planta y para todas las vigas que se apoyan en esos pilares.

b) En el caso de las vigas, se ha adoptado un único ancho y canto para todas las vigas de una misma planta.

- Si las vigas son planas, la única variable geométrica por planta referente a ellas es su ancho. Su canto pasa a ser un parámetro más que tendrá el mismo valor que el canto del forjado.

- En el armado de las vigas, se ha considerado una armadura base inferior y superior que recorre toda la longitud de las vigas de una misma planta. Adicionalmente se ha dispuesto en cada viga una armadura de refuerzo inferior (refuerzo de positivos) con una longitud de 0,8 veces su luz $(L)$ y una armadura de refuerzo superior (refuerzo de negativos) en cada uno de sus extremos con una longitud de $0,20 x L$ (véase la Figura 3.3). Estos valores son similares a los 
indicados por la EHE para la armadura a disponer en la banda de soportes de las placas sobre apoyos aislados (Art. 56.2 EHE).

L

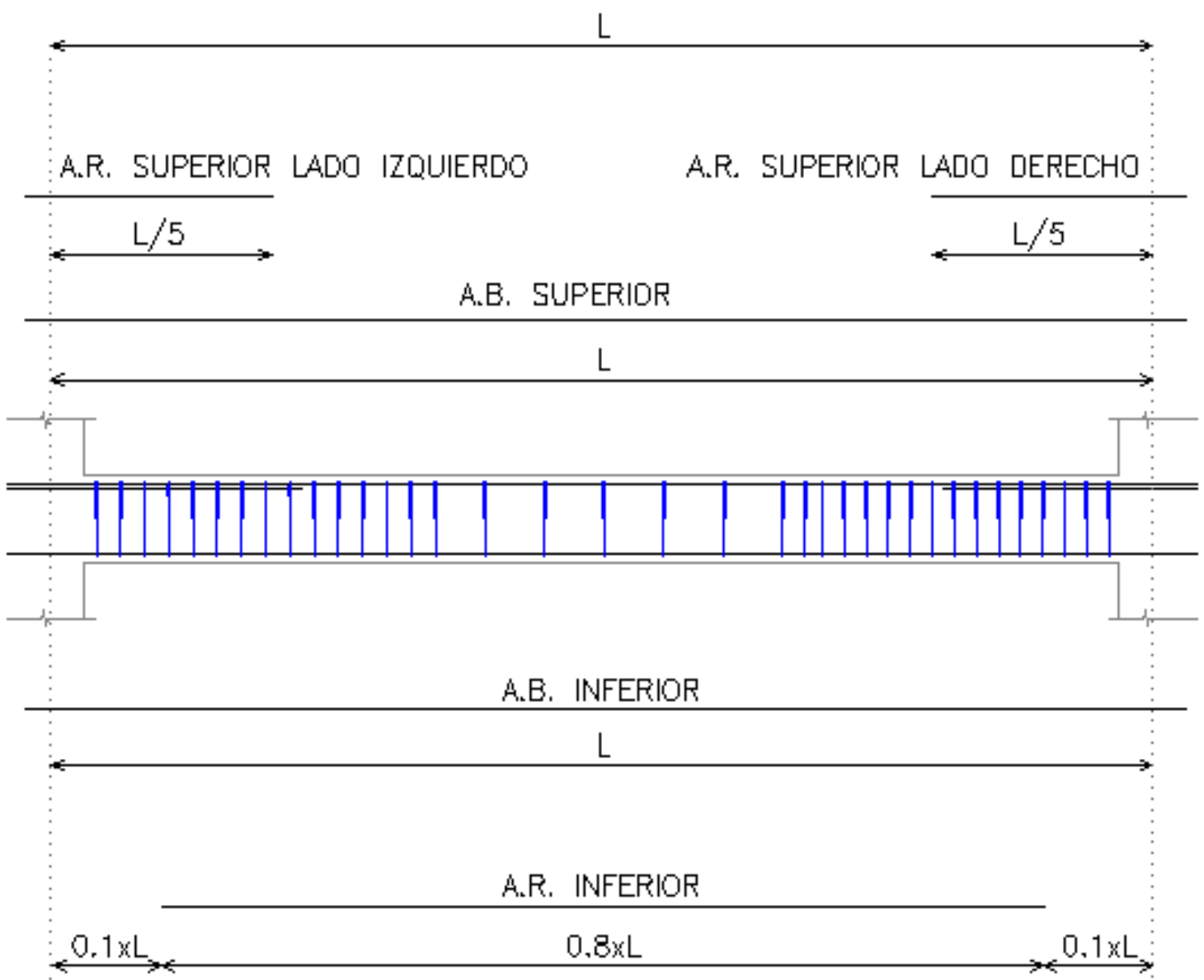

Figura 3.3. Armaduras longitudinales en vigas.

- Para definir la armadura de cortante de vigas, se han considerado divididas en tres zonas de igual longitud, en cada una de las cuales la armadura de cortante es la misma, pero pudiendo variar de una zona a otra dentro de cada viga (véase la Figura 3.4).

$\mathrm{L}$

\section{A.C. ZONA IZQUIERDA A.C. ZONA CENTRAL A.C. ZONA DERECHA}

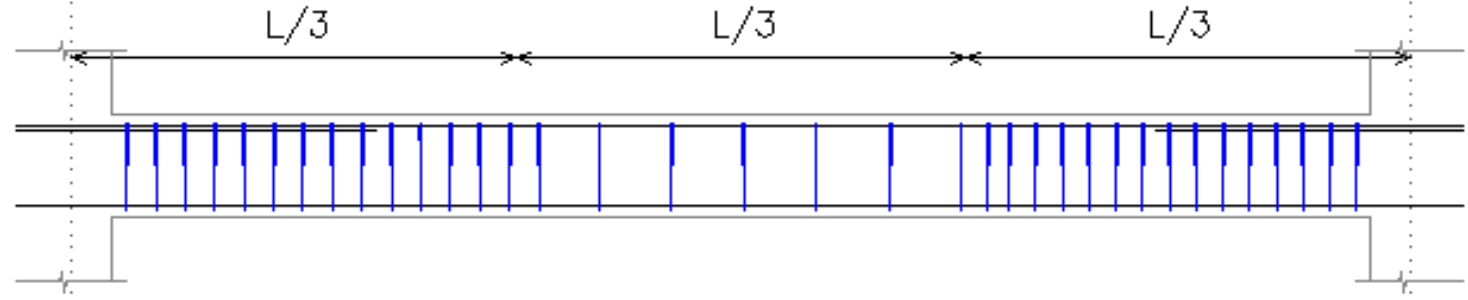

Figura 3.4. Armadura de cortante en vigas. 
- Reflejando la práctica constructiva habitual, los pilares se han armado con una armadura longitudinal y un estribado de cortante constantes en toda su altura.

Además, la Tabla 3.5 muestra:

- En la columna tercera, el número de valores diferentes que puede adoptar cada una de las variables (los valores concretos se proporcionan el Apéndice 1).

- En las columnas cuarta a sexta, el número de variables necesarias para definir un pórtico plano tipo de $N_{V}$ vanos y $N_{P}$ plantas, tanto si éste es simétrico como si no lo es.

El número total de variables (NumVar) para pórticos como el de la Figura 1.12 se obtiene directamente a partir de la tabla anteriormente citada y viene dado por las expresiones siguientes:

- Pórtico simétrico con vigas descolgadas, $N_{V}$ par.

NumVar $=1+10 N_{P}+4.5 N_{V} N_{P}$

- Pórtico simétrico con vigas planas, $N_{V}$ par.

NumVar $=1+9 N_{P}+4.5 N_{V} N_{P}$

- Pórtico simétrico con vigas descolgadas, $N_{V}$ impar.

NumVar $=1+8.5 N_{P}+4.5 N_{P} N_{V}$

- Pórtico simétrico con vigas planas, $N_{V}$ impar.

NumVar $=1+7.5 N_{P}+4.5 N_{P} N_{V}$

- Pórtico no simétrico con vigas descolgadas.

NumVar $=1+10 N_{P}+9 N_{V} N_{P}$

- Pórtico no simétrico con vigas planas.

NumVar $=1+9 N_{P}+9 N_{V} N_{P}$ 


\begin{tabular}{|c|c|c|c|c|c|}
\hline \multirow{2}{*}{ Descripción } & \multirow{2}{*}{ Variable } & \multirow{2}{*}{$\begin{array}{l}\text { Número de } \\
\text { valores }\end{array}$} & \multicolumn{2}{|c|}{ Estructura simétrica } & \multirow{2}{*}{$\begin{array}{l}\text { Estructura no } \\
\text { simétrica }\end{array}$} \\
\hline & & & Nv par & Nv impar & \\
\hline Límite elástico del acero en vigas y pilares $\left(\mathrm{N} / \mathrm{mm}^{2}\right)$ & $f_{y k}$ & 2 & 1 & 1 & 1 \\
\hline Resistencia característica del hormigón $\left(\mathrm{N} / \mathrm{mm}^{2}\right)$ & $f_{c k, v i g, i}$ & 6 & $\mathrm{~N}_{\mathrm{P}}$ & $\mathrm{N}_{\mathrm{P}}$ & $\mathrm{N}_{\mathrm{P}}$ \\
\hline Ancho de vigas $(\mathrm{m})$ & $b_{\text {vig, }, i}$ & 106 & $\mathrm{~N}_{\mathrm{P}}$ & $\mathrm{N}_{\mathrm{p}}$ & $\mathrm{N}_{\mathrm{p}}$ \\
\hline Canto de vigas (sólo si las vigas son descolgadas) (m) & $h_{\text {vig, }, i}$ & 20 & $\mathrm{~N}_{P}$ & $\mathrm{~N}_{\mathrm{P}}$ & $\mathrm{N}_{\mathrm{P}}$ \\
\hline Ancho de pilares (m) & $b_{\text {pil,i }}$ & 20 & $\left(\mathrm{~N}_{\mathrm{P}} \mathrm{XN} \mathrm{N}_{\mathrm{V}}\right) / 2+\mathrm{N}_{\mathrm{P}}$ & $N_{P} x\left(N_{V}+1\right) / 2$ & $N_{P} x\left(N_{V}+1\right)$ \\
\hline Canto de pilares (m) & $h_{\text {pil,i }}$ & 25 & $\left(\mathrm{~N}_{\mathrm{P}} \mathrm{XN} \mathrm{N}_{\mathrm{V}}\right) / 2+\mathrm{N}_{\mathrm{P}}$ & $\mathrm{N}_{\mathrm{P}} \mathrm{x}\left(\mathrm{N}_{\mathrm{V}}+1\right) / 2$ & $N_{P} x\left(N_{V}+1\right)$ \\
\hline Armadura base inferior en vigas $\left(\mathrm{cm}^{2}\right)$ & $A_{s, \text { base inf,vig, } i}$ & 23 & $\mathrm{~N}_{\mathrm{P}}$ & $\mathrm{N}_{\mathrm{P}}$ & $N_{p}$ \\
\hline Armadura de refuerzo inferior en vigas $\left(\mathrm{cm}^{2}\right)$ & $A_{s, \text { ref inf,vig,i }}$ & 25 & $\mathrm{~N}_{\mathrm{P}} \mathrm{xN} / 2$ & $N_{P} x\left(N_{V}+1\right) / 2$ & $\mathrm{~N}_{p} \times \mathrm{N}_{V}$ \\
\hline Armadura base superior en vigas $\left(\mathrm{cm}^{2}\right)$ & $A_{s, \text { base sup,vig, } i}$ & 23 & $N_{P}$ & $\mathrm{~N}_{\mathrm{P}}$ & $\mathrm{N}_{\mathrm{P}}$ \\
\hline Armadura de refuerzo superior en vigas $\left(\mathrm{cm}^{2}\right)$ & $A_{s, \text { ref sup,vig,i }}$ & 28 & $N_{P} x\left(N_{V} / 2+1\right)$ & $\mathrm{N}_{\mathrm{P}} \mathrm{X}\left(\mathrm{N}_{\mathrm{V}}+1\right) / 2$ & $N_{P} x\left(N_{V}+1\right)$ \\
\hline Armadura transversal en vigas & $A_{\text {st, cor, vig, }, i}$ & 330 & $3 \times N_{p} \times N_{V} / 2$ & $N_{P} x\left(3 x N_{V}+1\right) / 2$ & $3 \times N_{P} \times N_{V}$ \\
\hline Armadura longitudinal en pilares $\left(\mathrm{cm}^{2}\right)$ & $A_{s, l o n, p i l, i}$ & 21 & $\left(\mathrm{~N}_{\mathrm{P}} \mathrm{xN} \mathrm{N}_{\mathrm{V}}\right) / 2+\mathrm{N}_{\mathrm{P}}$ & $N_{P} x\left(N_{V}+1\right) / 2$ & $N_{P} X\left(N_{V}+1\right)$ \\
\hline Armadura transversal en pilares $\left(\mathrm{cm}^{2}\right)$ & $A_{s t, c o r, p i l, i}$ & 2 & $\left(\mathrm{~N}_{\mathrm{P}} \mathrm{xN} \mathrm{N}_{\mathrm{V}}\right) / 2+\mathrm{N}_{\mathrm{P}}$ & $\mathrm{N}_{\mathrm{P}} \mathrm{x}\left(\mathrm{N}_{\mathrm{V}}+1\right) / 2$ & $N_{P} x\left(N_{V}+1\right)$ \\
\hline
\end{tabular}




\subsection{RESTRICCIONES DE COMPORTAMIENTO Y DE DISEÑO.}

\subsubsection{Introducción.}

El cumplimiento de las restricciones permite asegurar que un pórtico plano solución del problema de optimización es construible porque tiene sentido físico y cumple las exigencias de seguridad estructural.

De acuerdo con el CTE la seguridad estructural de una estructura de hormigón se debe comprobar siguiendo la EHE, la cual emplea el "Método de los Estados Límites". Por ello, se explican a continuación los principios de esta metodología y su aplicación a los pórticos planos estudiados en este trabajo (apartados 3.4 .2 a 3.4.4). A continuación se indican otras restricciones necesarias pero no directamente contempladas en esta norma (epígrafe 3.4.5).

\subsubsection{Principios básicos del "Método de los Estados Límite".}

Esta técnica permite tener en cuenta de forma sencilla el carácter aleatorio de las solicitaciones, de las resistencias de los materiales y de las dimensiones de las piezas mediante el empleo de coeficientes de seguridad. Para ello, el proyecto de una estructura sigue los pasos siguientes:

1) Se definen las acciones que actúan sobre la estructura.

2) Se definen los Estados Límites que debe cumplir la estructura. Estos estados límites son situaciones cuya superación trae consigo que la estructura no cumpla alguna de las funciones para las que ha sido proyectada y pueden ser Estados Límites de Servicio (E.L.S.) o Últimos (E.L.U.).

3) Se comprueba cada uno de los Estados Límites, lo que conlleva:

a) Deducir por un lado los efectos de las acciones aplicadas a la estructura.

b) Deducir por otro lado la respuesta de la estructura para la situación límite en estudio. 
c) Verificar el cumplimiento del Estado Límite, lo que sucede si queda garantizado, con una fiabilidad aceptable, que la respuesta estructural no es inferior al efecto de las acciones aplicadas.

d) Definida una armadura, comprobar que cumple las disposiciones constructivas indicadas por la EHE.

\subsubsection{Acciones consideradas en el cálculo. Combinación de acciones.}

Las acciones consideradas para calcular la estructura son las indicadas en el punto 3.2.3 "Parámetros relativos a acciones". Estas acciones se combinan siguiendo las indicaciones de los artículos 12 y 13 de la EHE para obtener los valores de las acciones correspondientes a las combinaciones ELS y ELU.

En total se han considerado 48 diferentes combinaciones de los casos de carga elementales. Se indican en el Apéndice 3.

Señalamos que no se incluye en el modelo de optimización el proyecto del proceso de cimbrado-descimbrado. Su inclusión en el estudio se puede realizar siguiendo la metodología descrita por Calavera (2002).

\subsubsection{Verificación de Estados Límites.}

La determinación de los efectos de las acciones se realiza mediante un análisis estructural elástico y lineal. Este se basa en el comportamiento elástico-lineal de los materiales y en la consideración del equilibrio de la estructura en su geometría sin deformar. Este tipo de análisis, empleando la sección bruta de las piezas de hormigón para la obtención de las solicitaciones, está avalado por el Artículo 19.2 de la EHE.

Señalamos aquí que en la bibliografía (Calavera, 1999; Jiménez Montoya et al., 2000) se encuentra abundante documentación sobre cómo proceder a la verificación de estos estados límites, por lo que en este trabajo nos limitaremos a enumerar los estados límites comprobados realizando algunas aclaraciones sobre las hipótesis adoptadas. 


\subsubsection{ELS de fisuración en vigas.}

La verificación de este estado límite se realiza siguiendo el Artículo 49 de la EHE. En cada viga se verifican las tres secciones siguientes:

- Sección de centro luz (considerando como armadura de tracción la armadura base y de refuerzo inferior de la viga y como armadura de compresión la armadura base superior de la viga).

- Secciones situadas en los extremos de la vigas (empotramiento con los pilares). En estos casos la armadura de tracción es la armadura base y de refuerzo superior en cada uno de los extremos de la viga y la armadura de compresión es su armadura base inferior.

\subsubsection{ELS de deformación en vigas.}

Se verifica este estado límite de acuerdo con el método simplificado de la EHE (Art. 50.2.2), efectuándose siempre el cálculo independientemente de las cuantías de armadura y cantos de las vigas.

Se comprueban las flechas activas y las flechas totales a tiempo infinito. La flecha activa respecto a un elemento dañable es la producida en el elemento estructural en el que se apoya el elemento dañable a partir del momento en que se construye dicho elemento. La flecha total a tiempo infinito de un elemento estructural es la que experimenta debido a la totalidad de las cargas actuantes. La comprobación realizada sigue la metodología indicada por Corres et al. (1998) y precisa de la definición de un historial de cargas. Este historial se representa mediante las tres cargas siguientes (véase también la Figura 3.5) :

- $\quad q_{1}$ que indica las cargas permanentes que actúan en el momento de descimbrar $\left(t_{\text {desc }}\right)$. Representa, por tanto, el peso propio de los elementos estructurales y de una fracción de la carga permanente debida a los elementos no estructurales.

- $\quad q_{2}$ que indica la carga permanente y la parte de la sobrecarga que se considera actuando permanentemente - sobrecarga cuasi-permanente-. Se considera que a la edad $t_{c m}$ actúa la totalidad de la carga permanente y la parte cuasipermanente de la sobrecarga e, inmediatamente después, una sobrecarga de construcción equivalente a la sobrecarga total menos su parte cuasipermanente, de forma que en el momento $t_{c m}+d t$, se alcanza el grado de fisuración máximo. 
- $\quad q_{3}$ que representa la parte no cuasi-permanente de la sobrecarga. Esta carga actúa además de en el instante $t_{c m}+d t$, en el instante $t$ en el que se evalúan las flechas (normalmente a tiempo infinito).

Como valores admisibles de las flechas se adoptan los indicados en los comentarios del Artículo 50 de la EHE.

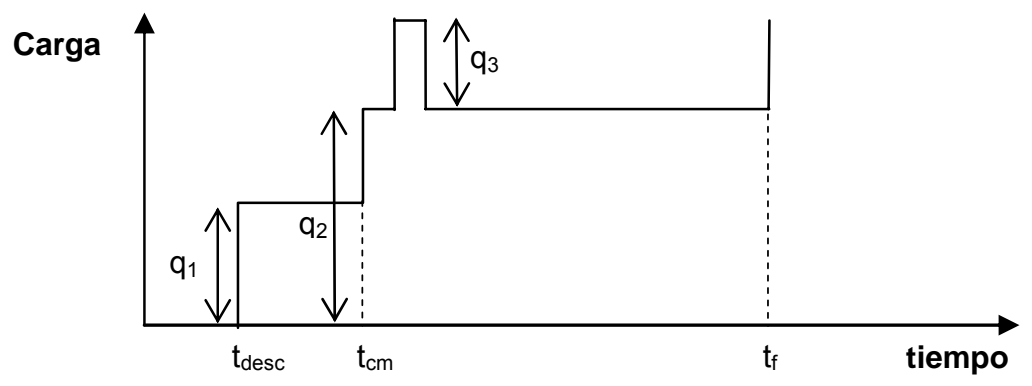

Figura 3.5. Historial de cargas considerado.

\subsubsection{ELU de agotamiento frente a solicitaciones normales en vigas y pilares.}

La verificación de este estado límite se realiza siguiendo el Artículo 42 de la EHE. En el caso de vigas, y de forma análoga a como se realizaba en la verificación del ELS de fisuración, se han verificado las secciones de apoyos y de máximo momento flector positivo. En el caso de los pilares se han comprobado las secciones de arranque y cabeza bajo las combinaciones de acciones que proporcionaban los axiles máximo y mínimo (considerando flectores concomitantes) y los momentos flectores máximo y mínimo (considerando sus axiles concomitantes).

La verificación se realiza obteniendo el diagrama de interacción de cada sección de hormigón armado y comprobando que la pareja de esfuerzos de cálculo (axil-momento flector) queda en el interior del diagrama de interacción. La Figura 3.6 muestra un ejemplo de uno de los diagramas de interacción obtenidos por el programa desarrollado. Como diagramas de cálculo tensión - deformación se han empleado el parábola rectángulo para el hormigón y el bilineal para el acero pasivo como se describen en los artículos 38.4 y 39.5 de la EHE. 


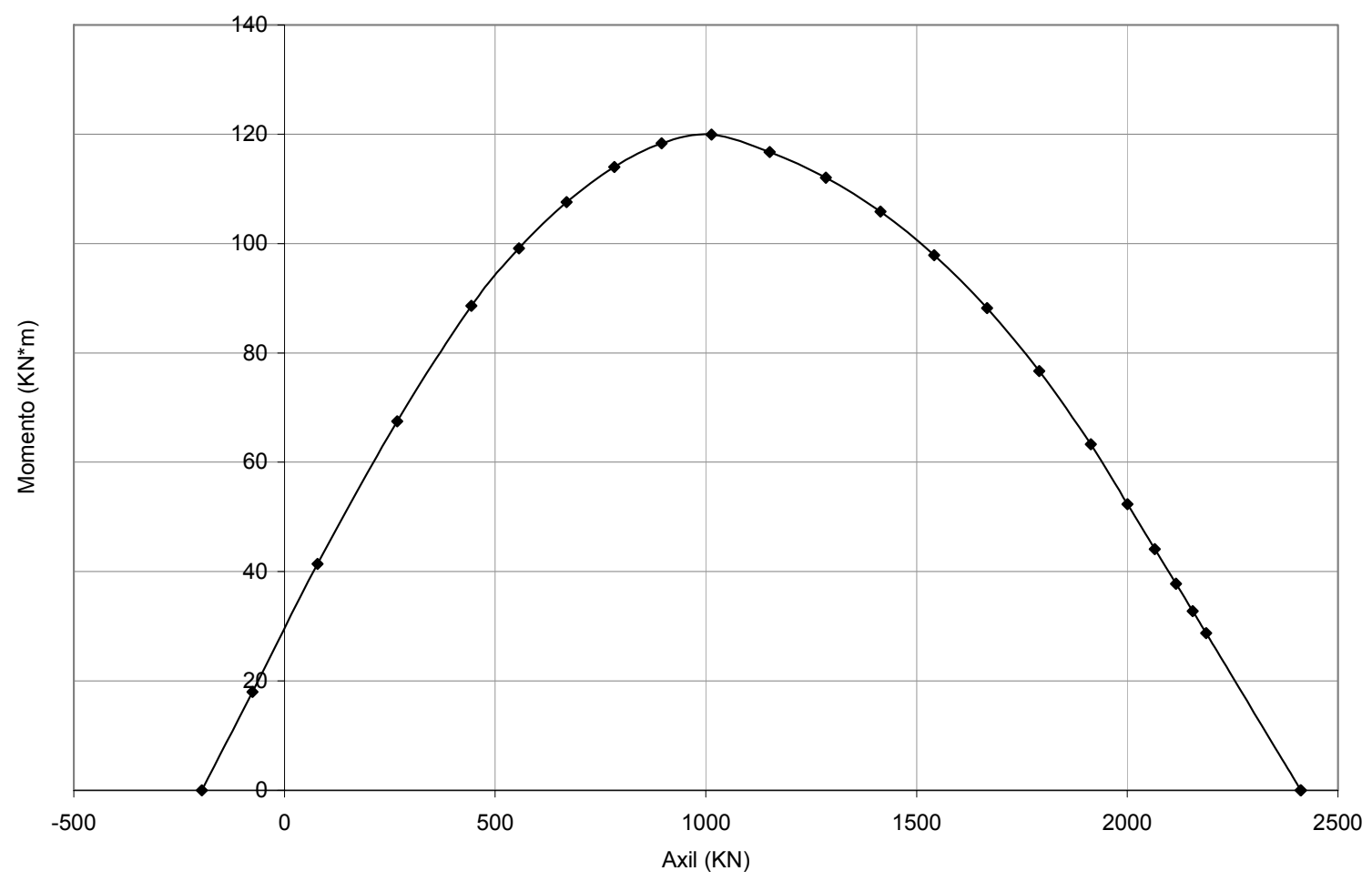

Figura 3.6. Ejemplo de diagrama de interacción de la sección de un pilar.

\subsubsection{EL de Agotamiento frente a cortante.}

La verificación de este estado límite sigue lo indicado por el Art. 44 de la EHE con las siguientes observaciones:

- En el caso de vigas, la verificación se hace a) en las secciones situadas a un tercio y a dos tercios de la luz y considerando como armadura de tracción toda la armadura inferior y b) en las secciones situadas en el empotramiento de las vigas con los pilares y a un canto útil de este empotramiento, adoptando que la armadura traccionada es toda la armadura superior.

\subsubsection{EL de Inestabilidad.}

Se verifican los pilares de los pórticos siguiendo el artículo 43 de la EHE con las siguientes consideraciones:

- Los pilares están perfectamente arriostrados en el plano perpendicular al del pórtico, por lo que sólo se verifica la posible inestabilidad en su plano.

- Se asume que los pórticos son traslacionales y que la verificación del estado límite puede hacerse siguiendo el método simplificado expuesto en el Art. 43.1 de la EHE. Para ello, y siguiendo lo indicado por los comentarios al Art. 43.4 de la EHE, se comprueba que el pórtico tiene menos de 15 plantas y que el 
desplazamiento horizontal máximo bajo las acciones horizontales características y con las rigideces correspondientes a las secciones brutas no supera $1 / 750$ de la altura total del edificio.

- Se comprueban las secciones de arranque y cabeza de cada pilar bajo las mismas combinaciones de esfuerzos -modificados éstos para tener en cuenta el efecto del pandeo- con que se verifican las secciones en el EL de Agotamiento frente a Solicitaciones Normales.

\subsubsection{Disposiciones relativas a las armaduras.}

Se comprueban también los aspectos siguientes:

- Cuantías mecánicas mínimas y máximas de la armadura longitudinal de acuerdo con los artículos 42.3 .2 (vigas) y 42.3 .3 (pilares) de la EHE.

- Cuantías geométricas mínimas de la armadura longitudinal de vigas y pilares (Art. 42.3.5 de la EHE).

- Separación máxima entre armaduras longitudinales en vigas (Art. 42.3.1) y pilares (Art. 55 de la EHE).

- Cuantía mínima de la armadura transversal en pilares y vigas (Art. 44.2.3.4.1 de la EHE).

- Diámetro mínimo de la armadura transversal en función del diámetro máximo de la armadura comprimida en pilares (Art. 42.3.1 de la EHE).

- Separaciones máximas entre estribos (Artículos 42.3.1, 44.2.3.4.1 y 49.3 de la EHE).

- Se lleva hasta el apoyo al menos un tercio de la armadura necesaria para resistir el máximo momento positivo en el caso de apoyos extremos de las vigas y al menos un cuarto en el caso de los apoyos intermedios (Art. 66.5.1 de la EHE).

- Sujeción eficaz mediante familias de cercos de la armadura longitudinal en pilares (comentarios al Art. 42.3.1 de la EHE).

- En el caso de existir más de $30 \mathrm{~cm}$. de separación entre la armadura superior y la inferior de una viga se coloca armadura de piel de $12 \mathrm{~mm}$ de diámetro de modo que la separación máxima entre dos armaduras sea de $30 \mathrm{~cm}$. 


\subsubsection{Otras comprobaciones.}

- En el caso de vigas planas (véase la Figura 3.7), se comprueba que el ancho de la viga es inferior a 3 veces el canto del forjado más la dimensión del pilar perpendicular al eje de la viga (Comentarios al artículo 7 de la "Instrucción para el proyecto y ejecución de forjados unidireccionales de hormigón estructural hechos con elementos prefabricados" -EFHE-, 2002; Calavera, 1993).

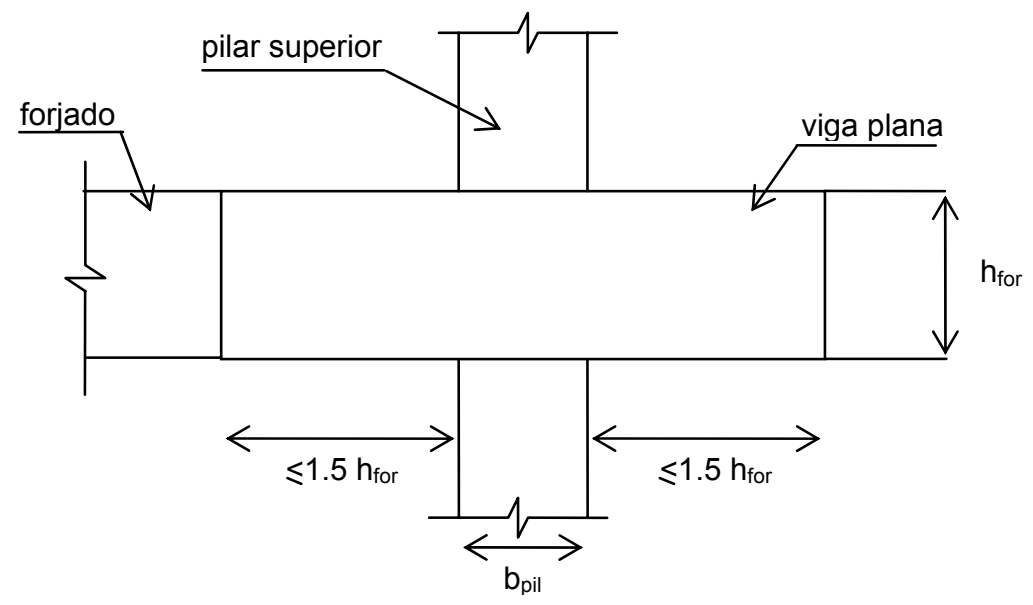

Figura 3.7. Sección de un forjado con viga plana. Dimensiones máximas de la viga.

- Derivas. Se ha comprobado que las derivas o diferencia de los desplazamientos horizontales de dos plantas consecutivas -véase la Fgura 3.8, bajo la combinación de acciones características más desfavorable es inferior a 1/250 de la altura de planta (capítulo 4.3.3.2 del Documento Básico Seguridad Estructural del CTE).

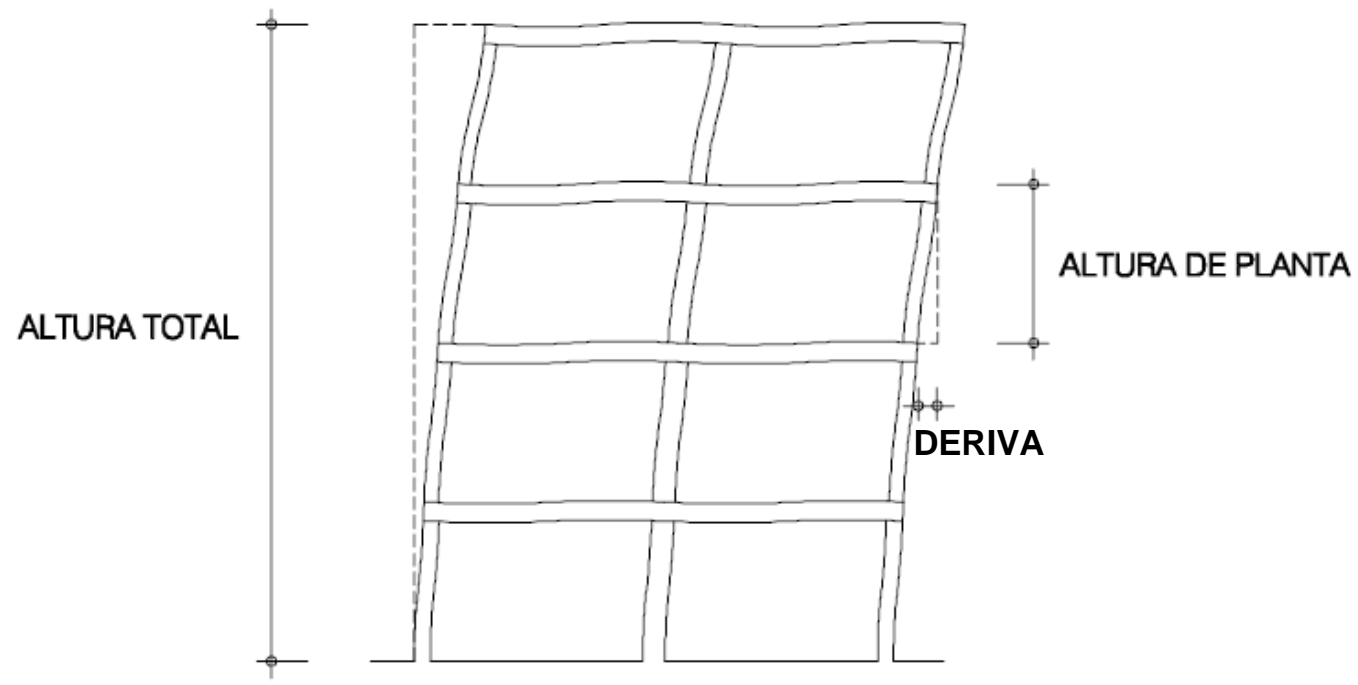

Figura 3.8. Definición de derivas (Tomada del CTE). 
- Habitabilidad. Se comprueba la máxima distancia entre la cara superior del forjado de una planta y la cara inferior de las vigas del forjado de la planta superior.

- Construibilidad. Se verifica que a) la escuadría de los pilares y las calidades del hormigón se mantienen o son decrecientes al aumentar las plantas del edificio y b) los máximos incrementos de cada uno de los lados de la sección de dos pilares consecutivos, situados en una misma alineación, son inferiores o iguales a $5 \mathrm{~cm}$.

\subsection{FUNCIONES OBJETIVO.}

En este trabajo se han considerado un total de cuatro funciones objetivo diferentes relacionadas con el coste económico de la construcción del pórtico, su facilidad constructiva, su sostenibilidad medioambiental y su seguridad global. Se explica a continuación cómo se han evaluado estas funciones.

\subsubsection{Coste económico.}

Para evaluar el coste de la estructura, se va a seguir el procedimiento habitual en obra civil y edificación de descomponer la misma en las diferentes unidades de obra que permiten ejecutarla. De este modo, el coste final se obtendrá como suma de los productos de la medición de cada unidad de obra por su precio unitario. Señalamos aquí que una descripción detallada sobre los criterios para realizar la medición y valoración de una obra se encuentra en De Fuentes (2002).

\subsubsection{Definición de las unidades de obra existentes.}

En el caso de un pórtico plano de edificación, las unidades de obra a considerar son las indicadas en la Tabla 3.4. Estas unidades se obtienen como consecuencia del proceso constructivo que se prevé para la ejecución de un pórtico plano. Esta secuencia, que se repite cada vez que se ejecuta una nueva planta, es la siguiente:

1) Colocación de la armadura de los pilares, solapándose de forma adecuada con las esperas de la cimentación o de los pilares de la planta inferior.

2) Encofrado de los pilares. 
3) Vertido y vibrado del hormigón de los pilares.

4) Desencofrado de los pilares.

5) Cimbrado de las vigas y forjados de la planta superior.

6) Encofrado de las vigas (y en algunos procedimientos constructivos, también de los forjados) de la planta superior.

7) Ejecución de los elementos prefabricados del forjado de la planta superior si los hubiere.

8) Ferrallado de las vigas y forjados de la planta superior.

9) Vertido y vibrado del hormigón de vigas y forjados de la planta superior.

10) Desencofrado de las vigas (y en su caso, también de forjados) de la planta superior.

11) Descimbrado de las vigas y forjados de la planta superior.

En la secuencia anterior no se han incluido unidades de obra referentes a las cimentaciones pues estos elementos se han excluido del estudio.

\subsubsection{Función de coste económico.}

El coste de la estructura $\left(\mathrm{C}_{\mathrm{EST}}\right)$ puede ser obtenido mediante la expresión:

$$
C_{E S T}=C_{V I G}+C_{P I L}
$$

Donde $C_{V I G}$ y $C_{P I L}$, que representan el coste de la construcción de las vigas y de los pilares respectivamente, vienen dados por:

$$
\begin{aligned}
& C_{V I G}=\sum_{V I G A S}\left(M_{C I M B, V I G} \times P_{c v}+M_{E N C, V I G} \times P_{e v}+M_{A C, V I G} \times P_{a}+M_{H O R M, V I G} \times P_{h v i}\right) \\
& C_{P I L}=\sum_{\text {PILARES }}\left(M_{E N C, P I L} \times P_{e p}+M_{A C, P I L} \times P_{a}+M_{H O R M, P I L} \times P_{h p i}\right)
\end{aligned}
$$


Donde:

$M_{C I M B, V I G}, M_{E N C, V I G}, M_{A C, V I G}, M_{H O R M, V I G}$ representan la medición del cimbrado, encofrado, acero y hormigón empleado en vigas respectivamente. La forma en que se obtienen estos valores se expone de forma detallada en el Apéndice 2.

$P_{c v}, P_{e v}, P_{a}, P_{h v i}$ representan los precios unitarios de las unidades de obra relativas al cimbrado, encofrado y hormigonado de vigas (véase la Tabla 3.6). " $\mathrm{P}_{\mathrm{a}}$ " es el precio unitario del kilogramo de acero puesto en obra, el cual es independiente de si el elemento ferrallado es un pilar o una viga.

$M_{E N C, P I L}, M_{A C, P I L}, M_{H O R M, P I L}$ representan la medición del encofrado, acero y hormigón empleado en plares respectivamente. La forma en que se obtienen estos valores se expone de forma detallada en el Apéndice 2.

$P_{e p}, P_{h p i}$ representan los precios unitarios de las unidades de obra relativas al encofrado y hormigonado de pilares (véase la Tabla 3.6).

Señalamos que los precios proceden de una consulta a empresas constructoras realizada en octubre del año 2004 e incluyen:

a) Los costes de la mano de obra necesaria, los materiales, los medios mecánicos y los costes indirectos (costes de ejecución material).

b) Un coste adicional (obtenido como un porcentaje del precio de ejecución material de la unidad) en concepto de los gastos generales y del beneficio industrial de la empresa contratista que ejecuta la obra.

Estos precios pueden ser en el futuro objeto de un estudio de sensibilidad que determine cómo afectan a las características de las estructuras optimizadas variaciones en los mismos. Asimismo, en un futuro pueden considerarse diferentes precios para las barras de acero en función de su diámetro y posición. 
Tabla 3.6. Precios unitarios de las unidades de obra.

\begin{tabular}{|c|c|c|c|}
\hline Unidad & Descripción del precio & Parámetro & $\begin{array}{c}\text { Valor en este } \\
\text { estudio }(€)\end{array}$ \\
\hline $\mathrm{Kg}$. & Acero B-400 S & $P_{a 1}$ & 1.27 \\
\hline $\mathrm{Kg}$. & Acero B-500 S & $\mathrm{P}_{\mathrm{a} 2}$ & 1.30 \\
\hline $\mathrm{m}^{3}$ & Hormigón HA-25 en vigas & $P_{\text {hv1 }}$ & 78.40 \\
\hline $\mathrm{m}^{3}$ & Hormigón HA-30 en vigas & $\mathrm{P}_{\mathrm{hv} 2}$ & 82.79 \\
\hline $\mathrm{m}^{3}$ & Hormigón HA-35 en vigas & $P_{\text {hv3 }}$ & 98.47 \\
\hline $\mathrm{m}^{3}$ & Hormigón HA-40 en vigas & $P_{\text {hv4 }}$ & 105.93 \\
\hline $\mathrm{m}^{3}$ & Hormigón HA-45 en vigas & $P_{\text {hv5 }}$ & 112.13 \\
\hline $\mathrm{m}^{3}$ & Hormigón HA-50 en vigas & $P_{\text {hv6 }}$ & 118.60 \\
\hline $\mathrm{m}^{3}$ & Hormigón HA-25 en pilares & $\mathrm{P}_{\mathrm{hp} 1}$ & 77.80 \\
\hline $\mathrm{m}^{3}$ & Hormigón HA-30 en pilares & $\mathrm{P}_{\mathrm{hp2}}$ & 82.34 \\
\hline $\mathrm{m}^{3}$ & Hormigón HA-35 en pilares & $\mathrm{P}_{\mathrm{hp3}}$ & 98.03 \\
\hline $\mathrm{m}^{3}$ & Hormigón HA-40 en pilares & $\mathrm{P}_{\mathrm{hp} 4}$ & 105.17 \\
\hline $\mathrm{m}^{3}$ & Hormigón HA-45 en pilares & $\mathrm{P}_{\mathrm{hp5}}$ & 111.72 \\
\hline $\mathrm{m}^{3}$ & Hormigón HA-50 en pilares & $\mathrm{P}_{\mathrm{hp} 6}$ & 118.26 \\
\hline $\mathrm{m}^{2}$ & Encofrado-desencofrado de vigas & $\mathrm{P}_{\mathrm{ev}}$ & 25.05 \\
\hline $\mathrm{m}^{2}$ & Encofrado-desencofrado de pilares & $P_{e p}$ & 22.75 \\
\hline $\mathrm{m}^{2}$ & Cimbrado-descimbrado de vigas & $\mathrm{P}_{\mathrm{cv}}$ & 38.89 \\
\hline
\end{tabular}

\subsubsection{Facilidad constructiva.}

La optimización basada únicamente en un criterio de coste puede traducirse en estructuras difíciles de ejecutar, pues las estructuras más económicas tienden a tener un elevado número de barras de pequeño diámetro y con poca separación para ajustarse al máximo a las cuantías de acero necesarias. Por ello, el número de barras de la armadura longitudinal puede considerarse como un indicador de la facilidad constructiva de la estructura, pues menos barras implican diámetros mayores y separaciones entre armaduras 
más grandes. Además, reducir el número de barras permite disminuir los errores durante la ejecución, facilita el control de calidad de la estructura y baja los plazos de la construcción.

En esta Tesis la facilidad constructiva ya ha sido considerada de forma implícita en la definición de los esquemas de armado e imponiendo que la separación entre armaduras cumpla los valores mínimos indicados por la normativa. Un modo adicional de tenerla en cuenta es minimizando el número de barras de la armadura longitudinal de un pórtico. Este valor, al que se llama $N_{s}$ en adelante, se obtiene mediante la expresión (3.10).

$$
N_{s}=\sum_{\text {vigas }}\left(n_{s b}+n_{s e}\right)+\sum_{\text {pilares }} n_{s l}
$$

Donde $n_{s b}$ y $n_{s e}$ representan el número de barras de la armadura base y de refuerzo de una viga y $n_{s l}$ el número de barras de la armadura longitudinal de un pilar. Al evaluar $n_{s b}$ se ha considerado que la longitud máxima de una barra de acero es de $12 \mathrm{~m}$, pues valores superiores implican medios especiales de transporte hasta la obra e incrementos importantes de su precio.

\subsubsection{Sostenibilidad ambiental.}

Adeli (2002) ha señalado las razones para considerar la sostenibilidad en el diseño de infraestructuras y algunas de las tecnologías que pueden aplicarse con este fin. Para estimar objetiva y cuantitativamente la sostenibilidad de un producto se puede emplear la técnica de la Evaluación del Ciclo de Vida o Life Cycle Assessment (LCA). Este análisis permite evaluar el impacto potencial sobre el medioambiente de un producto, proceso o actividad a lo largo de todo su ciclo de vida mediante la cuantificación del uso de recursos ("entradas" como energía, materias primas, agua) y emisiones medioambientales ("salidas" al aire, agua y suelo) asociados con el sistema que se está evaluando. Mediante el LCA todos los efectos ambientales generados por un producto, desde la adquisición de sus materiales componentes hasta su eliminación final, son agregados en un único valor. Cuanto más elevado es este valor, menor es su sostenibilidad. Esto permite comparar objetivamente diferentes alternativas de un diseño y tomar decisiones desde un punto de vista medioambiental. LCA ha sido aplicado a estructuras prefabricadas de hormigón (Féderation Internationale du Béton, 2003), de aluminio (Radlbeq et al., 2006) o al diseño general de edificios (Asif et al., 2007). 
En este estudio se considera como un objetivo minimizar la puntuación ambiental (EC en adelante) de pórticos de hormigón armado ejecutados in situ. Su valor viene dado por la expresión:

$$
E C=\sum_{i=1, s} d_{i} m_{i}\left(x_{1}, \ldots, x_{n}\right)
$$

donde $d_{i}$ es la puntuación ambiental de un material $i$ constituyente del pórtico obtenida mediante la técnica del LCA y $m_{i}$ su medición. Los valores de $d_{i}$ juegan un papel análogo al de los costes de las unidades de obra en la minimización del coste de una estructura y, por tanto, su obtención es un paso previo al de la optimización ambiental. Los $d_{i}$ empleados se proporcionan en la Tabla 3.7 y fueron obtenidos por Hospitaler (2006) empleando el Ecoindicador 99 (Goedkoop y Spriensma, 2001). La puntuación ambiental de los hormigones ha requerido su dosificación previa que se ha realizado siguiendo la metodología descrita por Fernández-Canovas (2002).

\section{Tabla 3.7. Puntuación ambiental de los materiales empleados en un pórtico.}

\begin{tabular}{cc}
\hline Hormigón & $\begin{array}{c}\text { Unidades de impacto } \\
\text { ambiental }\end{array}$ \\
\hline HA-25 & 39100.0 \\
\hline HA-30 & 41400.0 \\
\hline HA-35 & 42000.0 \\
\hline HA-40 & 50200.0 \\
\hline HA-45 & 55600.0 \\
\hline HA-50 & 61300.0 \\
\hline Acero B-400, B-500 & 61.00 \\
\hline
\end{tabular}




\subsubsection{Seguridad estructural.}

La cuarta función objetivo considerada en este trabajo es un coeficiente de seguridad global de la estructura que sirve de herramienta para evaluar el coste de incrementar su seguridad. Esta función se define mediante el procedimiento max-min siguiente:

Maximiza $\gamma_{o s}$

Donde $\gamma_{o s}$, es el coeficiente de seguridad global de la estructura dado por:

$$
\gamma_{o s}=\text { Mínimo } \gamma_{i} \quad i=1, . ., l
$$

En (3.13) $\gamma_{i}$ es el coeficiente de seguridad ofrecido por la estructura respecto a un estado límite $i$ y $l$ es el número total de restricciones debidas a los ELS y ELU que la estructura debe cumplir. De acuerdo con el Método de los Estados Límite, los coeficientes $\gamma_{i}$ son obtenidos como el cociente entre la máxima respuesta que puede proporcionar la estructura frente a un estado límite y el efecto provocado por las acciones que actúan sobre la estructura. Para el cálculo de los $\gamma_{i}$, respuestas y acciones vienen afectadas por coeficientes de seguridad indicados en las normativas.

\subsection{PROBLEMAS ESTUDIADOS.}

En capítulos posteriores de esta Tesis se estudia la optimización mono y multiobjetivo de pórticos de dos vanos y diferente número de plantas con los parámetros, variables y restricciones indicados en los epígrafes 3.2, 3.3 y 3.4. No obstante, las siguientes puntualizaciones deben realizarse:

- En general las estructuras se han optimizado empleando vigas descolgadas y permitiendo que la calidad del hormigón sea variable dentro de un pórtico. No obstante, para estudiar la repercusión de estas consideraciones, se han planteado algunos casos en los que el hormigón es HA-25 y en otro, además, las vigas planas.

- Para estudiar la influencia de la disminución del número de variables, éstas se han agrupado en alguno de los problemas. Los detalles de la agrupación se explican en el Capítulo 7. 
La consideración de estos aspectos lleva a la definición de los problemas de optimización estudiados en esta investigación indicados en las Tablas 3.8 y 3.9.

Tabla 3.8. Definición de problemas de optimización monoobjetivo.

\begin{tabular}{|c|c|c|c|c|c|c|}
\hline $\begin{array}{l}\text { Nombre del } \\
\text { problema }\end{array}$ & $\begin{array}{l}\text { Función } \\
\text { objetivo }\end{array}$ & $\begin{array}{l}\text { Núm. de } \\
\text { vanos }\end{array}$ & $\begin{array}{l}\text { Núm. de } \\
\text { plantas }\end{array}$ & $\begin{array}{l}\text { Tipo de } \\
\text { hormigón }\end{array}$ & $\begin{array}{l}\text { Tipo de } \\
\text { Vigas }\end{array}$ & $\begin{array}{l}\text { Agrupación } \\
\text { de variables }\end{array}$ \\
\hline $\mathrm{P} \_2 \mathrm{v} 2 \mathrm{~h}-\mathrm{C}_{\mathrm{EST}}$ & $\begin{array}{c}\text { Coste } \\
\text { económico }\end{array}$ & 2 & 2 & Variable & Descolgadas & No \\
\hline$P \_2 v 4 h-C_{E S T}$ & $\begin{array}{c}\text { Coste } \\
\text { económico }\end{array}$ & 2 & 4 & Variable & Descolgadas & No \\
\hline P_2v4h-N & $\begin{array}{c}\text { Facilidad } \\
\text { constructiva }\end{array}$ & 2 & 4 & Variable & Descolgadas & No \\
\hline P_2v4h-EC & $\begin{array}{c}\text { Sostenibilidad } \\
\text { ambiental }\end{array}$ & 2 & 4 & Variable & Descolgadas & No \\
\hline$P \_2 v 6 h-C_{E S T}$ & $\begin{array}{c}\text { Coste } \\
\text { económico }\end{array}$ & 2 & 6 & Variable & Descolgadas & No \\
\hline P_2v8h.1-C & $\begin{array}{c}\text { Coste } \\
\text { económico }\end{array}$ & 2 & 8 & Variable & Descolgadas & No \\
\hline P_2v8h.2-C & $\begin{array}{c}\text { Coste } \\
\text { económico }\end{array}$ & 2 & 8 & HA-25 & Descolgadas & No \\
\hline P_2v8h.3-C & $\begin{array}{c}\text { Coste } \\
\text { económico }\end{array}$ & 2 & 8 & HA-25 & Descolgadas & Sí \\
\hline P_2v8h.4-C & $\begin{array}{c}\text { Coste } \\
\text { económico }\end{array}$ & 2 & 8 & $\mathrm{HA}-25$ & Planas & Sí \\
\hline
\end{tabular}

Tabla 3.9. Definición de problemas de optimización multiobjetivo³.

\begin{tabular}{lr}
\hline $\begin{array}{c}\text { Nombre del } \\
\text { problema }\end{array}$ & Funciones objetivo \\
\hline$P \_2 v 4 h-C_{E S T} / N_{S}$ & Coste económico y facilidad constructiva \\
\hline$P \_2 v 4 h-C_{E S T} / E C$ & Coste económico y sostenibilidad medioambiental \\
\hline$P \_2 v 4 h-C_{E S T} / \gamma_{o s}$ & Coste económico y coeficiente de seguridad global \\
\hline${ }^{3}$ En este caso se ha analizado una única estructura de dos vanos y cuatro plantas, con \\
posibilidad de emplear hormigones de calidades diferentes y sin agrupar ninguna variable.
\end{tabular}




\section{CAPÍTULO 4. MÉTODOS HEURÍSTICOS EMPLEADOS.}




\section{Objetivos.}

Tras definir los problemas de optimización en el capítulo anterior, se explican a continuación los procedimientos heurísticos empleados para su resolución. Además, se describe el criterio utilizado para evaluar los resultados proporcionados por diferentes estrategias y el programa de ordenador creado para realizar todos los ensayos numéricos. 


\subsection{INTRODUCCIÓN.}

Esta investigación estudia la optimización de pórticos planos habitualmente empleados en edificación. Una primera estimación de la dificultad de este problema la proporciona el tamaño del espacio de soluciones $S$, pues a ese conjunto pertenecerá la solución óptima. La magnitud de $S$ o, lo que es lo mismo, el número máximo de soluciones que se pueden plantear a partir de todos los valores posibles de las variables de diseño, puede obtenerse del modo siguiente. Si el vector de variables de diseño tiene por componentes $x_{1}, x_{2}, \ldots, x_{m} y$ cada una de ellas puede adoptar $n_{1}, n_{2}, \ldots, n_{m}$ valores diferentes, el número de soluciones posibles del problema viene dado por el producto $n_{1} \times n_{2} \times \ldots \times n_{m}$. En estas condiciones es inmediato obtener, a partir de la ecuación (3.1) y de los datos del Apéndice 1, que un problema aparentemente sencillo como es minimizar el coste económico de un pórtico simétrico de dos vanos y cuatro alturas con vigas descolgadas tiene del orden de $10^{116}$ soluciones distintas. Al aumentar el número de plantas o de vanos, el número de soluciones del problema crece de forma exponencial (Figura 4.1).

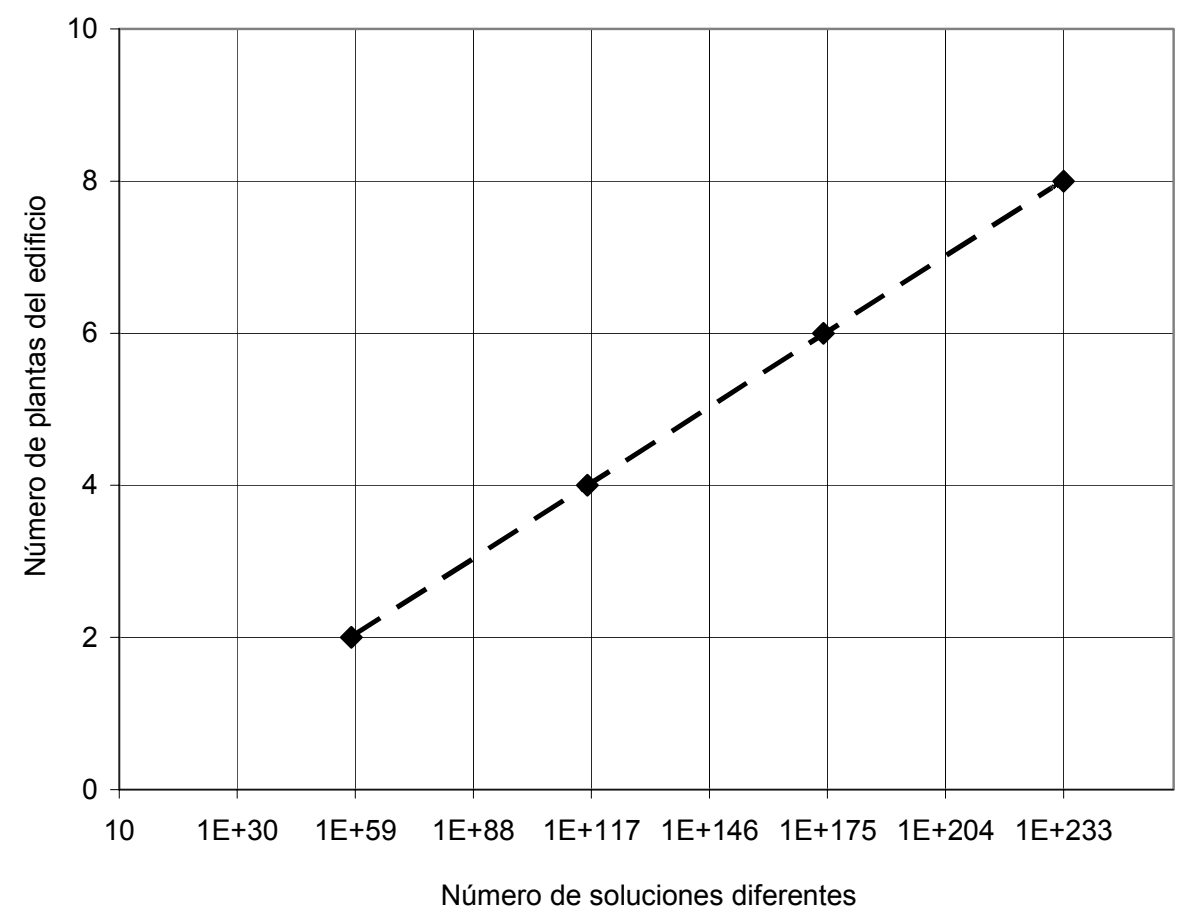

Figura 4.1. Número de soluciones distintas para un pórtico simétrico de dos vanos y dos, cuatro, seis y ocho plantas. El eje de abcisas está en escala logarítmica.

Una idea de lo que representan $10^{116}$ soluciones diferentes se puede obtener a partir de la consideración del volumen de agua existente en la Tierra. Este volumen es de $10^{21}$ litros (ver Yepes, 2003), por lo que el número de posibles soluciones es $10^{95}$ veces el 
volumen de agua en litros que hay en nuestro planeta. Habida cuenta que el programa de optimización desarrollado emplea 0.012 segundos en calcular una solución, el tiempo necesario para calcular todas ellas sería del orden de $10^{106}$ siglos. Por tanto, resolver el problema evaluando la función objetivo en todo el espacio de soluciones es inabordable y resulta necesario el empleo de alguna técnica más sofisticada de búsqueda de la solución óptima. En este trabajo de investigación, se ha optado por el empleo de:

- Los algoritmos FB, SA, TA y GA para el problema monoobjetivo (epígrafes 4.3 a 4.6).

- El algoritmo SMOSA para el problema multiobjetivo (epígrafe 4.7).

Estas técnicas precisan de una o varias soluciones iniciales del problema. Para obtenerla(s) se ha utilizado una estrategia de Saltos Múltiples Aleatorios o Random Walk (RW) cuyo funcionamiento se explica en el apartado 4.2. Finalmente, el epígrafe 4.8 describe la metodología empleada para comparar los resultados proporcionados por las diferentes heurísticas y el 4.9 el programa de ordenador desarrollado por el autor de esta Tesis para realizar los experimentos numéricos.

Es necesario resaltar que:

- La obtención del óptimo global precisa del cálculo de todas las posibles soluciones. Esto, como ha quedado demostrado anteriormente, resulta a día de hoy inviable. Por ello los diseños proporcionados por las heurísticas son siempre óptimos locales o soluciones optimizadas.

- Las versiones desarrolladas para esta Tesis de FB, SA, TA y SMOSA trabajan con soluciones factibles. La aplicación de GA maneja además soluciones no factibles gracias al empleo de funciones de penalización que empeoran el valor de la función objetivo cuando se incumplen algunas de las restricciones del problema.

- Las explicaciones sobre la aplicación de los algoritmos monoobjetivo se han particularizado para la minimización del coste económico de la estructura. No obstante, son válidas también para la minimización del número de barras de la estructura o de su impacto ambiental sin más que reemplazar $C_{E S T}$ por las funciones objetivo $N_{s}$ o $E C$ respectivamente. 


\subsection{ESTRATEGIA DE SALTOS MÚLTIPLES ALEATORIOS.}

\subsubsection{Introducción.}

Una primera aproximación para resolver cualquier problema de optimización consiste en seleccionar aleatoriamente un conjunto más o menos extenso de soluciones eligiendo posteriormente la solución factible de menor coste.

Esta estrategia, llamada Estrategia de Saltos Múltiples Aleatorios, presenta varios inconvenientes claros:

1) La obtención de una solución de calidad requiere la realización de un inventario exhaustivo del conjunto de soluciones, lo que, en general, resulta inaceptable por crecer exponencialmente el número de soluciones con la dimensión del problema.

2) Al obtenerse cada solución de forma totalmente independiente de las demás, no se aprovecha la información del espacio de soluciones generada durante el proceso.

3) En caso de no limitar los intervalos de variación de las variables y, en función de las características del problema, el número de soluciones factibles respecto del total planteadas puede llegar a ser muy reducido. Esto introduce una dificultad añadida en la obtención de soluciones de calidad.

A pesar de estos puntos débiles es interesante aplicar esta estrategia porque:

1) Proporciona diseños que se emplean como solución inicial en la aplicación de otras técnicas de optimización heurística.

2) Explora diferentes regiones del espacio de soluciones. Esto permite a las metaheurísticas que la emplean como método para conseguir soluciones iniciales a) aumentar su capacidad para encontrar soluciones iniciales diversas y b) valorar la dependencia del resultado de su aplicación de la solución inicial o del conjunto de soluciones iniciales. 
3) Permite valorar el total de soluciones factibles respecto del número total exploradas.

\subsubsection{Aplicación del algoritmo al problema planteado.}

\subsubsection{Descripción.}

La versión de la heurística RW utilizada en este trabajo se describe en la Figura 4.2. El proceso consiste en generar de forma aleatoria un pórtico, comprobar que es factible y, si lo es, calcular su coste económico. Encontrada la primera solución factible el proceso se invierte, de modo que se calcula primero el coste del pórtico y, si es menor que el del pórtico óptimo en ese momento, se calcula la estructura (de este modo se consiguen ahorros de tiempo de cálculo del orden del $40 \%$ ). Evaluados un número de pórticos fijado de antemano (parámetro "Nsol"), el proceso se detiene y se acepta como resultado el pórtico de menor coste.

La generación aleatoria de soluciones imponiéndoles como única condición que sus valores se encontraran entre los indicados en las tablas del Apéndice 1 proporciona un porcentaje de soluciones factibles respecto del total planteadas muy bajo (del orden del $1 \%$ para el problema $P_{\_} 2 v 4 h$ ), lo que resulta indeseable pues obliga a incrementar enormemente el número de pórticos a calcular ( $\mathrm{y}$ por tanto, los tiempos de cálculo) para tener una cantidad aceptable de soluciones factibles. Este problema se ha resuelto estableciendo intervalos de variación para algunas variables (horquillas), de modo que el proceso de construcción de cada solución garantice de forma automática el cumplimiento de algunas de las restricciones del problema. Estas horquillas se describen a continuación y permiten aumentar el número de soluciones factibles a un valor variable entre el $2 \%$ (en el $P \_2 v 6 h$ ) y el $40 \%$ (en el problema $P \_2 v 2 h$ ) en los problemas estudiados. Para la familia de problemas $P_{2} 2 v 8 h$ la heurística no fue capaz de proporcionar ninguna solución factible, por lo que RW se modificó según se indica en el apartado 6.1.4.

\subsubsection{Horquillas empleadas en la definición del armado de pilares.}

La armadura de los pilares se ha escogido de forma aleatoria, pero exigiendo el cumplimiento de las condiciones siguientes:

- La armadura longitudinal del pilar supera la cuantía mínima geométrica indicada por la EHE (Art. 42.3.5). 
4)

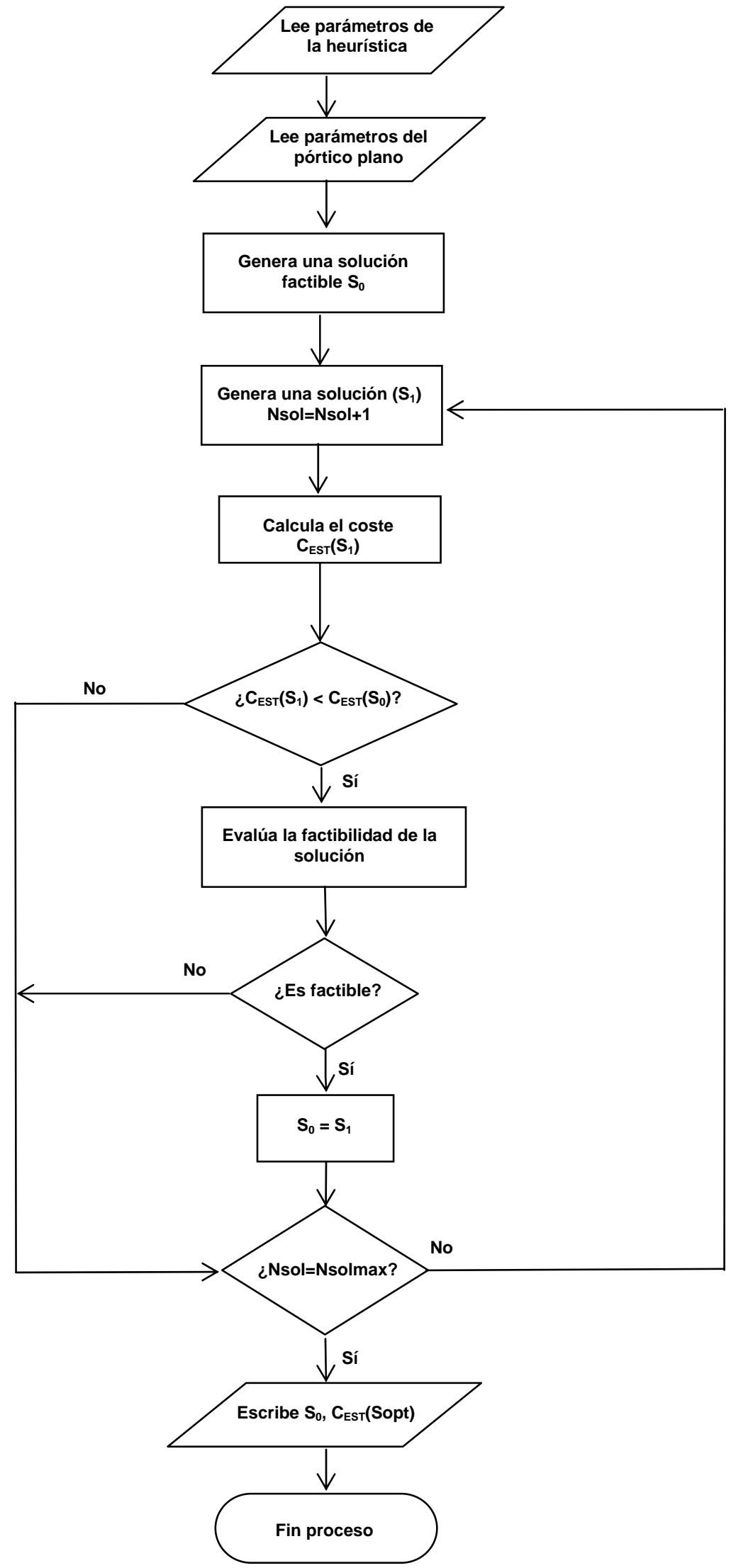

Figura 4.2. Diagrama de flujo de la heurística RW empleada en este trabajo. El diagrama sirve igualmente para la minimización de $N_{s}$ y EC reemplazando $C_{E S T}$ por estas funciones objetivo. 
- La separación entre armaduras es superior al valor mínimo indicado en la EHE (Art. 42.3.1).

- Las armaduras de cortante satisfacen los valores mínimos de la EHE (Art. 44.2.3.4.1 de la EHE).

\subsubsection{Horquillas empleadas en la definición del armado de vigas.}

Las armaduras de las vigas se han elegido de modo aleatorio, pero exigiendo el cumplimiento de las siguientes condiciones:

- La armadura base inferior debe ser superior a la cuantía mínima geométrica indicada por la EHE para la armadura de tracción en vigas (Art. 42.3.5).

- La armadura base superior debe ser superior a la cuantía mínima geométrica indicada por la EHE para la armadura de compresión en vigas (Art. 42.3.5).

- El total de la armadura inferior debe cumplir las cuantías mínimas mecánicas marcadas por la EHE (Art. 42.3.2).

- El total de la armadura superior debe cumplir las cuantías mecánicas mínimas establecidas por la EHE (Art. 42.3.2).

- La separación entre armaduras longitidinales es superior al valor mínimo indicado en la EHE (Art. 55 de la EHE).

- La armadura de cortante debe satisfacer los mínimos de la EHE (Art. 44.2.3.4.1 de la EHE).

\subsubsection{Otras horquillas.}

- El canto mínimo de las vigas es igual o superior al canto del forjado y el canto máximo es el máximo compatible con el gálibo necesario en interior del edificio.

- Las dimensiones de los pilares son escogidas de modo que, dada una alineación, no sean crecientes con la altura y que como máximo los lados de dos pilares consecutivos difieran en $5 \mathrm{~cm}$.

- La resistencia a compresión del hormigón se escoge de modo que la misma no sea creciente con la altura. 


\subsection{GRADIENTE FIRST BEST.}

\subsubsection{Introducción.}

Como ya se avanzó en el Capítulo 1, una forma de abordar la resolución del problema de optimización es modificar paulatinamente las variables que definen el pórtico a optimizar. Estas heurísticas, conocidas como estrategias de búsqueda secuencial por entornos, se basan en los conceptos de:

1) Movimiento o regla que permite transformar una solución en otra.

2) Entorno de una solución o conjunto de soluciones generables alterando esa solución con un movimiento.

De las diferentes estrategias que generalizan la búsqueda secuencial por entornos, se aplican en este apartado algoritmos de búsqueda local por gradiente, cuyo funcionamiento general se explicó en el epígrafe 1.3.2.2 para sus dos variantes FB y GB. Por ser los tiempos de computación empleados por GB superiores a los del FB, se ha empleado en esta investigación una versión de esta última heurística con las características y resultados que se indican en el punto siguiente.

\subsubsection{Aplicación del algoritmo.}

\subsubsection{Descripción.}

La versión de la heurística FB empleada en esta investigación se describe en la Figura 4.3. El método requiere de una solución factible de partida $S_{0}$ que se obtiene mediante RW. A partir de esta solución se aplican movimientos a los valores de las variables para obtener una nueva solución $S_{1}$. Esta solución es evaluada y, si mejora el coste de $S_{0}$, se comprueba su factibilidad. Si es factible se adopta como nueva solución de partida. El proceso es repetido hasta que no se produce ninguna mejora de la solución en un número de iteraciones fijado de antemano $\left(N_{\text {itsmmax }}\right)$.

Esta técnica precisa pues de la definición de a) las características de los movimientos y b) el parámetro $N_{\text {itsmmax }}$. Estos aspectos se tratan con detalle en el apartado siguiente. 


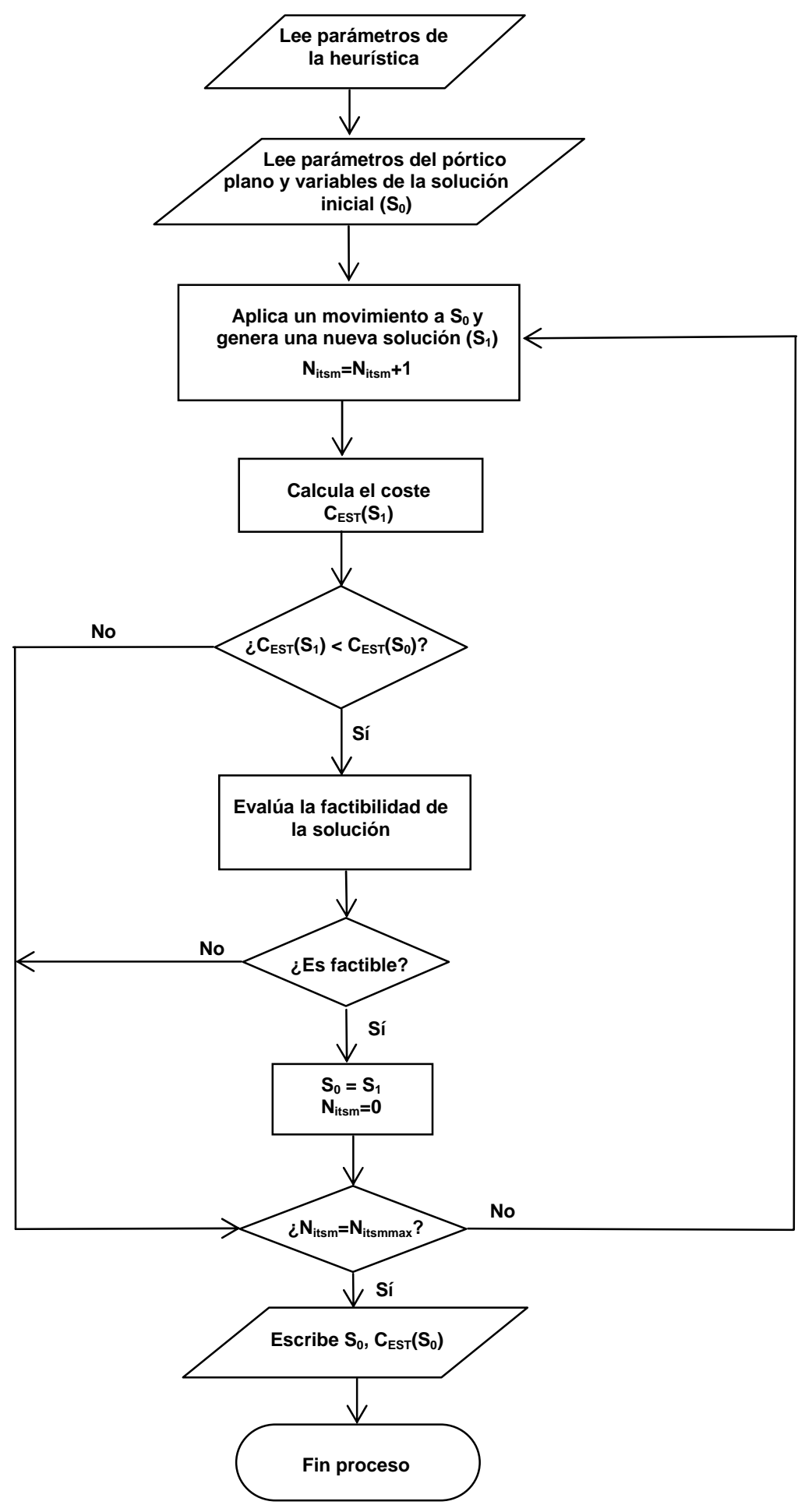

Figura 4.3. Diagrama de flujo de la heurística FB empleada en este trabajo. El diagrama sirve igualmente para la minimización de $N_{S}$ y $E C$ reemplazando $C_{E S T}$ por estas funciones objetivo. 


\subsubsection{Parámetros.}

a) Movimientos.

Aplicar un movimiento a una solución consiste en modificar un número de variables elegidas de forma aleatoria dentro de pequeños intervalos, pudiendo ser tanto el número de las variables como la amplitud del intervalo del movimiento constante o cambiante a lo largo del proceso. Por ello, caracterizar los movimientos implica decidir:

- Los intervalos de variación de cada una de las variables.

- El número de variables que se modifican en cada movimiento (parámetro Nvar).

- Qué variables se modifican en cada movimiento.

En este trabajo:

- Los intervalos de variación de cada una de las variables se han decidido tras una experimentación numérica y se reflejan en la Tabla 4.1.

Tabla 4.1. Intervalos máximos de variación de las variables.

\begin{tabular}{lc}
\hline Variable & $\begin{array}{c}\text { Intervalo máximo } \\
\text { de variación }\end{array}$ \\
\hline Límite elástico del acero en vigas y pilares $\left(\mathrm{N} / \mathrm{mm}^{2}\right)$ & \pm 100 \\
\hline Resistencia característica del hormigón en vigas y pilares $\left(\mathrm{N} / \mathrm{mm}^{2}\right)$ & \pm 5 \\
\hline Ancho de vigas $(\mathrm{m})$ & \pm 0.03 \\
\hline Canto de vigas $(\mathrm{m})$ & \pm 0.03 \\
\hline Ancho de pilares $(\mathrm{m})$ & \pm 0.15 \\
\hline Canto de pilares $(\mathrm{m})$ & \pm 0.15 \\
\hline Armadura base inferior en vigas $\left(\mathrm{cm}^{2}\right)$ & \pm 4.9 \\
\hline Armadura de refuerzo inferior en vigas $\left(\mathrm{cm}^{2}\right)$ & \pm 4.1 \\
\hline Armadura base superior en vigas $\left(\mathrm{cm}^{2}\right)$ & \pm 4.9 \\
\hline Armadura de refuerzo superior $\mathrm{en}$ vigas $\left(\mathrm{cm}^{2}\right)$ & \pm 4.12 \\
\hline Armadura transversal en vigas $\left(\mathrm{cm}^{2} / \mathrm{m}\right)$ & \pm 7.6 \\
\hline Armadura longitudinal en pilares $\left(\mathrm{cm}^{2}\right)$ & \pm 11.3 \\
\hline Armadura transversal en pilares $\left(\mathrm{cm}^{2} / \mathrm{m}\right)$ & \pm 6.1 \\
\hline
\end{tabular}


- El valor de Nvar se ha fijado mediante ensayos numéricos efectuados con cada problema particular según se detalla en el Capítulo 5.

- Las variables que se cambian en cada movimiento se escogen de modo totalmente aleatorio.

b) Número máximo de iteraciones sin mejora Nitsmmax.

El parámetro Nitsmmax es calibrado mediante ensayos numéricos para cada caso particular como se muestra en el Capítulo 5.

\subsection{CRISTALIZACIÓN SIMULADA.}

\subsubsection{Introducción.}

Como se mostró en el apartado 1.3.2.3 los métodos que realizan una aceptación voraz de nuevas soluciones como el FB o el GB corren el riesgo de quedar prematuramente atrapados en un óptimo local. Para evitar este problema, se han desarrollado estrategias que permiten una degradación acotada de la solución actual como SA o TA. El funcionamiento general de estas técnicas se explicó en el Capítulo 1, por lo que en los puntos siguientes se describirá la versión de las mismas desarrollada para este trabajo.

\subsubsection{Aplicación del algoritmo.}

La versión implementada en este trabajo de SA parte de una solución inicial factible $S_{0}$ obtenida mediante RW de la que se evalúa su coste económico. A partir de ella y, adoptada una temperatura inicial $T=T_{0}$, comienza el proceso iterativo siguiente:

1) Se aplica un movimiento a $S_{0}$ para obtener una nueva solución $S_{1}$ de forma análoga a como se explicó en 4.3.2.

2) Si $S_{1}$ cumple el criterio de aceptación explicado en 1.3.2.3 se comprueba su factibilidad. Si $S_{1}$ es factible:

a) Reemplaza a $S_{0}$ como solución actual. 
b) Si su coste es inferior al de la solución factible más económica encontrada hasta ese momento $\left(S_{o p t}\right)$, la sustituye como solución optimizada.

3) Se contabiliza el número de iteraciones $\left(N_{i t}\right)$ realizadas para la temperatura $T$. Si es igual a un valor fijado de antemano (longitud de la Cadena de Markov, $L m$ ), entonces se disminuye $T$. Si el número de iteraciones es inferior, el algoritmo vuelve al punto 1).

4) Se evalúa el criterio de parada.

a) Si no se satisface, el proceso iterativo comienza de nuevo en el punto 1) con una temperatura más baja.

b) Si se satisface, $S_{o p t}$ es la solución localmente óptima.

Para aplicar esta heurística es necesario definir:

1) Las características de los movimientos a aplicar.

2) El valor de la temperatura inicial.

3) La longitud de la cadena de Markov y la velocidad de enfriamiento.

4) El criterio de parada.

Estos aspectos se desarrollan a continuación.

- Movimientos a aplicar.

El número de variables modificadas en cada movimiento se ha determinado mediante ensayos numéricos efectuados con cada problema particular. Las variables a cambiar se han escogido aleatoriamente. Los intervalos máximos de variación son los mismos que se emplean para la estrategia FB y que se indicaron en la Tabla 4.1.

- Valor de la temperatura inicial.

El valor de $T_{0}$ puede tener influencia en el resultado del proceso de optimización (ver Yepes, 2002). Una temperatura inicial alta hace que la búsqueda sea errática al principio, 
pasando por regiones muy diferentes del espacio de soluciones al ser muy fácil aceptar soluciones de mayor coste que la solución de partida. Al contrario, si la temperatura inicial es demasiado baja, la búsqueda se centra únicamente en una parte muy restringida del espacio de soluciones. Por ello, son deseables métodos robustos capaces de decidir por sí mismos el valor de la temperatura inicial. En este trabajo se ha optado por emplear el algoritmo propuesto por Medina (2001) tal y como se describe a continuación:

1) Se selecciona una temperatura inicial $T_{0}$ de forma arbitraria.

2) Se aplica el algoritmo para la longitud de la cadena de Markov seleccionada y se contabiliza el número de soluciones aceptadas, obteniéndose la relación $A$ entre el número de soluciones aceptadas y el total de soluciones planteadas que suponen un empeoramiento del coste.

$$
A(\%)=\frac{\text { Número de soluciones aceptadas empleando el factor de Boltzmann }}{\text { Número total de soluciones con } \Delta>0}
$$

3) Si $A$ está comprendido entre dos límites fijados de antemano se acepta el valor de $T_{0}$ como temperatura inicial. Tras varios tanteos se ha adoptado un límite inferior del $20 \%$ y uno superior del $40 \%$. Los mismos valores han sido empleados por otros autores en la optimización de estructuras de hormigón armado con SA (Alcalá et al., 2005).

4) Si $A>$ límite superior se repite el proceso adoptando un nuevo valor de temperatura inicial igual a la mitad del $T_{0}$ seleccionado en el punto 1 ).

5) Si $A$ < límite inferior se repite el proceso adoptando una nuevo valor de temperatura inicial igual al doble del $T_{0}$ seleccionado en el punto 1).

Para los problemas objeto de estudio, los valores finales de la temperatura inicial obtenidos empleando este procedimiento ha oscilado entre 48 y 6.

\section{- $\quad$ Longitud de la cadena de Markov $(\mathrm{Lm})$ y la velocidad de enfriamiento.}

El parámetro $T$ se reduce a lo largo del proceso para disminuir progresivamente la probabilidad de elegir peores soluciones. Para cada temperatura se ensayan un número de movimientos igual a la longitud de la cadena de Markov elegida. Cadenas largas y velocidades de enfriamiento lentas conducen con mayor probabilidad a soluciones mejores, 
pero los tiempos de cálculo se incrementan (ver Yepes, 2002). Por tanto, una adecuada elección de $L m$ y del esquema de enfriamiento es de gran importancia.

Siguiendo las recomendaciones de Dowsland (1993), se ha adoptado una velocidad geométrica de decrecimiento de $T$ según el esquema siguiente:

$$
T_{i+1}=r \times T_{i} \quad \operatorname{con} r \in[0.8,0.99]
$$

La elección de los valores de $L m$ y $r$ se realiza mediante ensayos numéricos como se muestra en los capítulos 5 y 6 .

Algunos autores han propuesto incrementar $T$ cuando se alcanza un óptimo local (ver Yepes, 2002) para intentar escapar de él. Esta estrategia, conocida como "recalentamiento", no ha sido aplicada en este trabajo.

\section{- $\quad$ Criterio de parada.}

Existen dos posibilidades para determinar cuando debe detenerse la ejecución del algoritmo:

1) Un criterio basado en la temperatura final.

En teoría, el proceso se detendría cuando la temperatura final fuera nula. Sin embargo, mucho antes de llegar a este valor de temperatura, la probabilidad de encontrar una solución mejor es prácticamente nula, por lo que suele optarse por detener el proceso cuando la temperatura final sea un porcentaje prefijado de la temperatura inicial (por ejemplo un $1 \%)$.

2) Un criterio basado en la no existencia de mejoras de la solución optimizada después de un determinado número de cadenas de Markov.

El primer criterio tiene el inconveniente de fijar de antemano el número de iteraciones totales, lo que no resulta muy consistente. En este trabajo se ha optado por la segunda opción, ensayando heurísticas con una y con dos cadenas de Markov sin mejora como criterio de parada (parámetro "NCMsmmax").

El diagrama de flujo de esta estrategia se muestra en la Figura 4.4. 


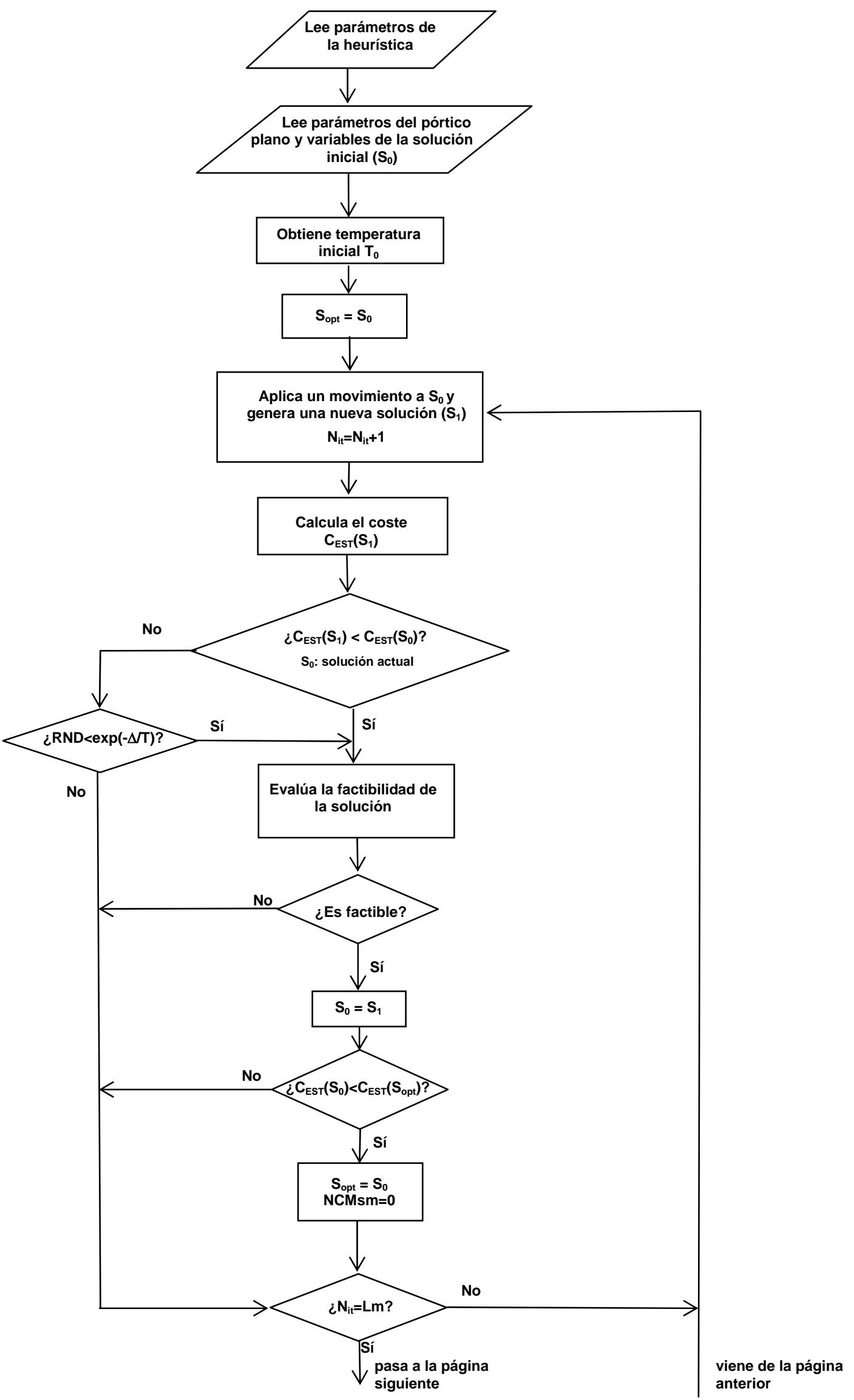

Figura 4.4. Diagrama de flujo de la heurística SA empleada en este trabajo (continua en la página siguiente). RND es un número aleatorio con una distribución uniforme de probabilidad entre 0 y 1. 


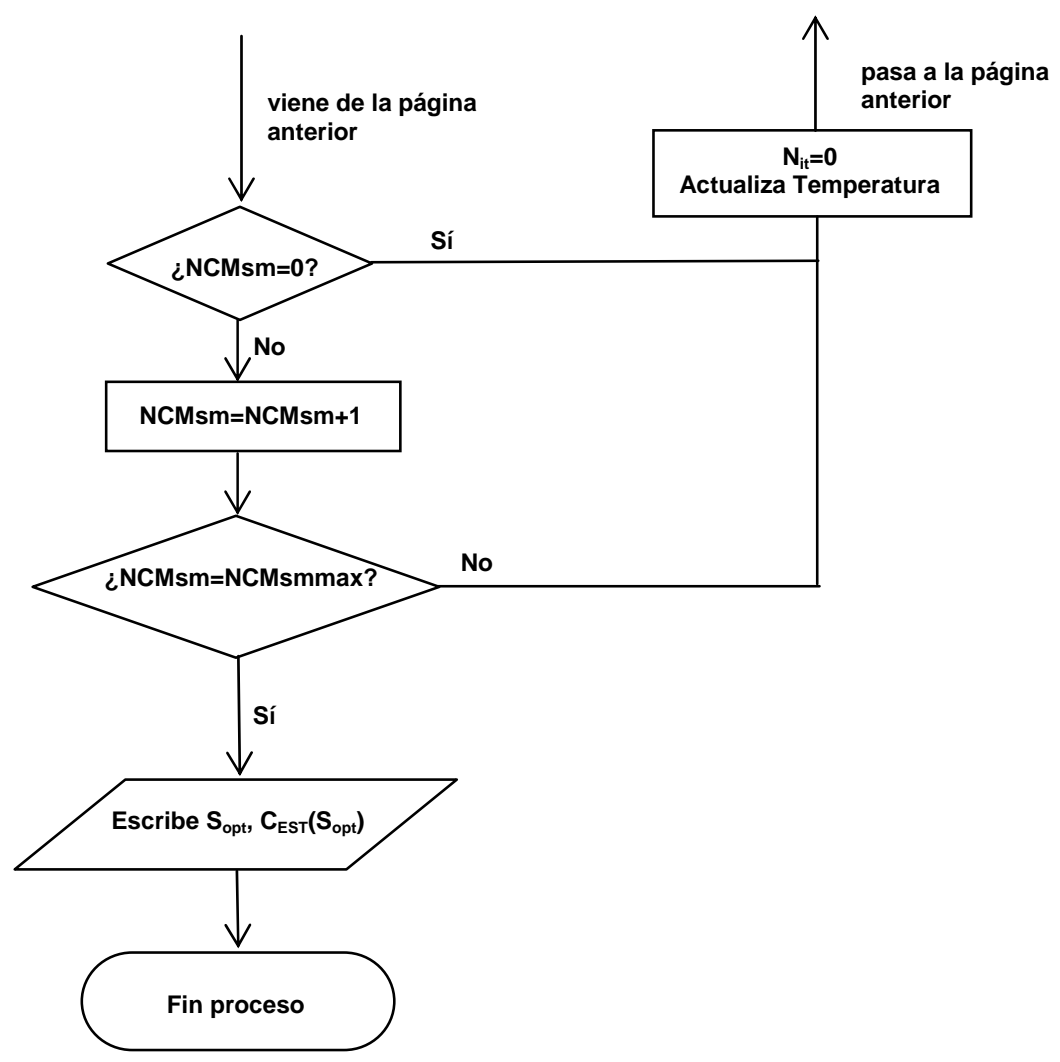

Figura 4.4. Diagrama de flujo de la heurística SA empleada en este trabajo (viene de la página anterior). El diagrama sirve igualmente para la minimización de $N_{S}$ y EC reemplazando $C_{E S T}$ por estas funciones objetivo.

\subsection{ACEPTACIÓN POR UMBRALES.}

La versión de TA desarrollada para este trabajo parte de una solución inicial factible $S_{0}$ obtenida mediante RW de la que se obtiene su coste económico. A partir de ella y, adoptado un umbral inicial $U_{0}$, comienza el proceso iterativo siguiente:

1) Se aplica un movimiento a $S_{0}$ para obtener una nueva solución $S_{1}$ como se explicó en el epígrafe 4.3.2.

2) Si $S_{1}$ cumple el criterio de aceptación explicado en 1.3.2.3 se comprueba su factibilidad. Si $S_{1}$ es factible:

a) Reemplaza a $S_{0}$ como solución actual.

b) Si su coste es inferior al de la solución factible más económica encontrada hasta ese momento $\left(S_{o p t}\right)$, la sustituye como solución optimizada. 
3) Se contabiliza el número de iteraciones (Nit) realizadas para el umbral $U$. Si es igual a un valor fijado de antemano (Nitc), entonces $U$ se disminuye. En caso contrario el algoritmo vuelve al punto 1).

4) Se evalúa el criterio de parada.

a) Si no se satisface, el proceso iterativo comienza de nuevo con un umbral más bajo.

b) Si se satisface, se adopta $S_{o p t}$ como diseño optimizado.

La aplicación de esta heurística precisa la definición de:

1) Las características de los movimientos a aplicar.

2) El número de iteraciones en las que el umbral de aceptación permanece constante (parámetro Nitc).

3) El valor del umbral inicial de aceptación (parámetro $U_{0}$ ).

4) La velocidad de reducción del umbral.

5) El criterio de parada.

Se aclaran seguidamente los parámetros anteriores.

- $\quad$ Valor del umbral inicial.

El umbral inicial se obtiene empleando el método desarrollado por Yepes (2002), quien extendió el método propuesto por Medina (2001) para SA a la estrategia TA. El algoritmo sigue los pasos siguientes:

1) Se selecciona un umbral inicial $U_{0}$ de forma arbitraria.

2) Se aplica TA partiendo de la solución inicial obtenida con RW y, para un ciclo de iteraciones, se contabiliza el número de soluciones aceptadas. Se determina la relación $A$ entre el número de soluciones aceptadas y el total de soluciones planteadas que suponen un empeoramiento del coste. 


$$
A(\%)=\frac{\text { Número de soluciones aceptadas }}{\text { Número total de soluciones con } \Delta>0}
$$

3) Si $A$ está comprendido entre dos límites fijados de antemano (en este caso entre el 20 y el $40 \%$ ) se acepta el valor de $U_{0}$.

4) Si $A>$ límite superior se repite el proceso adoptando un nuevo valor de umbral inicial igual a la mitad del $U_{0}$ seleccionado en el punto 1).

5) Si $A$ < límite inferior se repite el proceso adoptando una nuevo valor de temperatura inicial igual al doble del $U_{0}$ seleccionado en el punto 1 ).

Para los problemas a los que se ha aplicado este algoritmo el valor del umbral inicial ha oscilado entre 24 y 11.

- $\quad$ Velocidad de reducción del umbral.

La velocidad de reducción del umbral se puede definir de dos maneras (ver Yepes, 2002):

1) Se considera una velocidad geométrica de reducción del umbral en cada ciclo de iteraciones de modo que:

$$
U_{i+1}=r \times U_{i} \quad \text { con } r \in[0.8,0.99]
$$

Valores de $r$ altos hacen que el umbral baje muy lentamente, conduciendo a tiempos de cálculo elevados. Por otro lado, valores de $r$ bajos disminuyen los tiempos de cálculo, pero suelen conducir a óptimos locales de peor calidad.

2) Se considera una velocidad exponencial de reducción del umbral en cada ciclo de iteraciones.

En este trabajo se ha optado por la opción 1.

Señalar que en las aplicaciones iniciales de este algoritmo (Dueck y Scheuert, 1990) y en otras posteriores (Yepes, 2002) se disminuía el umbral de aceptación en cada ciclo desde un valor inicial hasta un valor final nulo. En cambio, en este trabajo el umbral se ha mantenido constante en todo el ciclo (véase la Figura 4.5). 

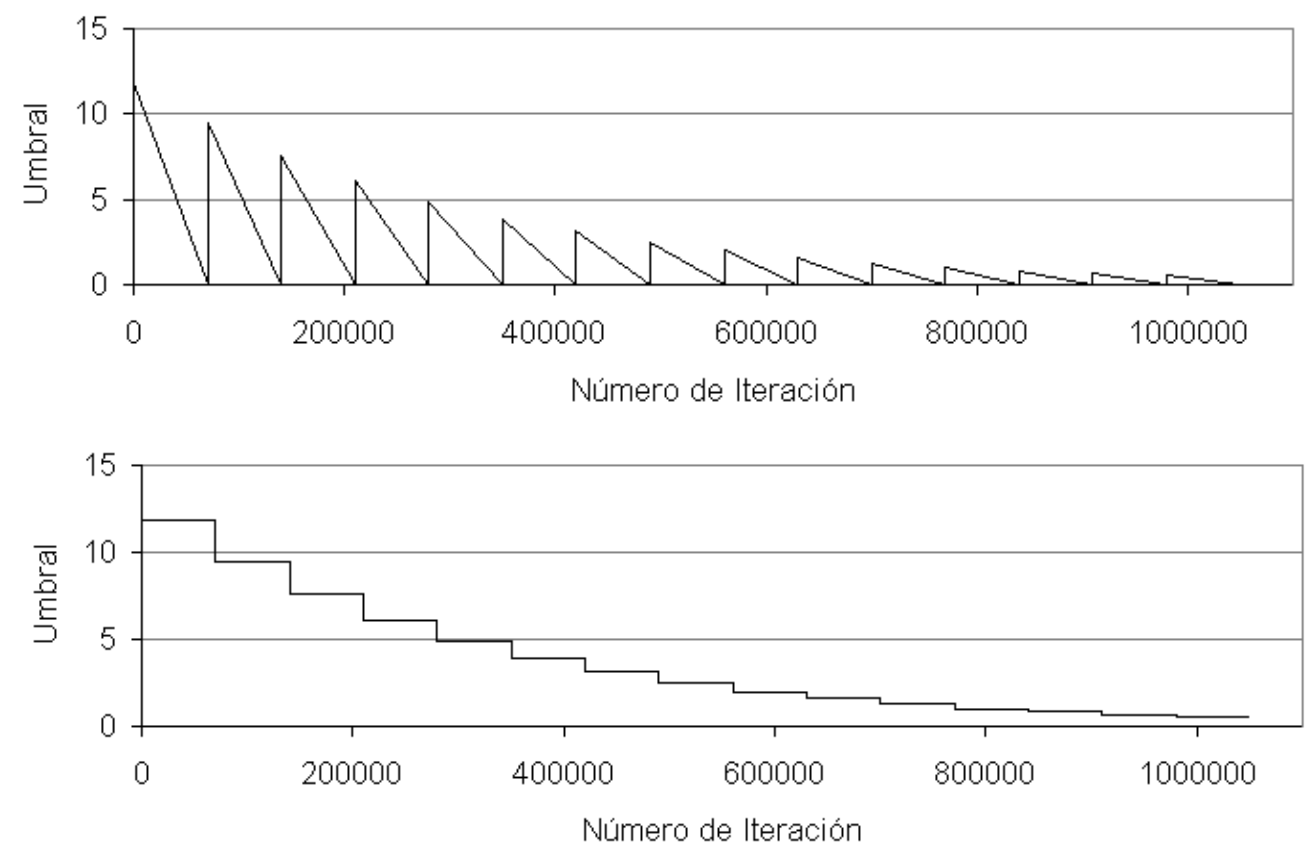

Figura 4.5. Esquemas de reducción del umbral empleados por Dueck y Scheuert (1991) - parte superior - $y$ en este trabajo - parte inferior -

- $\quad$ Criterio de parada.

Existen dos posibilidades para determinar cuando se detiene el algoritmo:

1) Emplear un criterio basado en el umbral final.

En teoría, el proceso se detendría cuando el umbral final fuera nulo. Sin embargo, mucho antes de llegar a este valor del umbral, la probabilidad de encontrar una solución mejor es prácticamente nula, por lo que suele determinarse que el proceso se detenga cuando el umbral final sea un determinado porcentaje del umbral inicial (por ejemplo un $1 \%)$.

2) Un criterio basado en la no existencia de mejoras después de un determinado número de ciclos.

En este trabajo se ha optado por este segundo tipo de criterio, habiéndose ensayado heurísticas con una y con dos ciclos sin mejora como criterio de parada (parámetro NCsmmax).

El diagrama de flujo del algoritmo se muestra en la Figura 4.6. 


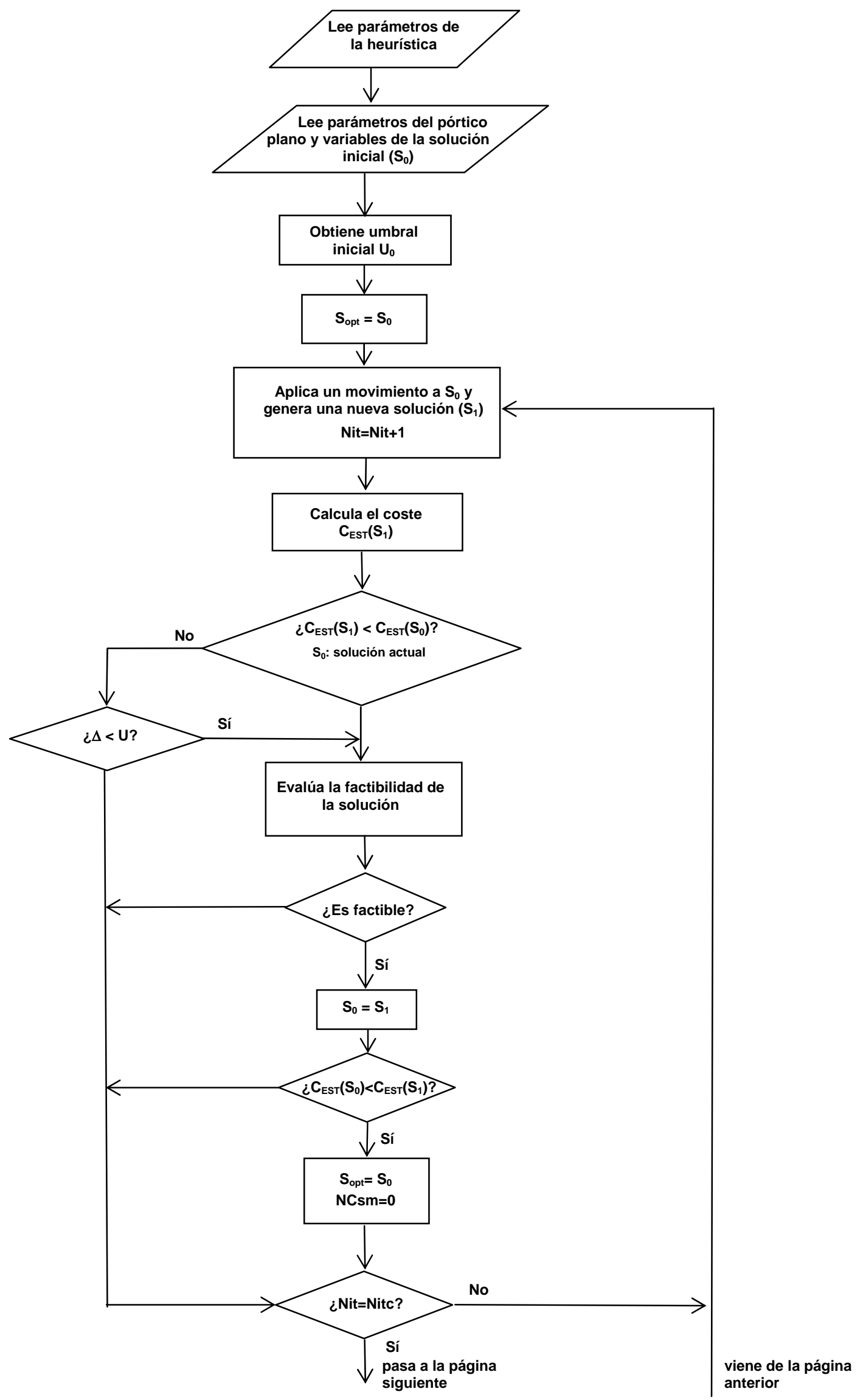

Figura 4.6. Diagrama de flujo de la heurística TA empleada en este trabajo (continua en la página siguiente). 


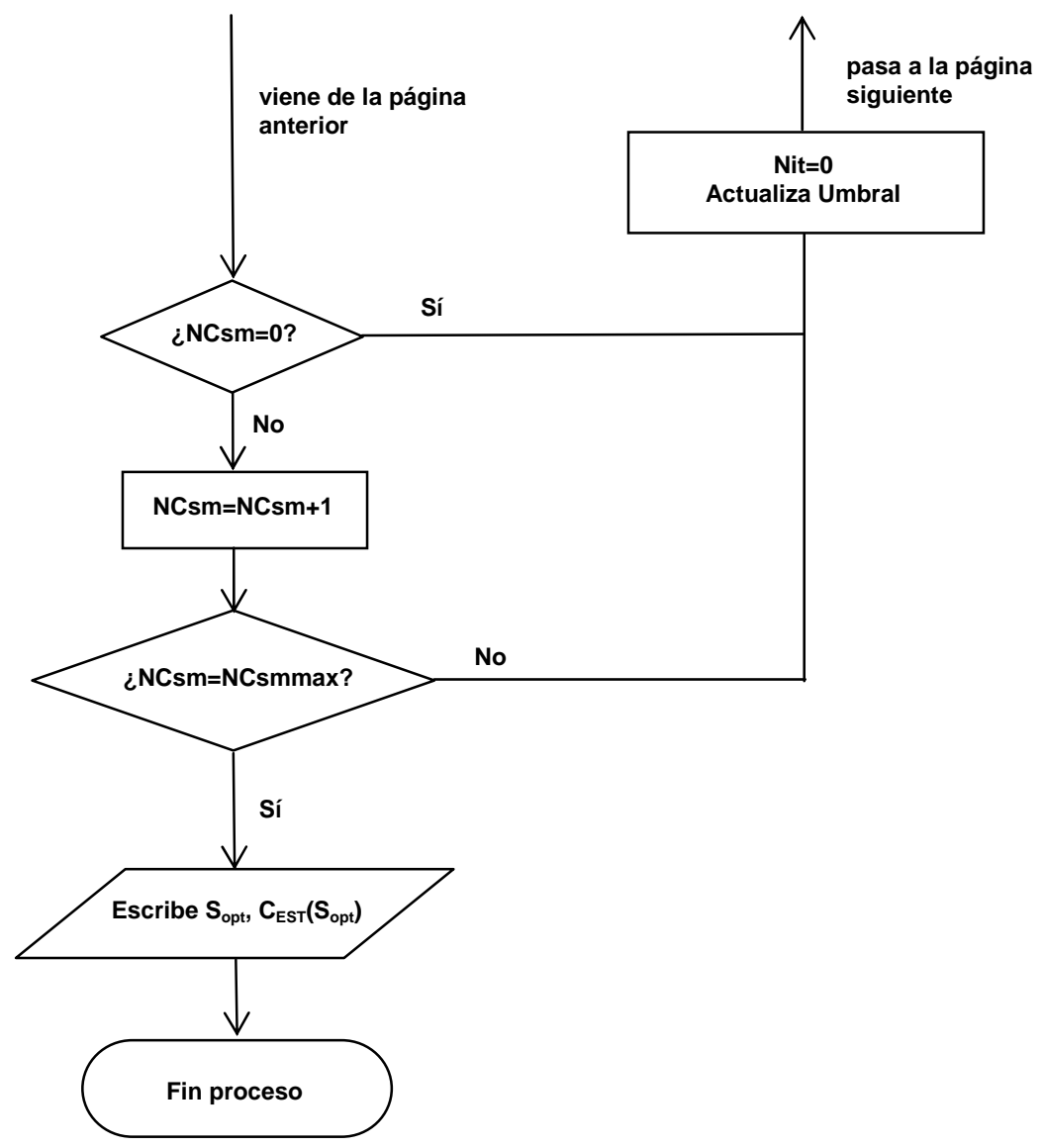

Figura 4.6. Diagrama de flujo de la heurística TA empleada en este trabajo (viene de la página anterior). El diagrama sirve igualmente para la minimización de $N_{s}$ y EC reemplazando $C_{E S T}$ por estas funciones objetivo.

\subsection{ALGORITMOS GENÉTICOS.}

\subsubsection{Introducción.}

En apartados anteriores se explicaron técnicas heurísticas que generalizan la búsqueda secuencial por entornos y que trabajan con una única solución. Se describen seguidamente los GA, que hacen evolucionar una población de individuos hacia zonas mejores del espacio de soluciones. Para ello se inspiran en los principios de la mecánica de la selección natural y en la genética. La aplicación de esta estrategia precisa de:

- Una representación de las soluciones del problema.

- Una forma de crear las soluciones potenciales.

- Una función de evaluación que juega el papel del ambiente, calificando a las soluciones producidas en términos de su "aptitud". 
- Unos operadores genéticos que permiten generar nuevos individuos a partir de una población inicial y hacerla evolucionar.

- Valores para los parámetros del algoritmo.

Una descripción detallada del funcionamiento de los GA y de algunas de sus aplicaciones puede encontrarse en Goldberg (1989), Coley (1999) y Coello (2004).

\subsubsection{Descripción general.}

La estructura básica de un GA se muestra en la Figura 4.7.

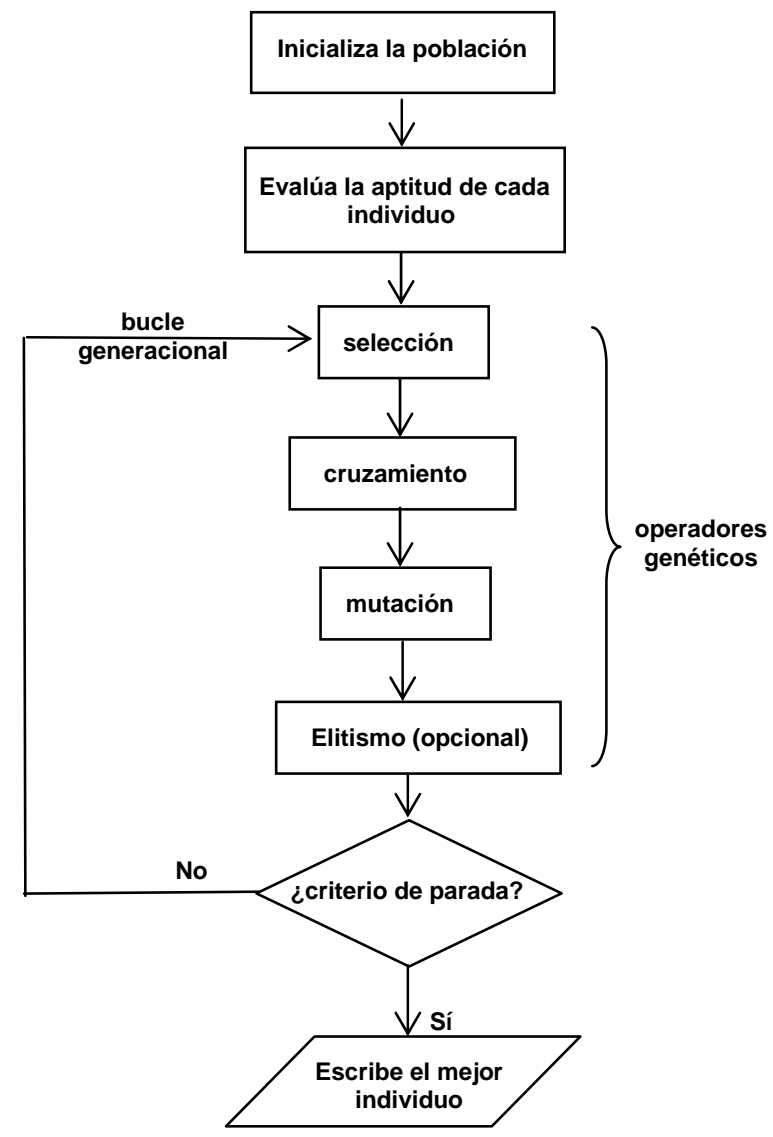

Figura 4.7. Diagrama de flujo de un GA básico.

El algoritmo se inicia con la creación de una población inicial de individuos (normalmente de un modo aleatorio, pero podrían usarse métodos determinísticos). Posteriormente se asigna una aptitud a cada uno de los elementos de la población dependiendo de su valor de la función de la función objetivo y del grado de cumplimiento de las restricciones del problema. Seguidamente, individuos de esta población son seleccionados de acuerdo con ciertas reglas (operador "selección") y combinados entre sí 
(operador "crossover" o "cruzamiento") para crear una nueva población. Finalmente, se emplean los operadores a) "mutación" para introducir nuevas características en la población, de modo que su evolución no se estanque prematuramente y, opcionalmente, b) "elitismo" que asegura que un determinado número de los mejores individuos de una generación pasen a la siguiente. Este proceso se repite hasta que se cumple el criterio de convergencia del algoritmo. Esta estrategia básica, sin el operador elitismo, se conoce en la literatura como el Simple Genetic Algorithm (SGA). Se explican a continuación con más detalle algunos de los elementos de esta estrategia.

\subsubsection{Terminología genética y representación de soluciones.}

En GA se llama cromosoma a una cadena de datos que contiene la información de una de las variables que definen una solución del problema. A cada posición de la cadena se le llama gen y, a su vez, un alelo es el valor particular que adopta un gen.

El modo tradicional de codificar una solución es el sistema binario, según el cual el cromosoma es una cadena de la forma $\left(b_{1}, \ldots, b_{m}\right)$ donde cada $b_{i}$ adopta un valor de " 0 " ó " 1 ". Holland (1975) y Goldberg (1989) justifican teóricamente las razones del empleo de esta representación. Otras formas son los códigos de Gray (una variante de la codificación binaria), la representación de punto flotante (en la que cada alelo puede tomar un valor entre 0 y 9) o la representación natural (en la que cada gen tiene un único alelo que es un número natural). Según el tipo de representación escogida, puede ser necesaria una función para codificar y decodificar los valores de las variables de diseño. Diferentes modos de realizar este proceso pueden consultarse en Coello (2004).

La figura 4.8 contiene una explicación de los conceptos anteriormente explicados.

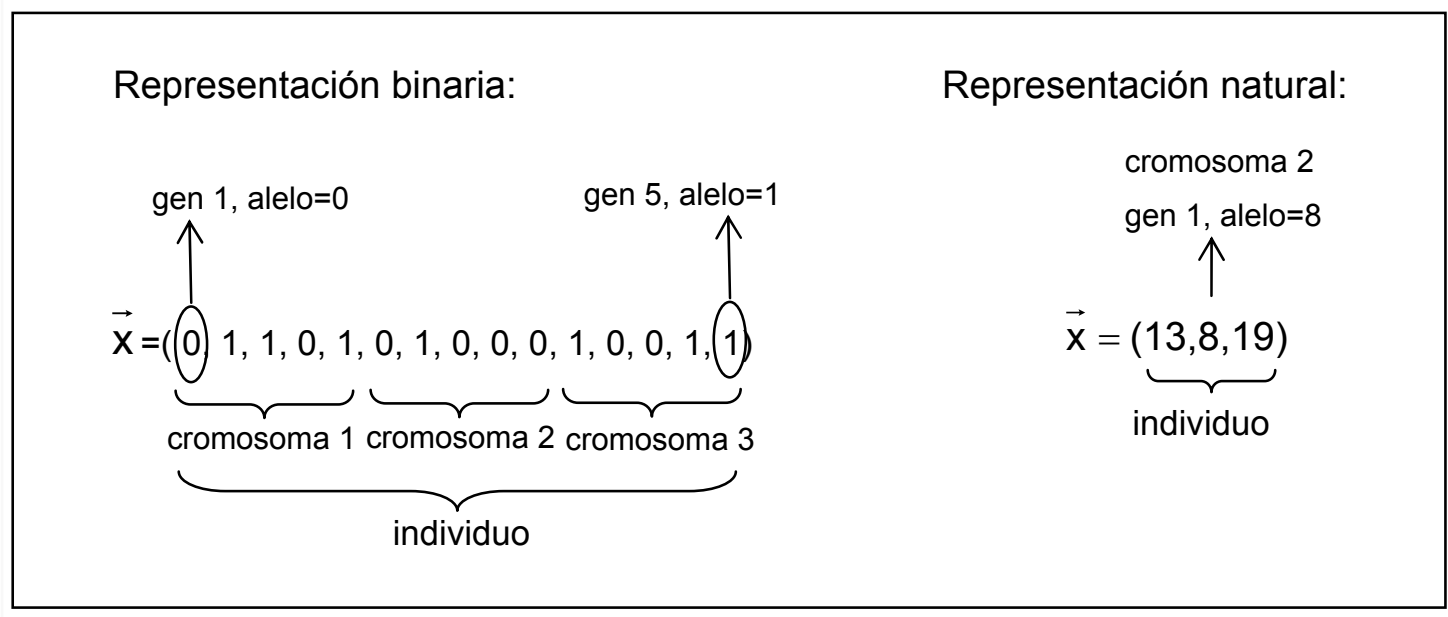

Figura 4.8. Terminología y ejemplos de representación de soluciones en GA. Se supone que una solución viene definida por un vector de tres componentes cada una de las cuales puede ser representada por un número binario de cinco dígitos. 


\subsubsection{Funciones de penalización y aptitud de un individuo.}

Los GA estaban inicialmente pensados para problemas en los que no hubiera restricciones, lo que no sucede en muchos casos prácticos. Por ello es necesario diseñar un elemento que permita incorporar información sobre la violación de las restricciones al valor de la función objetivo $f$. Estos elementos son las funciones de penalización y, suponiendo un problema de minimización, incrementan el valor de $f$ evaluada para una solución particular de forma variable en función del grado de violación de las restricciones. Así, en el caso de la optimización estructural, una solución que solamente incumple muy ligeramente un estado límite recibirá una penalización mucho menor que otra que lo incumpla en una elevada magnitud. A su vez, un mismo grado de violación puede ser penalizado de forma diferente en un ELS que en un ELU. Por ello, el valor de la función objetivo penalizada f' puede obtenerse como:

$$
f^{\prime}=f \times\left\{1+\sum_{i=1}^{i=k}\left[\Phi_{i}(\vec{x})\right]^{n_{i}}\right\}
$$

Donde $k$ es el número de restricciones del problema, $\Phi_{i}$ es la función de penalización asociada a la restricción número $i$ y $n_{i}$ es un exponente que determina la forma en la que se aplica la penalización (lineal, cuadrática y según la raíz cuadrada son las formas más habituales).

Una alternativa a las funciones de penalización es diseñar mecanismos reparadores de soluciones que faciliten la factibilidad de un individuo.

\subsubsection{Evaluación de la aptitud de un individuo.}

La aptitud o "fitness" $(F)$ de un miembro de la población se obtiene a través de la expresión (4.4) como:

$$
F=C-f^{\prime}\left(x_{1}, \ldots, x_{n}, p_{1}, \ldots p_{m}\right)
$$

Donde $C$ es una constante lo suficientemente grande como para que no se produzcan valores negativos del fitness y $f^{\prime}$ es el valor de la función objetivo penalizada para la solución considerada. 


\subsubsection{El operador "Selección".}

El mecanismo de la selección permite orientar la búsqueda hacia los puntos más prometedores del espacio de soluciones. La selección genera, a partir de una población de partida, otra intermedia reproduciendo con un mayor número de copias a los individuos más aptos y eliminando o asignando un menor número de copias a los individuos menos aptos. Este operador no produce soluciones nuevas, sino que únicamente determina quiénes van a dejar descendencia y en qué cantidad en la siguiente generación, en definitiva, decide quienes serán los padres de esa generación.

Los métodos de selección pueden clasificarse en tres grandes grupos: la selección proporcional, por torneo y de estado uniforme, siendo una técnica de selección proporcional, la de la ruleta, la más empleada (Coello, 2004). Esta estrategia fue propuesta por De Jong (1975) y la probabilidad de elección de un individuo es proporcional a su aptitud. Su algoritmo se muestra en la Figura 4.9 y ejemplos ilustrativos de su aplicación pueden encontrarse en Coello (2004) y Goldberg (1989).

1) Calcular el número de copias que se espera obtener de cada uno de los miembros de la población $\left(V_{e i}\right)$ :

$v_{e i}=\frac{F_{i}}{\sum_{j=1}^{j=n} F_{j}}$

2) Repetir "n" veces:

- Generar un número " $m$ " aleatorio entre 0 y $\sum_{j=1}^{j=n} F_{j}$.

- Ciclar a través de los individuos de la población sumando los valores esperados hasta que la suma sea mayor o igual a " $m$ ".

- $\quad$ El individuo que exceda esa suma será seleccionado.

Figura 4.9. Algoritmo de aplicación de la técnica de selección de la ruleta. Se supone una población de " $n$ " individuos y que la aptitud del individuo "i" es $F_{i}$.

\subsubsection{El operador "Cruzamiento" o "Crossover".}

El operador "cruzamiento" es el más importante de los operadores genéticos, pues permite el intercambio de información entre dos o más padres para producir descendientes (o hijos) que normalmente difieren de ellos. La idea es que, segmentos distintos de padres 
diferentes con alta aptitud, deberían producir al combinarse nuevos individuos también muy aptos. Este operador actúa con una probabilidad $p_{c}$ de modo que, en caso de no producirse el cruzamiento, los hijos producidos serían una copia exacta de los padres. Sus principales variantes se describen en la Figura 4.10 y son:

- Cruzamiento de un punto ("one point crossover", Figura 4.10). En este caso, el operador funciona con dos padres, determinando un punto para realizar el cruzamiento e intercambiando a partir de ese punto las componentes de los padres.

Punto de cruzamiento escogido aleatoriamente $y_{1}$

Padre $1(50,23,12,1,7,9 ! 11,3,43,12)$

$(50,23,12,1,7,9,10,3,40,13)$ Hijo 1

Padre $2(38,20,12,6,11,4,10,3,40,13)$

$(38,20,12,6,11,4,11,3,43,12)$ Hijo 2

Figura 4.10. Cruzamiento de un punto.

- Cruzamiento de dos puntos ("two points crosover", Figura 4.11). Es análoga a la anterior sólo que los padres intercambian información teniendo como base dos puntos elegidos al azar y no uno sólo.

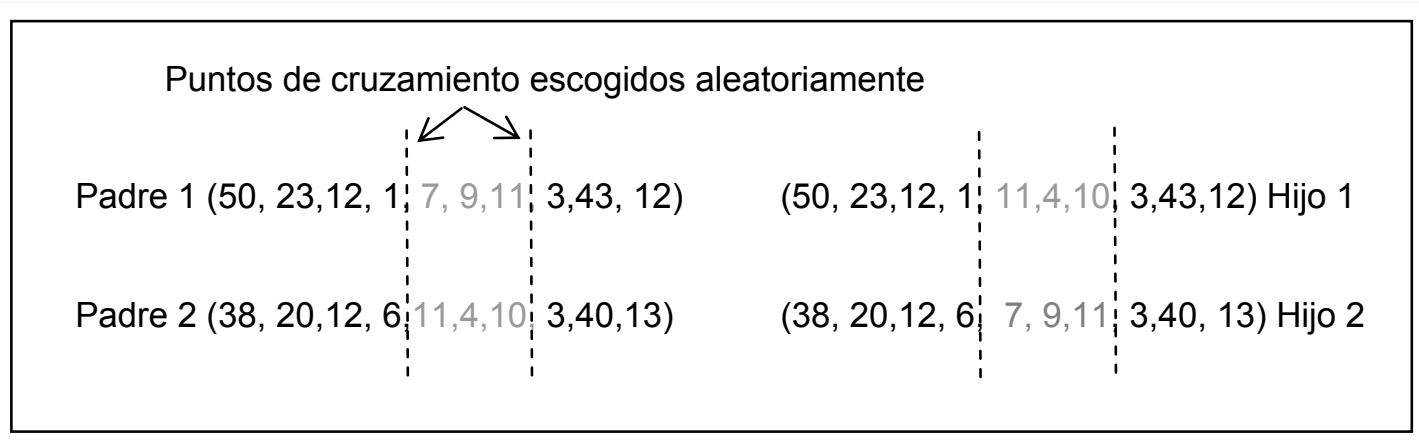

Figura 4.11. Cruzamiento de dos puntos.

- Cruzamiento uniforme ("uniform crossover", Figura 4.12). En este caso, es preciso definir previamente a la cruzamiento un vector $\vec{V}$ de la misma dimensión que los padres y en el que cada componente adopta un valor 0 o 1 de forma totalmente aleatoria. Para crear una componente del Hijo 1, se toma la componente del Padre 1 si la correspondiente componente del vector $\vec{V}$ es un 1 o la componente del Padre 2 si en esa posición encontramos un 0 en $\vec{V}$. Para 
definir el Hijo 2 se procede de forma recíproca: si en $\vec{V}$ hay un 0, el Hijo 2 toma la componente del Padre 1, y si hay un 1, del Padre 2.

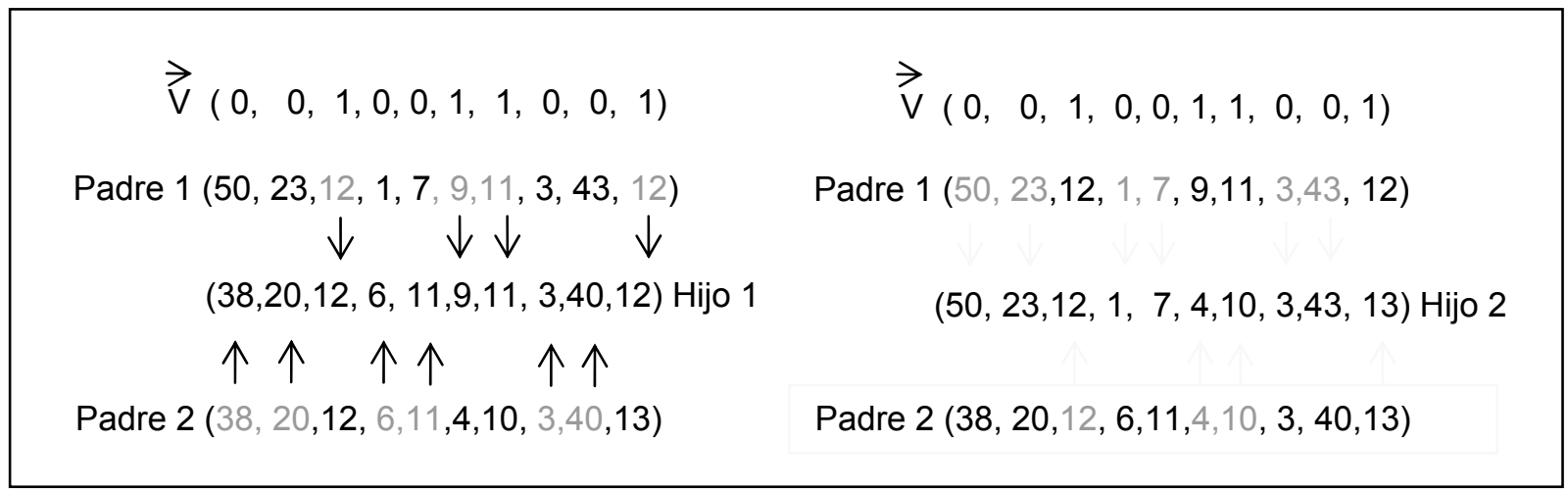

Figura 4.12. Cruzamiento uniforme.

Otros modos de realizar el cruzamiento y diferentes alternativas para definir el valor de $p_{c}$ pueden consultarse en Jenkins (1997).

\subsubsection{El operador "Mutación".}

En un SGA en el que se representan las soluciones mediante una cadena binaria, la mutación modifica ocasionalmente el valor de un bit, transformando un valor "0" en un " 1 " o la inversa. En el caso de representar un individuo con un vector de números enteros, la mutación se puede aplicar alterando en una magnitud aleatoria el valor de una de las componentes del vector. La mutación se produce con una probablilidad baja $p_{m}$ y tiene como misión evitar la pérdida de la diversidad en la población. Diferentes modos para definir el valor de $p_{m}$ pueden consultarse en Jenkins (1997).

\subsubsection{El operador "Elitismo".}

La aplicación del operador elitismo hace los miembros más aptos de una generación pasen a la siguiente sin verse alterados por los otros operadores genéticos. El elitismo asegura que la aptitud máxima de una población nunca se reducirá de una generación a la siguiente. Sin embargo, no necesariamente mejora la posibilidad de localizar el óptimo global de una función, aunque su uso se ha revelado vital para demostrar la convergencia de un GA (Rudolph, 1994). 


\subsubsection{Modificaciones del GA básico.}

\subsubsection{Introducción.}

EI GA mostrado en la Figura 4.7 con una estrategia elitista se utilizó para optimizar uno de los problemas planteados en esta investigación empleando una codificación de números naturales. Los resultados fueron un fracaso pues las únicas soluciones factibles producidas por el algoritmo eran las procedentes de generaciones anteriores por la aplicación del operador elitismo. Por ello se procedió a su mejora mediante la incorporación de:

- Una mejora de los individuos de una generación mediante una heurística de búsqueda local (apartado 4.6.3.2).

- Diseño de nuevas formas de aplicar el operador cruzamiento (apartado 4.6.3.3).

Otras técnicas han sido empleadas para mejorar el GA básico en aplicaciones a la ingeniería estructural. En estructuras de acero, Adeli y Cheng (1994a y b) introdujeron el método del Multiplicador Lagrangiano Aumentado y el procesado en paralelo, Rajev y Krishnamoorty (1997) emplearon cromosomas de longitud variable para incluir la topología en la optimización de celosías planas, Galante (1996) propuso un nuevo operador genético llamado "rebirth" que, cuando una búsqueda se estancaba, creaba una nueva población a partir de la solución óptima en un espacio de soluciones reducido y reiniciaba el algoritmo y Sarma y Adeli (2002) combinaron GA con la lógica difusa. También han sido propuestas mejoras en los operadores genéticos para asegurar un equilibrio entre la presión de selección y la exploración efectiva del espacio de soluciones (Leite y Topping, 1997) o para reducir su tamaño (Krishnamoorthy et al., 2002), de modo que se acelere la convergencia hacia la solución óptima. En hormigón armado, algunos autores han propuesto optimizaciones en varias etapas, combinando GA con una exploración intensiva del espacio de soluciones (Govindaraj y Ramasamy, 2005) o con el método de Hooke y Jeeves (Sahab et al., 2004, 2005).

\subsubsection{Algoritmos meméticos.}

Los algoritmos meméticos (MA) son una variante de los GA en la cada solución, antes de ser evaluada, es mejorada mediante una técnica de optimización apropiada al problema (ver Yepes, 2002). 
La versión de los MA empleada en este trabajo se muestra en la Figura 4.13. Cada individuo es optimizado mediante una heurística FB a la que se ha incorporado una función de penalización, de modo que es capaz de trabajar con soluciones factibles y no factibles.

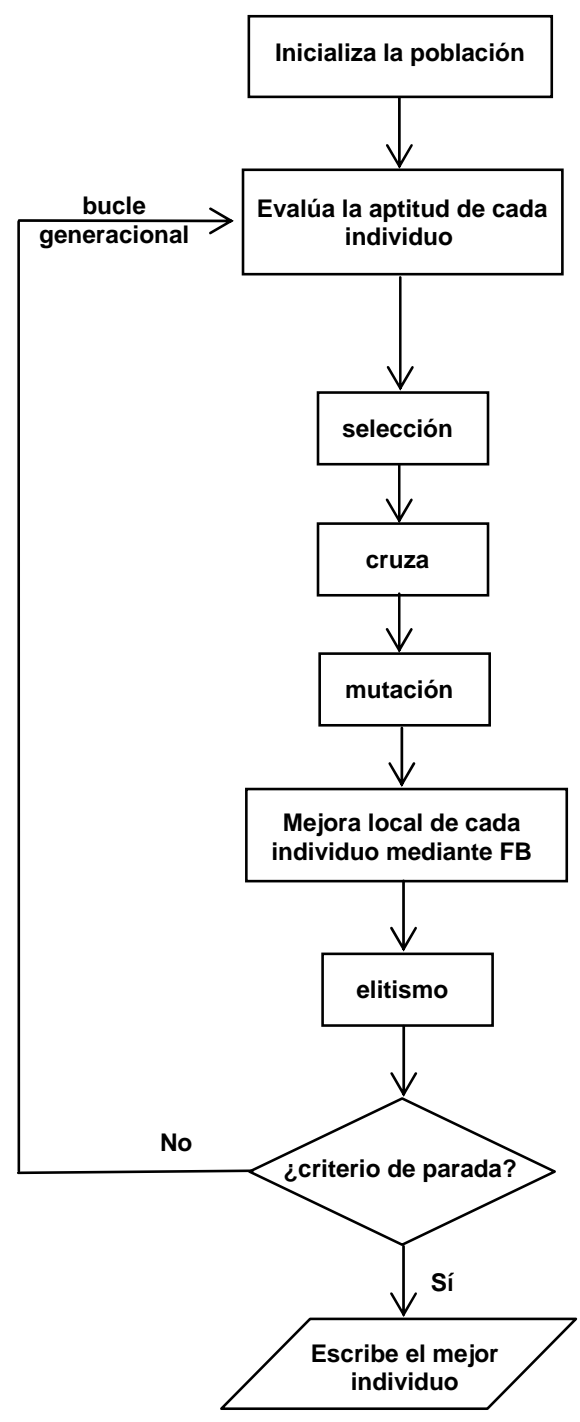

Figura 4.13. Diagrama de flujo de la heurística MA empleada en este trabajo.

\subsubsection{Nuevos modos de aplicar el operador "cruzamiento" .}

En trabajos anteriores se ha experimentado con diferentes tipos de cruzamientos pero en todos ellos el intercambio de variables se realizaba de forma aleatoria. En cambio, esta investigación experimenta con tres variantes diferentes de este operador:

1) Cruzamiento 1. 
Una posible desventaja de los cruzamientos aleatorios en problemas con muchas restricciones es que no tienen sentido físico. Por ello, en el cruzamiento es fácil perder la factibilidad de las soluciones de partida y generar soluciones de menor aptitud. En cambio, parece lógico pensar que, si dos individuos tienen una elevada aptitud, el resultado de intercambiar elementos homogéneos entre ellos será una pareja de individuos también muy aptos. Por ello, un pórtico genérico de los estudiados en este trabajo se ha dividido en unidades homogéneas (por ejemplo la armadura longitudinal de los pilares de una alineación o todos los elementos de las vigas de una planta) y las cruzamientos se han realizado en base a esas unidades. Se han definido 66 posibles cruces distintos que se indican en las figuras 4.14 y 4.15. $\mathrm{Si}$, por ejemplo, se escoge el número 5 unas vigas elegidas al azar intercambiarán su armadura de refuerzo inferior y su armadura transversal. Si se selecciona el cruce número 59, todos los pilares de dos pórticos de una alineación escogida aleatoriamente intercambiarán sus anchos y cantos. Este tipo de cruzamiento es una aportación original de esta Tesis.

2) Cruzamiento 2. Cruzamiento de un punto convencional .

3) Cruzamiento 3. Cruzamiento de " $m$ " puntos donde " $m$ " es un número aleatorio situado entre 2 y un valor máximo $m_{\max }$ definido por el usuario. En este estudio se ha tomado $m_{\max }=3$.

La elección del tipo de cruzamiento se realiza escogiendo un número de forma aleatoria entre 1 y 68 . Si el número está comprendido entre 1 y 66 se realiza el caso correspondiente del Cruzamiento 1, si es 67 se efectúa el Cruzamiento 2 y si es 68 el 3. 
Intercambios entre vigas

\begin{tabular}{|c|c|c|c|c|c|c|c|c|}
\hline & Número & $\mathbf{f}_{\mathrm{ck}}$ & b, h & $A_{\text {base,inf }}$ & $A_{\text {ref,inf }}$ & $A_{\text {base,sup }}$ & $A_{\text {ref,sup }}$ & $A_{\text {trans }}$ \\
\hline \multirow{7}{*}{ 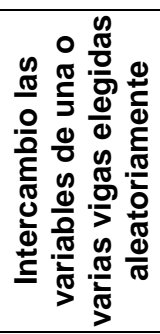 } & 1 & - & - & - & $x$ & - & & \\
\hline & 2 & & & & & & $x$ & \\
\hline & 3 & & & & & & & $x$ \\
\hline & 4 & & & & $x$ & & $x$ & \\
\hline & 5 & & & & $x$ & & & $x$ \\
\hline & 6 & & & & & & $x$ & $x$ \\
\hline & 7 & & & & $x$ & & $x$ & $x$ \\
\hline \multirow{33}{*}{ 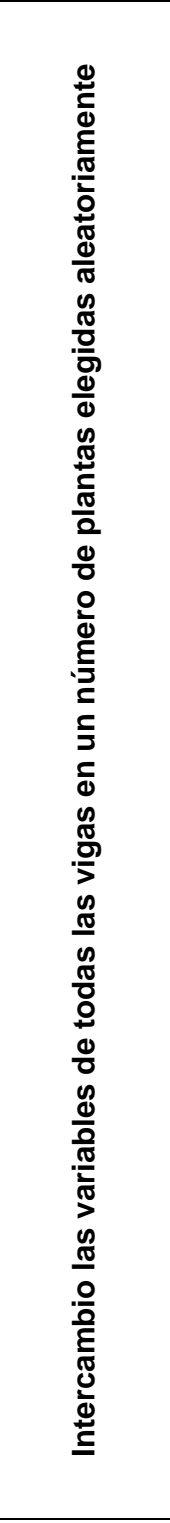 } & 8 & $x$ & & & & & & \\
\hline & 9 & & $x$ & & & & & \\
\hline & 10 & & & $x$ & & & & \\
\hline & 11 & & & & $x$ & & & \\
\hline & 12 & & & & & $x$ & & \\
\hline & 13 & & & & & & $x$ & \\
\hline & 14 & & & & & & & $x$ \\
\hline & 15 & $x$ & $x$ & & & & & \\
\hline & 16 & $x$ & & $x$ & & & & \\
\hline & 17 & $x$ & & & $x$ & & & \\
\hline & 18 & $x$ & & & & $x$ & & \\
\hline & 19 & $x$ & & & & & $x$ & \\
\hline & 20 & $x$ & & & & & & $x$ \\
\hline & 21 & & $x$ & $x$ & & & & \\
\hline & 22 & & $x$ & & $x$ & & & \\
\hline & 23 & & $x$ & & & $x$ & & \\
\hline & 24 & & $x$ & & & & $x$ & \\
\hline & 25 & & $x$ & & & & & $x$ \\
\hline & 26 & & & $x$ & $x$ & & & \\
\hline & 27 & & & $x$ & & $x$ & & \\
\hline & 28 & & & $x$ & & & $x$ & \\
\hline & 29 & & & $x$ & & & & $x$ \\
\hline & 30 & & & & $x$ & $x$ & & \\
\hline & 31 & & & & $x$ & & $x$ & \\
\hline & 32 & & & & $x$ & & & $x$ \\
\hline & 33 & & & & & $x$ & $x$ & \\
\hline & 34 & & & & & $x$ & & $x$ \\
\hline & 35 & & & & & & $x$ & $x$ \\
\hline & 36 & \multicolumn{7}{|c|}{ Intercambiar tres tipos de variables, elegidos éstos aleatoriamente } \\
\hline & 37 & \multicolumn{7}{|c|}{ Intercambiar cuatro tipos de variables, elegidos éstos aleatoriamente } \\
\hline & 38 & \multicolumn{7}{|c|}{ Intercambiar cinco tipos de variables, elegidos éstos aleatoriamente } \\
\hline & 39 & \multicolumn{7}{|c|}{ Intercambiar seis tipos de variables, elegidos éstos aleatoriamente } \\
\hline & 40 & $\mathrm{x}$ & $\mathrm{x}$ & $\mathrm{x}$ & $\mathrm{x}$ & $\mathrm{x}$ & $\mathrm{x}$ & $\mathrm{x}$ \\
\hline
\end{tabular}

Figura 4.14. Cruzamiento 1 en vigas. 
Intercambios entre pilares

\begin{tabular}{|c|c|c|c|c|c|}
\hline & Num & $f_{c k}$ & $\mathbf{b}, \mathbf{h}$ & $A_{\text {long }}$ & Atrans \\
\hline \multirow{7}{*}{ 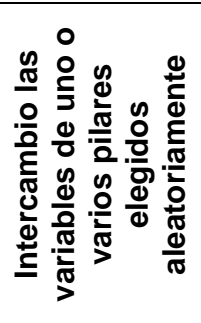 } & 41 & & $x$ & & \\
\hline & 42 & & & $x$ & \\
\hline & 43 & & & & $x$ \\
\hline & 44 & & $x$ & $x$ & \\
\hline & 45 & & $x$ & & $x$ \\
\hline & 46 & & & $x$ & $x$ \\
\hline & 47 & & $x$ & $x$ & $x$ \\
\hline \multirow{11}{*}{ 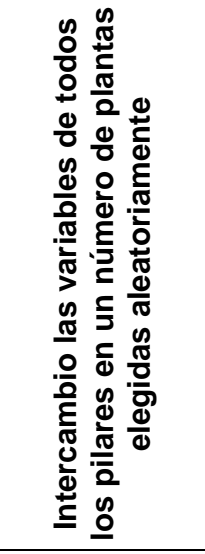 } & 48 & & $x$ & & \\
\hline & 49 & & & $x$ & \\
\hline & 50 & & & & $x$ \\
\hline & 51 & $x$ & $x$ & & \\
\hline & 52 & $x$ & & $x$ & \\
\hline & 53 & $x$ & & & $x$ \\
\hline & 54 & & $x$ & $x$ & \\
\hline & 55 & & $x$ & & $x$ \\
\hline & 56 & & & $x$ & $x$ \\
\hline & 57 & \multicolumn{4}{|c|}{$\begin{array}{l}\text { Intercambiar tres tipos de variables, elegidos estos } \\
\text { aleatoriamente }\end{array}$} \\
\hline & 58 & $x$ & $x$ & $x$ & $x$ \\
\hline \multirow{7}{*}{ 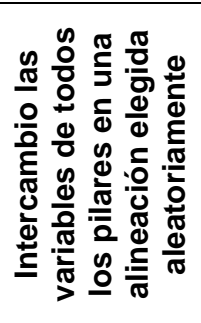 } & 59 & & $x$ & & \\
\hline & 60 & & & $x$ & \\
\hline & 61 & & & & $x$ \\
\hline & 62 & & $x$ & $x$ & \\
\hline & 63 & & $x$ & & $x$ \\
\hline & 64 & & & $x$ & $x$ \\
\hline & 65 & & $x$ & $x$ & $x$ \\
\hline
\end{tabular}

Otros posibles intercambios

\begin{tabular}{|c|c|}
\hline 66 & $\begin{array}{r}\text { Elegir aleatoriamente un movimiento de los de las vigas y } \\
\text { otro de los pilares y realizar ambos }\end{array}$ \\
\hline 67 & Cruzamiento 2 \\
\hline 68 & Cruzamiento 3 \\
\hline
\end{tabular}

Figura 4.15. Cruzamiento 1 en pilares y en pilares y vigas. Cruzamientos 2 y 3.

\subsubsection{Definición completa del algoritmo.}

Para poder aplicar esta heurística es necesario definir los elementos siguientes:

1) La forma de representar un individuo. 
2) El tamaño de la población (parámetro $n$ ).

3) La forma de las funciones de penalización.

4) La manera de realizar el operación selección.

5) El criterio de parada en la mejora local con FB (parámetro Nitsmmax).

6) La aplicación del elitismo.

7) El criterio de parada.

\subsubsection{Forma de representación.}

En este trabajo, una solución particular del problema es representada mediante un vector cuyas componentes son números naturales. Cada número natural se corresponde con un valor concreto de la variable que se obtiene de las tablas del Apéndice 1.

\subsubsection{Tamaño de la población.}

El tamaño de la población es uno de los parámetros más importantes del GA. Tamaños grandes permiten una mayor exploración del espacio de soluciones pero requieren tiempos mayores de cálculo. En este trabajo, se han empleado valores de 50 y 100 individuos. En otros trabajos (Sahab et al., 2005) $n$ no permanece constante a lo largo de la búsqueda sino que disminuye pues se agrupa a los individuos en función de su aptitud y se transmite una única copia de cada uno de los grupos a la siguiente generación.

\subsubsection{Funciones de penalización.}

La funciones de penalización $\Phi_{i}$ asociadas a una restricción $i$ vienen dadas por las expresiones siguientes:

$$
\begin{gathered}
\Phi_{i}(\vec{x})=0 \quad \text { si la solución no viola la restricción } i \\
\Phi_{i}(\vec{x})=\frac{\text { Magnitud de la violación de la restricción } i}{\text { Valor límite de la restricción }} \quad \text { en caso contrario }
\end{gathered}
$$

Así, si por ejemplo el momento último de una sección es de $94.58 \mathrm{KNm}$, y el momento de cálculo es de $98.30 \mathrm{KNm}$, el valor de la penalización asociada a ese incumplimiento será: 


$$
\Phi_{i}=\frac{98.30-94.58}{94.58}=0.0393
$$

En lo que respecta a los exponentes $n_{i}$, se han empleado diferentes valores en estudios anteriormente realizados con estructuras de hormigón. Govindaraj y Ramasamy (2005) han utilizado $n_{i}=2$ para todas las restricciones, Sahab et al. (2005) han realizado pruebas con $n_{i}=0.5, n_{i}=1, n_{i}=2$ también en todas las restricciones y no han apreciado diferencias significativas en los resultados. En este trabajo se ha adoptado $n_{i}=0.5$.

\subsubsection{Forma de aplicar el operador "selección".}

Como señala Coello (2004), la técnica de la ruleta no asegura que el número de copias transmitidas de un individuo a la siguiente generación coincida con el número esperado y, de hecho, el peor individuo puede ser seleccionado varias veces. En esta investigación, se ha implementado la técnica del torneo probabilístico cuyo funcionamiento se describe en la Figura 4.16. En cada torneo se han enfrentado dos individuos y se ha adoptado una probabilidad de que el vencedor del torneo sea el individuo más apto $p_{\text {tor }}=0.8$ (Coello -2004- recomienda valores entre 0.5 y 1). Esta estrategia se mostró más eficaz que la de la ruleta en el trabajo realizado por Sabah et al. (2005).

Repetir $p$ veces ( $p$ es el número de individuos que intervienen en el torneo).

1) Barajar los individuos de la población.

2) Escoger un número $p$ de individuos (normalmente $p=2$ ).

3) Comparar los $p$ individuos en base a su aptitud.

4) El ganador del torneo (padre para la siguiente generación) es el individuo más apto con una probabilidad $p_{\text {tor }}$. Si el individuo más apto pierde el torneo se escoge al menos apto.

Figura 4.16. Algoritmo de aplicación de la técnica de selección por torneo probabilístico.

\subsubsection{Criterio de parada en la mejora local con FB.}

Se han considerado valores de 100, 200, 400 y 1000 iteraciones sin mejora como criterios de parada en la heurística FB. 


\subsubsection{Elitismo.}

En cada generación se comprueba que la aptitud de su mejor individuo es superior a la del mejor individuo de la generación anterior. Si no es así, el individuo más apto de la generación anterior sustituye a un individuo de la generación actual tomado al azar.

\subsubsection{Criterio de parada.}

Diferentes criterios de parada son posibles. Éstos son relativos al número máximo de generaciones del algoritmo o al número máximo de generaciones en las cuales no se producen mejoras en la aptitud media o máxima de las soluciones factibles. En este estudio MA se detiene cuando se produce alguna de las condiciones siguientes:

1) Se alcanzan 1000 generaciones.

2) La aptitud media o máxima de las soluciones factibles no mejora en 20 generaciones.

\subsection{MÉTODO DE SUPPAPITNARM PARA LA OPTIMIZACIÓN MULTIOBJETIVO MEDIANTE LA CRISTALIZACIÓN SIMULADA.}

Para la optimización multicriterio se ha empleado una adaptación del algoritmo SMOSA propuesto por Suppapitnarm (2000). Como muestra el diagrama de flujo de la Figura 4.17, la estrategia se inicia con una solución inicial factible $S_{0}$ obtenida mediante la heurística RW. Posteriormente se procede a obtener una temperatura inicial para cada uno de los objetivos siguiendo el método propuesto por Medina para SA (2001) y explicado en el epígrafe 4.4.2.

Determinadas las temperaturas iniciales, se proporciona un movimiento a la solución inicial para obtener una nueva solución $S_{1}$ de modo análogo a como se realiza en la heurística SA. Llegados a este punto, el proceso depende de la función objetivo considerada:

- En caso de minimizar $N_{s} \circ E C$ se comprueba si $S_{1}$ pertenece a la Frontera de Pareto y, si pertenece, se comprueba su factibilidad. Si es factible se incorpora a dicho conjunto y sustituye a $S_{0}$ como solución actual.

- En caso de maximizar $\gamma_{o s}$ es necesario calcular y comprobar siempre la factibilidad de $S_{1}$, pues si no, no es posible obtener $\gamma_{\text {os. }}$ Si $S_{1}$ es factible y pertenece a la Frontera de Pareto se incorpora a dicho conjunto y sustituye a $S_{0}$ como solución actual. 


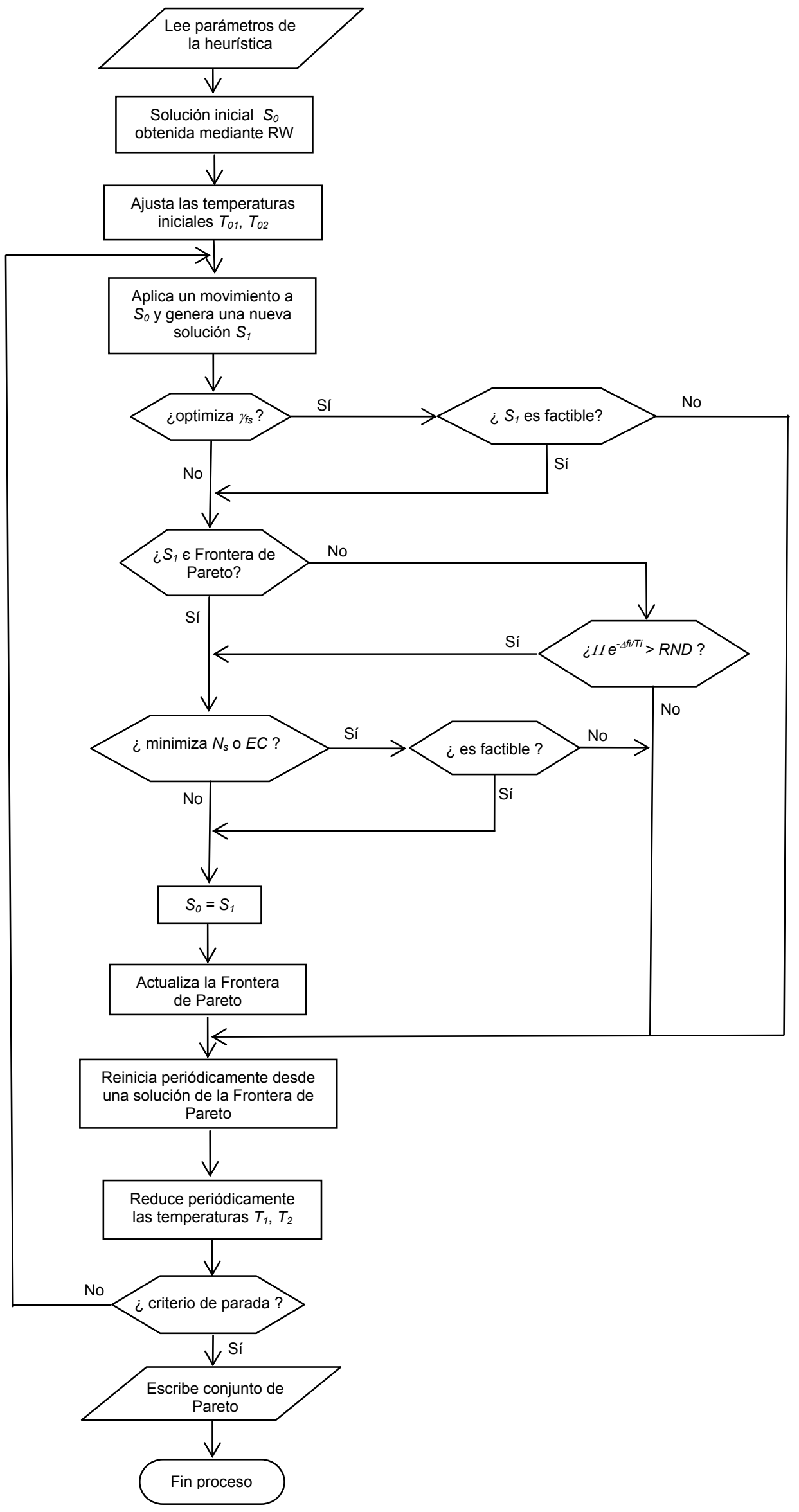

Figura 4.17. Diagrama de flujo del algoritmo SMOSA empleado en este trabajo. RND es un número aleatorio con una distribución uniforme de probabilidad entre 0 y 1. 
Si la solución $S_{1}$ es factible pero no pertenece a la Frontera de Pareto, no es descartada de forma automática como solución actual, sino que es aceptada como tal si el valor de la expresión (4.7) es superior a un número aleatorio con una distribución uniforme de probabilidad entre 0 y 1.

$$
\prod_{i=1}^{i=2} e^{-\frac{\Delta f_{i}}{T_{i}}}
$$

En la expresión (4.7) $T_{i}$ es el valor de la temperatura para el objetivo $i$. Para el valor de $\Delta f_{i}$ se han considerado las dos posibilidades indicadas en las expresiones (4.8) y (4.9):

$$
\begin{aligned}
& \Delta f_{i}=f_{i, 1}-f_{i, 0} \\
& \Delta f_{i}=\frac{f_{i, 1}-f_{i, 0}}{f_{i, 0}}
\end{aligned}
$$

Donde $f_{i, 1}$ and $f_{i, 0}$ son los valores de la función objetivo $i$ para $S_{1}$ y $S_{0}$ respectivamente. En la optimización del coste frente al número de barras y frente a la puntuación ambiental se han empleado ambas expresiones, mientras que en la optimización del coste frente al coeficiente de seguridad global solamente se ha empleado (4.8). En lo sucesivo se llama SMOSA1 a la versión del algoritmo SMOSA que emplea la expresión (4.8) y SMOSA2 a la que utiliza (4.9).

Las temperaturas de los objetivos se reducen como en SA transcurridos un número de iteraciones conocido como cadena de Markov. El algoritmo finaliza cuando tras un cierto número de cadenas de Markov, no se introduce ningún elemento nuevo en la Frontera de Pareto. Para evitar la estabilización prematura de la Frontera de Pareto y diversificar la exploración del espacio de soluciones, el algoritmo se reinicia periódicamente desde un punto de la misma. Este punto no se escoge de modo aleatorio, sino siguiendo el procedimiento expuesto por Suppapitnarm (2000). 


\subsection{EVALUACION DE HEURÍSTICAS.}

Previamente a la resolución de un problema de optimización por procedimientos heurísticos es necesario plantearse cómo se van a valorar los resultados obtenidos por las diferentes estrategias para decidir cual es la mejor. La comparación debería realizarse considerando los múltiples criterios que definen el comportamiento de las heurísticas como el tiempo empleado en el cálculo, la calidad de la solución encontrada, la facilidad de la codificación, la robustez... (Barr et al., 1995). Para abordarla, se emplea el concepto de Frontera de Pareto (véase el epígrafe 1.4.2).

En el caso de dos criterios (por ejemplo el tiempo necesario en obtener una solución y su coste económico), una estrategia es superior a otra si la supera en los dos criterios. En el caso de la Figura 4.18, los heurísticas que proporcionaron las soluciones "1" y "2" son mejores que la que obtuvo la solución " 5 " pues " 1 " y " 2 " tienen un coste inferior y precisaron de menos tiempo de cálculo. No obstante, no se puede afirmar que " 2 " es mejor que " 3 " o que "5" es mejor que " 3 " o "4", pues cada una de ellas es superior a la otra en uno de los criterios. En estos casos, es el decisor quien debe valorar las opciones posibles y escoger una de ellas (ver Yepes, 2002).

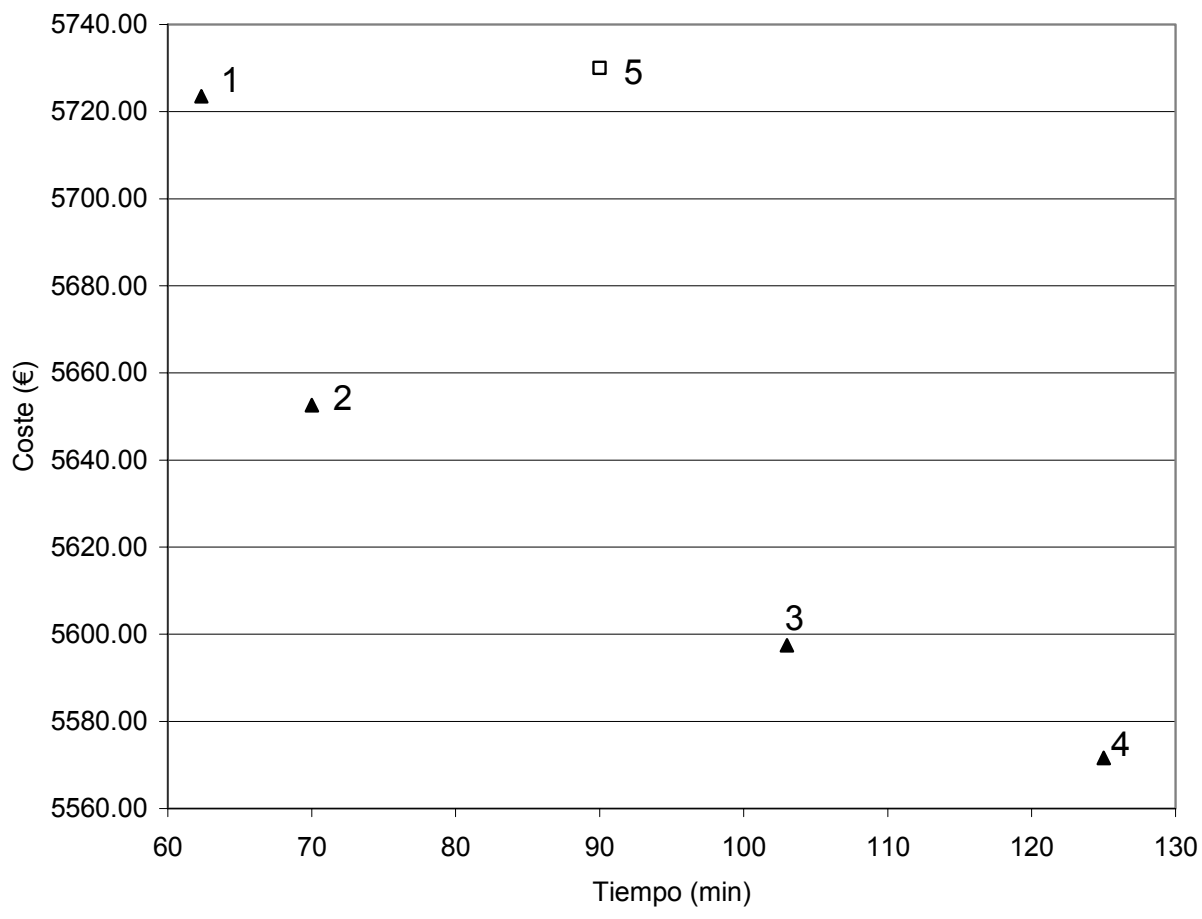

Figura 4.18. Comparación de heurísticas. 


\subsection{PROGRAMA PARA EL CÁlCULO Y OPTIMIZACIÓN DE PÓRTICOS PLANOS.}

La optimización de un pórtico definido por unos valores de sus parámetros y por otros de sus variables requiere de a) la comprobación de que el mismo cumple las restricciones o condiciones de diseño y b) la implementación de unas heurísticas capaces de resolver el problema de optimización.

Para efectuar estas tareas, el autor de esta Tesis ha desarrollado el programa “Optipor.2007" escrito en el lenguaje de programación Fortran y cuyo diagrama de flujo se muestra en la Figura 4.19. El programa tiene 27897 líneas de código y se ha implementado en un ordenador con un procesador Pentium IV con un procesador de $3.20 \mathrm{GHz}$ y $2 \mathrm{~GB}$ de memoria RAM y es capaz de realizar los siguientes trabajos:

1) Generar un fichero con los parámetros de un problema tipo, incluyendo la creación automática de las acciones de viento y de las distintas hipótesis de sobrecarga. El fichero generado tiene la extensión "*.opt”.

2) Proporcionar una o varias soluciones iniciales factibles del problema.

3) Obtener soluciones optimizadas empleando heurísticas previamente definidas por el usuario. Los valores de las variables correspondientes a cada óptimo local se escriben en otro fichero (extensión “*.ind”) que puede ser leído posteriormente.

Para ello, dados unos valores de los parámetros y de las variables de diseño, Optipor:

1) Realiza el cálculo de la estructura mediante un análisis estructural elástico y lineal por métodos de rigidez. Para ello, se ha creado un módulo específico que obtiene los esfuerzos y deformaciones correspondientes a los casos elementales de carga.

2) Obtiene las envolventes de esfuerzos y deformaciones (con los valores concomitantes) para cada una de las combinaciones indicadas por la EHE (ELS Poco Probable o Frecuente, ELS Cuasi-permanente y ELU).

3) Verifica el cumplimiento de las restricciones del problema comprobando la factibilidad o no factibilidad de la solución planteada (ficheros “*.res" con los resultados detallados de la comprobación de cada una de las restricciones y ficheros "*.fact" con un listado abreviado de los resultados relativos a la verificación de las restricciones y la conclusión final de si la solución es factible o no). 
4) Obtiene el valor de la función o funciones objetivos consideradas en el análisis, escribiendo los resultados en un fichero "*.med".

Señalar finalmente que:

1) Los ficheros "*.opt" y "*.ind" también pueden ser leídos por el programa para efectuar cálculos y comprobaciones.

2) Los ficheros “*.med", "*.res" y "*.fact" pueden ser generados o no a voluntad del usuario del programa.

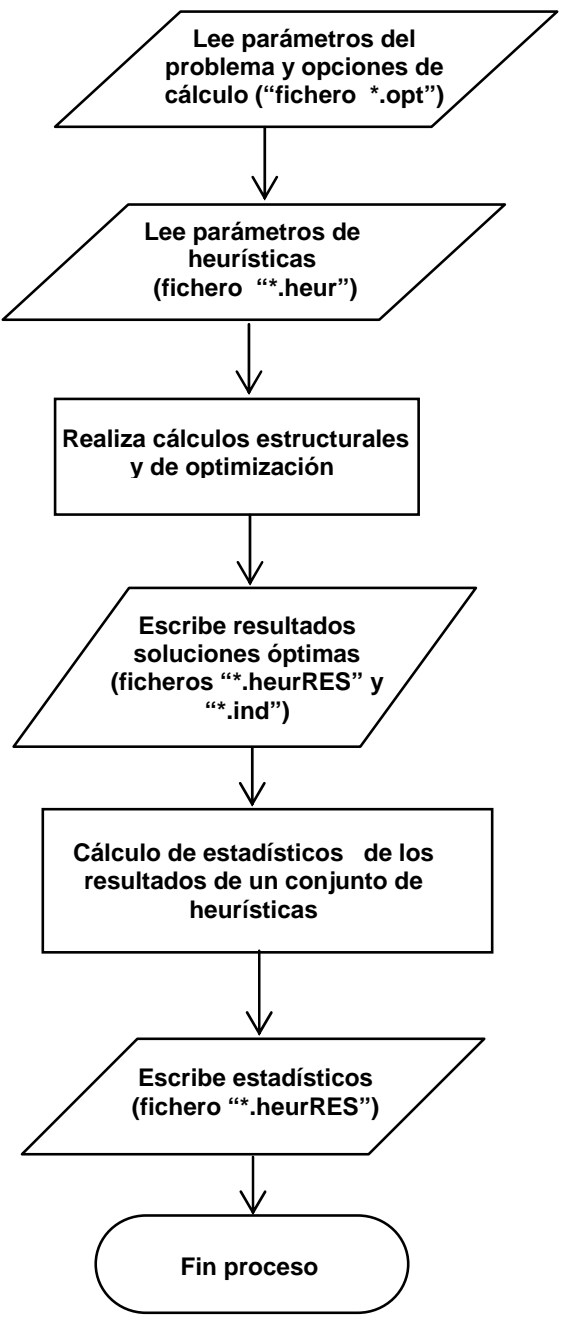

Figura 4.19. Diagrama de flujo del programa Optipor.2007. 
Página intencionadamente en blanco. 


\section{CAPÍTULO 5. RESULTADOS DE LA OTIMIZACIÓN ECONÓMICA DE UN PÓRTICO POR DIFERENTES PROCEDIMIENTOS HEURÍSTICOS.}




\section{Objetivos.}

Las heurísticas anteriormente descritas se emplean en este capítulo para minimizar el coste económico de un pórtico de dos vanos y cuatro plantas. Los resultados se comparan entre sí para determinar qué técnica proporciona los mejores resultados y, por lo tanto, se empleará para optimizar otras estructuras similares. 


\subsection{INTRODUCCIÓN.}

En este capítulo se aplican las heurísticas RW, FB, SA, TA y GA a la resolución del problema $P \_2 v 4 h-C_{E S T}$ (optimización económica del pórtico plano de dos vanos y cuatro alturas de la Figura 5.1). El problema tiene 77 variables que se detallan en la Tabla 5.1. Los resultados obtenidos mediante cada estrategia se proporcionan en los epígrafes 5.2 a 5.6 . Para obtener resultados estadísticamente representativos, cada experimento numérico consistente en obtener una estructura optimizada empleando unos determinados parámetros de una heurística, se repite nueve veces y se obtienen los estadísticos representativos de la muestra de resultados. La justificación del número de ensayos se efectúa en el apartado 5.7. Finalmente, el punto 5.8 contiene una discusión de los resultados obtenidos y determina cuál es el mejor procedimiento de los planteados para optimizar estas estructuras.

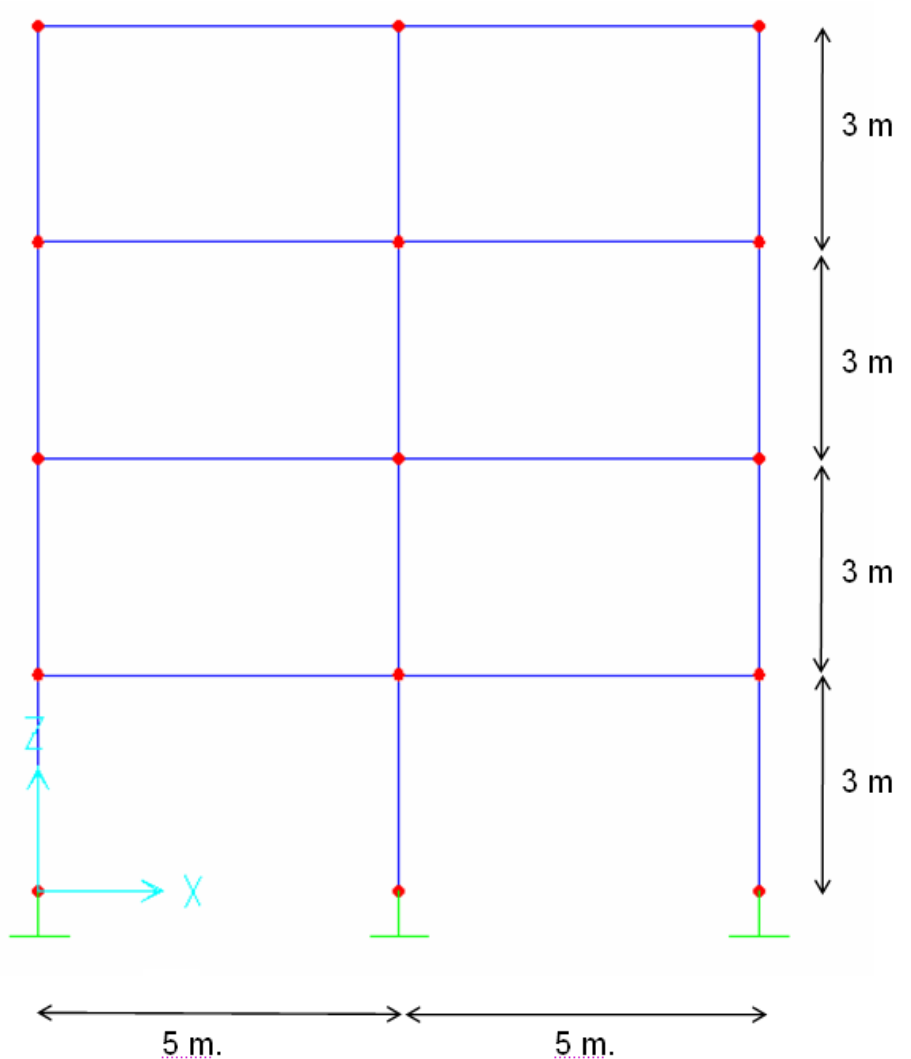

Figura 5.1. Definición geométrica del pórtico a optimizar. 
Tabla 5.1. Variables del pórtico simétrico de dos vanos y cuatro plantas (problema $P \_2 v 4 h-C_{E S T}$ ).

\begin{tabular}{lc}
\hline Descripción & $\begin{array}{c}\text { Número de } \\
\text { variables }\end{array}$ \\
\hline Límite elástico del acero en vigas y pilares $\left(\mathrm{N} / \mathrm{mm}^{2}\right)$ & 1 \\
\hline $\begin{array}{l}\text { Resistencia característica del hormigón en pilares y vigas } \\
\left(\mathrm{N} / \mathrm{mm}^{2}\right)\end{array}$ & 4 \\
\hline Ancho de vigas $(\mathrm{m})$ & 4 \\
\hline Canto de vigas $(\mathrm{m})$ & 4 \\
\hline Ancho de pilares $(\mathrm{m})$ & 8 \\
\hline Canto de pilares (m) & 8 \\
\hline Armadura base inferior en vigas $\left(\mathrm{cm}^{2}\right)$ & 4 \\
\hline Armadura de refuerzo inferior en vigas $\left(\mathrm{cm}^{2}\right)$ & 4 \\
\hline Armadura base superior en vigas $\left(\mathrm{cm}^{2}\right)$ & 4 \\
\hline Armadura de refuerzo superior en vigas $\left(\mathrm{cm}^{2}\right)$ & 8 \\
\hline Armadura transversal en vigas & 12 \\
\hline Armadura longitudinal en pilares $\left(\mathrm{cm}^{2}\right)$ & 8 \\
\hline Armadura transversal en pilares $\left(\mathrm{cm}^{2}\right)$ & 87 \\
\hline \multicolumn{1}{c}{ NúMERO TOTAL DE VARIABLES } & 8 \\
\hline
\end{tabular}

\subsection{ESTRATEGIA DE SALTOS MÚLTIPLES ALEATORIOS.}

Esta estrategia se ha aplicado con diferentes valores del parámetro $\mathrm{Nsol}$, mostrando los ensayos realizados que valores superiores a $N s o l=50000$ iteraciones no producen disminuciones en el coste de la solución optimizada encontrada (véase la Tabla 5.2). La Tabla 5.3 muestra los resultados detallados de la heurística que proporcionó la solución más económica y la Figura 5.2 los de una ejecución típica del algoritmo. 
Las características de la solución optimizada de menor coste se proporcionan en el Apéndice 5. El acero es B-400 S, el hormigón es HA-50 en la primera planta, HA-45 en la segunda y tercera y HA-40 en la cuarta. Su coste es de $7800.94 €$.

Tabla 5.2. Heurísticas RW ensayadas. Costes mínimos y tiempos de cálculo asociados.

\begin{tabular}{cccc}
\hline $\begin{array}{c}\text { Tipo de } \\
\text { heurística }\end{array}$ & Nsol & Coste mínimo $(€)$ & Tiempo (segs) \\
\hline RW1 & 500 & 12717.23 & 1 \\
\hline RW2 & 5000 & 7921.49 & 2 \\
\hline RW3 & 10000 & 7948.74 & 5 \\
\hline RW4 & 50000 & 7800.94 & 22 \\
\hline RW5 & 80000 & 7845.06 & 44 \\
\hline
\end{tabular}

Tabla 5.3. Resultados de la heurística RW4.

\begin{tabular}{ccc}
\hline $\begin{array}{c}\text { Número de } \\
\text { ejecución }\end{array}$ & Coste (€) & Tiempo (segs) \\
\hline 1 & 7985.99 & 23 \\
\hline 2 & 7800.94 & 22 \\
\hline 3 & 7956.26 & 20 \\
\hline 4 & 7940.97 & 21 \\
\hline 5 & 7870.87 & 23 \\
\hline 6 & 8261.32 & 21 \\
\hline 7 & 7946.70 & 22 \\
\hline 8 & 7950.09 & 24 \\
\hline 9 & 8042.88 & 23 \\
\hline Valor medio & 7972.89 & 22.11 \\
\hline Valor mínimo & 7800.94 & 20 \\
\hline Desviación típica & 127.84 & 1.27 \\
\hline Coef. de variación ${ }^{1}$ & 0.016 & 0.057 \\
\hline${ }^{1}$ Relación entre la desviación típica y el valor medio \\
\hline
\end{tabular}




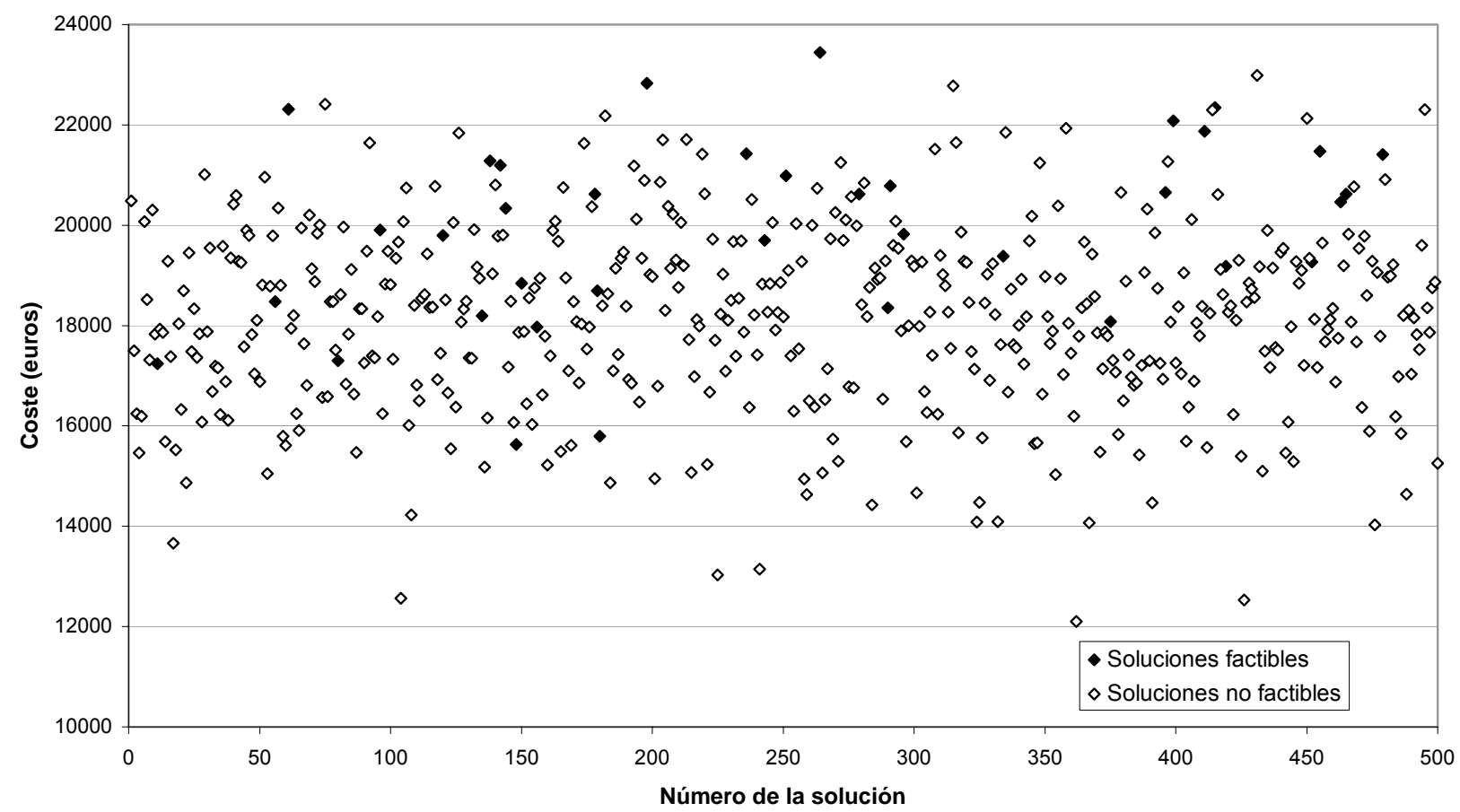

Figura 5.2. Ejemplo de los resultados de la aplicación de una heurística RW.

La estrategia RW no es una forma eficiente de abordar los problemas de optimización pues conduce a óptimos locales de baja calidad como se deduce de la comparación de los resultados obtenidos con los encontrados mediante otras heurísticas y que se muestran en el apartado 5.8. No obstante, es útil en tanto en cuanto proporciona:

1) Información sobre el espacio de soluciones (como por ejemplo el porcentaje de soluciones factibles respecto del total de soluciones posibles).

2) Soluciones que sirven de punto de partida a procedimientos heurísticos más complejos y eficientes.

\subsection{GRADIENTE FIRST BEST.}

El problema planteado se ha resuelto seguidamente mediante estrategias FB. Para calibrar los parámetros de la heurística (número de variables modificadas en cada movimiento - Nvar - y número máximo de iteraciones sin mejora de la solución actual Nitsmmax -) se han ensayado 62 combinaciones diferentes de los mismos (véase la Tabla 5.4). Como solución inicial se ha empleado la de menor coste obtenida mediante RW. La 
solución más económica se obtuvo modificando hasta un total de 3 variables en cada movimiento y adoptando Nitsmmax $=5000$.

Tabla 5.4. Parámetros definitorios de las heurísticas FB. Costes mínimos de las mejores soluciones encontradas.

\begin{tabular}{cccc}
\hline $\begin{array}{c}\text { Tipo de } \\
\text { heurística }\end{array}$ & Nvar & Nitsmmax & $\begin{array}{c}\text { Coste } \\
\text { mínimo (€) }\end{array}$ \\
\hline FB1 & 3 & $10,100,500,1000,3000,5000,7000,10000$ & 3803.36 \\
\hline FB2 & 6 & $10,100,500,1000,3000,5000$ & 4668.60 \\
\hline FB3 & 9 & $10,100,500,1000,3000,5000$ & 4941.68 \\
\hline FB4 & 12 & $10,100,500,1000,3000,5000$ & 6125.73 \\
\hline FB5 & 24 & $10,100,500,1000,3000,5000$ & 7800.94 \\
\hline FB6 & Hasta 3 & $10,100,500,1000,3000,5000$ & 3775.10 \\
\hline FB7 & Hasta 6 & $10,100,500,1000,3000,5000$ & 4483.93 \\
\hline FB8 & Hasta 9 & $10,100,500,1000,3000,5000$ & 4509.85 \\
\hline FB9 & Hasta 12 & $10,100,500,1000,3000,5000$ & 4462.33 \\
\hline FB10 & Hasta 24 & $10,100,500,1000,3000,5000$ & 4691.48 \\
\hline
\end{tabular}

La Figura 5.3 muestra la evolución del coste de la solución actual en una ejecución típica del algoritmo y permite observar cómo el coste de la solución actual es siempre decreciente. Por otro lado, la Tabla 5.5 resume los resultados obtenidos en las nueve ejecuciones de la estrategia que proporcionó la mejor solución optimizada (heurística FB6 con Nitsmmax=5000). Sus características se indican en los planos del Apéndice 4. El acero es B-500 S y el hormigón es HA-45 en todos los elementos. Su coste es de $3775.10 €$ y su cálculo ha requerido 3.3 minutos.

Es importante destacar que el empleo de la técnica FB6 ha permitido:

1) Reducir un $51.6 \%$ el coste de la mejor solución obtenida mediante RW.

2) Obtener el número de variables modificadas en cada movimiento que permite obtener mejores resultados. Este resultado se emplea posteriormente al aplicar las heurísticas SA y TA. 


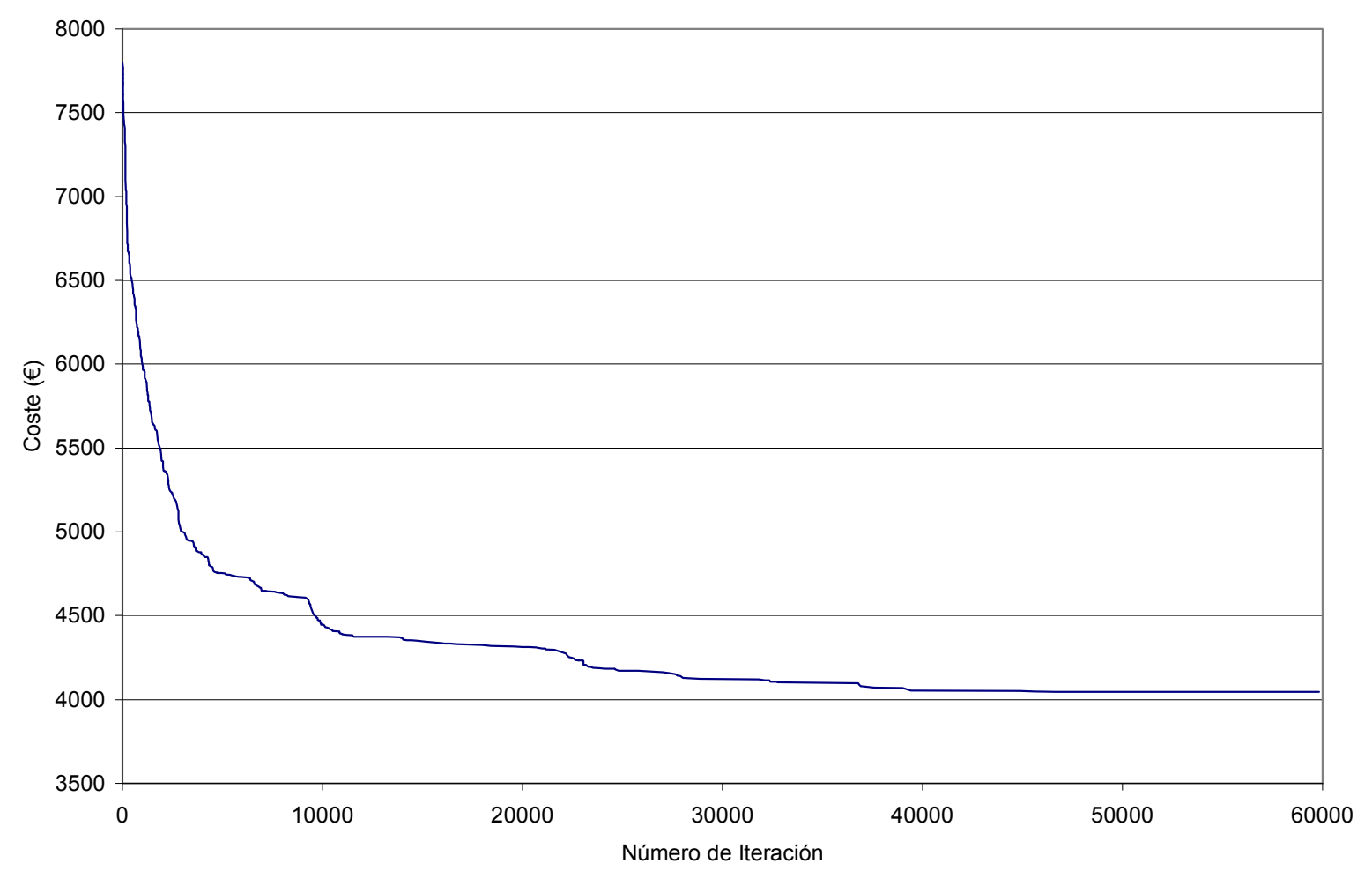

Figura 5.3. Evolución del coste de la solución actual en una ejecución típica del algoritmo FB.

\section{Tabla 5.5. Resultados de la heurística FB6 con Nitsmmax $=5000$.}

\begin{tabular}{ccc}
$\begin{array}{c}\text { Número de } \\
\text { ejecución }\end{array}$ & Coste $(€)$ & Tiempo (min) \\
\hline 1 & 3990.35 & 5.3 \\
\hline 2 & 3922.13 & 2.1 \\
\hline 3 & 4322.58 & 1.3 \\
\hline 4 & 4179.28 & 2.5 \\
\hline 5 & 3792.03 & 2.0 \\
\hline 6 & 3936.96 & 1.6 \\
\hline 7 & 3775.10 & 3.3 \\
\hline 8 & 4195.51 & 1.9 \\
\hline 9 & 4196.09 & 3.7 \\
\hline Valor medio & 4034.45 & 2.6 \\
\hline Valor mínimo & 3775.10 & 1.3 \\
\hline Desviación típica & 195.63 & 1.3 \\
\hline Coef. de variación & 0.048 & 0.5 \\
\hline
\end{tabular}




\subsection{CRISTALIZACIÓN SIMULADA.}

La solución del problema $P_{\_} 2 v 4 h-C_{E S T}$ se ha obtenido también mediante una heurística SA. En este caso, los parámetros de la heurística que hubo que calibrar son:

- El número de variables modificadas en cada movimiento (Nvar). Se han considerado únicamente los valores de "3" y "hasta 3" variables por ser los que proporcionaron soluciones de menor coste con la estrategia FB.

- La longitud de la cadena de Markov $(L m)$ o número de iteraciones en las que la temperatura permanece constante.

- El coeficiente de enfriamiento $(r)$.

- El número máximo de cadenas de Markov sin mejoras de la solución optimizada (NCMsmmax) que marcan el final de la ejecución del algoritmo.

Se han probado 63 combinaciones diferentes de valores de los parámetros para optimizar el pórtico propuesto (véase la Tabla 5.6). La estructura de menor coste se obtuvo mediante una heurística con los siguientes parámetros: Nvar=hasta 3 variables, $\quad r=0.8$ y NCMsmmax=2. La Tabla 5.7 contiene los resultados de las nueve ejecuciones de esta estrategia. Como solución de partida siempre se ha empleado la misma que para el FB.

El coste de la mejor solución encontrada ha sido de $3473.06 €$, empleándose un tiempo de cálculo de 18.9 minutos. Los planos de esta solución se dan en el Apéndice 4. El acero es B-500 S y el hormigón de todos los elementos es HA-45.

La Figura 5.4 muestra un ejemplo de la evolución de las temperaturas y de los costes de la solución actual en una ejecución típica del algoritmo. En ella se puede observar como:

- Se aceptan soluciones que empeoran el coste.

- A medida que el algoritmo avanza, las magnitudes de los incrementos del coste de la solución actual son menores. Ello es debido a la reducción de la temperatura. 
Tabla 5.6. Parámetros definitorios de heurísticas SA. Costes mínimos de las mejores soluciones encontradas.

\begin{tabular}{cccccc}
\hline $\begin{array}{c}\text { Tipo de } \\
\text { heurística }\end{array}$ & Nvar & Lm & $\mathbf{r}$ & NCMsmmax & $\begin{array}{c}\text { Coste } \\
\text { mínimo (€) }\end{array}$ \\
\hline SA1 & 3 & $\begin{array}{c}500,1000,3000, \\
5000\end{array}$ & 0.99 & 1,2 & 5391.48 \\
\hline SA2 & 3 & $\begin{array}{c}500,1000,3000, \\
5000\end{array}$ & $0.90,0.80$ & 1,2 & 4469.85 \\
\hline SA3 & 3 & 7000,10000 & $0.90,0.80$ & 2 & 3790.99 \\
\hline SA4 & 3 & 70000 & 0.80 & 2 & 3547.18 \\
\hline SA5 & Hasta 3 & $500,1000,3000$, & 0.99 & 1,2 & 4346.44 \\
\hline SA6 & Hasta 3 & $500,1000,3000$, & $0.90,0.80$ & 1,2 & 3941.8 \\
\hline SA7 & Hasta 3 & 70000,10000 & $0.90,0.80$ & 2 & 3473.06
\end{tabular}

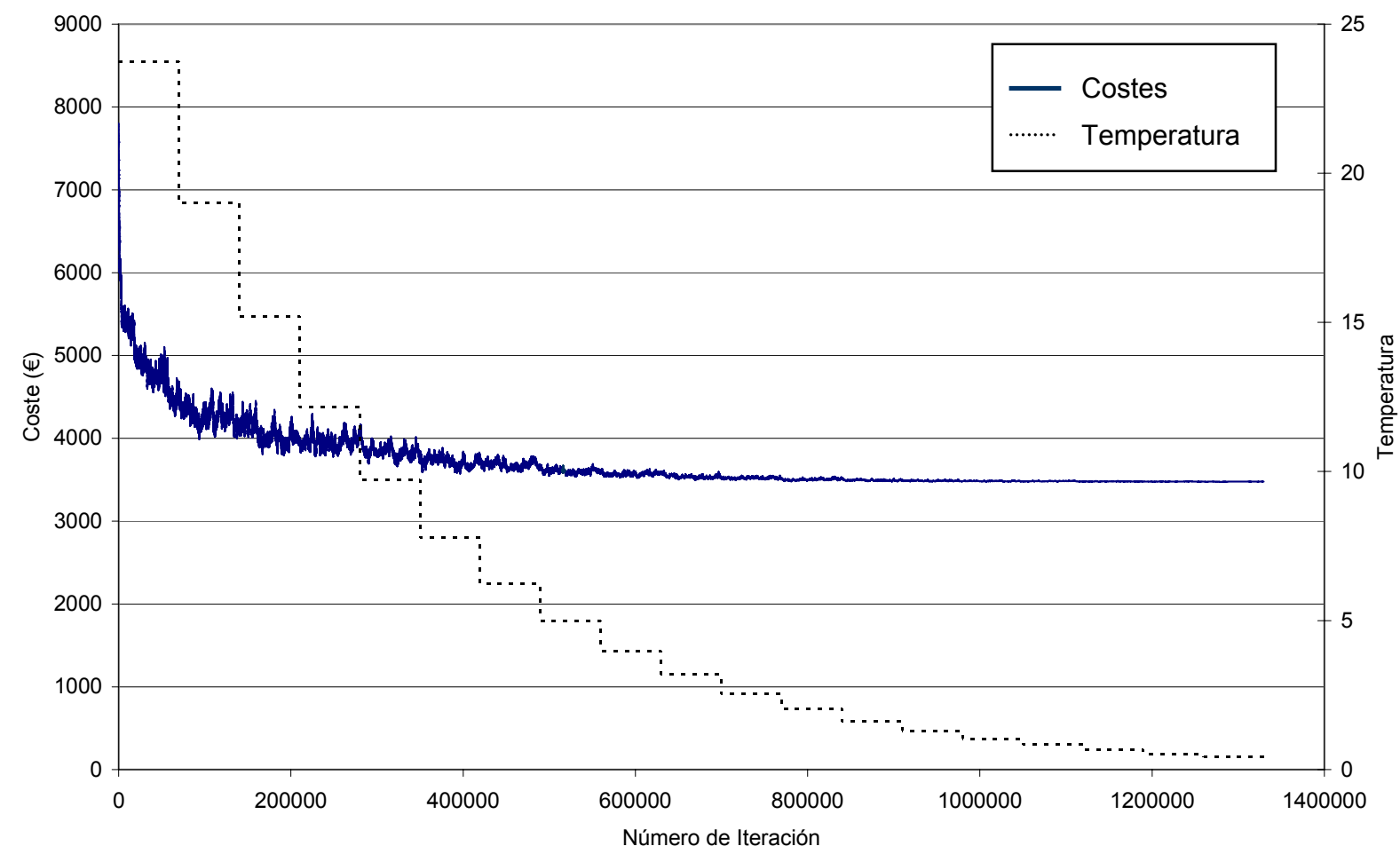


Figura 5.4. Evolución de la temperatura y del coste de la solución actual en una ejecución típica del algoritmo $S A$.

\begin{tabular}{ccc}
\hline \multicolumn{3}{c}{ Tabla 5.7. Resultados de la heurística SA7 con } \\
r=0.8.
\end{tabular}

\subsection{ACEPTACIÓN POR UMBRALES.}

El pórtico de la Figura 5.1 se ha optimizado empleando la versión del algoritmo TA propuesta en el epígrafe 4.5. Para ello fue necesario calibrar los parámetros siguientes:

- El número de variables modificadas en cada movimiento (Nvar). Se han considerado únicamente los valores de "3" y "hasta 3" variables por ser los que proporcionaron soluciones de menor coste con la estrategia FB.

- El número de iteraciones por ciclo en los que el umbral permanece constante (Nitc). 
- El coeficiente de enfriamiento $(r)$.

- El número máximo de ciclos sin mejoras de la solución optimizada (NCsmmax) que marcan el final de la ejecución del algoritmo.

Se han probado 52 combinaciones diferentes de valores de los parámetros para optimizar el pórtico propuesto (véase la Tabla 5.8), obteniéndose la solución de coste mínimo con Nvar=hasta 3 variables, $r=0.8$ y NCsmmax=2. Como solución de partida siempre se ha utilizado la mejor solución obtenida con RW.

Tabla 5.8. Parámetros definitorios de las heurísticas TA. Costes mínimos de las mejores soluciones encontradas.

\begin{tabular}{cccccc}
\hline $\begin{array}{c}\text { Tipo de } \\
\text { heurística }\end{array}$ & Nvar & Nitc & $\mathbf{r}$ & NCsmmax & $\begin{array}{c}\text { Coste } \\
\text { mínimo (€) }\end{array}$ \\
\hline TA1 & 3 & $\begin{array}{c}500,1000,3000,5000, \\
70000\end{array}$ & $0.99,0.90,0.80$ & 1,2 & 3545.08 \\
\hline TA2 & Hasta 3 & $\begin{array}{c}500,1000,3000,5000, \\
70000\end{array}$ & $0.99,0.90,0.80$ & 1,2 & 3491.31 \\
\hline
\end{tabular}

La Figura 5.5 muestra un ejemplo de la evolución del umbral de aceptación y de los costes de la solución actual en una ejecución típica. En ella se observa como se aceptan soluciones que empeoran el coste y como la tolerancia hacia diseños peores disminuye conforme avanza el algoritmo por la reducción del umbral.

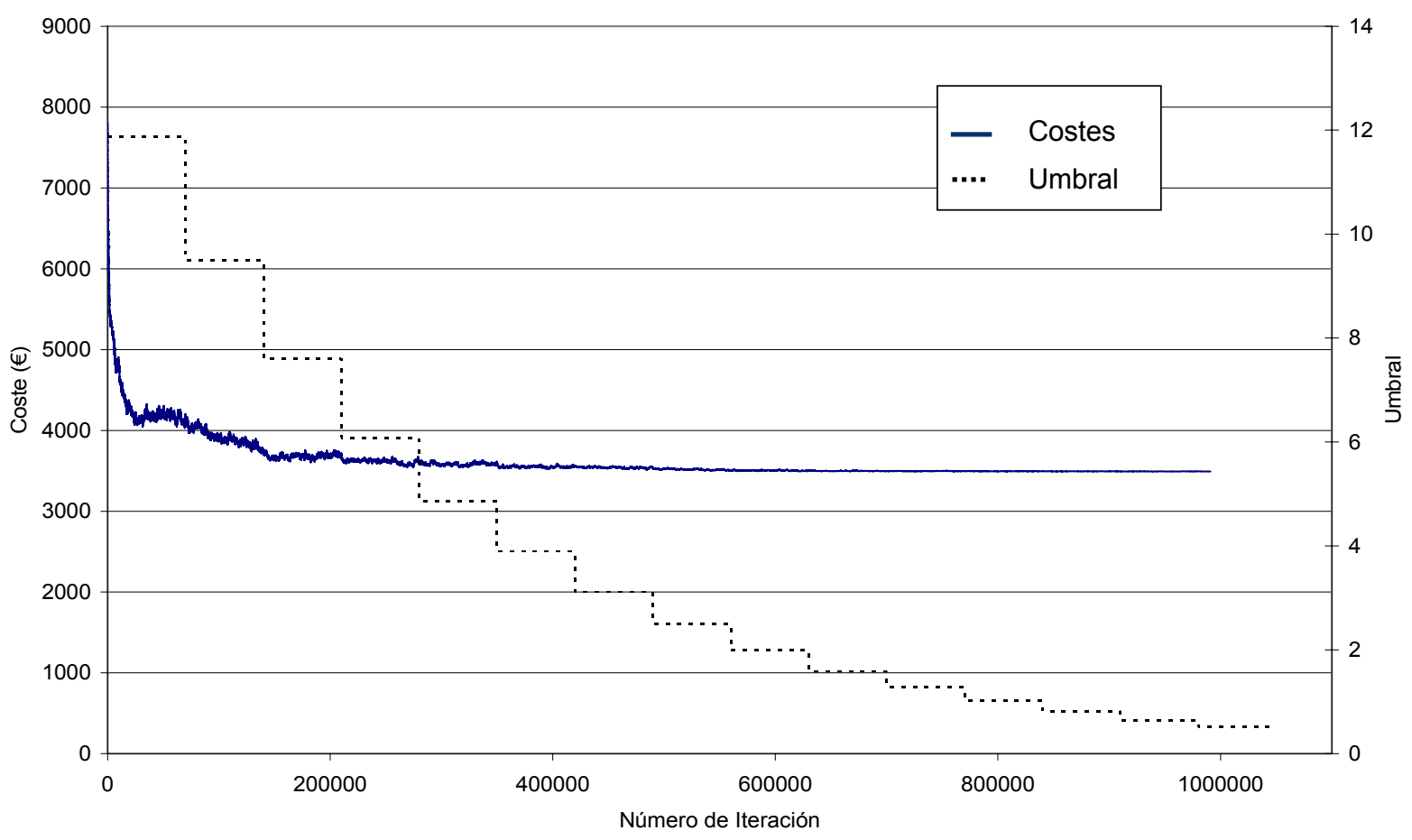

Figura 5.5. Evolución del umbral y del coste de la solución actual en una ejecución típica delalgoritmo TA. 
La Tabla 5.9 contiene los resultados de las nueve ejecuciones realizadas correspondientes a los parámetros del algoritmo que proporcionaron la solución optimizada de coste mínimo. Éste es de $3491.31 €$ y la estrategia empleó un tiempo de cálculo de 19.9 minutos. El Apéndice 5 contiene la definición completa de esta estructura. Su acero es B-500 S y el hormigón de todos los elementos es HA-45.

Tabla 5.9. Resultados de la heurística TA2 con Nvar=Hasta 3, $r=0.8$ y NCsmmax $=2$.

\begin{tabular}{ccc}
\hline $\begin{array}{c}\text { Número de } \\
\text { ejecución }\end{array}$ & Coste (€) & Tiempo (min) \\
\hline 1 & 3593.85 & 16.9 \\
\hline 2 & 3598.69 & 18.2 \\
\hline 3 & 3555.81 & 18.9 \\
\hline 4 & 3537.69 & 20.9 \\
\hline 5 & 3491.31 & 19.9 \\
\hline 6 & 3558.60 & 19.9 \\
\hline 7 & 3562.16 & 18.9 \\
\hline 8 & 3509.33 & 29.8 \\
\hline 9 & 3511.91 & 21.6 \\
\hline Valor medio & 3546.59 & 20.6 \\
\hline Valor mínimo & 3491.31 & 16.9 \\
\hline Desviación típica & 37.33 & 3.7 \\
\hline Coef. de variación & 0.0095 & 0.18 \\
\hline
\end{tabular}




\subsection{ALGORITMOS GENÉTICOS.}

Para poder aplicar la versión del GA explicada en el epígrafe 4.6 es necesario definir:

- El número de individuos de la población inicial $(n)$.

- El número de iteraciones sin mejora de la solución actual (Nitsm) que marca el final de la mejora local mediante FB que se aplica a cada individuo.

Los valores empleados en este trabajo de $n$ y de Nitsm junto con los principales resultados obtenidos se indican en la Tabla 5.10. La población inicial se ha obtenido mediante RW con un parámetro Nsol=50000. Por su parte, la Tabla 5.11 contiene los resultados de las nueve ejecuciones de la heurística que proporcionó la solución de menor coste (GA4, con $n=50$ individuos y Nitsm=1000 iteraciones) y la Figura 5.6 muestra la evolución de la solución factible de coste mínimo a lo largo de una ejecución típica del algoritmo.

Tabla 5.10. Parámetros definitorios de las heurísticas GA. Resultados obtenidos.

\begin{tabular}{ccccccccc}
\hline $\begin{array}{c}\text { Tipo de } \\
\text { heurística }\end{array}$ & $\mathbf{n}$ & Nitsm & $\begin{array}{c}\text { Coste } \\
\text { mínimo }(€)\end{array}$ & $\begin{array}{c}\text { Tiempo } \\
\text { mínimo } \\
(\mathbf{m i n})\end{array}$ & $\begin{array}{c}\text { Coste } \\
\text { medio }(€)\end{array}$ & $\begin{array}{c}\text { Tiempo } \\
\text { medio } \\
(\mathbf{m i n})\end{array}$ & $\sigma_{\text {coste }}$ & $\sigma_{\text {tiempo }}$ \\
\hline GA1 & 50 & 100 & 4006.49 & 21.6 & 4196.82 & 31.6 & 161.8 & 7.6 \\
\hline GA2 & 50 & 200 & 3860.12 & 33.4 & 4090.03 & 45.6 & 151.1 & 10.3 \\
\hline GA3 & 50 & 400 & 3693.97 & 27.6 & 4029.62 & 63.8 & 252.3 & 26.8 \\
\hline GA4 & 50 & 1000 & 3672.91 & 79.6 & 3879.69 & 121.4 & 136.8 & 36.7 \\
\hline GA5 & 100 & 200 & 3723.28 & 63.7 & 3911.45 & 97.6 & 157.4 & 26.4 \\
\hline
\end{tabular}


Tabla 5.11. Resultados de la heurística GA4.

\begin{tabular}{ccc}
\hline $\begin{array}{c}\text { Número de } \\
\text { ejecución }\end{array}$ & Coste $(€)$ & Tiempo (min) \\
\hline 1 & 3990.07 & 95.5 \\
\hline 2 & 4028.86 & 104.8 \\
\hline 3 & 3937.77 & 151.3 \\
\hline 4 & 3850.71 & 161.2 \\
\hline 5 & 3785.65 & 179.0 \\
\hline 6 & 3791.67 & 95.1 \\
\hline 7 & 4082.61 & 86.1 \\
\hline 8 & 3776.92 & 79.6 \\
\hline 9 & 3672.91 & 140.2 \\
\hline Valor medio & 3879.69 & 121.4 \\
\hline Valor mínimo & 3672.91 & 79.6 \\
\hline Desviación típica & 136.81 & 36.7 \\
\hline Coef. de variación & 0.0352 & 0.302 \\
\hline
\end{tabular}

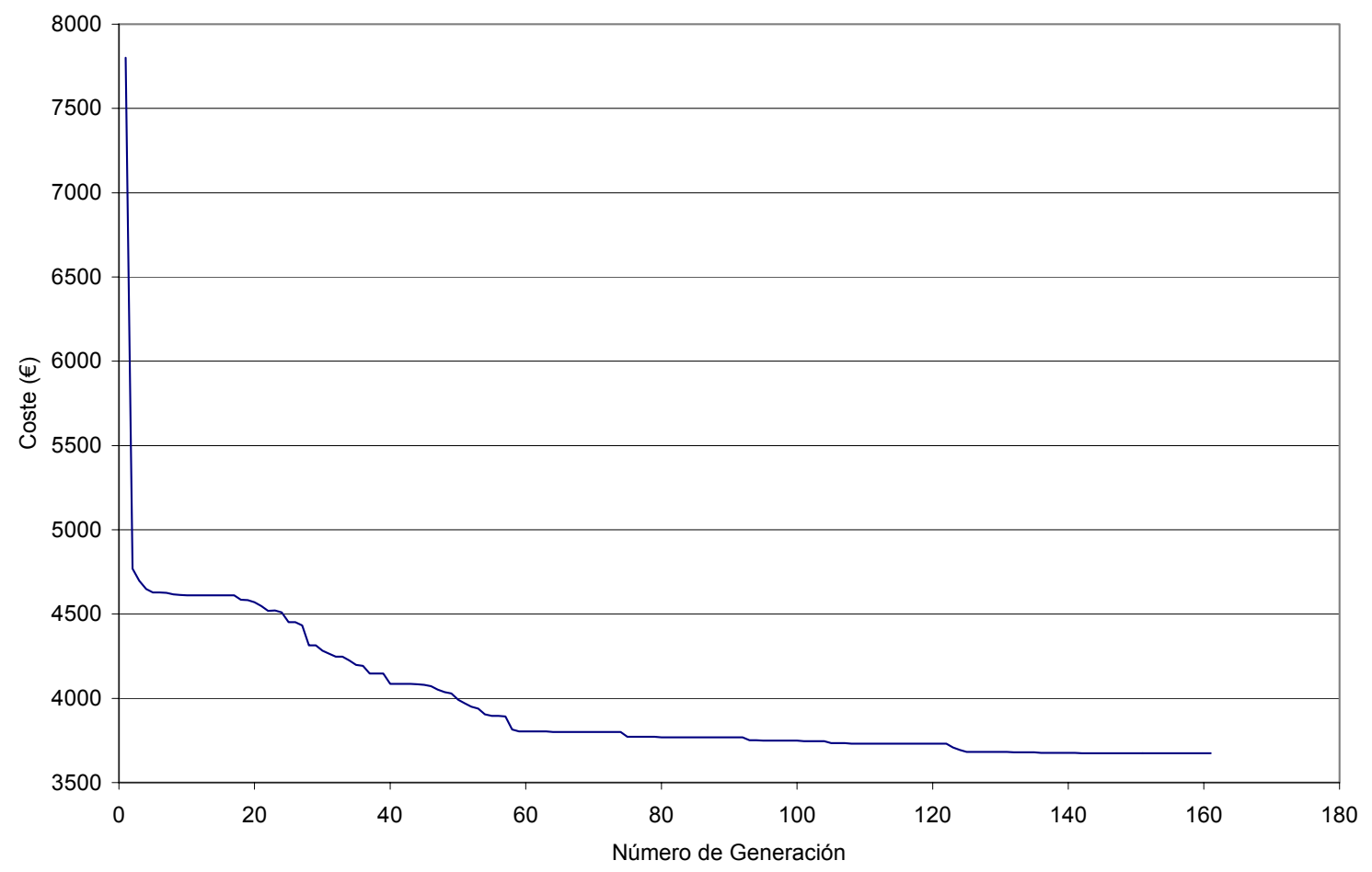

Figura 5.6. Evolución del coste de la solución óptima con el número de generaciones en una ejecución típica del GA modificado. 


\subsection{JUSTIFICACIÓN DEL NÚMERO DE ENSAYOS REALIZADOS CON CADA HEURÍSTICA.}

Para que los resultados obtenidos al optimizar un pórtico con unos parámetros de una heurística sean representativos de la misma, cada experimento debe repetirse un cierto número de veces, obteniéndose los estadísticos de la muestra de resultados. La pregunta por tanto es ¿cuántos ensayos deben realizarse?.

Para responderla, se han realizado 99 ejecuciones de la heurística que proporcionó la solución de menor coste del problema $P_{2} 2 v 4 h-C_{E S T}(S A 7$ con $r=0.8)$ y los resultados se han comparado con los ya obtenidos en el apartado 4.4 (véase la Tabla 5.12).

Tabla 5.12. Problema $P \_2 v 4 h-C_{E S T}$. Comparación de los resultados obtenidos con 9 y con 99 ensayos de la heurística SA7 con $r=0.8$.

\begin{tabular}{ccc}
\hline & 9 ensayos & 99 ensayos \\
\hline Coste mínimo $(€)$ & 3473.06 & 3460.45 \\
\hline $\begin{array}{c}\text { Tiempo de la solución de } \\
\text { coste mínimo }(\mathrm{min})\end{array}$ & 18.9 & 59.5 \\
\hline Coste medio $(€)$ & 3497.98 & 3502.04 \\
\hline$\sigma_{\text {coste }}(€)$ & 21.80 & 42.32 \\
\hline Coef. de variación del coste & 0.0062 & 0.0121 \\
\hline Tiempo total de cálculo & 253 & 2430 \\
\hline
\end{tabular}

Del estudio de la Tabla 5.12 se concluye que incrementar el número de pruebas de 9 a 99 disminuye muy ligeramente el coste de la solución optimizada ( un $0.36 \%$ ) pero incrementa muy notablemente los tiempos totales de cálculo (se multiplican por 9.6). Por ello, la mejora obtenida en los costes se considera despreciable y se considera validado el método de ejecutar 9 ensayos con cada estrategia. 


\subsection{COMPARACIÓN DE LOS RESULTADOS. CONCLUSIONES.}

Para comparar RW, SA, TA y GA se adjunta la Figura 5.7 con los resultados de las mejores ejecuciones y la 5.8 con la misma información filtrada al mostrar únicamente los Óptimos de Pareto. Además, los resultados más representativos se muestran en la Tabla 5.13.

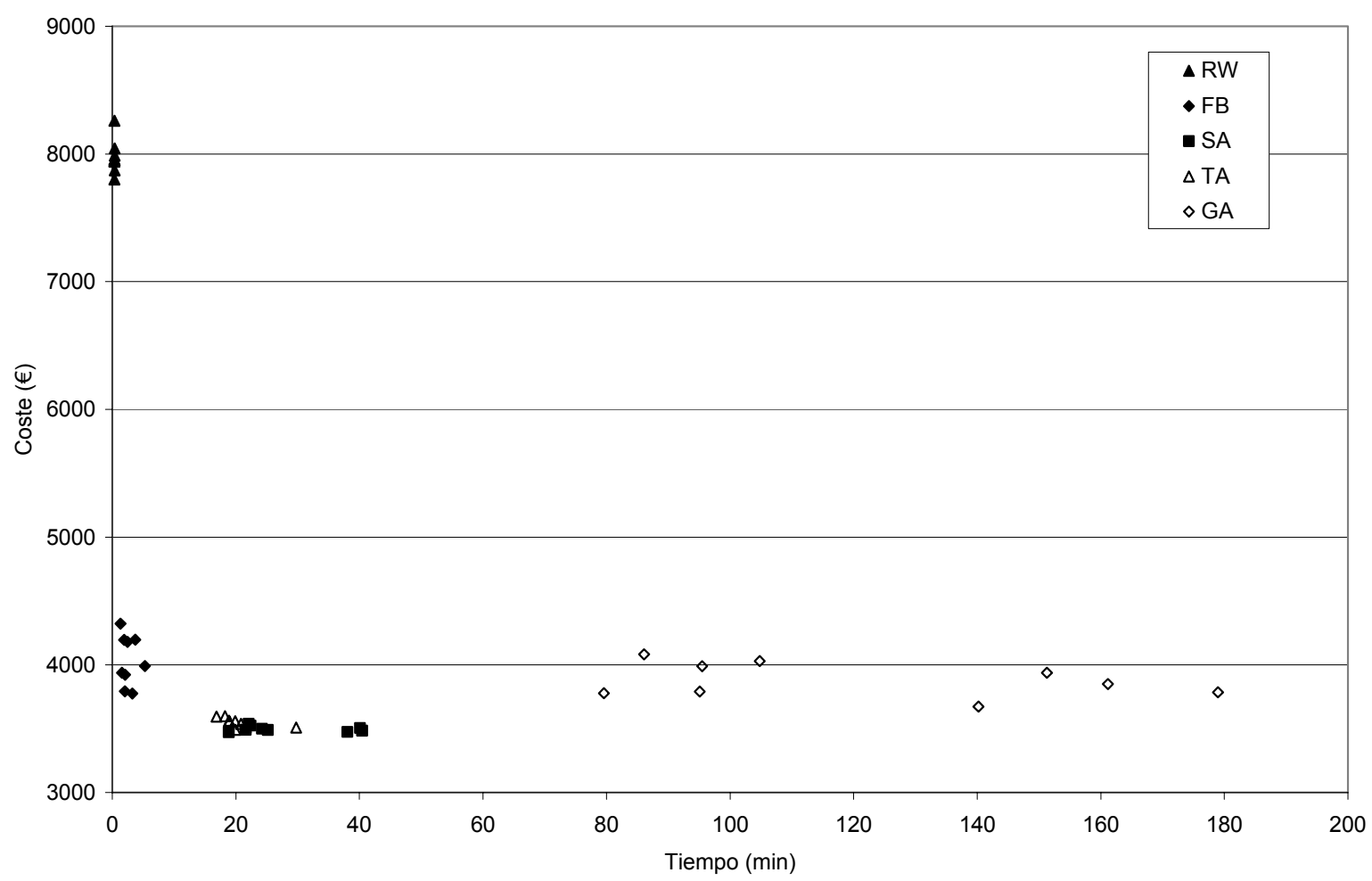

Figura 5.7. Resultados de las mejores heurísticas RW, FB, SA, TA y GA.

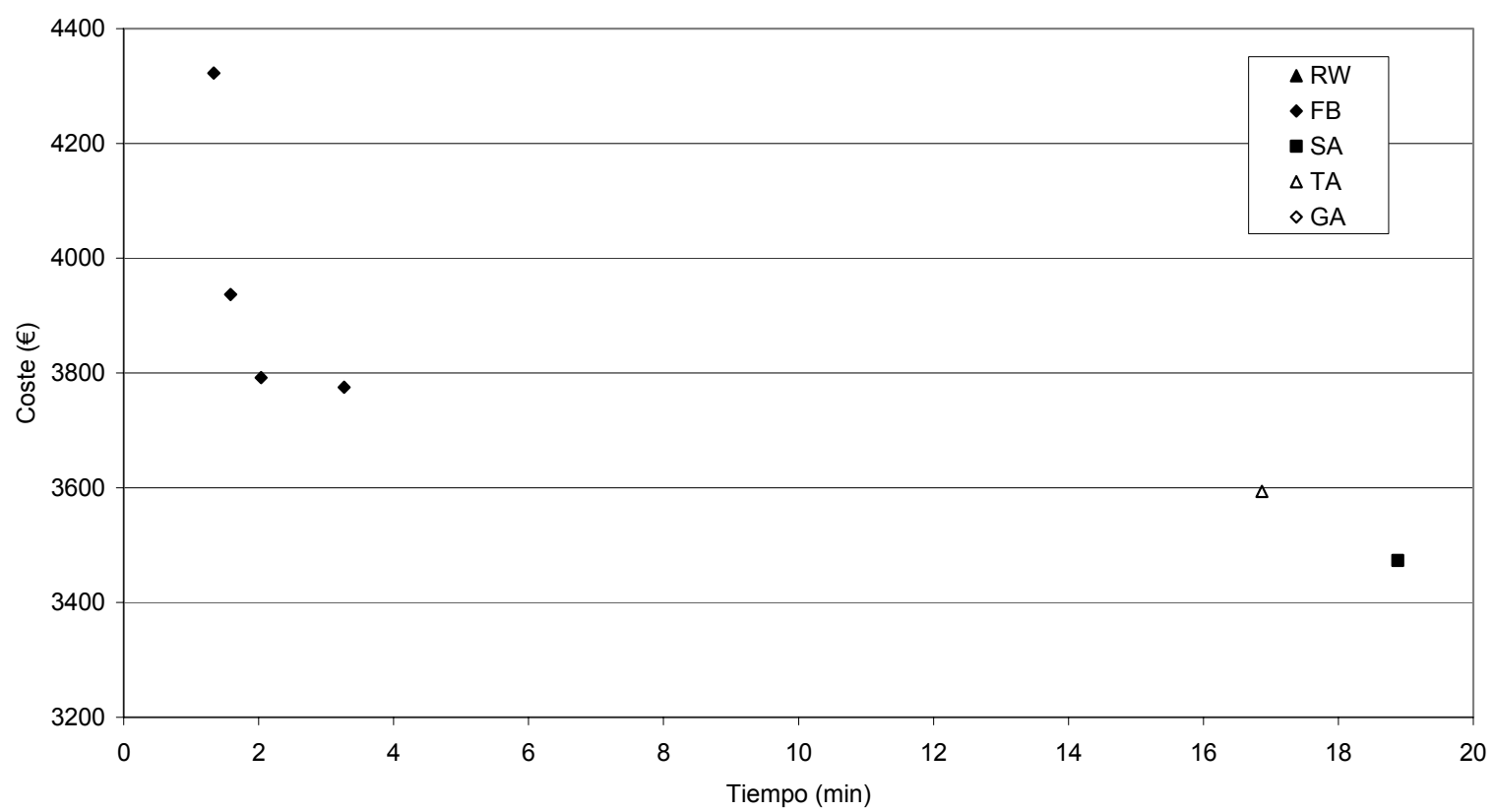

Figura 5.8. Óptimos de Pareto correspondientes a los resultados de las mejores heurísticas RW, FB, SA, TA y $G A$. 
Tabla 5.13. Comparación de heurísticas

\begin{tabular}{cccccccc}
\hline Heurística & $\begin{array}{c}\text { Coste } \\
\text { mínimo }(€)\end{array}$ & $\begin{array}{c}\text { Tiempo del } \\
\text { Coste mínimo } \\
(\mathbf{m i n})\end{array}$ & $\begin{array}{c}\text { Coste } \\
\text { medio }(€)\end{array}$ & $\begin{array}{c}\text { Tiempo } \\
\text { medio (min) }\end{array}$ & \multicolumn{2}{c}{ Coef. variación } \\
\hline RW & 7800.94 & 0.3 & 7972.89 & 0.3 & 0.016 & 0.057 \\
\hline FB & 3775.10 & 3.3 & 4034.45 & 2.6 & 0.048 & 0.5 \\
\hline SA & 3473.06 & 18.9 & 3497.98 & 28.1 & 0.0062 & 0.313 \\
\hline TA & 3491.31 & 19.9 & 3546.59 & 20.6 & 0.0095 & 0.18 \\
\hline GA & 3672.91 & 79.6 & 3879.69 & 121.4 & 0.0352 & 0.302 \\
\hline
\end{tabular}

A la vista de toda esta información se puede afirmar que la versión propuesta de SA es la heurística más eficaz y robusta para resolver el problema planteado pues:

1) Obtiene la solución de menor coste. Las soluciones más económicas obtenidas con TA, GA, FB y RW son, respectivamente, un $0.52 \%$, un $5.74 \%$, un $8.69 \%$ y un $124.6 \%$ más caras.

2) El coste medio de las soluciones óptimas es el mínimo. Los obtenidos mediante TA, GA y FB son superiores en, respectivamente, un $1.39 \%$, un $10.91 \%$ y un $15.3 \%$. Además, el coeficiente de variación del coste mínimo es también el menor del de todas las heurísticas probadas, lo que indica una mayor facilidad para proporcionar la mejor solución optimizada.

3) Los tiempos de cálculo son superiores a los de TA pero tienen un orden de magnitud razonable habida cuenta de la dificultad del problema. En valor medio, TA tardó un $26.7 \%$ menos que SA en encontrar la solución localmente óptima, sin embargo, el mejor óptimo fue encontrado por SA en un $5.2 \%$ menos de tiempo que por TA.

Señalamos que los resultados obtenidos mediante GA no contradicen el amplio uso de esta técnica de optimización pues son debidos a la elevada complejidad del problema tratado (dimensión del espacio de soluciones y número de restricciones). Para hacer más competitivo GA deberían introducirse nuevas mejoras en el algoritmo encaminadas, por 
ejemplo, a disminuir el tamaño del espacio de soluciones lo que bajaría los tiempos de cálculo y mejoraría la calidad de las soluciones encontradas.

Por todo ello, la Cristalización Simulada se emplea en los próximos capítulos para minimizar el coste de pórticos de dos vanos y dos, seis y ocho alturas y para estudiar la optimización multicriterio de estas estructuras. Para este último objetivo no se utiliza SA directamente sino un algoritmo en él basado. 
Página intencionadamente en blanco. 


\section{CAPÍTULO 6. ESTUDIO PARAMÉTRICO CON PÓRTICOS DE VARIAS ALTURAS.}




\section{Objetivos.}

En el capítulo anterior, la resolución del problema $P \_2 v 4 h-C_{E S T}$ permitió determinar que la versión de SA propuesta es la heurística más idónea para optimizar económicamente estas estructuras. A continuación se emplea SA para realizar un estudio paramétrico en el que se modifica el número de plantas del pórtico estudiado anteriormente y se mantienen el resto de parámetros.

Los resultados obtenidos en este capítulo permiten: a) definir los parámetros de la heurística para optimizar un pórtico cualquiera y b) analizar las características de las estructuras óptimas. 


\subsection{OPTIMIZACIÓN DE PÓRTICOS DE DOS VANOS Y DOS, CUATRO, SEIS Y OCHO ALTURAS.}

\subsubsection{Introducción.}

Se aborda a continuación la optimización de pórticos simétricos de dos vanos y diferente número de plantas. Los parámetros del problema se indicaron en el epígrafe 3.2. El número de variables, obtenido a partir de la expresión (3.1), y la dimensión del espacio de soluciones se proporcionan en la Tabla 6.1.

Tabla 6.1. Número de variables y dimensión del espacio de soluciones en pórticos simétricos de dos vanos.

\begin{tabular}{cccc}
\hline Problema & $\begin{array}{c}\text { Número de } \\
\text { plantas }\end{array}$ & Variables & Dimensión \\
\hline P_2v2h-C & 2 & 39 & $2.92 \times 10^{58}$ \\
\hline P_2v4h-C & 4 & 77 & $4.28 \times 10^{116}$ \\
\hline P_2v6h-C & 6 & 115 & $6.25 \times 10^{174}$ \\
\hline P_2v8h.1-C & 8 & 153 & $9.15 \times 10^{232}$ \\
\hline
\end{tabular}

Para minimizar el coste de estas estructuras, se ha empleado una estrategia SA cuyos parámetros se han calibrado para cada problema. Los rangos de variación de cada variable siguen lo indicado en la Tabla 4.1. Como en el capítulo anterior, cada ensayo numérico se ha repetido nueve veces para obtener valores estadísticamente representativos.

\subsubsection{Pórtico de dos vanos y dos alturas. Problema $P \_2 v 2 h-C_{E S T}$}

Para la resolución de este problema se han probado seis heurísticas diferentes (llamadas SA1 a SA6) con los parámetros indicados en la Tabla 6.2. La Tabla 6.3 proporciona los resultados obtenidos y la Figura 6.1 los Óptimos de Pareto. Se recuerda que Nvar es un parámetro de la heurística que indica el número máximo de variables modificadas en cada movimiento, $L m$ la longitud de la cadena de Markov y NCMsmmax el número de cadenas de Markov sin mejora que se adopta como criterio de parada. La 
solución inicial es la mejor de las obtenidas con nueve ejecuciones del algoritmo RW con 10000 iteraciones. Su coste es de $3131.65 €$.

Tabla 6.2. Problema $P \_2 v 2 h-C_{E S T}$. Parámetros definitorios de heurísticas SA.

\begin{tabular}{ccccc}
\hline $\begin{array}{c}\text { Tipo de } \\
\text { heurística }\end{array}$ & Nvar & Lm & $\mathbf{r}$ & NCMsmmax \\
\hline SA1 & Hasta 2 & 10000 & 0.8 & 2 \\
\hline SA2 & Hasta 3 & 10000 & 0.8 & 2 \\
\hline SA3 & Hasta 2 & 20000 & 0.8 & 2 \\
\hline SA4 & Hasta 3 & 20000 & 0.8 & 2 \\
\hline SA5 & Hasta 3 & 20000 & 0.9 & 2 \\
\hline SA6 & Hasta 3 & 35000 & 0.8 & 2 \\
\hline
\end{tabular}

Tabla 6.3. Problema $P \_2 v 2 h-C_{E S T}$. Resultados de heurísticas

\begin{tabular}{cccccccc}
\hline Heurística & $\begin{array}{c}\text { Coste } \\
\text { mínimo (€) }\end{array}$ & $\begin{array}{c}\text { Tiempo del } \\
\text { Coste mínimo } \\
(\mathbf{m i n})\end{array}$ & $\begin{array}{c}\text { Coste } \\
\text { medio }(€)\end{array}$ & $\begin{array}{c}\text { Tiempo } \\
\text { medio } \\
(\mathbf{m i n})\end{array}$ & $\sigma_{\text {coste }}$ & $\mathbf{C V}_{\text {coste }}$ & $\sigma_{\text {tiempo }}$ \\
\hline SA1 & 1654.99 & 1.9 & 1686.96 & 2.7 & 27.18 & 0.016 & 1.0 \\
\hline SA2 & 1643.36 & 2.5 & 1680.12 & 2.6 & 28.79 & 0.017 & 0.8 \\
\hline SA3 & 1643.36 & 7.0 & 1672.46 & 6.0 & 31.83 & 0.019 & 1.1 \\
\hline SA4 & 1648.50 & 5.3 & 1675.28 & 4.3 & 24.1 & 0.014 & 1.93 \\
\hline SA5 & 1670.48 & 10.6 & 1787.54 & 5.2 & 99.00 & 0.055 & 2.46 \\
\hline SA6 & 1645.87 & 5.8 & 1665.55 & 10.1 & 18.44 & 0.011 & 2.6 \\
\hline
\end{tabular}


Los resultados muestran que SA2 y SA3 proporcionan la solución más económica. Su coste es un $47.5 \%$ inferior al proporcionado por RW. Ambas heurísticas pueden considerarse aptas para resolver el problema y emplean $r=0.8$, Nvar="Hasta 3" y NCMsmmax=2 . El Apéndice 4 contiene los planos con las características de la solución más optimizada. El acero es B-500S y el hormigón de todos los elementos es HA-30.

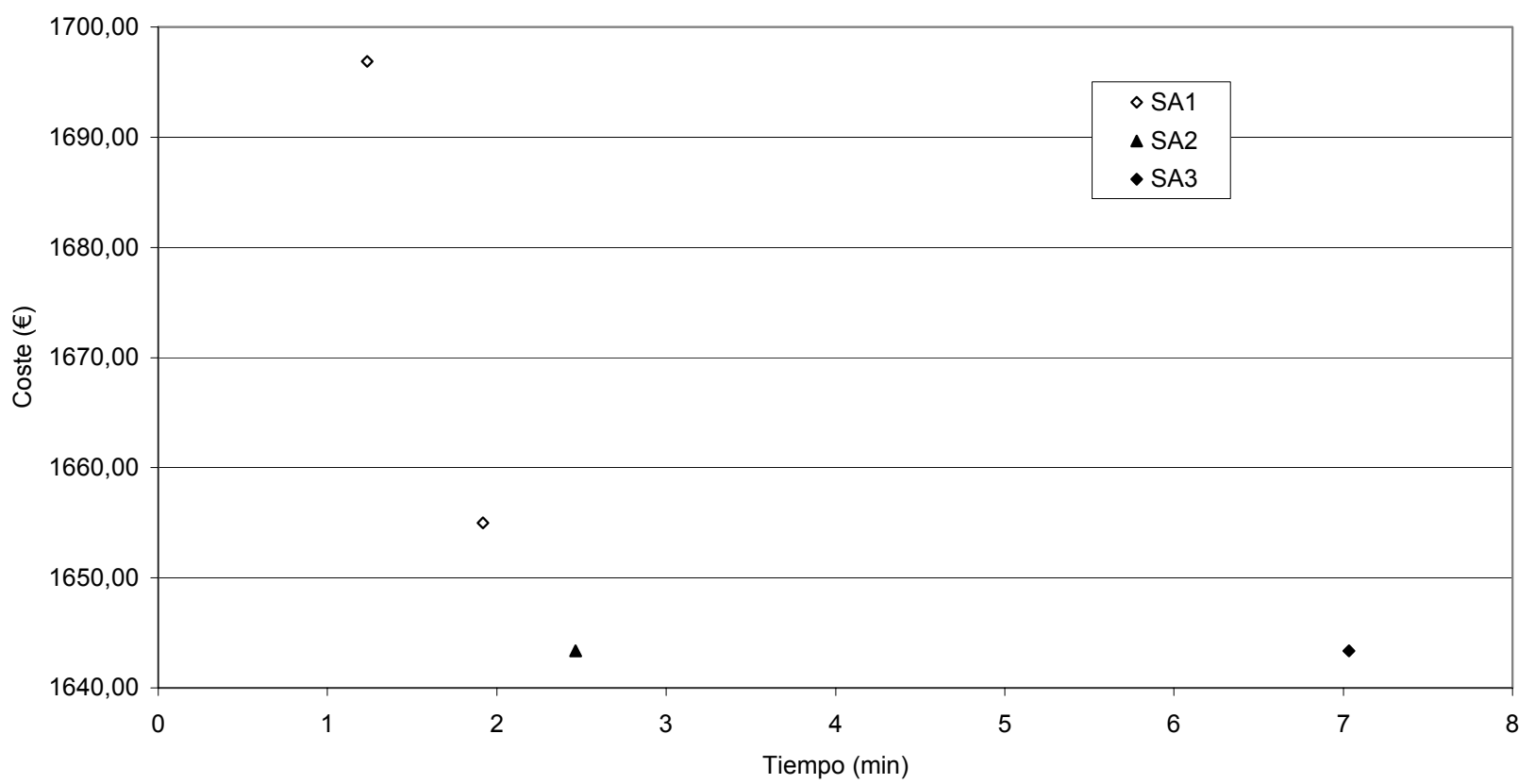

Figura 6.1. Problema P_2v2h-C $C_{E S T}$. Óptimos de Pareto y punto de coste mínimo proporcionado por SA3. Las heurísticas no representadas no aportan ningún Optimo de Pareto.

\subsubsection{Pórtico de dos vanos y seis alturas. Problema $P \_2 v 6 h-C_{E S T}$.}

Para la resolución de este problema se han probado seis heurísticas diferentes (SA7 a SA12) con los parámetros indicados en la Tabla 6.4. La Tabla 6.5 proporciona los resultados obtenidos y la Figura 6.2 los Óptimos de Pareto. La solución inicial es la mejor de las obtenidas con nueve ejecuciones del algoritmo RW con 100000 iteraciones. Su coste es de $14419.56 €$.

Las solución de coste mínimo se obtiene con SA11 (5561.81 €) y mejora la solución obtenida por RW un $61.4 \%$. SA12 proporciona un coste mínimo un $0.2 \%$ superior y un coste medio un $0.38 \%$ menor. Ambas heurísticas se consideran buenas para resolver el problema y utilizan $r=0.8$, Nvar="Hasta 3" y NCMsmmax=2. El Apéndice 4 contiene los planos de la solución optimizada de menor coste. El acero es B-500S y el hormigón de todos los elementos es HA-45. 
Tabla 6.4. Problema $P \_2 v 6 h-C_{E S T}$. Parámetros definitorios de heurísticas SA.

\begin{tabular}{ccccc}
\hline $\begin{array}{c}\text { Tipo de } \\
\text { heurística }\end{array}$ & Nvar & Lm & $\mathbf{r}$ & NCMsmmax \\
\hline SA7 & Hasta 3 & 80000 & 0.8 & 2 \\
\hline SA8 & Hasta 3 & 100000 & 0.8 & 2 \\
\hline SA9 & Hasta 3 & 100000 & 0.9 & 2 \\
\hline SA10 & Hasta 6 & 100000 & 0.8 & 2 \\
\hline SA11 & Hasta 3 & 135000 & 0.8 & 2 \\
\hline SA12 & Hasta 3 & 150000 & 0.8 & 2 \\
\hline
\end{tabular}

Tabla 6.5. Problema $P \_2 v 6 h-C_{E S T}$. Resultados de heurísticas

\begin{tabular}{cccccccc}
\hline Heurística & $\begin{array}{c}\text { Coste } \\
\text { mínimo }(€)\end{array}$ & $\begin{array}{c}\text { Tiempo del } \\
\text { Coste mínimo } \\
(\mathbf{m i n})\end{array}$ & $\begin{array}{c}\text { Coste } \\
\text { medio }(€)\end{array}$ & $\begin{array}{c}\text { Tiempo } \\
\text { medio } \\
(\mathbf{m i n})\end{array}$ & $\sigma_{\text {coste }}$ & $\mathbf{C V}_{\text {coste }}$ & $\sigma_{\text {tiempo }}$ \\
\hline SA7 & 5597.49 & 103.0 & 5667.82 & 93.1 & 38.16 & 0.007 & 17.51 \\
\hline SA8 & 5572.82 & 137.1 & 5670.18 & 106.4 & 69.78 & 0.012 & 24.05 \\
\hline SA9 & 5610.02 & 161.2 & 6000.96 & 134.8 & 287.29 & 0.047 & 53.69 \\
\hline SA10 & 5656.12 & 89.6 & 5769.03 & 109.5 & 19.6 & 0.003 & 19.55 \\
\hline SA11 & 5561.81 & 184.8 & 5633.69 & 157.6 & 41.70 & 0.007 & 32.42 \\
\hline SA12 & 5571.58 & 125.02 & 5612.05 & 176.9 & 33.68 & 0.006 & 49.01 \\
\hline
\end{tabular}




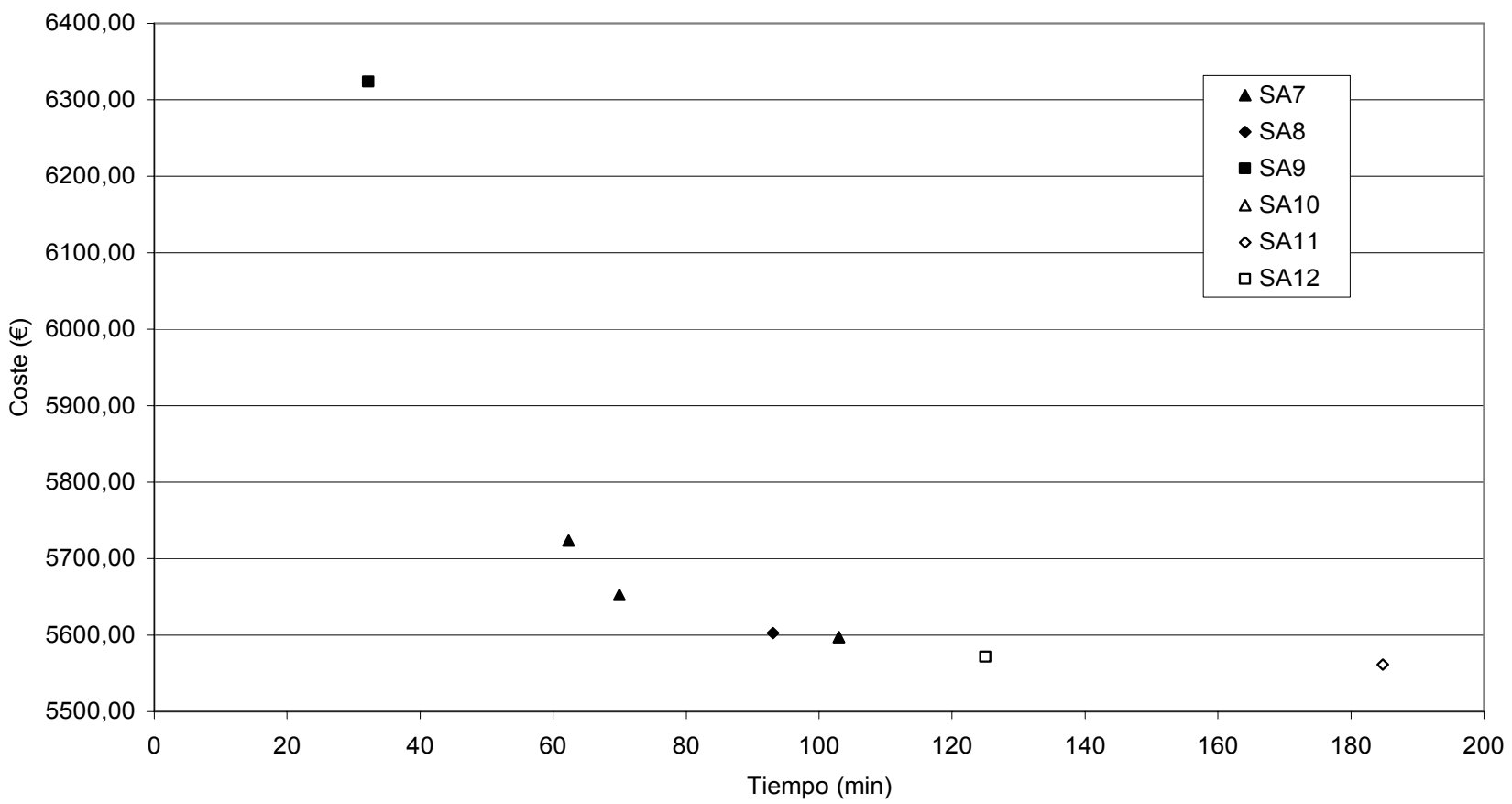

Figura 6.2. Problema P_2v6h-C EST. Óptimos de Pareto.

\subsubsection{Pórtico de dos vanos y ocho alturas. P_2v8h.1- $C_{E S T}$.}

Para la resolución de este problema se han probado cuatro heurísticas diferentes que se muestran en la Tabla 6.6. En la Tabla 6.7 se dan los resultados obtenidos, en la Figura 6.4 los Óptimos de Pareto y en el Apéndice 4 los planos del mejor óptimo local cuyo coste es de $8242.23 €$. El hormigón es HA-45 en los elementos situados hasta la planta séptima y HA-35 en los de la planta octava; el acero es B-500S.

Para definir la solución inicial no se ha utilizado un RW puro porque, dada la complejidad del problema, esta heurística no fue capaz de encontrar ninguna solución factible a pesar de emplearse valores del parámetro Nsol de hasta 500000 soluciones. Por ello, como diseño inicial se empleó el mejor de los obtenidos en nueve ejecuciones del algoritmo descrito en la Figura 6.3 y que se resume seguidamente. A partir de una solución no factible generada de forma aleatoria se obtuvo una factible empleando las funciones de penalización explicadas en el punto 4.8.3 y aplicando un FB en el que el objetivo no era minimizar el coste de la solución sino el valor del sumatorio de las funciones de penalización. El coste de la mejor solución inicial obtenida con este procedimiento es de $18440.62 €$. 


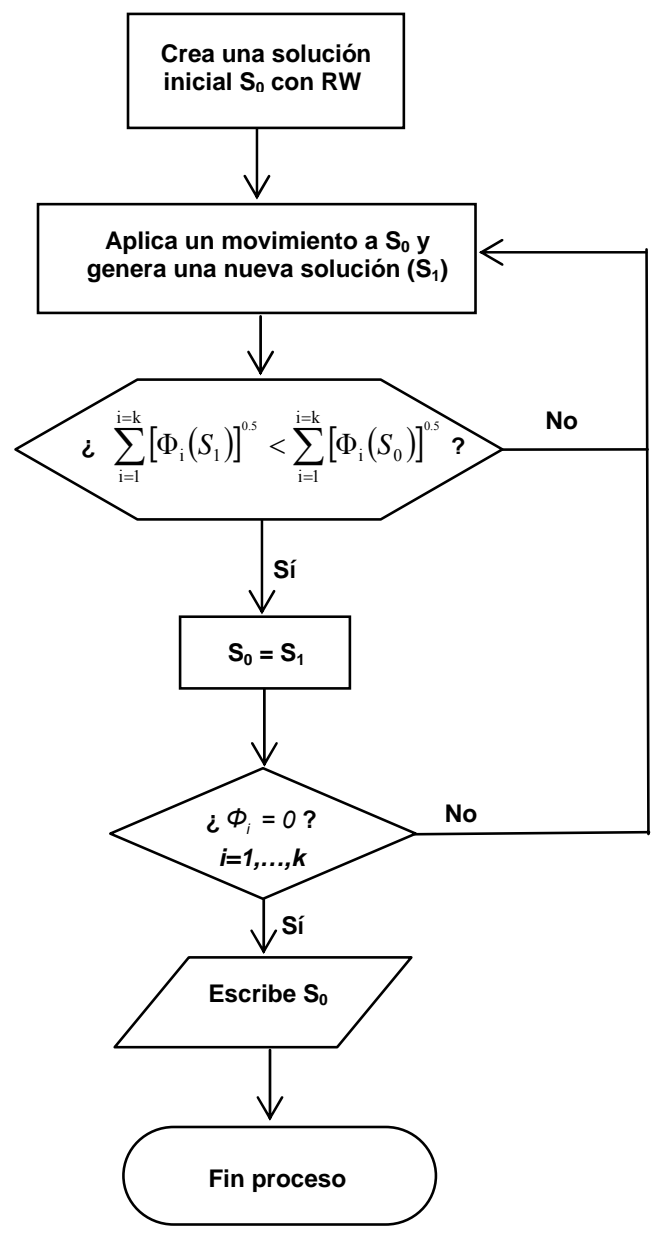

Figura 6.3. Heurística híbrida RW-FB empleada para la obtención de soluciones factibles en el problema $P_{-} 2 v 8 h .1-C_{E S T}$.

Tabla 6.6. P_2v8h.1-C $\mathrm{EST}_{\text {. }}$ Parámetros definitorios de heurísticas SA.

\begin{tabular}{ccccc}
\hline $\begin{array}{c}\text { Tipo de } \\
\text { heurística }\end{array}$ & Nvar & Lm & $\mathbf{r}$ & NCMsmmax \\
\hline SA13 & Hasta 3 & 200000 & 0.8 & 2 \\
\hline SA14 & Hasta 3 & 200000 & 0.9 & 2 \\
\hline SA15 & Hasta 6 & 200000 & 0.8 & 2 \\
\hline SA16 & Hasta 3 & 300000 & 0.8 & 2 \\
\hline
\end{tabular}


Tabla 6.7. Problema P_2v8h- $C_{E S T}$. Resultados de heurísticas.

\begin{tabular}{cccccccc}
\hline Heurística & $\begin{array}{c}\text { Coste } \\
\text { mínimo }(€)\end{array}$ & $\begin{array}{c}\text { Tiempo del } \\
\text { Coste mínimo } \\
(\mathbf{m i n})\end{array}$ & $\begin{array}{c}\text { Coste } \\
\text { medio }(€)\end{array}$ & $\begin{array}{c}\text { Tiempo } \\
\text { medio } \\
(\text { min) }\end{array}$ & $\sigma_{\text {coste }}$ & $\mathbf{C V}_{\text {coste }}$ & $\sigma_{\text {tiempo }}$ \\
\hline SA13 & 8242.23 & 403.2 & 8334.44 & 361.1 & 52.54 & 0.006 & 53.22 \\
\hline SA14 & 8280.61 & 686.0 & 8705.60 & 357.3 & 315.84 & 0.036 & 196.80 \\
\hline SA15 & 8303.61 & 318.6 & 8433.99 & 342.3 & 90.88 & 0.011 & 24.19 \\
\hline SA16 & 8244.73 & 487.6 & 8375.95 & 521.8 & 315.4 & 0.038 & 315.41 \\
\hline
\end{tabular}

SA13 y SA16 obtienen una familia de soluciones de coste mínimo y medio similar. No obstante, SA16 necesita tiempos medios superiores en un $46.2 \%$ a los de SA13 y su coeficiente de variación del coste también es muy superior. Por ello no se considera tan adecuada para resolver el problema como SA13.

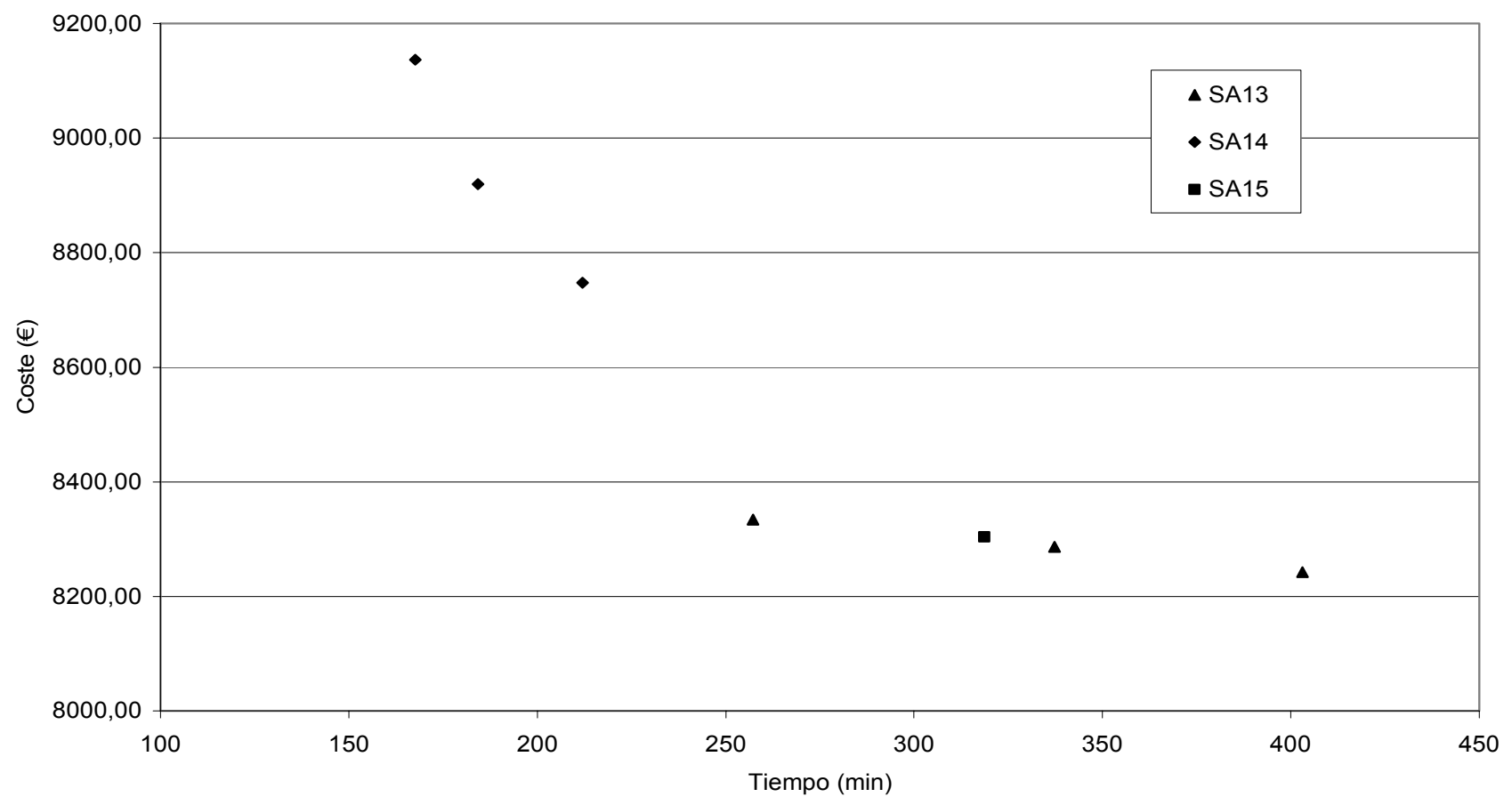

Figura 6.4. Problema P_2v8h.1-C EST. Óptimos de Pareto. Las heurísticas no representadas no aportan ningún Óptimo de Pareto. 


\subsection{ANÁLISIS DE LAS HEURÍSTICAS QUE PROPORCIONAN LAS ESTRUCTURAS ÓPTIMAS.}

Uno de los factores limitantes de la aplicación de las heurísticas es la necesidad de calibrar sus parámetros, pues éste es un proceso de prueba-error que puede consumir mucho tiempo de cálculo. No obstante, los experimentos realizados hasta ahora permiten solventar este problema. La Tabla 6.8 muestra los parámetros empleados en heurísticas que proporcionaron los óptimos locales de menor coste.

Tabla 6.8. Parámetros de las heurísticas SA que proporcionan las estructuras optimizadas de coste mínimo.

\begin{tabular}{ccccc}
\hline Problema & Nvar & Lm & $\mathbf{r}$ & NCMsmmax \\
\hline P_2v2h -C EST & Hasta 3 & 20000 & 0.8 & 2 \\
\hline P_2v4h -C EST & Hasta 3 & 70000 & 0.8 & 2 \\
\hline P_2v6h -C EST & Hasta 3 & 135000 & 0.8 & 2 \\
\hline P_2v8h.1-C EST & Hasta 3 & 200000 & 0.8 & 2 \\
\hline
\end{tabular}

En esta tabla los parámetros $r=0.8$, Nvar="Hasta 3 variables" y NCMsmmax=2 son comunes a todas las heurísticas. Queda por tanto determinar el valor de la Longitud de la Cadena de Markov, Lm. Para ello, se ha realizado un ajuste de $L m$ en función del número de variables del pórtico (NumVar) por el método de mínimos cuadrados cuyo resultado se muestra en la Figura 6.5 y en la ecuación (6.1). El ajuste muestra una relación lineal entre ambas variables con un Coeficiente de Correlación $R^{2}$ de 0.9963 , por lo que puede calificarse como de muy buena calidad.

$$
L m=1592.105 \text { NumVar }-46592.11
$$

Por tanto, podemos decir que, problemas como el planteado pueden resolverse mediante una heurística SA con parámetros $r=0.8$, Nvar="Hasta 3 variables" , $N C M s m m a x=2$ y un valor de $L m$ dado por la expresión (6.1). Este resultado es de gran importancia pues permite automatizar el diseño óptimo de estas estructuras. 


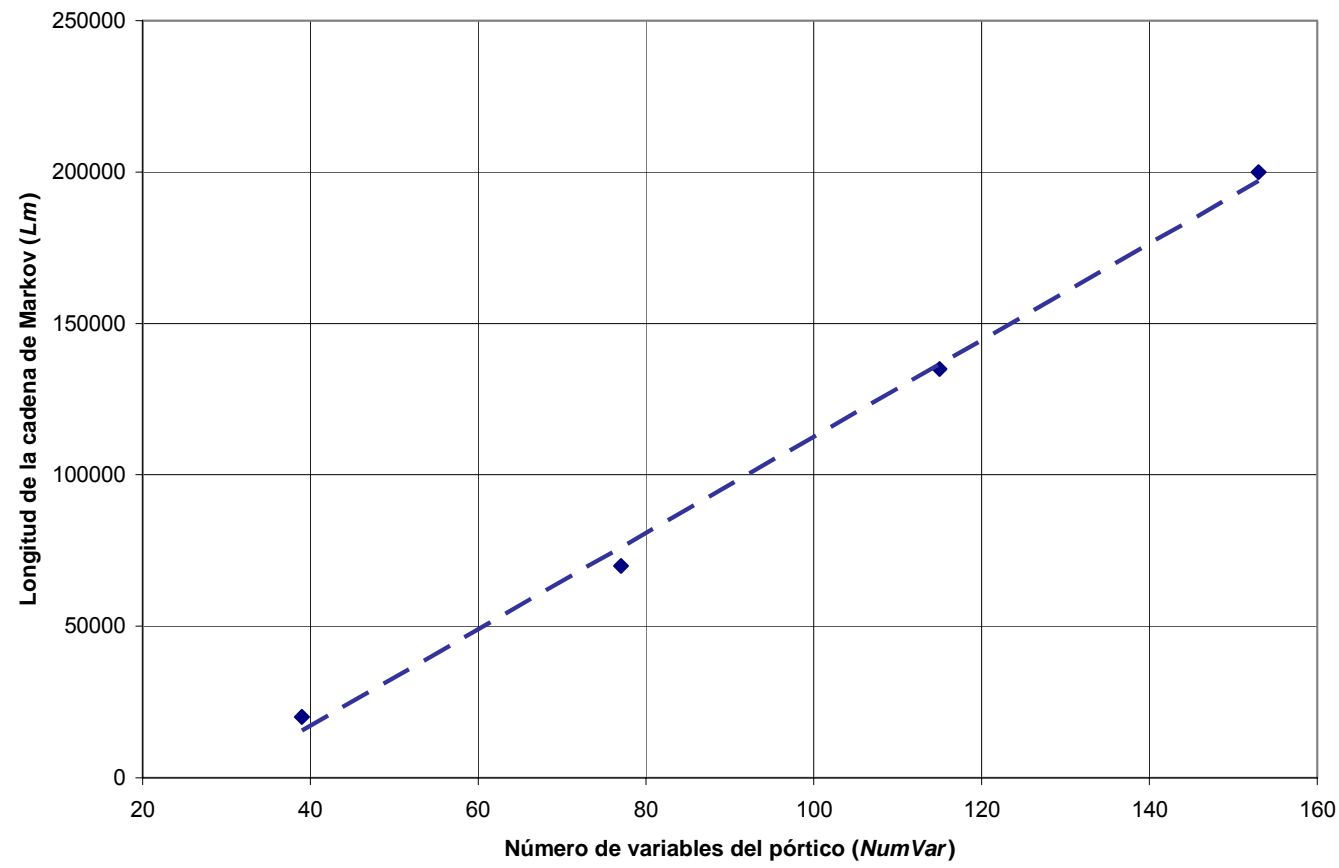

Figura 6.5. Relación entre el número de variables que definen un pórtico y la Longitud de la cadena de Markov.

Una estimación del tiempo medio de cálculo -en minutos- de una ejecución del algoritmo que proporciona la solución óptima $\left(t_{m e d}\right)$ se ha obtenido ajustando la relación entre NumVar y los tiempos medios empleados por las heurísticas de la Tabla 6.8 por mínimos cuadrados. Los resultados se muestran en la Figura 6.6 y reflejan una relación exponencial entre ambas variables dada por la expresión (6.2). El coeficiente $R^{2}$ es de 0.9814 por lo que el ajuste puede ser clasificado también como muy bueno.

$$
t_{m e d}=1.637 e^{0.0369 \text { Numvar }}
$$

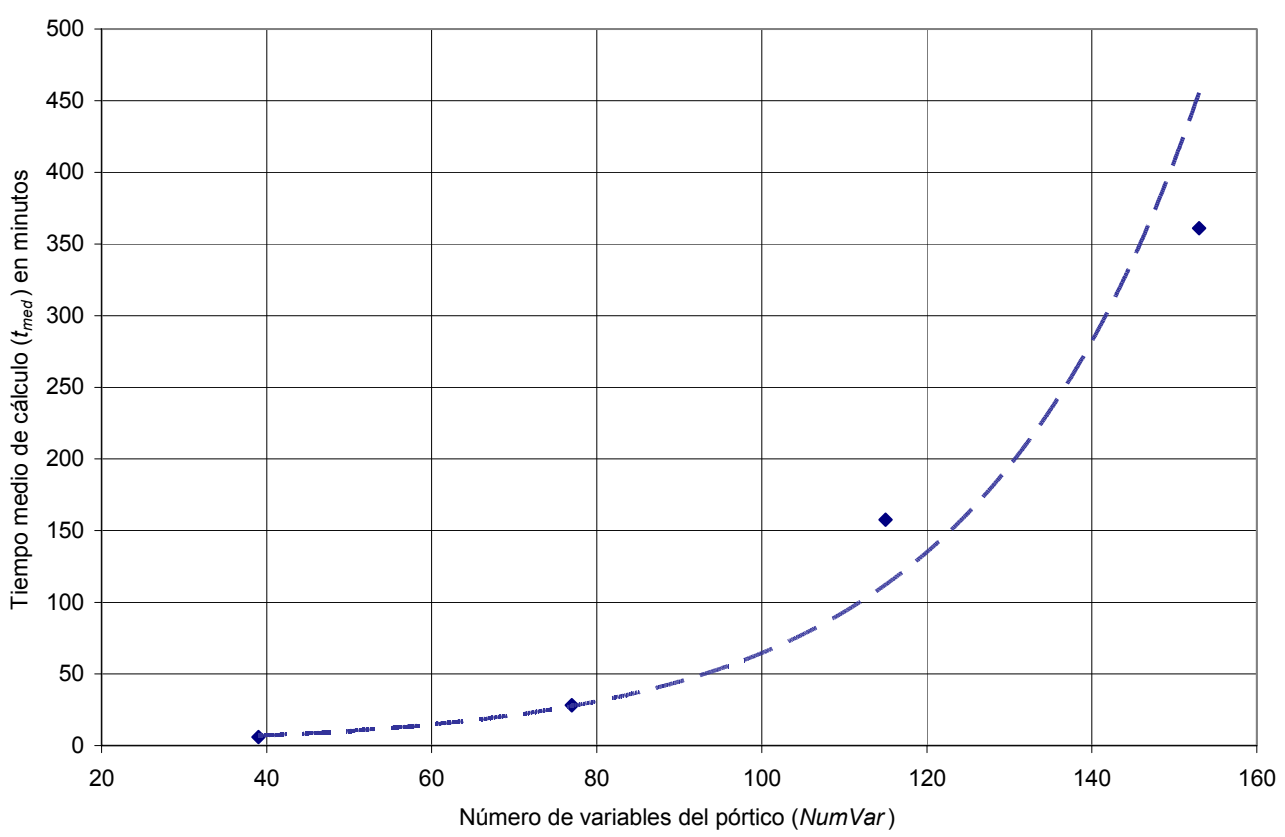

Figura 6.6. Relación entre el número de variables que definen un pórtico y el tiempo medio de cálculo de una ejecución del algoritmo que proporciona el mejor óptimo local. 


\subsection{ANÁLISIS DE LOS RESULTADOS DE LAS ESTRUCTURAS OPTIMIZADAS.}

Se presenta a continuación el análisis de las estructuras óptimas realizado a partir de:

- Los planos del Apéndice 4.

- Los resultados de los cálculos de hormigón armado (tablas 6.9 a 6.21).

- Las cuantías de armadura.

\subsubsection{Vigas.}

Se presentan seguidamente diferentes tablas con un resumen de los resultados obtenidos. La simetría de la estructura se ha tenido en cuenta a la hora de representar esos datos, por lo que cuando se escribe "extremo izquierdo de una viga" se refiere tanto al extremo izquierdo de las vigas del primer vano como, por la simetría, al extremo derecho de las vigas del segundo vano (ver Figura 6.7). Análogamente, cuando se escribe "extremo derecho" nos estamos refiriendo al extremo derecho de las vigas del primer vano y al izquierdo de las del segundo.

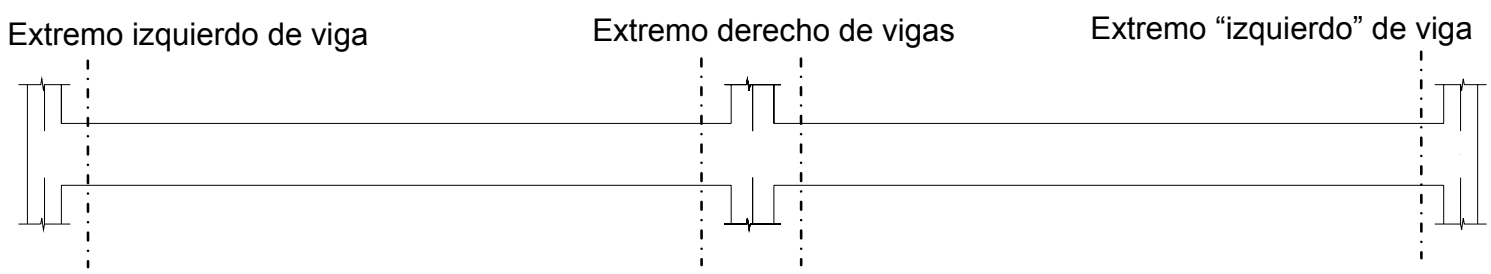

Figura 6.7. Nomenclatura empleada en la exposición de los resultados de las vigas optimizadas.

Tras estas puntualizaciones, se señala que las vigas de la estructuras optimizadas, se caracterizan porque:

- Como muestran las tablas 6.9 y 6.10 , los estados límites que condicionan su diseño son el ELS de deformaciones (en concreto la verificación de las flechas activas) y el ELU de agotamiento por solicitaciones normales. Para ellos, los coeficientes de seguridad ajustados por Optipor son muy próximos a la unidad de modo que incluso pequeñas alteraciones de la rigidez de vigas o pilares (como por ejemplo una modificación de la resistencia característica a compresión del hormigón) implican su incumplimiento. Por este motivo las vigas óptimas tienen los anchos mínimos permitidos (entre 20 y $23 \mathrm{~cm}$ ) y cantos 
significativamente altos (entre $0.40 \mathrm{~m}$. y $0.55 \mathrm{~m}$.), pues es la forma de conseguir que el funcionamiento estructural de un elemento que trabaja a flexión sea eficiente. Sólo en un caso el ancho de la viga es sensiblemente superior a los $20 \mathrm{~cm}$ que es la viga de cubierta del pórtico de ocho alturas. Este ancho está justificado por la imposibilidad de incrementar el canto por alcanzar éste su valor máximo compatible con las condiciones de habitabilidad en el interior del edificio.

Tabla 6.9. Coeficientes de seguridad ${ }^{1}$ mínimos en estructuras optimizadas correspondientes a la verificación del ELS de deformaciones en vigas.

\begin{tabular}{ccc}
\hline $\begin{array}{c}\text { Número de } \\
\text { plantas }\end{array}$ & CS flecha total & CS flecha activa \\
\hline 2 & 1.80 & 1.01 \\
\hline 4 & 1.70 & 1.01 \\
\hline 6 & 1.57 & 1.00 \\
\hline 8 & 1.52 & 1.00 \\
\hline $\begin{array}{l}\text { 1 Como CS se ha tomado la relación entre la flecha máxima admisible y la flecha } \\
\text { existente. }\end{array}$ \\
\hline
\end{tabular}

Tabla 6.10. Coeficientes de seguridad ${ }^{2}$ en estructuras optimizadas correspondientes a la verificación del ELU de agotamiento por solicitaciones normales en vigas.

\begin{tabular}{cccc}
\hline $\begin{array}{c}\text { Número de } \\
\text { plantas }\end{array}$ & CS centro luz & $\begin{array}{c}\text { CS extremo } \\
\text { izquierdo }\end{array}$ & $\begin{array}{c}\text { CS extremo } \\
\text { derecho }\end{array}$ \\
\hline 2 & 1.04 & 1.24 & 1.01 \\
\hline 4 & 1.02 & 1.03 & 1.03 \\
\hline 6 & 1.00 & 1.01 & 1.02 \\
\hline 8 & 1.00 & 1.15 & 1.01 \\
\hline $\begin{array}{l}2 \text { Se evalúa el CS mediante la relación } \mathrm{M}_{\mathrm{u}} / \mathrm{M}_{\mathrm{d}} \text {, donde } \mathrm{M}_{\mathrm{u}} \text { es el momento último de la sección y } \\
\mathrm{M}_{\mathrm{d}} \text { el momento de cálculo que la solicita. }\end{array}$ & & \\
\hline
\end{tabular}


- La armadura de compresión en vigas es generalmente necesaria tanto por el cálculo del ELS de deformaciones como por el cálculo del ELU de agotamiento por solicitaciones normales. Esto se ha comprobado modificando ligeramente a la baja las armaduras de compresión en las estructuras óptimas y comprobando el no cumplimiento de estos estados límites.

- La Tabla 6.11, en la que se ha representado la relación entre la abertura de fisura máxima permitida por la EHE y la máxima abertura de fisura característica en cada viga, muestra que el ELS de fisuración es un estado límite sin influencia en el dimensionado de las vigas.

Tabla 6.11. Coeficientes de seguridad ${ }^{3}$ mínimos en estructuras optimizadas correspondientes a la verificación del ELS de fisuración en vigas.

\begin{tabular}{cccc}
\hline $\begin{array}{c}\text { Número } \\
\text { de plantas }\end{array}$ & CS centro luz & $\begin{array}{c}\text { CS extremo } \\
\text { izquierdo }\end{array}$ & $\begin{array}{c}\text { CS extremo } \\
\text { derecho }\end{array}$ \\
\hline 2 & 2.02 & 1.64 & 1.47 \\
\hline 4 & 2.46 & 2.43 & 1.85 \\
\hline 6 & 2.03 & 1.98 & 1.62 \\
\hline 8 & 1.94 & 1.81 & 1.57 \\
\hline $\begin{array}{l}{ }^{3} \text { Llamamos CS a la relación entre } \mathrm{w}_{\text {adm }} / \mathrm{w} \text { donde } \mathrm{w}_{\text {adm }} \\
\text { permitida por la EHE y } \mathrm{w}_{\mathrm{k}} \text { la abertura característica de fisura. }\end{array}$ \\
\hline
\end{tabular}

- Las armaduras longitudinales necesarias por el cálculo son, en general, superiores a las cuantías geométricas y mecánicas mínimas (véase la Tabla 6.12). Solamente en la armadura de negativos izquierda de algunas vigas de cubierta las armaduras dispuestas son próximas a las mínimas. Por tanto, en estructura optimizadas, el armado a flexión de las vigas no viene condicionado por el cumplimiento de los mínimos reglamentarios. 
Tabla 6.12. Coeficientes de seguridad mínimos ${ }^{4}$ en estructuras optimizadas correspondientes a la verificación de las cuantías geométricas y mecánicas mínimas de la armadura longitudinal en vigas.

\begin{tabular}{|c|c|c|c|c|c|c|}
\hline \multirow{2}{*}{$\begin{array}{l}\text { Número } \\
\text { de } \\
\text { plantas }\end{array}$} & \multicolumn{2}{|c|}{ Extremo izquierdo } & \multicolumn{2}{|c|}{ Extremo derecho } & \multicolumn{2}{|c|}{ Sección central } \\
\hline & $\begin{array}{l}\text { CS Min. } \\
\text { Tracción }\end{array}$ & $\begin{array}{l}\text { CS Mín. } \\
\text { Compresión }\end{array}$ & $\begin{array}{l}\text { CS Mín. } \\
\text { Tracción }\end{array}$ & $\begin{array}{l}\text { CS Mín. } \\
\text { Compresión }\end{array}$ & $\begin{array}{l}\text { CS Mín. } \\
\text { Tracción }\end{array}$ & $\begin{array}{l}\text { CS Mín. } \\
\text { Compresión }\end{array}$ \\
\hline 2 & 1.06 & 2.42 & 2.32 & 2.42 & 1.46 & 1.39 \\
\hline 4 & 2.65 & 2.26 & 2.65 & 2.26 & 1.85 & 1.47 \\
\hline 6 & 1.19 & 2.29 & 1.78 & 2.29 & 1.46 & 2.29 \\
\hline 8 & 1.11 & 1.42 & 1.79 & 1.42 & 1.18 & 2.84 \\
\hline
\end{tabular}

${ }^{4}$ Llamamos CS en este caso a la relación entre la armadura realmente dispuesta y la mínima indicada por la EHE.

- Las armaduras de cortante de las vigas en sus zonas extremas, vienen condicionadas por el cumplimiento de la condición de no superar el esfuerzo cortante que produce el agotamiento por tracción del alma de la viga $\left(V_{u 2}\right)$. En la parte central de la jácena, la armadura de cortante viene definida por el cumplimiento de las cuantías mínimas y las disposiciones constructivas prescritas por la EHE (véanse los valores de los CS correspondientes a las verificaciones propias del estado límite para las zonas izquierda, derecha y central de las vigas en las tablas $6.13,6.14$ y 6.15 ).

Tabla 6.13. Coeficientes de seguridad ${ }^{5}$ mínimos en estructuras optimizadas correspondientes a la verificación del ELU de agotamiento frente a cortante. Zona izquierda de las vigas.

\begin{tabular}{cccc}
\hline $\begin{array}{c}\text { Número de } \\
\text { plantas }\end{array}$ & CS V $_{\mathrm{u} 1}$ & CS V $_{\mathrm{u} 2}$ & CS A $_{\alpha, \min }$ \\
\hline 2 & 4.49 & 1.02 & 1.88 \\
\hline 4 & 6.09 & 1.01 & 1.06 \\
\hline 6 & 5.63 & 1.00 & 1.01 \\
\hline 8 & 4.41 & 1.00 & 1.04 \\
\hline${ }^{5}$ Llamamos CS $\mathrm{V}_{\mathrm{u} 1}, \mathrm{CS} \mathrm{V}_{\mathrm{u} 2}$ y CS A & \\
\hline
\end{tabular}


Tabla 6.14. Coeficientes de seguridad mínimos en estructuras optimizadas correspondientes a la verificación del ELU de agotamiento frente a cortante. Zona derecha de las vigas.

\begin{tabular}{cccc}
\hline $\begin{array}{c}\text { Número de } \\
\text { plantas }\end{array}$ & CS V $_{\mathrm{u} 1}$ & CS V $_{\mathrm{u} 2}$ & CS A $_{\alpha, \min }$ \\
\hline 2 & 3.47 & 1.06 & 2.28 \\
\hline 4 & 4.90 & 1.00 & 1.60 \\
\hline 6 & 4.96 & 1.00 & 1.14 \\
\hline 8 & 4.12 & 1.01 & 1.17 \\
\hline
\end{tabular}

Tabla 6.15. Coeficientes de seguridad mínimos en estructuras optimizadas. ELU de agotamiento frente a cortante. Zona central de las vigas.

\begin{tabular}{cccc}
\hline $\begin{array}{c}\text { Número de } \\
\text { plantas }\end{array}$ & CS V V1 $_{\mathrm{u}}$ & CS V $_{\mathrm{u} 2}$ & CS A $_{\alpha, \min }$ \\
\hline 2 & 7.90 & 1.14 & 1.13 \\
\hline 4 & 10.33 & 1.25 & 1.06 \\
\hline 6 & 10.41 & 1.26 & 1.01 \\
\hline 8 & 9.01 & 1.07 & 1.04 \\
\hline
\end{tabular}

\subsubsection{Pilares.}

Respecto a los pilares de la estructura óptima indicamos que:

- Tienen una esbeltez mecánica lo suficientemente alta como para que los fenómenos del pandeo deban ser tenidos en cuenta, si bien es lo suficientemente reducida como para poder verificar el ELU de Inestabilidad según el método simplificado de la EHE (véase la Tabla 6.16) y, de hecho, están muy lejos de la esbeltez mecánica por encima de la cual es necesario aplicar el método general del Artículo 43.5.1 de la EHE.

- Sus dimensiones y armado longitudinal vienen condicionados por: 
a) El cumplimiento del EL de Inestabilidad, en los pilares de fachada y de las plantas altas. Este hecho viene corroborado por los bajos CS para este estado límite indicados en las tablas 6.16 y 6.17 para estos elementos y por sus cuantías de armadura longitudinal muy superiores a las mínimas geométricas. Este fenómeno es debido a la importancia que en estos pilares tienen los esfuerzos de flexión, lo que hace necesaria una contribución importante de la armadura pasiva a la resistencia del pilar.

b) El cumplimiento de las cuantías geométricas mínimas en los pilares centrales y de las plantas más bajas. Este hecho lo demuestra los coeficientes de seguridad de la Tabla 6.18 y es debido a que en estos pilares los esfuerzos de compresión son más importantes que los de flexión, resultando más eficiente hacer trabajar al hormigón a compresión que al acero.

c) En algunos casos por motivos constructivos (dimensiones mínimas de $25 \mathrm{x}$ $25 \mathrm{~cm}$, distancias máximas entre armaduras o armado mínimo de 4\$12).

d) Por su influencia en los esfuerzos y deformaciones en vigas, como se deriva de lo ajustado de los CS referentes a los EL de deformaciones y agotamiento por solicitaciones normales en vigas.

Tabla 6.16. Coeficientes de seguridad mínimos en estructuras optimizadas. ELU agotamiento por solicitaciones normales, EL Inestabilidad.

\begin{tabular}{cccc}
\hline Número de plantas & $\begin{array}{c}\text { Esbeltez } \\
\text { mecánica } \\
\text { máxima }\end{array}$ & $\begin{array}{c}\text { CS mínimo ELU } \\
\text { Agot. Sol. } \\
\text { Normales }\end{array}$ & CS EL Inestab. ${ }^{6}$ \\
\hline 2 & 46.9 & 1.25 & 1.02 \\
\hline 4 & 50.2 & 1.23 & 1.00 \\
\hline 6 & 46.3 & 1.41 & 1.01 \\
\hline 8 & 48.5 & 1.31 & 1.01 \\
\hline
\end{tabular}

${ }^{6}$ Para cada pilar se indica el CS más desfavorable entre los correspondientes a la verificación del Estado Límite en el nudo superior e inferior. 
Tabla 6.17. Coeficientes de seguridad mínimos en estructuras optimizadas.

ELU agotamiento por solicitaciones normales, EL Inestabilidad y cumplimiento de cuantías geométricas mínimas. Pórtico de ocho plantas.

Pilares de fachada.

\begin{tabular}{ccccc}
\hline Planta & $\begin{array}{c}\text { Esbeltez } \\
\text { mecánica }\end{array}$ & $\begin{array}{c}\text { CS mínimo } \\
\text { ELU Agot. Sol. } \\
\text { Normales }\end{array}$ & $\begin{array}{c}\text { CS EL } \\
\text { Inestabilidad }\end{array}$ & $\begin{array}{c}\text { CS Mínimos } \\
\text { geométricos }\end{array}$ \\
\hline $0 / 1$ & 40.5 & 1.40 & 1.07 & 1.29 \\
\hline $1 / 2$ & 48.7 & 1.45 & 1.02 & 2.94 \\
\hline $2 / 3$ & 44.6 & 1.47 & 1.01 & 1.29 \\
\hline $3 / 4$ & 46.2 & 1.62 & 1.02 & 1.29 \\
\hline $4 / 5$ & 44.9 & 1.60 & 1.01 & 1.94 \\
\hline $5 / 6$ & 48.5 & 1.64 & 1.01 & 2.26 \\
\hline $6 / 7$ & 47.1 & 1.60 & 1.04 & 2.26 \\
\hline $7 / 8$ & 42.3 & 1.31 & 1.10 & 2.68 \\
\hline
\end{tabular}

Tabla 6.18. Coeficientes de seguridad mínimos en estructuras optimizadas. ELU agotamiento por solicitaciones normales, EL Inestabilidad y cumplimiento de cuantías geométricas mínimas. Pórtico de ocho plantas.

Pilares centrales.

\begin{tabular}{ccccc}
\hline Planta & $\begin{array}{c}\text { Esbeltez } \\
\text { mecánica }\end{array}$ & $\begin{array}{c}\text { CS mínimo } \\
\text { ELU Agot. Sol. } \\
\text { Normales }\end{array}$ & $\begin{array}{c}\text { CS EL } \\
\text { Inestabilidad }\end{array}$ & $\begin{array}{c}\text { CS Mínimos } \\
\text { geométricos }\end{array}$ \\
\hline $0 / 1$ & 28.8 & 1.75 & 1.39 & 1.07 \\
\hline $1 / 2$ & 47.0 & 2,25 & 1,6 & 1,17 \\
\hline $2 / 3$ & 39.2 & 2.32 & 1.89 & 1.34 \\
\hline $3 / 4$ & 40.6 & 2.53 & 1.89 & 1.47 \\
\hline $4 / 5$ & 39.4 & 2.3 & 1.7 & 1.26 \\
\hline $5 / 6$ & 38.3 & 2.52 & 1.95 & 1.41 \\
\hline $6 / 7$ & 39.1 & 2.7 & 2.07 & 1.29 \\
\hline $7 / 8$ & 39.7 & 3.08 & 2.36 & 1.51 \\
\hline
\end{tabular}


- Las cuantías máximas y mínimas mecánicas no son en dimensionantes, pues sus respectivos coeficientes de seguridad son superiores a los de los mínimos geométricos (véase la Tabla 6.19).

Tabla 6.19. Coeficientes de seguridad mínimos de las armaduras mínimas y máximas.

\begin{tabular}{cccc}
\hline $\begin{array}{c}\text { Número de } \\
\text { plantas }\end{array}$ & $\begin{array}{c}\text { CS mínimo } \\
\text { Mínimos } \\
\text { geométricos }\end{array}$ & $\begin{array}{c}\text { CS mínimo } \\
\text { Mínimos } \\
\text { mecánicos }\end{array}$ & $\begin{array}{c}\text { CS mínimo } \\
\text { Máximos } \\
\text { mecánicos }\end{array}$ \\
\hline 2 & 1.51 & 2.65 & 4.61 \\
\hline 4 & 1.13 & 1.35 & 5.83 \\
\hline 6 & 1.26 & 1.14 & 5.46 \\
\hline 8 & 1.07 & 1.20 & 5.83 \\
\hline
\end{tabular}

- En lo que respecta a las armaduras de cortante, los pilares van armados con la armadura más próxima a la armadura mínima que resulte compatible con los armados tipo disponibles y con el correcto arriostramiento de la armadura longitudinal del pilar (comentarios al Art. 42.3.1 de la EHE). Esto puede comprobarse en la Tabla 6.20 .

Tabla 6.20. Coeficientes de seguridad mínimos en estructuras optimizadas correspondientes a la verificación del ELU de agotamiento frente a cortante. Pilares.

\begin{tabular}{cccc}
\hline Número de plantas & CS V $_{\mathrm{u} 1}$ & CS V $_{\mathrm{u} 2}$ & CS A $_{\alpha, \min }$ \\
\hline 2 & 14.65 & 3.32 & 1.50 \\
\hline 4 & 16.44 & 3.20 & 1.00 \\
\hline 6 & 13.73 & 2.55 & 1.00 \\
\hline 8 & 12.49 & 2.89 & 1.00 \\
\hline
\end{tabular}

- La Figura 6.8 proporciona la cuantías de armadura longitudinal de pilares del pórtico optimizado de seis plantas. A la vista de ellas, se puede señalar que, 
generalmente, las cuantías de armadura longitudinal en pilares son mayores en los pilares extremos que en los interiores situados a una misma altura. Además, la cuantía de armadura de los pilares se incrementa al aumentar la planta en la que nace el pilar. Ello es debido a la mayor importancia de los esfuerzos de flexión frente a los axiles en los pilares más armados. Similares conclusiones se obtienen si se estudian las otras estructuras.

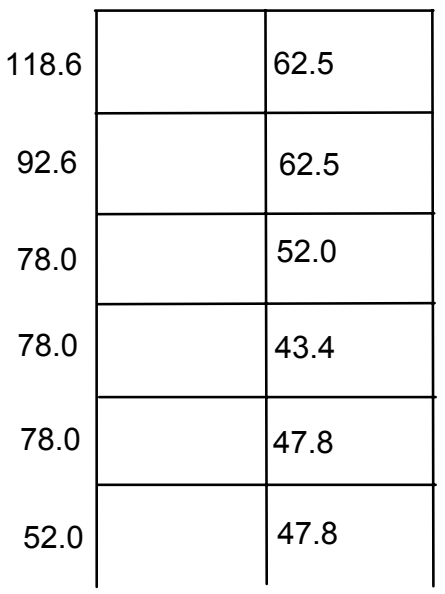

Figura 6.8. Cuantías de armadura longitudinal (en $\mathrm{Kg} / \mathrm{m}^{3}$ de hormigón) en los pilares de la estructura optimizada de seis plantas de menor coste.

\subsubsection{Estructura.}

Todas las estructuras emplean acero B-500S y hormigones con resistencia a compresión entre 30 y $45 \mathrm{MPa}$, superior a la normalmente empleada en este tipo de estructuras. Estos resultados confirman la idoneidad de considerar la resistencia del hormigón como una variable más en el análisis.

Además, los coeficientes de seguridad frente a derivas y desplazamientos horizontales máximos del pórtico (véase la Tabla 6.21) muestran que, estructuras aporticadas optimizadas relativamente esbeltas, son capaces de resistir las acciones horizontales consideradas (viento para una situación topográfica normal) sin necesidad de ningún elemento de rigidización especial. No obstante, se aprecia una clara disminución de los coeficientes de seguridad al aumentar el número de plantas del pórtico. 
Tabla 6.21. Coeficientes de seguridad mínimos relativos al cumplimiento de las derivas y desplazamientos horizontales máximos.

\begin{tabular}{ccc}
\hline $\begin{array}{c}\text { Número de } \\
\text { plantas }\end{array}$ & CS Derivas & $\begin{array}{c}\text { CS Desp } \\
\text { horizontal }\end{array}$ \\
\hline 2 & 11.4 & 4.91 \\
\hline 4 & 5.9 & 2.71 \\
\hline 6 & 3.9 & 1.78 \\
\hline 8 & 3.4 & 1.66 \\
\hline
\end{tabular}

\subsubsection{Comparación con los resultados obtenidos o empleados por otros autores.}

Los resultados de este trabajo de optimización confirman las conclusiones de Moragues en su tesis doctoral (1980) en lo referente a:

- La importancia de la armadura de compresión en las secciones que condicionan el diseño a flexión de las vigas de los pórticos.

- Las cuantías de armadura longitudinal en pilares.

Sin embargo, las resultados muestran la necesidad de verificar varios estados límites por él no considerados: el EL de deformaciones en vigas, el EL de Inestabilidad en pilares y los estados límites relativos a la resistencia frente al esfuerzo cortante.

Se han intentado comparar los resultados de esta investigación con los proporcionados por Gómez (1998), si bien esto no es posible por ser las condiciones de su estudio muy diferentes a las de esta investigación pues:

- Considera en el coste de la estructura el precio del espacio ocupado por los pilares, lo que favorece el incremento de las cuantías de acero en pilares y la disminución de su escuadría. De hecho, emplea como criterio de armado en pilares el de adoptar una cuantía de armado igual a los máximos mecánicos de la instrucción de hormigón, lo que no se corresponde con los resultados obtenidos en esta investigación. 
- Emplea como marco normativo la Instrucción de hormigón EH-91 que en algunos casos, como en la verificación del Estado Límite de Agotamiento frente a Cortante, es menos restrictiva que la actual EHE.

- Incluye en su estudio solamente hormigones con resistencia característica a compresión inferior a $25 \mathrm{MPa}$.

- Tiene en cuenta la influencia de la existencia de huecos, brochales y voladizos en la edificación y la existencia de estos elementos supone modificaciones al alza de las cuantías de acero.

Finalmente indicar que, a diferencia de lo que sucedió en las investigaciones de Alcalá (2004), Carrera (2004) y Perea (2004), las características de las soluciones optimizadas no hacen necesaria la introducción de modificaciones en los métodos habituales de diseño de pórticos de edificación de hormigón armado.

Otros autores (Rajev y Krishnamoorty, 1998; Camp et. al., 2003; Lee y Ahn, 2003; Sahab et al., 2004, 2005) que han empleado métodos heurísticos se han limitado a aplicar un método a una estructura concreta, sin aportar conclusiones generales de las estructuras optimizadas, por lo que no ofrecen resultados comparables.

\subsection{ANÁLISIS DEL COSTE DE LAS ESTRUCTURAS OPTIMIZADAS.}

Las Figuras 6.9, 6.10 y 6.11 muestran cómo depende 1) el coste material, 2) el coste por metro cuadrado de forjado y 3 ) el coste de las diferentes unidades de obra de las estructuras optimizadas de su número de plantas.

A la vista de las mismas, pueden extraerse las siguientes conclusiones:

1) El coste total de los pórticos óptimos (Figura 6.9) aumenta linealmente con el número de plantas de acuerdo con la expresión:

$$
C_{E S T}=1094.3 N p-741.23 \quad\left(R^{2}=0.9923\right)
$$




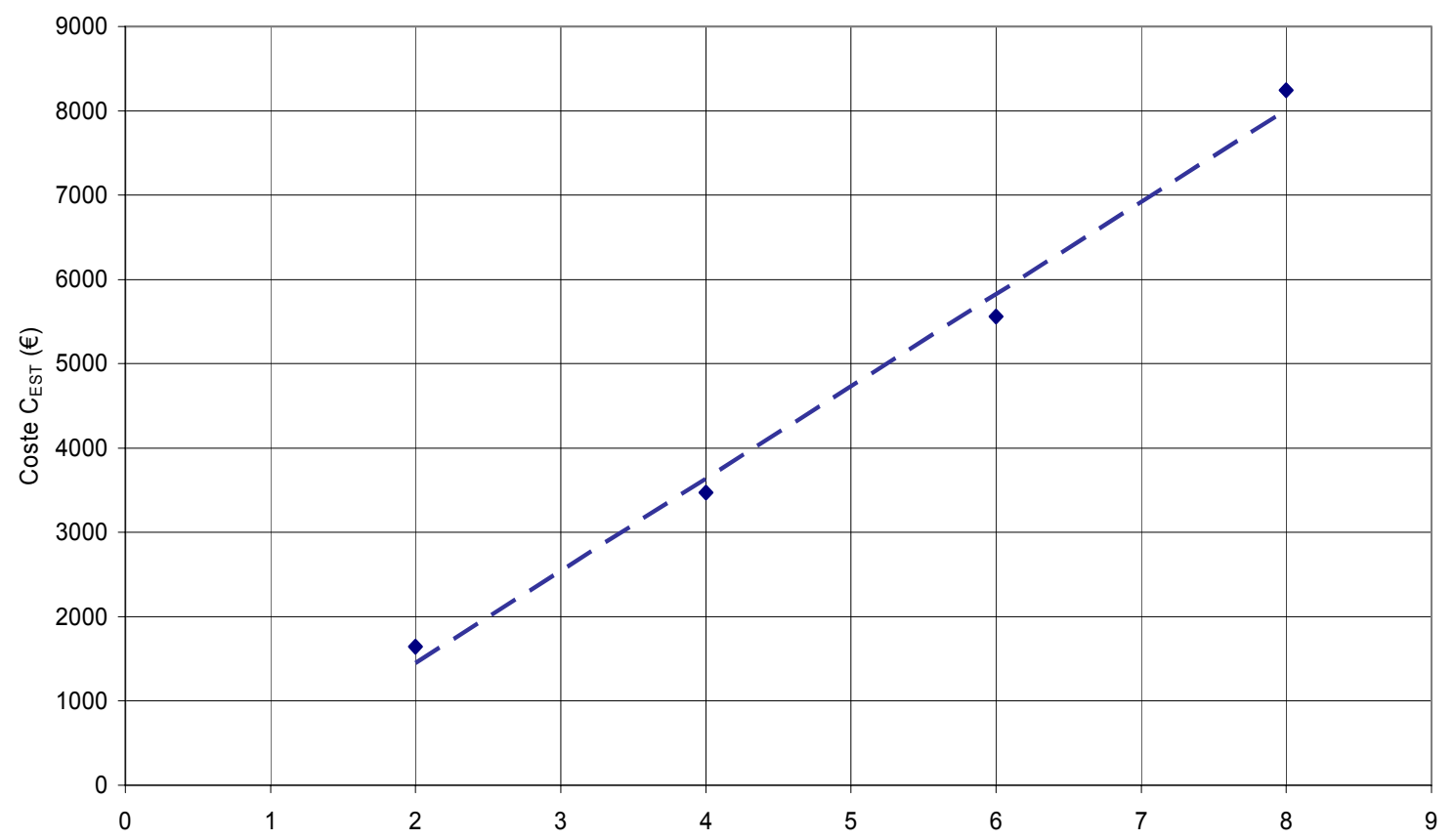

Figura 6.9. Relación entre el número de plantas de la estructura y el coste total del pórtico optimizado.

2) El coste de los pórticos por metro cuadrado de forjado construido varía de forma cuadrática con el número de plantas (Figura 6.10) según la expresión:

$$
C_{E S T, M 2}=0.0713 N p^{2}-0.0265 N p+16.23 \quad\left(R^{2}=0.9979\right)
$$

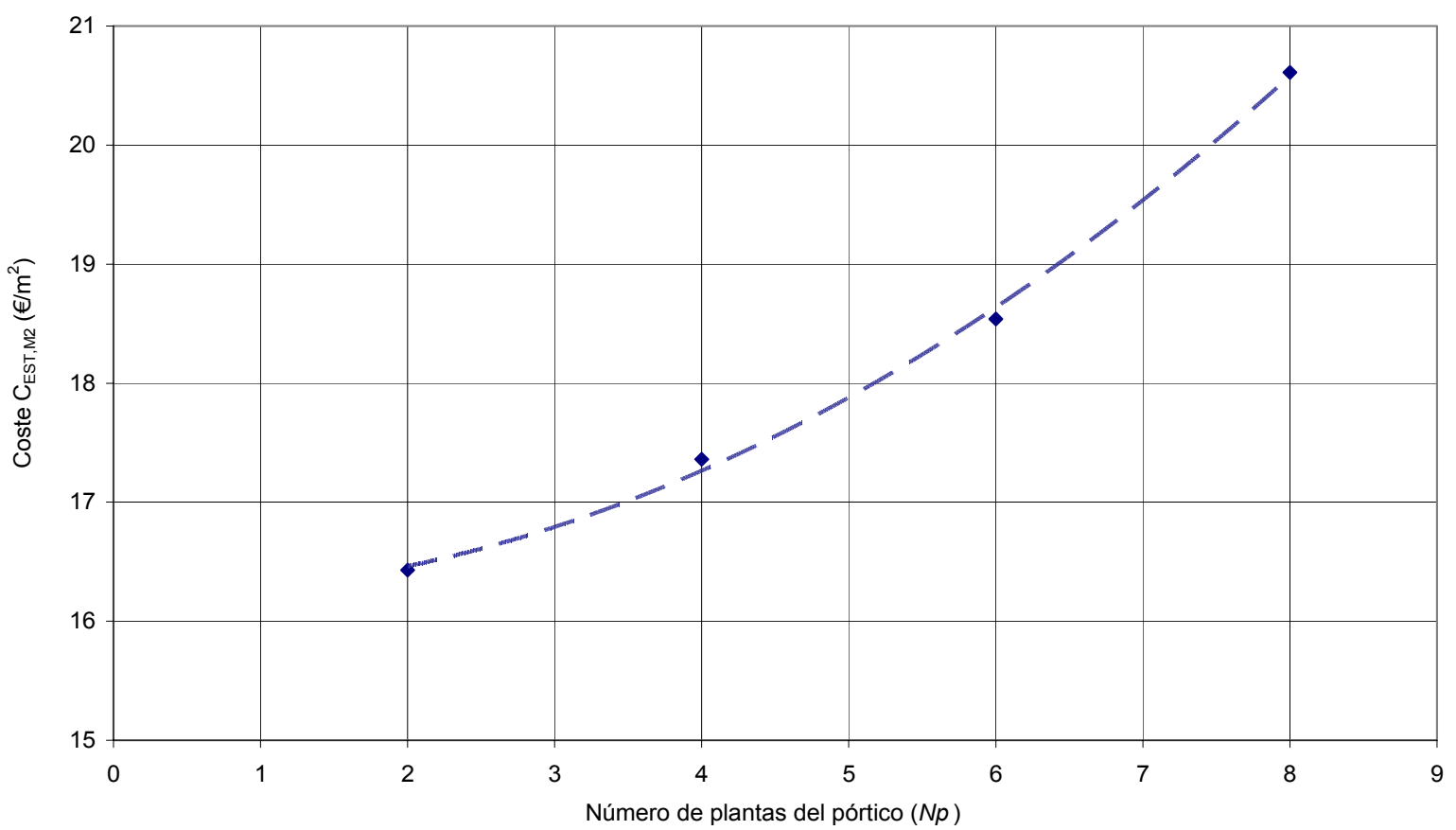

Figura 6.10. Relación entre el número de plantas de la estructura y el coste total del pórtico por $\mathrm{m}^{2}$ de forjado en estructuras optimizadas. 
3) Los porcentajes del coste total que representa cada una de las unidades de obra (Figura 6.11) no experimentan grandes variaciones con el número de plantas. Los encofrados suponen la partida más importante (entre un 39.88 y un $46.07 \%$ del coste), seguidos por los hormigones (entre un 27.02 y un $30.62 \%$ ), la armadura pasiva (entre un 16.98 y un $21.36 \%$ ) y el cimbrado de vigas (entre un 8.20 y un $9.92 \%$ ). Al aumentar el número de plantas del edificio disminuye la importancia relativa del coste de los encofrados y aumenta la del acero para armar.

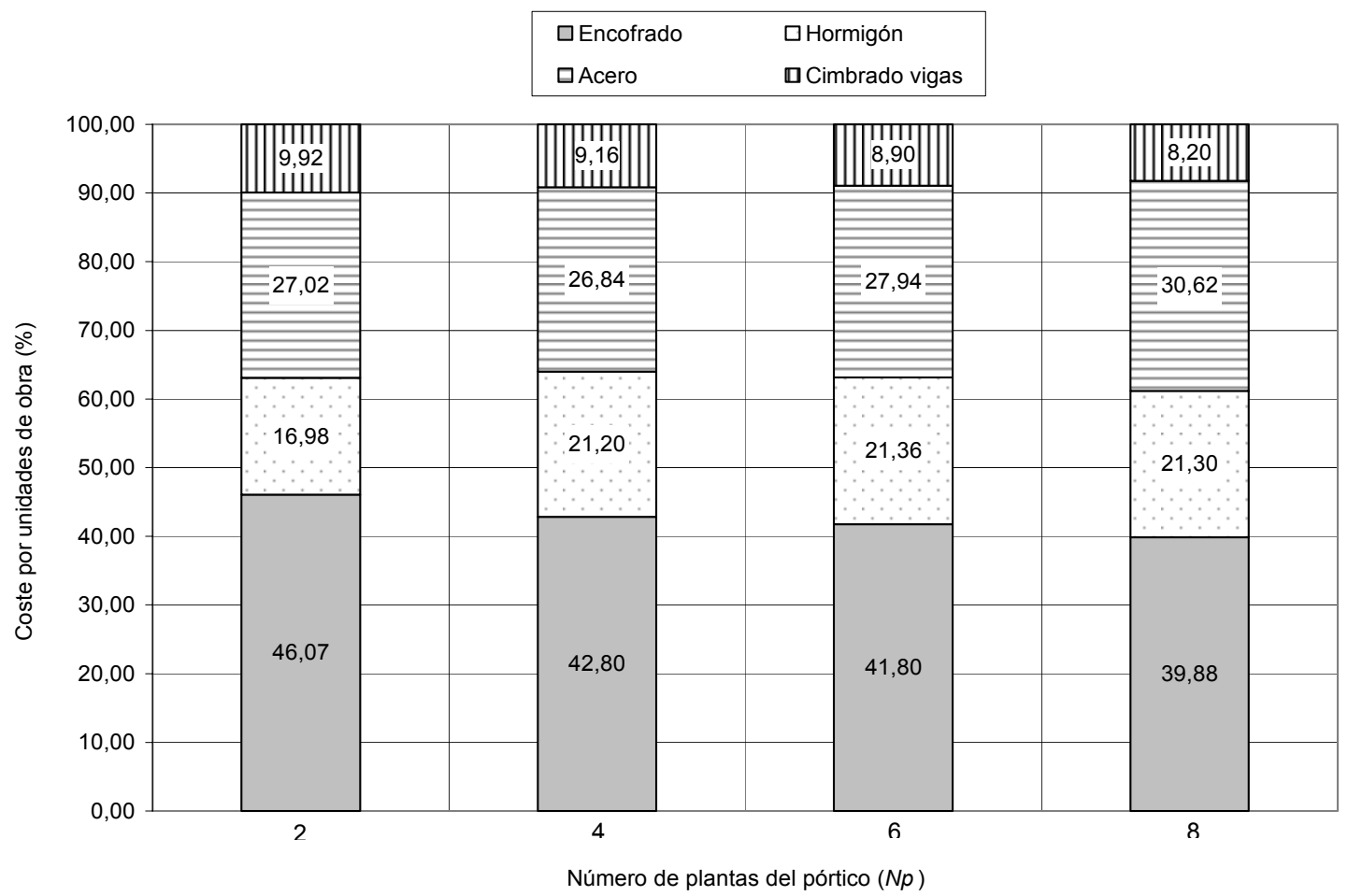

Figura 6.11. Porcentaje del coste total que representa cada una de las unidades de obra en los pórticos optimizados.

4) Las armaduras longitudinales de las vigas suponen más de la mitad del peso total del acero del pórtico (el porcentaje exacto varía entre el $54.79 \%$ y el $57.11 \%$ ). Le siguen en importancia las longitudinales de los pilares (porcentaje entre el $22.19 \%$ y el $25.24 \%$ ) y las transversales de vigas (porcentaje entre el $10.13 \%$ y el $13.46 \%$ ) y pilares (porcentaje entre el $6.64 \%$ y el $8.13 \%$ ). El total de la armadura transversal supone entre un $18.25 \%$ y un $20.10 \%$ del total de la armadura del pórtico y, por tanto, su influencia debe ser considerada en la búsqueda de la solución óptima a diferencia de lo realizado en algunos estudios anteriores. Estos resultados se visualizan en la Figura 6.12. 


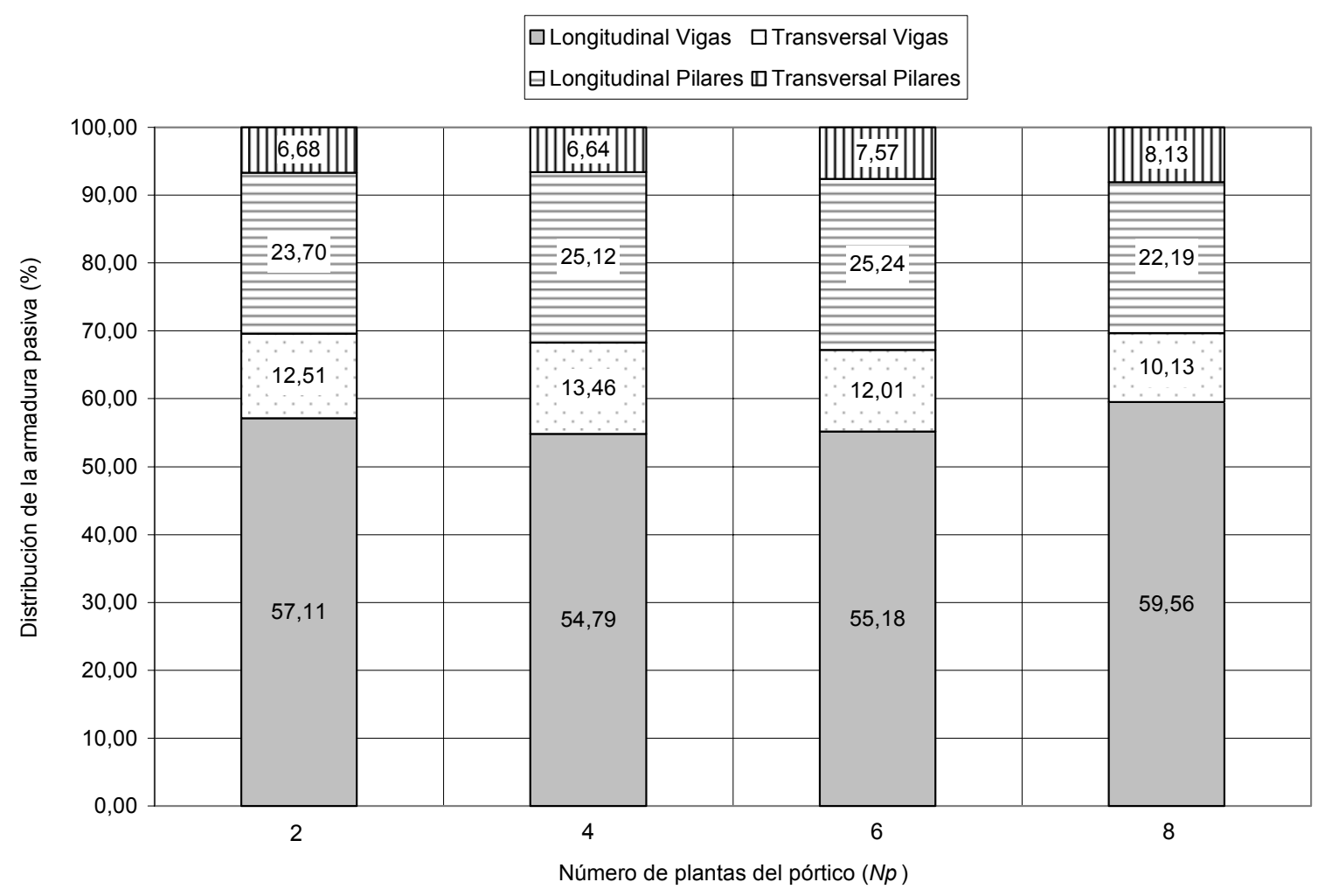

Figura 6.12. Distribución de la armadura pasiva en los pórticos optimizados (porcentajes referidos al peso total de las armaduras).

Señalamos finalmente que estas conclusiones son válidas para estructuras con dos vanos y con el resto de parámetros considerados en este estudio, precisándose más experimentación para ampliar su campo de validez. 
Página intencionadamente en blanco. 


\section{CAPÍTULO 7. RESULTADOS ADICIONALES DE OPTIMIZACIÓN ECONÓMICA.}




\section{Objetivos.}

Los resultados de los capítulos anteriores muestran que los hormigones de las estructuras óptimas tienen calidades superiores a la habitual en edificación en España (HA25). Esto puede plantear problemas de facilidad constructiva al emplearse normalmente HA25 en la capa de compresión de los forjados unidireccionales.

Por ello, se estudian en este capítulo las características de las soluciones óptimas considerando únicamente HA-25. Además, puesto que la gran mayoría de las estructuras en España se construyen con vigas planas, se aborda aquí la optimización de esta tipología estructural. Asimismo, se proporcionan reglas para el predimensionamiento de estas estructuras y se comparan con las propuestas de autores de gran renombre. 


\subsection{INTRODUCCIÓN.}

La gran mayoría de los edificios construidos en España con un máximo de ocho plantas emplean hormigón HA-25 y vigas con un canto igual al de los forjados (vigas planas). Por otro lado, interesa, desde el punto de vista constructivo, igualar dimensiones y elementos de diferentes miembros estructurales, pues ello simplifica la ejecución de la obra. Esto también es deseable desde el punto de vista de la optimización, pues disminuye el número de variables del problema y, por tanto, los tiempos de cálculo. La agrupación de variables es una práctica adoptada en trabajos anteriores (Rajeev y Krishnamoorthy, 1998; Lee et al., 2003), cuya repercusión económica no se ha estudiado.

Para cuantificar la influencia de los aspectos anteriores, el estudio paramétrico realizado en el capítulo 6 se completa ahora con la optimización del pórtico de dos vanos y ocho alturas con las condiciones indicadas en la Tabla 7.1.

Tabla 7.1. Ampliación del estudio de pórticos de dos vanos y ocho plantas.

\begin{tabular}{|c|c|c|c|}
\hline Nombre del problema & Tipo de hormigón & Tipo de Vigas & $\begin{array}{l}\text { Agrupación de } \\
\text { variables }\end{array}$ \\
\hline P_2v8h.1- $\mathrm{C}_{\mathrm{EST}}{ }^{1}$ & Variable & Descolgadas & No \\
\hline P_2v8h.2-C EST $_{\text {I }}$ & HA-25 & Descolgadas & No \\
\hline P_2v8h.3- $C_{\text {EST }}$ & $\mathrm{HA}-25$ & Descolgadas & Sí (Figura 7.1) \\
\hline P_2v8h.4-C $C_{\text {EST }}$ & HA-25 & Planas & Sí (Figura 7.1) \\
\hline
\end{tabular}

${ }^{1}$ El problema $P \_2 v 8 h .1-C_{E S T}$ ya fue resuelto en el apartado 6.1 .4

Para igualar variables se han definido cuatro grupos en las vigas (véase la Figura 7.1) de modo que todos los elementos pertenecientes a un mismo grupo tienen idénticas dimensiones y armados.

Los pórticos se han optimizado mediante SA, modificando un máximo de tres variables en cada movimiento y empleando un coeficiente $r=0.8$. El empleo de las expresiones (6.1) y (6.2) permite determinar los valores de la longitud de la Cadena de Markov ( $\mathrm{Lm}$ ) y los tiempos medios de cálculo esperados. En el caso del problema $P \_2 v 8 h .3-C_{E S T}$ se ha empleado para $L m$ el valor dado por $(6.1)$ y otro superior $(L m=135000)$ para comprobar la 
idoneidad de la expresión (6.1). Los datos necesarios y los tiempos medios de cálculo esperados se indican en la Tabla 7.2.

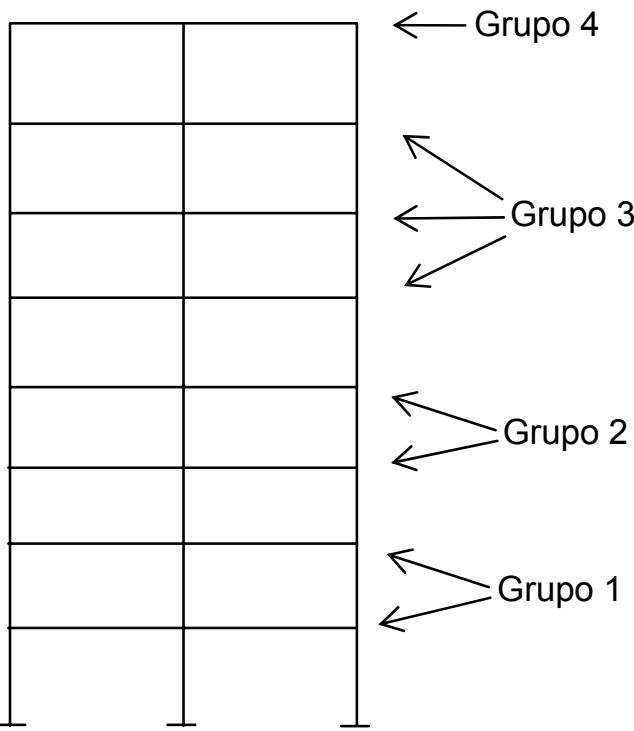

Figura 7.1. Definición de grupos.

Tabla 7.2. Optimización de pórticos de dos vanos y ocho plantas. Datos.

\begin{tabular}{|c|c|c|c|}
\hline Nombre del Problema & Nvar & $L m$ & $t_{\text {med }}(\min )$ \\
\hline P_2v8h.1-C $C_{E S T}$ & 153 & 200000 & - \\
\hline P_2v8h.2-C $C_{E S T}$ & 145 & 185000 & 345 \\
\hline P_2v8h.3-C $C_{E S T}$ & 105 & 121000 & 78 \\
\hline P_2v8h.3bis- $C_{E S T}$ & 105 & 135000 & - \\
\hline P_2v8h.4-C $C_{E S T}$ & 101 & 115000 & 68 \\
\hline
\end{tabular}

\subsection{RESULTADOS.}

\subsubsection{Tiempos y costes.}

Como en casos anteriores, cada uno de los algoritmos se ejecutó nueve veces. Los principales resultados referentes a costes y tiempos de cálculo se proporcionan en la Tabla 7.3. Las características de las soluciones óptimas se encuentran en los planos del Apéndice 4. 
Tabla 7.3. Optimización de pórticos de dos vanos y ocho plantas. Resultados.

\begin{tabular}{|c|c|c|c|c|c|c|c|}
\hline $\begin{array}{l}\text { Familia de } \\
\text { pórticos }\end{array}$ & $\begin{array}{c}\text { Coste } \\
\text { mínimo }(€)\end{array}$ & $\begin{array}{l}\text { Tiempo del } \\
\text { Coste mínimo } \\
\text { (min) }\end{array}$ & $\begin{array}{c}\text { Coste } \\
\text { medio }(€)\end{array}$ & $\begin{array}{c}\text { Tiempo } \\
\text { medio } \\
\text { (min) }\end{array}$ & $\sigma_{\text {coste }}$ & $\mathrm{CV}_{\text {coste }}$ & $\sigma_{\text {tiempo }}$ \\
\hline P_2v8h.1-C $C_{\text {EST }}$ & 8242.23 & 403.2 & 8334.44 & 361.1 & 52.54 & 0.0063 & 53.22 \\
\hline P_2v8h.2-C $C_{\text {EST }}$ & 8491.51 & 406.76 & 8534.03 & 337.9 & 39.87 & 0.0047 & 73.88 \\
\hline P_2v8h.3-C $C_{\text {EST }}$ & 8536.01 & 167.9 & 8659.32 & 184.1 & 135.95 & 0.016 & 22.87 \\
\hline $\begin{array}{c}\text { P_2v8h.3bis- } \\
C_{\text {EST }}\end{array}$ & 8609.76 & 208.5 & 8713.85 & 199.4 & 26.07 & 0.003 & 26.07 \\
\hline P_2v8h.4-C $C_{E S T}$ & 12095.04 & 176.2 & 12267.78 & 151.2 & 121.0 & 0.0099 & 31.06 \\
\hline
\end{tabular}

7.2.2 Características de las vigas de las estructuras óptimas para los pórticos de los problemas $\mathbf{P} \_2 v 8 h .3-C_{E S T}$ y $P \_2 v 8 h .4-C_{E S T}$. Reglas de predimensionado.

Las características de las vigas de estas estructuras se muestran en las tablas $7.4 \mathrm{y}$ 7.5 .

Tabla 7.4. Dimensiones de vigas en estructuras optimizadas ${ }^{2}$.

\begin{tabular}{ccccccc}
\hline \multirow{2}{*}{$\begin{array}{c}\text { Grupo de } \\
\text { vigas }\end{array}$} & \multicolumn{3}{c}{ Vigas descolgadas } & \multicolumn{2}{c}{ Vigas planas } \\
\cline { 2 - 7 } & Ancho $\boldsymbol{b}$ & L/b & Canto $\boldsymbol{h}$ & L/h & Ancho $\boldsymbol{b}$ & L/b \\
\hline 1 & 0.23 & 21.7 & 0.49 & 10.2 & 0.34 & 14.7 \\
\hline 2 & 0.23 & 21.7 & 0.54 & 9.3 & 0.40 & 12.5 \\
\hline 3 & 0.24 & 20.8 & 0.55 & 9.1 & 0.45 & 11.1 \\
\hline 4 (Cubierta) & 0.39 & 12.8 & 0.55 & 9.1 & 0.61 & 8.2 \\
\hline
\end{tabular}

${ }^{2}$ Las dimensiones de las vigas están en metros. $L$ es la luz del vano del pórtico $(5 \mathrm{~m}$. $)$.

A la vista de estos datos, las siguientes reglas de predimensionamiento pueden ser enunciadas: 
"1. La relación luz/canto de las vigas descolgadas de pórticos óptimos sometidos a acciones verticales y horizontales varía entre 9 y 10.5. La relación luz/ancho de las vigas de estos elementos varía entre 20 y 22 en las plantas tipo y entre 12 y 13 en la planta de cubierta. Las vigas pueden ser de menores dimensiones si las acciones horizontales son de escasa importancia".

"2. La relación luz/ancho de las vigas planas de pórticos óptimos sometidos a acciones verticales y horizontales varía entre 11 y 14 para la planta tipo y adopta valores próximos a 8 en la planta de cubierta. Estos valores han sido obtenidos con un canto de las vigas y de los forjados que es igual a 1/17 de la luz de las vigas, por lo que valores superiores o inferiores de los anchos son empleables si disminuye o aumenta el canto de los forjados. Las vigas pueden ser de menores dimensiones si las acciones horizontales son de escasa importancia".

Tabla 7.5. Cuantías de las vigas óptimas ( $\mathrm{Kg}$ acero $/ \mathrm{m}^{3}$ de hormigón).

\begin{tabular}{cccccccc}
\hline \multirow{2}{*}{$\begin{array}{c}\text { Grupo de } \\
\text { vigas }\end{array}$} & \multicolumn{3}{c}{ Vigas descolgadas } & & \multicolumn{3}{c}{ Vigas planas } \\
\cline { 2 - 4 } \cline { 7 - 8 } & Flexión & Cortante & Total & & Flexión & Cortante & Total \\
\hline 1 & 102 & 25 & 127 & & 253 & 39 & 292 \\
\hline 2 & 90 & 21 & 111 & & 262 & 38 & 300 \\
\hline 3 & 90 & 18 & 108 & & 259 & 36 & 295 \\
\hline 4 (Cubierta) & 50 & 15 & 65 & & 276 & 23 & 299 \\
\hline
\end{tabular}

A la vista de los resultados anteriores, puede decirse que:

“3. Las cuantías totales de armadura en las vigas descolgadas de pórticos óptimos sometidos a acciones verticales y horizontales varían entre 100 y $130 \mathrm{Kg} / \mathrm{m}^{3}$ en la planta tipo y entre 60 y $70 \mathrm{Kg} / \mathrm{m}^{3}$ en la planta de cubierta. La armadura de cortante supone un porcentaje entre el 20 y el $25 \%$ de la armadura total".

Señalamos que las vigas de cubierta tienen una cantidad de acero (expresada en $\mathrm{Kg}$ ) similar a las de las vigas de la planta tipo, pero, al ser superiores sus dimensiones, la cuantía finalmente resultante es inferior. 
"4. Las cuantías totales de armadura en las vigas planas de pórticos óptimos sometidos a acciones verticales y horizontales varían entre 290 y $300 \mathrm{Kg} / \mathrm{m}^{3}$ en la planta tipo y entre 90 y $110 \mathrm{Kg} / \mathrm{m}^{3}$ en la planta de cubierta. La armadura de cortante supone un porcentaje entre el 7 y $15 \%$ de la armadura total ".

\subsubsection{Características de los pilares de las estructuras óptimas.}

Las tablas $7.6,7.7$ y 7.8 presentan las escuadrías de los pilares de las estructuras optimizadas y sus cuantías de armadura.

Tabla 7.6. Escuadrías de pilares en estructuras optimizadas ${ }^{3}$.

\begin{tabular}{ccccc}
\hline \multirow{2}{*}{ Planta } & \multicolumn{2}{c}{ Vigas descolgadas } & \multicolumn{2}{c}{ Vigas planas } \\
\cline { 2 - 5 } & $\begin{array}{c}\text { Pilares } \\
\text { interiores }\end{array}$ & $\begin{array}{c}\text { Pilares } \\
\text { exteriores }\end{array}$ & $\begin{array}{c}\text { Pilares } \\
\text { interiores }\end{array}$ & $\begin{array}{c}\text { Pilares } \\
\text { exteriores }\end{array}$ \\
\hline Cubierta & $30 \times 35$ & $25 \times 35$ & $35 \times 30$ & $25 \times 55$ \\
\hline Inmediata inferior & $35 \times 40$ & $25 \times 35$ & $40 \times 35$ & $25 \times 55$ \\
\hline Inmediata inferior & $40 \times 40$ & $25 \times 35$ & $45 \times 40$ & $25 \times 55$ \\
\hline Inmediata inferior & $40 \times 40$ & $25 \times 35$ & $50 \times 45$ & $25 \times 55$ \\
\hline Inmediata inferior & $40 \times 45$ & $25 \times 40$ & $55 \times 50$ & $25 \times 60$ \\
\hline Inmediata inferior & $45 \times 50$ & $25 \times 40$ & $55 \times 55$ & $25 \times 65$ \\
\hline Inmediata inferior & $50 \times 55$ & $25 \times 40$ & $55 \times 60$ & $30 \times 70$ \\
\hline Inmediata inferior & $55 \times 60$ & $25 \times 40$ & $60 \times 60$ & $30 \times 70$
\end{tabular}

${ }^{3}$ El primer número de la escuadría indica el ancho del pilar y el segundo su canto 
Tabla 7.7. Cuantías mecánicas de la armadura longitudinal $(\omega)$ de los pilares de las estructuras optimizadas ${ }^{4}$.

\begin{tabular}{ccccc}
\hline \multirow{2}{*}{ Planta } & \multicolumn{2}{c}{ Vigas descolgadas } & \multicolumn{2}{c}{ Vigas planas } \\
\cline { 2 - 5 } & $\begin{array}{c}\text { Pilares } \\
\text { interiores }\end{array}$ & $\begin{array}{c}\text { Pilares } \\
\text { exteriores }\end{array}$ & $\begin{array}{c}\text { Pilares } \\
\text { interiores }\end{array}$ & $\begin{array}{c}\text { Pilares } \\
\text { exteriores }\end{array}$ \\
\hline Cubierta & 0.113 & 0.202 & 0.112 & 0.229 \\
\hline Inmediata inferior & 0.107 & 0.135 & 0.126 & 0.129 \\
\hline Inmediata inferior & 0.105 & 0.135 & 0.131 & 0.172 \\
\hline Inmediata inferior & 0.131 & 0.202 & 0.105 & 0.238 \\
\hline Inmediata inferior & 0.111 & 0.177 & 0.107 & 0.249 \\
\hline Inmediata inferior & 0.111 & 0.315 & 0.108 & 0.274 \\
\hline Inmediata inferior & 0.126 & 0.491 & 0.167 & 0.340 \\
\hline Inmediata inferior & 0.112 & 0.315 & 0.107 & 0.112 \\
\hline${ }^{2} \omega=A_{s} f_{y d} /\left(f_{c d} b h\right)$ donde $\mathrm{A}_{s}$ es el área de armadura y b, h son el ancho y el canto del pilar
\end{tabular}

Tabla 7.8. Cuantías geométricas de la armadura longitudinal de los pilares de las estructuras optimizadas ( $\mathrm{Kg}$ acero $/ \mathrm{m}^{3}$ de hormigón).

\begin{tabular}{|c|c|c|c|c|}
\hline \multirow{2}{*}{ Planta } & \multicolumn{2}{|c|}{ Vigas descolgadas } & \multicolumn{2}{|c|}{ Vigas planas } \\
\hline & $\begin{array}{c}\text { Pilares } \\
\text { interiores }\end{array}$ & $\begin{array}{c}\text { Pilares } \\
\text { exteriores }\end{array}$ & $\begin{array}{l}\text { Pilares } \\
\text { interiores }\end{array}$ & $\begin{array}{c}\text { Pilares } \\
\text { exteriores }\end{array}$ \\
\hline Cubierta & 37 & 67 & 37 & 76 \\
\hline Inmediata inferior & 42 & 45 & 42 & 43 \\
\hline Inmediata inferior & 37 & 45 & 43 & 57 \\
\hline Inmediata inferior & 37 & 67 & 35 & 79 \\
\hline Inmediata inferior & 43 & 59 & 36 & 82 \\
\hline Inmediata inferior & 35 & 104 & 36 & 91 \\
\hline Inmediata inferior & 35 & 163 & 55 & 113 \\
\hline Inmediata inferior & 37 & 104 & 36 & 37 \\
\hline $\begin{array}{l}\text { Cuantía media de } \\
\text { todos los pilares }\end{array}$ & \multicolumn{2}{|c|}{60} & \multicolumn{2}{|c|}{59} \\
\hline
\end{tabular}




\subsection{ANÁLISIS DE LOS RESULTADOS.}

\subsubsection{Tiempos y costes.}

Los resultados confirman la idoneidad de la expresión (6.1) para determinar el valor de longitud de la cadena de Markov Lm en la heurística SA propuesta. Ello es debido a que la estrategia con $L m=145000$ no ha sido capaz de proporcionar mejores resultados que la que empleó $L m=121000$.

La disminución del número de variables permite bajar significativamente los tiempos medios de cálculo. Por ejemplo, pasar de 153 variables (problema $P \_2 v 8 h .1-C_{E S T}$ ) a 105 $\left(P \_2 v 8 h .3-C_{E S T}\right)$ redujo los tiempos de cálculo de 361 minutos a 168 , lo que supone un $49 \%$ menos de tiempo. Esta disminución no es tan grande como la prevista - 78 minutos es el $t_{\text {med }}$ estimado por la expresión (6.2) -. Ello se debe a que la gran mayoría del tiempo de cálculo se emplea en la inversión de la matriz de rigidez de la estructura y éste tiempo depende de las dimensiones del pórtico y no de su número de variables. Al agrupar variables, reducimos el tamaño del espacio de soluciones, por lo que el algoritmo converge antes, pero el tiempo necesario para calcular una estructura sigue siendo el mismo. Por ello, la ecuación (6.2) sirve como una cota superior del tiempo medio de cálculo si se entra en ella con el número de variables correspondiente a la no agrupación de ninguna variable, pero debería corregirse si se quisiera generalizar para afinar los tiempos de cálculo cuando se igualan variables. Ello requeriría la realización de más experimentos numéricos.

Emplear exclusivamente HA-25 en la estructura supone incrementar su coste un 3.02 $\%$. Agrupar variables implica un incremento adicional del $0.54 \% \mathrm{y}$, si además se emplean vigas planas, la estructura optimizada resultante es un $46.74 \%$ más cara que el pórtico más económico del problema $P_{2} 2 v 8 h .1-C_{E S T}$ y un $41.7 \%$ más cara que su equivalente con vigas descolgadas (problema $P_{-} 2 v 8 h .3-C_{E S T}$ ).

Una descomposición de los costes por unidades de obra se muestra en la Figura 7.2. En ella se aprecia como en las estructuras con vigas descolgadas el mayor porcentaje del coste se debe al encofrado (un $44.54 \%$ ) seguido del acero (un $27.85 \%$ ). Estos porcentajes se invierten en la estructura con vigas planas en la que el acero es responsable de la mayor parte del coste (un $42.33 \%$ ) y el encofrado representa un $31.51 \%$ del coste total. 


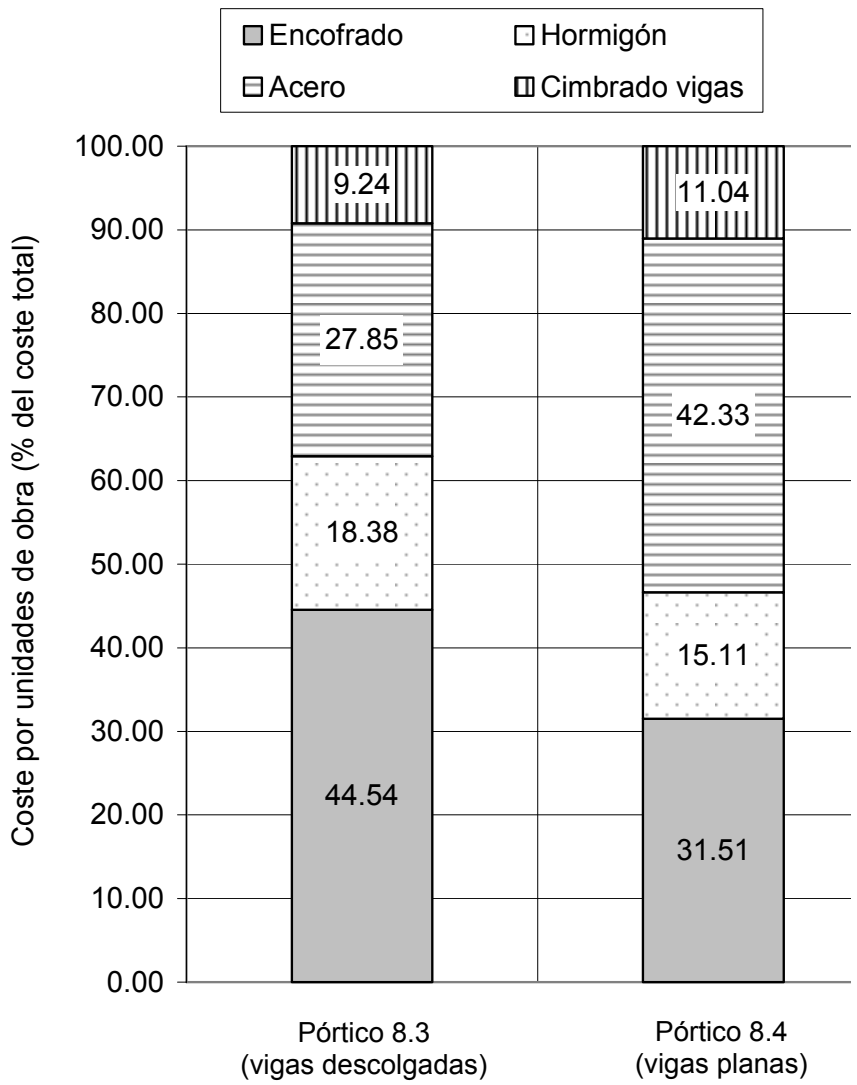

Figura 7.2. Descomposición del coste de los pórticos optimizados que emplean HA-25.

\subsubsection{Reglas de predimensionamiento.}

Calavera (1999) proporciona recomendaciones para predimensionar los elementos de un pórtico. Al hablar de dinteles de pórticos sometidos exclusivamente a acciones verticales, indica que "una relación luz/canto usual es del orden de 10 a 15 con vigas descolgadas (...). En vigas planas la relación puede oscilar de 18 a 22". No establece ningún valor para las escuadrías de los pilares pero recomienda cuantías próximas a la mínima $(\omega=0.1$ a 0.15$)$ a menos que motivos funcionales aconsejen reducir las dimensiones de los pilares.

Los valores obtenidos en este estudio se aproximan a estas recomendaciones pues se proponen relaciones luz/canto entre 9 y 11 para vigas descolgadas y un valor de 17 para las vigas planas. En lo que respecta a las cuantías, todos los pilares interiores tienen cuantías entre 0.1 y 0.15 y solamente los de medianería, en los que los esfuerzos de flexión son más importantes, tienen cuantías superiores. El análisis realizado completa los valores proporcionados por este autor al tener en cuenta las acciones horizontales y, además, proponer valores de anchos para las vigas y dar valores de cuantías en vigas y pilares. 
Rodríguez (2001), basándose en su experiencia, proporciona las recomendaciones siguientes pensadas para luces de $5.50 \mathrm{~m}$, hormigón HA-25 y carga total de $700 \mathrm{Kg} / \mathrm{m}^{2}$ :

- Dimensiones mínimas de vigas planas de luz $L$ :

- Cantos: $L / 30$ en vanos interiores y $L / 24$ en vanos extremos.

- Anchos: $L / 10$ en alineaciones que soportan paños por ambos lados.

- Dimensiones mínimas de vigas descolgadas de luz $L$ :

- Cantos: entre $L / 10$ y $L / 12$.

- Anchos: entre 20 y $35 \mathrm{~cm}$.

- Dimensiones de pilares. Se indican en la Tabla 7.9.

Tabla 7.9. Escuadrías de pilares recomendadas por Rodríguez (2001).

\begin{tabular}{ccc}
\hline Planta & Pilares interiores & $\begin{array}{c}\text { Pilares de } \\
\text { medianería }\end{array}$ \\
\hline Cubierta & $25 \times 25$ & $25 \times 25$ \\
\hline Inmediata inferior & $25 \times 25$ & $25 \times 25$ \\
\hline Inmediata inferior & $25 \times 30$ & $25 \times 25$ \\
\hline Inmediata inferior & $30 \times 30$ & $25 \times 30$ \\
\hline Inmediata inferior & $30 \times 35$ & $25 \times 30$ \\
\hline Inmediata inferior & $30 \times 35$ & $30 \times 30$ \\
\hline Inmediata inferior & $30 \times 35$ & $30 \times 30$ \\
\hline Inmediata inferior & $35 \times 40$ & $30 \times 35$ \\
\hline
\end{tabular}

Rodríguez indica además que un buen predimensionado conduce a cuantías de acero entre 100 y $120 \mathrm{Kg} / \mathrm{m}^{3}$, si bien no especifica si esas cuantías son totales o están referidas solamente a la armadura longitudinal del pilar.

La comparación de los resultados obtenidos en los puntos 7.2.2 y 7.2.3 con las propuestas de Rodríguez en lo que respecta a los pilares revela dos criterios de diseño diferentes. Rodríguez, por motivos arquitectónicos, prefiere pilares de menores dimensiones y con cuantías de armadura más altas de las aquí obtenidas por criterios estrictamente económicos. En lo que respecta a las vigas, los criterios son muy similares para las vigas 
descolgadas, pero difieren en las vigas planas, para las que Rodríguez recomienda valores de canto inferiores a los aquí obtenidos.

\subsection{CONCLUSIONES.}

Los resultados indican que:

- Emplear hormigones de resistencia variable reduce significativamente el coste de la estructura.

- El empleo de vigas planas es altamente ineficiente desde el punto de vista económico, pues conduce a estructuras mucho más caras que sus equivalentes con vigas descolgadas.

- Agrupar variables es recomendable pues permite reducir significativamente los tiempos de cálculo y simplificar la ejecución de la estructura con pequeños incrementos de su coste.

El estudio aquí realizado ha permitido obtener reglas para el predimensionado de vigas y pilares de estructuras aporticadas sometidas a acciones verticales y horizontales. Resulta especialmente relevante que las reglas han sido obtenidas por una aplicación directa de los procedimientos de inteligencia artificial desarrollados en esta Tesis y son, en líneas generales, coherentes con las propuestas de arquitectos e ingenieros de gran experiencia y prestigio. Además, se aporta información adicional a la suministrada por ellos. En definitiva, la inteligencia artificial ha sido capaz de descubrir lo que la experiencia de muchos trabajos puede enseñar a un buen técnico. 


\section{CAPÍTULO 8. OPTIMIZACIÓN MULTIOBJETIVO.}




\section{Objetivos.}

Se aborda en este capítulo la resolución de pórticos de edificación considerando varios criterios de modo simultaneo mediante un algoritmo de la familia del SA, el SMOSA. 


\subsection{INTRODUCCIÓN.}

En capítulos anteriores de esta investigación se abordó la optimización de estructuras aporticadas de edificación considerando una única función objetivo, su coste económico. No obstante, la práctica demuestra que otros criterios también deben considerarse además a la hora de proyectar una estructura como pueden ser su facilidad de construcción o su impacto ambiental y estos objetivos podrían estar en oposición con el coste económico. Por otro lado, muchos de los coeficientes de seguridad de las estructuras optimizadas son exactamente iguales a los valores mínimos admitidos por los códigos reglamentarios, por lo que resulta interesante conocer cuál es el coste económico de incrementar un determinado valor su seguridad. Por ello, este capítulo aborda la aplicación del algoritmo SMOSA a la optimización multiobjetivo del pórtico de dos vanos y cuatro plantas de la Figura 5.1 sometido a las acciones indicadas en el capítulo 3 .

La descripción de las funciones objetivo empleadas se realizó en el epígrafe 3.5, mientras que la versión de SMOSA desarrollada para este estudio se explicó en el apartado 4.7. Los parámetros adoptados son los ya empleados para resolver el problema monoobjetivo:

- Número de variables modificadas en cada movimiento: Nvar = hasta 3 variables.

- Longitud de la cadena de Markov, $L m=70000$.

- Coeficiente de enfriamiento, $r=0.8$.

Este capítulo se inicia directamente con los resultados numéricos obtenidos. No obstante, se recuerda que se han definido dos versiones del algoritmo (SMOSA1 y SMOSA2) en función de la expresión empleada para decidir si una solución que no pertenece a la Frontera de Pareto se acepta como solución actual o no. 


\subsection{RESULTADOS NUMÉRICOS.}

\subsubsection{Introducción.}

El pórtico a analizar se optimizó primeramente mediante SA considerando como criterios independientes la minimización de su número de barras y de su coste ambiental (la minimización de su coste económico se realizó en el capítulo 5). Esto permitió calibrar el algoritmo para cada uno de los objetivos y obtener valores de referencia para la evaluación de los resultados de SMOSA. Como solución inicial se empleó la misma utilizada en el capítulo 5, la cual tiene un coste de $7800.94 €, 203$ barras en su armadura longitudinal y un impacto ambiental de 849546.12 unidades.

Los mejores valores de las funciones objetivo obtenidos con SA fueron $3473.06 €, 74$ barras y 300809.53 unidades de impacto. Para cada objetivo se realizaron nueve ejecuciones del algoritmo empleando como parámetros $L_{m}=70000, r=0.8$ y Nvar $=$ hasta 3 . La Tabla 8.1 proporciona las características más representativas de las mejores soluciones encontradas. En lo sucesivo se llama $\mathrm{C}_{\mathrm{EST}}-\mathrm{SA}, \mathrm{N}_{\mathrm{S}}-\mathrm{SA}$ y EC-SA a los problemas monoobjetivo resueltos mediante $S A$ relativos al coste económico, número de barras e impacto ambiental respectivamente.

Tabla 8.1. Problemas $P \_2 v 4 h-C_{E S T}, P \_2 v 4 h-N_{S}$ y $P \_2 v 4 h-E C$. Solución inicial y resultados de la optimización monocriterio mediante SA

\begin{tabular}{ccccc}
\hline Solución & Coste $(€)$ & $\mathbf{N}_{\mathbf{s}}$ & EC & $\begin{array}{c}\text { Tiempo de } \\
\text { cálculo (min) }\end{array}$ \\
\hline Inicial & 7800.94 & 203 & 849546.12 & 0.36 \\
\hline Mínimo $C_{\mathrm{EST}}$ & 3473.06 & 118 & 409980.53 & 18.9 \\
\hline Mínimo N & 5582.78 & 74 & 551384.38 & 15.5 \\
\hline Mínimo EC & 3724.17 & 121 & 300809.53 & 94.5 \\
\hline
\end{tabular}

A la vista de los resultados se señala que:

- La solución con menor EC tiene un elevado número de barras (121) y una elevada cuantía de armadura longitudinal en las vigas (valor medio de 289.5 $\mathrm{Kg} / \mathrm{m}^{3}$ ), lo que podría ser perjudicial desde el punto de vista de la facilidad constructiva. 
- La solución con menor $N_{s}$ tiene un elevado impacto ambiental y coste económico. Sus valores de $C_{E S T}$ y $E C$ superan en un $60.74 \%$ y en un $83.3 \%$ respectivamente a los de las mejores soluciones optimizadas encontradas.

\subsubsection{Coste frente a facilidad constructiva.}

La Tabla 8.2 resume los resultados numéricos de la aplicación de SMOSA1 y SMOSA2 a la minimización de $C_{E S T}$ y $N_{s}$. Los tiempos medios de cálculo fueron de 14.3 minutos para SMOSA1 y de 20.3 minutos para SMOSA2.

La solución con un menor número de barras $\left(\mathrm{N}_{\mathrm{s}}-\mathrm{SMOSA} 2\right)$ tiene 78 y su coste es de $3670.38 €$, lo que supone un incremento del $5.4 \%$ de $N_{s}$ y del $5.7 \%$ de $C_{E S T}$ respecto a las 74 barras y los $3473.06 €$ de las mejores soluciones proporcionadas por SA al problema monoobjetivo. No obstante, la solución proporcionada por $\mathrm{N}_{\mathrm{s}}-\mathrm{SMOSA2}$ es claramente preferible a la dada por $\mathrm{N}_{\mathrm{s}} \mathrm{S} \mathrm{SA}$, pues el impacto ambiental y el coste de esta última solución son un $37.8 \%$ y un $52.1 \%$ superiores. Por otro lado, el incremento del coste del $5.7 \%$ de la solución $\mathrm{N}_{\mathrm{s}}$-SMOSA2 está claramente justificado por su facilidad constructiva, pues la mejor solución del problema $\mathrm{C}_{\mathrm{EST}} \mathrm{SA}$ tiene 118 barras, un $51.3 \%$ más.

Tabla 8.2. Problema $P \_2 v 4 h-C_{E S T} / N_{S}$. Resultados.

\begin{tabular}{|c|c|c|c|c|}
\hline Solución & Coste $(€)$ & $\mathbf{N}_{\mathbf{s}}$ & EC & $\begin{array}{c}\text { Tiempo de } \\
\text { cálculo (min) }\end{array}$ \\
\hline Mínimo $\mathrm{N}_{\mathrm{s}}$-SMOSA1 & 3844.92 & 78 & 342349.47 & 18.8 \\
\hline Mínimo $\mathrm{C}_{\mathrm{EST}}-\mathrm{SMOSA} 1$ & 3653.00 & 96 & 396187.81 & 19.9 \\
\hline Mínimo $\mathrm{N}_{\mathrm{s}}$-SMOSA2 & 3670.38 & 78 & 400138.68 & 12.9 \\
\hline Mínimo $\mathrm{C}_{\mathrm{EST}}-\mathrm{SMOSA} 2$ & 3537.07 & 99 & 413096.68 & 15.8 \\
\hline
\end{tabular}

Las características de la solución $\mathrm{N}_{\mathrm{s}}$-SMOSA2 se proporcionan en los planos del Apéndice 4. El acero es B-500 S y el hormigón tienen una resistencia característica a compresión de $45 \mathrm{MPa}$ en los elementos de la planta primera y segunda y de $40 \mathrm{MPa}$ en el resto.

Por otro lado, la solución más económica ( $\left.\mathrm{C}_{\mathrm{EST}}-\mathrm{SMOSA2}\right)$ tiene un coste de $3537.07 €$ y 99 barras de acero, lo que supone $1.8 \%$ más de coste y un $16.1 \%$ menos de barras que 
los valores de la solución $\mathrm{C}_{E S T}-\mathrm{SA}$. Las características de $\mathrm{C}_{\mathrm{EST}}-\mathrm{SMOSA} 2$ se dan en los planos del Apéndice 4. El acero es B-500 S y el hormigón de todos los elementos HA-45.

Las Figuras 8.1 y 8.2 muestran, respectivamente, la evolución de la Frontera de Pareto a lo largo de una ejecución típica de SMOSA2 y la Frontera de Pareto estabilizada junto con la parábola que la ajusta por mínimos cuadrados. Su expresión es:

$$
C_{E S T}=0.2703 N_{s}^{2}-53.902 N_{s}+6228.5 \quad\left(R^{2}=0.98\right)
$$

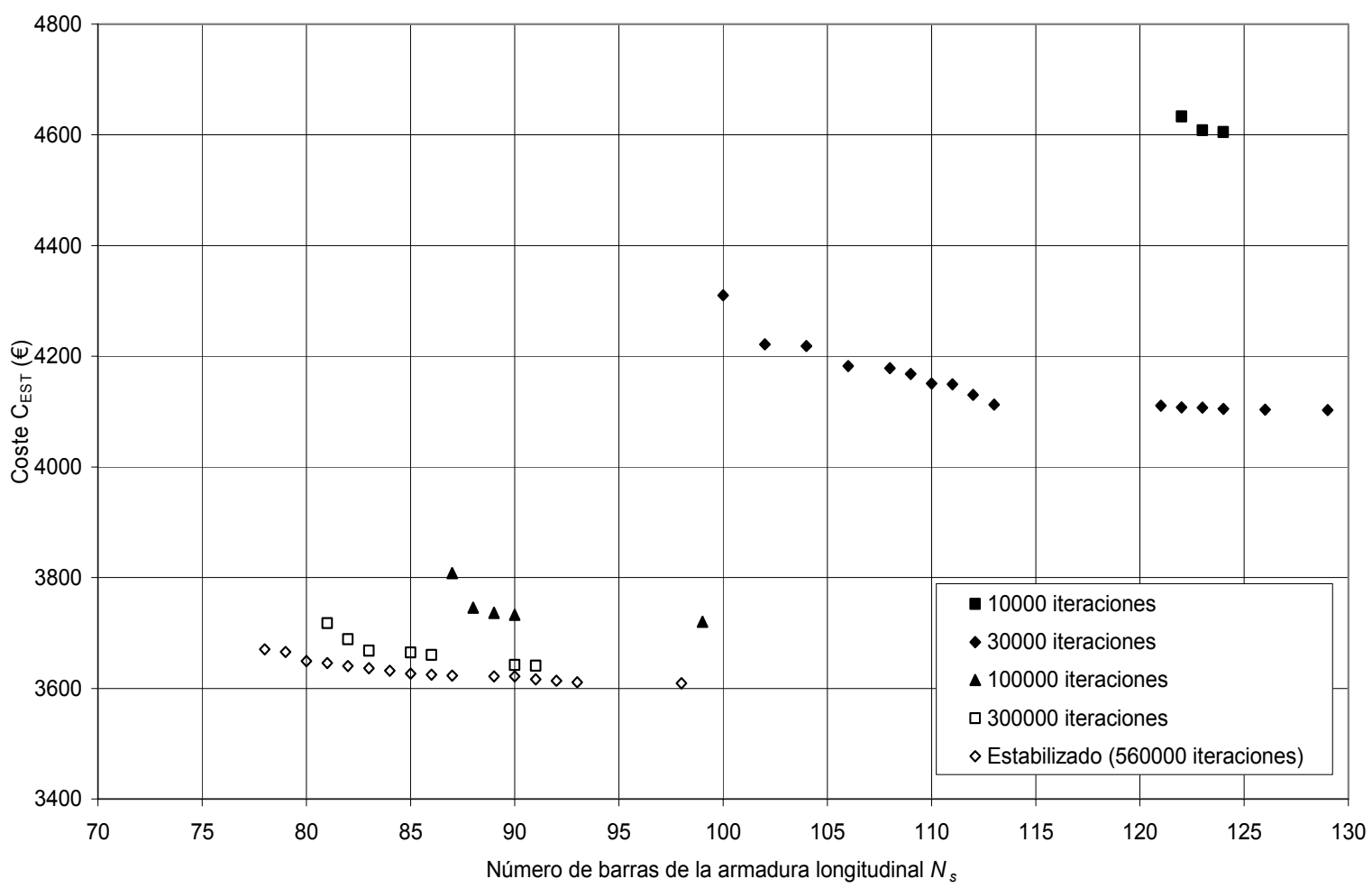

Figura 8.1. Problema $P_{-} 2 v 4 h-C_{E S T} / N_{S}$. Evolución de la Frontera de Pareto en una ejecución típica del algoritmo SMOSA2.

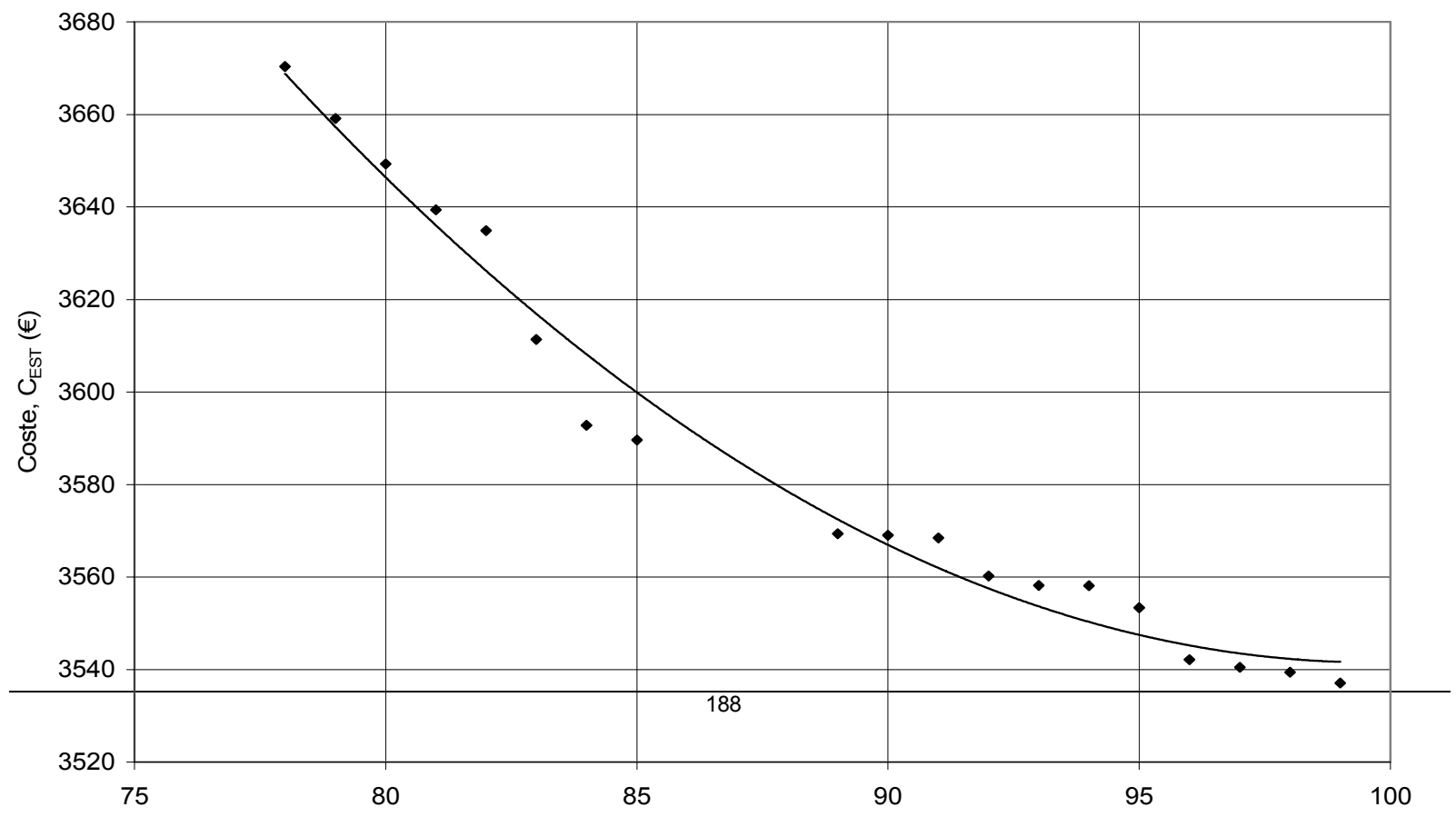




\subsubsection{Coste frente a sostenibilidad ambiental}

Los algoritmos SMOSA1 y SMOSA2 se han empleado para estudiar la relación entre el coste de la estructura y su sostenibilidad expresada a través de su puntuación ambiental EC. Los resultados se muestran en la Tabla 8.3, siendo los tiempos medios de cálculo de 17.6 y 13.8 minutos para SMOSA1 y SMOSA2 respectivamente.

Tabla 8.3. Problema $P \_2 v 4 h-C_{E S T} / E C$. Resultados.

\begin{tabular}{|c|c|c|c|c|}
\hline Solución & Coste $(€)$ & $\mathbf{N}_{\mathbf{s}}$ & EC & $\begin{array}{l}\text { Tiempo de } \\
\text { cálculo (min) }\end{array}$ \\
\hline Mínimo EC-SMOSA1 & 3670.36 & 134 & 310483.50 & 13.6 \\
\hline Mínimo $C_{E S T}-S M O S A 1$ & 3561.63 & 123 & 331011.72 & 23.0 \\
\hline Mínimo EC-SMOSA2 & 3663.50 & 104 & 321424.06 & 12.1 \\
\hline Mínimo $\mathrm{C}_{\mathrm{EST}}-\mathrm{SMOSA} 2$ & 3610.56 & 125 & 363396.84 & 15.6 \\
\hline
\end{tabular}

Los mejores resultados se han obtenido con el algoritmo SMOSA1 siendo el coste ambiental de la solución más sostenible, EC-SMOSA1, de 310483.50 unidades y su coste económico de $3670.36 €$. Estos valores son superiores en un $3.2 \%$ y en un $5.7 \%$ a los de los óptimos EC-SA y $\mathrm{C}_{\mathrm{EST}}-\mathrm{SA}$. Por otro lado, la solución de menor coste económico $\mathrm{C}_{\mathrm{EST}}$ SMOSA1 tiene un impacto ambiental de 331011.72 unidades y un coste de $3561.63 €$, lo que supone unos incrementos del $10.0 \%$ y del $2.5 \%$ respecto a los mejores valores obtenidos mediante EC-SA y $\mathrm{C}_{\mathrm{EST}}-\mathrm{SA}$. Estos resultados demuestran que soluciones de bajo coste se comportan relativamente bien en términos de sostenibilidad y a la inversa.

Las características de la solución EC-SMOSA1 se proporcionan en el Apéndice 5. El acero es B-500 S y el hormigón es HA-35 en la primera planta y HA-25 en el resto de la estructura. La Frontera de Pareto final se muestra en la Figura 8.3. El ajuste de la misma mediante una parábola por mínimos cuadrados viene dado por la expresión:

$$
C_{E S T}=\frac{2.12324}{10^{7}} E C^{2}-0.14055 E C+26825 \quad\left(R^{2}=0.91\right)
$$




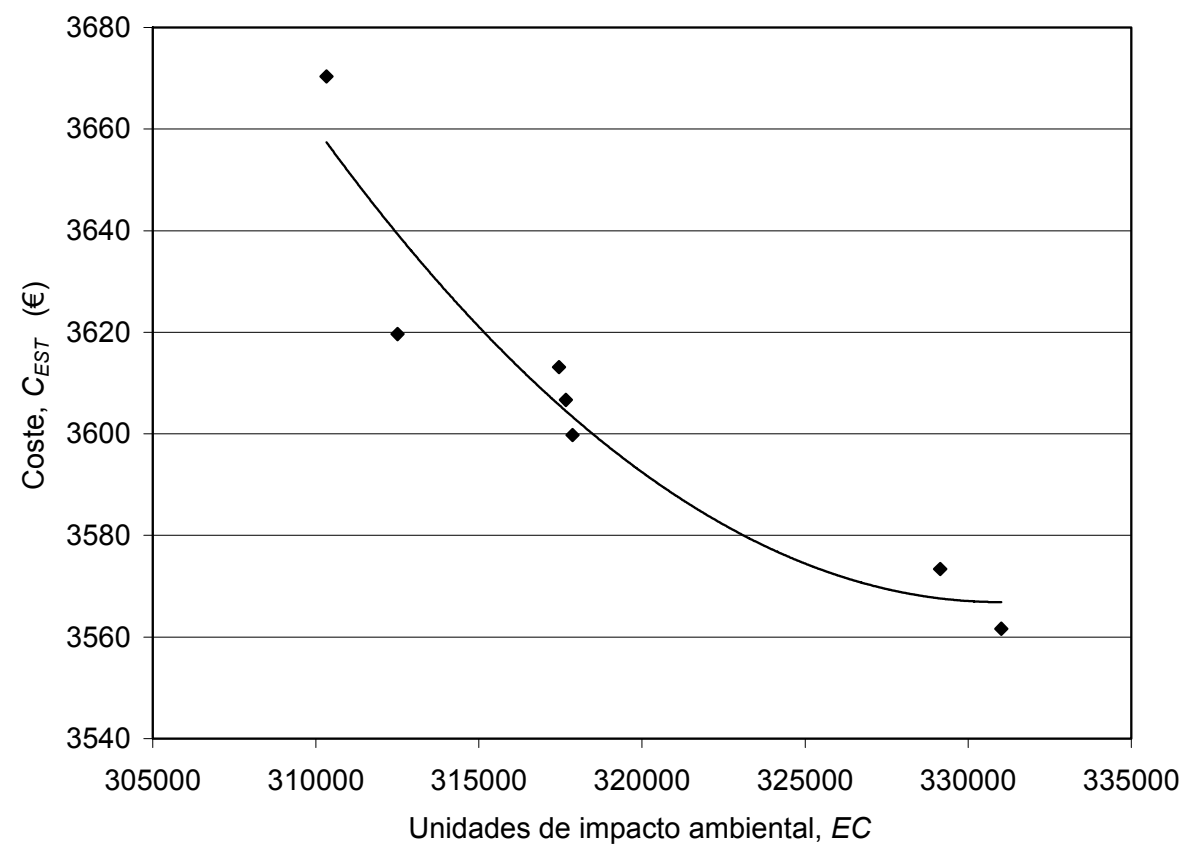

Figura 8.3. SMOSA1-EC y SMOSA2-EC. Frontera de Pareto final.

\subsubsection{Optimización simultanea de coste económico, facilidad constructiva y sostenibilidad ambiental.}

En los apartados anteriores se ha estudiado la optimización de diferentes objetivos por parejas, pero lo ideal sería proyectar una estructura económica, sencilla de construir y, además, ecológica, ¿es eso posible?.

Para responder a esta pregunta se ha confeccionado la Tabla 8.4 en la que, para los puntos extremos de las Fronteras de Pareto de las figuras 8.2 y 8.3 , se representan:

- Los porcentajes en que esas soluciones, incrementan los óptimos locales encontrados con SA - expresiones (8.3), (8.4) y (8.5) -.

$$
\begin{aligned}
& \Delta C_{E S T}=\frac{C_{E S T}(\text { solución_SMOSA })-3473.06}{3473.06} \times 100 \\
& \Delta N_{S}=\frac{N_{S}(\text { solución_SMOSA })-74}{74} \times 100 \\
& \Delta N_{S}=\frac{E C(\text { solución_SMOSA })-300809.53}{300809.53} \times 100
\end{aligned}
$$

- $\quad$ El valor medio de las expresiones anteriores y su desviación típica $\left(\sigma_{\Delta}\right)$. 
Tabla 8.4. Optimización simultanea de $\mathrm{C}_{\mathrm{EST}}, \mathrm{N}_{\mathrm{s}}$ y EC.

\begin{tabular}{|c|c|c|c|c|c|}
\hline Solución & $\Delta \mathrm{C}_{\mathrm{EST}}(\%)$ & $\Delta \mathrm{N}_{\mathrm{S}}(\%)$ & $\Delta \mathrm{EC}(\%)$ & $\frac{\Delta \mathbf{C}_{\mathrm{EST}}+\Delta \mathbf{N}_{\mathrm{S}}+\Delta \mathrm{EC}}{3}$ & $\sigma_{\Delta}$ \\
\hline Mínimo $\mathrm{N}_{\mathrm{s}}-\mathrm{SMOSA} 2$ & 5.7 & 5.4 & 33.0 & 14.7 & 15.9 \\
\hline Mínimo $\mathrm{C}_{\mathrm{EST}}$-SMOSA2 & 1.8 & 33.8 & 37.3 & 24.3 & 19.5 \\
\hline Mínimo EC-SMOSA1 & 5.7 & 81.1 & 3.2 & 30.0 & 44.3 \\
\hline Mínimo $\mathrm{C}_{\mathrm{EST}}-\mathrm{SMOSA} 1$ & 2.5 & 66.2 & 10.0 & 26.2 & 34.8 \\
\hline
\end{tabular}

Los resultados muestran que, en el entorno del óptimo económico, no se han encontrado soluciones sencillas de construir y a la vez sostenibles ambientalmente, pues valores pequeños de $\Delta N_{S}$ se traducen en $\triangle E C$ elevados y a la inversa.

\subsubsection{Coste frente a seguridad.}

Finalmente, SMOSA1 se empleó para minimizar el coste de la estructura y a la vez maximizar su coeficiente de seguridad global $\gamma_{o s}$. El coste de la solución más económica encontrada fue de $3473.57 €$, siendo su $\gamma_{o s}$ de 1.003. La Figura 8.4 muestra el conjunto de Pareto final (se han dibujado solamente las soluciones con un coste inferior a $3900 €$ ) y la parábola que ajusta $C_{E S T}$ y $\gamma_{o s}$. Su expresión viene dada por (8.6). Los resultados indican que es necesario un incremento del coste de la estructura del $13.2 \%$ para amentar su coeficiente de seguridad global un $10 \%$.

$$
C_{E S T}=52774 \gamma_{o s}^{2}-106276 \gamma_{o s}+56978 \quad\left(R^{2}=0.9835\right)
$$

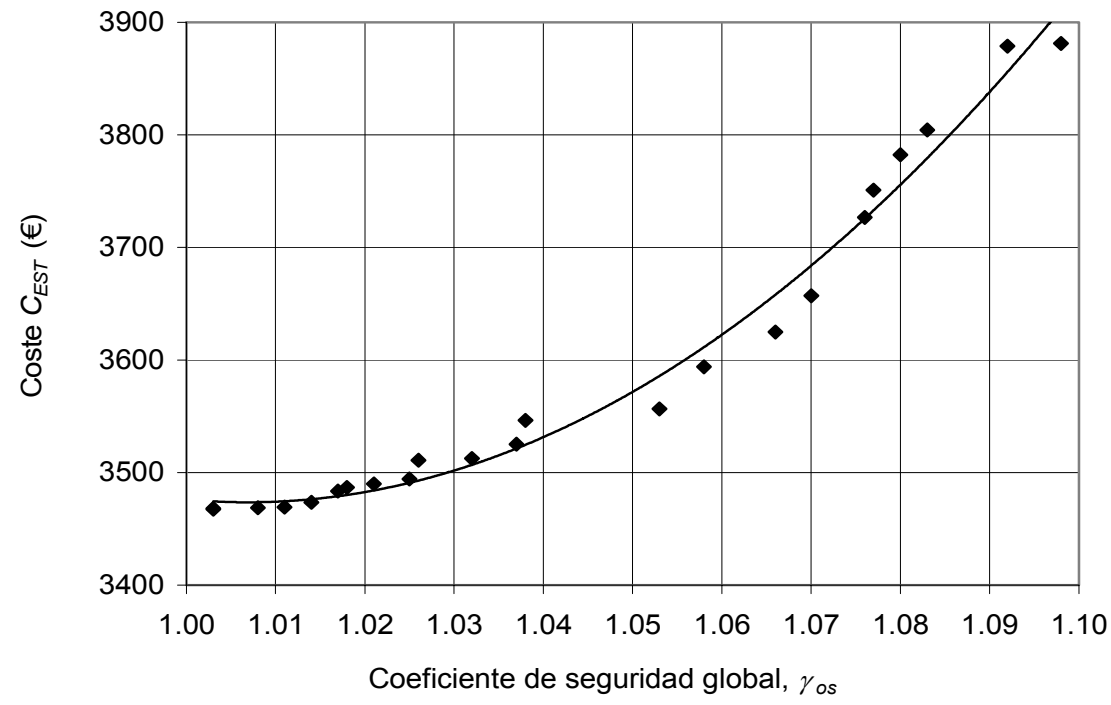

Figura 8.4. SMOSA- $\gamma_{\text {os. }}$ Frontera de Pareto final. 


\subsubsection{Estudio de sensibilidad.}

La Tabla 8.5 contiene un estudio de sensibilidad de las cuatro funciones objetivo, realizado a partir de las expresiones (8.1), (8.2) y (8.6). En ella se indica cómo repercute un cierto incremento porcentual del coste de la estructura en su facilidad constructiva, sostenibilidad y coeficiente de seguridad global. Todos los porcentajes están referidos a la mejor solución del problema $\mathrm{C}_{\mathrm{EST}}-\mathrm{SA}$.

Los resultados muestran que un incremento del coste de la estructura del $5 \%$ podría mejorar :

- En un $32.25 \%$ su facilidad constructiva.

- Ó en un $24.04 \%$ su sostenibilidad ambiental.

- Ó en un $6.11 \%$ su coeficiente de seguridad global.

Por tanto, son posibles en las cercanías del óptimo económico soluciones más fácilmente construibles, o con menos impacto ambiental o más seguras, con incrementos de coste asumibles. 


\section{Tabla 8.5. Estudio de sensibilidad.}

\begin{tabular}{|c|c|c|c|c|c|c|c|}
\hline$C_{E S T}(€)$ & $\frac{C_{E S T}}{C_{E S T \min }{ }^{1}}$ & $N_{s}$ & $E C$ & yos & $\frac{N_{s}\left(C_{E S T}\right)-N_{s}\left(C_{E S T \min }\right)}{N_{s}\left(C_{E S T \min }\right)} \times 100$ & $\frac{E C\left(C_{E S T}\right)-E C\left(C_{E S T \min }\right)}{E C\left(C_{E S T \min }\right)} \times 100$ & $\frac{V_{\text {os }}\left(C_{E S T}\right)-V_{\text {os }}\left(C_{E S T \min }\right)}{Y_{\text {os }}\left(C_{\text {ESTmin }}\right)} \times 100$ \\
\hline 3473.06 & 1.00 & 118 & 409980.53 & 1.0029 & 0. & 0. & 0. \\
\hline 3507.79 & 1.01 & - & - & 1.0324 & - & - & 2.94 \\
\hline 3542.52 & 1.02 & 97.27 & - & 1.0431 & 17.57 & - & 4.00 \\
\hline 3577.25 & 1.03 & 88.15 & 323502.93 & 1.0512 & 25.30 & 21.09 & 4.82 \\
\hline 3611.98 & 1.04 & 83.51 & 316165.24 & 1.0581 & 29.23 & 22.88 & 5.50 \\
\hline 3646.71 & 1.05 & 79.93 & 311408.37 & 1.0642 & 32.25 & 24.04 & 6.11 \\
\hline
\end{tabular}

${ }^{1} C_{\text {ESTmin }}$ es el coste económico de la solución SA-C $\mathrm{EST}_{\mathrm{T}}, 3473.06 €$. 


\subsection{CONCLUSIONES.}

La optimización multiobjetivo de pórticos de edificación de hormigón armado ha sido estudiada en este capítulo considerando como objetivos el coste económico, su facilidad constructiva, su sostenibilidad y un indicador de su seguridad global. Estos objetivos están en oposición entre sí, por lo que no existe una única solución sino un conjunto de ellas que definen lo que se conoce como Frontera de Pareto.

Para resolver el problema se ha empleado un algoritmo derivado del SA, el SMOSA, que ha demostrado ser una herramienta eficaz y robusta para proporcionar la Frontera de Pareto. Ésta incluye puntos próximos a los óptimos monoobjetivo, lo que valida el método y el hecho de emplear los mismos parámetros que en el SA para este caso concreto. No obstante, una mayor experimentación con otras configuraciones estructurales y objetivos sería deseable para generalizar este supuesto.

Ante la diversidad de diseños posibles, es responsabilidad del ingeniero escoger una solución de compromiso que tenga un buen comportamiento en los objetivos considerados en cada caso. Los resultados permiten afirmar que son posibles soluciones más fáciles de construir, más sostenibles y más seguras que la más económica con incrementos de coste aceptables en la práctica. Por otro lado, el análisis de sensibilidad indica que la facilidad constructiva es el objetivo más sensible a un incremento del coste en el entorno del óptimo económico. Finalmente, este estudio demuestra que SMOSA tiene un gran potencial para la optimización multiobjetivo de estructuras de hormigón armado. 


\section{CAPÍTULO 9. CONCLUSIONES Y FUTURAS LÍNEAS DE INVESTIGACIÓN.}




\section{Objetivos.}

Se proporcionan en este capítulo las conclusiones del trabajo realizado a la vez que se plantean futuras líneas de investigación. 


\subsection{CONCLUSIONES.}

El propósito de esta Tesis es doble. Por un lado, presentar un procedimiento general para la optimización automatizada, mono y multiobjetivo, de pórticos planos de hormigón armado que emplee tiempos de cálculo razonables. Por otro, describir las características principales de las estructuras optimizadas.

En el capítulo 1 se introducen de modo general los problemas de optimización y las técnicas que permiten resolverlos. Así mismo, se establecen los argumentos que motivan la Tesis, basándose en la importancia de los pórticos de hormigón armado en la edificación, la ausencia de un procedimiento general para minimizar su coste y la necesidad de considerar otros criterios además de la economía en su diseño, como su facilidad constructiva o su sostenibilidad ambiental.

La descripción del estado de arte en optimización estructural con una especial atención a la aplicación de los métodos heurísticos a las estructuras de hormigón se realiza en el capítulo 2. Del estudio efectuado se deduce que:

Conclusión 1: Los trabajos de optimización anteriores con pórticos de edificación de hormigón armado han sido versiones simplificadas del problema real por las acciones, hipótesis de cálculo, esquemas de armado y estados límites considerados. Además, no abordan el problema multiobjetivo y se limitan, en su práctica totalidad, a resolver casos particulares sin proporcionar conclusiones generales respecto a los métodos de optimización o las características de las estructuras óptimas.

(Epígrafe 2.8)

En el capítulo 3 se define el problema de la minimización del coste económico y de la maximización de la facilidad constructiva, sostenibilidad ambiental y seguridad global de un pórtico plano. Para ello se indican cuáles son los parámetros, variables y expresiones de las funciones objetivo adoptados en este estudio. El planteamiento efectuado es general e independiente del número de vanos y plantas del pórtico.

El capítulo 4 contiene la explicación de las versiones de los métodos heurísticos empleados en esta Tesis (Estrategia de Saltos Múltiples Aleatorios -RW-, Gradiente First Best -FB-, Cristalización Simulada -SA- , Aceptación por Umbrales -TA-, Algoritmos 
Genéticos -GA- y el Método de Suppapitnarm para la Optimización Multiobjetivo mediante la Cristalización Simulada-SMOSA-).

El capítulo 5 comienza demostrando que la minimización del coste económico de un pórtico tipo de dos vanos y cuatro alturas es inviable mediante una exploración intensiva del espacio de soluciones por los tiempos de cálculo que precisa. Por ello se obtienen soluciones iniciales mediante RW que posteriormente se optimizan mediante FB, SA, TA y GA. Los resultados se evalúan y comparan de acuerdo a dos criterios, tiempo de cálculo y coste, empleando el concepto de Óptimo de Pareto.

Conclusión 2: Los mejores resultados en la minimización del coste económico de un pórtico tipo de dos vanos y cuatro plantas se obtienen mediante la versión implementada de la Cristalización Simulada, por lo que se selecciona este heurística para realizar un estudio paramétrico de pórticos de edificación.

(Epígrafe 5.8)

En el capítulo 6 se optimizan pórticos de dos vanos y dos, cuatro, seis y ocho alturas mediante SA. A partir de los resultados obtenidos se determinan los parámetros de la heurística para optimizar un pórtico genérico plano de $N_{v}$ vanos y $N_{p}$ plantas y las características generales de las estructuras optimizadas.

Conclusión 3: El pórtico plano de $N_{v}$ vanos y $N_{p}$ plantas de coste mínimo se obtiene mediante una heurística Cristalización Simulada con los siguientes parámetros:

- Número de variables modificadas en cada movimiento: hasta 3.

- Coeficiente de enfriamiento: $r=0.8$.

- Número de cadenas de Markov sin mejora (criterio de parada): 2

- Longitud de la Cadena de Markov: $\quad L_{m}=1592.105$ NumVar - 46592.11 donde NumVar es el número de variables del problema que se obtiene mediante las expresiones (3.1) a (3.6).

(Epígrafe 6.2)

Conclusión 4: Las estructuras óptimas se caracterizan porque:

- El diseño de las vigas viene condicionado por: a) el cumplimiento del ELU de agotamiento frente a solicitaciones normales, b) las flechas activas y c) El ELU de agotamiento frente a cortante en sus secciones extremas. Además, la armadura de compresión es necesaria por cálculo.

- El diseño de los pilares viene impuesto por: a) la verificación del ELU de Inestabilidad en los pilares de fachada y de las plantas altas, b) el cumplimiento de las cuantías geométricas mínimas en los pilares de interiores y de las plantas más bajas, c) motivos constructivos (dimensiones y armados mínimos), d) por su influencia en los esfuerzos y deformaciones en vigas. Las armaduras de cortante son las mínimas indicadas por la normativa. 
Conclusión 4: (continuación)

- El empleo de acero B-500 S y de hormigones de resistencia superior a la mínima indicada por la normativa española (HA-25) y habitualmente empleada en edificación.

(Epígrafe 6.3)

\section{Conclusión 5:}

- Las características de las estructuras optimizadas no hacen necesaria la verificación de estados límites adicionales a los habitualmente estudiados en estas estructuras.

(Epígrafe 6.3)

Conclusión 6: Los costes de los pórticos óptimos aquí estudiados se caracterizan porque:

- El coste total por $m^{2}$ de forjado construído aumenta linealmente con el número de plantas de acuerdo con la expresión: $C_{E S T, M 2}=0.686 N_{p}+14.805$

- Los encofrados representan el porcentaje más importante del coste (entre un 39.9 y un $46.1 \%$ del total), seguidos de hormigones (entre un 27.02 y un $30.6 \%$ ), armadura pasiva (entre un 16.98 y un 21.4\%) y cimbrado de vigas (entre un 8.2 y un 9.9\%). Al aumentar el número de plantas del edificio disminuye la importancia relativa de los encofrados y aumenta la del acero.

- El coste de las armaduras de cortante no es despreciable y, a diferencia de lo realizado en numerosos estudios anteriores, debe considerarse en los estudios de optimización.

(Epígrafe 6.4)

En el capítulo 7 se estudia la repercusión en el coste del pórtico de dos vanos y ocho plantas de: a) emplear hormigón HA-25 en toda la estructura, b) igualar variables en vigas y c) utilizar vigas planas en lugar de descolgadas. Además se proponen reglas para el predimensionamiento de los pórticos y los resultados se comparan con las recomendaciones proporcionadas por otros autores.

Conclusión 8: La relación luz/canto de las vigas descolgadas de pórticos óptimos sometidos a acciones verticales y horizontales varía entre 9 y 10.5. La relación luz/ancho de las vigas de estos elementos varía entre 20 y 22 en las plantas tipo y entre 12 y 13 en la planta de cubierta. Las vigas pueden ser de menores dimensiones si las acciones horizontales son de escasa importancia. 
Conclusión 9: La relación luz/ancho de las vigas planas de pórticos óptimos sometidos a acciones verticales y horizontales varía entre 11 y 14 para la planta tipo y adopta valores próximos a 8 en la planta de cubierta. Estos valores han sido obtenidos con un canto de las vigas y de los forjados que es igual a 1/17 de la luz de las vigas, por lo que valores superiores o inferiores de los anchos son empleables si disminuye o aumenta el canto de los forjados. Las vigas pueden ser de menores dimensiones si las acciones horizontales son de escasa importancia.

(Epígrafe 7.2)

Conclusión 10: Las cuantías totales de armadura en las vigas descolgadas de pórticos optimizados sometidos a acciones verticales y horizontales varian entre 100 y $130 \mathrm{Kg} / \mathrm{m}^{3}$ en la planta tipo y entre 60 y $70 \mathrm{Kg} / \mathrm{m}^{3}$ en la planta de cubierta. La armadura de cortante supone un porcentaje entre el 20 y el $25 \%$ de la armadura total.

(Epígrafe 7.2.)

Conclusión 11: Las cuantías totales de armadura en las vigas planas de pórticos óptimos sometidos a acciones verticales y horizontales varian entre 290 y 300 $\mathrm{Kg} / \mathrm{m}^{3}$ en la planta tipo y entre 90 y $110 \mathrm{Kg} / \mathrm{m}^{3}$ en la planta de cubierta. La armadura de cortante supone un porcentaje entre el 7 y $15 \%$ de la armadura total.

(Epígrafe 7.2.)

Conclusión 12: Las escuadrías de los pilares optimizados se proporcionan en la Tabla 7.6. Valores menores son empleables si las acciones horizontales son de escasa importancia o si el número de plantas es inferior a ocho.

(Epígrafe

Conclusión 13: El empleo de hormigones de resistencia a compresión superior a 25 MPa conduce a ahorros considerables en la estructura (del $3 \%$ en el ejemplo estudiado). Utilizar vigas planas en lugar de descolgadas produce importantes incrementos de su coste (del $46.7 \%$ en el ejemplo estudiado).

(Epígrafe 7.3)

Conclusión 14: Agrupar variables es una estrategia conveniente para resolver un problema de optimización pues, con ligeros incrementos del coste de la estructura, se puede simplificar su ejecución y se disminuyen los tiempos de 
Conclusión 15: El procedimiento de optimización desarrollado en esta Tesis ha sido capaz de llegar a reglas de predimensionamiento similares a las propuestas por ingenieros $y$ arquitectos de gran prestigio a partir de su experiencia $y$, además, ampliar sus propuestas al incluir aspectos como las cuantías de acero en vigas y la influencia de las acciones horizontales.

(Epígrafe 7.3)

Finalmente, el capítulo 9 estudia la optimización multiobjetivo del pórtico de dos vanos y cuatro plantas considerando como criterios el coste económico, la facilidad constructiva, la sostenibilidad ambiental y un índice de la seguridad global de la estructura. Los objetivos entran en conflicto entre sí, por lo que se necesita el empleo de un algoritmo capaz de proporcionar un número suficientemente amplio de diseños entre las que el ingeniero pueda escoger la solución de compromiso. Para ello, se emplea una estrategia de la familia de la Cristalización Simulada, el SMOSA.

Conclusión 15: En el entorno de la solución optimizada desde el punto de vista del coste económico son posibles estructuras más fáciles de construir, más sostenibles y más seguras con incrementos de coste aceptables en la práctica.

(Epígrafe 8.2)

Conclusión 17: En el entorno de la solución más económica el objetivo más sensible a un incremento del coste es la facilidad constructiva.

(Epígrafe 8.2)

Conclusión 18: La versión propuesta de SMOSA, empleando los mismos parámetros que para una optimización monoobjetivo con Cristalización Simulada, es capaz de proporcionar una buena aproximación a la Frontera de Pareto en los problemas multiobjetivo estudiados.

(Epígrafe 8.3)

Conclusión 19: SMOSA tiene un gran potencial en la optimización multiobjetivo de estructuras de hormigón armado.

(Epígrafe 8.3) 


\subsection{FUTURAS LÍNEAS DE INVESTIGACIÓN.}

Esta Tesis demuestra la capacidad de los métodos heurísticos para resolver la optimización mono y multiobjetivo de pórticos planos de hormigón armado. Se comentan seguidamente las líneas por las que la investigación puede seguir avanzando.

\subsubsection{Métodos heurísticos y funciones objetivo.}

- Una experimentación con estructuras no simétricas y con valores de parámetros relativos a la geometría y las acciones diferentes a los aquí empleados se realizará para reafirmar la validez de la fórmula aquí propuesta para el diseño óptimo totalmente automatizado.

- Chen y Su (2002) han propuesto mejoras al algoritmo SA para limitar el número de análisis estructurales necesarios y mejorar su eficacia y las han aplicado a estructuras de acero. Se investigará su aplicación a las estructuras aquí estudiadas.

- En el análisis multicriterio se han estudiado las funciones objetivos agrupadas por parejas, lo que ha permitido: a) comprobar la validez y potencial de SMOSA y b) proporcionar una primera respuesta a la pregunta de si son posibles soluciones que sean al mismo tiempo económicas, sostenibles y fáciles de construir. Para profundizar en esta cuestión sería conveniente:

a) Ampliar la versión propuesta de SMOSA para que pueda trabajar con los tres objetivos simultáneamente.

b) Realizar un estudio paramétrico del problema multicriterio similar al descrito en el capítulo 7.

c) Estudiar la definición de una función de coste generalizada que, de modo sencillo, modifique el coste económico de una estructura en función de su facilidad de construcción y su impacto ambiental. De este modo, dos alternativas de diseño podrían compararse directamente a partir de su coste modificado, sin tener que realizar el análisis multicriterio para cada caso concreto.

- En este trabajo se ha adoptado un tiempo fijo de descimbrado y no se han estudiado diferentes alternativas para el proceso de construcción. En un futuro, se incluirán en el modelo de optimización las variables que permitan describir 
este proceso. Esto permitirá definir una nueva función objetivo, el tiempo total necesario para construir la estructura $t_{c}$, que podrá ser objeto de minimización y de un análisis multiobjetivo (considerando por ejemplo $C_{E S T}$ frente a $t_{c}$ ).

- En versiones futuras de Optipor, las longitudes de los refuerzos de la armadura longitudinal no sean parámetros sino variables. Se incluirá también el diseño detallado de los nudos de los pórticos.

\subsubsection{Modelos de cálculo adoptados.}

El cálculo se ha realizado empleando las leyes de momentos flectores que resultan de un análisis elástico y lineal. En el caso de las vigas, dos posibles mejoras pueden ser introducidas que conducirían a ahorros en las cuantías de armadura:

- Redondeo de las leyes de momentos flectores en apoyos, tal y como propone Bernárdez (1987) y como realizan algunos programas comerciales (Figura 9.1).

- Posibilidad de redistribuir esfuerzos en vigas, siguiendo los criterios fijados por la EHE y por otros códigos estructurales.

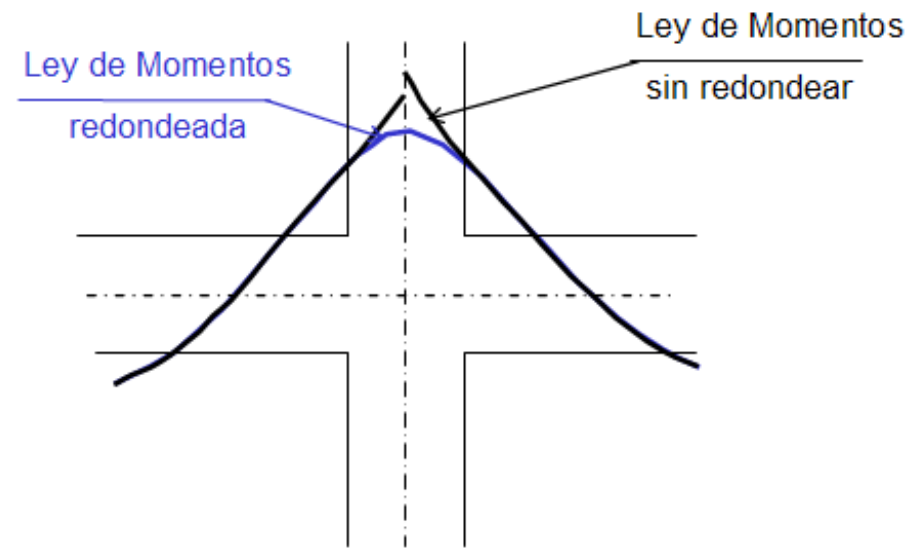

Figura 9.1. Redondeo de la ley de momentos flectores en un nudo de un pórtico (el nudo está representado en alzado).

\subsubsection{Tipología estructural y acciones.}

En esta Tesis se han optimizado pórticos planos, sin voladizos y en los que todos los pilares son continuos desde la cimentación del edificio hasta la planta de cubierta. Este caso particular debería extenderse a otros casos más generales que incluyeran:

- Pórticos tridimensionales.

- Pórticos con voladizos. 
- Pilares que arranquen en la cimentación y que no necesariamente terminen en la planta de cubierta.

En lo que respecta a las acciones consideradas, sería conveniente incluir el sismo y los asientos diferenciales.

\subsubsection{Difusión y aplicabilidad de los resultados.}

De cara a facilitar la difusión de las conclusiones obtenidas y su aplicabilidad, resulta conveniente implementar en próximas versiones del programa Optipor, además de la EHE, los códigos ACI (2002) y/o los Eurocódigos (2002).

\subsubsection{Otros aspectos.}

Sería conveniente efectuar un análisis de sensibilidad respecto de los parámetros de los precios unitarios y los niveles de control de la ejecución. De este modo se podría, por ejemplo, estudiar la repercusión económica y técnica de variaciones en los precios del hormigón y del acero o del paso de un nivel de control de la ejecución normal a uno intenso.

Finalmente se considera necesario señalar que los procedimientos heurísticos suponen tienen un gran potencial como estrategias para la optimización al permitir incluir en el análisis todas las restricciones del problema y un número indefinido de variables. Por ello, los límites vienen impuestos únicamente por los tiempos de cálculo, o lo que es lo mismo, por la potencia de los ordenadores disponibles. Puesto que esta potencia crece de año en año y sin límite aparente, no está muy lejano el día en que los programas de cálculo de estructuras puedan llevar incorporados módulos de optimización estructural y sean capaces de proporcionar planos de estructuras construibles. De este modo se verá realizado uno de los más viejos sueños del ingeniero, cumplir los encargos de la sociedad empleando los limitados recursos disponibles con la máxima eficacia y eficiencia. 


\section{Referencias.}

(1) Adeli, H; Yeh C. "Perceptron learning in engineering design". Microcomputers in Civil Engineering 4(4): 247-256. 1989.

(2) Adeli, H; Cheng N.T. "Augmented Lagrangian Genetic Algorithm for structural optimization”. ASCE Journal of Aerospace Engineering 7(1): 104-118. 1994a.

(3) Adeli, H; Cheng N.T. "Concurrent Genetic Algorithms for the optimization of large structures". ASCE Journal of Aerospace Engineering 7(3): 276-296. 1994b.

(4) Adeli H. "Neuronal Networks in Civil Engineering: 1989-2000". Computer-Aided Civil and Infraestructural Engineering, 16:126-142. 2001.

(5) Adeli H. "Sustainable infrastructures systems and environmentally-conscious design - a view for the next decade". ASCE Journal of Computing in Civil Engineering, 16(4): 231-233. 2002.

(6) Adeli, H; Sharma K. "Cost optimization of structures. Fuzzy, Genetic Algorithms and Parallel Computing". Ed. Wiley-VCH. 2006.

(7) Ahmadhkanlou, F.; Adeli, H.; "Optimum cost design of reinforced concrete slabs using neural dynamics model". Engineering Applications of Artificial Inteligence, 18: $65-72$.

(8) Alcalá J.; Carrera, M.; González Vidosa, F.; Yepes V. "Cristalización simulada aplicada a la optimización económica de muros ménsula de contención de hormigón armado". Hormigón y Acero 236:97-108. 2005.

(9) Ali, N.; Behdinan K., Fawaz, Z.; "Applicability and viability of a GA based finite element analysis architecture for structural design optimisation". Computer and Structures, 81: 2251 - 2271. 2003.

(10) American Institute of Steel Construction. "Manual of steel construction-allowable stress design". Chicago, 1989. 
(11) American Concrete Institute Comitee 318. "Building code requirements for structural concrete and commentaries". ACI 318-99. Detroit, 1999.

(12) American Concrete Institute Comitee 318. "Building code requirements for structural concrete and commentaries". ACI 318-02. Detroit, 2002.

(13) Asif M., Muneer T. y Kelley R.; "Life Cycle Assessment: A case study of a dwelling home in Scotland". Building and Environment, 42(3): 1391-1394. 2007.

(14) ANSYS Theory Reference 9.0. Canonsburg. ANSYS Inc. 2004.

(15) Atrek, E., Gallagher, R. H., Ragsdell, K.M. y Zienkiewicz, O. "New directions in optimal structural design". John Wiley. 1984.

(16) Balling, R.; "Optimal Steel frame design by Simulated Annealing". ASCE Journal of Structural Engineering 117(6): 1780-1795. 1991.

(17) Balling, R.; Yao, X.; "Optimization of reinforced concrete frames". ASCE Journal of Structural Engineering 123(2): 193-202. 1997.

(18) Barr, R.S.; Goleen, B.L.; Kelly, J.P.; Resende, M.G.C.; Stewart, W.R. (1995). "Designing and reporting on computacional experiments with Heuristics Methods". Jourmal of Heuristics 1: 9-32.

(19) Bennage W.A; Dhingra, A.K. "Single and multiobjective structural optimization in discrete-continuous variables using Simulated Annealing". International Journal for Numerical Methods in Engineering, 38: 2753-2773. 1995.

(20) Bernárdez, R. "Reajuste de la ley de momentos flectores de dinteles, en la zona de empotramiento en los soportes de hormigón”. Hormigón y Acero n¹65: 6176. 1987.

(21) Botello, S.; Hernández A.; Lizárraga G. "Un nuevo algoritmo para la optimización de una o varias funciones objetivo sujetas a restricciones". Revista Internacional de Métodos Numéricos para Cálculo y Diseño en Ingeniería 20(2): 139-167. 
(22) Cai, J.; Thierauf, G.; "A parallel evolution strategy for solving discrete structural optimization". Advances in Engineering Software 27: 91-96. 1996.

(23) Calavera, J. "Manual de detalles constructivos en obras de hormigón armado. Edificación y obras públicas". Instituto Técnico de Materiales y Construcciones. Madrid, 1993.

(24) Calavera, J. "Proyecto y cálculo de estructuras de hormigón". Instituto Técnico de Materiales y Construcciones. Madrid, 1999.

(25) Calavera, J. "Cálculo, construcción, patología y rehabilitación de forjados de edificación". Instituto Técnico de Materiales y Construcciones. Madrid, 2002.

(26) Camp, C.; Pezeshk, S.; Cao, G. "Optimized design of two dimensional structures using a genetic algorithm". ASCE Journal of Structural Engineering 124(5): 551-559. 1998.

(27) Camp, C.; Pezeshk, S.; Hansson, H. "Flexural design of reinforced concrete frames using a genetic algorithm". ASCE Journal of Structural Engineering 130 (5): 741-751. 2003.

(28) Camp, C.; Bichon, B.; Stovall, S. "Design of space trusses using Ant Colony optimization". ASCE Journal of Structural Engineering 131(3): 369-379. 2005.

(29) Camp, C.; Bichon, B.; Stovall, S. "Design of steel frames using Ant Colony Algorithm". ASCE Journal of Structural Engineering 131(3): 369-379. 2005.

(30) Carbonell, A. "Optimización heurística de bóvedas de paso de carretera". Trabajo de investigación CST/GPRC-05. 2005.

(31) Carrera, M. "Optimización heurística de pórticos de paso de carretera". Trabajo de investigación CST/GPRC-02. 2004.

(32) Carrera M.; Alcalá J.; Yepes V.; González Vidosa, F. "Optimización heurística de pórticos de paso de carretera de hormigón armado". Hormigón y Acero 236:85-95. 2005. 
(33) Carrol, D. "D.L. Carroll's Fortran genertic algorithm driver". Disponible en http://cuaerospace.com/carroll/ga.html. Consultada el 16 de noviembre de 2006.

(34) Ceracnic, B.; Fryer, C. "A Computer-Based, Interactive Genetic Algorithm Optimisation Design Tool (GENOD) for Reinforced Concrete Structures". International Journal of Design Computing, vol. 2. 1999. Disponible únicamente en http://wwwfaculty.arch.usyd.edu.au/kcdc/ijdc/. Consultada el 17 de diciembre de 2007.

(35) Ceracnic, B.; Fryer, C. and Bines, R. W. "An application of simulated annealing to the optimum design of reinforced concrete retaining structures". Computers and Structures, 79: 1569 - 1581. 2001.

(36) Chau, K. W.; Albermani, F.; "Knowledge-based system on optimum design of liquid retaining structures with Genetic Algorithms". ASCE Journal of Structural Engineering 129 (10): 1312-1321. 2003.

(37) Chen, T.; Su, J. "Efficiency improvement of simulated annealing in optimal structural designs". Advances in Engineering Software, 33: 675-680. 2002.

(38) Chen, G.; Bruno, R.; Salama, M. "Optimal placement of active/passive members in truss structures using simulated annealing". American Institut of Aeronautics and Astronautics Journal, 29(8): 1327-1334. 1991.

(39) Cladera, A. " Shear design of high strength concrete beams". Tesis Doctoral. 2003.

(40) Coello, C. "An empirical study of Evolutionary Techniques for Multiobjective Optimization in engineering design". PhD Tesis. Tulane University. 1996.

(41) Coello, C.; Santos, F.; Farrera, F. "Optimal design of reinforced concrete beams using Genetic Algorithms". Expert Systems with Applications, 12(1):101-108. 1997.

(42) Coello, C.; Christiansen, A. "MOSES: a multiobjective tool for engineering design". Engineering Optimization, 31, (3): 337-368. 1999. 
(43) Coello, C. "A comprehensive survey of evolutionary-based multiobjective optimization techniques". Knowledge and Information Systems, Vol. 1(3): 269308. 1999.

(44) Coello, C.; Christiansen, A. "Multiobjective optimization of trusses using Genetic Algorithms". Computers and Structures, 75(6): 647-660. 2000.

(45) Coello, C y Lamont G.B. Eds. "Advances in Natural Computation Vol. 1. Applications of Multi-objective evolutionary algorithms". Ed. World Scientific. 2004.

(46) Coello, C. "Introducción a la computación evolutiva (notas del curso)". 2004. Disponible en http://elavio2005.uniandes.edu.co. Consultada en noviembre 2006.

(47) Coello, C. "Introducción a la Optimización Multiobjetivo usando Metaheurísticas". Apuntes del curso celebrado en la XI Escuela Latinoamericana de Verano en Investigación de Operaciones. 2005. Disponible en http://elavio2005.uniandes.edu.co. Consultada en noviembre 2006 .

(48) Coello, C. "Búsqueda Tabú: evitando lo prohibido". 2007. Disponible en http://delta.cs.cinvestav.mx/ ccoello/revistas/tabu.pdf.gz. Consultada en marzo 2007.

(49) Cohn M.Z.; Dinovitzer A.S. "Application of structural optimization". ASCE Journal of Structural Engineering, 120(2):617-649. 1994.

(50) Colley, D. "An introduction to Genetic Algorithms for Scienticist and Engineers". World Scientific. Singapore. 1999.

(51) Comisión Permanente del Hormigón. "EH-91. Instrucción para el proyecto y la ejecución de obras de hormigón en masa o armado". Ministerio de Obras Públicas y Transportes. Madrid. 1991.

(52) Comisión Permanente del Hormigón. "Instrucción de hormigón estructural. EHE”. Ministerio de Fomento. Madrid. 1999. 
(53) Corres, H.; López, J.C.; Martínez, J. L.; Pérez, A. "Prontuario Informático del Hormigón Estructural”. Instituto Español del Cemento y sus Aplicaciones. 2001.

(54) Cziak, P.; Hapke, M.; Jaszkiewicz, A. "Application of the Pareto-Simulated Annealing to the multiple criteria shortest path problem". Technical Report, Politechnika Poznanska Instytut Informatyki. Polonia. 1994.

(55) Cziak, P.; Jaszkiewicz, A. "Pareto-Simulated Annealing: a metaheuristic technique for multiple-objective combinatorial optimization". Journal of Multicriteria Decision Analysis, 7(34). 1998.

(56) Deb, K. "Multi-objective Genetic Algorithms: problem difficulties and construction of test problems". Technical Report Cl-49/98. Department of Computer Science. University of Dortmound, Dortmound. 1998.

(57) Deb, K. "Evolutionary algorthms for multi-criterion optimization in engineering design". Proc. Evolutionary Algorithms in Engineering and Computer Science (EUROGEN'99): 135-161. 1999.

(58) Deb, K. "Multi-objective Optimization using Evolutionary Algorithms". Ed. John Wiley \& Sons. 2001.

(59) De Fuentes, G. "Valoración de obras en ingeniería civil". Servicio de Publicaciones de la Escuela Universitaria de Ingenieros Técnicos de Obras Públicas. 2002.

(60) De Jong, A.K. "An anlysis of the behavior of a class og Genetic Adaptative Systems". PhD Thesis. University of Michigan, 1975.

(61) Dirección General para la Vivienda y la Arquitectura. "NBE AE-88. Acciones en la edificación". Ministerio de Obras Públicas y Transportes. 1988.

(62) Dirección General para la Vivienda y la Arquitectura. "NTE ECV-88. Viento". Ministerio de Obras Públicas y Transportes. 1988. 
(63) Díaz, A.; Glover, F.; Ghaziri, H.M.; González, J.L.; Laguna, M.; Moscato, P. Tseng, F.T.. "Optimización heurística y redes neuronales en dirección de operaciones e ingeniería”. Editorial Paraninfo. 1996.

(64) Dorigo, M.; Maniezzo, V; Colorni, A. "Ant System: optimization by a colony of cooperating agents". IEEE Transactions on Systems, Man and Cybernetics Part B 26 (1): 29-41. 1996.

(65) Dorigo M.; Stützle T. "Ant colony optimization”. Cambridge, MIT Press. 2004.

(66) Dowsland, K.A. "Some experiments with Simulated Annealing Techniques for packing problems". European Journal of Operational Research 68:389-399. 1993.

(67) Dreo, J.; Petrowsky, A.; Siarry, P.; Taillard, E.; Chatterjee A. "Metaheuristics for hard optimization. Methods and case studies”. Berlin, Springer. 2006.

(68) Dueck, G.; Scheuert, T.. "Threshold Accepting: a genereal purpose optimization algorithm superior to Simulated Annealing". Journal of Computation Physics 90: 161-175. 1990.

(69) Ehrgott, M.; Gandibleux, X.. “ An annotated bibliography of multiobjective combinatorial optimization". International Series in Operations Research and Management Science, 52. Kluwer Academic Publishers. Boston. 2002.

(70) Elbehairy, H.; Elbeltagi, E.; Hegazy, T.; Soudki, K.. "Comparison of two evolutionary algorithms for optimization of bridge deck repairs". Computer-Aided Civil and Infraestructural Engineering 21: 561-572. 2006.

(71) Engrand, P. "A multiobjective approach based on Simulated Annealing and its application to nuclear fuel manegement". 5th Internacional Conference on Nuclear Engineering. Niza (Francia). 1997.

(72) European Committee for Standardization. "Eurocode 2: Design of Concrete Structures. Part 1: general rules and rules for buildings" (Draft for Stage 49). ENV-1992-1-1. 2002. 
(73) Evans, G.W. "An overwiew of techniques for solving multiobjective mathematical programs". Management Science, 30: 1268-1282. 1984.

(74) Fairbairn, E.; Silvoso, M; Toledo, R.; Alves, J.; Ebecken, N. "Optimization of mass concrete construction using genetic algorithms". Computers and Structures, 82: 281 - 299. 2004.

(75) Fédération Internationale du Béton "Environmental issues in prefabrication, state-of-art report”. Laussanne, Fédération Internationale du Béton. 2003.

(76) Fernandez-Canovas M.; "Hormigón". Madrid, Colegio de Ingenieros de Caminos, Canales y Puertos. 2002.

(77) Fletcher R. "Practical methods of optimization”. Chichester, Wiley. 2001.

(78) Fogel, L.J. "Artificial Intelligence through Simulated Evolution". New York, John Wiley. 1965.

(79) Franco, G.; Betti, R.; Lus, H. "Identification of structural systems using an evolutionary strategy ". ASCE Journal of Engineering Mechanics, 130(10): 1125-1139. 2004.

(80) Fuller, R.B. "Building Construction. Patent N²682235". 1954.

(81) Galante, M. "Genetic Algorithms as an approach to optimize real-world trusses". International Journal for Numerical Methods in Engineering, 39: 361382. 1996.

(82) Gasch, M. "Optimización de estructuras de forjados reticulares". Tesis doctoral. Universidad Politécnica de Valencia. 1991.

(83) Glover F. "Future paths for integer programming and links to artificial intelligence". Computers and Operations Research, 5:533-549. 1986.

(84) Glover F.; Laguna M. "Tabu search". Boston, Kluwer Academic Publishers. 1997. 
(85) González Vidosa, F.; Yepes, V.; Alcalá, J.; Carrera, M.; Perea, C. "Simulated annealing optimization of walls, portal and box reinforced concrete road structures". Proceedings of the 9th International Conference on Computer Aided Optimum Design in Engineering (OPTI-2005), Skiathos (Grecia). 2005.

(86) Gómez, J. "Análisis técnico económico de la influencia que presenta el empleo de diferentes materiales y tipologías estructurales en el proyecto de estructuras de edificios". Tesis doctoral. 1998.

(87) Greiner, D; Emperador, J.M.; Winter, G. "Single and multiobjective frame optimization by evolutionary algorithms and the auto-adaptative rebirth operator". Computer Methods in Applied Mechanichs and Engineering, 193: 3711-3743. 2004.

(88) Goldberg, D.E. "Computer-aided gas pipeline operation using genetic algorithms and rule learning". Dissertation Abstracts International, 44(10):3174. 1983.

(89) Goldberg, D.E. "Genetic Algorithms in search, optimization and machine learning". Addison-Wesley. Boston. 1989.

(90) Goldberg, D.E.; Samtani, M.P. “ Engineering optimization via genetic algorithms". In: ASCE Proceedings of the Ninth Conference on Electronic Computation :471-482. New York. 1986.

(91) Grierson D.E.; Pak, W.H. "Optimal sizing, geometrical and topological design using a genetic algorithm". Structural Optimization 6:151-159. 1993.

(92) Gasch, M.S. "Optimización de estructuras de forjados reticulares". Tesis doctoral. Universidad Politécnica de Valencia. 1991.

(93) Goedkoop, M.; Spriensma, R. "The Ecoindicator 99. A damage oriented method for Life Cycle Impact Assessment. Methodology Report". 2001. Disponible en http://www.pre.nl/eco-indicator99/ei99-reports.htm. Consultada en Diciembre 2006. 
(94) Gunel, M.H.; Ilgin, H.E. “ A proposal for the classification of structural systems of tall buildings". In Building and Environment. Mdoi:10.106/j.buildenv.2006.07.007. In press. 2006.

(95) Hasancebi, O.; Erbatur, F. "Layout optimization of trusses using Simulated Annealing". Advances in Engineering Software, 33: 681-696. 2002.

(96) Hajela, P. "Genetic algorithms in automated structural shynthesis" presented at the NATO Advanced Study Institute on Optimization and Decision Support Systems, June 26-July 7, 1989, Edinburgh, United Kingdom.1989.

(97) Hajela, P. "Genetic Search - An Approach to the Nonconvex Optimization Problem". AIAA Journal, 26(7):1205-1210. 1990.

(98) Hajela, P.; Lin, C.Y. "Genetic search strategies in multicriterion optimal design". Structural Optimization , 4: 99-107. 1992.

(99) Heitkötter, J; Beasley, D. "The Hitch-Hiker's Guide to Evolutionary Computation: A list of Frequently Asked Questions (FAQ)". Disponible en ftp://ftp.cerias.purdue.edu/pub/doc/EC/FAQ/www/top.htm. Consultada en noviembre de 2006. 1998.

(100) Hernández, S. "Métodos de diseño óptimo de estructuras". Colegio de Ingenieros de Caminos, Canales y Puertos. Madrid. 1990.

(101) Hernandez S.; Fontan A. "Practical Applications of Design Optimization". Southampton, WIT Press. 2002.

(102) Holland, J.H. "Adaptation in natural and artificial systems". University of Michigan Press. 1975.

(103) Hopfield, J.J.; Tank, D. "Neural computation of decision in optimization problems". Biological Cybernetics, 52: 141-152. 1985.

(104) Hornlein, H.E.R.M. "Take-off in optimal structural design". Computer Aided Optimal Design. NATO/ASI Seminar, 3: 176-200. 1986. 
(105) Hospitaler, A. "Puntuación ambiental de hormigones y aceros empleados en edificación mediante el Ecoindicador 99". Informe interno del Departamento de Ingeniería de la Construcción y Proyectos de Ingeniería Civil. 2006.

(106) Hrstka, O; Kucerová, A.; Leps M. Zeman, J. "A competitive comparison of different types of evolutionary algorithms". Computers and Structures, 81: 1979-1990. 2003.

(107) International Conference of Building Officials. "Uniform Building Code". Whittier, California. 1997.

(108) Jenkins, W. M. "Structural optimisation with the genetic algorithm". The Structural Engineer, 24: 418-422. 1991.

(109) Jenkins W. M. "Towards structural optimisation via the genetic algorithm". Computers and Structures, 40 (5): 1321-1327. 1991a.

(110) Jenkins W. M. "Structural optimisation with the genetic algorithm". The Structural Engineer, 69 (24): 418-422. 1991b.

(111) Jenkins W. M. "On the application of natural algorithms to structural design optimization". Engineering Structures 19 (4): 302-308. 1997.

(112) Jenkins W.M. "Improving structural design by genetic search". Computer-Aided Civil and Infraestructural Engineering, 13: 5-11. 1998.

(113) Jeong, H.; Quiao, J.; Abraham, D. "Minimizing the consequences of intentional attack on water infraestructure". Computer-Aided Civil and Infraestructural Engineering 21: 79-92. 2006.

(114) Jiménez Montoya, P.; Morán Cabré, F.; García Meseguer, A. "Hormigón Armado". Barcelona, Ed. Gustavo Gili. 2000.

(115) Jones M.T. "Artificial intelligence application programming". Hingham (Massachussets), Charles River Media. 2003. 
(116) Kaveh, A.; Shojaee S. "Optimal design of scissor-link foldable structures using Ant Colony Optimization algorithm". Computer-Aided Civil and Infraestructural Engineering, 22: 56-64. 2007.

(117) Kicinger, R.; Arciszewski, T.; De Jong, K. "Evolutionary computation and structural design: A survey of the state of the art". Computers and Structures, 83: 1943-1978. 2005.

(118) Kim, H.S.; Roschke, P. "Fuzzy control of base-isolation system using multiobjective Genetic Algorithm". Computer-Aided Civil and Infraestructural Engineering, 21: 436-449. 2006.

(119) Kiong, C.; Yang Y. "Genetic programming-based approach for structural optimization". Journal of Computing in Civil Engineering, 14(1): 31-37. 2000.

(120) Kirkpatrichk, S.; Gelatt, C. D.; Vecchi, M.P. "Optimization by simulated annealing". Science $n^{\circ}$ 220: 671-680. 1983.

(121) Kong, J. "Optimal drift design of tall reinforced concrete buildings using genetic algorithms". Proc. Computational Mechanics. WCCM VI in conjunction with APCOM’04. Beijing, China. 2004.

(122) Koumousis, V.K.; Arsenis, S.J. "Genetic Algorithms in optimal detailed design of reinforced concrete members". Computer-Aided Civil and Infraestructural Engineering, 13: 43-52. 1998.

(123) Krishnamoorthy, C.S.; Prasanna P.; Sudarshan R. "Object-oriented framework for Genetic Algorithms with application to space truss optimization". ASCE Journal of Computing in Civil Engineering 16(1): 66-75. 2002.

(124) L'Energie de l'Oest de Suisse. "Projet du pylon Tenseur Tt1 (Pylon 102-106)". Document $n^{\circ}$ HA007. Laussanne, Suiza.1997

(125) Lagaros, N.; Charmpis, D.; Tsompanakis, Y.; Papadrakakis M. "Structural design in the framework of casacade evolutionary optimization". Proc. 6th Congress of Structural and Multidisciplinary Optimization. Rio de Janeiro. 2005. 
(126) Laguna, M.; Feo, T.; Elrod, H. "A greedy randomized adaptive search procedures for the 2-partion problem". Operations Research 42 (4): 677-687. 1997.

(127) Lee, C.; Ahn, J.; "Flexural design of reinforced concrete frames by Genetic Algorithm". ASCE Journal of Structural Engineering 129(6): 762-773. 2003.

(128) Leps, M.; Sejnoha, M.; "New approach to optimization of reinforced concrete beams". Computers and Structures, 8: 1957-1966. 2003.

(129) Leps, M.; "Multi-objective optimization of reinforced concrete frames". Proc. 6th Congress of Structural and Multidisciplinary Optimization. Rio de Janeiro. 2005.

(130) Leite, J.; Topping, B.H.V. "Improved genetic operators for structural engineering optimization". Advances in Engineering Software, 29(7-9):529-562 .1998.

(131) Liu, C.; Hammad, A.; Itoh, Y. "Multiobjective optimization of bridge deck rehabilitation using a Genetic Algortihm". Computer-Aided Civil and Infraestructural Engineering, 12: 431-443. 1997.

(132) Liu, D.K.; Yang Y.L., Li, Q.S. "Optimum positioning of actuators in tall buildings using genetic algorithm". Computers and Structures 81:2823-2827. 2003.

(133) Liu, C.; Frangopol, D. "Bridge annual maintenance priorization under uncertainty by multiobjective combinatorial optimization". Computer-Aided Civil and Infraestructural Engineering, 20: 343-353. 2005.

(134) Liu, X; Begg, D. W.; Fishwick, R.J. "Genetic approach to optimal topology/controller design of adaptative structures". International Journal for Numerical Methods in Engineering, 41: 815-830. 1998.

(135) Lundy, M.; Mees, A. "Convergence of an Annealing Algorithm". Mathematical Programming, 34: 111- 124. 1986.

(136) Manoharan, S.; Shanmuganathan S. "A comparison of search mechanisms for structural optimization". Computers and Structures 73: 363-372. 2003. 
(137) Martínez, F.; Perea, C.; Yepes, V.; Hospitaler, A.; González Vidosa, A. "Optimización heurística de pilas rectangulares huecas de hormigón armado". Hormigón y Acero. Aceptado para su publicación en octubre del año 2006.

(138) Matlab's User Guide. The MathWorks Inc. Natick. 1999.

(139) Matous, K; Leps, M.; Zeman, J.; Sejnoha, M. “ Applying Genetic Algorithms to selected topics commonly encountered in engineering practice". Computer Methods Applied in Mechcanical Engineering 190(13-14):1629-1650. 2000.

(140) McCulloh, W.; Pitts, W. "A logical calculus of the ideas inmanent in nervous system". Bulletin of Mathematical Bhiophysics, 5: 115-133. 1946.

(141) Medina, J.R. "Estimation of incident and reflected waves using Simulated Annealing". ASCE Journal of Waterway, Port, Coastal and Ocean Engineering 127(4): 213-221. 2001.

(142) Metrópolis, N.; Rosenbluth, A. W.; Rosenbluth, M. N.; Teller, A.H.; Teler, E. "Equation of state calculation by Fast Computing Machines". Journal of Chemical Physics, 21: 1087-1092. 1953.

(143) Ministerio de Asuntos Exteriores. Secretaría de Estado para la Cooperación Internacional. "Metodología de Evaluación de la Cooperación Española II". Madrid 2001. Disponible en www.aeci.es.

(144) Ministerio de Fomento "Instrucción para el proyecto y ejecución de forjados unidireccionales de hormigón estructural hechos con elementos prefabricados". Ed. Ministerio de Fomento. Madrid 2002.

(145) Ministerio de Vivienda. "CTE. Código Técnico de la Edificación". Madrid 2006. Disponible en www.codigotecnico.org/index?id=29. Consultado en marzo 2007.

(146) Moharrami, H.; grierson D.E. "Reinforced concrete framework design optimization". Transactions on the Built Environment 2: 287-307. 1993.

(147) Moragues, J. "Diseño óptimo de estructuras aporticadas de hormigón armado". Tesis doctoral. Universidad Politécnica de Valencia. 1980. 
(148) Osman, I.H.; Kelly, J.P. (Eds.). "Meta-heuristics: theory and applications". Boston, Kluwer Academic Publishers. 1996.

(149) Pandia Raj, R.; Kalyanaraman V.; "GA optimal design of steel truss bridge". Proc. of the 6th World Congress of Structural and Multidisciplinary Optimization. Rio de Janeiro. 2005.

(150) Papadrakakis, M.; Lagaros, N.; Plevris, V. "Multi-objective optimization of skeletal structures under static and seismic loading conditions". Engineering Optimization, 34:645-669. 2005.

(151) Pareto, V. “Cours d'Economie Politique”, vol 1 y 2. F. Rouge. Lausanne. 1896.

(152) Park, K.W.; Grierson, D. "Pareto-optimal conceptual design of the structural layout of buidings using a multicriteria Genetic Algortihm". Computer-Aided Civil and Infraestructural Engineering, 14: 163-170. 1999.

(153) Payá, I. “Optimización heurística de pórticos de edificación de hormigón armado". Trabajo de investigación CST/GPRC-04 2005.

(154) Payá, I.; Yepes, V.; Clemente, J.; González Vidosa, F.“Optimización heurística de pórticos de edificación de hormigón armado".Revista Internacional de Métodos Numéricos para Cálculo y Diseño en Ingeniería, 22(3): 241-259. 2006.

(155) Payá, I.; Yepes, V.; González, F.; Hospitaler, A. "Multiobjective Simulated Annealing of concrete frames". Proc. of the Symposium "Responding to Tomorrow's Challenges in Structural Engineering" of the International Association for Bridge and Structural Engineering. Budapest. 2006.

(156) Perea, M. "Optimización heurística de marcos de carretera". Trabajo de investigación CST/GPRC-07. 2006.

(157) Perea, C; Payá, I; Yepes, V.; González Vidosa, F. "Heuristic optimization of reinforced concrete road bridges and frames". Proceedings 2th Congreso FIB, Nápoles (Italia). 2006a. 
(158) Perea, C.; Yepes, V.; Alcalá, J.; Hospitaler, A.; González-Vidosa, F. "Heuristic optimization of reinforced concrete road bridge frames". Proceedings 8th International Conference on Computational Structures Technology, Las Palmas de Gran Canaria (España). 2006b.

(159) Perea, C.; Alcalá, J.; Martínez, F.; Yepes, V.; González-Vidosa, F. "Optimum design of the box frame tunnel of the Underground Line Palma - UIB". Proceedings 10th International Conference on Project Engineering, Valencia. 2006c.

(160) Powell, M.J.D. "On the calculation of orthogonal vectors". Computers and Structures, 11: 302-304. 1968.

(161) Radlbeq, C.; Dienes, E.; Kosteas, D. "Aluminium structures, a sustainable future?" Structural Engineering International (4), 339-344. 2006.

(162) Rafiq, M.Y.; Sothcombe, C. "Geneti algorithms in optimal design and detailing of reinforced concrete columns supported by a declarative approach for capacity checking". Computers and Structures 69:443-457. 1998.

(163) Rajeev S. y Krishnamoorthy C.S. "Discrete optimization of structures using genetic algorithms". ASCE Journal of Structural Engineering, 118(5):1233-1250. 1992.

(164) Rajeev S. y Krishnamoorthy C.S. "Genetic algorithms based methodologies for design optimization of trusses". ASCE Journal of Structural Engineering, 123(3):350-358. 1997.

(165) Rajeev S. y Krishnamoorthy C.S. "Genetic algorithm-based methodology for design optimization of reinforced concrete frames". Computer-Aided Civil and Infrastructure Engineering 13: 63-74. 1998.

(166) Rechenberg, I. "Evolutionsstrategie: Optimierung technischer Systeme nach Prinzipen der biologischen Evolution". Ed. Frommann-Holzboog, Stuttgart, Alemania. 1973. 
(167) Rodrigues Junior, S.; Eloy Vaz, L.; Barbosa Guimaraes, G. "Optimum design of tall buildings in reinforced concrete subjected to wind forces". Proc. of the 6th Congress of Structural and Multidisciplinary Optimization. Rio de Janeiro. 2005.

(168) Rodriguez Martín, L.F. "Curso práctico Cypecad. Versión 2000.1”. Ed. Cype Ingenieros. Alicante, 2001.

(169) Rudolph G. "Convergence analysis of canonical genetic algorithms". IEEE Transactions on Neuronal Networks, 5: 96-101. 1994.

(170) Ruy W.; Yang Y.; Kim W.; Yeun Y. "Topology design of Truss Structures in a Multicriteria Environment". Computer-Aided Civil and Infraestructural Engineering, 16: 246-251. 2001.

(171) Salama, M.; Bruno R., Chen, G.; Garba, J. "Optimal placement of excitations and sensors by simulated annealing". Proceedings of NASA/Air Force Symposium on Recent Experiences in Multidisciplinary Analysis and Optimization. 2005.

(172) Sandgren, E. "Multicriteria design optimization by Goal Programming". Advances in Design Optimization: 225-265. Chapmann \& Hall, London, 1994.

(173) Sahab M.G.; Ashour A.F.; Toropov, V.V. "A hybrid genetic algorithm for reinforced concrete flat buildings". Computers and Structures, 83(8):551-559. 2004.

(174) Sahab M.G.; Ashour A.F.; Toropov, V.V. "Cost optimization of reinforced concrete flat buildings". Engineering structures, 27(3):313-322. 2005.

(175) Sarma K.; Adeli, H. "Cost optimization of concrete structures". ASCE Journal of Structural Engineering, 124(5): 570-578. 1998.

(176) Sarma K.; Adeli, H. "Cost optimization of steel structures". Engineering Optimization, 32(6): 777-802. 2000a. 
(177) Sarma K.; Adeli, H. "Fuzzy discrete multicriteria cost optimization of steel structures". ASCE Journal of Structural Engineering, 126(11): 1339-1347. $2000 b$

(178) Sarma K.; Adeli, H. "Life-cycle cost optimization of steel structures". Journal for Numerical Methods in Engineering, 55(12): 1451-1462. 2002.

(179) Sarma K.; Adeli, H. "Comparative study of optimum designs of steel high rise building structures using Allowable Stress Design and Load and Resistance design Factor Codes". ASCE Practice Periodical on Structural Design and Construction, 10(1): 12-17. 2005.

(180) Savic, D.; Evans, K.; Silberhorn, T. "A Genetic Algorithm-based system for the optimal design of laminates". Computer-Aided Civil and Infraestructural Engineering, 14: 187-197. 1999.

(181) Schwefel, H.P. "Kybernetische evolution als strategie der experimentellen Forschung in der Ströomungstechnik". Dipl.-Ing. Arbeit. 1965.

(182) Serafini, P. "Mathematics of multiobjective optimization problems". CISM Courses and Lectures, vol. 289. Ed. Spring Verlag. Berlín (Alemania). 1984.

(183) Serra, M.; Venini, P. "On some applications of Ant Colony Optimization metaheuristic to structural optimization problems". Proc. of the 6th Congress of Structural and Multidisciplinary Optimization. Rio de Janeiro. 2005.

(184) Shelokar, P.; Adhikari, S.; Vakil, R.; Jayaraman V.K.; Kulkarni, B.D." Multiobjective Ant Algorithm for continuous function optimization: combination of strength Pareto fitness assignment and thermodynamic clustering". Foundations of Computing and Decision Sciences, 25: 213-230. 2000.

(185) Shea, K.; Cagan J. "Innovative dome design: applying geodesic patterns with shape annealing". Artificial Intelligence for Engineering Design, Analysis and Manufacturing 11: 379-394. 1997. 
(186) Shea, K.; Zaho, X. "A novel noon mark cantilever support: from design generation to realization". Proc. of IASS 2004 Shell and Spatial Structures from Models to Realization. Montpellier, Francia. 2004.

(187) Shea, K.; Smith F.C. "Improving full-scale transmission tower design through topology and shape optimization". ASCE Journal of Structural Engineering 132(5): 781-790. 2006.

(188) Shelokar, P.; Jayaraman V.K.; Kulkarni, B.D." Ant Algorithm for simple and multiobjective reliability optimization problems". Quality Reliability in Engineering International,18 : 497-514. 2002.

(189) Shrestha, S.; Ghaboussi, J. "Evolution of optimum shapes using Genetic Algorithm". ASCE Journal of Structural Engineering, 124(11): 1331-1338. 1998.

(190) Schmidt, H.; Thierauf, G. "A combined optimization technique". Advances in Engineering Software, 36: 11-19. 2005.

(191) Suman, B. "Study of Simulated Annealing based algorithms for multiobjective optimization of a constrained problem". Computers and Chemical Engineering, 28: 1849-1871. 2004.

(192) Suppapitnarm, A.; Seffen K.A.; Parks, G.T.; Clarkson P.J.." A Simulted Annealing for multiobjective optimization". Engineering Optimization, 33: 59-85. 2000.

(193) Taranath, B. "Steel, concrete and composite design of tall buildings". New York, McGraw-Hill. 1997.

(194) Torrano, M. "Diseño óptimo de secciones y elementos estructurales de hormigón armado". Tesis Doctoral. Universidad Politécnica de Cartagena. 2003.

(195) Tzan, S.R.; Pantelides, C. "Annealing Strategy for optimal structural design". ASCE Journal of Structural Engineering, 122(7): 815-827. 1996. 
(196) Tzan, S.R.; Pantelides, C. "Annealing Strategy for optimal structural design". Advances in Engineering Software, 31: 391-400. 2000.

(197) Ulungu, L.; Teghem, J.; Fortemps, P. "Heuristics for multiobjective combinatorial optimization problems by Simulated Annealing" en "MCDM: Theory and applications". In J. Gu, G. Chen, Q. Wei and S. Wang (Eds.). Winsor: Sci-Tech. 1995.

(198) Venanzi, I.; Materazzi, A.L. "Multi-objective optimization of wind-excited structures". Engineering Structures. Doi:10.1016/j.engstruct.2006.07.007. 2006.

(199) Xu, L.; Gong, Y. "Preliminary design of Long-Span King Post Trust Systems with a Genetic Algorithm". Computer-Aided Civil and Infraestructural Engineering, 16: 94-105. 2001.

(200) Yepes, V. “Optimización heurística económica aplicada a las redes de transporte del tipo VRPTW". Tesis doctoral. Universidad Politécnica de Valencia. 2002.

(201) Yepes, V. “Apuntes de optimización en ingeniería”. Servicio de Publicaciones de la Universidad Politécnica de Valencia Ref.: 2003.249. 2003, Valencia, 2003.

(202) Yepes, V. "La inteligencia artificial aplicada al diseño económico de muros ménsula de hormigón armado". Trabajo de investigación para la prueba de habilitación nacional al Cuerpo de Profesores Titulares de Universidad. 2006.

(203) Yepes, V.; Alcalá, J.; Perea, C.; González-Vidosa, F. "Estudio paramétrico de muros óptimos de contención de hormigón armado". Ingeniería Civil 142:129139. 2006. 


\section{APÉNDICES:}

APÉNDICE 1. VALORES DE LAS VARIABLES.

APÉNDICE 2. MEDICIONES DEL PÓRTICO TIPO.

APÉNDICE 3. COMBINACIÓN DE ACCIONES.

APÉNDICE 4. PLANOS DE LAS ESTRUCTURAS OPTIMIZADAS. 
Página intencionadamente en blanco. 


\section{APÉNDICE 1. VALORES DE LAS VARIABLES.}


Página intencionadamente en blanco. 
Tipos del acero.

\begin{tabular}{|c|c|c|}
\hline Núm. acero & Tipo & $\mathbf{f}_{\mathbf{y k}}$ (Mpa) \\
\hline 1 & B 400 S & 400 \\
\hline 2 & B 500 S & 500 \\
\hline
\end{tabular}

$\underline{\text { Resistencia característica del hormigón en vigas y pilares. }}$

\begin{tabular}{|c|c|}
\hline Núm. hormigón & $\mathbf{f}_{\mathbf{c k}}$ (Mpa) \\
\hline 1 & 25 \\
\hline 2 & 30 \\
\hline 3 & 35 \\
\hline 4 & 40 \\
\hline 5 & 45 \\
\hline 6 & 50 \\
\hline
\end{tabular}

\section{Ancho y canto de vigas.}

\begin{tabular}{|c|c|c|c|c|c|c|c|}
\hline Número & Ancho/Canto & Número & Ancho/Canto & Número & Ancho/Canto & Número & Ancho/Canto \\
\hline 1 & 0.15 & 31 & 0.45 & 61 & 0.75 & 91 & 1.05 \\
\hline 2 & 0.16 & 32 & 0.46 & 62 & 0.76 & 92 & 1.06 \\
\hline 3 & 0.17 & 33 & 0.47 & 63 & 0.77 & 93 & 1.07 \\
\hline 4 & 0.18 & 34 & 0.48 & 64 & 0.78 & 94 & 1.08 \\
\hline 5 & 0.19 & 35 & 0.49 & 65 & 0.79 & 95 & 1.09 \\
\hline 6 & 0.2 & 36 & 0.5 & 66 & 0.8 & 96 & 1.1 \\
\hline 7 & 0.21 & 37 & 0.51 & 67 & 0.81 & 97 & 1.11 \\
\hline 8 & 0.22 & 38 & 0.52 & 68 & 0.82 & 98 & 1.12 \\
\hline 9 & 0.23 & 39 & 0.53 & 69 & 0.83 & 99 & 1.13 \\
\hline 10 & 0.24 & 40 & 0.54 & 70 & 0.84 & 100 & 1.14 \\
\hline 11 & 0.25 & 41 & 0.55 & 71 & 0.85 & 101 & 1.15 \\
\hline 12 & 0.26 & 42 & 0.56 & 72 & 0.86 & 102 & 1.16 \\
\hline 13 & 0.27 & 43 & 0.57 & 73 & 0.87 & 103 & 1.17 \\
\hline 14 & 0.28 & 44 & 0.58 & 74 & 0.88 & 104 & 1.18 \\
\hline 15 & 0.29 & 45 & 0.59 & 75 & 0.89 & 105 & 1.19 \\
\hline 16 & 0.3 & 46 & 0.6 & 76 & 0.9 & 106 & 1.2 \\
\hline 17 & 0.31 & 47 & 0.61 & 77 & 0.91 & & \\
\hline 18 & 0.32 & 48 & 0.62 & 78 & 0.92 & & \\
\hline 19 & 0.33 & 49 & 0.63 & 79 & 0.93 & & \\
\hline 20 & 0.34 & 50 & 0.64 & 80 & 0.94 & & \\
\hline 21 & 0.35 & 51 & 0.65 & 81 & 0.95 & & \\
\hline 22 & 0.36 & 52 & 0.66 & 82 & 0.96 & & \\
\hline 23 & 0.37 & 53 & 0.67 & 83 & 0.97 & & \\
\hline 24 & 0.38 & 54 & 0.68 & 84 & 0.98 & & \\
\hline 25 & 0.39 & 55 & 0.69 & 85 & 0.99 & & \\
\hline 26 & 0.4 & 56 & 0.7 & 86 & 1 & & \\
\hline 27 & 0.41 & 57 & 0.71 & 87 & 1.01 & & \\
\hline 28 & 0.42 & 58 & 0.72 & 88 & 1.02 & & \\
\hline 29 & 0.43 & 59 & 0.73 & 89 & 1.03 & & \\
\hline 30 & 0.44 & 60 & 0.74 & 90 & 1.04 & & \\
\hline
\end{tabular}




\section{Ancho y canto de pilares.}

\begin{tabular}{|c|c|c|c|}
\hline Número & Ancho/Canto & Número & Ancho/Canto \\
\hline 1 & 0.25 & 11 & 0.75 \\
\hline 2 & 0.3 & 12 & 0.8 \\
\hline 3 & 0.35 & 13 & 0.85 \\
\hline 4 & 0.4 & 14 & 0.9 \\
\hline 5 & 0.45 & 15 & 0.95 \\
\hline 6 & 0.5 & 16 & 1 \\
\hline 7 & 0.55 & 17 & 1.05 \\
\hline 8 & 0.6 & 18 & 1.1 \\
\hline 9 & 0.65 & 19 & 1.15 \\
\hline 10 & 0.7 & 20 & 1.2 \\
\hline
\end{tabular}




\section{Armadura base superior e inferior en vigas.}

\begin{tabular}{|c|c|c|c|}
\hline Número & $\mathbf{n}^{\mathbf{0}}$ redondos & $\phi \mathbf{( m m})$ & As $\mathbf{( c m}^{\mathbf{2}} \mathbf{~}$ \\
\hline 1 & 2 & 10 & 1.57 \\
\hline 2 & 2 & 12 & 2.26 \\
\hline 3 & 3 & 10 & 2.36 \\
\hline 4 & 4 & 10 & 3.14 \\
\hline 5 & 3 & 12 & 3.39 \\
\hline 6 & 2 & 16 & 4.02 \\
\hline 7 & 4 & 12 & 4.52 \\
\hline 8 & 5 & 12 & 5.65 \\
\hline 9 & 3 & 16 & 6.03 \\
\hline 10 & 2 & 20 & 6.28 \\
\hline 11 & 6 & 12 & 6.79 \\
\hline 12 & 7 & 12 & 7.92 \\
\hline 13 & 4 & 16 & 8.04 \\
\hline 14 & 3 & 20 & 9.42 \\
\hline 15 & 2 & 25 & 9.82 \\
\hline 16 & 5 & 16 & 10.05 \\
\hline 17 & 6 & 16 & 12.06 \\
\hline 18 & 4 & 20 & 12.57 \\
\hline 19 & 3 & 25 & 14.73 \\
\hline 20 & 5 & 20 & 15.71 \\
\hline 21 & 8 & 16 & 16.09 \\
\hline 22 & 6 & 20 & 18.85 \\
\hline 23 & 4 & 25 & 19.64 \\
\hline 24 & 7 & 20 & 21.99 \\
\hline 25 & 5 & 25 & 24.54 \\
\hline & & & \\
\hline
\end{tabular}

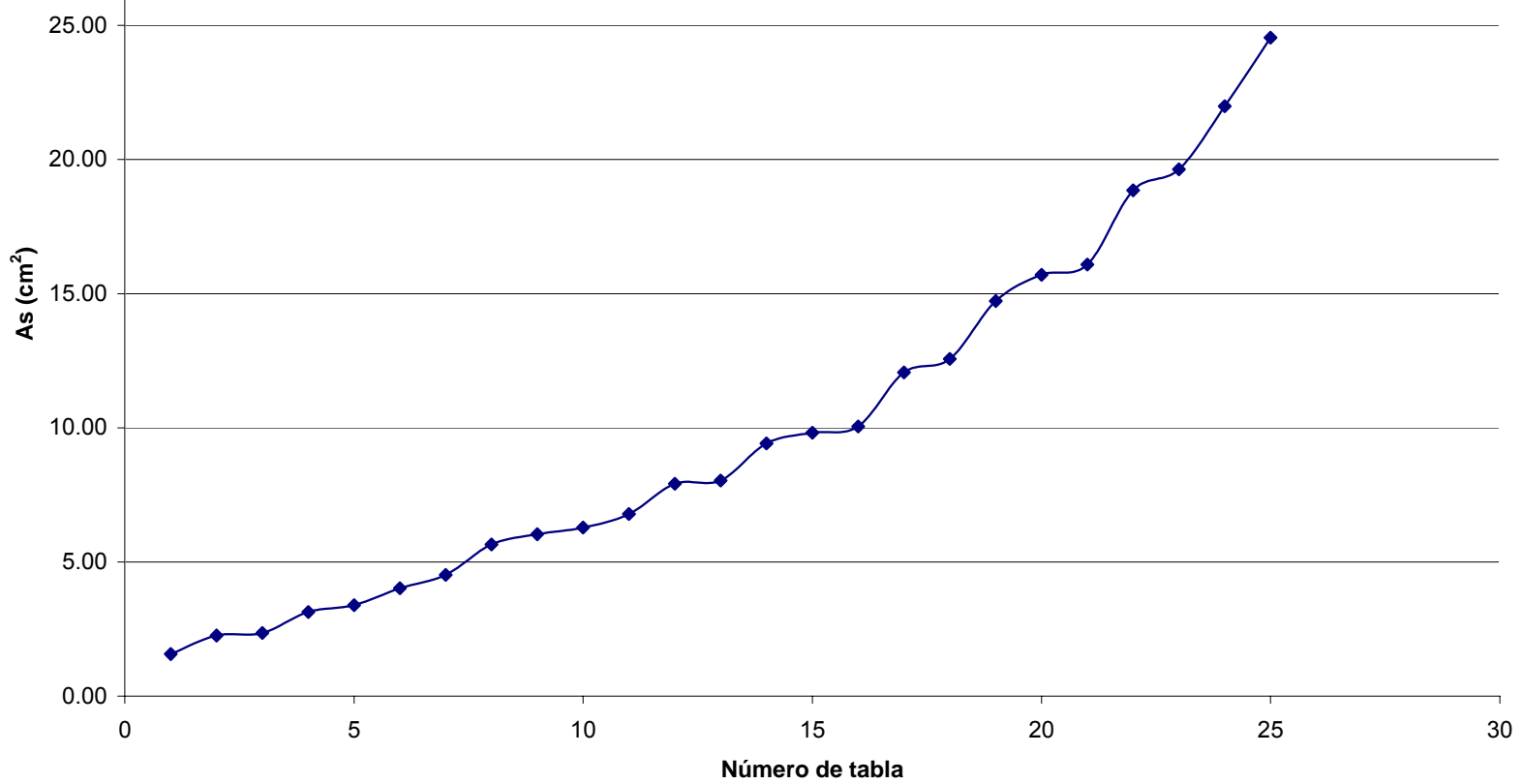




\section{Armadura de refuerzo superior e inferior en vigas.}

\begin{tabular}{|c|c|c|c|}
\hline Número & $\mathbf{n}^{\mathbf{o}}$ redondos & $\boldsymbol{\phi} \mathbf{( m m})$ & As $\mathbf{( c m}^{\mathbf{2}} \mathbf{~}$ \\
\hline 1 & 0 & 0 & 0.00 \\
\hline 2 & 1 & 10 & 0.79 \\
\hline 3 & 1 & 12 & 1.13 \\
\hline 4 & 2 & 10 & 1.57 \\
\hline 5 & 1 & 16 & 2.01 \\
\hline 6 & 2 & 12 & 2.26 \\
\hline 7 & 1 & 20 & 3.14 \\
\hline 8 & 3 & 12 & 3.39 \\
\hline 9 & 2 & 16 & 4.02 \\
\hline 10 & 1 & 25 & 4.91 \\
\hline 11 & 3 & 16 & 6.03 \\
\hline 12 & 2 & 20 & 6.28 \\
\hline 13 & 6 & 12 & 6.79 \\
\hline 14 & 4 & 16 & 8.04 \\
\hline 15 & 3 & 20 & 9.42 \\
\hline 16 & 2 & 25 & 9.82 \\
\hline 17 & 5 & 16 & 10.05 \\
\hline 18 & 6 & 16 & 12.06 \\
\hline 19 & 4 & 20 & 12.57 \\
\hline 20 & 3 & 25 & 14.73 \\
\hline 21 & 5 & 20 & 15.71 \\
\hline 22 & 6 & 20 & 18.85 \\
\hline 23 & 4 & 25 & 19.64 \\
\hline & & & \\
\hline
\end{tabular}

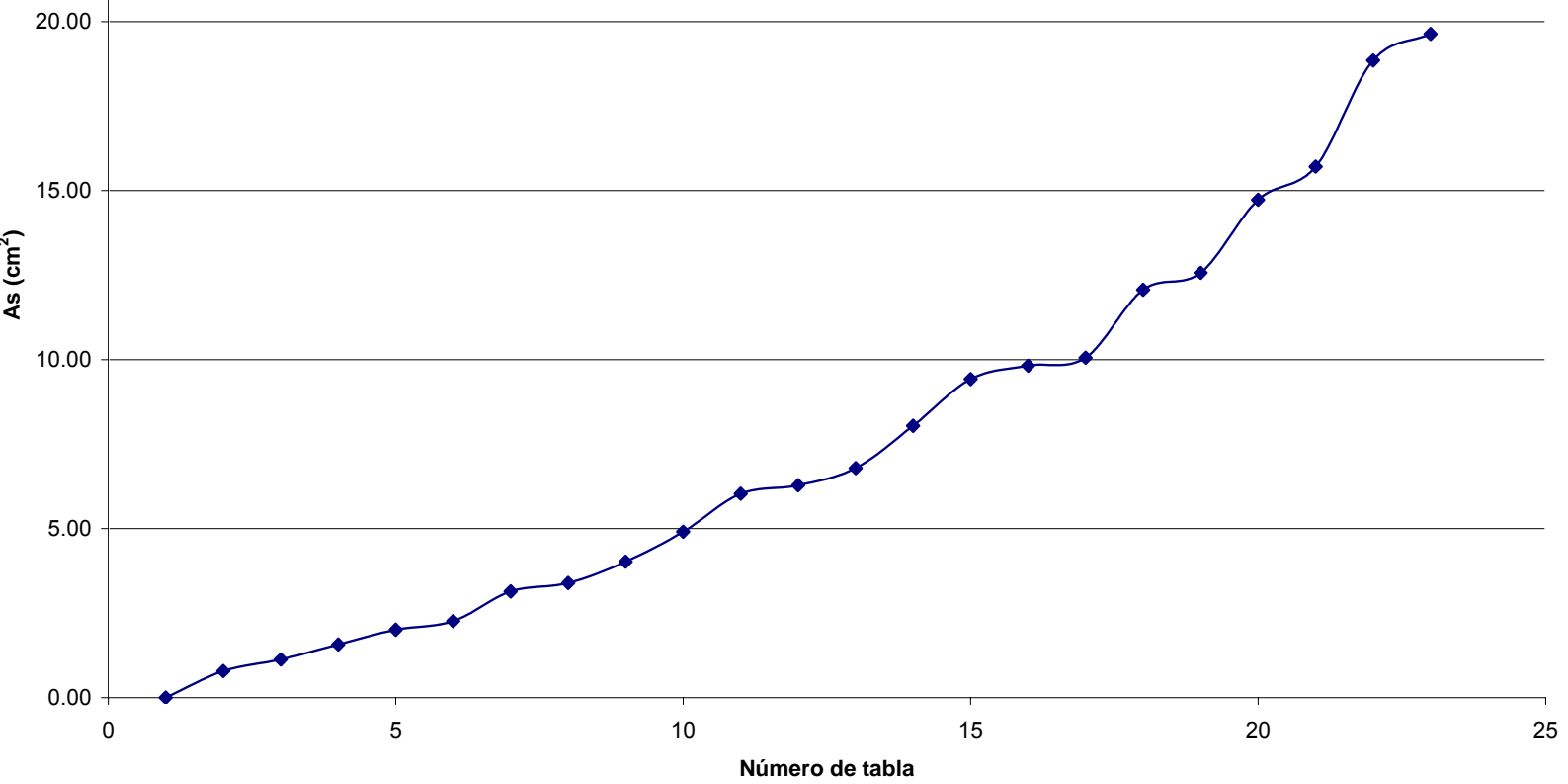




\section{Armadura transversal en vigas.}

\begin{tabular}{|c|c|c|c|c|}
\hline $\begin{array}{l}\text { NUM } \\
\text { CASO }\end{array}$ & diámetro & $\begin{array}{l}\text { separación } \\
\text { transversal } \\
\text { (m) }\end{array}$ & $\begin{array}{l}\text { Número de } \\
\text { cercos }\end{array}$ & $\operatorname{At}\left(\mathrm{cm}^{2} / \mathrm{m}\right)$ \\
\hline 1 & 6 & 0.3 & 1 & 1.88 \\
\hline 2 & 6 & 0.25 & 1 & 2.26 \\
\hline 3 & 6 & 0.2 & 1 & 2.83 \\
\hline 4 & 8 & 0.3 & 1 & 3.35 \\
\hline 5 & 6 & 0.15 & 1 & 3.77 \\
\hline 6 & 8 & 0.25 & 1 & 4.02 \\
\hline 7 & 8 & 0.2 & 1 & 5.03 \\
\hline 8 & 10 & 0.3 & 1 & 5.24 \\
\hline 9 & 10 & 0.25 & 1 & 6.28 \\
\hline 10 & 8 & 0.15 & 1 & 6.70 \\
\hline 11 & 12 & 0.3 & 1 & 7.54 \\
\hline 12 & 10 & 0.2 & 1 & 7.85 \\
\hline 13 & 12 & 0.25 & 1 & 9.05 \\
\hline 14 & 10 & 0.15 & 1 & 10.47 \\
\hline 15 & 12 & 0.2 & 1 & 11.31 \\
\hline 16 & 16 & 0.3 & 1 & 13.40 \\
\hline 17 & 12 & 0.15 & 1 & 15.08 \\
\hline 18 & 10 & 0.2 & 2 & 15.71 \\
\hline 19 & 16 & 0.25 & 1 & 16.08 \\
\hline 20 & 12 & 0.25 & 2 & 18.10 \\
\hline 21 & 16 & 0.2 & 1 & 20.11 \\
\hline 22 & 10 & 0.15 & 2 & 20.94 \\
\hline 23 & 12 & 0.2 & 2 & 22.62 \\
\hline 24 & 16 & 0.15 & 1 & 26.81 \\
\hline 25 & 12 & 0.15 & 2 & 30.16 \\
\hline 26 & 16 & 0.25 & 2 & 32.17 \\
\hline 27 & 16 & 0.2 & 2 & 40.21 \\
\hline 28 & 16 & 0.15 & 2 & 53.62 \\
\hline
\end{tabular}

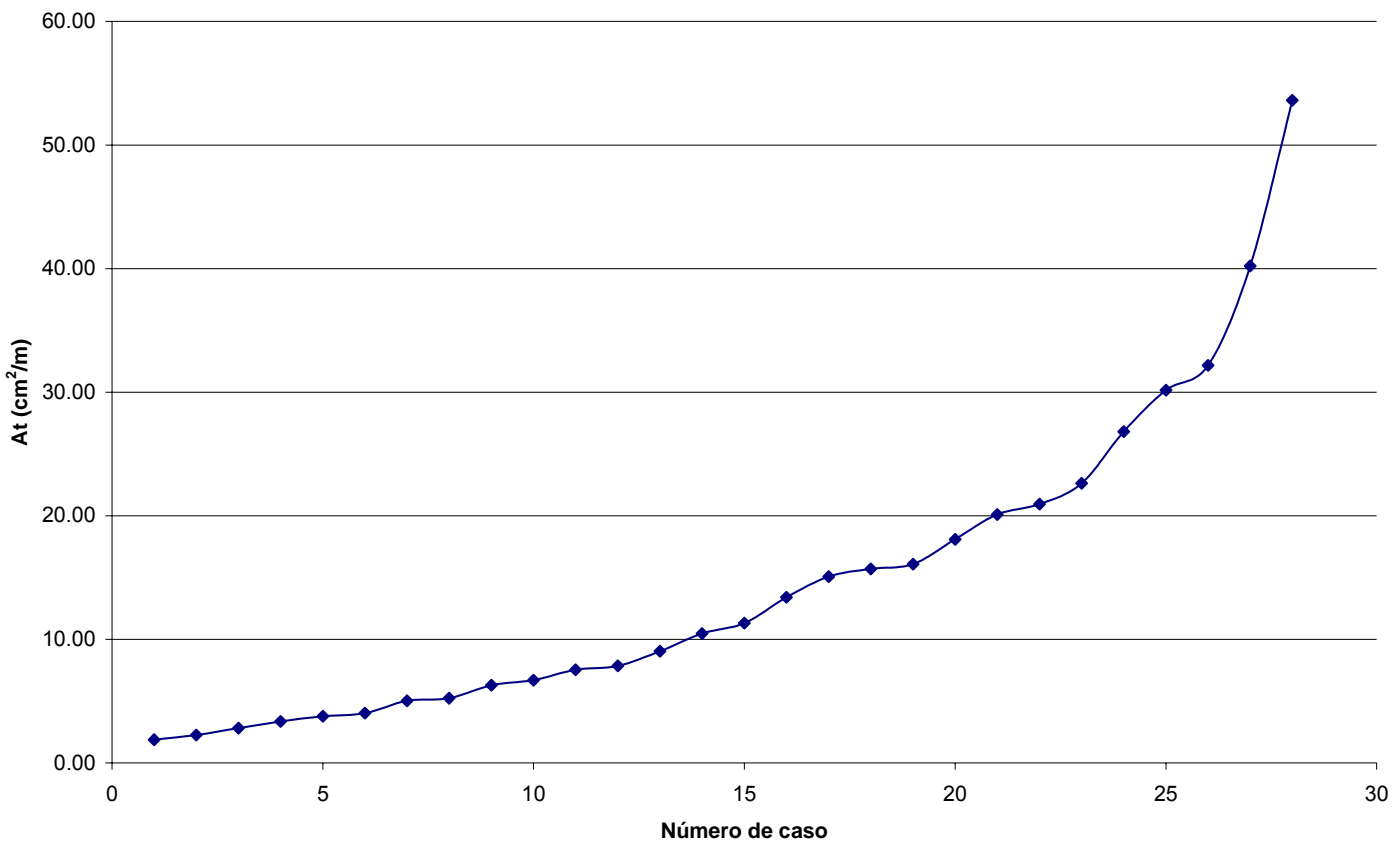




\section{Armadura longitudinal en pilares.}

Se han considerado un total de 331 casos diferentes de armado longitudinal de pilares. Cada caso se caracteriza por:

1) Armadura en las esquinas (número de redondos " $n$ ” y diámetro en mm “ $\phi ”)$.

2) Armadura de refuerzo - adicional a la situada en las esquinas- situada en las caras "a" del pilar -caras paralelas al eje de las vigas del pórtico-. Esta armadura se caracteriza por el número total de redondos " $\mathrm{n}$ ” y por su diámetro en mm “ $\phi$ ").

3) Armadura de refuerzo situada en las caras "b" del pilar -caras perpendiculares al eje de las vigas del pórtico-. Esta armadura se caracteriza por el número total de redondos " $\mathrm{n}$ " y por su diámetro en $\mathrm{mm}$ " $\phi$ ").

\begin{tabular}{|c|c|c|c|c|c|c|c|}
\hline \multirow{2}{*}{ Número } & \multicolumn{2}{|c|}{ ESQUINAS } & \multicolumn{2}{|c|}{ CARA "a" } & \multicolumn{2}{|c|}{ CARA "b" } & \multirow{2}{*}{ As } \\
\hline & $\mathbf{n}$ & $\phi$ & $\mathbf{n}_{\text {ref, TOTAL }}$ & $\phi$ & $\mathbf{n}_{\text {ref, TOTAL }}$ & $\phi$ & \\
\hline 1 & 4 & 12 & 0 & 0 & 0 & 0 & 4.52 \\
\hline 2 & 4 & 12 & 2 & 12 & 0 & 0 & 6.78 \\
\hline 3 & 4 & 12 & 0 & 0 & 2 & 12 & 6.78 \\
\hline 4 & 4 & 16 & 0 & 0 & 0 & 0 & 8.04 \\
\hline 5 & 4 & 12 & 2 & 12 & 2 & 12 & 9.04 \\
\hline 6 & 4 & 12 & 0 & 0 & 4 & 12 & 9.04 \\
\hline 7 & 4 & 16 & 2 & 12 & 0 & 0 & 10.30 \\
\hline 8 & 4 & 16 & 0 & 0 & 2 & 12 & 10.30 \\
\hline 9 & 4 & 12 & 4 & 12 & 2 & 12 & 11.30 \\
\hline 10 & 4 & 12 & 2 & 12 & 4 & 12 & 11.30 \\
\hline 11 & 4 & 12 & 0 & 0 & 6 & 12 & 11.30 \\
\hline 12 & 4 & 16 & 2 & 16 & 0 & 0 & 12.06 \\
\hline 13 & 4 & 16 & 0 & 0 & 2 & 16 & 12.06 \\
\hline 14 & 4 & 16 & 2 & 12 & 2 & 12 & 12.56 \\
\hline 15 & 4 & 16 & 0 & 0 & 4 & 12 & 12.56 \\
\hline 16 & 4 & 20 & 0 & 0 & 0 & 0 & 12.56 \\
\hline 17 & 4 & 12 & 4 & 12 & 4 & 12 & 13.56 \\
\hline 18 & 4 & 12 & 2 & 12 & 6 & 12 & 13.56 \\
\hline 19 & 4 & 12 & 0 & 0 & 8 & 12 & 13.56 \\
\hline 20 & 4 & 16 & 2 & 16 & 2 & 12 & 14.32 \\
\hline 21 & 4 & 16 & 2 & 12 & 2 & 16 & 14.32 \\
\hline 22 & 4 & 16 & 4 & 12 & 2 & 12 & 14.82 \\
\hline 23 & 4 & 16 & 2 & 12 & 4 & 12 & 14.82 \\
\hline 24 & 4 & 16 & 0 & 0 & 6 & 12 & 14.82 \\
\hline 25 & 4 & 20 & 2 & 12 & 0 & 0 & 14.82 \\
\hline 26 & 4 & 20 & 0 & 0 & 2 & 12 & 14.82 \\
\hline 27 & 4 & 12 & 4 & 12 & 6 & 12 & 15.83 \\
\hline 28 & 4 & 12 & 2 & 12 & 8 & 12 & 15.83 \\
\hline 29 & 4 & 12 & 0 & 0 & 10 & 12 & 15.83 \\
\hline 30 & 4 & 16 & 2 & 16 & 2 & 16 & 16.08 \\
\hline
\end{tabular}




\begin{tabular}{|c|c|c|c|c|c|c|c|}
\hline \multirow{2}{*}{ Número } & \multicolumn{2}{|c|}{ ESQUINAS } & \multicolumn{2}{|c|}{ CARA "a" } & \multicolumn{2}{|c|}{ CARA "b" } & \multirow{2}{*}{ As } \\
\hline & $\mathbf{n}$ & $\phi$ & $\mathbf{n}_{\text {ref, TOTAL }}$ & $\phi$ & $\mathbf{n}_{\text {ref, Тот }}$ & $\phi$ & \\
\hline 31 & 4 & 16 & 0 & 0 & 4 & 16 & 16.08 \\
\hline 32 & 4 & 16 & 4 & 12 & 2 & 16 & 16.58 \\
\hline 33 & 4 & 16 & 2 & 16 & 4 & 12 & 16.58 \\
\hline 34 & 4 & 20 & 2 & 16 & 0 & 0 & 16.58 \\
\hline 35 & 4 & 20 & 0 & 0 & 2 & 16 & 16.58 \\
\hline 36 & 4 & 16 & 4 & 12 & 4 & 12 & 17.08 \\
\hline 37 & 4 & 16 & 2 & 12 & 6 & 12 & 17.08 \\
\hline 38 & 4 & 16 & 0 & 0 & 8 & 12 & 17.08 \\
\hline 39 & 4 & 20 & 2 & 12 & 2 & 12 & 17.08 \\
\hline 40 & 4 & 20 & 0 & 0 & 4 & 12 & 17.08 \\
\hline 41 & 4 & 12 & 4 & 12 & 8 & 12 & 18.09 \\
\hline 42 & 4 & 12 & 2 & 12 & 10 & 12 & 18.09 \\
\hline 43 & 4 & 12 & 0 & 0 & 12 & 12 & 18.09 \\
\hline 44 & 4 & 12 & 6 & 12 & 6 & 12 & 18.09 \\
\hline 45 & 4 & 16 & 4 & 16 & 2 & 12 & 18.34 \\
\hline 46 & 4 & 16 & 2 & 12 & 4 & 16 & 18.34 \\
\hline 47 & 4 & 16 & 2 & 16 & 6 & 12 & 18.84 \\
\hline 48 & 4 & 20 & 2 & 20 & 0 & 0 & 18.84 \\
\hline 49 & 4 & 20 & 0 & 0 & 2 & 20 & 18.84 \\
\hline 50 & 4 & 16 & 4 & 12 & 6 & 12 & 19.34 \\
\hline 51 & 4 & 16 & 2 & 12 & 8 & 12 & 19.34 \\
\hline 52 & 4 & 16 & 0 & 0 & 10 & 12 & 19.34 \\
\hline 53 & 4 & 20 & 4 & 12 & 2 & 12 & 19.34 \\
\hline 54 & 4 & 20 & 2 & 12 & 4 & 12 & 19.34 \\
\hline 55 & 4 & 20 & 0 & 0 & 6 & 12 & 19.34 \\
\hline 56 & 4 & 25 & 0 & 0 & 0 & 0 & 19.63 \\
\hline 57 & 4 & 16 & 4 & 16 & 2 & 16 & 20.10 \\
\hline 58 & 4 & 16 & 2 & 16 & 4 & 16 & 20.10 \\
\hline 59 & 4 & 16 & 0 & 0 & 6 & 16 & 20.10 \\
\hline 60 & 4 & 12 & 4 & 12 & 10 & 12 & 20.35 \\
\hline 61 & 4 & 16 & 4 & 16 & 4 & 12 & 20.60 \\
\hline 62 & 4 & 16 & 4 & 12 & 4 & 16 & 20.60 \\
\hline 63 & 4 & 20 & 2 & 16 & 2 & 16 & 20.60 \\
\hline 64 & 4 & 20 & 0 & 0 & 4 & 16 & 20.60 \\
\hline 65 & 4 & 16 & 2 & 16 & 8 & 12 & 21.10 \\
\hline 66 & 4 & 20 & 2 & 20 & 2 & 12 & 21.10 \\
\hline 67 & 4 & 20 & 2 & 12 & 2 & 20 & 21.10 \\
\hline 68 & 4 & 16 & 6 & 12 & 6 & 12 & 21.60 \\
\hline 69 & 4 & 16 & 4 & 12 & 8 & 12 & 21.60 \\
\hline 70 & 4 & 16 & 2 & 12 & 10 & 12 & 21.60 \\
\hline 71 & 4 & 16 & 0 & 0 & 12 & 12 & 21.60 \\
\hline 72 & 4 & 20 & 4 & 12 & 4 & 12 & 21.60 \\
\hline 73 & 4 & 20 & 0 & 0 & 8 & 12 & 21.60 \\
\hline 74 & 4 & 16 & 2 & 12 & 6 & 16 & 22.36 \\
\hline 75 & 4 & 12 & 0 & 0 & 16 & 12 & 22.61 \\
\hline 76 & 4 & 16 & 4 & 16 & 6 & 12 & 22.86 \\
\hline 77 & 4 & 20 & 2 & 20 & 2 & 16 & 22.86 \\
\hline 78 & 4 & 20 & 2 & 16 & 2 & 20 & 22.86 \\
\hline 79 & 4 & 16 & 2 & 16 & 10 & 12 & 23.36 \\
\hline 80 & 4 & 20 & 2 & 20 & 4 & 12 & 23.36 \\
\hline
\end{tabular}




\begin{tabular}{|c|c|c|c|c|c|c|c|}
\hline \multirow{2}{*}{ Número } & \multicolumn{2}{|c|}{ ESQUINAS } & \multicolumn{2}{|c|}{ CARA "a" } & \multicolumn{2}{|c|}{ CARA "b" } & \multirow{2}{*}{ As } \\
\hline & $\mathbf{n}$ & $\phi$ & $\mathbf{n}_{\text {ref, TOTAL }}$ & $\phi$ & $\mathbf{n}_{\text {ref, TOTAL }}$ & $\phi$ & \\
\hline 81 & 4 & 20 & 4 & 12 & 2 & 20 & 23.36 \\
\hline 82 & 4 & 25 & 2 & 16 & 0 & 0 & 23.64 \\
\hline 83 & 4 & 25 & 0 & 0 & 2 & 16 & 23.64 \\
\hline 84 & 4 & 16 & 4 & 12 & 10 & 12 & 23.86 \\
\hline 85 & 4 & 20 & 0 & 0 & 10 & 12 & 23.86 \\
\hline 86 & 4 & 16 & 4 & 16 & 4 & 16 & 24.12 \\
\hline 87 & 4 & 16 & 2 & 16 & 6 & 16 & 24.12 \\
\hline 88 & 4 & 16 & 0 & 0 & 8 & 16 & 24.12 \\
\hline 89 & 4 & 16 & 4 & 12 & 6 & 16 & 24.62 \\
\hline 90 & 4 & 20 & 4 & 16 & 2 & 16 & 24.62 \\
\hline 91 & 4 & 20 & 0 & 0 & 6 & 16 & 24.62 \\
\hline 92 & 4 & 20 & 2 & 16 & 4 & 16 & 24.62 \\
\hline 93 & 4 & 12 & 0 & 0 & 18 & 12 & 24.87 \\
\hline 94 & 4 & 16 & 4 & 16 & 8 & 12 & 25.12 \\
\hline 95 & 4 & 20 & 2 & 20 & 2 & 20 & 25.12 \\
\hline 96 & 4 & 20 & 0 & 0 & 4 & 20 & 25.12 \\
\hline 97 & 4 & 20 & 2 & 20 & 6 & 12 & 25.62 \\
\hline 98 & 4 & 25 & 2 & 20 & 0 & 0 & 25.91 \\
\hline 99 & 4 & 25 & 0 & 0 & 2 & 20 & 25.91 \\
\hline 100 & 4 & 16 & 0 & 0 & 16 & 12 & 26.12 \\
\hline 101 & 4 & 20 & 0 & 0 & 12 & 12 & 26.12 \\
\hline 102 & 4 & 16 & 2 & 12 & 8 & 16 & 26.38 \\
\hline 103 & 4 & 25 & 0 & 0 & 6 & 12 & 26.41 \\
\hline 104 & 4 & 16 & 6 & 16 & 6 & 12 & 26.88 \\
\hline 105 & 4 & 16 & 6 & 12 & 6 & 16 & 26.88 \\
\hline 106 & 4 & 20 & 4 & 16 & 2 & 20 & 26.88 \\
\hline 107 & 4 & 20 & 2 & 20 & 4 & 16 & 26.88 \\
\hline 108 & 4 & 16 & 4 & 16 & 10 & 12 & 27.38 \\
\hline 109 & 4 & 20 & 4 & 20 & 2 & 12 & 27.38 \\
\hline 110 & 4 & 20 & 2 & 12 & 4 & 20 & 27.38 \\
\hline 111 & 4 & 25 & 0 & 0 & 4 & 16 & 27.66 \\
\hline 112 & 4 & 25 & 2 & 16 & 2 & 16 & 27.66 \\
\hline 113 & 4 & 20 & 2 & 20 & 8 & 12 & 27.88 \\
\hline 114 & 4 & 16 & 4 & 16 & 6 & 16 & 28.13 \\
\hline 115 & 4 & 16 & 0 & 0 & 10 & 16 & 28.13 \\
\hline 116 & 4 & 16 & 2 & 16 & 8 & 16 & 28.13 \\
\hline 117 & 4 & 16 & 0 & 0 & 18 & 12 & 28.39 \\
\hline 118 & 4 & 16 & 4 & 12 & 8 & 16 & 28.64 \\
\hline 119 & 4 & 20 & 4 & 16 & 4 & 16 & 28.64 \\
\hline 120 & 4 & 20 & 0 & 0 & 8 & 16 & 28.64 \\
\hline 121 & 4 & 25 & 0 & 0 & 8 & 12 & 28.67 \\
\hline 122 & 4 & 20 & 4 & 20 & 2 & 16 & 29.14 \\
\hline 123 & 4 & 20 & 2 & 16 & 4 & 20 & 29.14 \\
\hline 124 & 4 & 12 & 0 & 0 & 22 & 12 & 29.39 \\
\hline 125 & 4 & 25 & 2 & 25 & 0 & 0 & 29.44 \\
\hline 126 & 4 & 25 & 0 & 0 & 2 & 25 & 29.44 \\
\hline 127 & 4 & 20 & 4 & 20 & 4 & 12 & 29.64 \\
\hline 128 & 4 & 20 & 4 & 12 & 4 & 20 & 29.64 \\
\hline 129 & 4 & 20 & 2 & 20 & 10 & 12 & 30.14 \\
\hline 130 & 4 & 16 & 2 & 12 & 10 & 16 & 30.40 \\
\hline
\end{tabular}




\begin{tabular}{|c|c|c|c|c|c|c|c|}
\hline \multirow{2}{*}{ Número } & \multicolumn{2}{|c|}{ ESQUINAS } & \multicolumn{2}{|c|}{ CARA "a" } & \multicolumn{2}{|c|}{ CARA "b" } & \multirow{2}{*}{ As } \\
\hline & $\mathbf{n}$ & $\phi$ & $\mathbf{n}_{\text {ref, TOTAL }}$ & $\phi$ & $n_{\text {ref, TOT }}$ & $\phi$ & \\
\hline 131 & 4 & 20 & 0 & 0 & 16 & 12 & 30.65 \\
\hline 132 & 4 & 20 & 2 & 20 & 6 & 16 & 30.90 \\
\hline 133 & 4 & 25 & 0 & 0 & 10 & 12 & 30.93 \\
\hline 134 & 4 & 20 & 2 & 20 & 4 & 20 & 31.40 \\
\hline 135 & 4 & 20 & 4 & 20 & 2 & 20 & 31.40 \\
\hline 136 & 4 & 20 & 0 & 0 & 6 & 20 & 31.40 \\
\hline 137 & 4 & 25 & 4 & 16 & 2 & 16 & 31.68 \\
\hline 138 & 4 & 25 & 2 & 16 & 4 & 16 & 31.68 \\
\hline 139 & 4 & 25 & 0 & 0 & 6 & 16 & 31.68 \\
\hline 140 & 4 & 20 & 4 & 20 & 6 & 12 & 31.90 \\
\hline 141 & 4 & 16 & 6 & 16 & 6 & 16 & 32.15 \\
\hline 142 & 4 & 16 & 4 & 16 & 8 & 16 & 32.15 \\
\hline 143 & 4 & 16 & 2 & 16 & 10 & 16 & 32.15 \\
\hline 144 & 4 & 16 & 0 & 0 & 12 & 16 & 32.15 \\
\hline 145 & 4 & 25 & 4 & 20 & 0 & 0 & 32.19 \\
\hline 146 & 4 & 25 & 2 & 20 & 2 & 20 & 32.19 \\
\hline 147 & 4 & 25 & 0 & 0 & 4 & 20 & 32.19 \\
\hline 148 & 4 & 16 & 4 & 12 & 10 & 16 & 32.66 \\
\hline 149 & 4 & 20 & 0 & 0 & 10 & 16 & 32.66 \\
\hline 150 & 4 & 16 & 0 & 0 & 22 & 12 & 32.91 \\
\hline 151 & 4 & 20 & 0 & 0 & 18 & 12 & 32.91 \\
\hline 152 & 4 & 20 & 4 & 20 & 4 & 16 & 33.16 \\
\hline 153 & 4 & 20 & 4 & 16 & 4 & 20 & 33.16 \\
\hline 154 & 4 & 25 & 0 & 0 & 12 & 12 & 33.19 \\
\hline 155 & 4 & 25 & 2 & 25 & 2 & 16 & 33.46 \\
\hline 156 & 4 & 25 & 2 & 16 & 2 & 25 & 33.46 \\
\hline 157 & 4 & 20 & 2 & 12 & 6 & 20 & 33.66 \\
\hline 158 & 4 & 12 & 0 & 0 & 26 & 12 & 33.91 \\
\hline 159 & 4 & 20 & 4 & 20 & 8 & 12 & 34.16 \\
\hline 160 & 4 & 20 & 2 & 20 & 8 & 16 & 34.92 \\
\hline 161 & 4 & 20 & 2 & 16 & 6 & 20 & 35.42 \\
\hline 162 & 4 & 25 & 4 & 16 & 4 & 16 & 35.70 \\
\hline 163 & 4 & 25 & 0 & 0 & 8 & 16 & 35.70 \\
\hline 164 & 4 & 25 & 2 & 25 & 2 & 20 & 35.72 \\
\hline 165 & 4 & 25 & 2 & 20 & 2 & 25 & 35.72 \\
\hline 166 & 4 & 20 & 4 & 12 & 6 & 20 & 35.92 \\
\hline 167 & 4 & 16 & 4 & 16 & 10 & 16 & 36.17 \\
\hline 168 & 4 & 25 & 2 & 25 & 6 & 12 & 36.22 \\
\hline 169 & 4 & 20 & 4 & 20 & 10 & 12 & 36.42 \\
\hline 170 & 4 & 20 & 6 & 16 & 6 & 16 & 36.68 \\
\hline 171 & 4 & 20 & 0 & 0 & 12 & 16 & 36.68 \\
\hline 172 & 4 & 20 & 4 & 20 & 6 & 16 & 37.18 \\
\hline 173 & 4 & 16 & 0 & 0 & 26 & 12 & 37.43 \\
\hline 174 & 4 & 20 & 0 & 0 & 22 & 12 & 37.43 \\
\hline 175 & 4 & 25 & 2 & 25 & 4 & 16 & 37.48 \\
\hline 176 & 4 & 25 & 4 & 16 & 2 & 25 & 37.48 \\
\hline 177 & 4 & 20 & 4 & 20 & 4 & 20 & 37.68 \\
\hline 178 & 4 & 20 & 2 & 20 & 6 & 20 & 37.68 \\
\hline 179 & 4 & 20 & 0 & 0 & 8 & 20 & 37.68 \\
\hline 180 & 4 & 25 & 0 & 0 & 16 & 12 & 37.71 \\
\hline
\end{tabular}




\begin{tabular}{|c|c|c|c|c|c|c|c|}
\hline \multirow{2}{*}{ Número } & \multicolumn{2}{|c|}{ ESQUINAS } & \multicolumn{2}{|c|}{ CARA "a" } & \multicolumn{2}{|c|}{ CARA "b" } & \multirow{2}{*}{ As } \\
\hline & $\mathbf{n}$ & $\phi$ & $\mathbf{n}_{\text {ref, TOTAL }}$ & $\phi$ & $\mathbf{n}_{\text {ref, TOTAL }}$ & $\phi$ & \\
\hline 181 & 4 & 20 & 6 & 20 & 6 & 12 & 38.18 \\
\hline 182 & 4 & 20 & 6 & 12 & 6 & 20 & 38.18 \\
\hline 183 & 4 & 12 & 0 & 0 & 30 & 12 & 38.43 \\
\hline 184 & 4 & 25 & 4 & 20 & 2 & 20 & 38.47 \\
\hline 185 & 4 & 25 & 2 & 20 & 4 & 20 & 38.47 \\
\hline 186 & 4 & 25 & 0 & 0 & 6 & 20 & 38.47 \\
\hline 187 & 4 & 25 & 2 & 25 & 8 & 12 & 38.48 \\
\hline 188 & 4 & 20 & 2 & 20 & 10 & 16 & 38.94 \\
\hline 189 & 4 & 25 & 4 & 25 & 0 & 0 & 39.25 \\
\hline 190 & 4 & 25 & 2 & 25 & 2 & 25 & 39.25 \\
\hline 191 & 4 & 25 & 0 & 0 & 4 & 25 & 39.25 \\
\hline 192 & 4 & 20 & 4 & 16 & 6 & 20 & 39.44 \\
\hline 193 & 4 & 25 & 0 & 0 & 10 & 16 & 39.72 \\
\hline 194 & 4 & 20 & 2 & 12 & 8 & 20 & 39.94 \\
\hline 195 & 4 & 25 & 0 & 0 & 18 & 12 & 39.97 \\
\hline 196 & 4 & 16 & 0 & 0 & 16 & 16 & 40.19 \\
\hline 197 & 4 & 12 & 0 & 0 & 32 & 12 & 40.69 \\
\hline 198 & 4 & 25 & 2 & 25 & 10 & 12 & 40.74 \\
\hline 199 & 4 & 20 & 4 & 20 & 8 & 16 & 41.20 \\
\hline 200 & 4 & 25 & 2 & 25 & 6 & 16 & 41.50 \\
\hline 201 & 4 & 20 & 2 & 16 & 8 & 20 & 41.70 \\
\hline 202 & 4 & 16 & 0 & 0 & 30 & 12 & 41.95 \\
\hline 203 & 4 & 20 & 0 & 0 & 26 & 12 & 41.95 \\
\hline 204 & 4 & 25 & 4 & 20 & 2 & 25 & 42.00 \\
\hline 205 & 4 & 25 & 2 & 25 & 4 & 20 & 42.00 \\
\hline 206 & 4 & 20 & 4 & 12 & 8 & 20 & 42.20 \\
\hline 207 & 4 & 25 & 2 & 16 & 4 & 25 & 43.27 \\
\hline 208 & 4 & 25 & 4 & 25 & 2 & 16 & 43.27 \\
\hline 209 & 4 & 20 & 6 & 20 & 6 & 16 & 43.46 \\
\hline 210 & 4 & 20 & 6 & 16 & 6 & 20 & 43.46 \\
\hline 211 & 4 & 25 & 6 & 16 & 6 & 16 & 43.74 \\
\hline 212 & 4 & 25 & 0 & 0 & 12 & 16 & 43.74 \\
\hline 213 & 4 & 25 & 4 & 25 & 4 & 12 & 43.77 \\
\hline 214 & 4 & 20 & 4 & 20 & 6 & 20 & 43.96 \\
\hline 215 & 4 & 20 & 2 & 20 & 8 & 20 & 43.96 \\
\hline 216 & 4 & 20 & 0 & 0 & 10 & 20 & 43.96 \\
\hline 217 & 4 & 16 & 0 & 0 & 18 & 16 & 44.21 \\
\hline 218 & 4 & 16 & 0 & 0 & 32 & 12 & 44.21 \\
\hline 219 & 4 & 25 & 0 & 0 & 22 & 12 & 44.49 \\
\hline 220 & 4 & 20 & 0 & 0 & 16 & 16 & 44.71 \\
\hline 221 & 4 & 25 & 4 & 20 & 4 & 20 & 44.75 \\
\hline 222 & 4 & 25 & 0 & 0 & 8 & 20 & 44.75 \\
\hline 223 & 4 & 25 & 2 & 20 & 6 & 20 & 44.75 \\
\hline 224 & 4 & 20 & 4 & 20 & 10 & 16 & 45.22 \\
\hline 225 & 4 & 25 & 2 & 25 & 8 & 16 & 45.51 \\
\hline 226 & 4 & 25 & 4 & 25 & 2 & 20 & 45.53 \\
\hline 227 & 4 & 25 & 2 & 20 & 4 & 25 & 45.53 \\
\hline 228 & 4 & 20 & 4 & 16 & 8 & 20 & 45.72 \\
\hline 229 & 4 & 25 & 4 & 25 & 6 & 12 & 46.03 \\
\hline 230 & 4 & 20 & 2 & 12 & 10 & 20 & 46.22 \\
\hline
\end{tabular}




\begin{tabular}{|c|c|c|c|c|c|c|c|}
\hline \multirow{2}{*}{ Número } & \multicolumn{2}{|c|}{ ESQUINAS } & \multicolumn{2}{|c|}{ CARA "a" } & \multicolumn{2}{|c|}{ CARA "b" } & \multirow{2}{*}{ As } \\
\hline & $\mathbf{n}$ & $\phi$ & $\mathbf{n}_{\text {ref, TOTAL }}$ & $\phi$ & $\mathbf{n}_{\text {ref, TOTA }}$ & $\phi$ & \\
\hline 231 & 4 & 20 & 0 & 0 & 30 & 12 & 46.47 \\
\hline 232 & 4 & 25 & 4 & 25 & 4 & 16 & 47.29 \\
\hline 233 & 4 & 25 & 4 & 16 & 4 & 25 & 47.29 \\
\hline 234 & 4 & 20 & 2 & 16 & 10 & 20 & 47.98 \\
\hline 235 & 4 & 25 & 2 & 25 & 6 & 20 & 48.28 \\
\hline 236 & 4 & 25 & 4 & 25 & 8 & 12 & 48.29 \\
\hline 237 & 4 & 20 & 4 & 12 & 10 & 20 & 48.48 \\
\hline 238 & 4 & 20 & 0 & 0 & 18 & 16 & 48.73 \\
\hline 239 & 4 & 20 & 0 & 0 & 32 & 12 & 48.73 \\
\hline 240 & 4 & 25 & 0 & 0 & 26 & 12 & 49.02 \\
\hline 241 & 4 & 25 & 4 & 25 & 2 & 25 & 49.06 \\
\hline 242 & 4 & 25 & 2 & 25 & 4 & 25 & 49.06 \\
\hline 243 & 4 & 25 & 0 & 0 & 6 & 25 & 49.06 \\
\hline 244 & 4 & 25 & 2 & 25 & 10 & 16 & 49.53 \\
\hline 245 & 4 & 20 & 4 & 20 & 8 & 20 & 50.24 \\
\hline 246 & 4 & 20 & 2 & 20 & 10 & 20 & 50.24 \\
\hline 247 & 4 & 20 & 0 & 0 & 12 & 20 & 50.24 \\
\hline 248 & 4 & 20 & 6 & 20 & 6 & 20 & 50.24 \\
\hline 249 & 4 & 25 & 4 & 25 & 10 & 12 & 50.55 \\
\hline 250 & 4 & 25 & 2 & 20 & 8 & 20 & 51.03 \\
\hline 251 & 4 & 25 & 0 & 0 & 10 & 20 & 51.03 \\
\hline 252 & 4 & 25 & 4 & 25 & 6 & 16 & 51.31 \\
\hline 253 & 4 & 25 & 0 & 0 & 16 & 16 & 51.78 \\
\hline 254 & 4 & 25 & 4 & 25 & 4 & 20 & 51.81 \\
\hline 255 & 4 & 25 & 4 & 20 & 4 & 25 & 51.81 \\
\hline 256 & 4 & 20 & 4 & 16 & 10 & 20 & 52.00 \\
\hline 257 & 4 & 16 & 0 & 0 & 22 & 16 & 52.25 \\
\hline 258 & 4 & 25 & 2 & 16 & 6 & 25 & 53.08 \\
\hline 259 & 4 & 25 & 0 & 0 & 30 & 12 & 53.54 \\
\hline 260 & 4 & 25 & 4 & 12 & 6 & 25 & 53.58 \\
\hline 261 & 4 & 25 & 2 & 25 & 8 & 20 & 54.56 \\
\hline 262 & 4 & 25 & 4 & 25 & 8 & 16 & 55.33 \\
\hline 263 & 4 & 25 & 2 & 20 & 6 & 25 & 55.34 \\
\hline 264 & 4 & 25 & 0 & 0 & 18 & 16 & 55.80 \\
\hline 265 & 4 & 25 & 0 & 0 & 32 & 12 & 55.80 \\
\hline 266 & 4 & 25 & 6 & 25 & 6 & 12 & 55.84 \\
\hline 267 & 4 & 20 & 4 & 20 & 10 & 20 & 56.52 \\
\hline 268 & 4 & 20 & 0 & 0 & 22 & 16 & 56.77 \\
\hline 269 & 4 & 25 & 4 & 16 & 6 & 25 & 57.10 \\
\hline 270 & 4 & 25 & 2 & 20 & 10 & 20 & 57.31 \\
\hline 271 & 4 & 25 & 6 & 20 & 6 & 20 & 57.31 \\
\hline 272 & 4 & 25 & 0 & 0 & 12 & 20 & 57.31 \\
\hline 273 & 4 & 25 & 4 & 25 & 6 & 20 & 58.09 \\
\hline 274 & 4 & 25 & 4 & 25 & 4 & 25 & 58.88 \\
\hline 275 & 4 & 25 & 2 & 25 & 6 & 25 & 58.88 \\
\hline 276 & 4 & 25 & 0 & 0 & 8 & 25 & 58.88 \\
\hline 277 & 4 & 25 & 4 & 25 & 10 & 16 & 59.35 \\
\hline 278 & 4 & 16 & 0 & 0 & 26 & 16 & 60.29 \\
\hline 279 & 4 & 25 & 2 & 25 & 10 & 20 & 60.84 \\
\hline 280 & 4 & 25 & 6 & 25 & 6 & 16 & 61.12 \\
\hline
\end{tabular}




\begin{tabular}{|c|c|c|c|c|c|c|c|}
\hline \multirow{2}{*}{ Número } & \multicolumn{2}{|c|}{ ESQUINAS } & \multicolumn{2}{|c|}{ CARA "a" } & \multicolumn{2}{|c|}{ CARA "b" } & \multirow{2}{*}{ As } \\
\hline & $\mathbf{n}$ & $\phi$ & $\mathbf{n}_{\text {ref, TOTAL }}$ & $\phi$ & $\mathbf{n}_{\text {ref, TOTAL }}$ & $\phi$ & \\
\hline 281 & 4 & 25 & 6 & 16 & 6 & 25 & 61.12 \\
\hline 282 & 4 & 25 & 4 & 20 & 6 & 25 & 61.62 \\
\hline 283 & 4 & 20 & 0 & 0 & 16 & 20 & 62.80 \\
\hline 284 & 4 & 25 & 2 & 16 & 8 & 25 & 62.89 \\
\hline 285 & 4 & 25 & 4 & 12 & 8 & 25 & 63.40 \\
\hline 286 & 4 & 25 & 0 & 0 & 22 & 16 & 63.84 \\
\hline 287 & 4 & 25 & 4 & 25 & 8 & 20 & 64.37 \\
\hline 288 & 4 & 20 & 0 & 0 & 26 & 16 & 64.81 \\
\hline 289 & 4 & 25 & 2 & 20 & 8 & 25 & 65.16 \\
\hline 290 & 4 & 25 & 4 & 16 & 8 & 25 & 66.91 \\
\hline 291 & 4 & 25 & 6 & 25 & 6 & 20 & 67.90 \\
\hline 292 & 4 & 25 & 6 & 20 & 6 & 25 & 67.90 \\
\hline 293 & 4 & 16 & 0 & 0 & 30 & 16 & 68.33 \\
\hline 294 & 4 & 25 & 4 & 25 & 6 & 25 & 68.69 \\
\hline 295 & 4 & 25 & 2 & 25 & 8 & 25 & 68.69 \\
\hline 296 & 4 & 25 & 0 & 0 & 10 & 25 & 68.69 \\
\hline 297 & 4 & 20 & 0 & 0 & 18 & 20 & 69.08 \\
\hline 298 & 4 & 25 & 0 & 0 & 16 & 20 & 69.87 \\
\hline 299 & 4 & 25 & 4 & 25 & 10 & 20 & 70.65 \\
\hline 300 & 4 & 25 & 4 & 20 & 8 & 25 & 71.44 \\
\hline 301 & 4 & 25 & 0 & 0 & 26 & 16 & 71.87 \\
\hline 302 & 4 & 16 & 0 & 0 & 32 & 16 & 72.35 \\
\hline 303 & 4 & 25 & 2 & 16 & 10 & 25 & 72.71 \\
\hline 304 & 4 & 20 & 0 & 0 & 30 & 16 & 72.85 \\
\hline 305 & 4 & 25 & 4 & 12 & 10 & 25 & 73.21 \\
\hline 306 & 4 & 25 & 2 & 20 & 10 & 25 & 74.97 \\
\hline 307 & 4 & 25 & 0 & 0 & 18 & 20 & 76.15 \\
\hline 308 & 4 & 25 & 4 & 16 & 10 & 25 & 76.73 \\
\hline 309 & 4 & 20 & 0 & 0 & 32 & 16 & 76.87 \\
\hline 310 & 4 & 25 & 6 & 25 & 6 & 25 & 78.50 \\
\hline 311 & 4 & 25 & 4 & 25 & 8 & 25 & 78.50 \\
\hline 312 & 4 & 25 & 2 & 25 & 10 & 25 & 78.50 \\
\hline 313 & 4 & 25 & 0 & 0 & 12 & 25 & 78.50 \\
\hline 314 & 4 & 25 & 0 & 0 & 30 & 16 & 79.91 \\
\hline 315 & 4 & 25 & 4 & 20 & 10 & 25 & 81.25 \\
\hline 316 & 4 & 20 & 0 & 0 & 22 & 20 & 81.64 \\
\hline 317 & 4 & 25 & 0 & 0 & 32 & 16 & 83.93 \\
\hline 318 & 4 & 25 & 4 & 25 & 10 & 25 & 88.31 \\
\hline 319 & 4 & 25 & 0 & 0 & 22 & 20 & 88.71 \\
\hline 320 & 4 & 20 & 0 & 0 & 26 & 20 & 94.20 \\
\hline 321 & 4 & 25 & 0 & 0 & 16 & 25 & 98.13 \\
\hline 322 & 4 & 25 & 0 & 0 & 26 & 20 & 101.27 \\
\hline 323 & 4 & 20 & 0 & 0 & 30 & 20 & 106.76 \\
\hline 324 & 4 & 25 & 0 & 0 & 18 & 25 & 107.94 \\
\hline 325 & 4 & 20 & 0 & 0 & 32 & 20 & 113.04 \\
\hline 326 & 4 & 25 & 0 & 0 & 30 & 20 & 113.83 \\
\hline 327 & 4 & 25 & 0 & 0 & 32 & 20 & 120.11 \\
\hline 328 & 4 & 25 & 0 & 0 & 22 & 25 & 127.56 \\
\hline 329 & 4 & 25 & 0 & 0 & 26 & 25 & 147.19 \\
\hline 330 & 4 & 25 & 0 & 0 & 30 & 25 & 166.81 \\
\hline
\end{tabular}




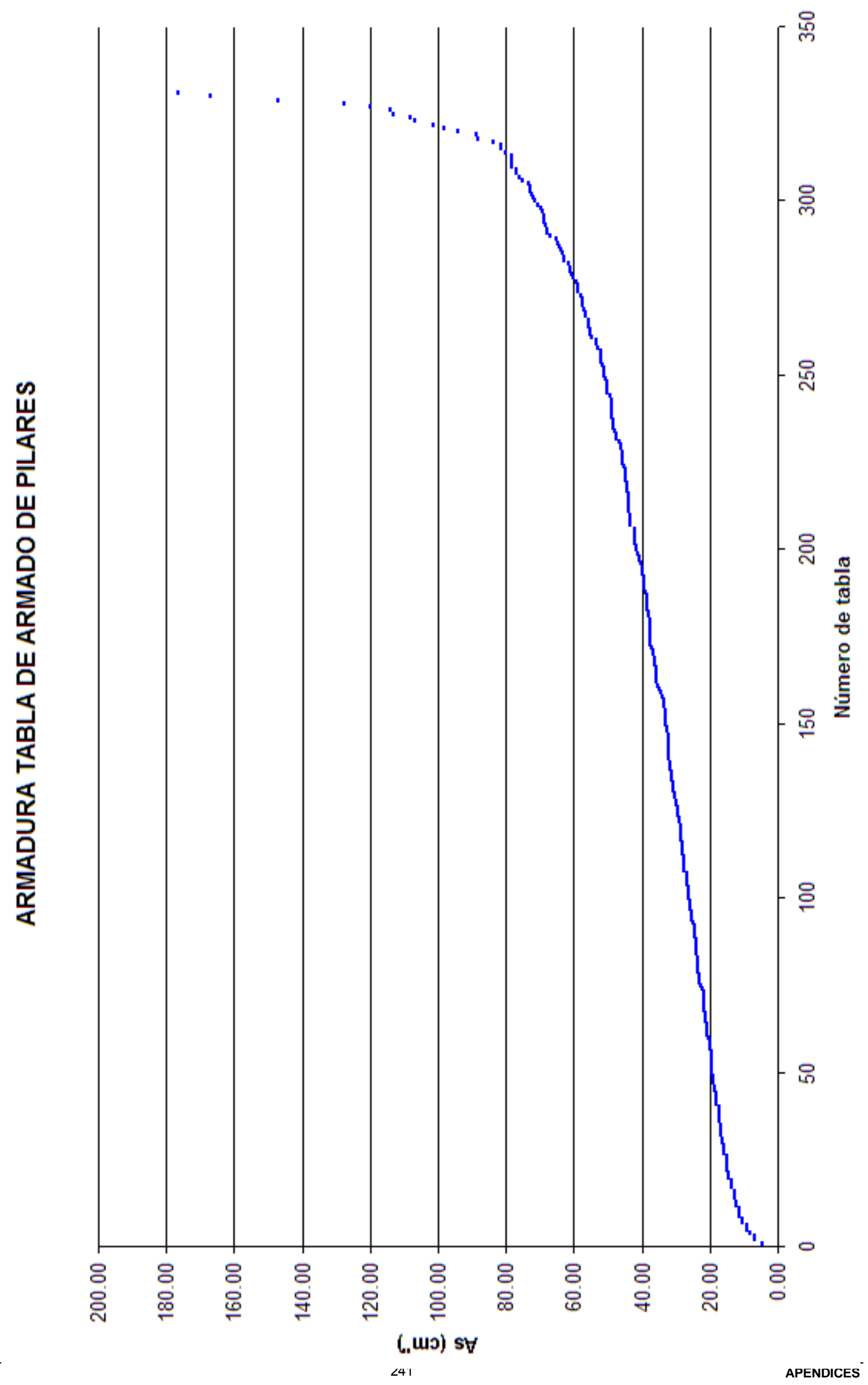




\section{Armadura transversal en pilares.}

Tabla "Armadura Transv. Pilares".

\begin{tabular}{|c|c|c|c|c|}
\hline $\begin{array}{l}\text { Núm. } \\
\text { Caso }\end{array}$ & $\begin{array}{c}\text { Diámetro } \\
\phi(\mathrm{mm})\end{array}$ & $\begin{array}{c}\text { Separación } \\
\text { transversal } \\
S_{t}(\mathrm{~mm})\end{array}$ & $\begin{array}{l}\text { Número de } \\
\text { ramas } \\
\text { adosadas }\end{array}$ & $\begin{array}{l}\text { At }\left(\mathrm{cm}^{2} / \mathrm{m}\right. \\
\text { rama) }\end{array}$ \\
\hline 1 & 6 & 0.3 & 1 & 0.94 \\
\hline 2 & 6 & 0.25 & 1 & 1.13 \\
\hline 3 & 6 & 0.2 & 1 & 1.41 \\
\hline 4 & 8 & 0.3 & 1 & 1.68 \\
\hline 5 & 6 & 0.15 & 1 & 1.88 \\
\hline 6 & 8 & 0.25 & 1 & 2.01 \\
\hline 7 & 8 & 0.2 & 1 & 2.51 \\
\hline 8 & 10 & 0.3 & 1 & 2.62 \\
\hline 9 & 10 & 0.25 & 1 & 3.14 \\
\hline 10 & 8 & 0.15 & 1 & 3.35 \\
\hline 11 & 12 & 0.3 & 1 & 3.77 \\
\hline 12 & 10 & 0.2 & 1 & 3.93 \\
\hline 13 & 12 & 0.25 & 1 & 4.52 \\
\hline 14 & 10 & 0.15 & 1 & 5.24 \\
\hline 15 & 12 & 0.2 & 1 & 5.65 \\
\hline 16 & 12 & 0.15 & 1 & 7.54 \\
\hline 17 & 10 & 0.2 & 2 & 7.85 \\
\hline 18 & 12 & 0.25 & 2 & 9.05 \\
\hline 19 & 10 & 0.15 & 2 & 10.47 \\
\hline 20 & 12 & 0.2 & 2 & 11.31 \\
\hline 21 & 12 & 0.15 & 2 & 15.08 \\
\hline
\end{tabular}

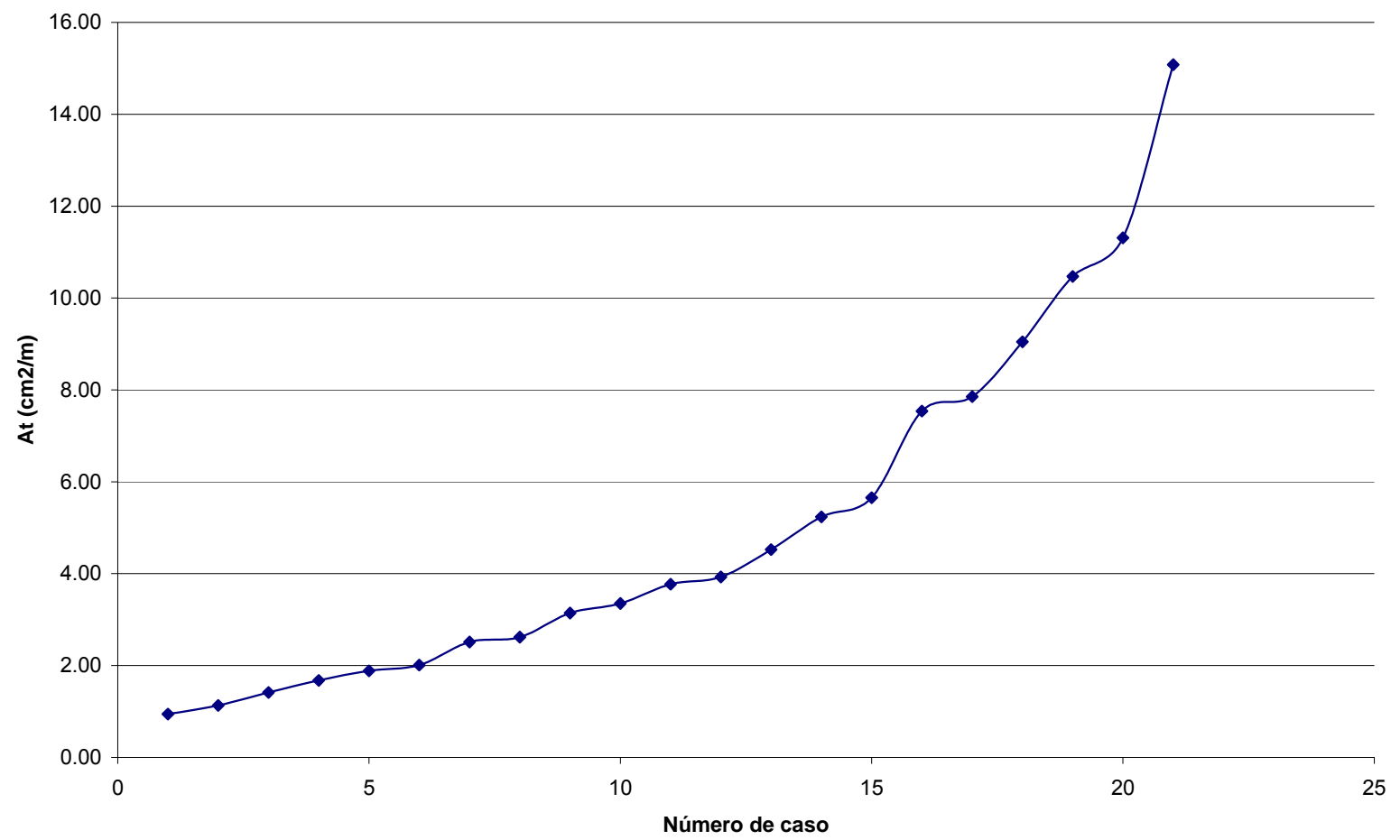




\section{Tabla "Armado Estribos Pil".}

El área total de armadura transversal depende del número de ramas de cortante que tenga el pilar. Este número depende de las dimensiones del pilar y del número de armaduras longitudinales que haya en cada cara, calculándose de modo que se cumplan las prescripciones del Artículo 42.3 de la EHE relativas a la separación máxima entre dos armaduras longitudinales consecutivas no cogidas mediante cercos estribos.

Se proporcionan a continuación los casos posibles de disposición de la armadura transversal del pilar en función del número y disposición de su armadura longtudinal. 
TABS DBAMET ALN.

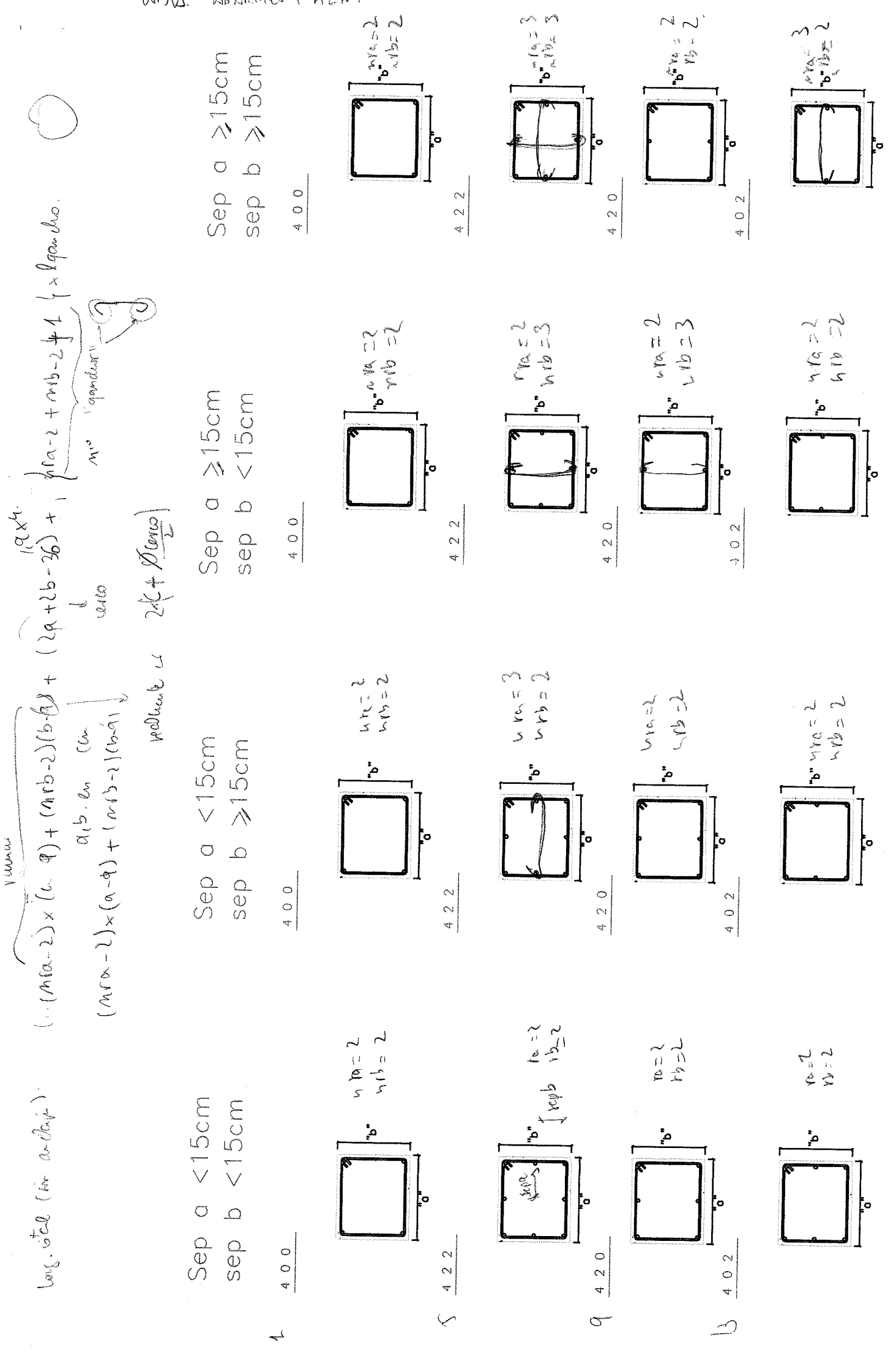



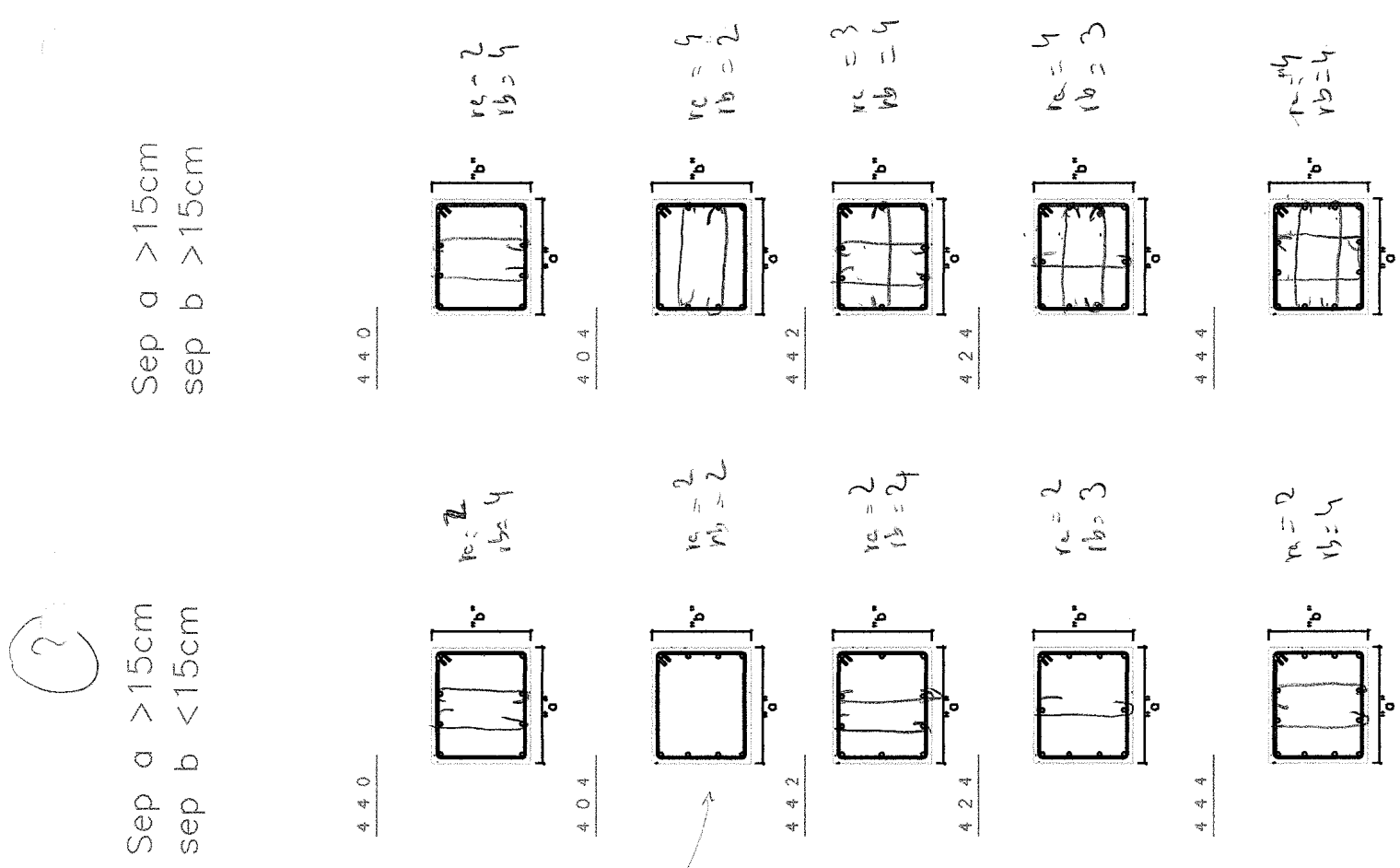

$\begin{array}{ll}E & E \\ 0 & 0 \\ 0 & \omega \\ V & A \\ 0 & 0 \\ 0 & 0 \\ 0 & 0\end{array}$
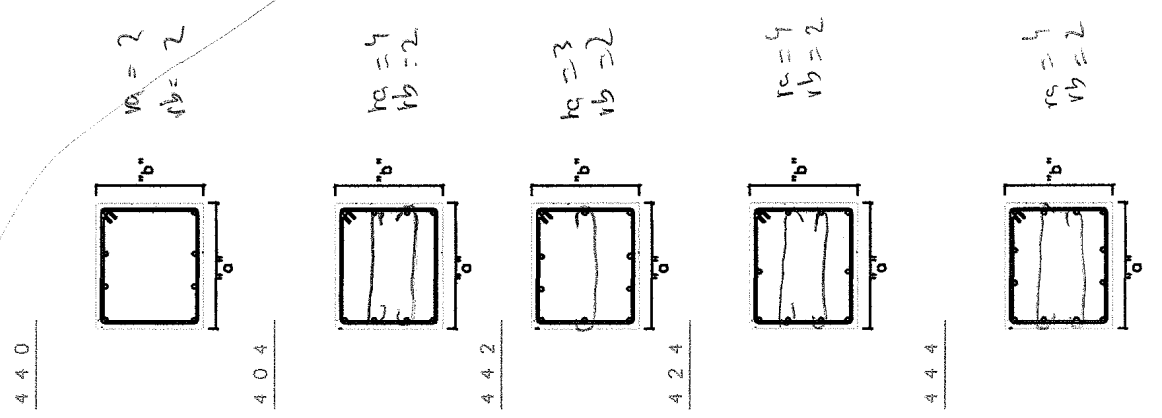

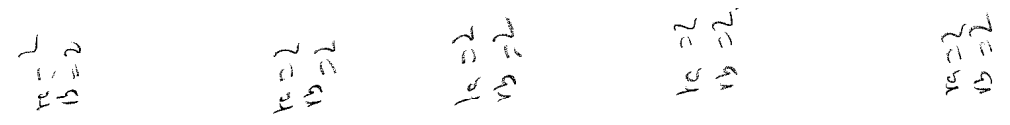
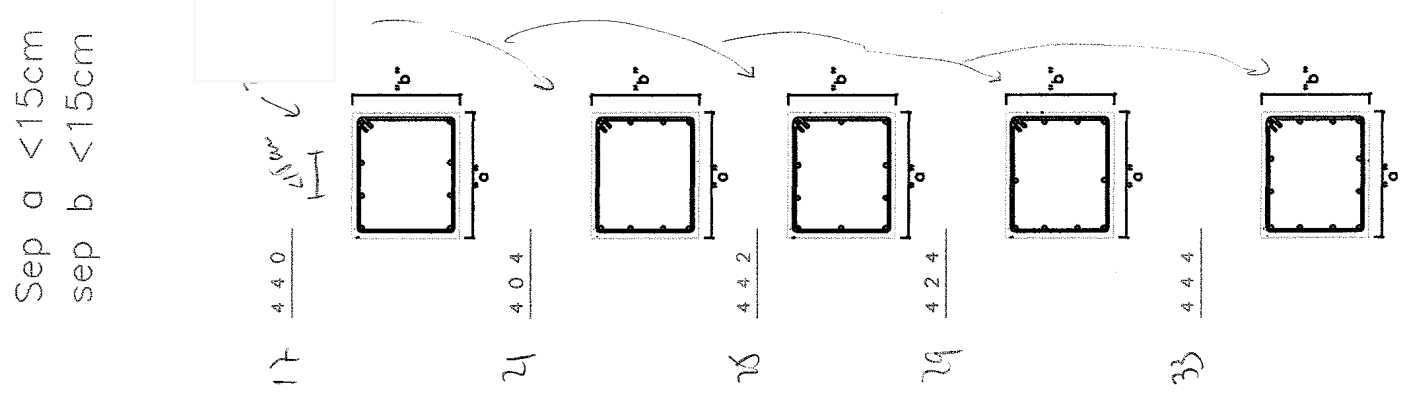

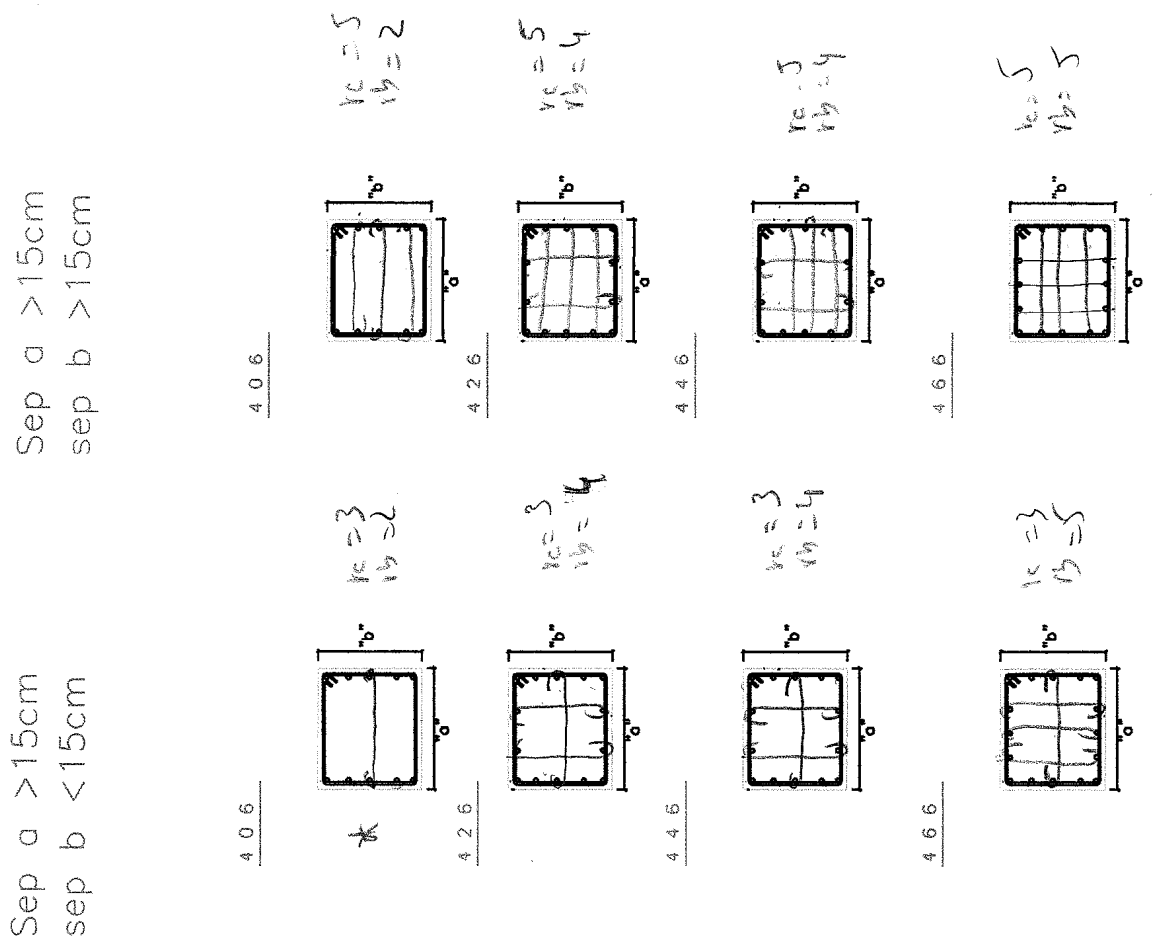

$\pi n$

$\pm 0$

$\begin{array}{lll}E & E \\ \vdots & \\ \frac{n}{V} & \\ 0 & 0 \\ 0 & 0 \\ 0 & 0 \\ \cdots & 0\end{array}$

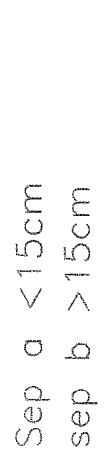

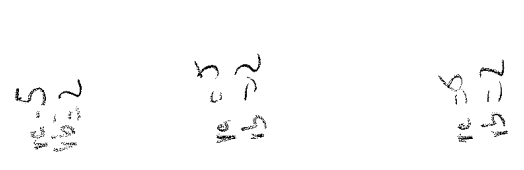$$
\eta n
$$$$
+3
$$

$\begin{array}{ll}\frac{8}{0} & \frac{E}{U} \\ 0 & 0 \\ 7 & 0 \\ 0 & 0 \\ 0 & 0 \\ 0 & 0 \\ 0 & 0\end{array}$
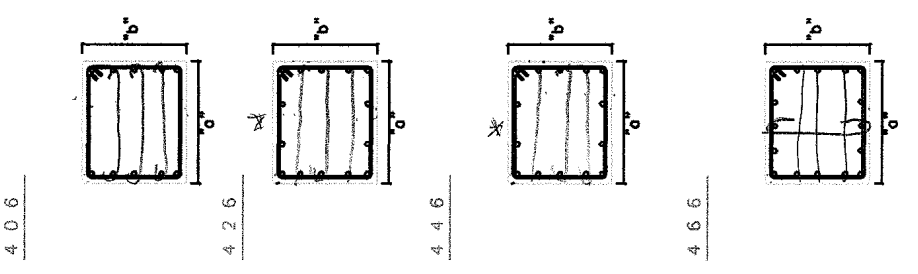

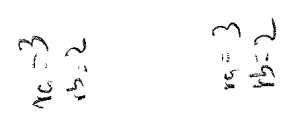

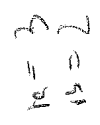

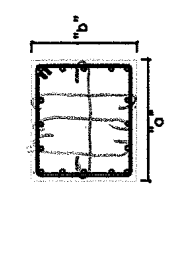

0
6
4

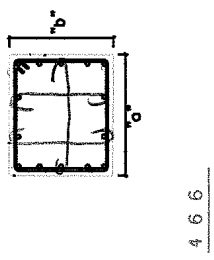

$\begin{array}{ll}E & E \\ 0 & 0 \\ \frac{1}{2} & \bar{V} \\ V & 0 \\ 0 & 0 \\ 0 & 0 \\ 0 & 0\end{array}$

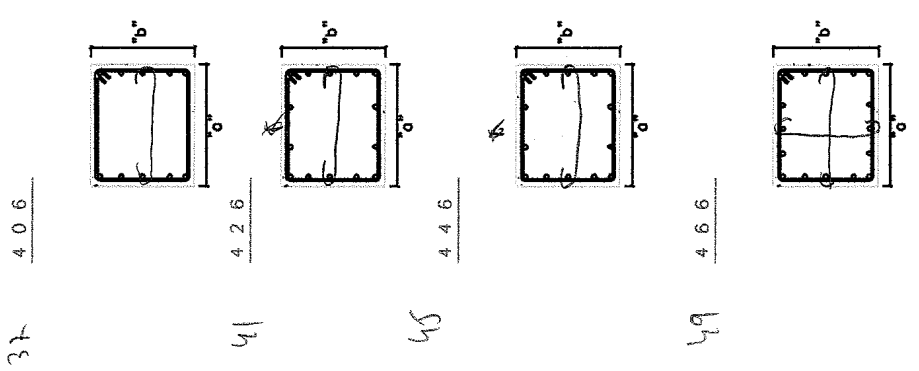



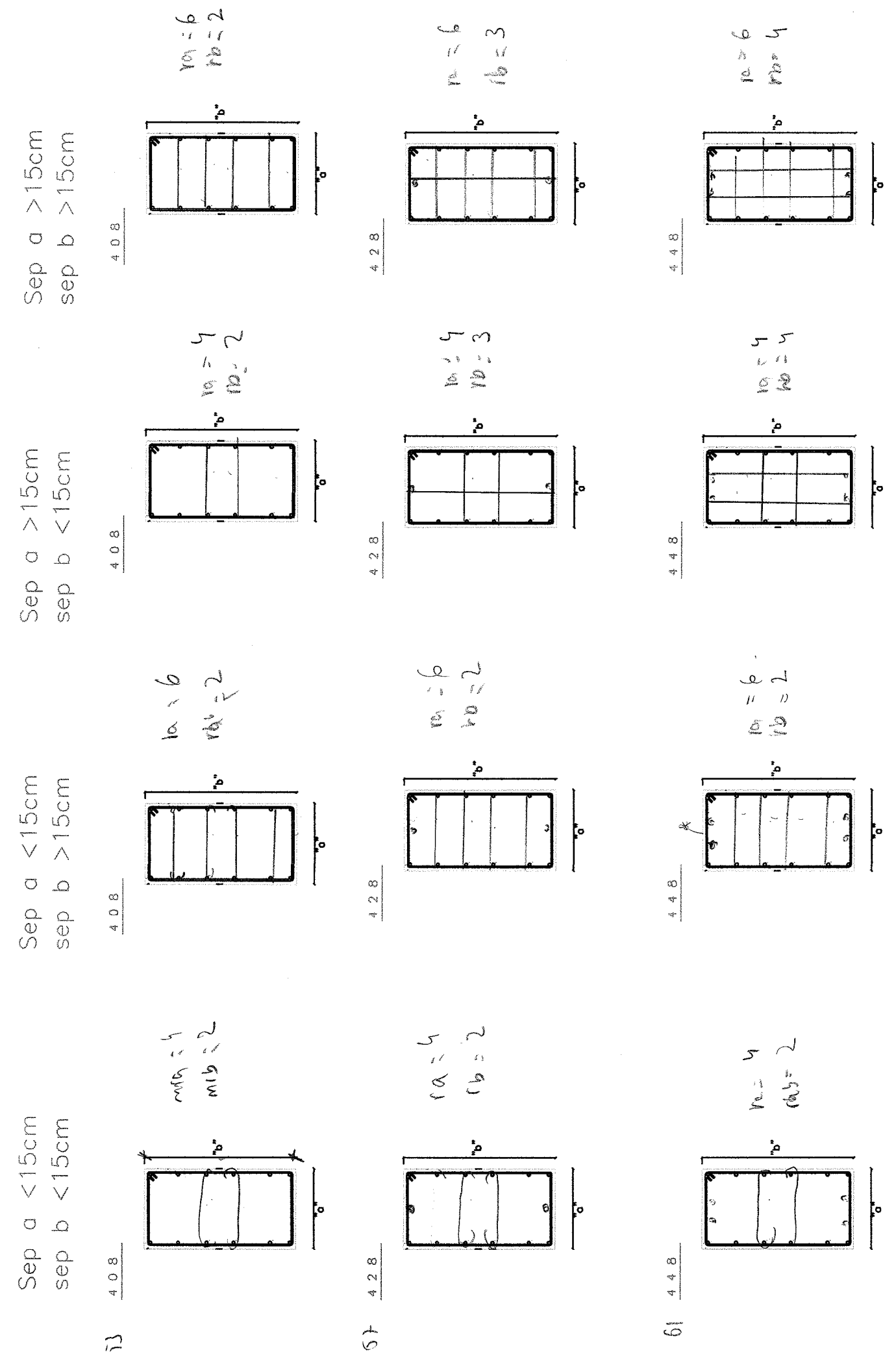

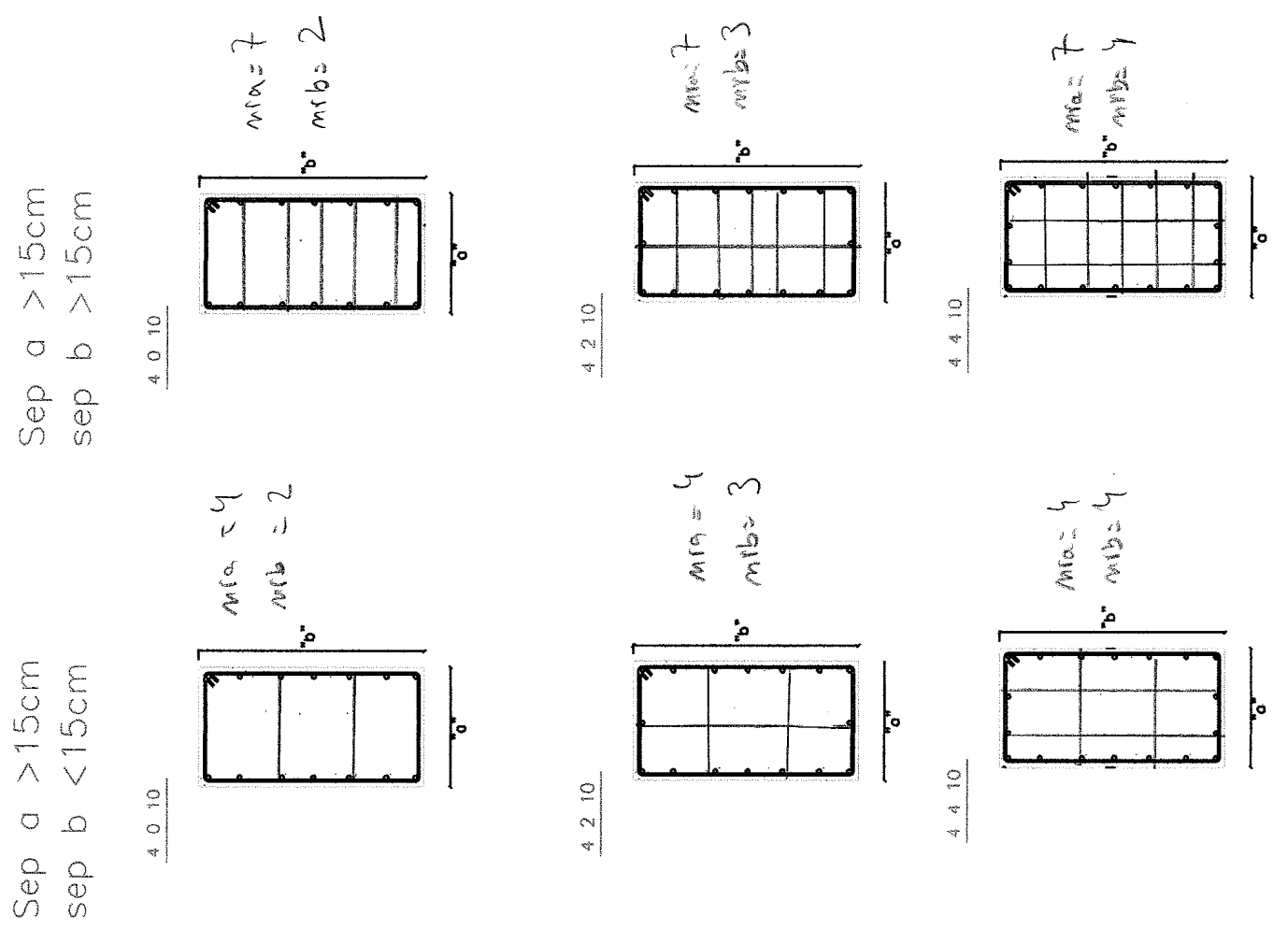

5
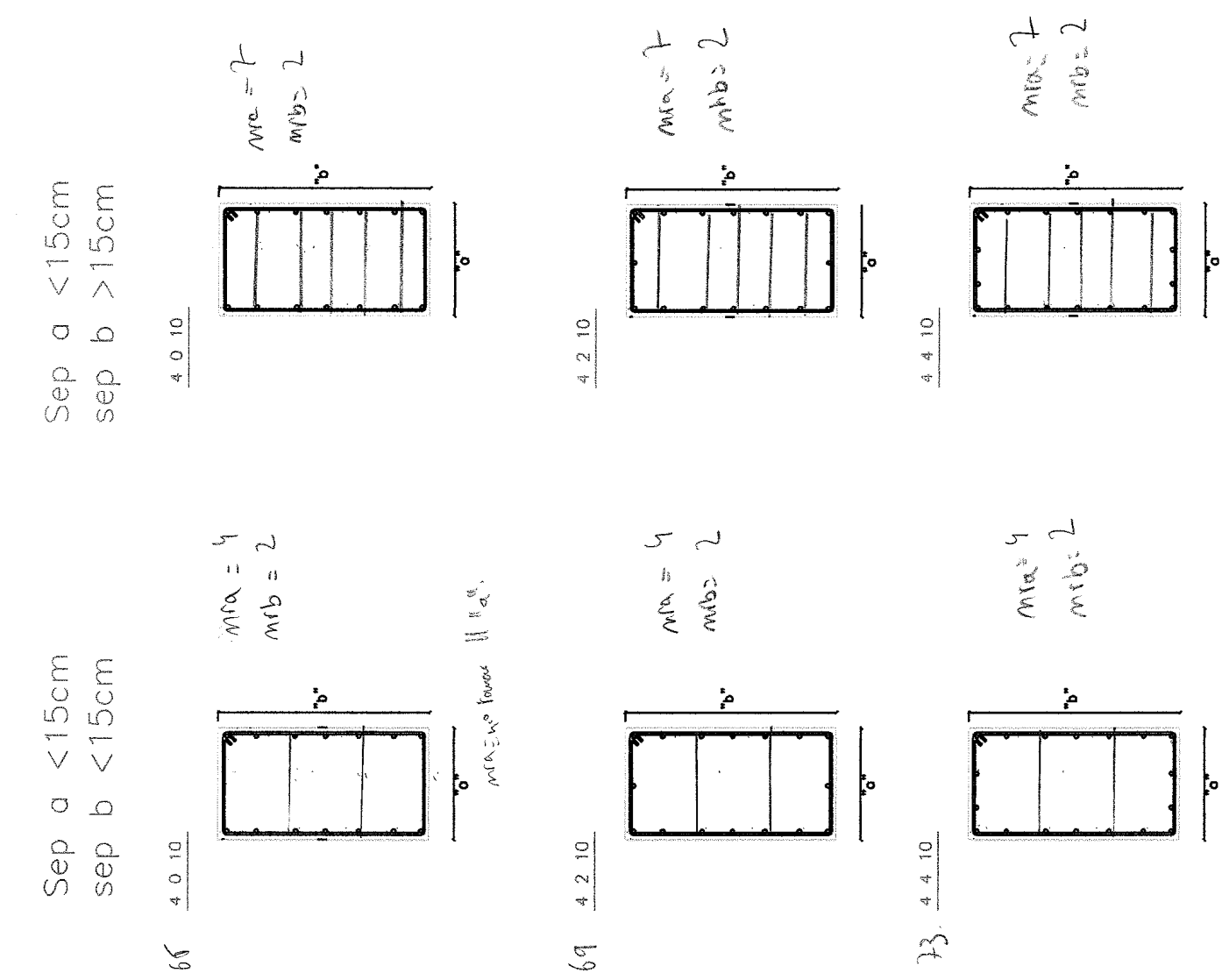
D
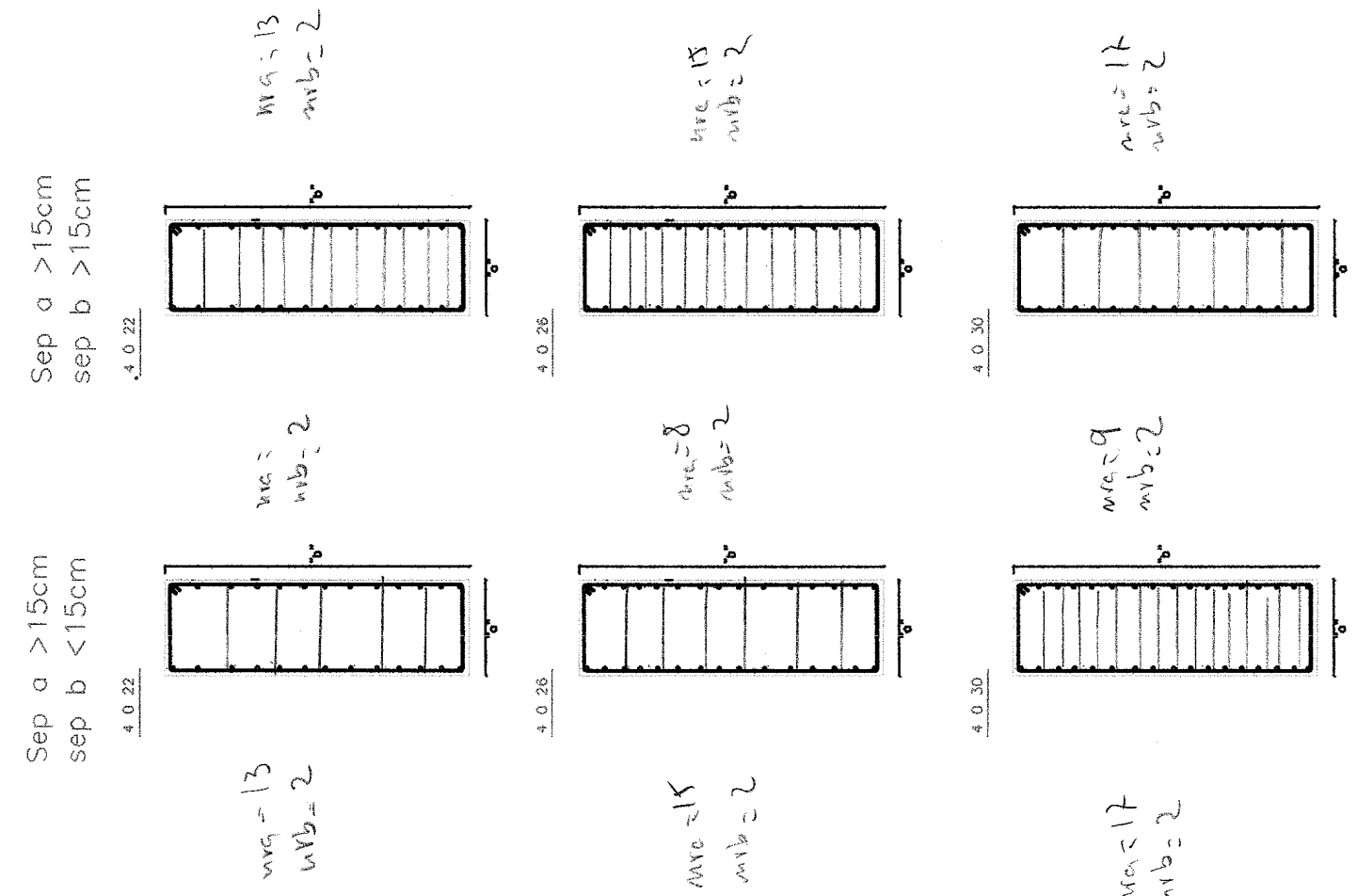

$$
\frac{m}{4}
$$
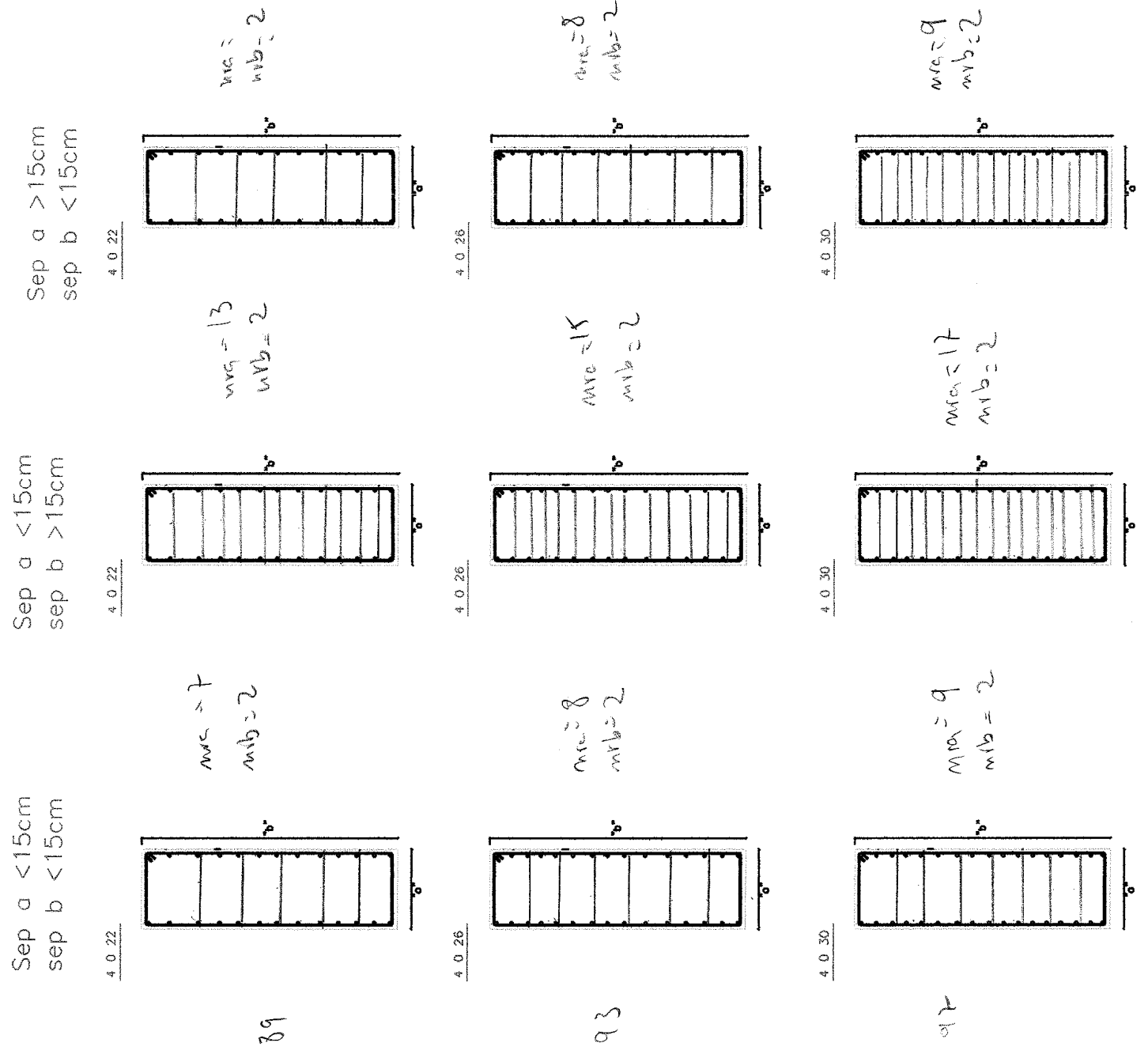

:

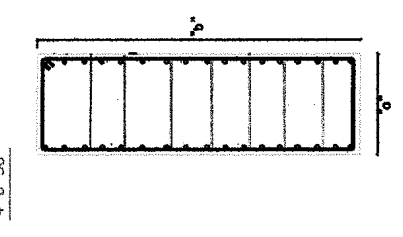

$\frac{1}{3}$ 
$\infty$

$\infty^{-5}$

4

3

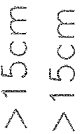

○。

웅웅

a)

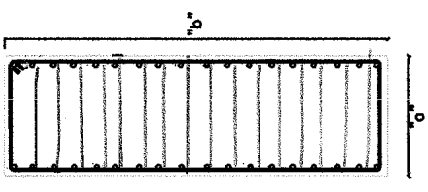

요

ON

:

$\begin{array}{ll}E & E \\ 0 & 0 \\ \triangle & \end{array}$

0.0

(1) 0

인.

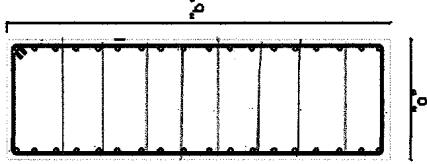

$: 1$

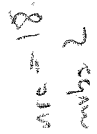

$\begin{array}{ll}E & E \\ 0 & 0 \\ V & A \\ 0 & 0 \\ 0 & 0 \\ 0 & 0\end{array}$

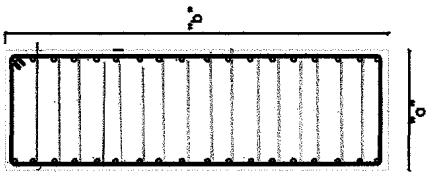

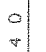

$$
\begin{aligned}
& Q \\
& i \\
& \frac{j}{3}
\end{aligned}
$$

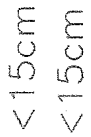

$0 \Omega$

(4)

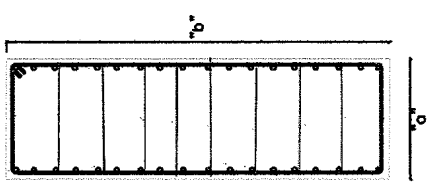

ō 


\section{APÉNDICE 2. MEDICIONES DEL PÓRTICO TIPO.}


Página intencionadamente en blanco. 


\section{Introducción.}

Se detalla en este Apéndice cómo se obtienen las mediciones de las unidades de obra que permiten valorar la construcción de una viga y de un pilar. Para obtener las mediciones del conjunto de la estructura basta con sumar las mediciones de cada unidad de obra en todos y cada uno de sus vigas y pilares.

Las tablas A.2.1 y A.2.2 muestran la nomenclatura empleada en este Apéndice.

Tabla A.2.1. Nomenclatura empleada. Vigas.

\begin{tabular}{|c|c|}
\hline Descripción & Abreviatura \\
\hline Luz de la viga "i” (m) & $L_{v, i}$ \\
\hline Ancho de la viga "i" (m) & $b_{\text {vig,i }}$ \\
\hline Canto de la viga “i” (m) & $\mathrm{h}_{\text {vig,i }}$ \\
\hline Canto del forjado (m) & $\mathrm{h}_{\text {for }}$ \\
\hline Armadura base inferior en la viga "i" $\left(\mathrm{cm}^{2} / \mathrm{m}\right)$ & $A_{s, \text { base inf,vig,i }}$ \\
\hline Armadura base superior en la viga “i” $\left(\mathrm{cm}^{2} / \mathrm{m}\right)$ & $A_{s, \text { base sup,vig,i }}$ \\
\hline Armadura de piel en la viga “i” $\left(\mathrm{cm}^{2} / \mathrm{m}\right)$ & $A_{s, p i e l, v i g, i}$ \\
\hline Armadura de refuerzo inferior en la viga “i” $\left(\mathrm{cm}^{2} / \mathrm{m}\right)$ & $A_{s, \text { ref inf,vig,i }}$ \\
\hline Armadura de refuerzo superior en el extremo izquierdo de la viga "i”" $\left(\mathrm{cm}^{2} / \mathrm{m}\right)$ & $\mathrm{A}_{\mathrm{s}, \text { ref sup,vig, i, izqda }}$ \\
\hline Armadura de refuerzo superior en el extremo derecho de la viga "i” $\left(\mathrm{cm}^{2} / \mathrm{m}\right)$ & $A_{s, \text { ref sup,vig,l,der }}$ \\
\hline Armadura de cortante en el tercio izquierdo de la viga "i" & $A_{\text {st,cor,vig, }, \text { i,izqda }}$ \\
\hline Armadura de cortante en el tercio central de la viga "i" & $A_{\text {st,cor,vig,i,centro }}$ \\
\hline Armadura de cortante en el tercio derecho de la viga "i” & $\mathrm{A}_{\mathrm{st}, \mathrm{cor}, \mathrm{vig}, \mathrm{i}, \mathrm{cender}}$ \\
\hline
\end{tabular}


Tabla A.2.2. Nomenclatura empleada. Pilares.

\section{Descripción}

Altura de la planta en la que se encuentra el pilar "i" (m)

Ancho del pilar "i" (m)

Canto de pilar "i" (m)

Armadura longitudinal del pilar "i" $\left(\mathrm{cm}^{2} / \mathrm{m}\right)$

Armadura transversal del pilar "i” $\left(\mathrm{cm}^{2} / \mathrm{m}\right)$
Abreviatura

$\mathrm{H}_{\mathrm{p}, \mathrm{i}}$

$b_{\text {pil,i }}$

$\mathrm{h}_{\text {pil,i }}$

$A_{s, \text { lon, pil,i }}$

$A_{\text {st,cor,pil,i }}$

\section{Mediciones de una viga.}

1) Cimbrado. Esta unidad se mide por metros cuadrados $\left(\mathrm{m}^{2}\right)$ de superficie horizontal del elemento a cimbrar. Por tanto, la medición para la viga "i", es:

$$
M_{C I M B, V I G, i}=L_{v, i} \times b_{v i g, i}
$$

2) Encofrado. Esta unidad se mide por metros cuadrados $\left(\mathrm{m}^{2}\right)$ de superficie del elemento a encofrar,.por lo que su medición para la viga "i" tiene por valor:

- Vigas planas: se encofra únicamente la cara inferior de la viga, pues en los laterales de la viga se apoyan los elementos del forjado.

$$
M_{E N C, V I G, i}=L_{v, i} \times b_{v i g, i}
$$

- Vigas descolgadas: se encofra la cara inferior de la viga y una altura en cada una de las caras laterales de valor hvig, i - hfor.

$$
M_{E N C, V I G, i}=L_{v, i} \times\left(b_{v i g, i}+\left(h_{v i g, i}-h_{f o r}\right)\right)
$$


3) Acero. Esta unidad se mide en kilogramos $(\mathrm{Kg})$ de acero colocado en obra, multiplicándose el valor teórico obtenido por un coeficiente de 1.10 para tener en cuenta las pérdidas de acero producidas por los despuntes de las armaduras y los solapes y anclajes no considerados en los despieces tipo de las armaduras. La medición total de acero en la viga "i” $\left(\mathrm{M}_{\mathrm{AC}, \mathrm{VIG}, \mathrm{i}}\right)$ se puede expresar como:

$\left.M_{A C, V I G, i}=M_{A C, A B A S E, V I G, i}+M_{A C, A P I E L, V I G, i}+M_{A C, A R E F, I N F, V I G, i}+M_{A C, A R E F, S U P, V I G, i}+M_{A C, C O R, V I G, i}\right) \times 1,10$

Donde:

$M_{A C, A B A S E, V I G, i}$ : es la armadura base superior e inferior de la viga "i".

$M_{A C, A P I E L, V I G, i}$ : es la armadura de piel de la viga “i”.

$\mathrm{M}_{\mathrm{AC}, \mathrm{AREF}, \mathrm{INF}, \mathrm{VIG}, \mathrm{i}}$ : es la armadura de refuerzo inferior de la viga "i".

$\mathrm{M}_{\mathrm{AC}, \mathrm{COR}, \mathrm{VIG}, \mathrm{i}}$ : es la armadura de cortante de la viga "i".

Exponemos a continuación cómo se obtiene cada uno de los sumandos.

- Armadura base (superior e inferior) y armadura de piel:

$M_{A C, A B A S E, V I G, i}=L_{v, i} \times\left(A_{s, \text { base }, \text { inf }, \text { vig }, i}+A_{s, \text { base }, \text { sup }, v i g, i}\right) \times F P$

$M_{A C, A P I E L, V I G, i}=L_{v, i} \times A_{s, p i e l, v i g, i} \times F P$

"FP" es un factor de paso de valor 0,785 que permite pasar de un área de acero expresada en $\mathrm{cm}^{2}$ a un peso expresado en $\mathrm{Kg}$.

- Armadura de refuerzo inferior (armadura de refuerzo de positivos).

$M_{A C, A R E F, I N F, V I G, i}=0,80 \times L_{v, i} \times A_{s, r e f \text { inf }, v i g, i} \times F P$

- Armadura de refuerzo superior (armadura de refuerzo de negativos). 
$M_{A C, A R E F, S U P, V I G, i}=0,20 \times L_{v, i} \times\left(A_{s, r e f \text { sup }, \text { vig, }, i, i z q d a}+A_{s, r e f \text { sup }, \text { vig }, i, d e r}\right) \times F P$

- Armadura de cortante.

$$
M_{A C, C O R, V I G, i}=\frac{L_{v, i}}{3} \times\left(A_{s t, c o r, v i g, i, i z q d a}+A_{s t, c o r, v i g, i, c e n t r o}+A_{s t, c o r, v i g, i, d e r}\right) \times F P
$$

4) Hormigón. Esta unidad se mide en metros cúbicos $\left(\mathrm{m}^{3}\right)$, obteniéndose su medición a partir de la expresión siguiente:

$$
M_{\mathrm{HORM}, V I G, i}=L_{v, i} \times b_{v i g, i} \times h_{v i g, i}
$$

\section{Mediciones de un pilar.}

1) Encofrado. Esta unidad se mide por metros cuadrados $\left(m^{2}\right)$ de superficie del elemento a encofrar, .por lo que su medición para el pilar "i” tiene por valor:

$$
M_{E N C, P I L, i}=H_{p, i} \times 2 \times\left(b_{p i l, i}+h_{p i l, i}\right)
$$

2) Acero en pilares. Esta unidad se mide en kilogramos $(\mathrm{Kg})$, multiplicándose el valor teórico obtenido por un coeficiente de 1.10 para tener en cuenta las pérdidas de acero por solapes, despuntes y anclajes. La medición total de acero en el pilar "i" $\left(\mathrm{M}_{\mathrm{AC}, \mathrm{PI}, \mathrm{i}}\right)$ se puede expresar como:

$$
\left.M_{A C, P I L, i}=M_{A C, L O N G, V I G, i}+M_{A C, C O R, P I L, i}\right) \times 1,10
$$

Donde:

$M_{A C, L O N G, P I L, i}$ : es la armadura longitudinal del pilar "i".

$\mathrm{M}_{\mathrm{AC}, \mathrm{COR}, \mathrm{PL}, \mathrm{i}}$ : es la armadura de cortante del pilar "i".

Exponemos a continuación cómo se obtiene cada uno de los sumandos.

- Armadura longitudinal: 
$M_{A C, L O N G, P I L, i}=H_{p, i} \times A_{s, \text { long }, p i l, i} \times F P$

"FP" es un factor de paso de valor 0,785 que permite pasar de un área de acero expresada en $\mathrm{cm}^{2}$ a un peso expresado en $\mathrm{Kg}$.

- Armadura de cortante.

$M_{A C, C O R, P I L, i}=H_{p, i} \times A_{s t, c o r, p i l, i} \times F P$

3) Hormigón en pilares. Esta unidad se mide en metros cúbicos $\left(\mathrm{m}^{3}\right)$, obteniéndose su medición a partir de la expresión siguiente

$$
M_{H O R M, P I L, i}=H_{p, i} \times b_{p i l, i} \times h_{p i l, i}
$$


Página intencionadamente en blanco. 


\section{APÉNDICE 3. COMBINACIÓN DE ACCIONES.}


Página intencionadamente en blanco. 


\section{Combinación de acciones.}

En esta investigación se han considerado los siguientes casos de carga elementales:

1) Cargas permanentes.

2) Sobrecarga de uso en vanos pares de vigas.

3) Sobrecarga de uso en vanos impares de vigas.

4) Sobrecarga de uso en todos los vanos.

5) Viento en una dirección.

6) Viento en la dirección opuesta.

La nieve se debe añadir en la sobrecarga de uso actuante en la planta de cubierta para tenerla en cuenta en el cálculo.

Las acciones se combinan de acuerdo a las prescripciones del Artículo 13 de la EHE dando lugar a las 48 combinaciones de casos de carga elementales que se muestran en la tabla de la página siguiente. En esta tabla, G representa las cargas permanentes, SC1, la sobrecarga de uso en vanos impares, SC2, la sobrecarga de uso en los vanos pares, W1, el viento de izquierda a derecha y W2, el viento de derecha a izquierda. 
CUADRO DE COMBINACIONES (Art. 13.2 y 13.3 EHE)

\begin{tabular}{|c|c|c|c|c|c|c|c|c|}
\hline & COMB. & $G$ & $\mathrm{SC} 1$ & $\mathrm{SC} 2$ & SCTOT & W1 & W2 & \\
\hline \multirow{12}{*}{ 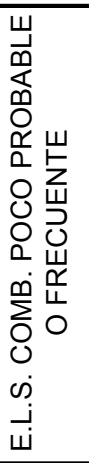 } & 1 & 1 & 0 & 0 & 0 & 0 & 0 & \multirow{6}{*}{ 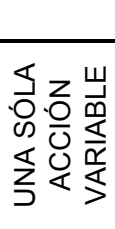 } \\
\hline & 2 & 1 & 1 & 0 & 0 & 0 & 0 & \\
\hline & 3 & 1 & 0 & 1 & 0 & 0 & 0 & \\
\hline & 4 & 1 & 0 & 0 & 1 & 0 & 0 & \\
\hline & 5 & 1 & 0 & 0 & 0 & 1 & 0 & \\
\hline & 6 & 1 & 0 & 0 & 0 & 0 & 1 & \\
\hline & 7 & 1 & .9 & 0 & 0 & .9 & 0 & \multirow{6}{*}{ 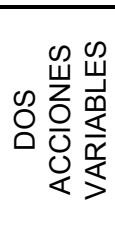 } \\
\hline & 8 & 1 & 0 & .9 & 0 & .9 & 0 & \\
\hline & 9 & 1 & 0 & 0 & .9 & .9 & 0 & \\
\hline & 10 & 1 & .9 & 0 & 0 & 0 & .9 & \\
\hline & 11 & 1 & 0 & .9 & 0 & 0 & .9 & \\
\hline & 12 & 1 & 0 & 0 & .9 & 0 & .9 & \\
\hline \multirow{12}{*}{ 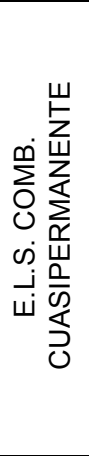 } & 13 & 1 & 0 & 0 & 0 & 0 & 0 & \\
\hline & 14 & 1 & .6 & 0 & 0 & 0 & 0 & \multirow{5}{*}{ 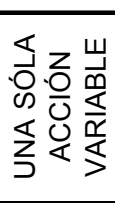 } \\
\hline & 15 & 1 & 0 & .6 & 0 & 0 & 0 & \\
\hline & 16 & 1 & 0 & 0 & .6 & 0 & 0 & \\
\hline & 17 & 1 & 0 & 0 & 0 & .6 & 0 & \\
\hline & 18 & 1 & 0 & 0 & 0 & 0 & .6 & \\
\hline & 19 & 1 & .6 & 0 & 0 & .6 & 0 & \multirow{6}{*}{ 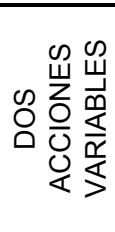 } \\
\hline & 20 & 1 & 0 & .6 & 0 & .6 & 0 & \\
\hline & 21 & 1 & 0 & 0 & .6 & .6 & 0 & \\
\hline & 22 & 1 & .6 & 0 & 0 & 0 & .6 & \\
\hline & 23 & 1 & 0 & .6 & 0 & 0 & .6 & \\
\hline & 24 & 1 & 0 & 0 & .6 & 0 & .6 & \\
\hline \multirow{12}{*}{ 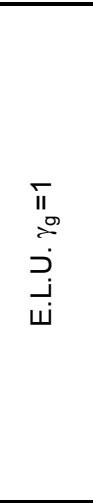 } & 25 & 1 & 0 & 0 & 0 & 0 & 0 & \\
\hline & 26 & 1 & $\gamma_{q}$ & 0 & 0 & 0 & 0 & \multirow{5}{*}{ 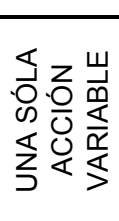 } \\
\hline & 27 & 1 & 0 & $\overline{\gamma_{q}}$ & 0 & 0 & 0 & \\
\hline & 28 & 1 & 0 & 0 & $\overline{\gamma_{q}}$ & 0 & 0 & \\
\hline & 29 & 1 & 0 & 0 & 0 & $\gamma_{q}$ & 0 & \\
\hline & 30 & 1 & 0 & 0 & 0 & 0 & $\gamma_{q}$ & \\
\hline & 31 & 1 & $0.9^{*} \gamma_{\mathrm{q}}$ & 0 & 0 & $0.9^{*} \gamma_{\mathrm{q}}$ & 0 & \multirow{6}{*}{ 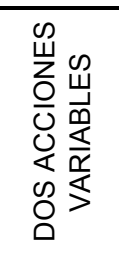 } \\
\hline & 32 & 1 & 0 & $0.9^{*} \gamma_{q}$ & 0 & $0.9^{*} \gamma_{\mathrm{q}}$ & 0 & \\
\hline & 33 & 1 & 0 & 0 & $0.9^{*} \gamma_{q}$ & $0.9^{*} \gamma_{\mathrm{q}}$ & 0 & \\
\hline & 34 & 1 & $0.9^{*} \gamma_{\mathrm{q}}$ & 0 & 0 & 0 & $0.9^{*} \gamma_{q}$ & \\
\hline & 35 & 1 & 0 & $0.9^{*} \gamma_{q}$ & 0 & 0 & $0.9^{*} \gamma_{q}$ & \\
\hline & 36 & 1 & 0 & 0 & $0.9^{*} \gamma_{q}$ & 0 & $0.9^{*} \gamma_{q}$ & \\
\hline \multirow{12}{*}{ 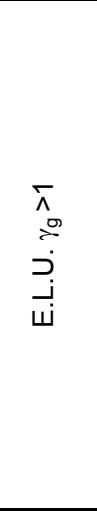 } & 37 & $\gamma_{g}$ & 0 & 0 & 0 & 0 & 0 & \multirow{6}{*}{ 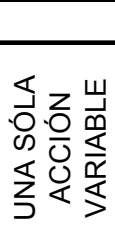 } \\
\hline & 38 & $\gamma_{g}$ & $\gamma_{q}$ & 0 & 0 & 0 & 0 & \\
\hline & 39 & $\gamma_{\mathrm{g}}$ & 0 & $\gamma_{q}$ & 0 & 0 & 0 & \\
\hline & 40 & $\gamma_{\mathrm{g}}$ & 0 & 0 & $\overline{\gamma_{q}}$ & 0 & 0 & \\
\hline & 41 & $\gamma_{g}$ & 0 & 0 & 0 & $\gamma_{q}$ & 0 & \\
\hline & 42 & $\gamma_{\mathrm{g}}$ & 0 & 0 & 0 & 0 & $\gamma_{q}$ & \\
\hline & 43 & $\gamma_{g}$ & $0.9^{*} \gamma_{q}$ & 0 & 0 & $0.9^{*} \gamma_{\mathrm{q}}$ & 0 & \multirow{6}{*}{ 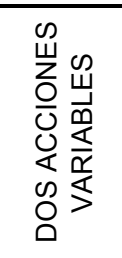 } \\
\hline & 44 & $\gamma_{g}$ & 0 & $0.9^{*} \gamma_{\mathrm{q}}$ & 0 & $0.9^{*} \gamma_{\mathrm{q}}$ & 0 & \\
\hline & 45 & $\gamma_{\mathrm{g}}$ & 0 & 0 & $0.9^{*} \gamma_{q}$ & $0.9^{*} \gamma_{\mathrm{q}}$ & 0 & \\
\hline & 46 & $\gamma_{g}$ & $0.9^{*} \gamma_{\mathrm{q}}$ & 0 & 0 & 0 & $0.9^{*} \gamma_{q}$ & \\
\hline & 47 & $\gamma_{\mathrm{g}}$ & 0 & $0.9^{*} \gamma_{\mathrm{q}}$ & 0 & 0 & $0.9^{*} \gamma_{\mathrm{q}}$ & \\
\hline & 48 & $\gamma_{\mathrm{g}}$ & 0 & 0 & $0.9^{*} \gamma_{q}$ & 0 & $0.9^{*} \gamma_{q}$ & \\
\hline
\end{tabular}




\section{APÉNDICE 4. PLANOS DE LAS ESTRUCTURAS OPTIMIZADAS.}


Página intencionadamente en blanco. 


\section{Índice de planos.}

PLANO N01. Problema P_2v4h-C EST.

PLANO N¹.1. Mejor solución obtenida con una Estrategia de Saltos Múltiples Aleatorios.

PLANO N01.2. Mejor solución obtenida con Gradiente First Best.

PLANO N¹.3. Mejor solución obtenida con Cristalización Simulada.

PLANO N¹.4. Mejor solución obtenida con Aceptación por Umbrales.

PLANO N01.5. Mejor solución obtenida con Algoritmos Genéticos.

PLANO N². Problema P_2v2h-CEST.

PLANO N².1. Mejor solución obtenida con Cristalización Simulada.

PLANO N³. Problema P_2v6h-CEST.

PLANO N³.1. Mejor solución obtenida con Cristalización Simulada.

PLANO No4. Problema P_2v8h.1-C EST.

PLANO N4.1. Mejor solución obtenida con Cristalización Simulada.

PLANO N05. Problema P_2v8h.2-C EST $_{\text {. }}$

PLANO N5.1. Mejor solución obtenida con Cristalización Simulada.

PLANO N6. Problema P_2v8h.3-C EST $_{\text {. }}$

PLANO N6.1. Mejor solución obtenida con Cristalización Simulada.

PLANO N07. Problema P_2v8h.4-C EST.

PLANO N7.1. Mejor solución obtenida con Cristalización Simulada.

PLANO No8. Problema P_2v4h-C $\mathrm{EST}_{\mathrm{S}} / \mathrm{N}_{\mathrm{s}}$.

PLANO N8.1. Solución de coste mínimo.

PLANO N8.2. Solución de máxima construibilidad.

PLANO No9. Problema P 2v4h-C EST/EC.

PLANO N9.1. Solución de máxima sostenibilidad ambiental.

Nota: se recuerda que la definición de los problemas de optimización se realizó en el epígrafe 3.6. 
ágina intencionadamente en blanco. 


\section{Esquemas de armado de vigas.}
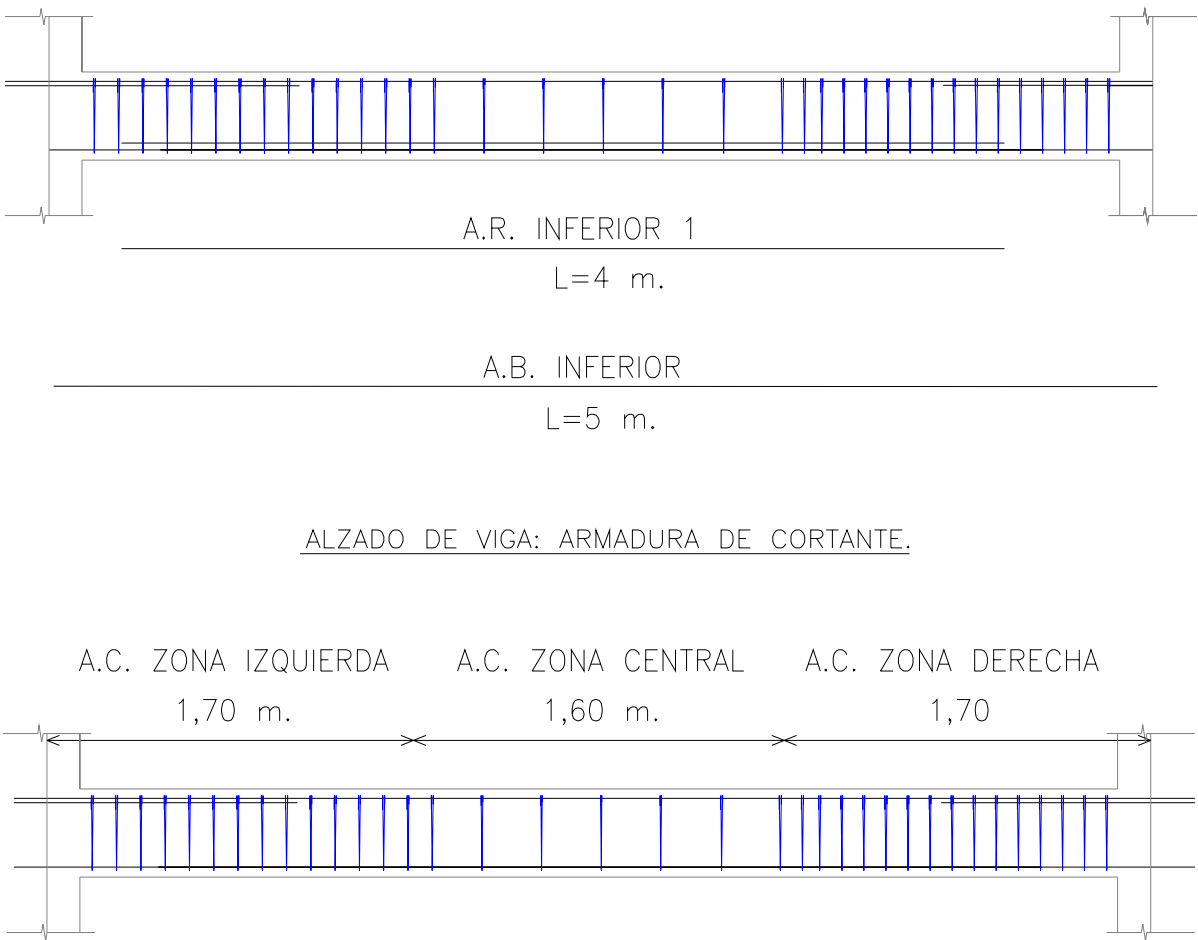

\section{Esquemas de armado de pilares.}

ALZADO

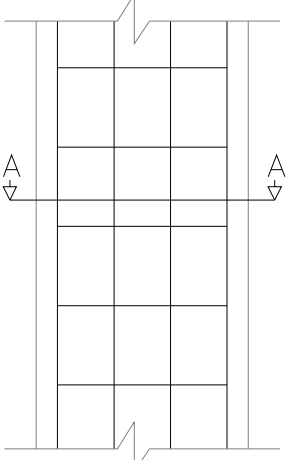

Notas:

- El suplemento de la armadura de cortante en pilares sólo figura en las tablas cuando es necesario. 


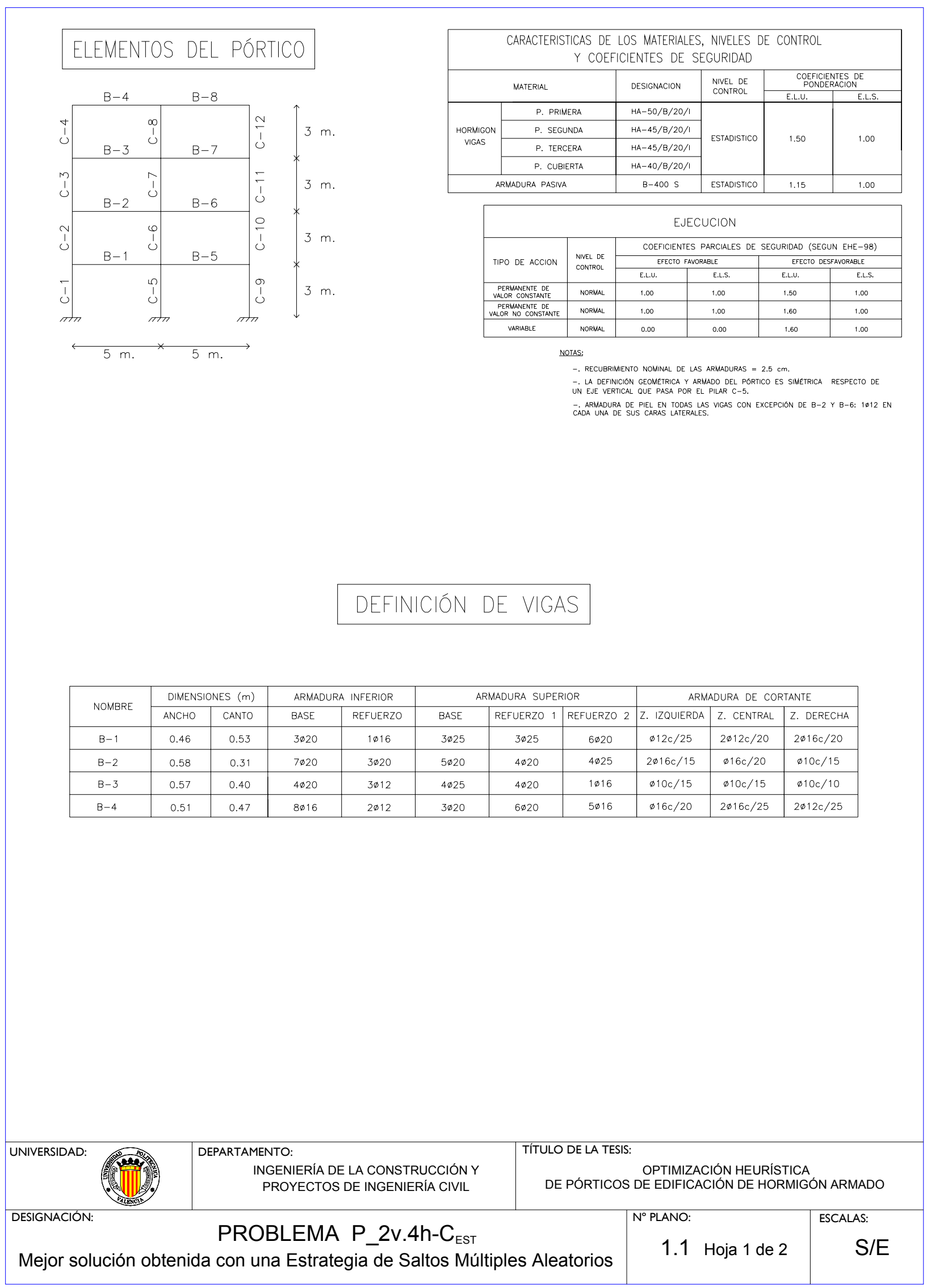




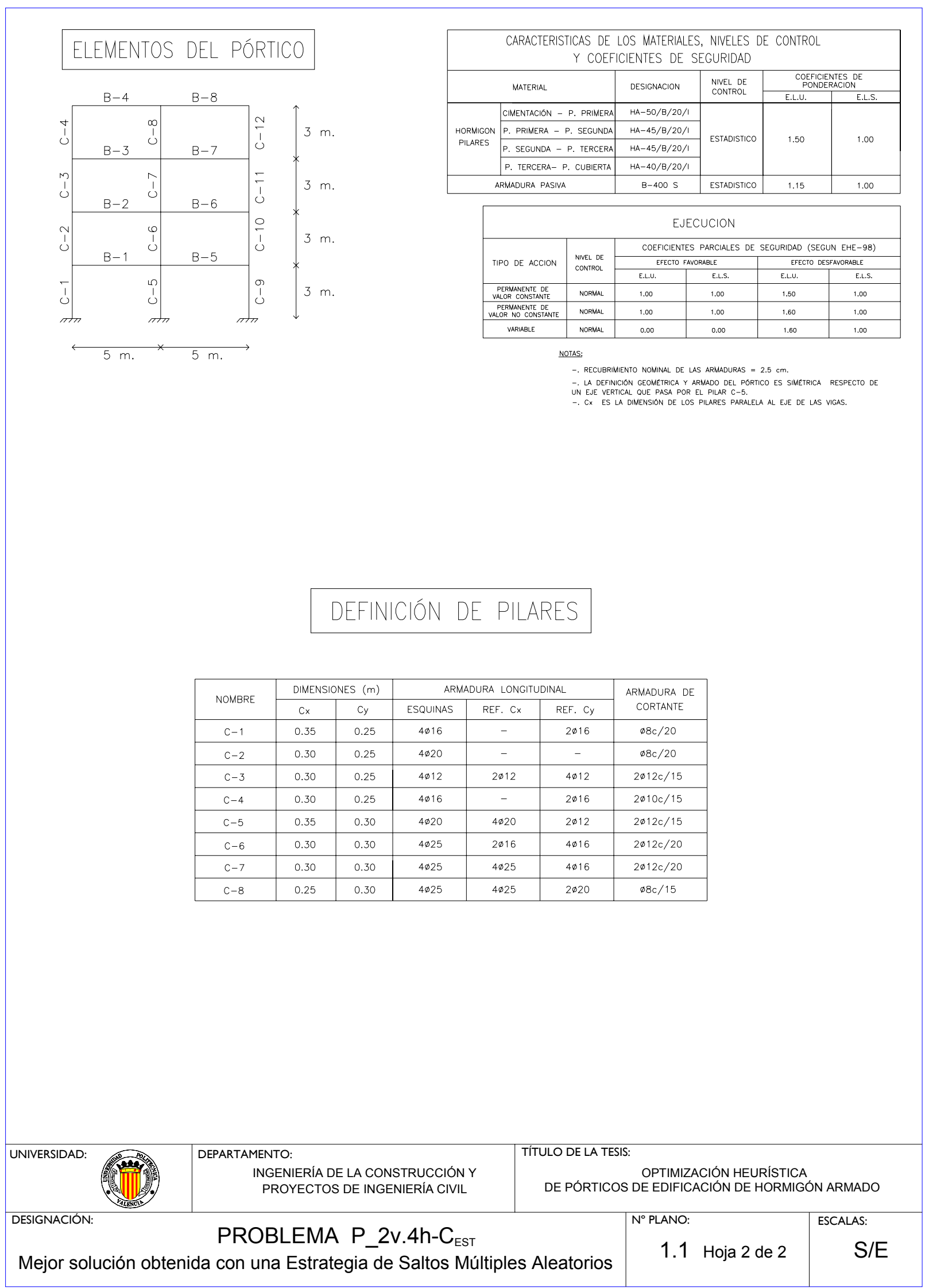




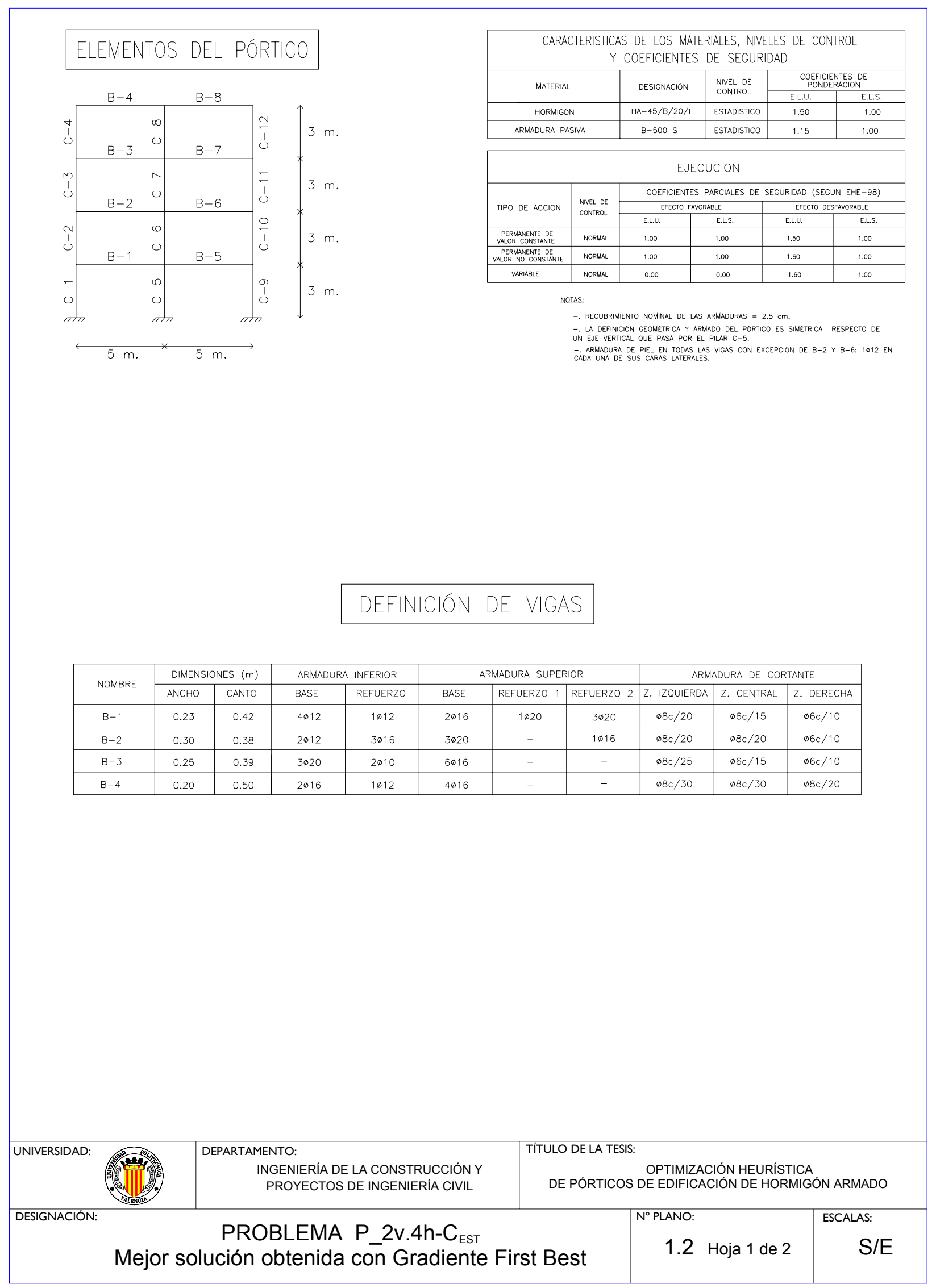




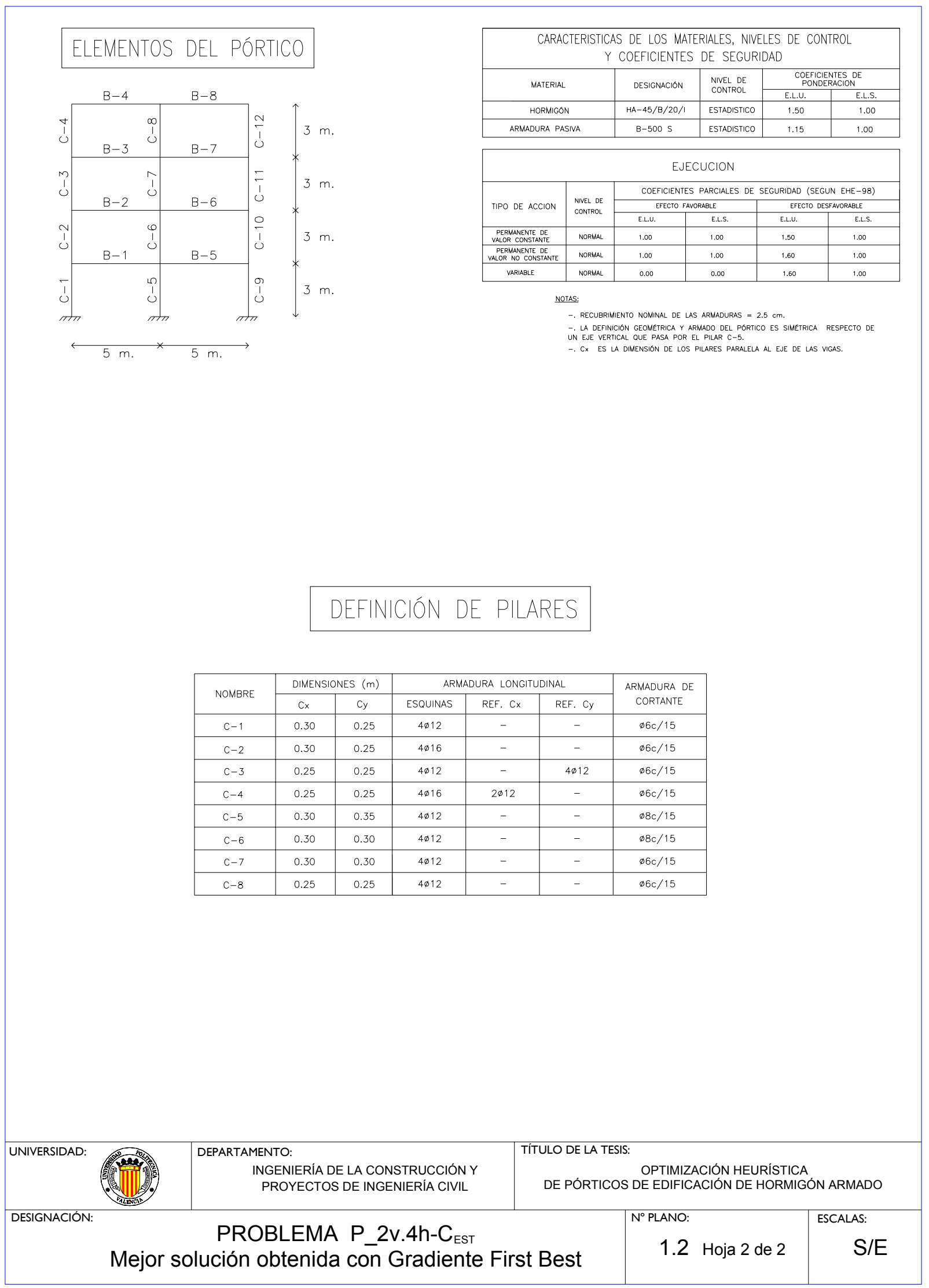




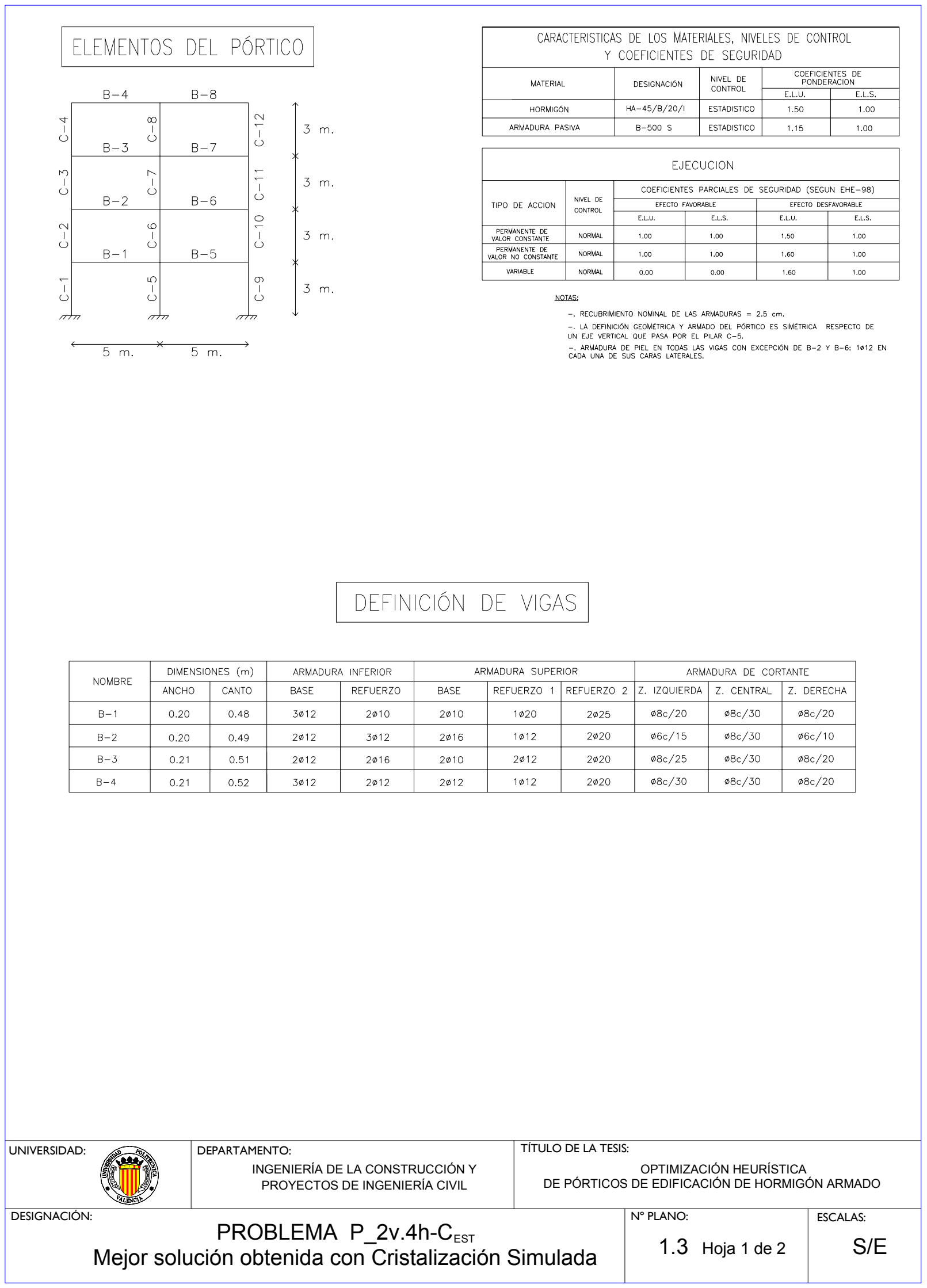




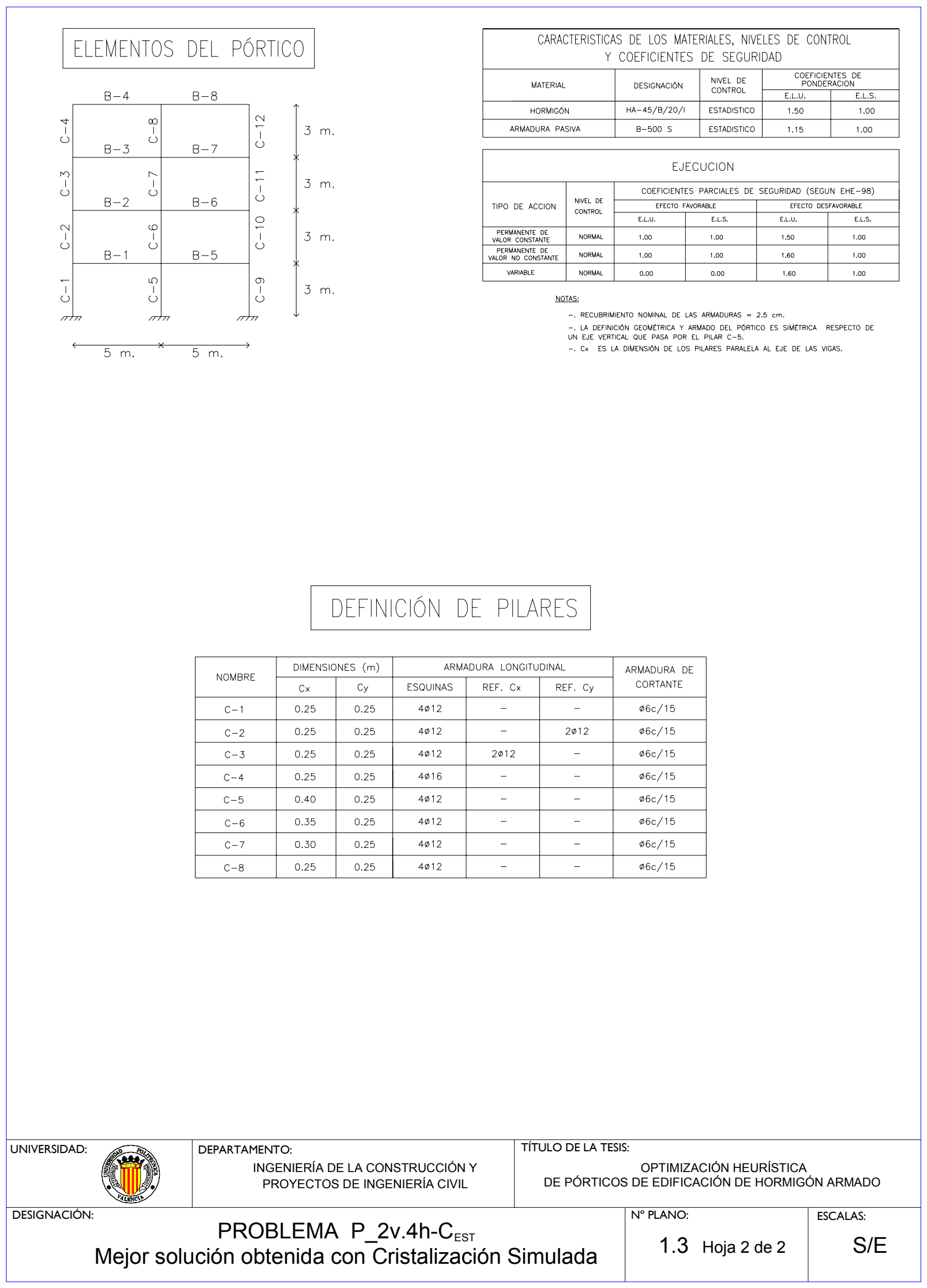




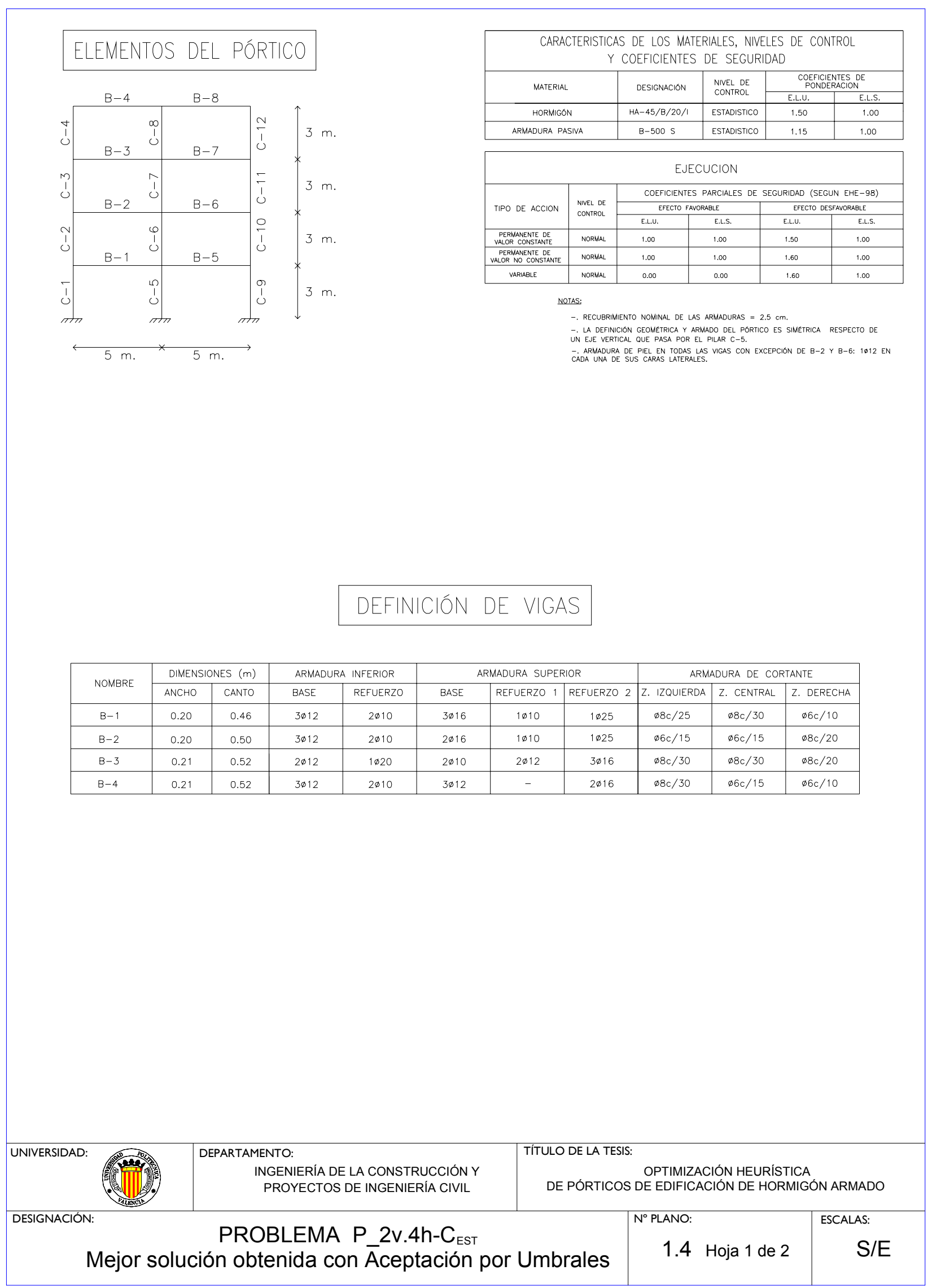




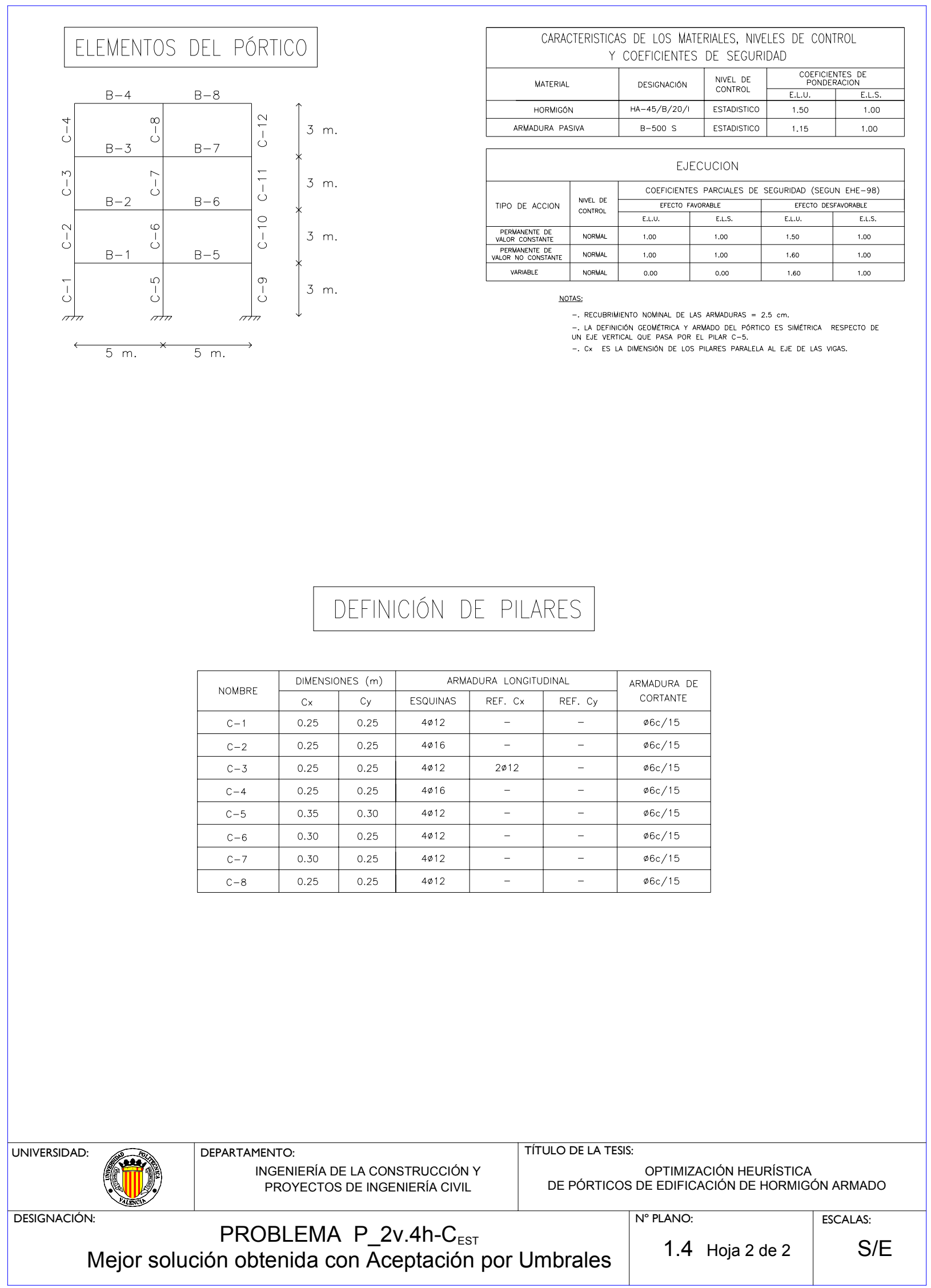




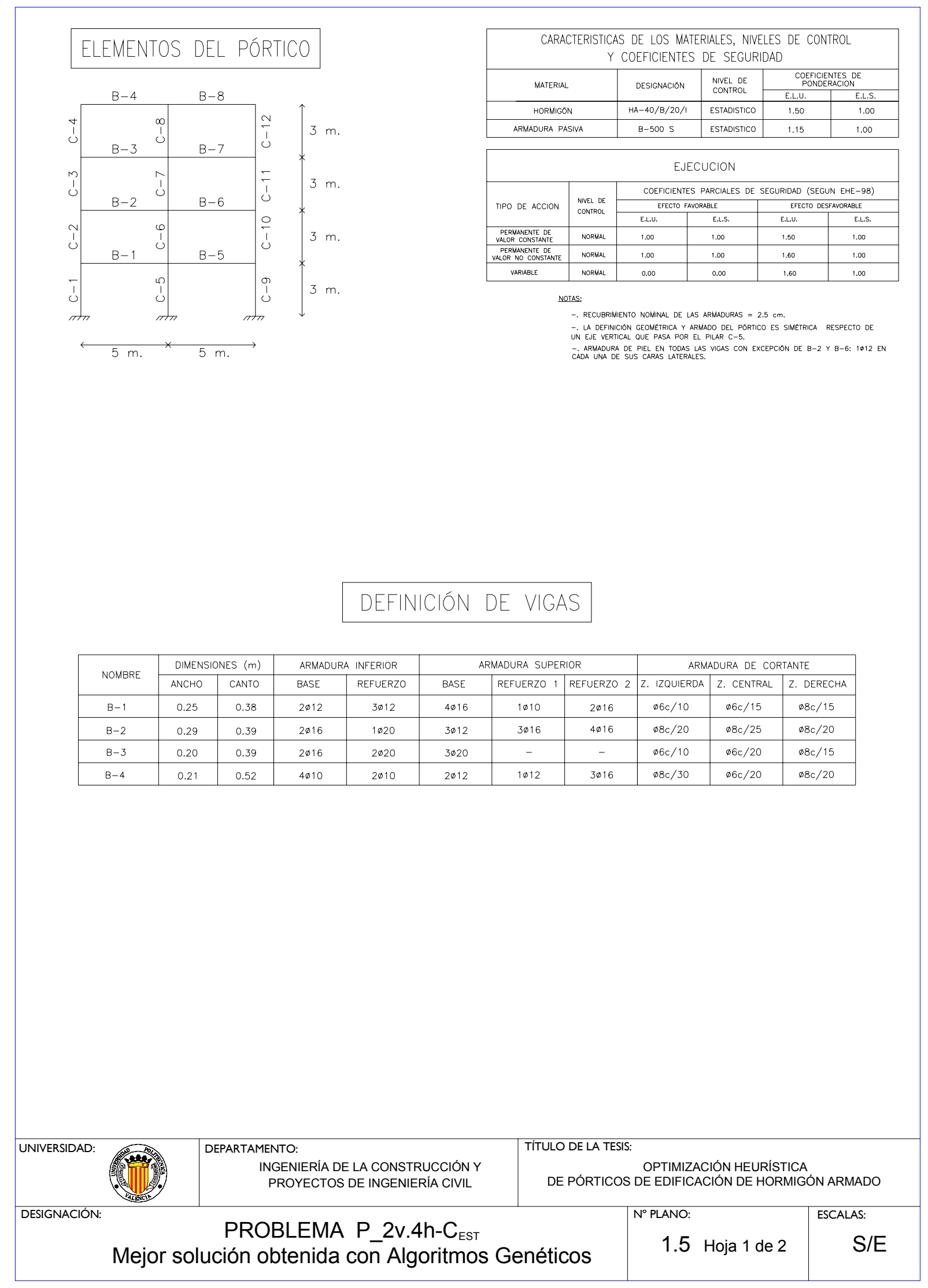




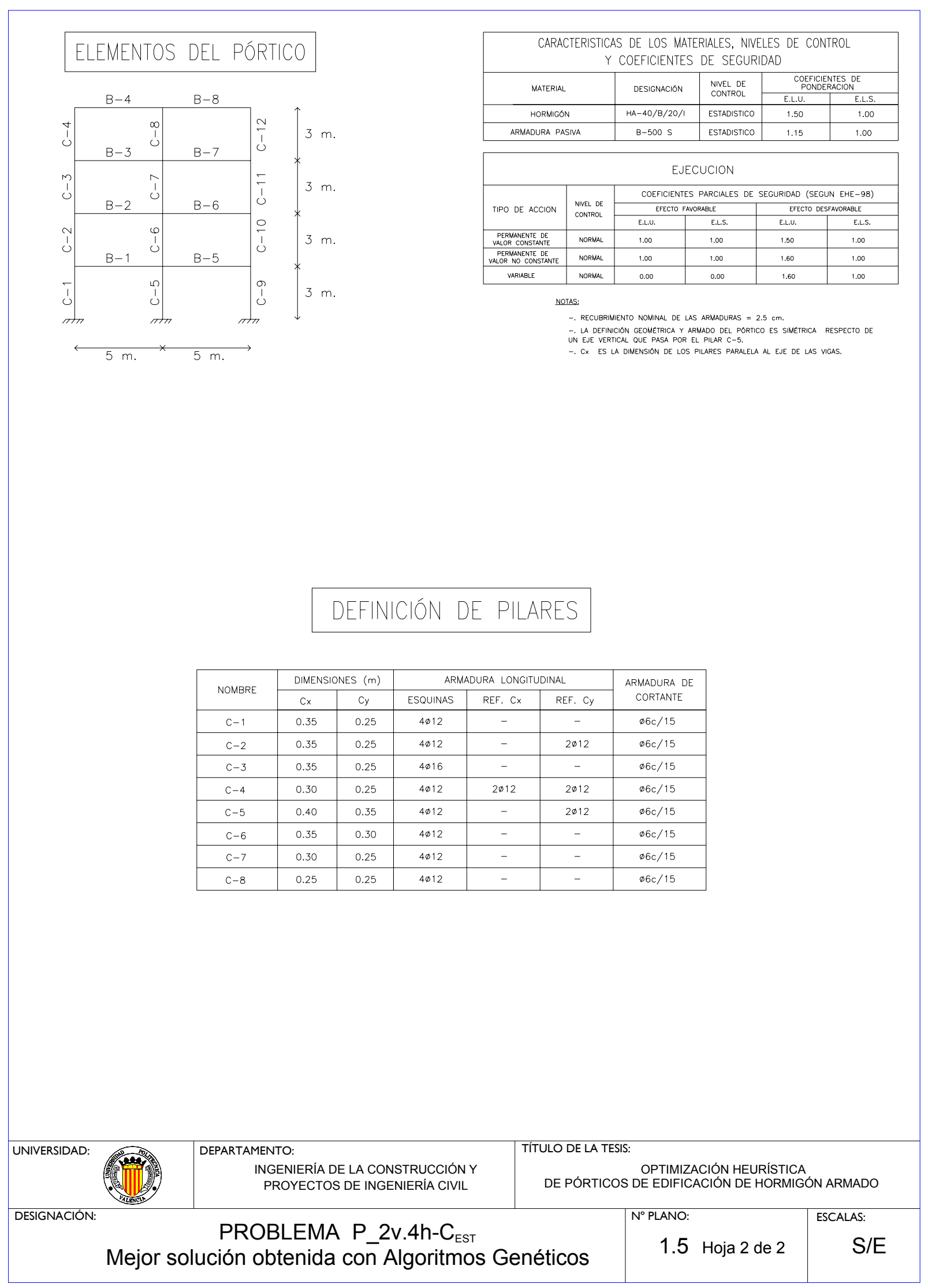




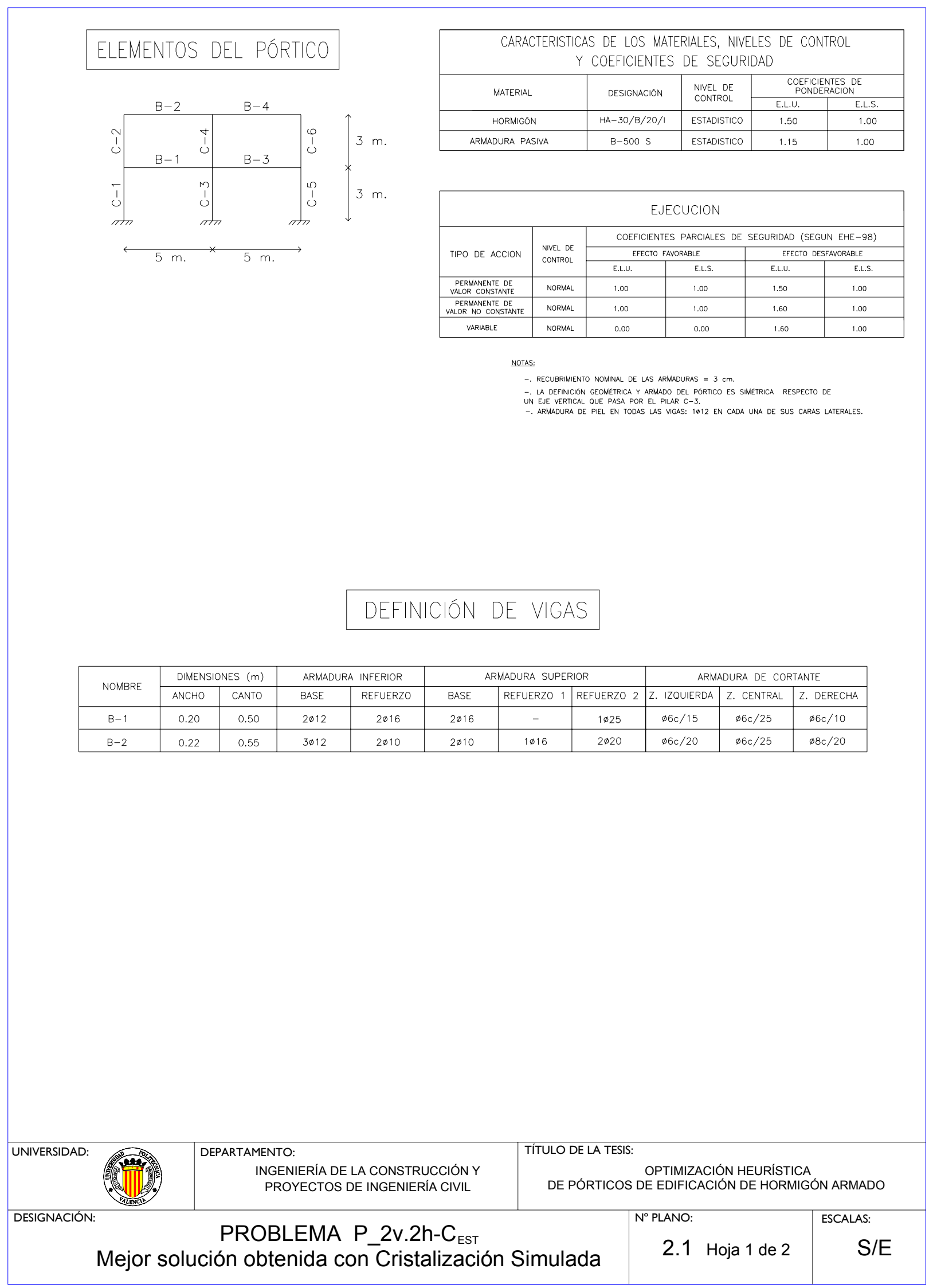




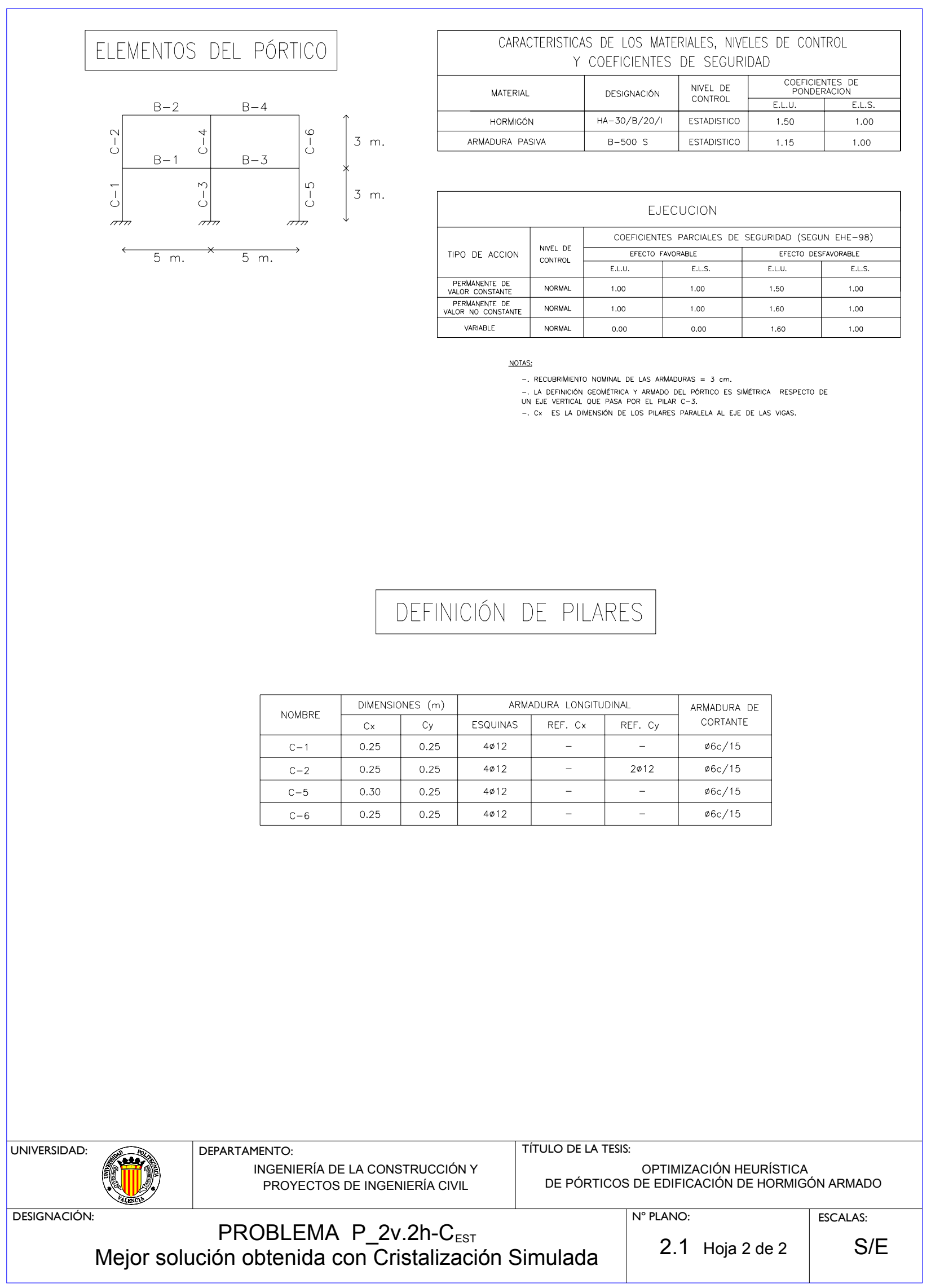




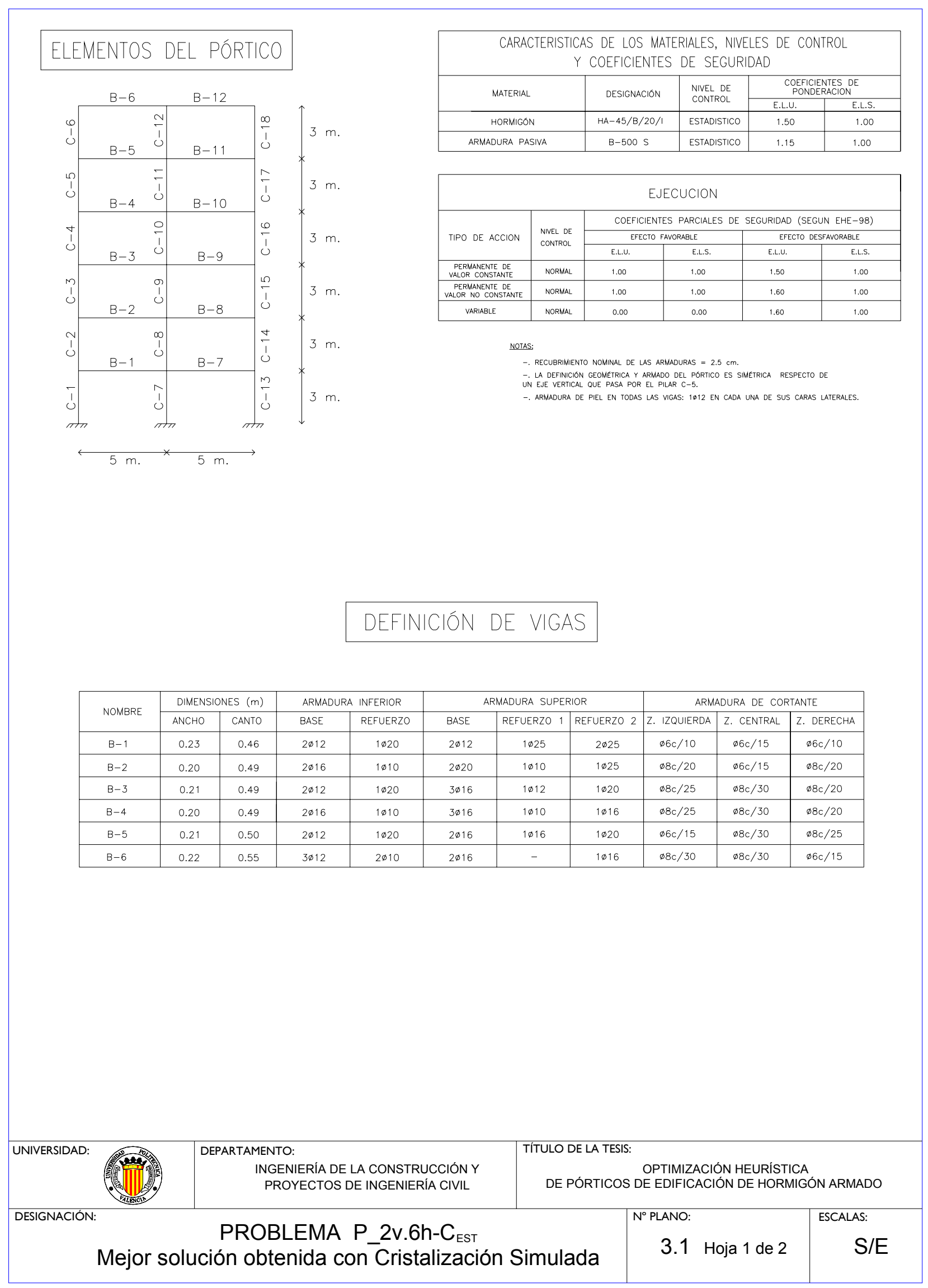




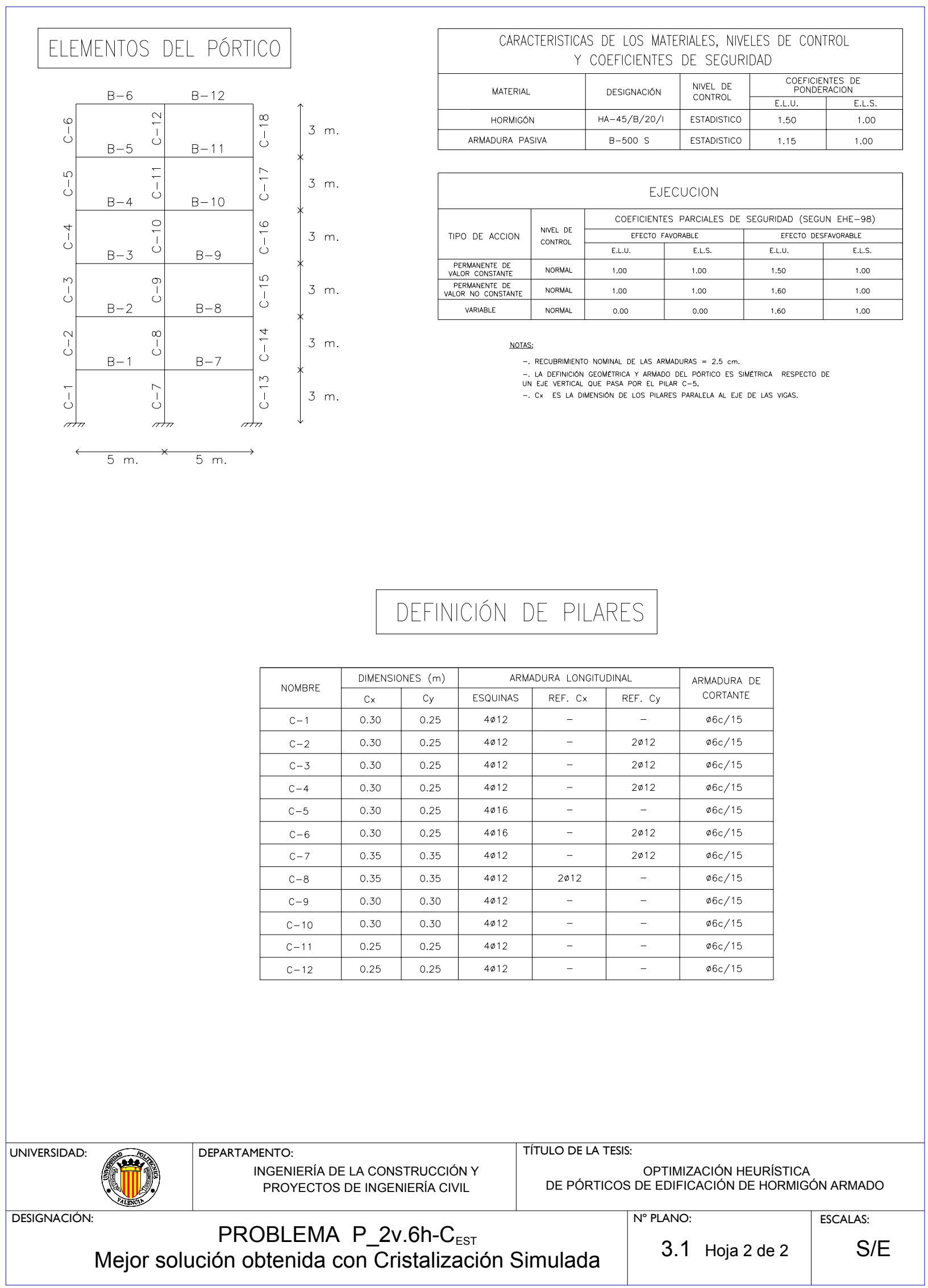




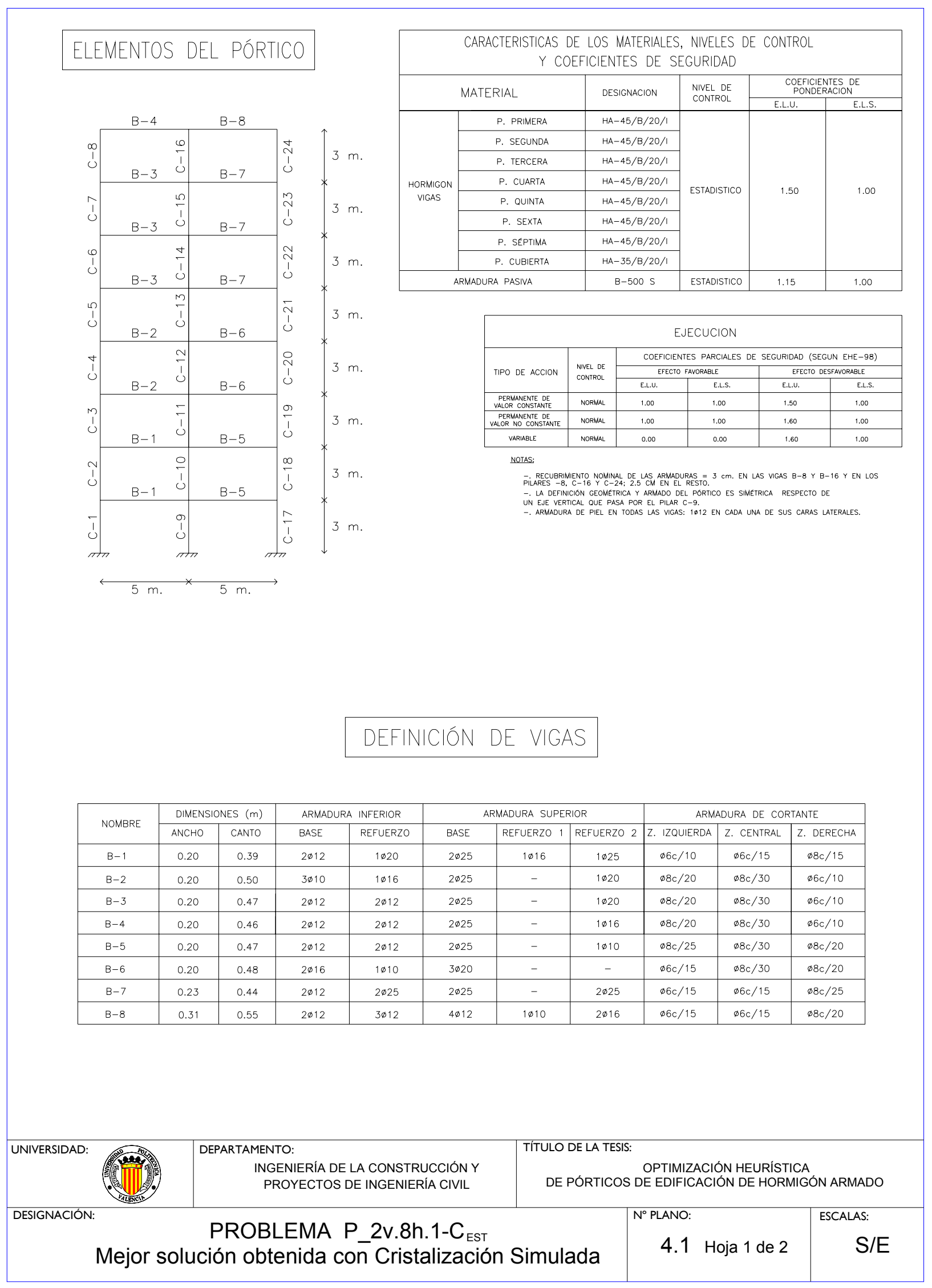




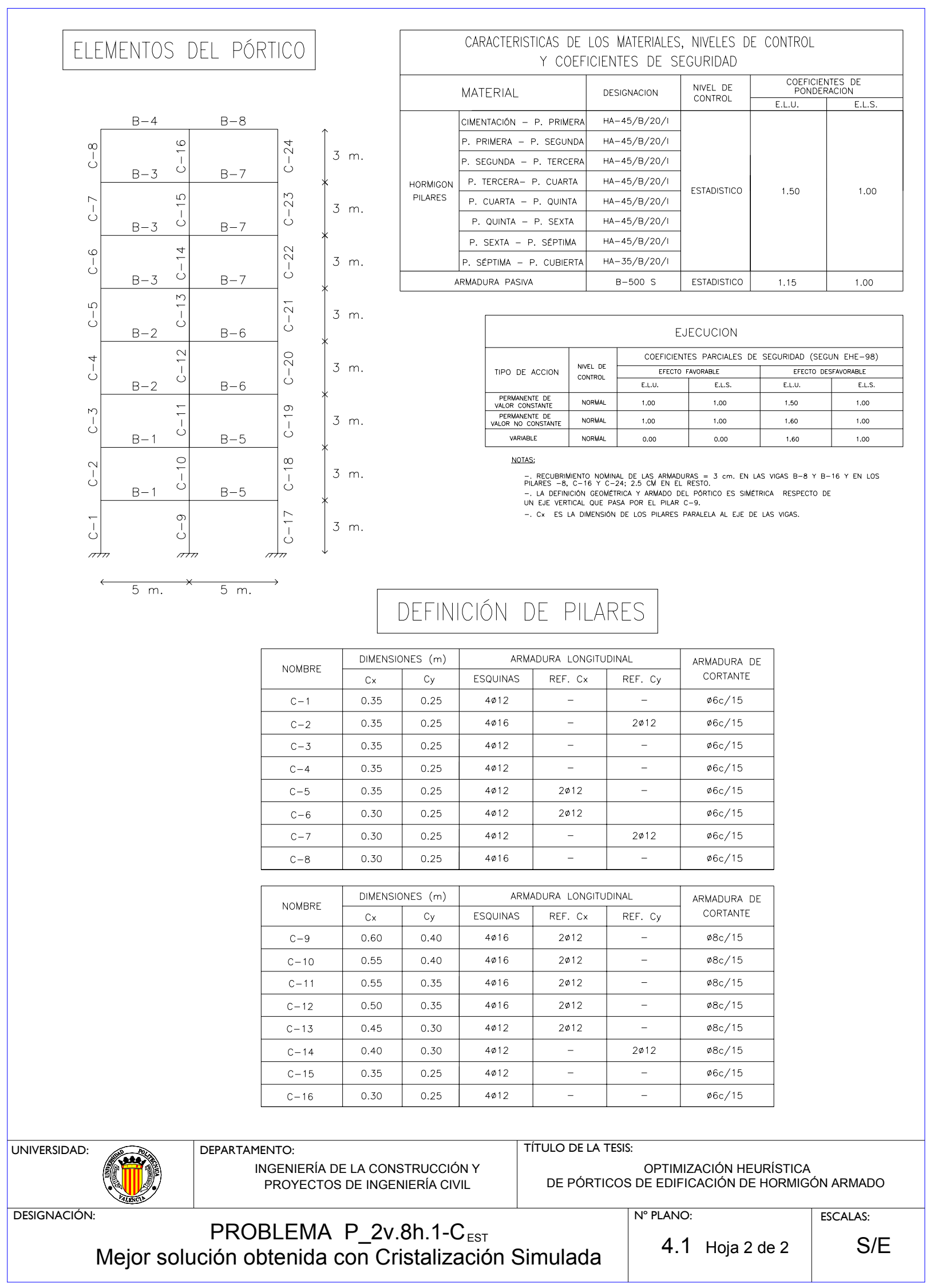




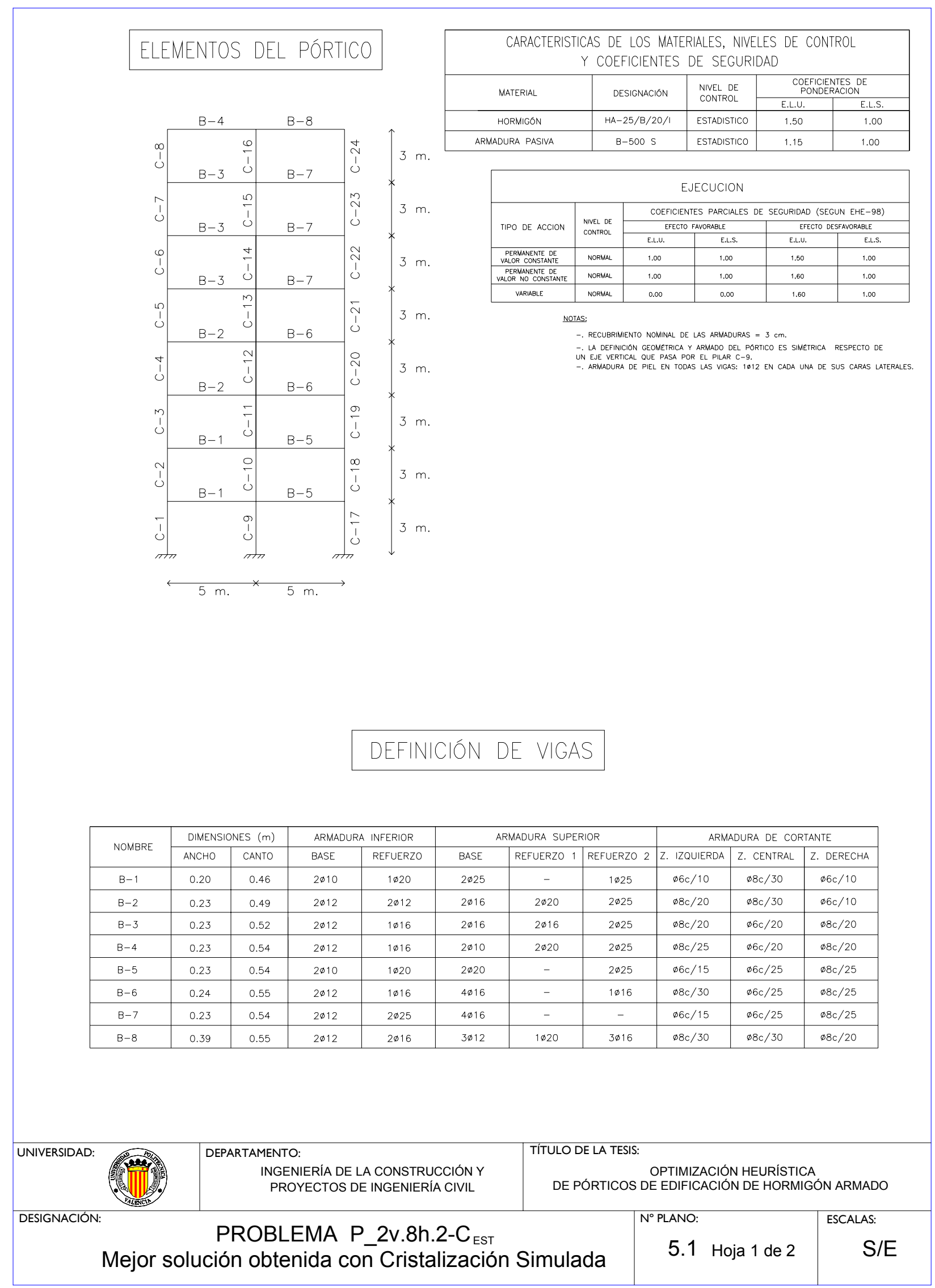




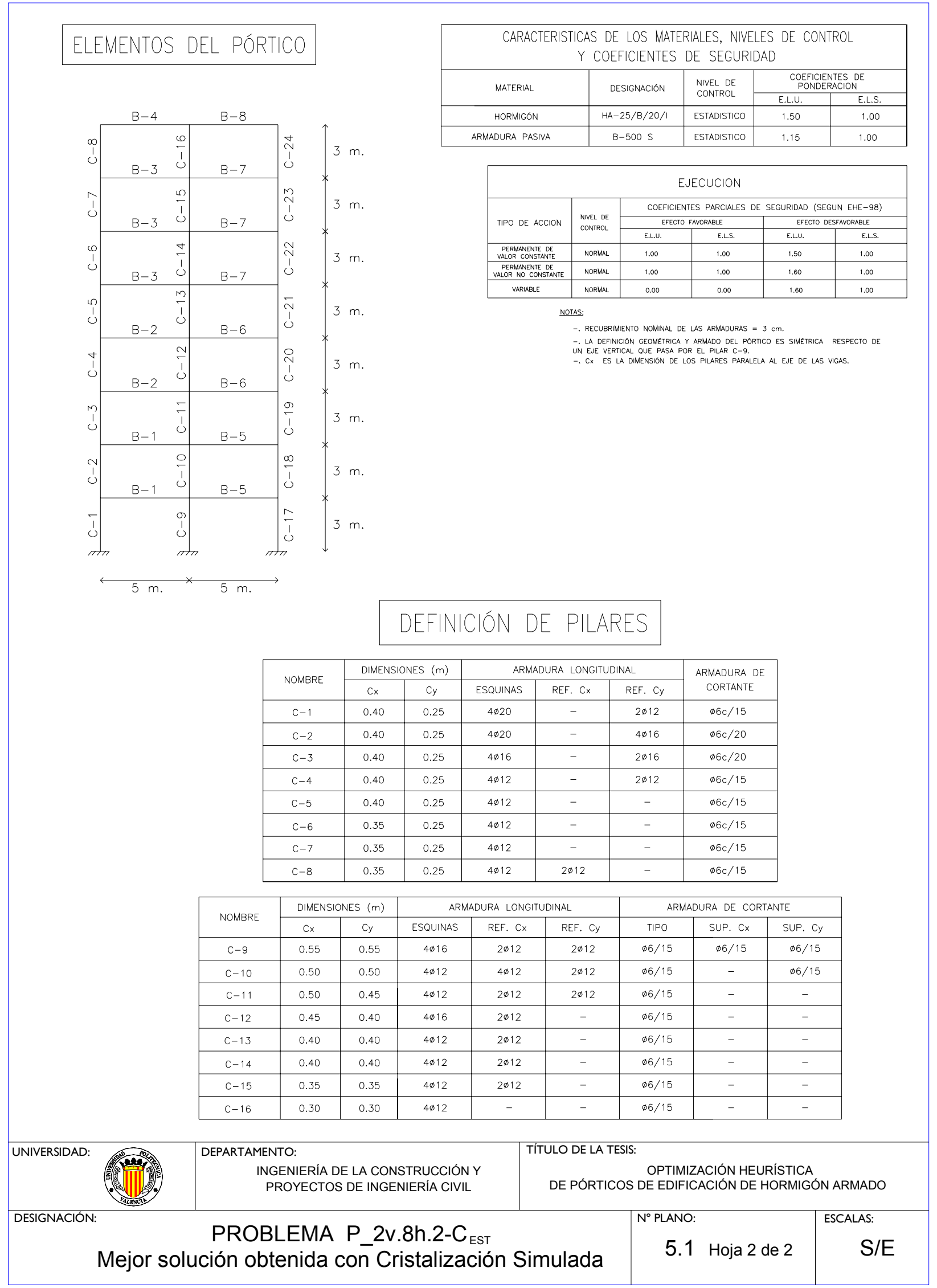




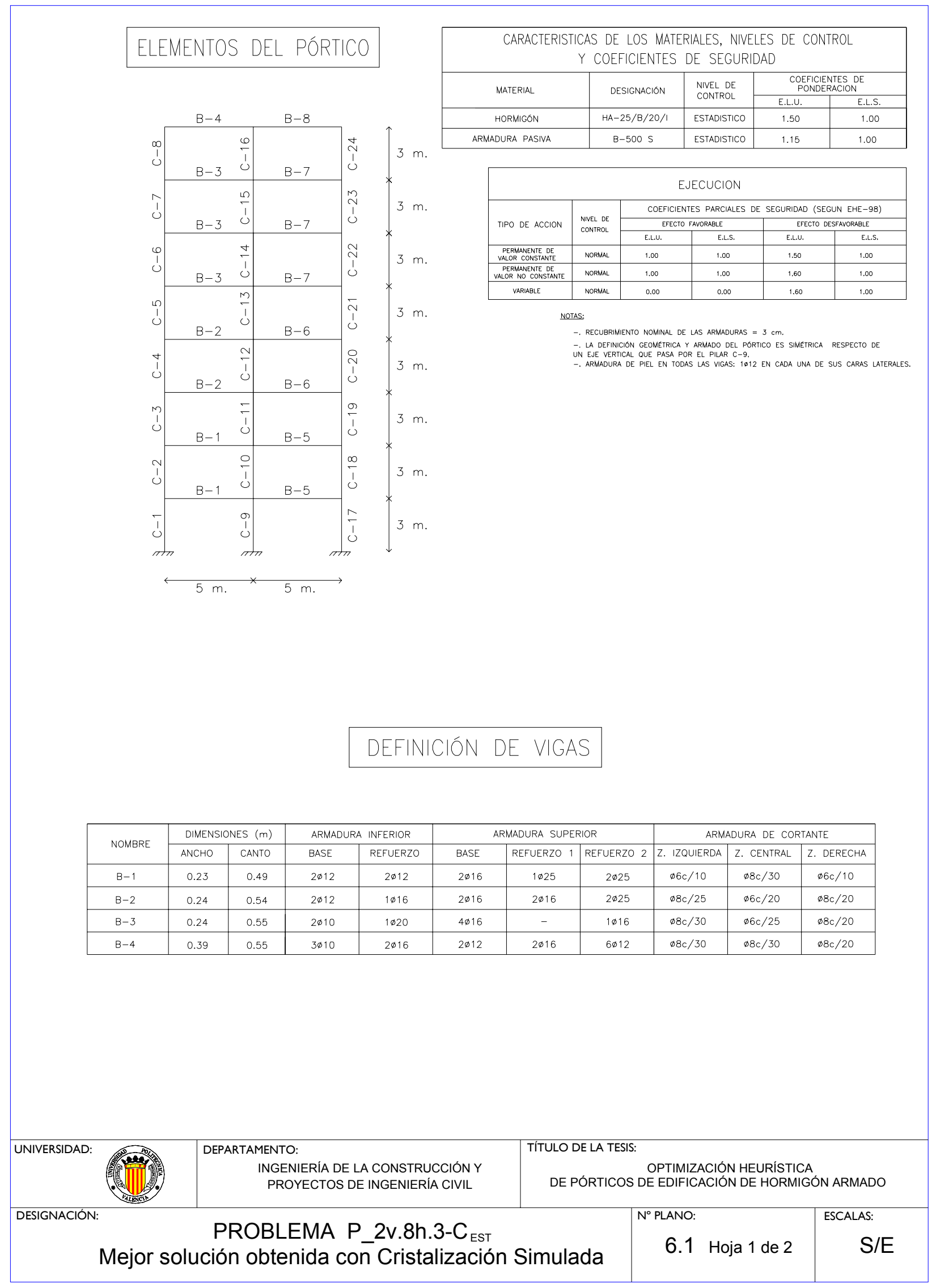




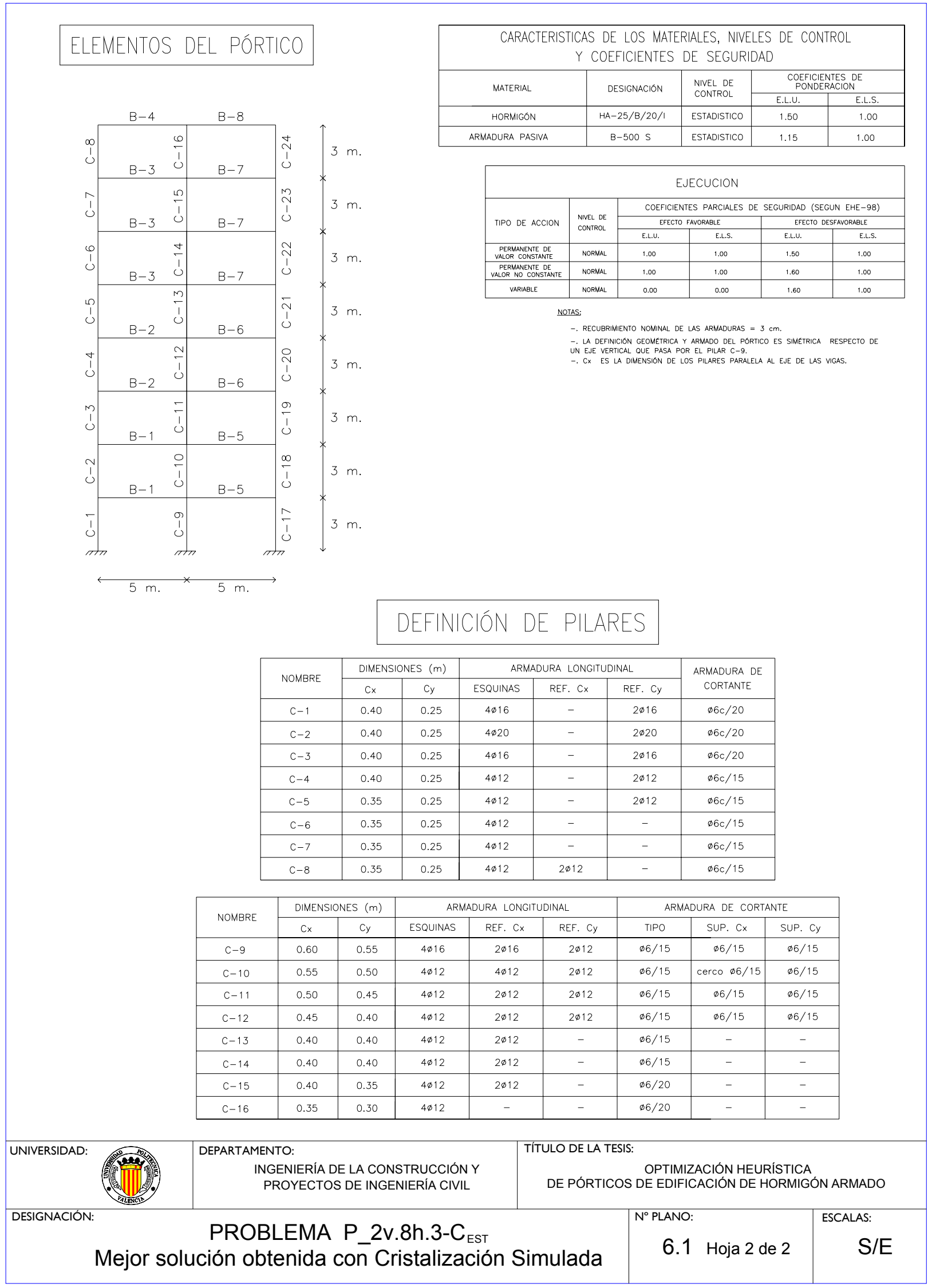




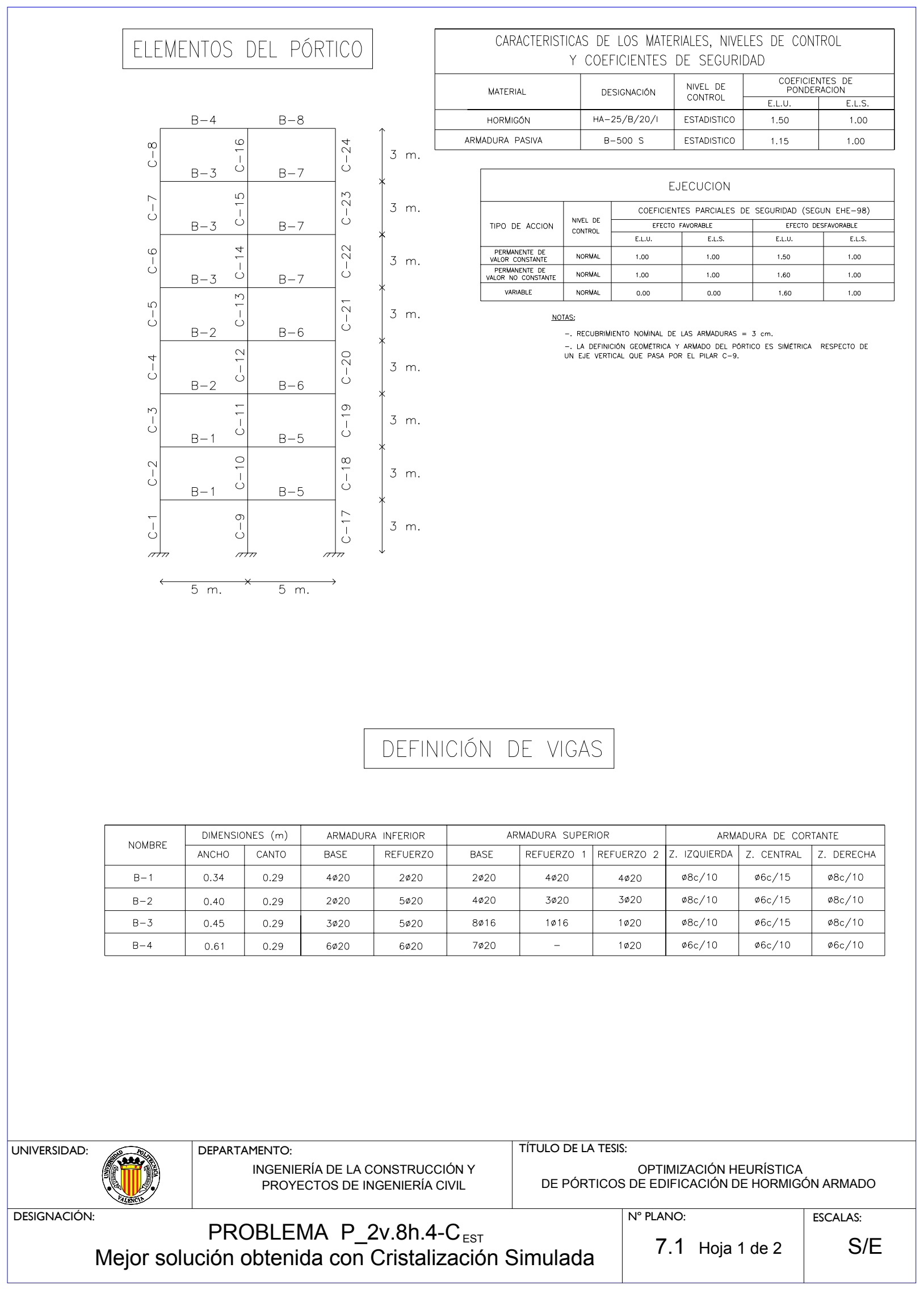




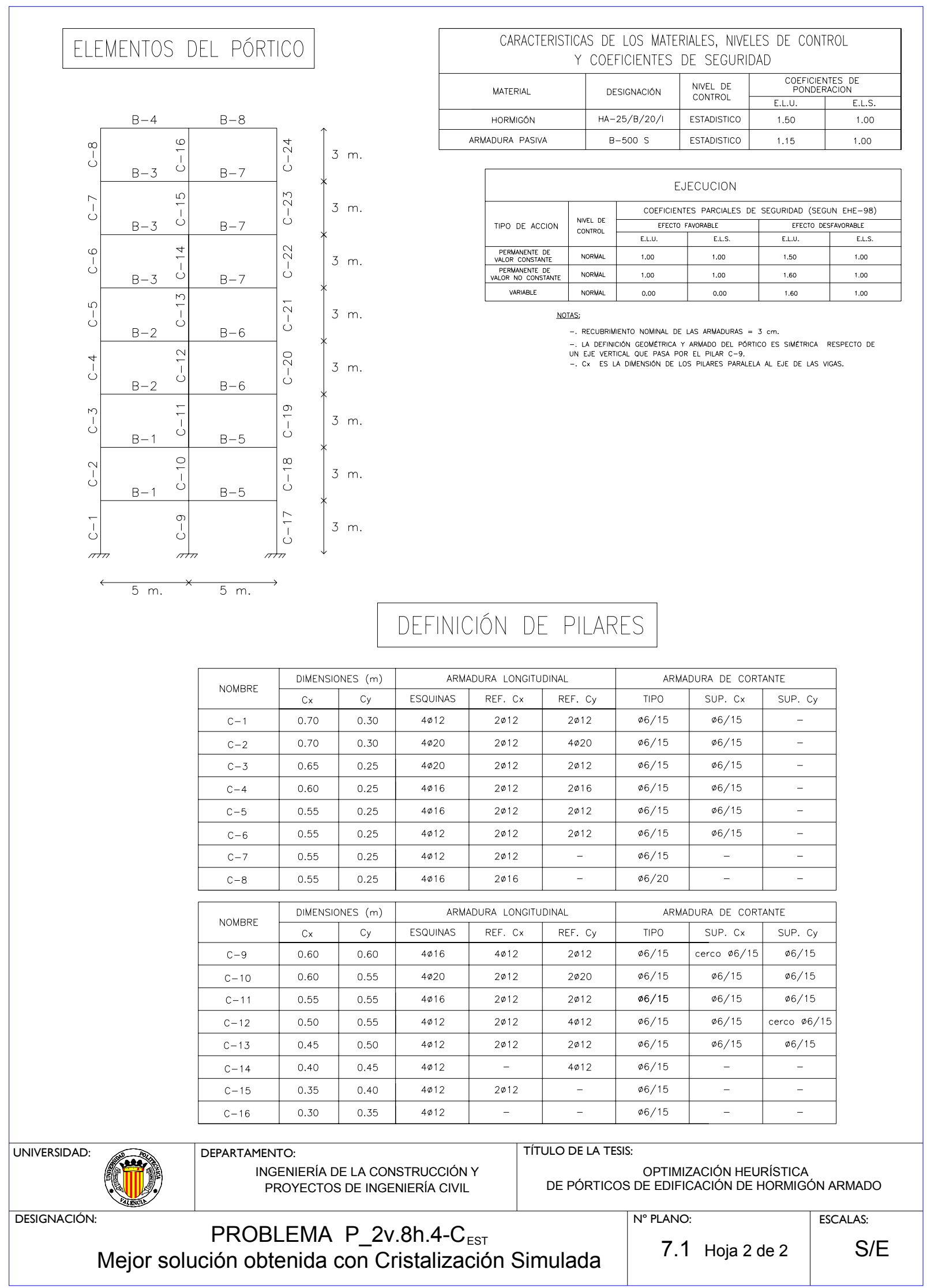




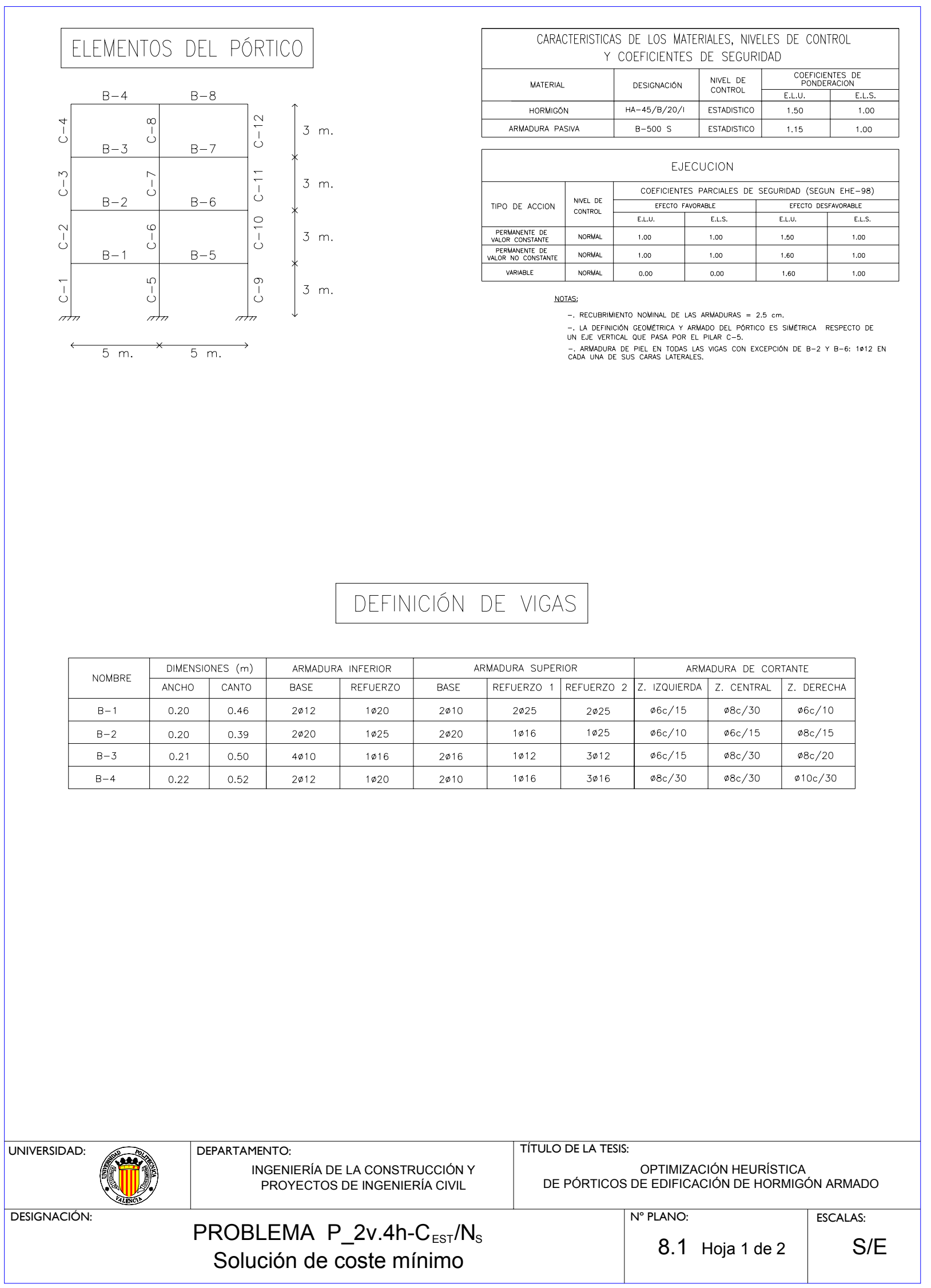




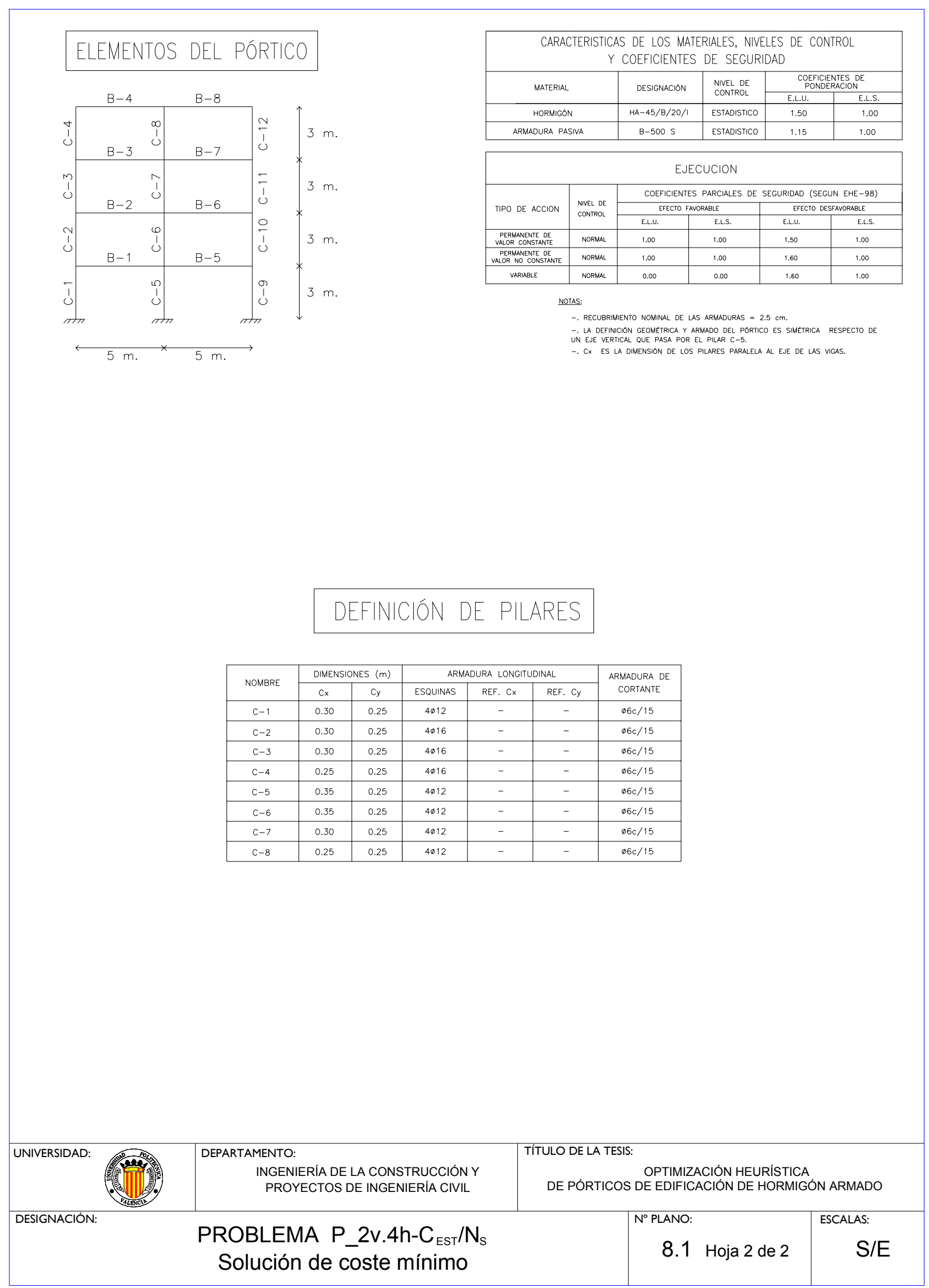




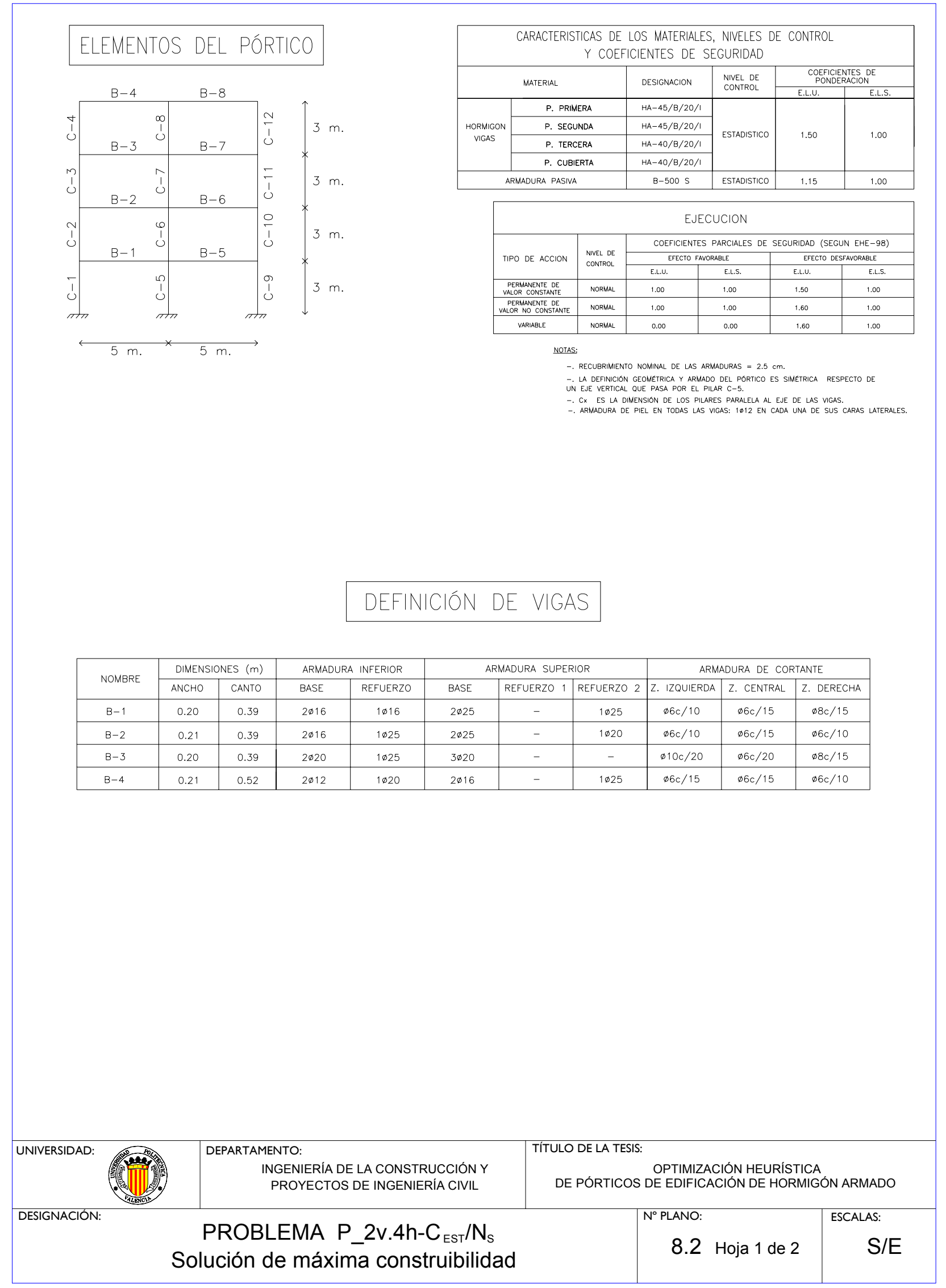




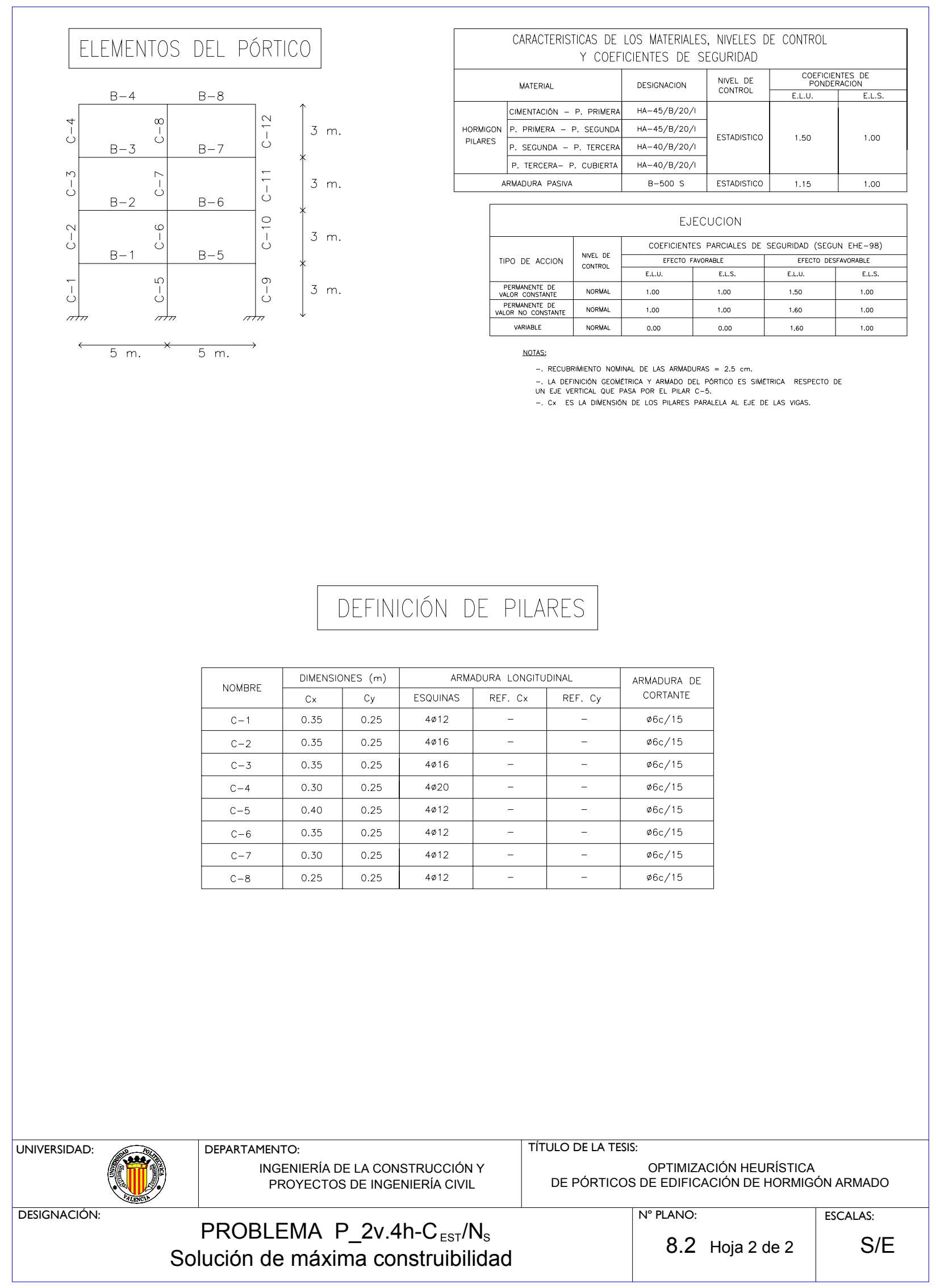




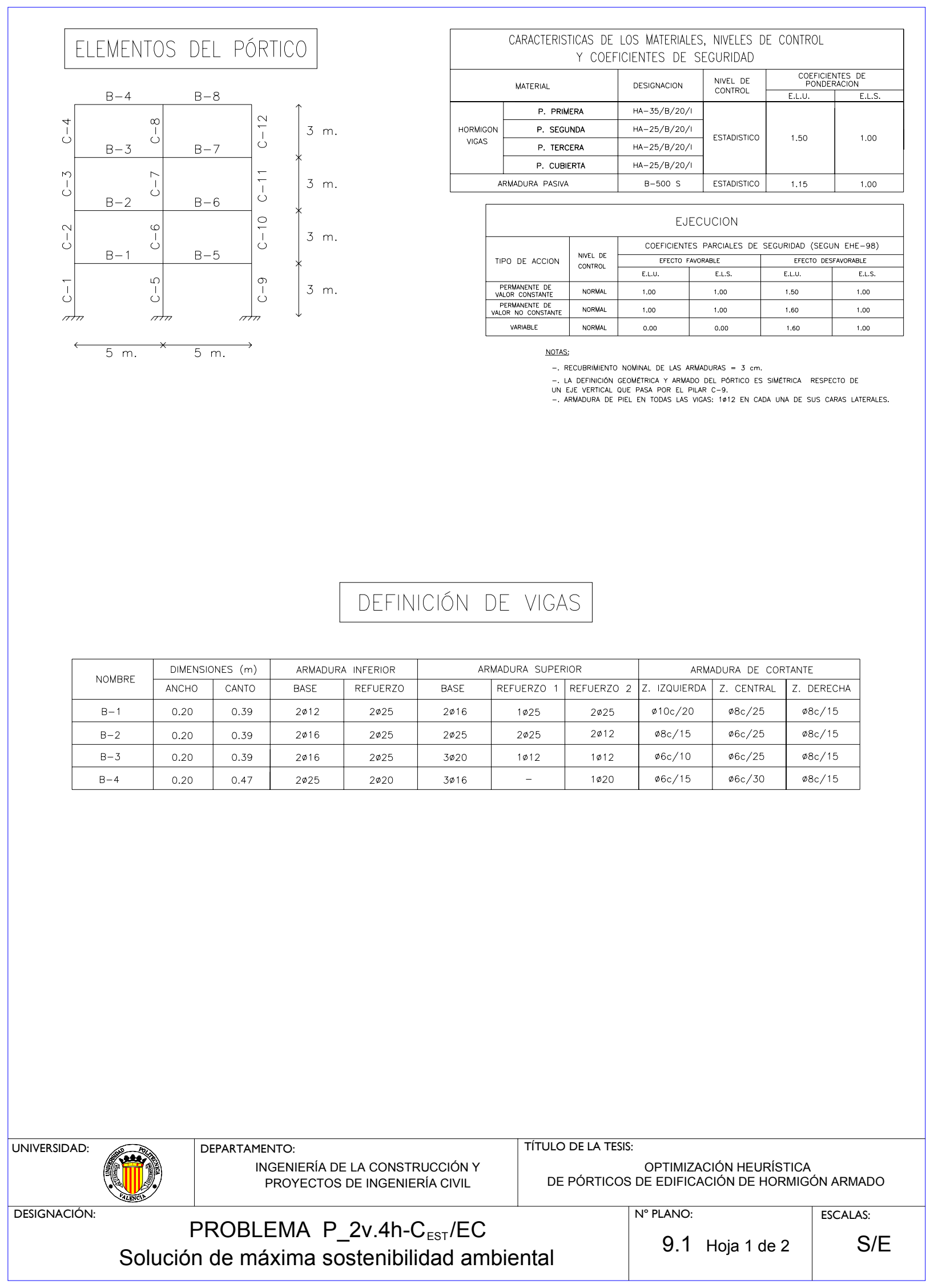




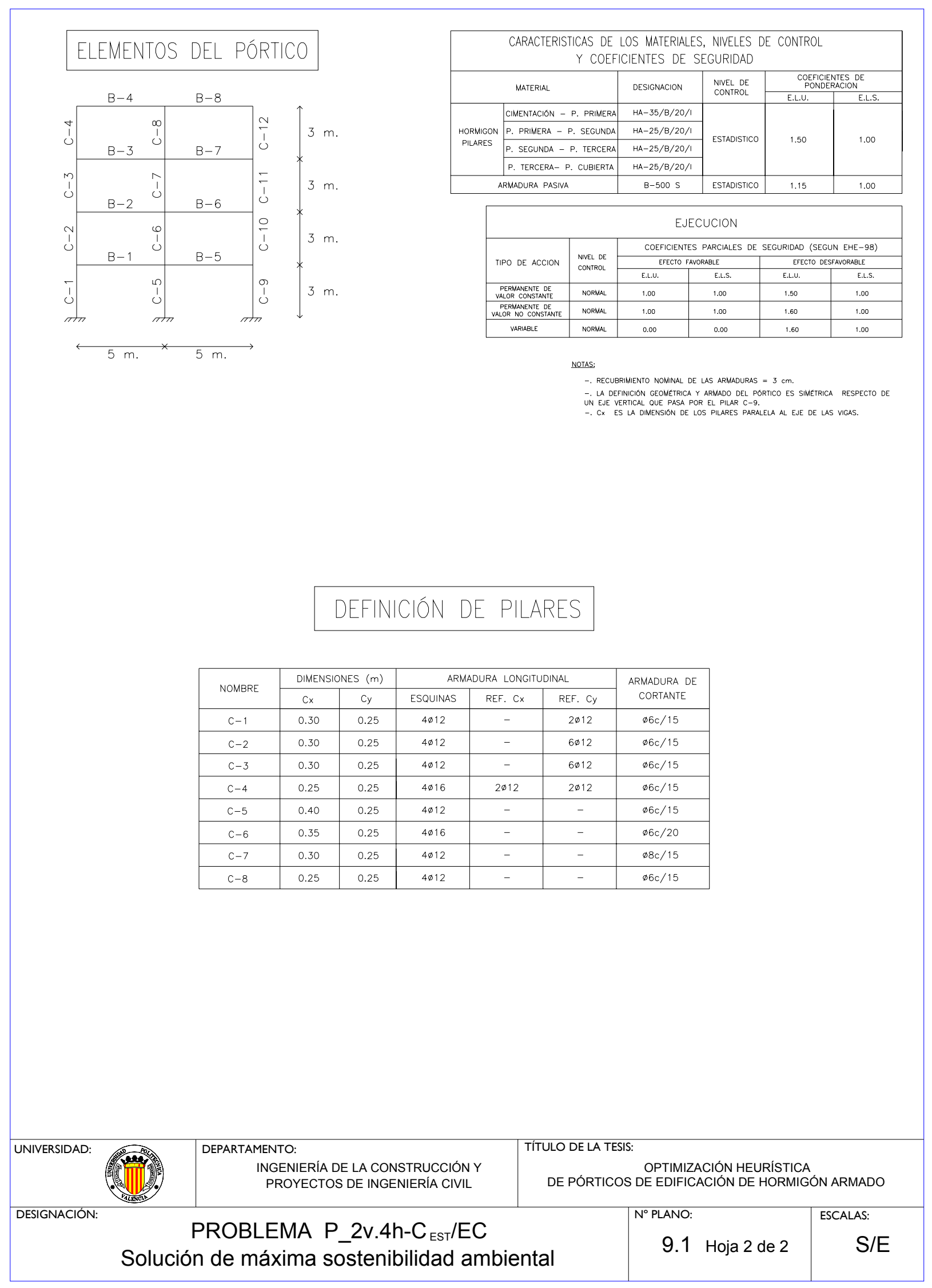

